\title{
Final Report on Experimental Validation of Stratified Flow Phenomena, Graphite Oxidation, and Mitigation Strategies of Air Ingress Accidents
}

Chang H. Oh Eung S. Kim Hee C. No Nam Z. Cho

January 2011
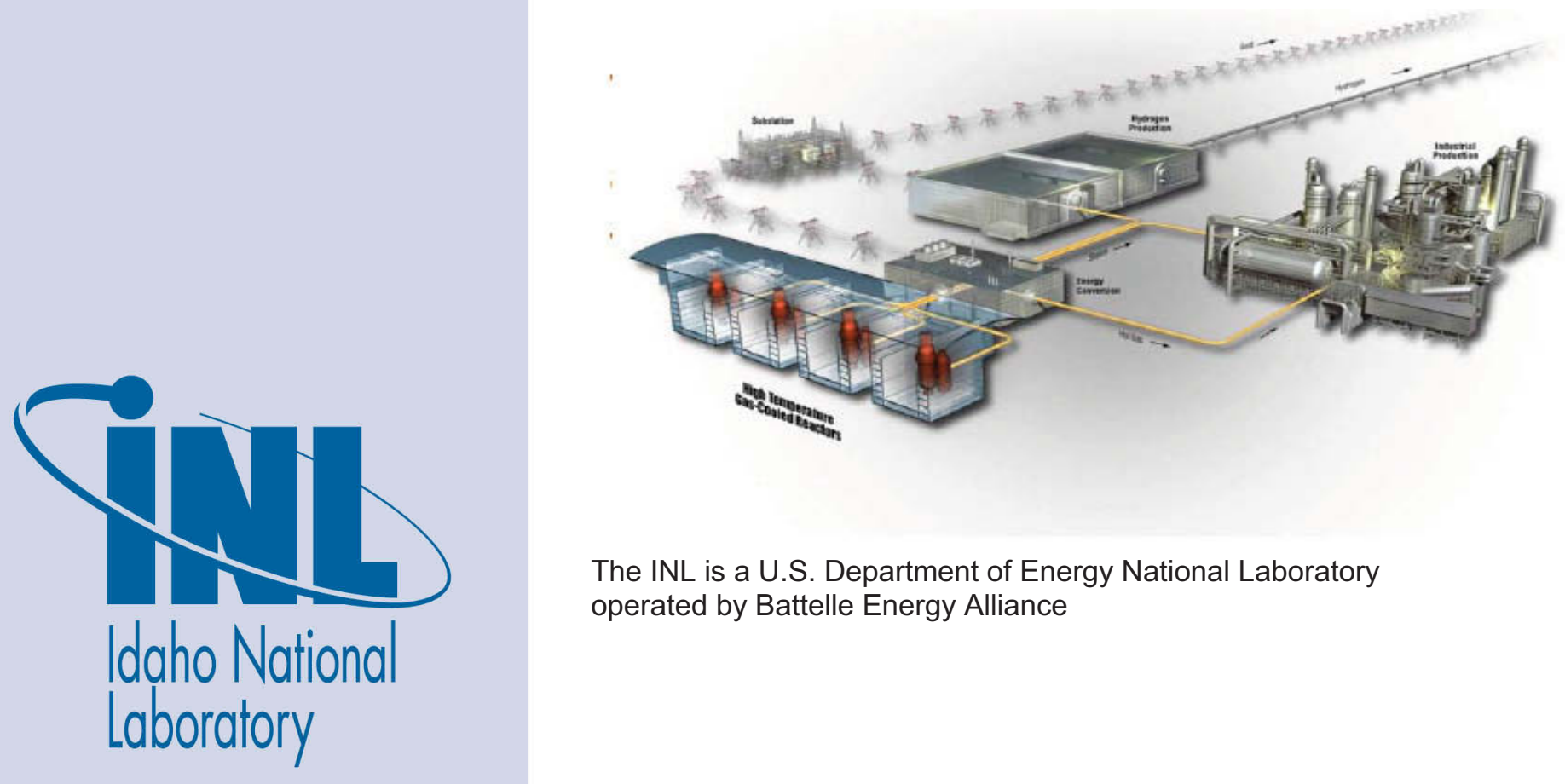

The INL is a U.S. Department of Energy National Laboratory operated by Battelle Energy Alliance 
INL/EXT-10-20759

\title{
Final Report on Experimental Validation of Stratified Flow Phenomena, Graphite Oxidation, and Mitigation Strategies of Air Ingress Accidents
}

\author{
Chang H. Oh \\ Eung S. Kim \\ Hee C. No \\ Nam Z. Cho
}

January 2011

\author{
Idaho National Laboratory \\ Next Generation Nuclear Plant Project \\ Idaho Falls, Idaho 83415
}

http://www.inl.gov

Prepared for the

U.S. Department of Energy

Office of Nuclear Energy

Under DOE Idaho Operations Office

Contract DE-AC07-05ID14517 


\section{DISCLAIMER}

This information was prepared as an account of work sponsored by an agency of the U.S. Government. Neither the U.S. Government nor any agency thereof, nor any of their employees, makes any warranty, expressed or implied, or assumes any legal liability or responsibility for the accuracy, completeness, or usefulness, of any information, apparatus, product, or process disclosed, or represents that its use would not infringe privately owned rights. References herein to any specific commercial product,

process, or service by trade name, trade mark, manufacturer, or otherwise, does not necessarily constitute or imply its endorsement, recommendation, or favoring by the U.S. Government or any agency thereof. The views and opinions of authors expressed herein do not necessarily state or reflect those of the U.S. Government or any agency thereof. 

Next Generation Nuclear Plant Project

\section{Final Report on Experimental Validation of Stratified Flow Phenomena, Graphite Oxidation, and Mitigation Strategies of Air Ingress Accidents}

INL/EXT-10-20759

January 2011

Approved by:
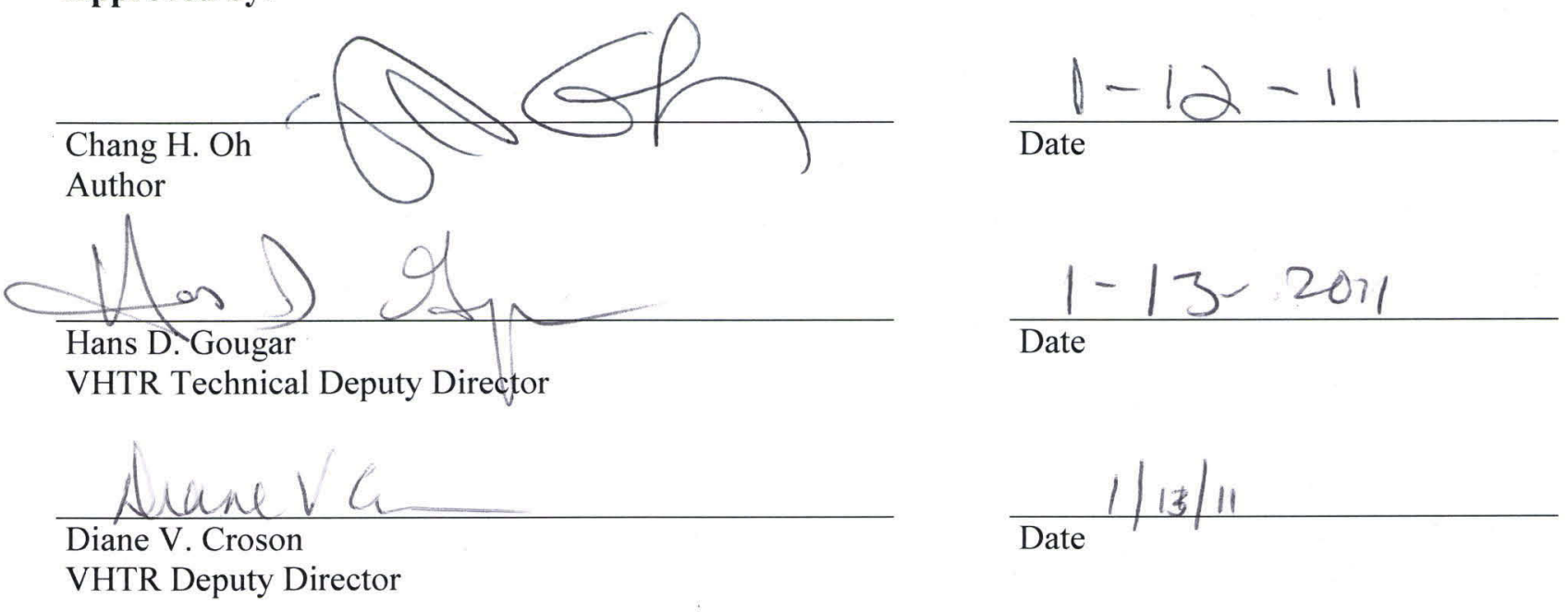

Diane V. Croson
VHTR Deputy Director 



\section{EXECUTIVE SUMMARY}

The U.S. Department of Energy is performing research and development focused on key phenomena important during challenging scenarios that may occur in the Next Generation Nuclear Plant (NGNP)/Generation IV very high temperature reactor (VHTR). Phenomena Identification and Ranking studies to date have identified the air ingress event, following on the heels of a VHTR depressurization, as being very important. Consequently, the development of advanced air ingress-related models and verification and validation are a very high priority for the NGNP Project.

Following a loss of coolant and system depressurization incident, air ingress will occur through the break, leading to oxidation of the in-core graphite structure and fuel. This study indicates that depending on the location and the size of the pipe break, the air ingress phenomena are different. In an effort to estimate the proper safety margin, experimental data and tools, including accurate multidimensional thermal-hydraulic and reactor physics models, a burn-off model and a fracture model are required. It will also eventually require effective strategies to mitigate the effects of oxidation.

This 3-year project (FY 2008 to FY 2010) focused on various issues related to the VHTR air-ingress accident, including (a) analytical and experimental study of air ingress caused by density-driven, stratified, countercurrent flow, (b) advanced graphite oxidation experiments, (c) experimental study of burn-off in the core bottom structures, (d) structural tests of the oxidized core bottom structures, (e) implementation of advanced models developed during the previous tasks into the GAMMA code, (f) full air ingress and oxidation mitigation analyses, (g) development of core neutronic models, (h) coupling of the core neutronic and thermal hydraulic models, and (i) verification and validation of the coupled models.

\section{E-1. RESEARCH OBJECTIVES}

The main objectives of this 3-year project were to investigate air-ingress related phenomena in the VHTRs and perform modeling and experiments for better understanding on the accident consequences. The major phenomena, targeted in this study, were (1) density gradient driven stratified flow, (2) graphite oxidation and structural degradation, (3) thermal hydraulics and neutronics coupling, and (4) development of air ingress mitigation and computational fluid dynamics (CFD) calculations

\section{E-2. REPORT CONTENT AND ORGANIZATION}

This report, which highlights key accomplishments achieved from FY 2008 to FY 2010, consists of the following sections:

1. Introduction: Section 1 is introductory information about this project with objectives and strategies.

2. Density Gradient Driven Stratified Flow Analyses: Section 2 is CFD and analytical calculations on the density gradient driven stratified flow and air-ingress accident in the VHTRs.

3. Experimental Study on the Stratified Flow: Section 3 is the experimental works conducted to support air-ingress analyses and validate computational methods.

4. Advanced Graphite Oxidation Study: Section 4 is graphite oxidation experiments and modeling conducted by INL, which correlate graphite oxidation, internal surface density, mechanical strength, and fracture.

5. Air Ingress Mitigation Study: Section 5 is basic air-ingress mitigation concepts and validation studies.

6. Experiment on Burn-off in the Bottom Reflector: Section 6 is KAIST experiments on the oxidation characteristics in the VHTR core supporting graphite structures. 
7. Structure Test of Burn-off Reflector: Section 7 is experiment and modeling on the graphite oxidation and graphite structure degradation.

8. Coupling Neutronic-Thermal Hydraulic Tools: Section 8 is the coupled GAMMA/COREDAX code with coupling methods and some verification results.

9. Core Neutronic Model: Section 9 is the core neutroncs model, based on analytic function expansion method (AFEN), used in the GAMMA/COREDAX code.

10. Coupled Core Model V\&V: Section 10 is some calculations conducted for validation of the GAMMA/COREDAX code.

11. Summary and Conclusions: Section 11 summarizes the accomplishments in this project and discusses some remaining issues.

Figure E-1 shows more details of tasks involved in this project.

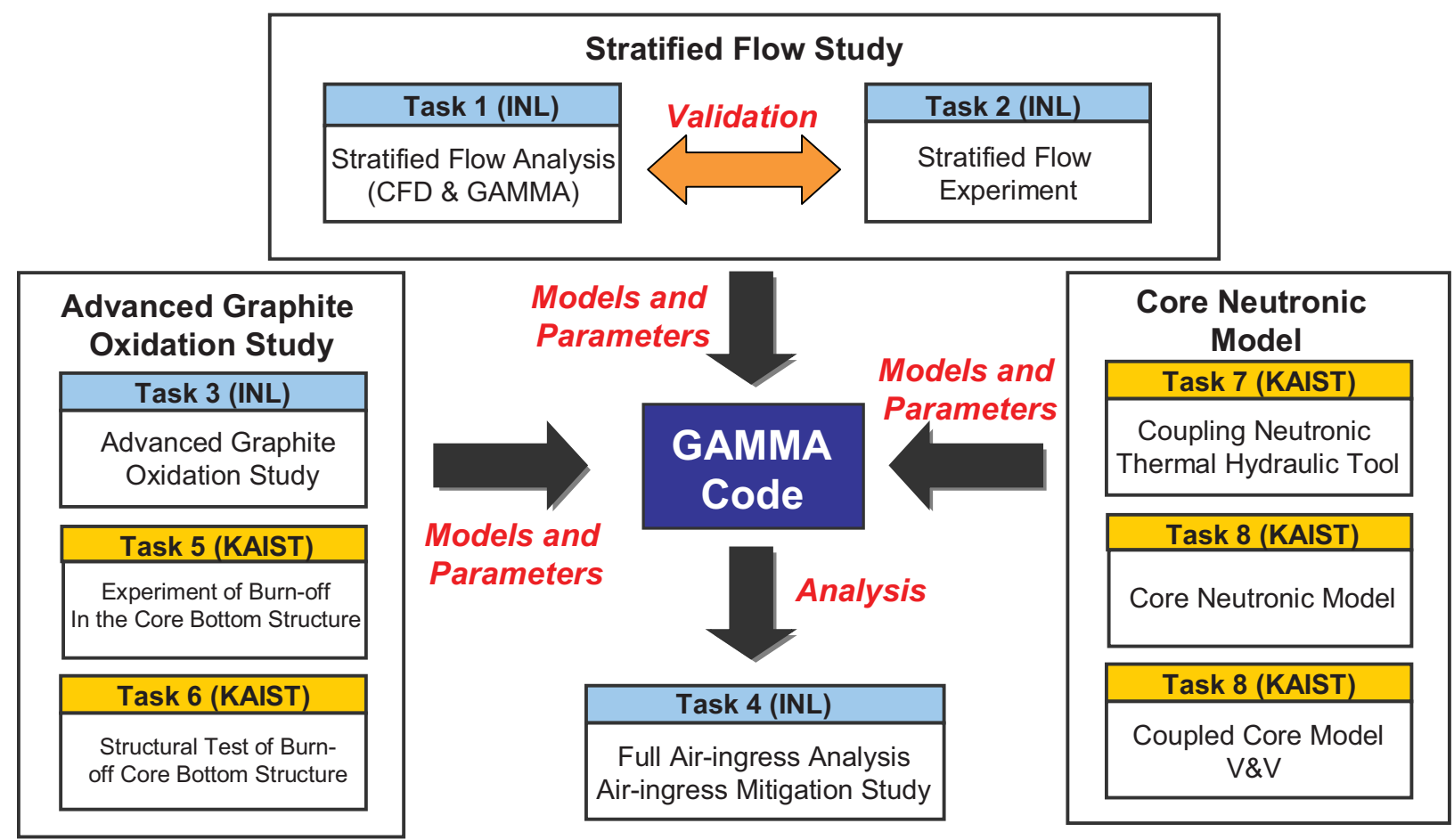

Figure E-1. Schematic diagram of all tasks involved.

\section{E-3. KEY PROGRAM ACCOMPLISHMENTS}

Highlights of key accomplishments are summarized. These accomplishments include items required to meet task objectives outlined in the original proposal for this project and items that meet overall International Nuclear Energy Research Initiative objectives. As indicated below, this project not only advanced state-of-the-art research pertaining to the VHTR, it also helped prepare graduate students to join the nuclear engineering workforce. 


\section{E-3.1 Task 1-Density-difference Induced Stratified Flow Analysis (INL)}

In Task 1, INL performed various analyses using computational fluid dynamic (CFD) and analytical methods focused on the density gradient driven stratified flow expected in the VHTR air-ingress accident. First, this task reviewed some previous studies on the lock exchange phenomena that are physically similar to what happens in the VHTR air-ingress accident. Based on those reviews, the analytical study was performed, which focused on identification and comparison of major air-ingress mechanisms (molecular diffusion versus density gradient driven flow). The results clearly showed that density gradient driven stratified flow is a dominant air-ingress mechanism in the VHTR loss-of-coolant-accident (LOCA). In parallel with the analytical studies, various CFD simulations were conducted using simplified 2-D and detailed 3-D models. The $600 \mathrm{MWth}$ gas turbine modular helium reactor (GTMHR) was selected as the reference reactor. As a result, analytical models agree very well with those of 2-D and 3-D CFD simulations in terms of time scales and the recirculation pattern in the lower plenum. Detailed 3-D calculations also confirm the VHTR air-ingress accident, which is based on the density-gradient driven stratified flow. Heterogeneous chemical reaction effects and small break accident were also studied by CFD analyses. These analyses showed that the air-ingress in the small break is dependent on break size, orientation, and density ratios. In most of the postulated small break conditions, the air-ingress was dominated by density gradient driven flow. It indicated that the density gradient driven flow is important not only for double-ended-guillotine-break (DEGB) but also for small break situations. Finally, natural circulation pattern in the post onset natural circulation was investigated by CFD analyses. The main objective of this study was to validate the previous 1-D natural circulation pattern, which has been assumed in earlier air-ingress studies. In this analysis, the 3-D CFD simulation showed very different flow configurations and predicted much faster air-ingress speed, compared to the previous 1-D simulations because of temperature gradient between the inside and outside of the reactor, which causes density gradient driven flow. Finally, Task 1 concludes from all of the reviews and analyses that the density gradient driven flow is major phenomena that control the air-ingress process. Therefore, the airingress analyses needs multi-D simulations, and 1-D modeling cannot represent the correct phenomena.

\section{E-3.2 Task 2-Experimental Study on the Stratified Flow (INL)}

In Task 2, INL set up the experimental facility and obtained the experimental data to understand stratified flow phenomena in the VHTR and to provide experimental data for validating computational methods. The experiment was focused on the stratified flow in the horizontal pipe and expansion at the pipe and vessel junction. Brine and sucrose were used as heavy fluids and water was used as the light fluid. The density ratios were changed between 0.99 and 0.7 . The experiment shows clear stratified flow between heavy and light fluids, even for the low-density differences. The stratified flow experimental data based on the circular pipe was compared with the previous theoretical model based on the rectangular channel. Results are in good agreement with the experimental data within a 10\% deviation. Some blind CFD calculations were carried out for comparisons with the experimental data. A grid sensitivity study was also performed based on Richardson extrapolation and grid convergence index for modeling confidence. As a result, the simulation result shows very good agreement with the experimental data, indicating that the current CFD code and physical models are appropriate for predicting stratified flow phenomena. As a part of the CFD method validation, other experimental results obtained by Grobelbauer et al. (1993) in ETH Zurich were also used. The experimental results cover the full range of gas intrusions, including helium and air, for the gravity current flows in the lock-exchange situations. In this study, the current speed estimated by the CFD simulation showed very good agreement with the experimental data for both heavy current and light current intrusions. Especially, the realizable k-e model showed the best predictions among the Reynolds-averaged turbulence models based on two equations. 


\section{E-3.3 Task 3-Advanced Graphite Oxidation Study (INL)}

In Task 3, INL investigated graphite oxidation characteristics in the air-ingress accident by considering three main characteristics: (1) effect of oxidation degree on the graphite strength, (2) effect of oxidized graphite density on the oxidation rate, and (3) surface area density in the graphite internal pores. An experimental methodology was newly developed to validate the previous correlations related to the oxidized graphite strength, which is essential for analysis of graphite structure fracture. Following the graphite experiment, fracture of the graphite structure was estimated for the reference VHTR by two computer codes: GAMMA code (system analysis code) and ABAQUS code (stress analysis code). The graphite oxidation and corrosion were predicted by the GAMMA code, and the information was implemented into the ABAQUS code to estimate the facture time. Aside from this analysis, further computations were performed to conservatively estimate the maximum allowable burn-off to maintain graphite structural integrity using MATLAB. Finally, advanced graphite oxidation models and algorithm, including graphite corrosion and facture, were constructed and implemented in the GAMMA code.

\section{E-3.4 Task 4-Air Ingress Mitigation Study (INL)}

In Task 4, air-ingress mitigation methods were discussed. This study used root-cause analyses to figure out important factors closely associated with air-ingress consequences. Based on the derived basic concepts, several air-ingress mitigation methods were developed and proposed. Among them, the following two methods were strongly recommended: helium injection in the lower plenum, and reactor enclosure opened at the bottom. The former method injects helium into the lower plenum. The injected helium replaces the air in the core and the lower plenum upper part by buoyancy force. It significantly reduces graphite oxidation by reducing oxygen concentration and reaction temperature in the inside of the reactor. The later method encloses the reactor by a nonpressure boundary. Some design modifications on the preexisting cavity can be used for this. This enclosure has an opening at the bottom. After depressurization, the air-ingress rate is controlled by molecular diffusion through this opening. Validation of the air-ingress mitigation methods was conducted by CFD simulations. The results show that both methods can effectively mitigate the air-ingress process. This study also reviewed a well-known previous air-ingress mitigation idea proposed by JAEA that injects very small helium flow at the top of the reactor vessel for mitigating air-ingress. From the review process, we found that this idea may not work in the real situation for several reasons. One of the main reasons is that this method does not provide sufficient energy to prevent buoyancy force generated by heating in the lower plenum because it was originally designed to use the counter-diffusion mechanism for mitigating air diffusion into the reactor.

\section{E-3.5 Task 5-Experiment of Burn-off in the Core Bottom Structures (KAIST)}

In Task 5, graphite oxidation tests for IG-110 and IG-430 were performed. Kinetics parameters, dimensional effect, effect of burn-off, and effect of moisture were investigated.

\section{E-3.6 Task 6-Structural Tests of Oxidized Core Bottom Structure (KAIST)}

In Task 6, KAIST investigated the graphite oxidation and mechanical behaviors for the selected graphite materials (IG-110, IG-430, and NBG-10). According to the experiment, the graphite mechanical fracture was mainly affected by slenderness ratio and oxidation burn-off. In this study, the following two correlations were finally suggested for predicting graphite fracture in the VHTRs: 
$\sigma_{0}=A-B \frac{L}{r}, \frac{\sigma}{\sigma_{0}}=\exp (-k d), d=1-\frac{M+\left\{\left(V_{0}-V\right) \rho_{0}\right\}}{M_{0}}$

This task also estimated allowable total burn-offs for IG-110, IG-430, and NBG-10 graphite, which can be used for conservative graphite fracture criteria. This estimation showed that the allowable burn-off is predicted when the reaction is dominated by internal pore reactions ( $f$-value $=0$ ).

This task also implemented graphite oxidation models into the GAMMA code and conducted various air-ingress analyses using 1-D and 2-D modeling. These analyses showed that core maximum temperature is not affected by the onset natural circulation and type of models (1-D or 2-D). However, predications of bottom reflector temperature and oxidation patterns were significantly different between 1-D and 2-D modeling because of different flow patterns. Validation on the GAMMA code 2-D simulation for density driven stratified flow was performed.

Finally, a one-eighth scaled down bottom structure was tested. Main characteristics of the test are that heating and loading are applied at once. It is confirmed that the bottom structure is safe for at least $40 \%$ of the local burn-off.

\section{E-3.7 Task 7-Coupling Neutronic-Thermal Hydraulic Tools (KAIST)}

In Task 7, thermal power distributions in the reactor core were provided to GAMMA code by COREDAX (nodal diffusion code) for realistic thermal-hydraulics analysis. Thermal feedback from GAMMA affected the cross-section, and the effects were reflected on the next time step neutronics calculation by COREDAX. This procedure was included in coupled code GAMMA/COREDAX and tested on GTMHR-600 core with various transient situations.

\section{E-3.8 Task 8-Core Neutronic Model (KAIST)}

In Task 8, advanced neutronics code development was completed based on analytic function expansion method (AFEN).This code provides accurate results compared with well known PARCS code as shown in the VVER-440 benchmark problem test result.

\section{E-3.9 Task 9-Coupled Core Model V\&V (KAIST)}

In Task 9, homogenized cross-sections of GTMHR-600 assemblies were generated by MCNP calculation for analysis of realistic situations in nuclear reactors and tabulated for representative temperatures. From this data, cross-sections values were interpolated for required temperature and provided to GAMMA/COREDAX code.

An air-ingress accident situation was analyzed by GAMMA/COREDAX code, and the important parameters were calculated and compared with the result of GAMMA, which used point kinetics method for power generation feedback. The fuel compact temperature progressed in different directions in GAMMA and GAMMA/COREDAX code results.

\section{E-4. STUDENT PARTICIPATION}

United States:

- Seung-Jun Kim (University of Illinois, Urbana Champaign)

- Jong Bin Lim (University of Wisconsin, Madison)

- Nathaniel Salpeter (Texas A\&M) 
- Thibaut Simond (Ecole des Mines de Paris-Paristech)

Republic of Korea:

- $\quad$ Byung Ha Park (KAIST)

- Hyung Gon Jin (KAIST)

- Ho Joon Yoon (KAIST)

- Jong Woon Kim (KAIST)

- Jae-Jun Lee (KAIST)

- Han Jong Ryu (KAIST)

- Young Soo Kim (KAIST)

- Sung-Hwan Yoon (KAIST)

- Jae Hoon Song (KAIST) 


\section{CONTENTS}

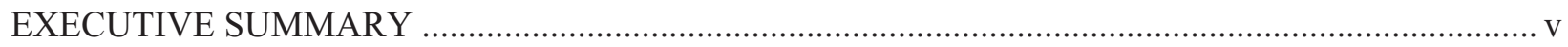

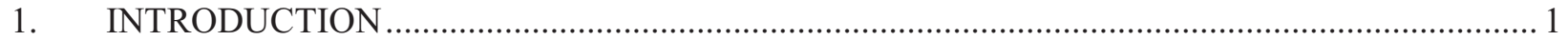

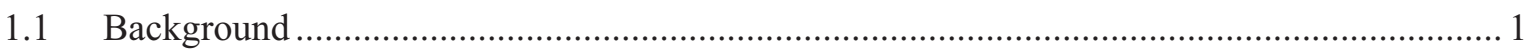

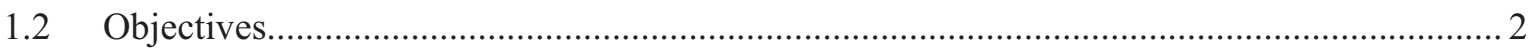

1.3 Research and Development Plan.......................................................................... 2

1.3.1 Task 1: Density-Difference Induced Stratified Flow Analysis - FY 2008 and

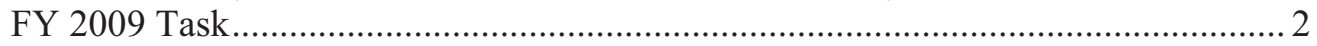

1.3.2 Task 2: Experimental Study on the Stratified Flow and Validation of CFD Models - FY 2009 and FY 2010 Task .................................................................... 3

1.3.3 Task 3: Advanced Graphite Oxidation Study - FY 2008 and FY 2009 Task.............. 3

1.3.4 Task 4: Air Ingress Mitigation Study - FY 2010 Task ........................................... 3

1.3.5 Task 5: Experiment of Burn-off in the Core Bottom Structures (KAIST) -

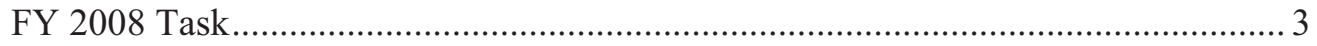

1.3.6 Task 6: Structural Tests of Oxidized Core Bottom Structures (KAIST) - FY 2009 and FY 2010 Task ...................................................................................... 3

1.3.7 Task 7: Coupling Neutronic-Thermal Hydraulic Tools (KAIST) - FY 2009

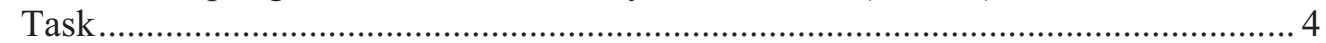

1.3.8 Task 8: Core Neutronic Model (KAIST) - FY 2008 and FY 2009 Task.................... 4

1.3.9 Task 9: Coupled Core Model V\&V (KAIST) - FY 2010 Task .................................. 4

2. TASK 1: DENSITY-DIFFERENCE INDUCED STRATIFIED FLOW ANALYSIS .................... 5

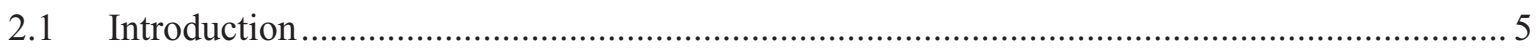

2.2 Review of Previous Gravity Current Flow Studies ............................................................. 7

2.3 Analytical Estimations for VHTR Air-ingress Accident .................................................. 20

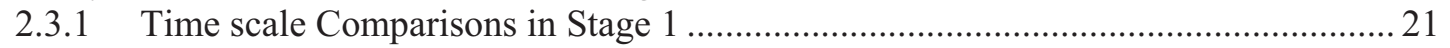

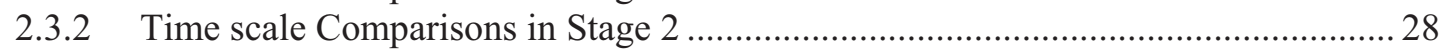

2.4 Discussions on Stage-2 and Early Onset of Natural Circulation............................................ 36

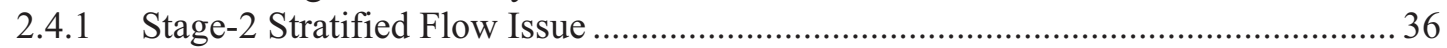

2.4.2 Estimation of Energy Barrier in Stage-2 Stratified Flow ........................................ 37

2.5 Preliminary 2-D CFD Analysis for Density-Difference Driven Stratified Flow in

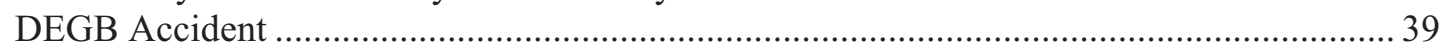

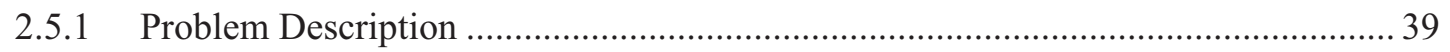

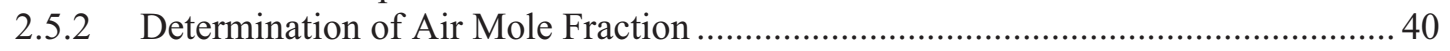

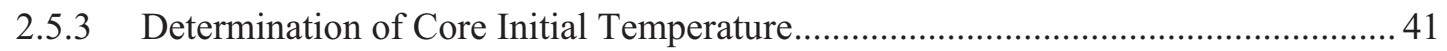

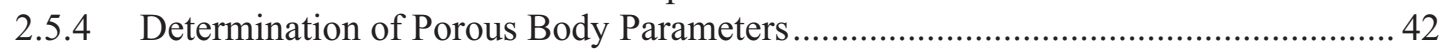

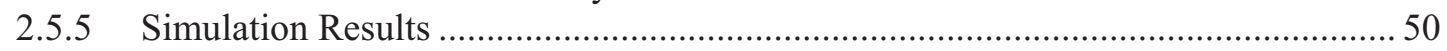

2.6 Preliminary 3-D CFD Analyses for DEGB Air-ingress Accident ...................................... 51

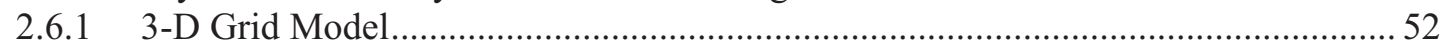

2.6.2 Initial, Boundary, Porous Media Conditions and Properties....................................... 54

2.6.3 Flow Field models and Numerical Models for the 3-D CFX Analysis.......................57

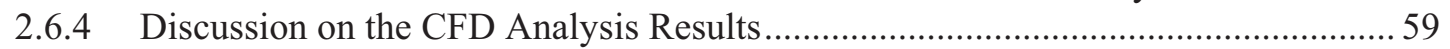

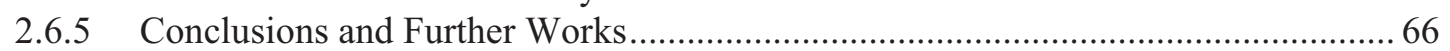

2.7 2-D Preliminary Analyses on the Effect of Chemical Reaction in DEGB Air-ingress

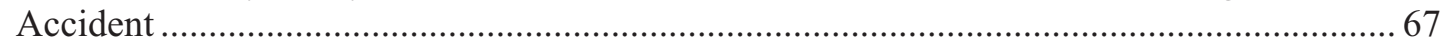

2.8 Preliminary 2-D CFD Analyses on the Small Break Air-ingress Accident ......................... 71 
2.9 Analyses on Natural Circulation Patterns in VHTR Air-ingress Accident.......................... 79

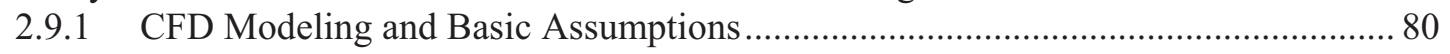

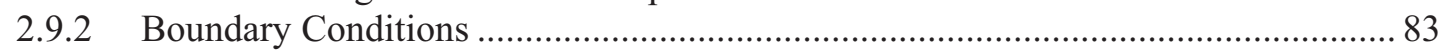

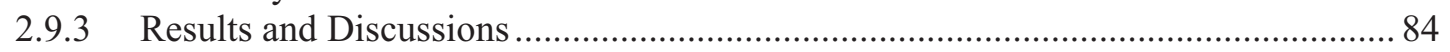

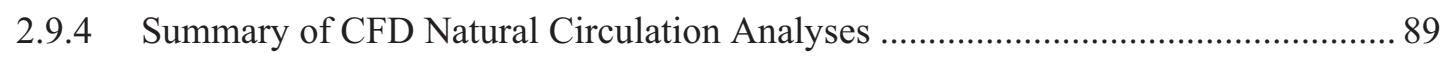

3. TASK 2: EXPERIMENTAL STUDY ON THE STRATIFIED FLOW ….................................... 90

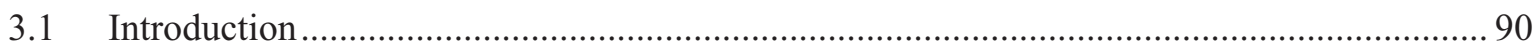

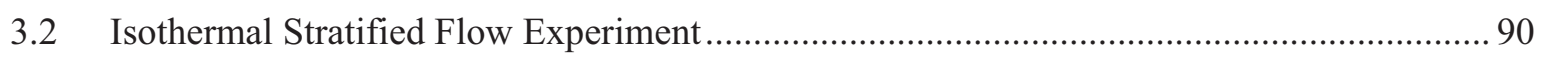

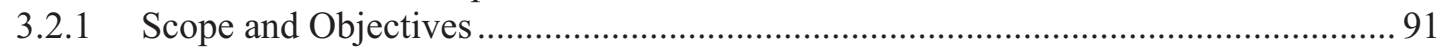

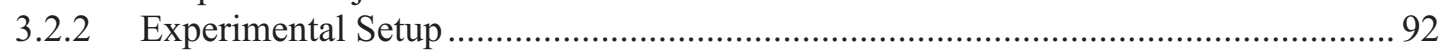

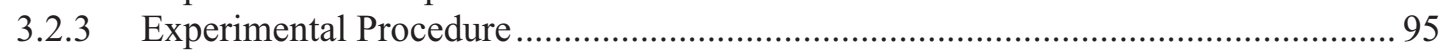

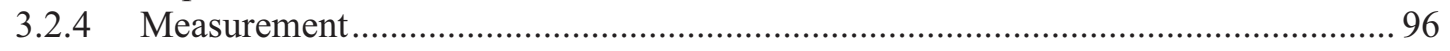

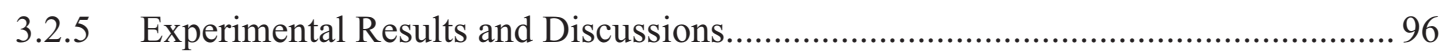

3.3 Validation of CFD Code for Stratified Flow (Water/Brine(Sucrose)) ................................. 98

3.3.1 Preliminary CFD Result and Validation Study with Experiment ............................. 98

3.4 Validations on the CFD code for Air/Helium Stratified Flow .......................................... 111

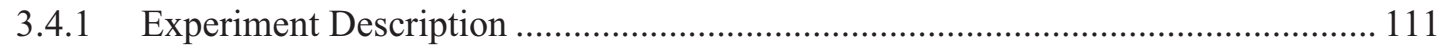

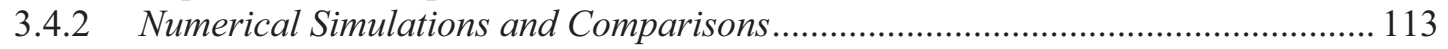

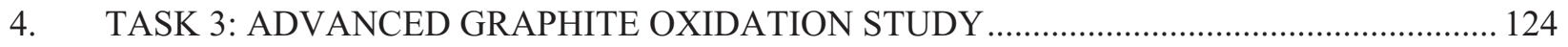

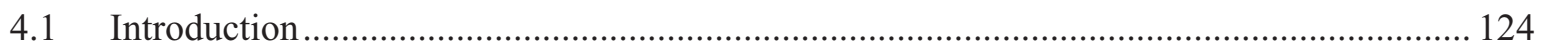

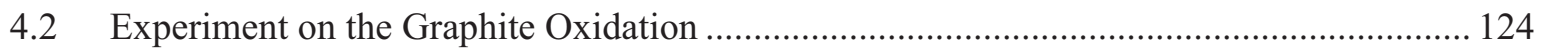

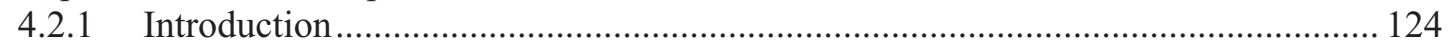

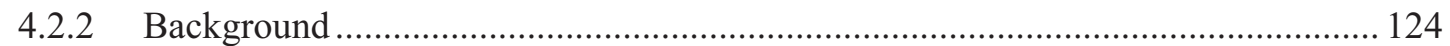

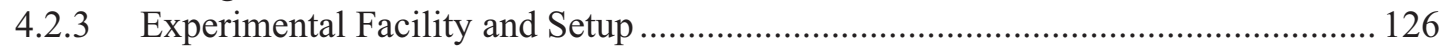

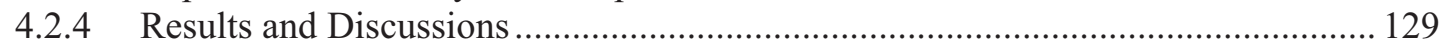

4.3 Modeling of Graphite Oxidation and Fracture in Air-ingress ............................................ 143

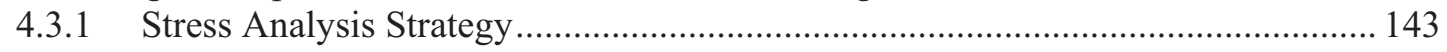

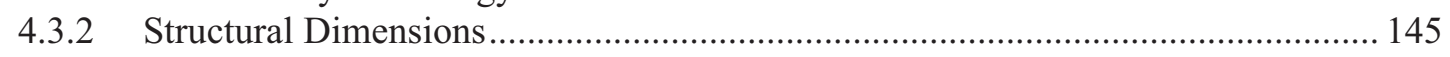

4.3.3 Change of Temperature, Burn-off, and Corrosion Thickness ................................ 147

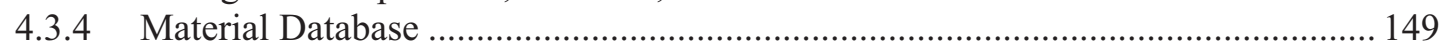

4.3.5 Treatment of Material Properties during Analysis................................................. 152

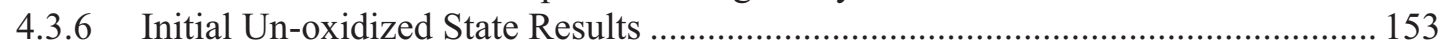

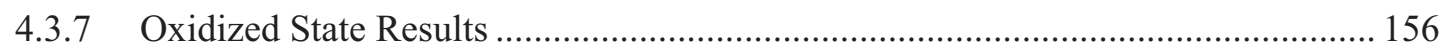

4.4 Estimation of Conservative Burn-off Criteria for Graphite Structure.................................. 160

4.5 Implementation of Advanced Graphite Oxidation Model into GAMMA code and

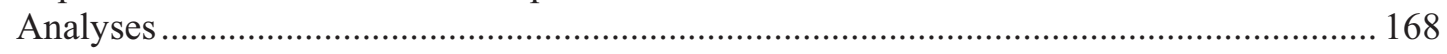

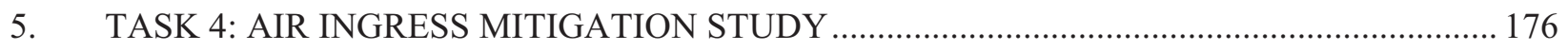

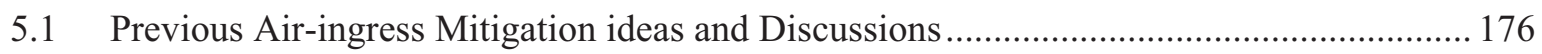

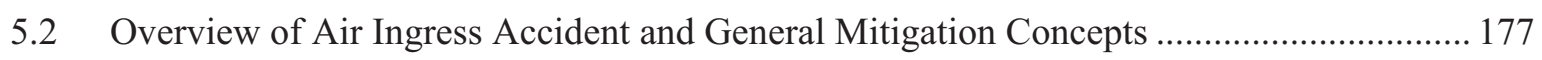

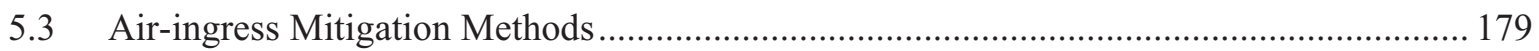

5.3.1 Prevention (or Mitigation) of Graphite Fracture ................................................... 180

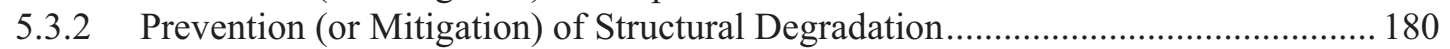

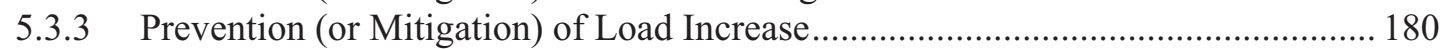

5.3.4 Prevention (or Mitigation) of Oxidation ........................................................... 181

5.3.5 Prevention (or Mitigation) of Air Ingress ........................................................... 182 
5.3.6 Prevention (or Mitigation) of Density Gradient Driven Flow................................ 183

5.3.7 Prevention (or Mitigation) of Density Gradient ...................................................... 185

5.3.8 Prevention (or Mitigation) of Molecular Mass Differences.................................... 185

5.3.9 Prevention (or Mitigation) of Temperature Gradient ............................................. 185

5.3.10 Prevention (or Mitigation) of High Temperature Inside the Reactor ...................... 186

5.4 Validation of Air-ingress Mitigation Methods on Helium Injection and Reactor

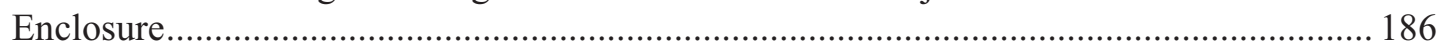

5.4.1 Validation of Air-ingress Mitigation using Lower Plenum Helium Injection ......... 186

5.4.2 Validation of Air-ingress Mitigation using Reactor Enclosure Concept ................ 195

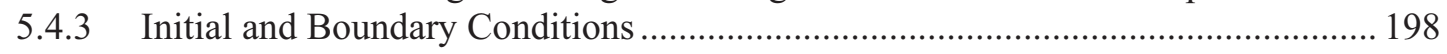

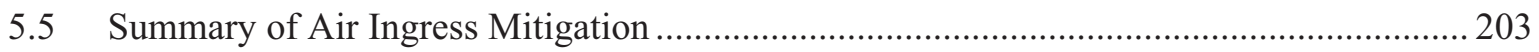

6. TASK 5: EXPERIMENT OF BURN-OFF IN THE BOTTOM REFLECTOR (KAIST).............. 205

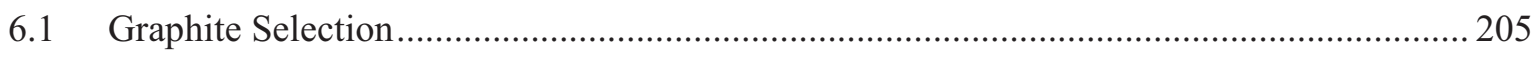

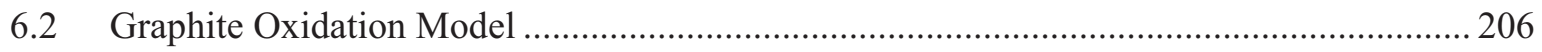

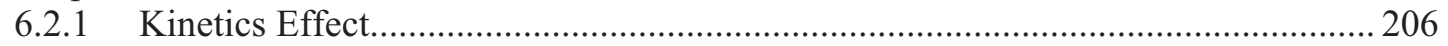

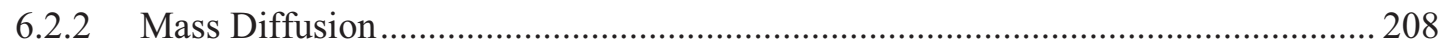

6.2.3 Combined Effect of Kinetics and Mass Diffusion ..................................................209

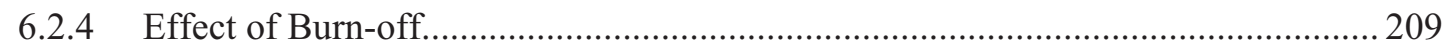

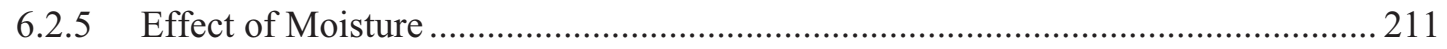

7. TASK 6: Structural Tests of Burn-off Bottom reflector.............................................................. 214

7.1 Graphite Oxidation and Fracture Mechanisms............................................................... 215

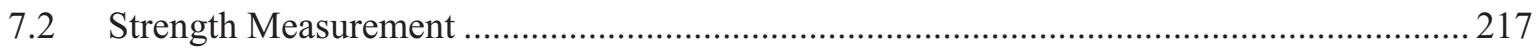

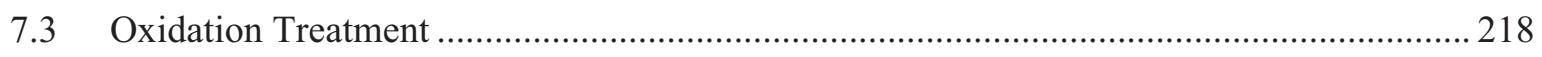

7.4 Measurements of Compressive and Buckling Strength of Fresh Graphite Column ............ 219

7.5 Compressive and Buckling Strength Degradation of Graphite Column Oxidized in

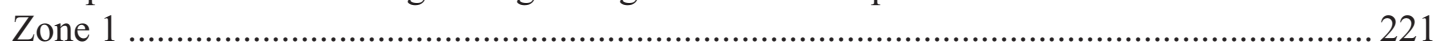

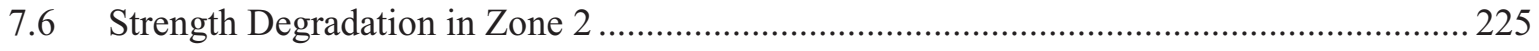

7.7 Buckling Strength Graphite Column Oxidized in Zone 3 ….......................................... 227

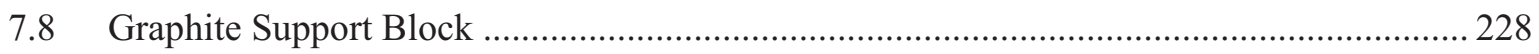

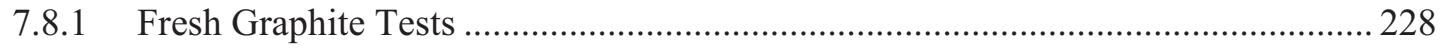

7.8.2 Strength Degradation of Various Columns Oxidized In Zone 1 ............................. 229

7.9 Minimum Burn-off to Lead Failure of Structural Component............................................2 230

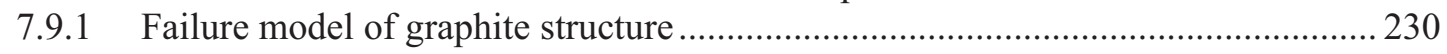

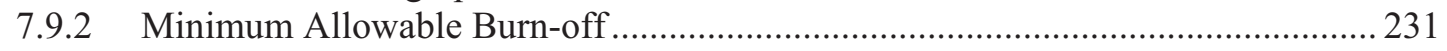

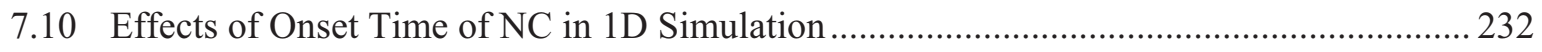

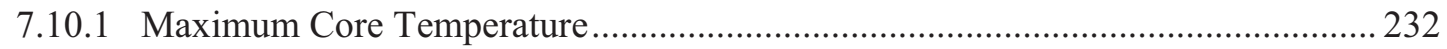

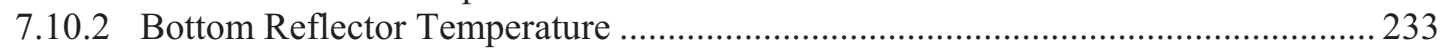

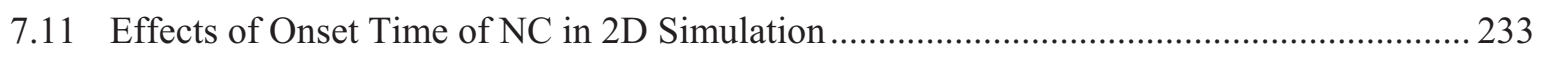

7.12 Comparison of Minimum Burn-off with GAMMA 1D and 2D Results ........................... 235

7.13 Validation on the GAMMA Code 2D Simulation for Density Driven Stratified Flow ....... 235

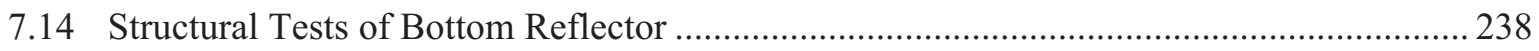

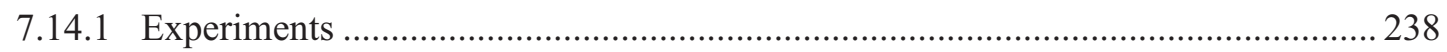

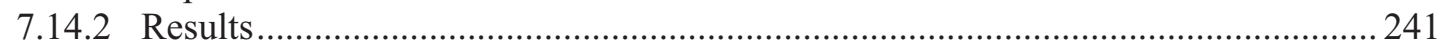

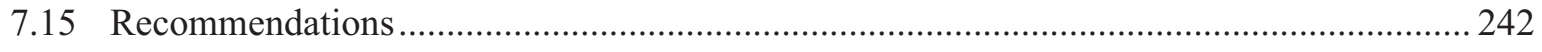




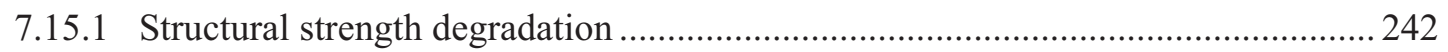

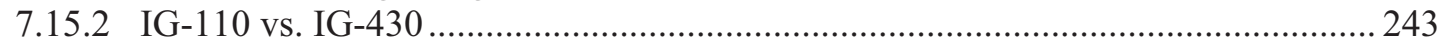

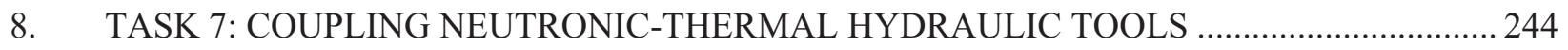

8.1 Setup Parameters for Neutronics/Thermal-Hydraulics Code Coupling .............................. 244

8.2 Calculation Node Mapping Between the COREDAX and GAMMA Code ....................... 244

8.3 Code Coupling of COREDAX with GAMMA …............................................................. 246

8.4 Calculation Procedure in a Coupling System...................................................................... 246

8.5 The GAMMA/COREDAX Test Calculation .................................................................. 248

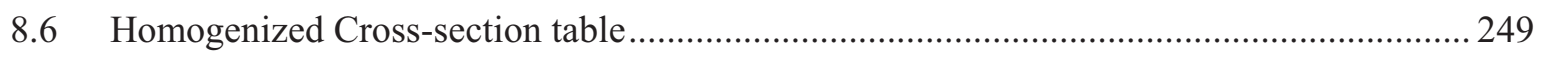

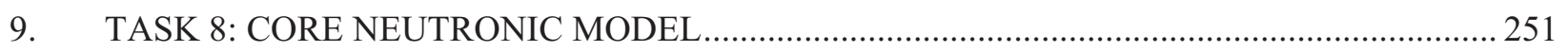

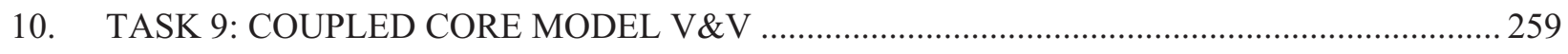

10.1 V\&V of COREDAX (Steady State/Transient Calculation) ............................................... 259

10.2 Generation of Cross-Section Table Set for the GTMHR-600 ............................................ 261

10.3 Calculation of Steady-State by the GAMMA/COREDAX Code with the Generated

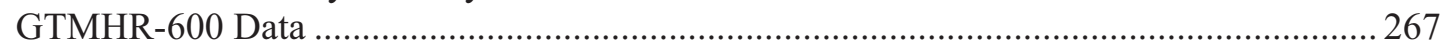

10.4 Calculation of 'Air-ingress' Accident by GAMMA/COREDAX ….................................. 269

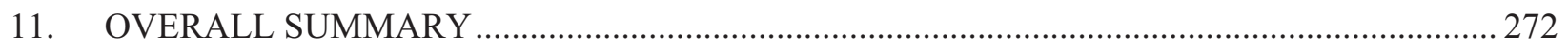

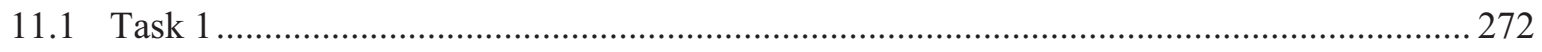

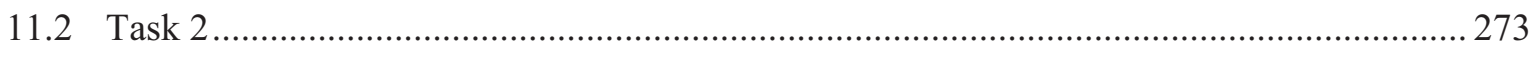

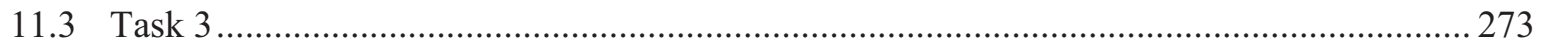

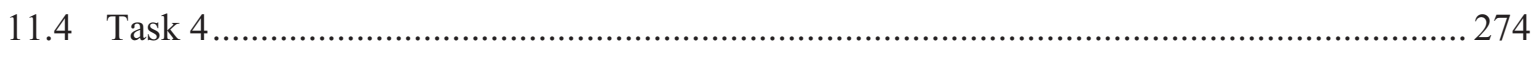

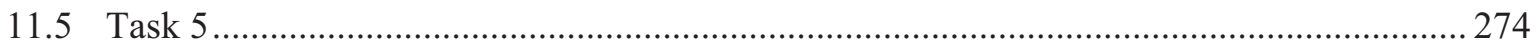

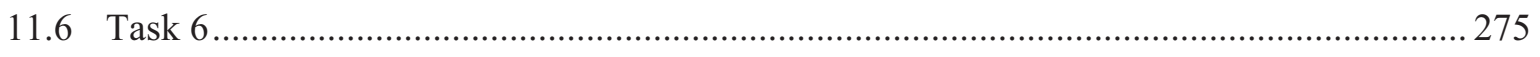

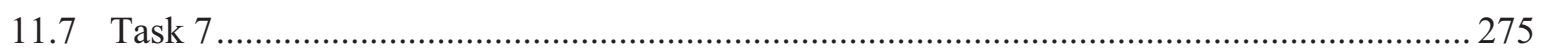

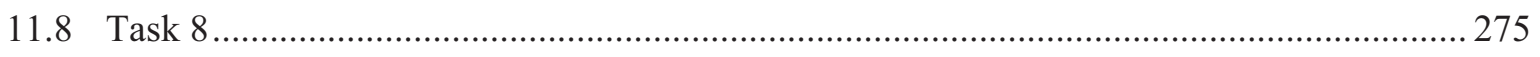

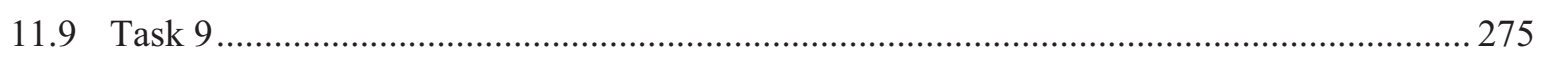

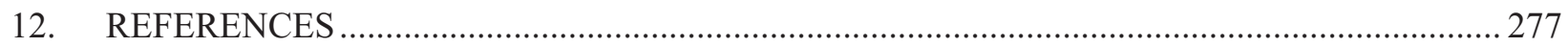

Appendix A-Isothermal Stratified Flow Experiment Facility Schematics ......................................283

Appendix B —Knife Valve Supporter Load Analysis.................................................................... 287

\section{FIGURES}

Figure E-1. Schematic diagram of all tasks involved. ............................................................................ vi

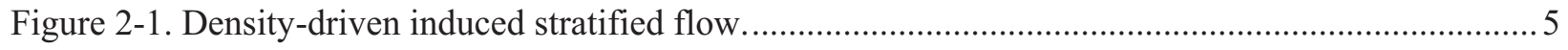

Figure 2-2. Gravity current produced by lock-exchange in a rectangular channel (Shin et al. 2004).

Figure 2-3. Diagram of idealized gravity current in the rest frame of the current (Shin et al. 2004), .........9 
Figure 2-4. Dimensionless net energy flux plotted against dimensionless current depth $h / H$ (Shin et al. 2004).

Figure 2-5. Wave speed plotted versus density ratios $\left(r=\rho_{B} / \rho_{A}\right)$ (Keller and Chyou 1991)..................... 11

Figure 2-6. Gravity current of low-density fluid penetrating into a channel that initially contained high-density fluid (Keller and Chyou 1991).

Figure 2-7. Loss-free gravity current of high density fluid penetrating into a horizontal channel originally filled with low density fluid (Keller and Chyou 1991). 12

Figure 2-8. Fractional depth ( $\xi$ ) versus density ratio (r) (Keller and Chyou 1991). 12

Figure 2-9. Typical gravity current front advancing along a horizontal plane (Mok et al. 2003). .13

Figure 2-10. Full depth lock exchange experiment with the lock gate at an angle (Shin et al. (2004))......

Figure 2-11. Schematic of a partial-depth lock exchange in a channel (a) before release and (b) after release (Shin et al. 2004)...... 15

Figure 2-12. Lock exchange experiment for non-Boussinesq cases $(\gamma=0.681)$................................. 16

Figure 2-13. Schematic of two lock exchange configuration for non-Boussinesq flow............................ 17

Figure 2-14. Speed of the light current for various density ratios (Lowe et al. (2005)). .......................... 17

Figure 2-15. Speeds of the heavy currents (Lowe et al. 2005). ................................................................ 18

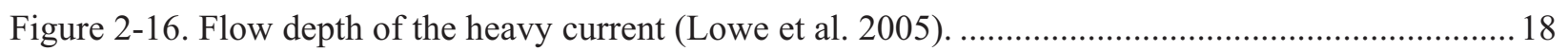

Figure 2-17. Nondimensional vorticity maps for the steady flow by DNS (Etienne et al. 2005).............. 19

Figure 2-18. Air-ingress scenario (Idaho National Laboratory's [INL's] current understanding). ............ 20

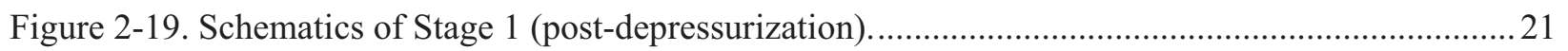

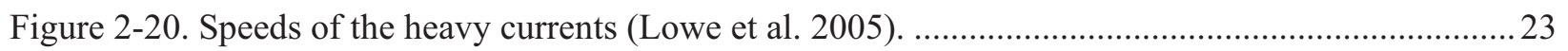

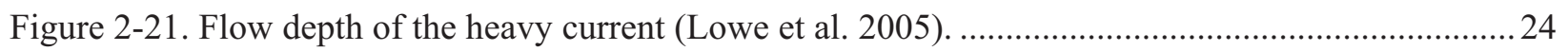

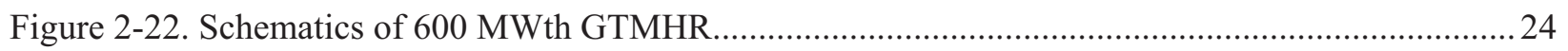

Figure 2-23. Correlation between length scale and relative time scales. ..................................................2 27

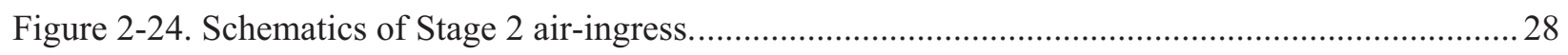

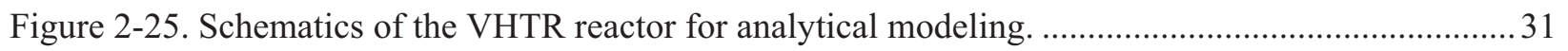

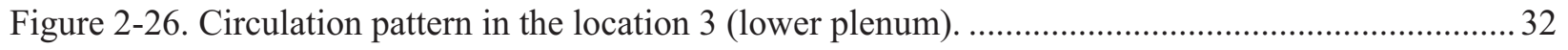

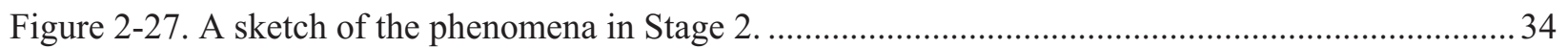

Figure 2-28. Calculated y-velocity profile in the reactor core during Stage 2 (FLUENT 6.3).................. 35

Figure 2-29. Stability concept of Stage-2 in air-ingress accident.......................................................... 37

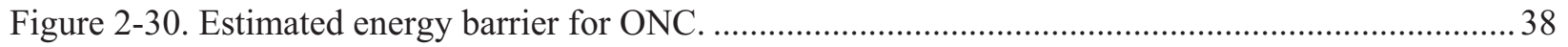

Figure 2-31. Reference reactor (GTMHR 600 MWth) and mesh of FLUENT 6.3 .................................3

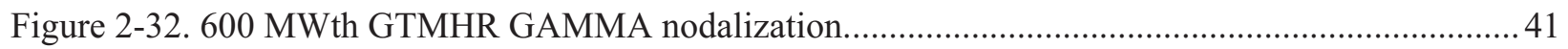

Figure 2-33. Initial condition of temperature and air mole fraction for CFD analysis (non-

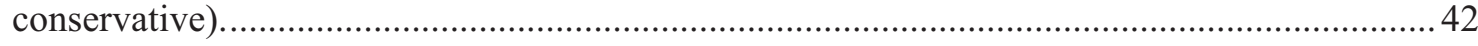


Figure 2-34. Time variations of the temperatures in the reactor core during an air-ingress accident.

Figure 2-35. Core pattern $(\mathrm{d}=1.58 \mathrm{~cm}, \mathrm{p}=3.27 \mathrm{~cm})$. (Internal Design Review Presentation

Material, General Atomics, San Diego, 1997)

Figure 2-36. Typical geometry of lower plenum $\left(\mathrm{d}_{\mathrm{LP}}=0.212 \mathrm{~m}, \mathrm{P}_{\mathrm{LP}}=0.36 \mathrm{~m}\right)$. (The red circle represents rod.) (McEligot and McCreery 2004)................................................................ 44

Figure 2-37. Detail view of lower plenum in 600 MWth GTMHR........................................................ 44

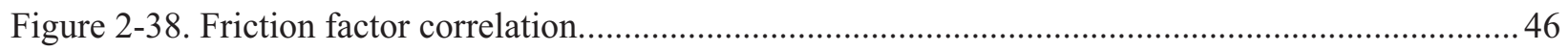

Figure 2-39. Equally spaced triangular tube array (Vassallo and Symolon 2007). .................................4 47

Figure 2-40. Cross-flow friction factor data for staggered tube arrays (Vassallo and Symolon 2007).

Figure 2-41. CFD analysis results (air mole fraction) of stratified flow in VHTR

(nonconservative).

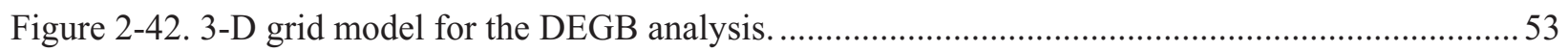

Figure 2-43. Initial air mass fraction, temperature, and pressure conditions for 3-D CFX analysis...........55

Figure 2-44. Wall temperature conditions for the core blocks, support blocks, and reactor vessel............55

Figure 2-45. Pressure distribution results with the porous conditions under the normal operation

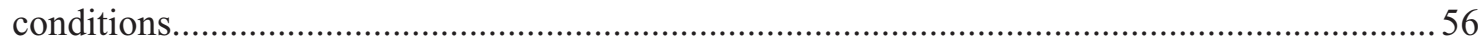

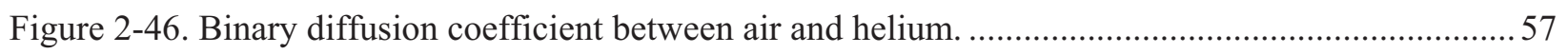

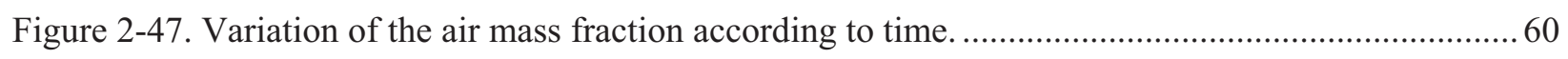

Figure 2-48. Velocity profile, density, and pressure distribution at 0.0 seconds and 0.18 seconds............61

Figure 2-49. Air mass fraction of upper plenum, coolant riser, cold duct header, and reactor

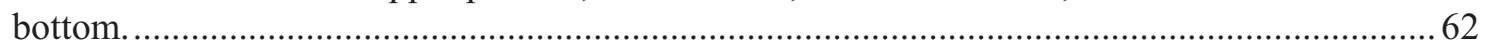

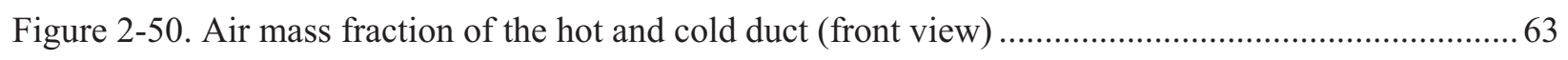

Figure 2-51. Air mass fraction and velocity profile in the lower plenum at 5.96 seconds.......................63

Figure 2-52. Volume averaged air mass fraction of the lower plenum, the reactor bottom, the cold duct header and the coolant riser (maximum value of the air mass fraction is 0.5 ). ................. 64

Figure 2-53. Volume averaged velocity of the core blocks.

Figure 2-54. Volume and area averaged air mass fraction of the lower plenum, core blocks, and core inlet (maximum value of the air mass fraction is 0.5 ).

Figure 2-55. Volume and area averaged air temperature of the lower plenum, core blocks, and core inlet.

Figure 2-56. Temperature distribution on the plane of $\mathrm{z}=6.7 \mathrm{~m}$ in the lower plenum (LP bottom: $\mathrm{z}=7.624 \mathrm{~m}, \mathrm{LP}$ top: $\mathrm{z}=7.624 \mathrm{~m}$, temperature and air mass fraction are averaged over the area of the plane at $\mathrm{z}=6.7 \mathrm{~m}$ ). 66

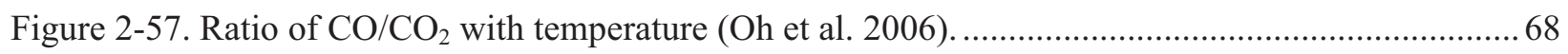

Figure 2-58. Effect of temperature on the graphite oxidation rates and oxidation regimes........................ 68

Figure 2-59. Species concentration profiles in the VHTR at 13 seconds. ............................................. 70 
Figure 2-60. Comparisons of with and without chemical reaction models............................................ 71

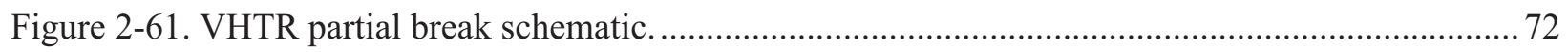

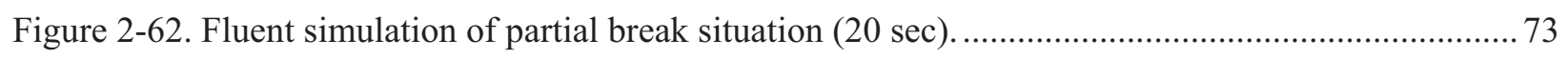

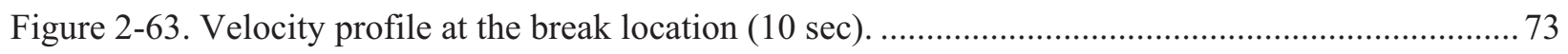

Figure 2-64. Air-mass fractions in the VHTR in the partial break situation (FLUENT results). .............. 74

Figure 2-65. Average air mass fraction in the core and the lower plenum. .......................................... 75

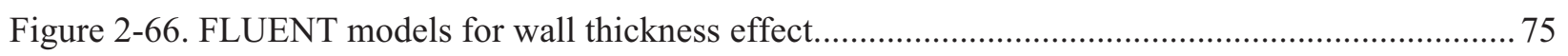

Figure 2-67. Effect of wall thickness in the partial break. .................................................................. 76

Figure 2-68. Three different regimes created depending on the break angle of the hole........................... 76

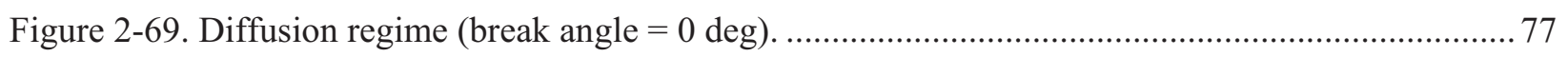

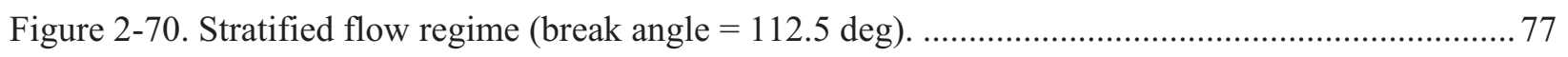

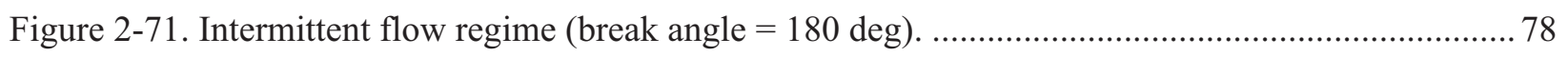

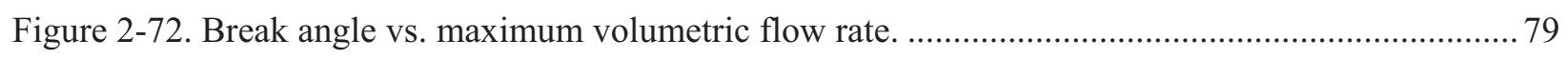

Figure 2-73. Previous natural circulation pattern assumed in 1-D simulation........................................ 79

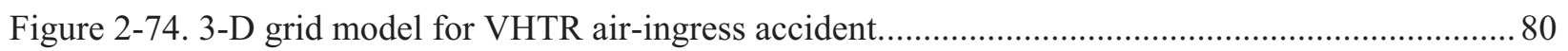

Figure 2-75. Geometry and mesh of the 3 -D grid at the lower part of the reactor................................... 81

Figure 2-76. Temperature variations with time during air-ingress accident ( $\mathrm{Oh}$ et al. 2008)..................8 83

Figure 2-77. Reference boundary conditions for the CFD simulation...................................................... 83

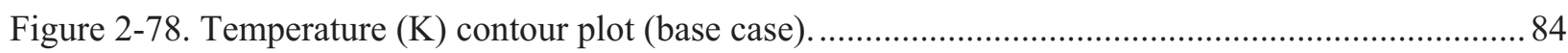

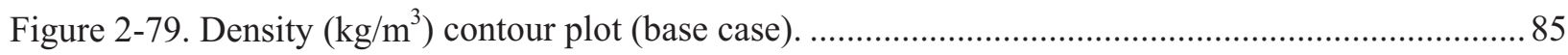

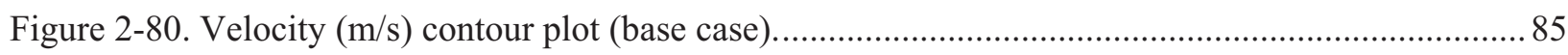

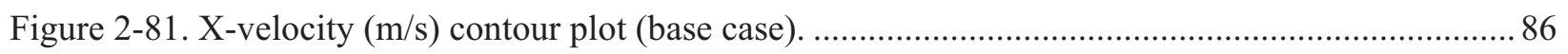

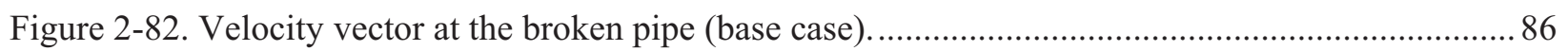

Figure 2-83. Comparisons of natural circulation flow patterns between 1-D assumption and multi-

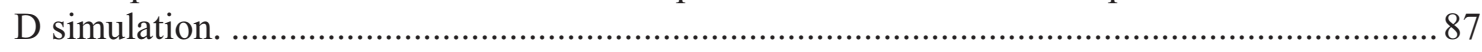

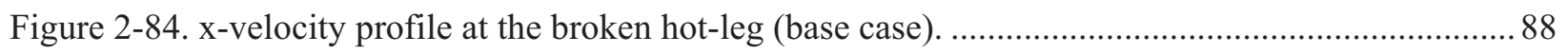

Figure 3-1. Flows of interest in the isothermal full-break experiment.................................................91

Figure 3-2. Comparisons between previous gravity current studies and VHTR air-ingress

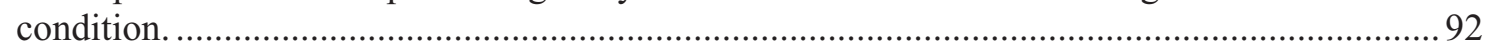

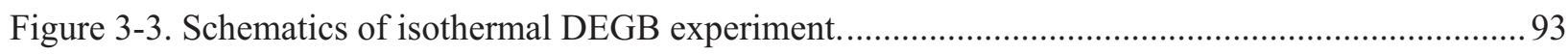

Figure 3-4. Isothermal stratified flow experimental setup for brine-water........................................... 93

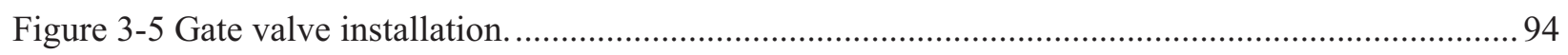

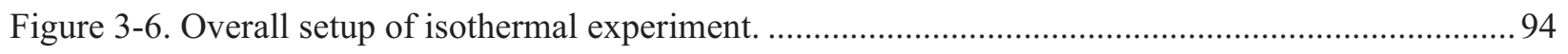

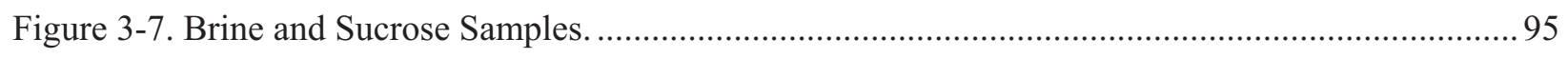

Figure 3-8. Progress of gravity current and stratified flow (water-sucrose, density ratio $=0.866$ (Case 8)). 
Figure 3-9. Experimental results (traveling distance vs. time). ...................................................... 97

Figure 3-10. Overall geometry with normal mesh and side view from symmetry surface....................... 98

Figure 3-11. Initial Brine-water mass fraction (Red: brine, Blue: water) ............................................. 100

Figure 3-12. Visualization of brine water intrusion from tilted top view at marching time frame........... 101

Figure 3-13. The correlation of density and ratio of sucrose viscosity to water viscosity...................... 102

Figure 3-14. Brine intrusion simulation with coarse mesh. ............................................................... 103

Figure 3-15. Brine intrusion simulation with normal mesh. .............................................................. 103

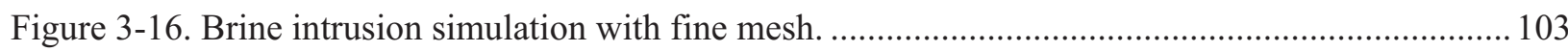

Figure 3-17. Brine mass fraction at the bottom as time marches until 7 seconds (normal, coarse

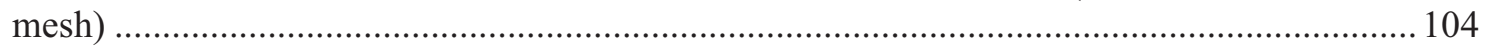

Figure 3-18. Front speed calculation in different mesh (coarse, normal, and fine). ............................. 105

Figure 3-19. Correlation of grid spacing and prediction value in grid convergence study...................... 106

Figure 3-20. Simulated front head speed and calculated front head speed at zero grid spacing with

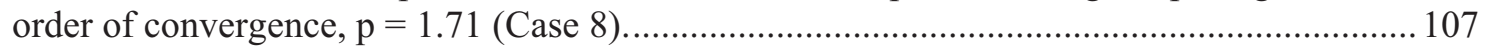

Figure 3-21. Comparison of CFD result and experiment measurement of front head speed................... 108

Figure 3-22. Plot of experimental data with uncertainty band and CFD prediction value with code uncertainty band comparison between experiment measurement and previous model (Simpson model (1979) and Benjamin model (1968))

Figure 3-23. Measured front head speed U, scaled with $\left(\boldsymbol{g}^{\prime} \boldsymbol{d} \mathbf{1}\right) \mathbf{1} / \mathbf{2}$, as a function of $\boldsymbol{d} \mathbf{1} / \boldsymbol{D}$ of

INL experimental data and Britter and Simpson's data (1979).......................................... 111

Figure 3-24. Experimental arrangement (Grobelbauer et al. 1993).................................................... 112

Figure 3-25. Propagation velocities of dense gas and light gases (Grobelbauser et al. 1993)................ 112

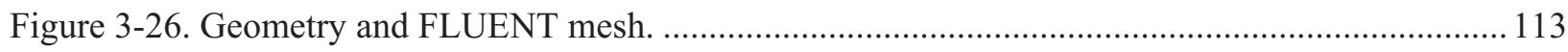

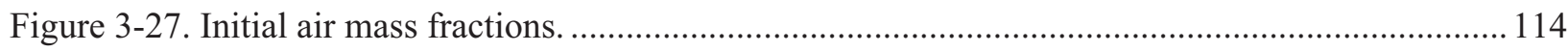

Figure 3-28. FLUENT simulation (air mass fraction (fine mesh)) ................................................... 115

Figure 3-29. FLUENT simulation (air mass fraction (normal mesh)) …............................................ 115

Figure 3-30. FLUENT simulation (air mass fraction [coarse mesh]) …............................................. 116

Figure 3-31. Air mass fraction at the bottom plate (current locations) ................................................ 116

Figure 3-32. Comparisons between different mesh sizes.................................................................. 117

Figure 3-33. Simulated front speeds and estimated front speed at zero grid spacing............................ 118

Figure 3-34. FLUENT simulation (air mass fraction, k-w model, fine mesh)...................................... 119

Figure 3-35. FLUENT simulation (air mass fraction, standard k-e model, fine mesh). ........................ 119

Figure 3-36. FLUENT simulation (air mass fraction, RSM model, fine mesh). ................................... 120

Figure 3-37. Comparisons between CFD results and experimental data (front location)...................... 120

Figure 3-38. Initial air mass fraction for light gas intrusion (helium)................................................ 121 
Figure 3-39. FLUENT simulation for light gas intrusion (air mass fraction, realizable k-e model, fine mesh). 122

Figure 3-40. Helium mass fraction at the upper plane for light gas intrusion. 122

Figure 3-41. Comparisons between CFD results and experimental data (light gas intrusion)................. 123

Figure 4-1. Experimental data of compressive strength with burn-off (Eto and Growcock 1983).......... 125

Figure 4-2. Basic idea on the prediction of graphite compressive strength. ......................................... 126

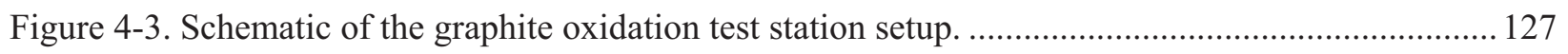

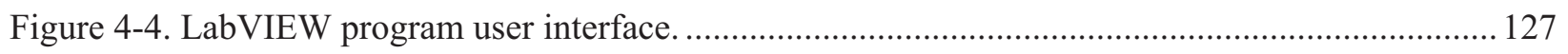

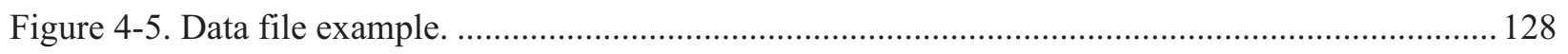

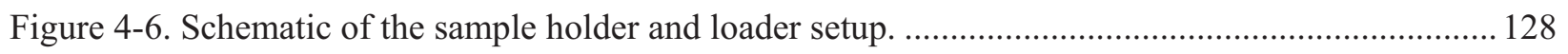

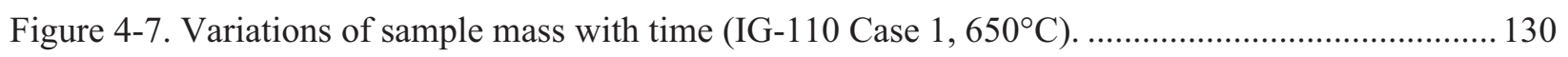

Figure 4-8. Variations of sample mass with time (IG-1 10 Case $\left.2,650^{\circ} \mathrm{C}\right)$. ......................................... 131

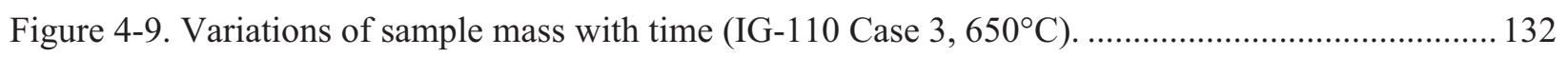

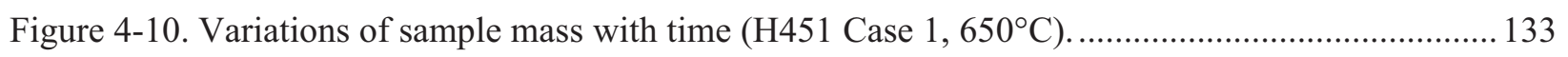

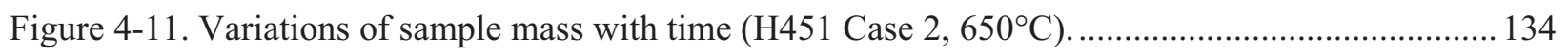

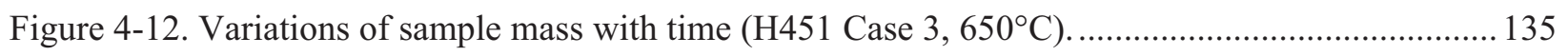

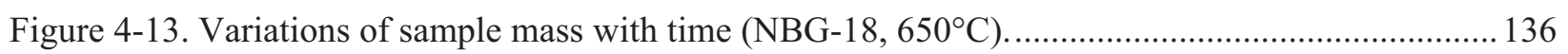

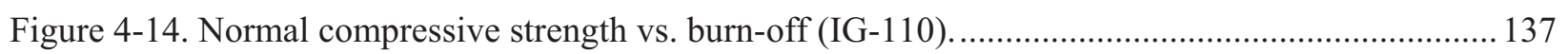

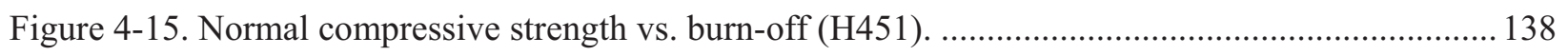

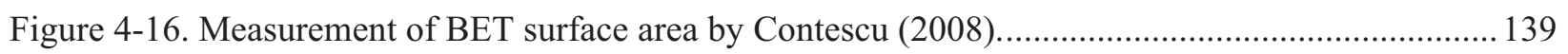

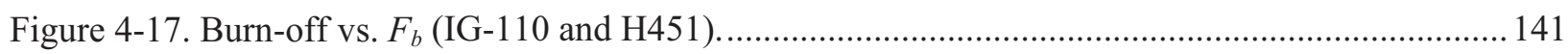

Figure 4-18. Comparisons of surface area density and oxidation rate between IG-110 and H451.......... 141

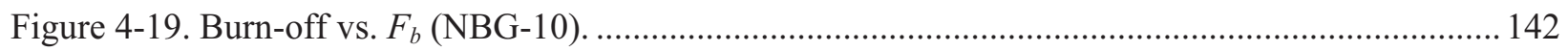

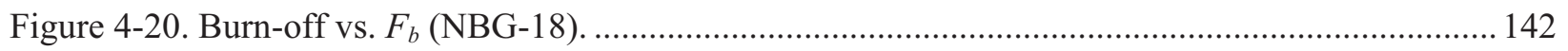

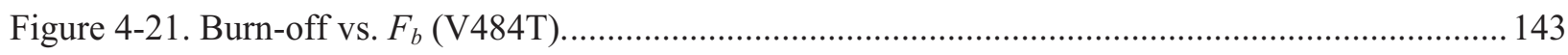

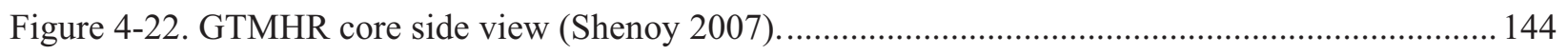

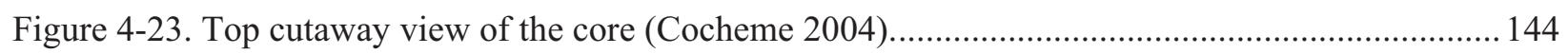

Figure 4-24. ABAQUS generated whole and cut view of the support block and plenum...................... 145

Figure 4-25. The dimensions of a General Atomics' GTMHR fuel block. (GA 1997, Cocheme

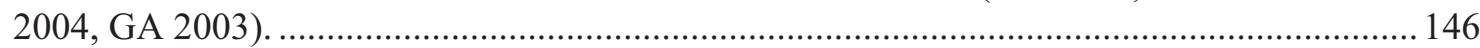

Figure 4-26. Plenum (a) side views (with dimensions), and (b) wireframe top view............................ 147

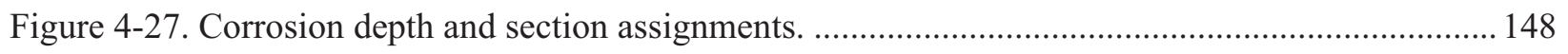

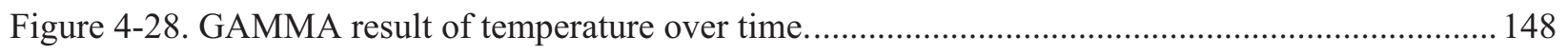

Figure 4-29. GAMMA result of burn-off over time. ......................................................................... 149

Figure 4-30. (a) Tensile strength as function of bun-off; (b) compressive strength as function of burn-off (Ishihara et al. 2004). 150 
Figure 4-31. Tensile strength as function of temperature (Eto et al. 1986).

Figure 4-32. (a) The change in Young's modulus because of oxidation as a function of temperature (best fit of experiment data), and (b) the correlations for the Young's modulus as a function of temperature and burn off (Eto et al. 1986).

Figure 4-33. Young's modulus (a) and mechanical strength (b) as function of dpa caused by irradiation (Burchell et al. 1996).

Figure 4-34. (a) Compressive stress-strain curve for UNS31803 Steel (Rasmussen 2001), and (b) Compressive stress-strain curve for IG-110 (Fuji et al. 1997). 153

Figure 4-35. Compressive stress distribution on an unoxidized support block and plenum. 154

Figure 4-36. The principal stress components (Beer et al. 2006). 155

Figure 4-37. (a) Upper part of support block with triangular prism; (b) Mesh and temperature distribution; (c) Compressive stress; and (d) Tensile stress. 156

Figure 4-38. Modification of plenum head's height block by $25 \mathrm{~cm}$. 157

Figure 4-39. Assumed 1/6 cyclic symmetry units of the modified plenum head for each day..... 157

Figure 4-40. Maximum compressive stress over time. 158

Figure 4-41. Maximum compressive tensile stress over time. 159

Figure 4-42. Compressive stress distribution of non-uniform corrosion model, 12.25 days after LOCA. 159

Figure 4-43. Tensile stress distribution of non-uniform corrosion model, 12.25 days after LOCA......... 160

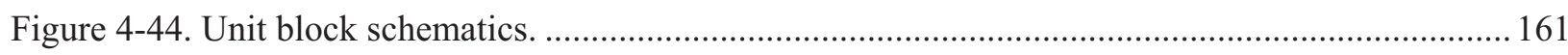

Figure 4-45. Relation between local burn-off and normalized compressive strength (IG-110).............. 164

Figure 4-46. Relation between local burn-off and normalized compressive strength (H451)................. 164

Figure 4-47. Modeling of graphite fracture by corrosion damage (constant f)..................................... 165

Figure 4-48. Modeling of graphite fracture by corrosion damage (randomly sampled f [uniform]) ........ 165

Figure 4-49. Modeling of graphite fracture by corrosion damage (randomly sampled $\mathrm{f}$ [Gaussian])..... 166

Figure 4-50. Relations between f-value $(f)$ vs. fractured total burn-off (IG-110)................................ 167

Figure 4-51. Fractured burn-off (Random f) vs. fractured burn-off (constant f) - IG-110.................... 167

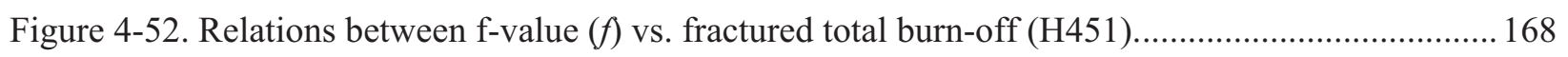

Figure 4-53. Fractured burn-off (random f) vs. fractured burn-off (constant f) - H451 ......................... 168

Figure 4-54. Overall graphite oxidation rate (Oh et al. 2006). ............................................................... 169

Figure 4-55. Relationship between burn-off (\%) and multiplication factor (Oh et al. 2008)................. 170

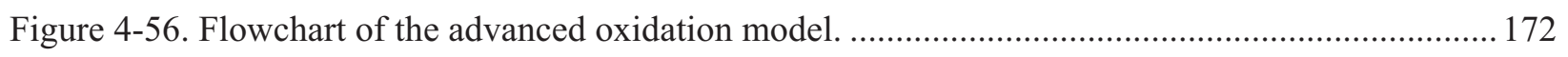

Figure 4-57. 600 MWth GTMHR code nodalization (GAMMA) ....................................................... 173

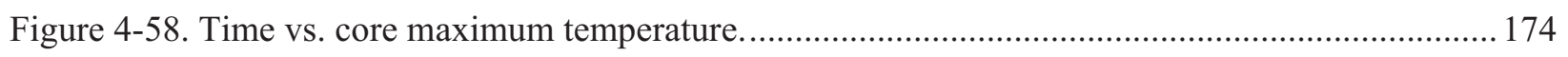

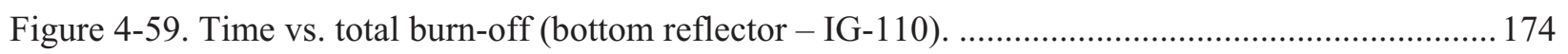

Figure 4-60. Time vs. total burn-off (lower plenum - IG-110). ...................................................... 175 
Figure 4-61. Comparisons of IG-110 and H-451 for fracture at the bottom reflector. 175

Figure 5-1. GTHTR300 geometry and CFD model (Yan et al. 2008) ................................................. 177

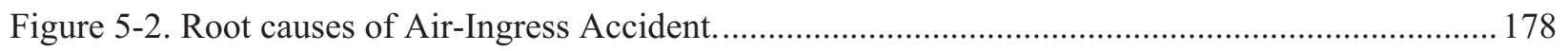

Figure 5-3. Concept of using supporting structure for preventing core weakening.............................. 180

Figure 5-4. Air-ingress mitigation method at the lower plenum........................................................ 181

Figure 5-5. Concept of reactor enclosure with opening at the bottom................................................. 182

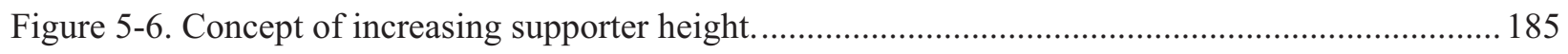

Figure 5-7. Air-ingress mitigation method by helium injection at the lower plenum............................. 186

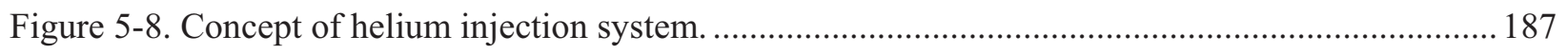

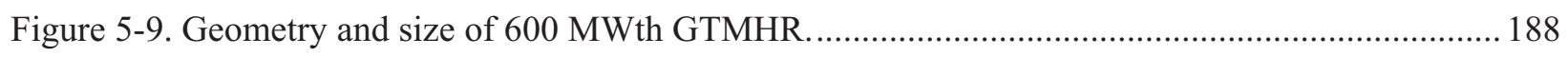

Figure 5-10. 2-D FLUENT model (geometry and grid model). ........................................................ 189

Figure 5-11. Initial conditions (air mass fraction, temperature) .......................................................... 191

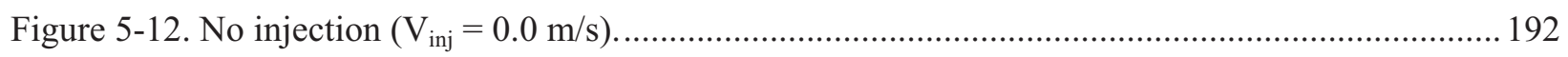

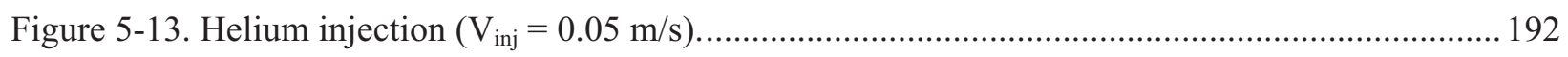

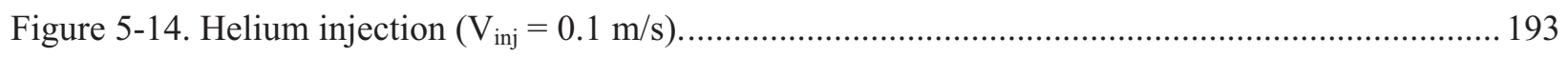

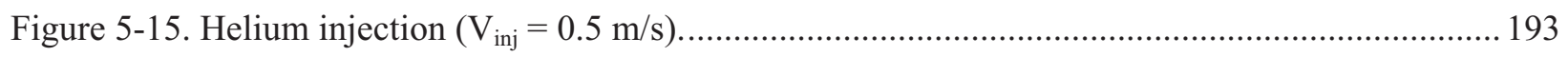

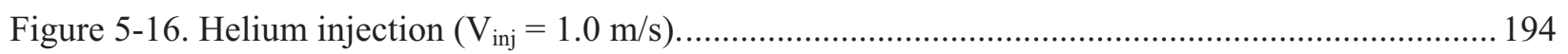

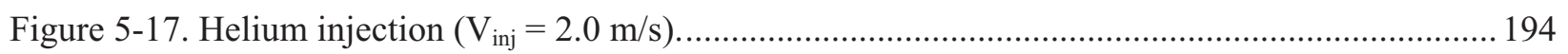

Figure 5-18. Comparisons on the air distributions for the different injection speed $(\mathrm{t}=200 \mathrm{sec})$.......... 195

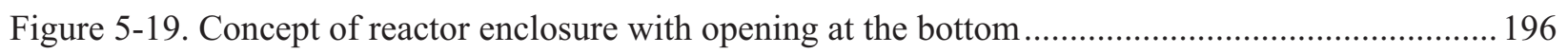

Figure 5-20. 2D FLUENT Model (Geometry and Grid Model). ......................................................... 197

Figure 5-21. Initial conditions (air mass fraction, temperature). ........................................................ 199

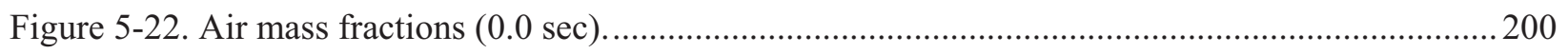

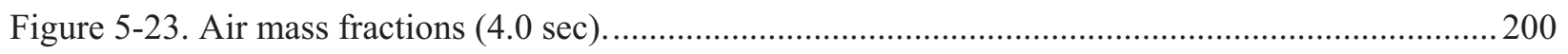

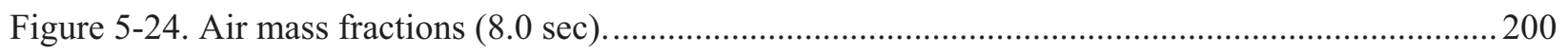

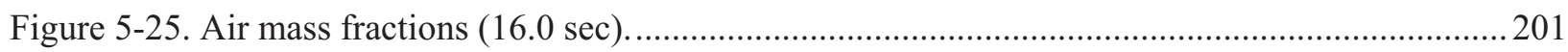

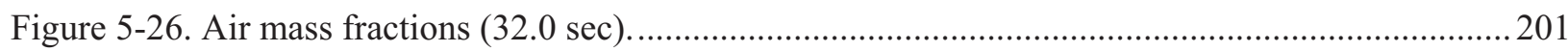

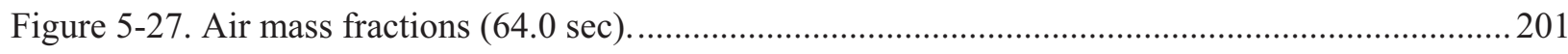

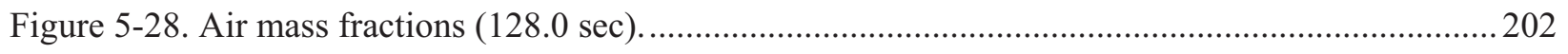

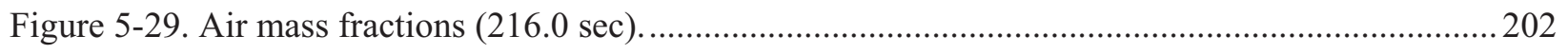

Figure 5-30. Air-ingress mechanism in the proposed air-ingress mitigation method $(\mathrm{t}=216 \mathrm{sec}) \ldots \ldots \ldots . .203$

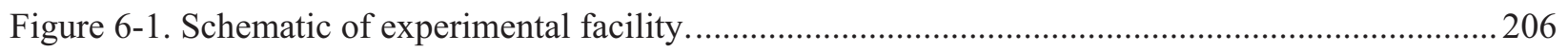

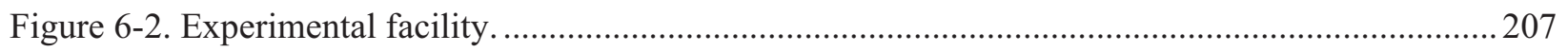

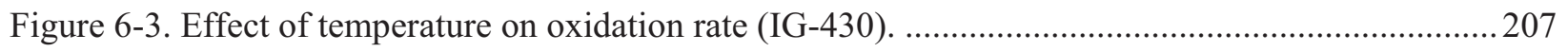

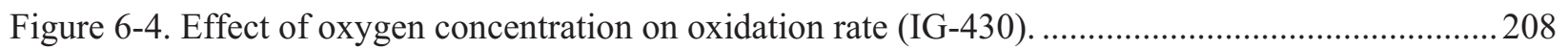




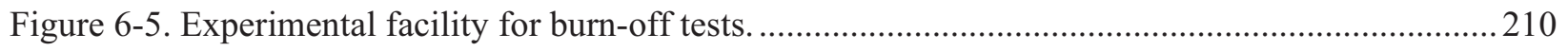

Figure 6-6. Changes of burn-off with time for different conditions. .................................................... 210

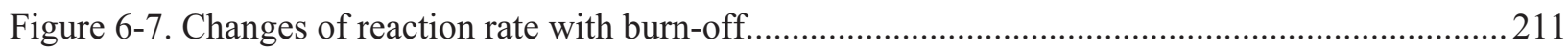

Figure 6-8. Relation between burn-off and ratio of reaction rate.......................................................212

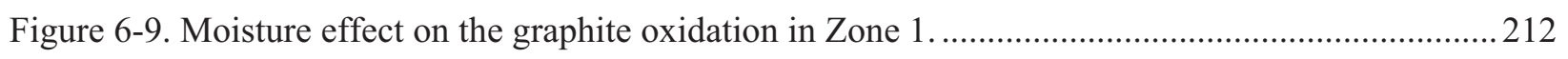

Figure 6-10 Moisture effect on the graphite oxidation in Zone 3 .................................................... 213

Figure 7-1. Schematics of 600 MWth GTMHR and graphite support column in the lower plenum........214

Figure 7-2 Schematic of oxidation in a graphite column................................................................... 215

Figure 7-3. Compression test facility, INSTRON Model 4204.......................................................... 218

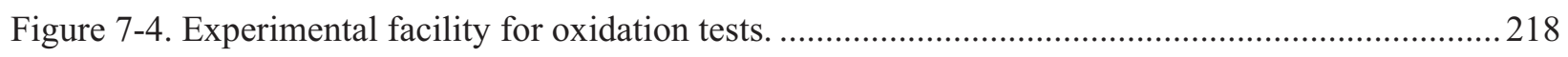

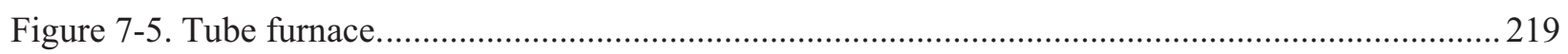

Figure 7-6. Compressive and buckling strength of IG-110 graphite columns..................................... 219

Figure 7-7. Compressive and buckling strength of IG-430 graphite columns...................................... 220

Figure 7-8. Compressive and buckling strength of NBG-10 graphite columns.................................. 221

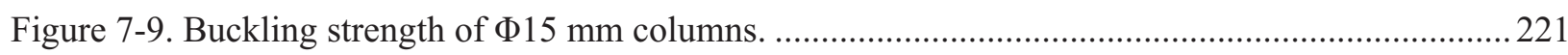

Figure 7-10. Normalized compressive strength of IG-110 oxidized in Zone 1. .....................................222

Figure 7-11. Normalized compressive and buckling strength of graphite columns oxidized in

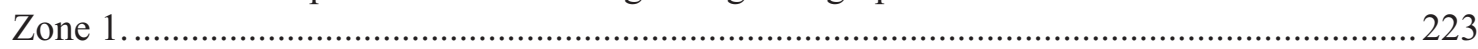

Figure 7-12. Comparison of the experimental results for the compressive strength degradation............223

Figure 7-13. Normalized compressive strength of IG-430 oxidized in Zone 1. ....................................224

Figure 7-14. Normalized compressive strength of NBG-10 oxidized in Zone 1. ..................................224

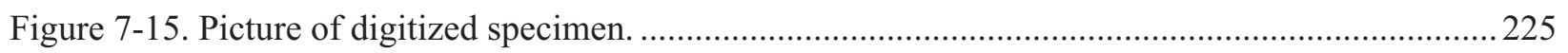

Figure 7-16. Fraction of surface reaction to total reaction (5 SLPM) .................................................225

Figure 7-17. Normalized strength of graphite oxidized in Zones 1 and 2 versus total burn-off..............226

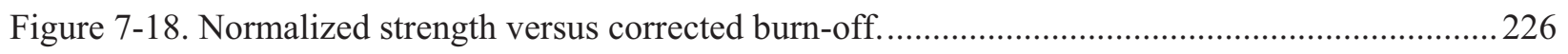

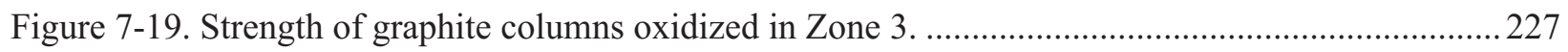

Figure 7-20. One-sixth of the graphite support block and its simplified model. ...................................228

Figure 7-21. Normalized compressive and buckling strength of IG-430 columns. ............................... 230

Figure 7-22. Relation between allowable total burn-off and f-value.................................................. 231

Figure 7-23. Comparison of allowable total burn-offs for graphite structure........................................2232

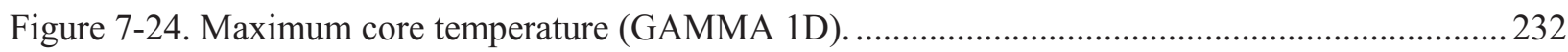

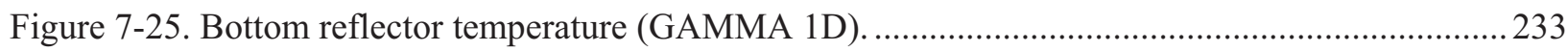

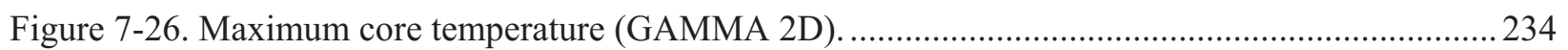

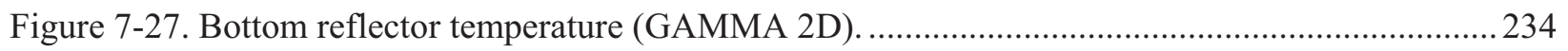

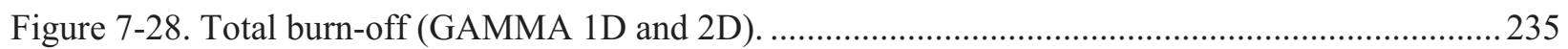




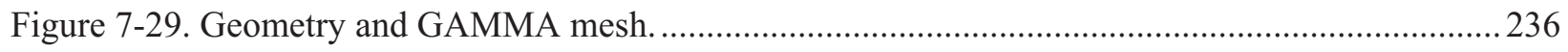

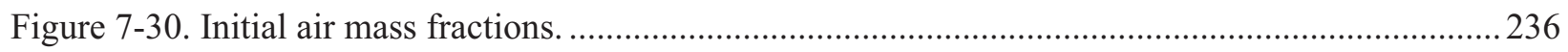

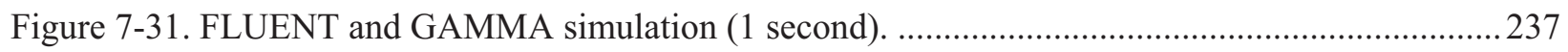

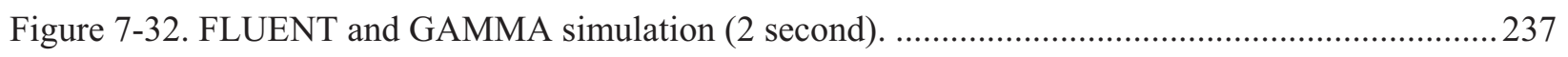

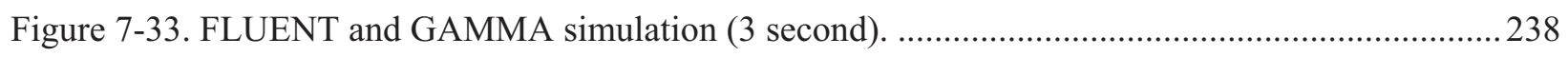

Figure 7-34. Velocity of front location (experimental data, Fluent, GAMMA code).............................239

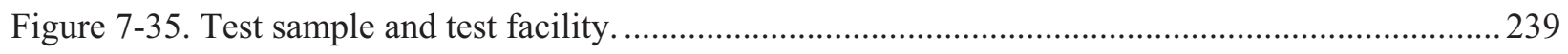

Figure 7-36. Test section without the mirror and FLUENT calculation result......................................240

Figure 7-37. Test section with the mirror and FLUENT calculation result. .......................................... 240

Figure 7-38. Relation between fractured burn-off and f-value .........................................................2. 241

Figure 7-39. Oxidized graphite structure in the case with initial temperature of $650^{\circ} \mathrm{C} \ldots \ldots \ldots \ldots \ldots \ldots \ldots . . . . . . . . .242$

Figure 7-40. Oxidized graphite structure in the case with initial temperature of $1050^{\circ} \mathrm{C} \ldots \ldots \ldots \ldots \ldots \ldots \ldots . . . . . . . .242$

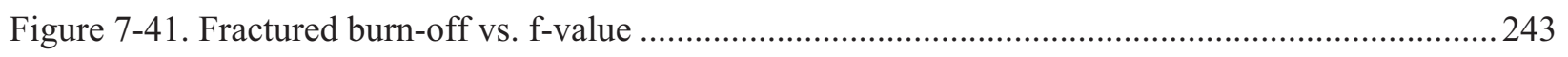

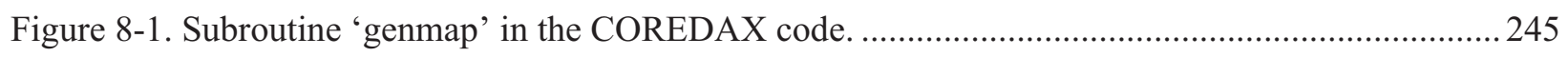

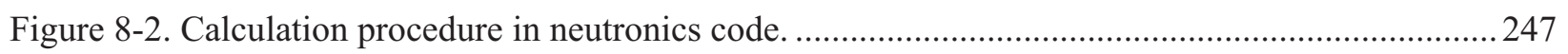

Figure 8-3: Simplified GTMHR 600 benchmark problem in the GAMMA/COREDAX code................248

Figure 8-4. Calculation results of GAMMA/COREDAX code .............................................................. 249

Figure 8-5. Homogenized cross-section form in the COREDAX code.................................................. 249

Figure 8-6. Homogenized cross-section sample in the COREDAX code...............................................250

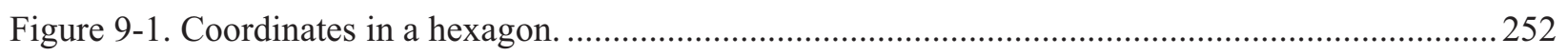

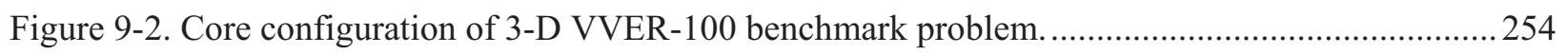

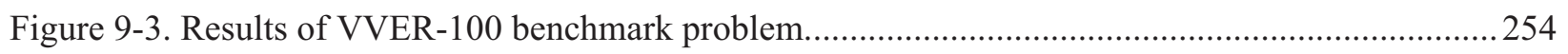

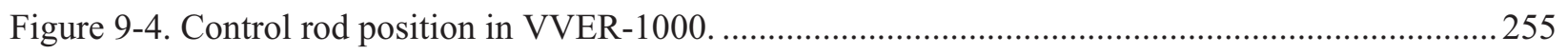

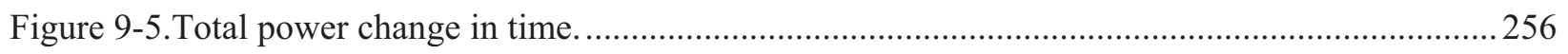

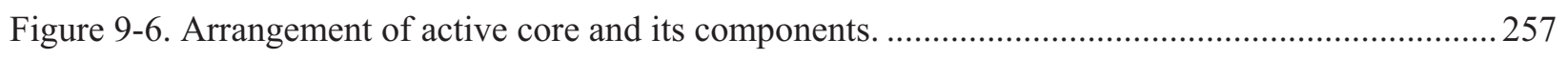

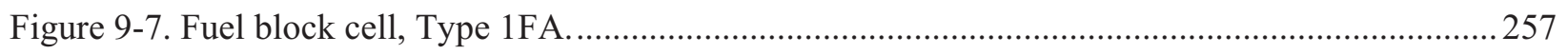

Figure 9-8. Fuel block with cavity for control rod or reserve shutdown system, Type 2FA...................258

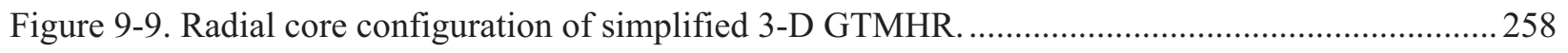

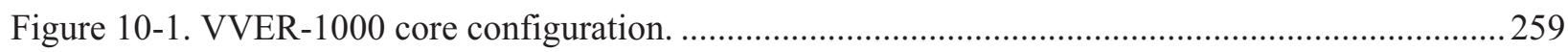

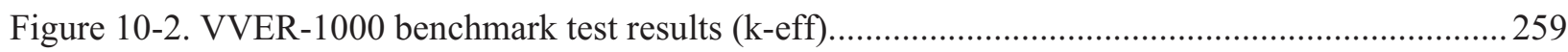

Figure 10-3. Comparisons of total power change between COREDAX and other codes........................260

Figure 10-4. Comparisons of core average fuel temperature between COREDAX and other codes. ...... 260

Figure 10-5. Radial configuration of type 1 fuel assembly model.......................................................262

Figure 10-6. Radial configuration of type 2 fuel assembly with control rod model .............................. 262

Figure 10-7. Radial configuration of type 2 fuel assembly without control rod model..........................262 
Figure 10-8. Radial configuration of type 1 moderator assembly model..............................................263

Figure 10-9. Radial configuration of type 2 moderator assembly with control rod model.....................263

Figure 10-10. Radial configuration of type 2 moderator assembly without control rod model............... 263

Figure 10-11. Axial configuration of type 1 moderator assembly model. ............................................264

Figure 10-12. $k_{\text {inf }}$ for various fuel and moderator temperatures in type 1 fuel assembly.......................265

Figure 10-13. Generated fast-group capture cross-section for various temperature calculation in

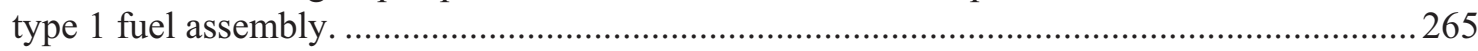

Figure 10-14. Generated thermal-group capture cross-section for various temperature calculation

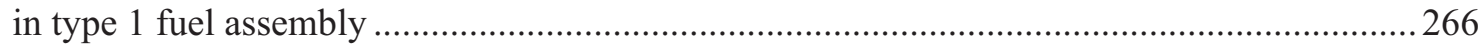

Figure 10-15. Generated fast-group $v \Sigma_{f}$ cross-section for various temperature calculation in type

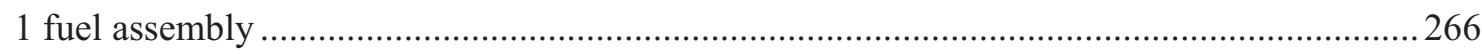

Figure 10-16. Generated thermal-group $v \sum_{f}$ cross-section for various temperature calculation in

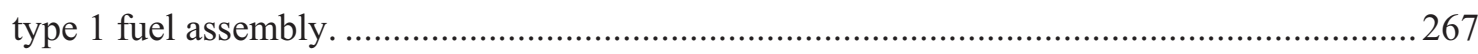

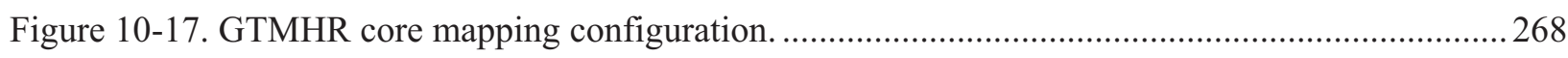

Figure 10-18. Block power distribution compared to GAMMA for all rods in (ARI) case. ................... 268

Figure 10-19. Block power distribution compared to GAMMA for all rods out (ARO) case................. 269

Figure 10-20. Block power distribution compared to GAMMA for rod half insertion case. .................. 269

Figure 10-21. GAMMA nodal scheme of 600 MWtg GTMHR........................................................ 270

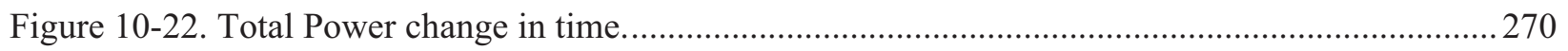

Figure 10-23. Comparisons of Parameters between GAMMA and GAMMA/COREDAX code. ........... 271

Figure A-1. Double-ended guillotine break facility assembly with knife gate valve. ............................. 284

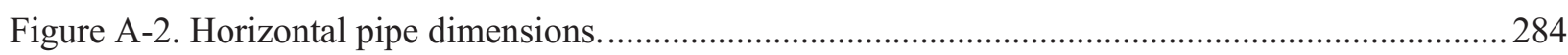

Figure A-3. Flanged tank dimensions. Tanks have covers on both top and bottom to facilitate

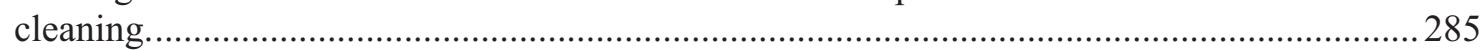

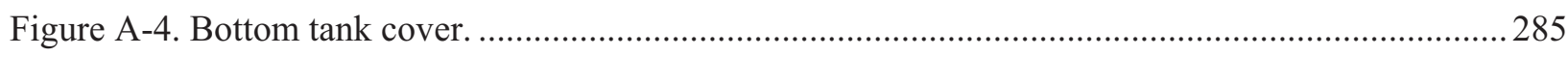

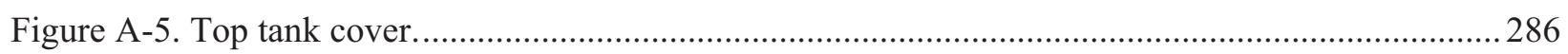

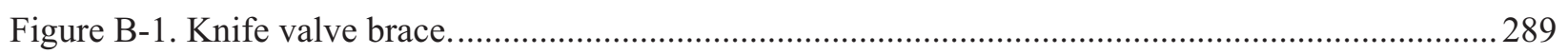

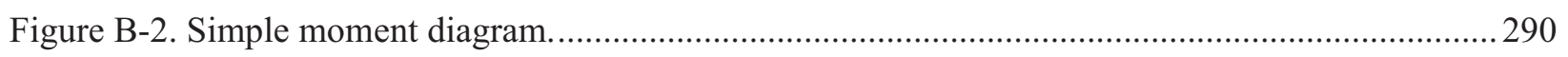

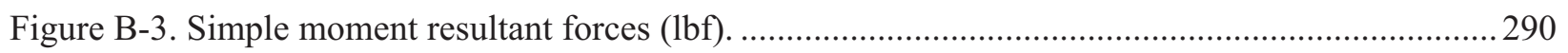

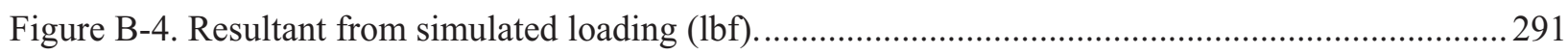

Figure B-5. Local Frame Stresses from Simulated Load (psi)............................................................. 292

\section{TABLES}

Table 2-1. Comparisons of pressure build-up and static head for GTMHR and NACOK

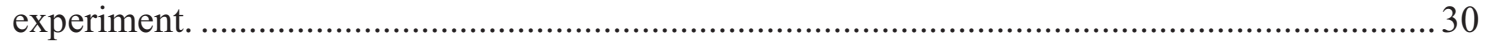

Table 2-2. Comparisons of time scales for the GTMHR (Stage 2)........................................................ 35 


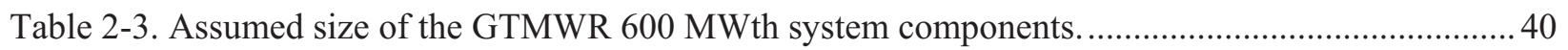

Table 2-4. Correlation for the friction factor and their mean absolute errors. (Kosar et al. 2005) .............49

Table 2-5. Number of mesh and volume data for each region in the 3-D grid model. .............................5 54

Table 2-6. Pressure drop results using the porous media conditions. ......................................................56

Table 2-7. NASA format correlation for specific heat of helium. ......................................................... 57

Table 2-8. Number of mesh and volume data for each region in the 3-D grid model. ..............................81

Table 2-9. Reference FLUENT model setup. ....................................................................................... 82

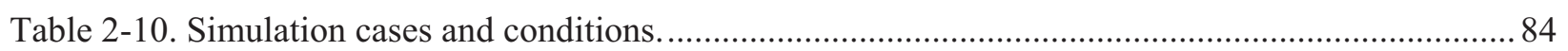

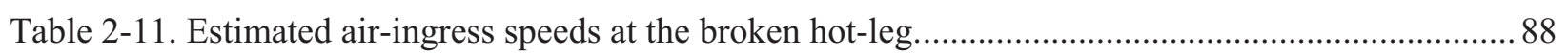

Table 3-1. Previous and current validation data for air-ingress analyses................................................. 90

Table 3-2. Experimental conditions and case summary.................................................................... 95

Table 3-3. Experimental results (traveling distance $[\mathrm{x}]$ vs. time) ........................................................ 97

Table 3-4. Comparisons of current speeds between experiment and Benjamin's theoretical model..........98

Table 3-5.Front head speed calculation at different normalized spacing............................................... 106

Table 3-6. Uncertainty of front head speeds from experiments and CFD code calculations.................... 109

Table 3-7. Gas combinations used for Grobelbauser et al. (1993)'s experiment..................................... 113

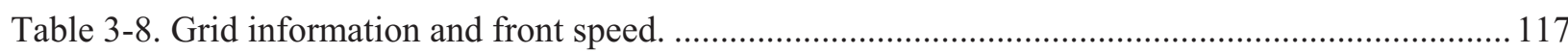

Table 3-9. Comparisons between CFD results and experimental data (current speed). ......................... 120

Table 3-10. Comparisons between CFD results and experimental data (light gas intrusion).................. 123

Table 4-1. Parameters in Equation (4-1) for various graphite materials (Eto and Growcock 1983)........ 125

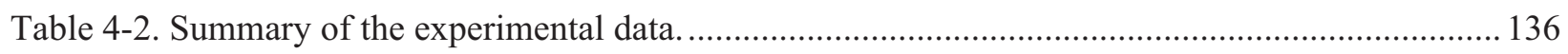

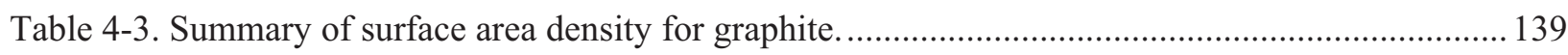

Table 4-4. Basic thermo mechanical properties of IG-110 at standard conditions. (Ishihara et al.

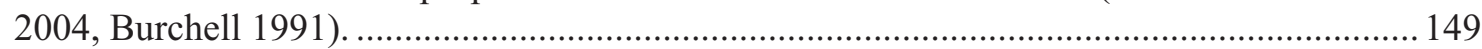

Table 4-5. Comparison of the analytical solution to the ABAQUS result.............................................. 155

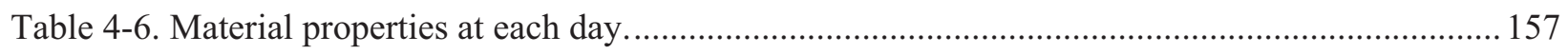

Table 4-7. Input parameters for graphite oxidation and structural integrity......................................... 166

Table 5-1. Input variables and reference values for the sensitivity analyses. ........................................ 184

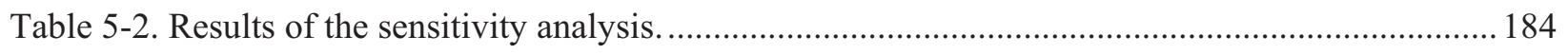

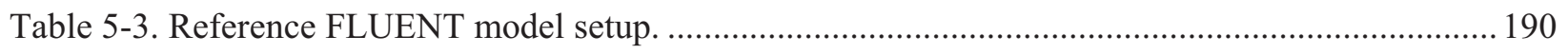

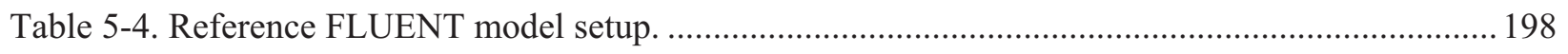

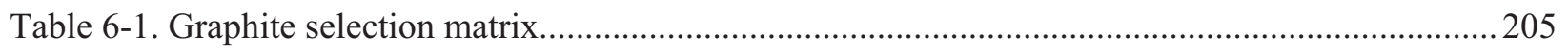

Table 6-2. Properties of IG-110 and IG-430 manufactured by Toyo Tanso in Japan..............................206

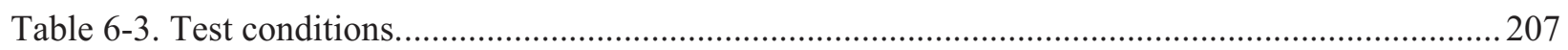

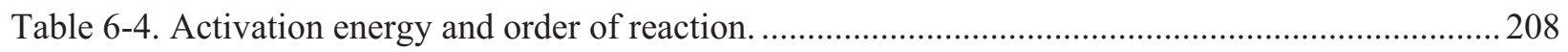


Table 6-5. Experimental results on kinetics parameters for IG-430 .................................................208

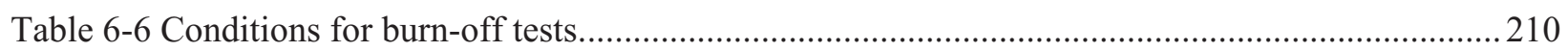

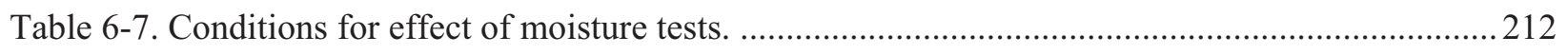

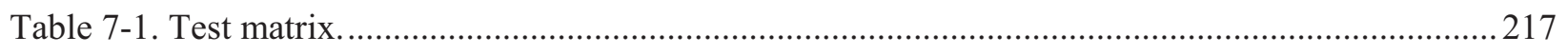

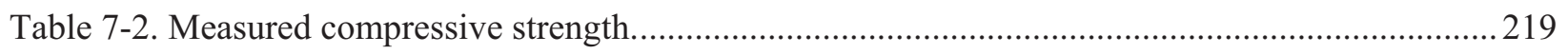

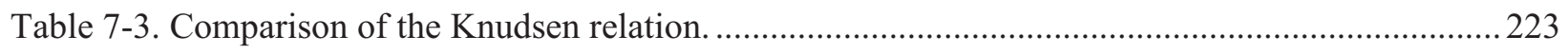

Table 7-4. Dimensions of graphite specimens for fresh graphite tests. .................................................228

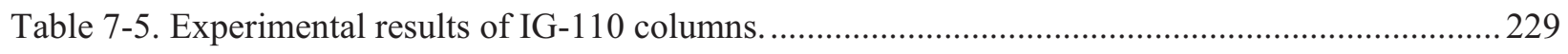

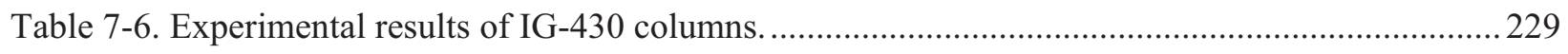

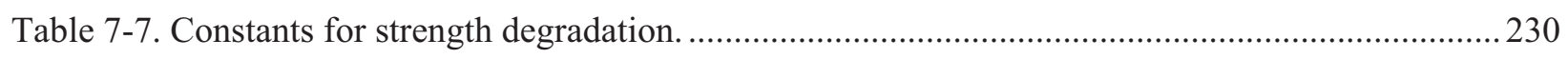

Table 7-8. Test condition and local burn-off of graphite structure. ....................................................... 241

Table 9-1. Results of VVER-440 benchmark problem. ' ........................................................................ 252

Table 9-2. Results on $k_{\text {eff }}$ of simplified VVER-1000 benchmark problem. ..........................................253

Table 9-3. Results of SNR-300 benchmark problem............................................................................. 253

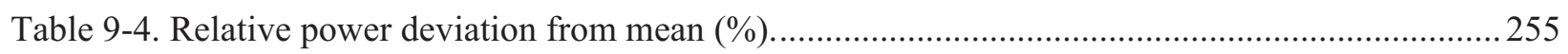

Table 9-5. Results on $k_{\text {eff }}$ of simplified GTMHR benchmark problem. ................................................2 258

Table 10-1. $k_{\text {inf }}$ of type 1 fuel assembly in air-ingress accident calculation...........................................267 


\section{ACRONYMS}

ASTM American Society for Testing and Materials

BET Brunaur-Emmett-Teller

CFD computational fluid dynamics

DEGB double-ended guillotine break

GCI Grid Convergence Index

GTHTR Gas Turbine High Temperature Reactor

GTMHR gas-turbine modular helium reactor

HTGR high temperature gas-cooled reactor

INL Idaho National Laboratory

LOCA loss-of-coolant accident

NGNP Next Generation Nuclear Plant

ONC onset of natural circulation

ORNL Oak Ridge National Laboratory

RSM Reynold's Stress Model

VHTR very high temperature gas-cooled reactor 
xxviii 


\title{
Final Report on Experimental Validation of Stratified Flow Phenomena, Graphite Oxidation, and Mitigation Strategies of Air Ingress Accidents
}

\author{
1. INTRODUCTION
}

A loss-of-coolant accident (LOCA) is considered a critical event for a very high temperature reactor (VHTR). Following helium depressurization, it is anticipated that, unless countermeasures are taken, air will enter the core through the break leading to oxidation of the in-core graphite structure. Thus, without mitigation features, a LOCA will lead to an air ingress event, which will result in exothermic chemical reactions of graphite with oxygen, mechanical graphite strength degradation, and toxic gas releases, potentially having significant safety implications.

The U.S. Department of Energy is supporting research and development that focuses on key phenomena important during challenging scenarios that may occur in the Next Generation Nuclear Plant (NGNP)/Generation IV VHTR. Phenomena Identification and Ranking Table studies to date have identified the air ingress event, following on the heels of a VHTR depressurization, as very important (Schultz et al. 2006). Consequently, the development of advanced air ingress-related models and verification and validation $(V \& V)$ requirements are high priorities for the NGNP Program.

\subsection{Background}

The VHTR is a graphite-moderated, uranium-fueled, helium-cooled reactor using a direct or indirect gas cycle to convert the heat generated by nuclear fission into electrical energy by means of a helium turbo-generator. High temperature gas-cooled reactor (HTGR) technology has been researched and developed since the 1950s. The VHTR produces a higher outlet temperature than the HTGR. VHTRs work on the principle of passing a cooling gas through the core, then running the heated gas directly to a steam generator or a gas turbine. Japan and China have constructed and are using VHTRs for their nuclear research. Some advantages VHTRs have over existing light water reactors include: fuel integrity, proliferation resistance, a relatively simple fuel cycle, easy refueling, and modularity to supply electricity to remote areas and energy-starved underdeveloped countries with a smaller power generation infrastructure. The VHTR is a helium cooled reactor with an outlet temperature above $900^{\circ} \mathrm{C}$ and a modularity of $600 \mathrm{MWth}$. Benefits of the VHTR concept are a higher thermal efficiency than other concepts, process heat for hydrogen production, and a higher degree of passive safety. However, the VHTR faces some technical and economical challenges, particularly reactor safety and production costs. The plant design should be streamlined to be technically sound, robust, proliferation-resistant, and economical. Even though gas reactors have been developed in the past with limited success, the innovations of modularity and integrated state-of-the-art safety systems make the VHTR design attractive from a technical and economic perspective.

The very high temperatures of the VHTR concept can be detrimental if a LOCA occurs that results in the loss of coolant through the break and coolant depressurization, allowing air to enter the core through the break by molecular diffusion and ultimately by natural convection. Such a LOCA would lead to the oxidation of the in-core graphite structure and fuel, which would accelerate heat-up of the reactor core and the subsequent release of toxic gasses $\left(\mathrm{CO}\right.$ and $\left.\mathrm{CO}_{2}\right)$ and fission products. Without effective countermeasures, a pipe break may lead to significant fuel damage and fission product release.

To resolve these potential concerns, a well-validated safety and design analysis tool is needed. As the first step, the GAMMA code, which has a capability to analyze the air-ingress accident under a VHTR LOCA, was successfully developed as part of the International Nuclear Energy Research Initiative 
program (Oh et al. 2006). To complete the mission of solving technical challenges, the GAMMA code will be further improved so it can deal with all the possible transient situations expected during air ingress.

Two important new issues are associated with air-ingress in a VHTR. One concerns the potential graphite strength degradation caused following oxidation. The other is associated with stratified flow caused by density differences that will accelerate the air-ingress into the lower plenum of the reactor. It is obvious that both phenomena have the potential to cause some serious physical consequences to VHTR safety; however, little attention has been paid to either one until recently. It is therefore necessary to significantly investigate these phenomena to better estimate VHTR safety.

\subsection{Objectives}

The major objective of this 3-year study was to perform air-ingress-related experiments and validate the computer codes, such as computational fluid dynamics (CFD) and system analysis code, in order to make them reliable for predicting air-ingress accident consequences in the VHTRs. The information from this research was needed for the codes used to model the important phenomena during air-ingress accidents. The following were sought for in the research:

- Understanding effects of density-driven stratified flow on air ingress accident and developing models

- Identifying air ingress controlling phenomena

- Providing experimental data and validating CFD methods

- Developing air ingress mitigation methods and validation

- Characterizing graphite oxidation versus structural degradations and modeling

- Understanding coupling effects of thermal hydraulics and core neutronics and developing GAMMA/COREDAX code.

\subsection{Research and Development Plan}

The research and development plan of the whole 3-year project is summarized in this section.

\subsubsection{Task 1: Density-Difference Induced Stratified Flow Analysis - FY 2008 and FY 2009 Task}

This task involves computational analyses to validate the density-difference induced air ingress phenomena expected in the VHTR following air-ingress. After the hypothesized break in the hot duct of the VHTR, air present in the reactor cavity will enter the reactor vessel via a density-driven stratified flow. Because of the significantly higher molecular weight and lower initial temperature of the reactor cavity air, the air-helium mixture in the cavity is heavier than the helium discharging through the break. In the later stages of the helium blow-down, the momentum of the helium flow decreases enough that the heavier cavity air can flow into the reactor vessel lower plenum through the lower portion of the broken hot duct while helium flows in the opposite direction in the upper portion of the hot duct. Once air enters the reactor, the heavier gas will pool at the bottom of the lower plenum and begin to diffuse upwards into the core. This condition (based on the countercurrent stratified flow assumption) is considerably different from the standard assumption used in calculations to date where the air is assumed to diffuse into the lower plenum through the hot duct. The following activities were planned for this task:

- Understanding density gradient driven stratified flow characteristics in the air-ingress accident

- Understanding air-ingress accident scenarios from analytical or computational analyses

- Performing various air-ingress accident analyses based on the new scenarios. 


\subsubsection{Task 2: Experimental Study on the Stratified Flow and Validation of CFD Models - FY 2009 and FY 2010 Task}

This task involves experimental data collection to provide a baseline for validation of computer codes based on experimental results. To accomplish this task, the test loop was set up for mimicking the density-difference-induced air-ingress phenomena. The following activities were planned for this task:

- Developing and performing experiment for visualizing density-difference-induced air-ingress phenomena and for validation of current computational methods

- Validating current computational methods by comparing the experimental results with the codes.

\subsubsection{Task 3: Advanced Graphite Oxidation Study - FY 2008 and FY 2009 Task}

This task measures the transient graphite oxidation with burn-off and the internal pore surface area density of nuclear-grade graphite, a parameter found to be a very important in the early stage of graphite oxidation. The results of these measurements are implemented into the upgraded GAMMA code. The following activities were planned for this task:

- Measuring surface area density of nuclear graphite using Brunaur-Emmett-Teller (BET) method

- Measuring transient graphite oxidation with burn-off

- Implementing the advanced graphite material parameters and oxidation models into GAMMA code.

\subsubsection{Task 4: Air Ingress Mitigation Study - FY 2010 Task}

This task develops potential methods of air-ingress mitigation and evaluates those methods by computational methods. The following activities were planned for this task:

- Reviewing previous air-ingress mitigation concepts

- Understanding air-ingress accident consequences and their mechanisms

- Developing air-ingress mitigation concepts

- Validating those air-ingress concepts by using computational methods.

\subsubsection{Task 5: Experiment of Burn-off in the Core Bottom Structures (KAIST) - FY 2008 Task}

This task measures the oxidation rate and density of the nuclear graphite and developed oxidation models of the core bottom structures which would be exposed to air in a LOCA. The main parameters that affect the rate of oxidation and density of the graphite of the core bottom are: kinetics; mass diffusion; combined effect of kinetics and mass diffusion; moisture, shape, and size; and degree of burn-off. The following activities were planned for this task:

- Measurement of oxidation rate of nuclear graphite

- Measurement of density of nuclear graphite with burn-off.

\subsubsection{Task 6: Structural Tests of Oxidized Core Bottom Structures (KAIST) - FY 2009 and FY 2010 Task}

This task involves the fracture model regarding the oxidation of the nuclear graphite. Because of density changes in the nuclear graphite, structural characteristics will be investigated and the fracture model of the core bottom structures will be developed by fracture testing, including the internal pressure 
test, uniaxial compression test, diametrical compression test, and fracture toughness test. The following activities were planned for this task:

- Fracture test of nuclear graphite with burn-off

- Development of fracture model of burnt-off bottom reflector.

\subsubsection{Task 7: Coupling Neutronic-Thermal Hydraulic Tools (KAIST) - FY 2009 Task}

This task involves the enhancement of thermal-hydraulic capability of GAMMA code. Thermal power distribution in the reactor core is needed to improve the reliability of thermal-hydraulic analysis. The knowledge of accurate thermal distribution is also needed to generate exact cross-section of nuclei. Neutronics/thermal-hydraulics feedback effects will be investigated and the feedback parameters implemented into an upgraded GAMMA code. The following activities were planned for this task:

- Set up parameters for neutronics/thermal-hydraulics code coupling

- Code coupling of COREDAX with GAMMA.

\subsubsection{Task 8: Core Neutronic Model (KAIST) - FY 2008 and FY 2009 Task}

This task involves an advanced neutronics code for both steady and transient analysis of a VHTR core. Korea Advanced Institute of Science and Technology (KAIST) developed COREDAX code to analyze the hexagonal-z three-dimensional (3-D) geometry. The COREDAX code is based on analytic function expansion nodal (AFEN) method, which does not use the transverse-integration procedure but uses analytic basis functions to represent the solution with uppermost accuracy. The COREDAX and GAMMA coupled code will provide accurate analysis of initial condition power distribution of VHTR by feedback calculation with each other. In this task, the COREDAX code will be developed to deal with the hexagonal-z 3-D geometry. COREDAX code will be used to investigate the feedback between neutronics and thermal-hydraulics. The following activities were planned in this task:

- Development of a VHTR core neutronics analysis code in hexagonal 3-D geometry

- Investigation of neutronics/thermal-hydraulics feedback effects.

\subsubsection{Task 9: Coupled Core Model V\&V (KAIST) - FY 2010 Task}

This task involves the verification of GAMMA code coupled with COREDAX code and the validation of prediction results of thermal power distribution in the hexagonal reactor core. The following activities were planned in this task:

- Verification of GAMMA and COREDAX coupling

- Validation of coupled code with reference data. 


\section{TASK 1: DENSITY-DIFFERENCE INDUCED STRATIFIED FLOW ANALYSIS}

\subsection{Introduction}

The potential for air to ingress into the VHTR vessel stems from consideration of postulated LOCAs. The VHTR has a reactor cavity that is filled with air under normal operational conditions. If a LOCA occurs, air may be able to move into the reactor vessel. It is presently thought that the worst-case scenario will occur if a double-ended guillotine break (DEGB) is postulated in the hot duct. The hot duct is a large pipe (exact dimensions are presently not defined, but the outer diameter is over a meter) that connects the reactor vessel with the vessel housing the power conversion equipment.

For a double-ended guillotine rupture, the transient will commence with a depressurization from operating pressure (assumed to be approximately 7 to $9 \mathrm{MPa}$ ) as helium is discharged into the reactor cavity. During the depressurization phase, hot helium from the vessel will mix with the air in the reactor cavity. Hence a helium-laced air mixture will be available to move into the reactor vessel once the pressure gradient across the break has been equalized; thus changing the flow behavior at the break from momentum-driven flow out of the reactor vessel into the reactor cavity to a density-gradient driven stratified countercurrent flow with helium moving out of the reactor vessel into the cavity while heliumlaced air moves into the reactor vessel from the reactor cavity.

The potential for density-gradient governed stratified air to ingress into the VHTR following a large-break LOCA was first described in the NGNP Methods Technical Program (Schultz et al. 2006) based on a stratified flow study performed with liquid (Liou Schultz, and Kukita 1997, Liou et al. 2005). Studies on density-gradient-driven stratified flow in advanced reactor systems has been the subject of active research for well over a decade because density-gradient dominated stratified flow is an inherent characteristic of passive systems used in advanced reactors.

The work done on Generation 3+ systems, although for light water reactors, is conceptually identical and directly applicable to the phenomenological behavior that occurs in the NGNP. Even though the earlier studies were based on Generation 3+ systems using water as the working fluid, the governing equations are identical. The boundary conditions are changed to reflect the differences in the working fluid and the reactor vessel geometry. Computational fluid dynamic calculations were recently made to mimic the LOCA between two tanks filled with helium and oxygen, respectively. The scenario postulated by Oh et al. (2008) is depicted in Figure 2-1.

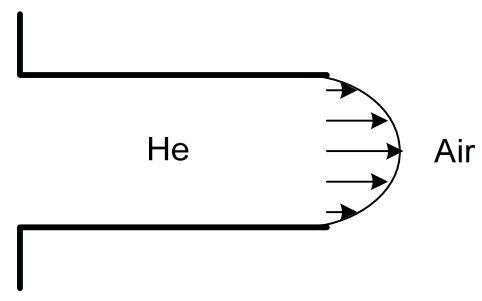

(a) Depressurization.

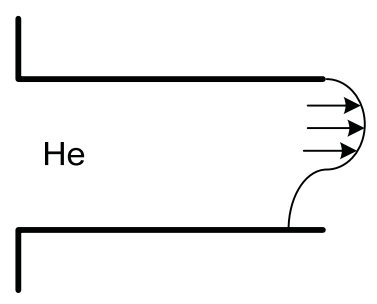

(b) Onset of density driven flow (no flow at the bottom of the break).

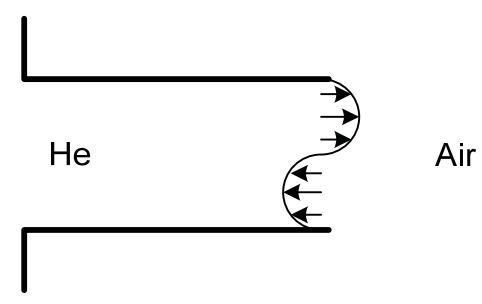

(c) Density driven flow (Reverse flow at the bottom of the pipe).

\section{Figure 2-1. Density-driven induced stratified flow.}

Earlier studies of the mechanisms leading to air ingress into the reactor vessel focused on diffusion as described by Fick's Law (Takeda 1997, Takeda and Hishida 1996, Oh et al. 2006, Kim et al. 2007, NO et al. 2007) and ignored the effects of density gradients on the interactions between helium (low density) and air or helium-laced air (high density) flow. 
Air ingress into the reactor vessel stemming from density-gradient-driven stratified flow occurs in a much quicker time scale than diffusion, resulting in a depressurized conduction cooling scenario with a different set of boundary conditions than previously assumed. Hence, experiments are needed to study these phenomena as noted in the NGNP Methods Thermal-Fluids Experiment Plan (Schultz et al. 2006). Subsequent to the break in the hot duct hypothesized in depressurized conduction cool-down, air present in the reactor cavity will enter the reactor vessel. Because of the significantly higher molecular weight and lower initial temperature of the reactor cavity air, the air-helium mixture in the cavity is always heavier than the helium discharging from the reactor vessel via the break into the reactor cavity. Once the air-helium mixture enters the reactor vessel, it will pool at the bottom of the lower plenum. It will move from the lower plenum into the core via diffusion and the density-gradient induced by heating. When density-gradient-driven stratified flow is considered as a contributing phenomena for air ingress into the reactor vessel, the following factors contribute to a much earlier natural circulation-phase in the reactor vessel: (a) density-gradient-driven stratified flow is a much more rapid mechanism (at least one order of magnitude) for moving air into the reactor vessel lower plenum than diffusion, and consequently, (b) the diffusion dominated phase begins with a much larger flow area and a much shorter distance for air to move into the core than earlier scenarios that attribute all air ingress from the reactor cavity into the core to diffusion only.

In essence, the stratified flow assumption is based on the formation of a wedge of air at the lower portion of the hot duct break that will advance into the reactor vessel as a function of the density-gradients once the blow down has depressurized. Such flows are well characterized by the densimetric Froude number F, which correlates the densities of helium and the air mixture to a constant value representative of the flow condition at different times in the scenario.

$$
F=\frac{u}{\sqrt{g^{\prime} d}}
$$

where $u=$ discharge velocity of air, $d=$ hydraulic depth of air, and $g^{\prime}=$ reduced gravity defined by

$$
g^{\prime}=\frac{g\left(\rho_{2}-\rho_{1}\right)}{\frac{\rho_{2}+\rho_{1}}{2}} \text {. }
$$

The buoyancy induced by the density difference of the two fluids necessitates the use of reduced gravity $(g$ ') instead of the standard gravity $(g)$. The magnitude of $F$ indicates the magnitude of inertia force relative to the buoyancy created by stratification, and is a controlling parameter in stratified flows. This idea and experimental confirmation can be found in Liou, Schultz, and Kukita (1997) and Yih (1980).

A stratified flow experiment is required to better understand this phenomenon and provide data for validating codes that will be used in conjunction with systems analysis codes to model this inherently multidimensional phenomenon. It is expected the densimetric Froude number will be found to be a function of

$$
F=f\left(\alpha, L / D, \frac{V_{\text {vessel }}}{V_{\text {vault }}}, P_{R}, R\right)
$$

where $\alpha=$ orientation of the break with respect to the vertical, $L=$ length of the separated hot duct on the reactor vessel side, $D=$ diameter of the hot duct, $V=$ volume, $\operatorname{Pr}=$ Pressure coefficient, and $R=$ Reynolds number. 
Thus, as shown in Figure 2-1(a), outward flow of helium into the reactor cavity from the reactor vessel continues until the reactor pressure is sufficiently reduced such that the blowdown flow subsides. Thereafter, air starts to intrude into the pipe through the lower portion of the break as depicted in Figures 2-1(b) and 2-1(c). In a rectangular flow cross section, it can be shown theoretically that the volumetric flow rates of the two fluids through the break are the same (Liou, Schultz, and Kukita 1997). The helium volumetric flow and air volumetric flow are therefore assumed to be equal. The heavy air will enter the vessel and collect (allowing dome turbulent mixing) at the bottom of the VHTR in the lower plenum. The air in the lower plenum will heat up and create a density gradient that causes a buoyancy force that drives the air further up into the reactor core. This density gradient will trigger a natural circulation in the reactor, resulting in graphite oxidation, which will be detrimental to VHTR safety. If the stratified air flow induces the natural circulation flow to begin earlier than previously thought, the time frame for graphite oxidation will occur earlier and at a more rapid rate. Earlier predictions from the GAMMA code (NO et al. 2007) predict oxidation between 150 and 200 hours following pipe rupture, depending on the initial air volume in the containment. Calculations using MELCOR predict that oxidation begins at 220 hours (Yih 1980) following pipe rupture. However, recent CFD calculations (Oh et al. 2008) using the stratified flow approach predict that natural circulation commences much earlier than 150 hours. It is therefore important that this phenomena and its effect on scenario progression are clarified.

\subsection{Review of Previous Gravity Current Flow Studies}

This section summarizes previous investigations of the gravity current, which appears to have similar flow mechanisms to those of air-ingress accidents. The gravity current, also known as density gradient driven stratified flow, is the flow of one fluid through another by density differences that are caused by temperature differences, dissolved materials, or suspended particles. This type of stratified flow happens when a heavy fluid intrudes into a lighter fluid or vice versa. This gravity current flow is easily seen in our natural surroundings. Thermal stratification during the emergency coolant injection in advanced reactors, thunderstorm outflows, growth of lava domes, and avalanches are widely known natural examples of gravity currents. Wastewater discharge into rivers, oil spills in the ocean, accidental release of toxic industrial gases, and smoke movement are some examples of manmade gravity current (Simpson 1999). Figure 2-2 depicts the controlled lock-exchange experiment performed by Shin et al. (2004) for small density differences (Boussinesq flow). This figure clearly shows that a dense gravity current of salted water (dark side) travels to the right along the lower boundary while the lighter current of pure water (light side) travels to the left along the upper boundary.

The study of gravity currents has a long history. The first modeling for the gravity current flow was carried out by von Karman (1940), who was one of the pioneers in the fluid dynamics field. He considered energy conserving current in his derivation, which is propagated in an ambient fluid of infinite depth, and proposed a theoretical correlation predicting the frontal speed $(U)$ of the current flow as

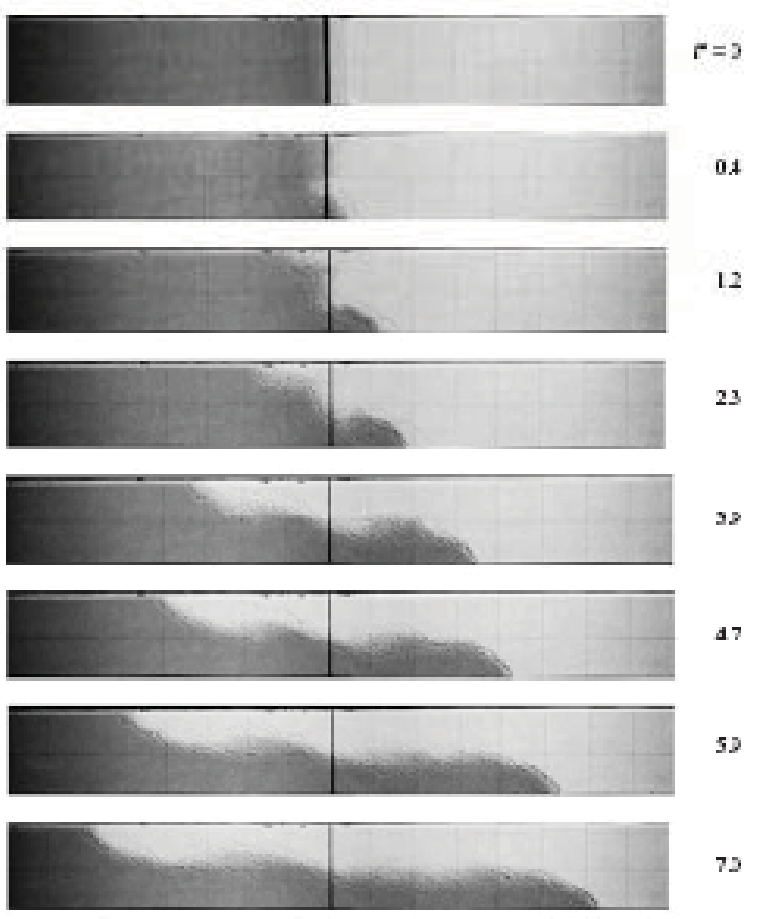

Figure 2-2. Gravity current produced by lockexchange in a rectangular channel (Shin et al. 2004). 


$$
F_{h}=\frac{U}{\sqrt{g^{\prime} h}}=\frac{\sqrt{2}}{\gamma}
$$

where

$$
\begin{aligned}
& g^{\prime}=\text { reduced gravity }\left(g^{\prime}=g(1-\gamma)\right) \\
& h=\text { depth of the current }(\mathrm{m}) \\
& \gamma=\text { density ratio }\left(\rho_{1} / \rho_{2}, \text { the low density/the higher density fluid }\right) .
\end{aligned}
$$

In 1958, Keulengan indicated that the speed of the current was independent of the ratio of the channel width $(w)$ and depth $(h)$ from his lock-exchange experiment. He also observed a small increase of Froude number $\left(F_{H}\right)$ with an increase of Reynolds number $(R e)$. Based on his experiment, he proposed the correlation

$$
\begin{aligned}
& F_{H}=\frac{U}{\sqrt{g^{\prime} H}}=0.42(\text { at } R e=600) \\
& F_{H}=\frac{U}{\sqrt{g^{\prime} H}}=0.48(\text { at } R e>150000)
\end{aligned}
$$

where

$$
H=\text { channel depth (m). }
$$

The Reynolds number in Eqs. (2-5) and (2-6) was defined by

$$
\operatorname{Re}=\frac{U H}{v}
$$

where

$$
v=\text { kinematic viscosity }\left(\mathrm{m}^{2} / \mathrm{s}\right) \text {. }
$$

Yih (1965) proposed that the depths of the two currents are equal and have the value of half the channel depth along their entire lengths, and that the speeds of both gravity currents are the same for the Boussinesq flow $(\gamma \sim 1)$ where the density difference between two fluids is very small, like water and salted water.

Barr (1967) carried out experiments for both a free and a rigid upper surface. In separate tests, temperature and salinity were used to provide a density difference. His results showed that $F_{H}$ increases with Reynolds number. The variations were significant for low Re numbers between 200 and 1,000, but the change was slight for higher Re numbers $(\operatorname{Re}>1,000)$. He also found that the free-surface cases have higher values of $F_{H}$.

Benjamin (1968) first developed a theory for the propagation of a steadily advancing current by using conservation of mass and momentum flux in a frame of reference moving with the current (Figure 2-3). Therefore, the front was set to be at rest in this reference frame. His derivation assumed inviscid flow where the Grasshof number $(G r)$ is infinite. His derivation showed that there were various possible solutions, depending on the depth of the current as follows: 


$$
\frac{U^{2}}{g H}=\frac{h(2 H-h)(H-h)\left(\rho_{2} / \rho_{1}-1\right)}{H^{2}(H+h)}=\left(\frac{1-\gamma}{\gamma}\right)\left(\frac{h(2 H-h)(H-h)}{H^{2}(H+h)}\right) .
$$

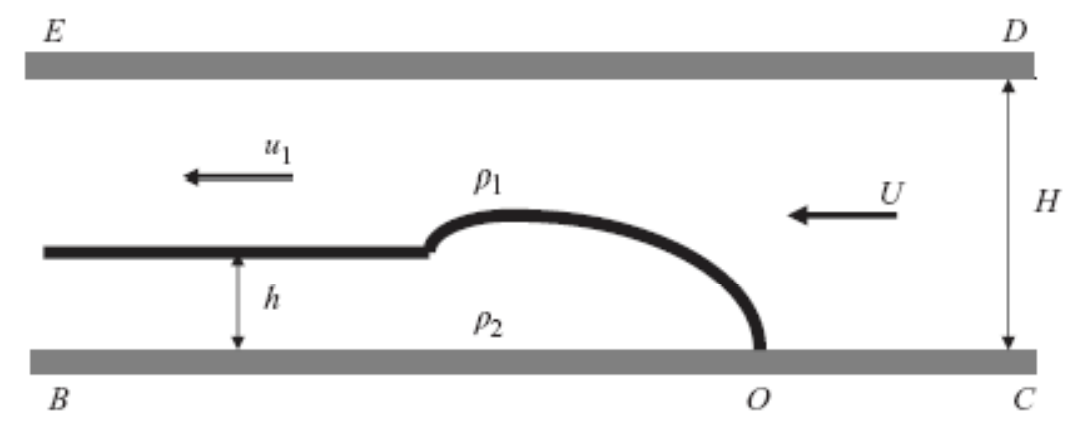

Figure 2-3. Diagram of idealized gravity current in the rest frame of the current (Shin et al. 2004).

Benjamin (1968) also showed that if there is no dissipation in the flow (if the energy fluxes into and out of the control volume is the same), the solution reduced to

$$
\frac{h}{H}=0 \text { or } \frac{h}{H}=\frac{1}{2} \text {. }
$$

The first solution is reduced to the exact same solution derived by von Karman (Eq. (2-4)) in the limit $\mathrm{h} / \mathrm{H} \rightarrow 0$ obtained as $\mathrm{H} \rightarrow 0$. The second solution leads to the nondimensional front speed correlation from Eq. (2-8)

$$
\frac{U^{2}}{g H}=\frac{1}{4} \frac{(1-\gamma)}{\gamma}
$$

For Boussinesq case $(\gamma \sim 1)$, Eq. (2-9) shows the Froude number defined in terms of the reduced gravity, as Eq. (2-5) is

$$
F_{H}=\frac{U}{\sqrt{g^{\prime} H}}=\frac{1}{2}
$$

He also argued that the gravity current occupying less than half the channel depth do not conserve energy and the maximum energy flux is reached when $h=0.347 H$. Figure 2-4 shows the dimensionless net energy flux based on Benjamin's theory. This figure shows that the energy flux increases from 0 with $h$, reaches a maximum when $h=0.347$, and then decreases to 0 when $h=0.5 \mathrm{H}$. For the case $\mathrm{h}>0.5 \mathrm{H}$, the energy leaving the downstream section is greater than that entering from upstream. This flow is clearly impossible unless there is an alternative energy supply within the control volume. Therefore, Benjamin (1968) argued that the depth of the gravity current should be less than half of the channel depth $(H)$. 


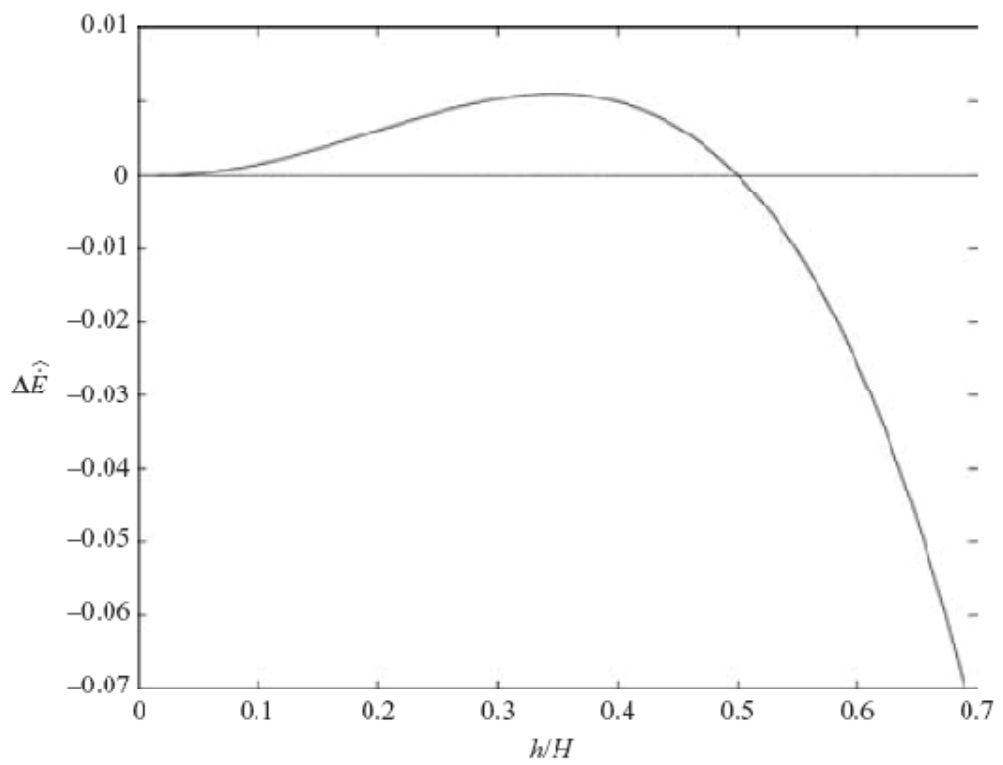

Figure 2-4. Dimensionless net energy flux plotted against dimensionless current depth $h / H$ (Shin et al. 2004).

Britter and Simpson (1978) observed that most of the mixing took place along the interface between the two fluids behind the head, and the Kelvin-Helmholtz billows were shed behind the head. Hupper and Simpson (1980) developed an empirical model that incorporates mixing at the front. The correlations suggested from their experiments are

$$
F_{h}=\frac{U}{\sqrt{g^{\prime} h}}=1.19(\mathrm{~h} / \mathrm{H}<0.075)
$$

and

$$
F_{h}=\frac{U}{\sqrt{g^{\prime} h}}=\frac{1}{2} \cdot\left(\frac{h}{H}\right)^{1 / 3} \quad(0.075<\mathrm{h} / \mathrm{H}<0.5)
$$

Gardner and Crow (1970) and Wilkinson (1982) showed the existence of half-depth by their experiments with air cavities intruding into a water filled channel (large density differences and immiscible flow). They also observed that the cavities could occupy half the depth and the free surface is smooth without energy loss, when the surface tension effects are small. They extended Benjamin's analysis to account for surface tension effects and showed that surface tension slows the cavity.

Linden and Simpson (1986) showed that mixing behind the head significantly affects the dynamics of the current.

Keller and Chyou (1991) formulated a hydraulic theory for the entire density ratio range (from 0 to 1.0). They assumed that for the small density differences, both gravity currents are energy conserving and are connected by a combination of a long wave of expansion and an internal bore. But, they assumed that for large density differences, the light current is energy conserving, the heavy current dissipative, and the gravity currents connected only by a long wave of expansion. Figure 2-5 summaries their models for various density ratios. 


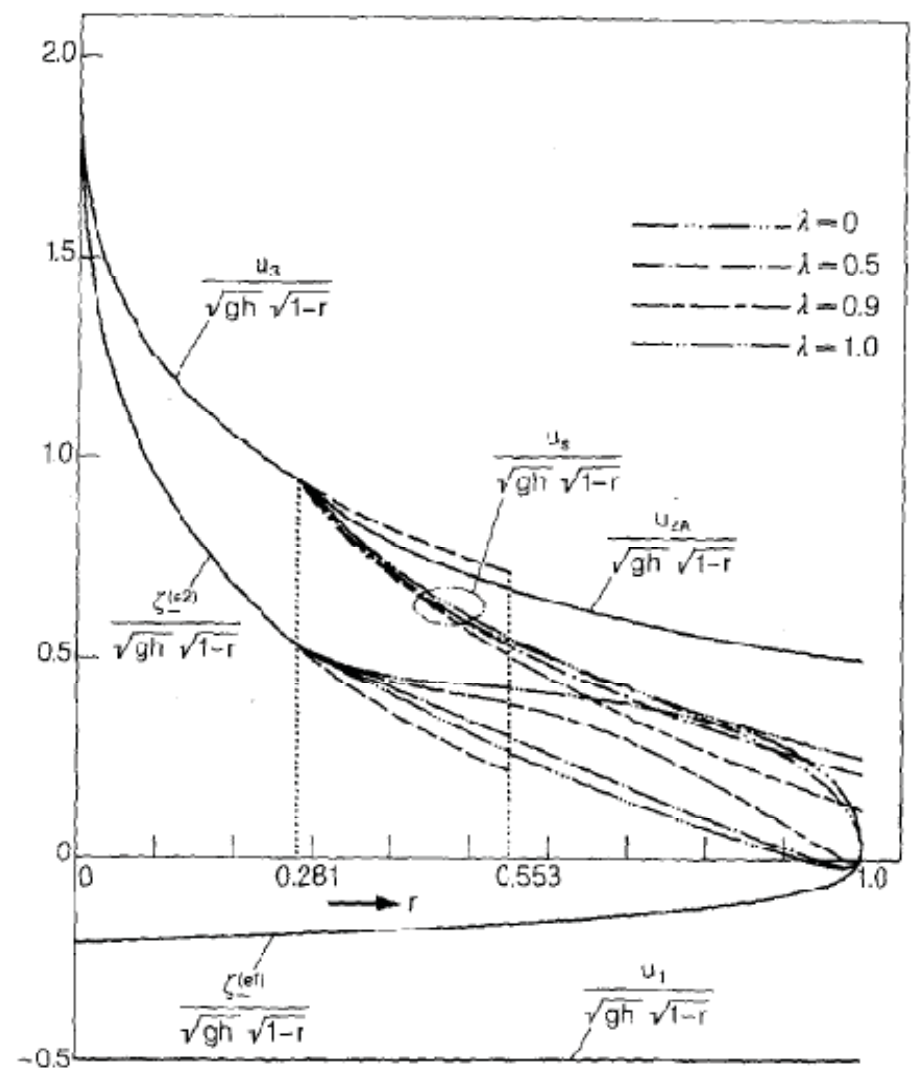

Figure 2-5. Wave speed plotted versus density ratios $\left(r=\rho_{B} / \rho_{A}\right)$ (Keller and Chyou 1991).

For density ratio $(r>0.281)$, Keller and Chyou (1991) described the complete lock exchange flow as shown in Figures 2-6 and 2-7. The velocities of relevant waves were expressed as:

(a) Front speed of the left-running gravity (loss-free) current $\left(u_{1}\right.$ : See Figure 2-6)

$$
F_{1}=\left(\frac{u_{1}}{\sqrt{g^{\prime} h}}\right)=\frac{1}{2}
$$

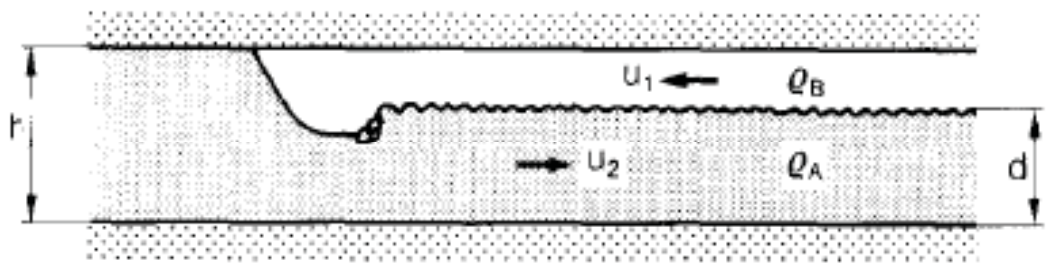

Figure 2-6. Gravity current of low-density fluid penetrating into a channel that initially contained high-density fluid (Keller and Chyou 1991).

(b) Front of the right running (dissipative) gravity current $\left(u_{2 A}\right.$ : See Figure $\left.2-7\right)$

$$
F_{2 A}=\left(\frac{u_{2 A}}{\sqrt{g^{\prime} h}}\right)=\left(\frac{\xi \cdot(1-\xi)^{2}}{2-\xi}\right) \cdot \frac{1}{\sqrt{r}}
$$


Where $\xi=\quad$ fractional depth $(h / H)$

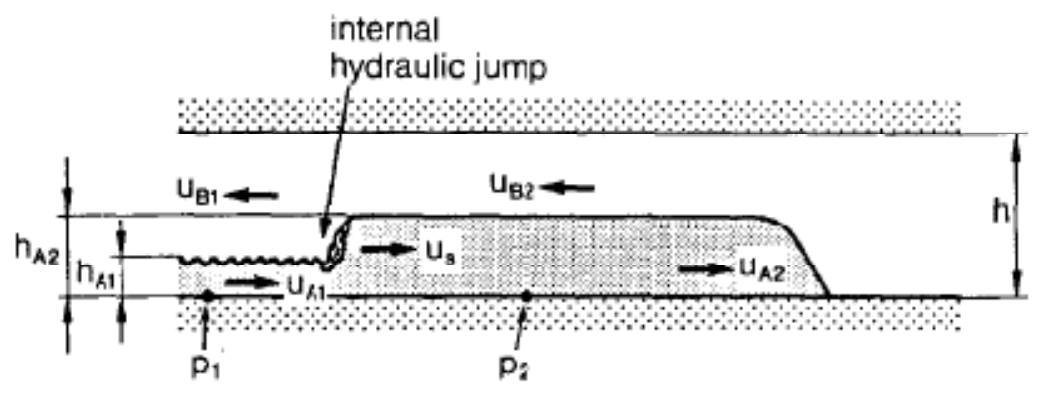

Figure 2-7. Loss-free gravity current of high density fluid penetrating into a horizontal channel originally filled with low density fluid (Keller and Chyou 1991).

In the Eq. (2-13) and (2-14), the value of $\xi$ is determine by Figure 2-8, where the coefficient $\lambda$ represents the relative amount of energy loss. The mathematical representation, where Case $\lambda=1$ corresponds to a loss-free flow in the lower layer and case $\lambda=0$ to the special case of no recovery of kinetic energy, is

$p_{2}-p_{1}=\lambda \cdot \frac{\rho_{A}}{2}\left(\left(u_{1 A}-u_{s}\right)^{2}-\left(u_{2 A}-u_{s}\right)^{2}\right)$.

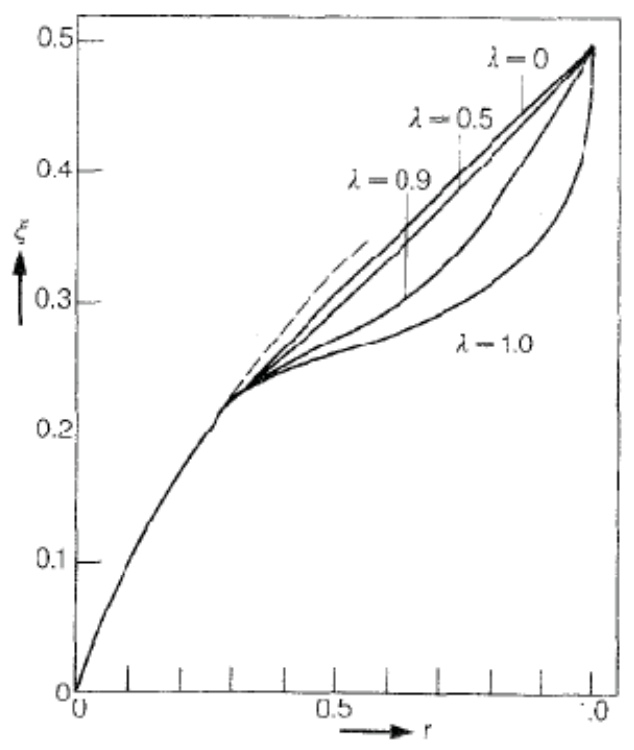

Figure 2-8. Fractional depth ( $\xi$ ) versus density ratio ( $r$ ) (Keller and Chyou 1991).

Grobelbauer et al. (1993) conducted lock-exchange flow experiments with gases of density ratios down to 0.046 . They used an unevenly divided horizontal channel of half height $(\mathrm{h}=0.15 \mathrm{~m})$ with various combinations of helium, air, argon, and $\mathrm{CO}_{2}$ gases for their experiment.

Klemp et al. (1994) calculated the behavior of lock exchange gravity current using both shallowwater theory and two-dimensional (2-D) numerical simulation. They argued that dissipation must be included in the modeling and an energy conserving gravity current cannot be physically realized based on their theory. They also argued that the maximum achievable depth of the heavier current is $0.347 \mathrm{H}$, which 
Benjamin's (1968) theory gives for the current with the maximum speed and the maximum dissipation. They also explained that the inviscid gravity depth can never be greater than 0.347 of the channel depth. However, Gardner and Crow (1970), Wilkinson (1982), and Keller and Chyou (1991) clearly showed that the air cavity has both the shape and speed predicted by Benjamin's energy conserving gravity current. They argued about the fact that the differences in speeds between the fastest allowable current and Benjamin's energy conserving current are too small to discriminate in an experiment. However, the measurement of the current depth showed that their observations are much closer to the energy conserving value than to the fastest allowable gravity current. In addition, recent research by Shin et al. (2004) showed that there is very slight dissipation in the gravity current.

Parson and Garcia (1998) showed the importance of the Grashof/Reynolds' numbers on the evolution of the gravity current. They found that mixing is intensified at large Reynolds numbers.

Chen and Lee (1999) used Reynolds-averaged Navier-Stokes (RANS) model for lock release flows. The challenge related to the use of RANS models to the gravity current is that practically all these models (RANS) are calibrated for fully developed turbulent flow and are not capable of accurately predicting transition and re-laminarization, which is essential for simulating gravity current flow.

Simpson (1999), who extensively reviewed the gravity current, explains that the current moves at an almost constant speed, depending on the depth of the water and the density difference. According to his summary, as the gravity current advances, the current front is formed at the leading edge of the flow and is slightly raised above the bottom surface with intense mixing between the front of the current and its surrounding. He also explained that the head of the current is approximately two times as deep as the following flow depth. He also said that the characteristic head would control the mixing behavior, current velocity, and current profile. The current induced mixing is considered to be caused by two types of instabilities: billows, and lobes and clefts as shown in Figure 2-9. Billows roll up in the region of velocity shear above the front of the dense fluid, and lobes and clefts are formed by the influence of the ground on the lower part of the leading edge.

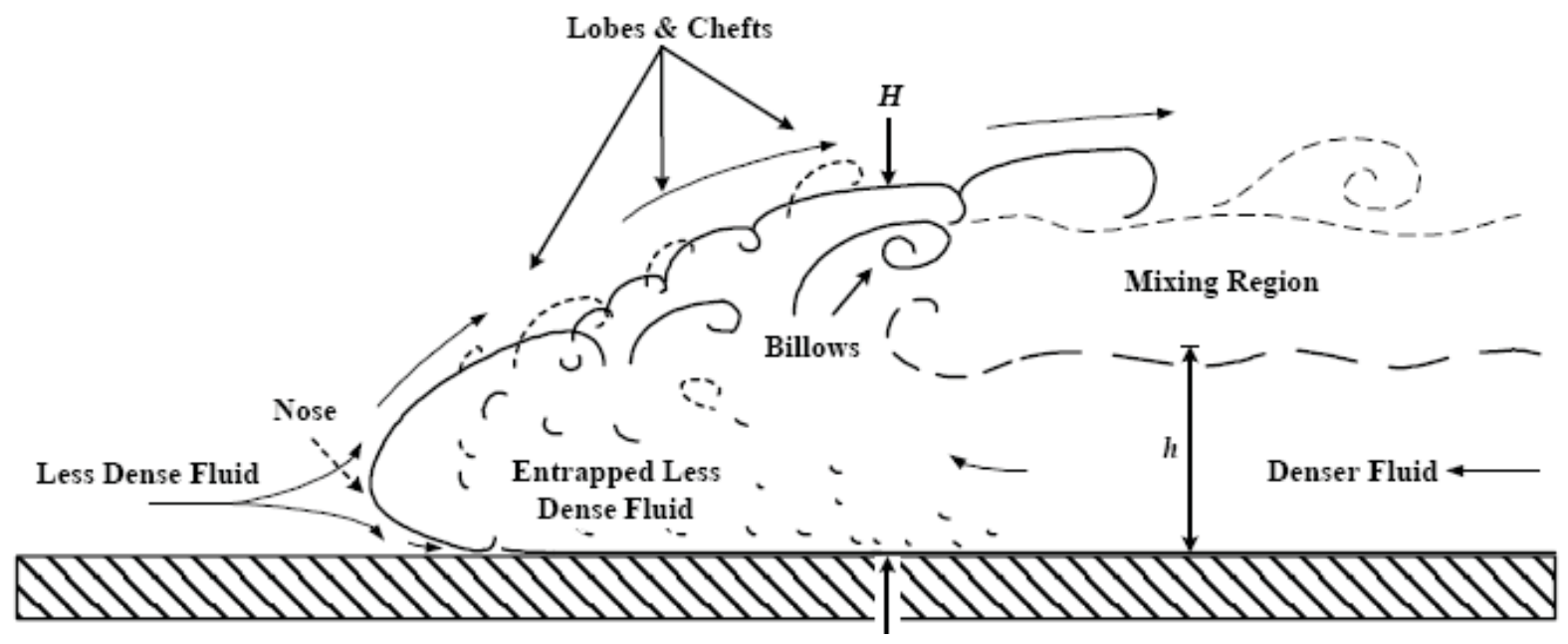

Figure 2-9. Typical gravity current front advancing along a horizontal plane (Mok et al. 2003).

Hartel et al. (2000) showed that the current can be explained using shallow-water theory if sufficiently accurate front conditions are prescribed for the nonhydrostatic flow at the head of the current.

Recently, Lowe et al. (2002) repeated experiments on symmetric intrusions propagating along a sharp density interface conducted by Britter and Simpson (1981). They observed in their experiment that the 
shape and speed of the intrusion were in good agreement with Benjamin's theory. These experiments suggest that Benjamin's energy conserving gravity currents are observed for Boussinesq, miscible fluids.

Shin et al. (2004) performed an experiment with freshwater and sodium chloride for both full and partial lock exchange $(\operatorname{Re}>1,000, \gamma>0.9,0.11<\mathrm{h} / \mathrm{H}<1)$. In the experiment, they found that the speed of the front head is constant and the shapes of the light and heavy currents are symmetric about the centerline. They measured the speed of the current head and current depth and concluded that Benjamin's energy conserving theory predicts their experiments very well. The measured $F_{H}$ for the full depth experiment was 0.5 within 5-10\% maximum error. The depth of the current ranged between 0.35 and $0.5 \mathrm{H}$. The $0.35 \mathrm{H}$ represents the maximum dissipation depth predicted by Benjamin's theory, which was obtained by changing gate-valve slope and giving initial disturbance in the flow (See Figure 2-10). Their experiment showed lots of evidence that Benjamin's previous theory describes the phenomena satisfactorily.

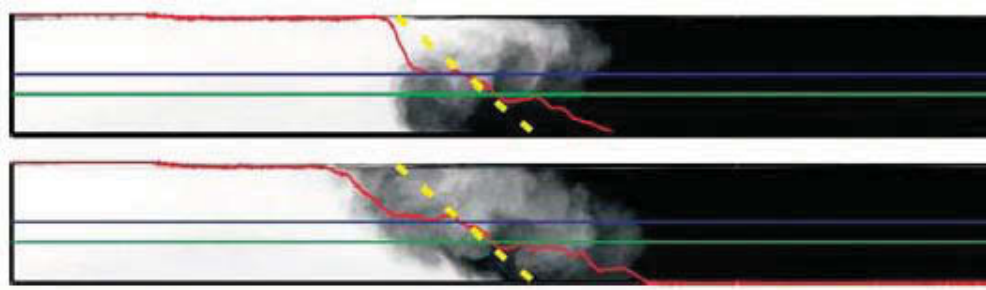
$r^{*}=1.9$
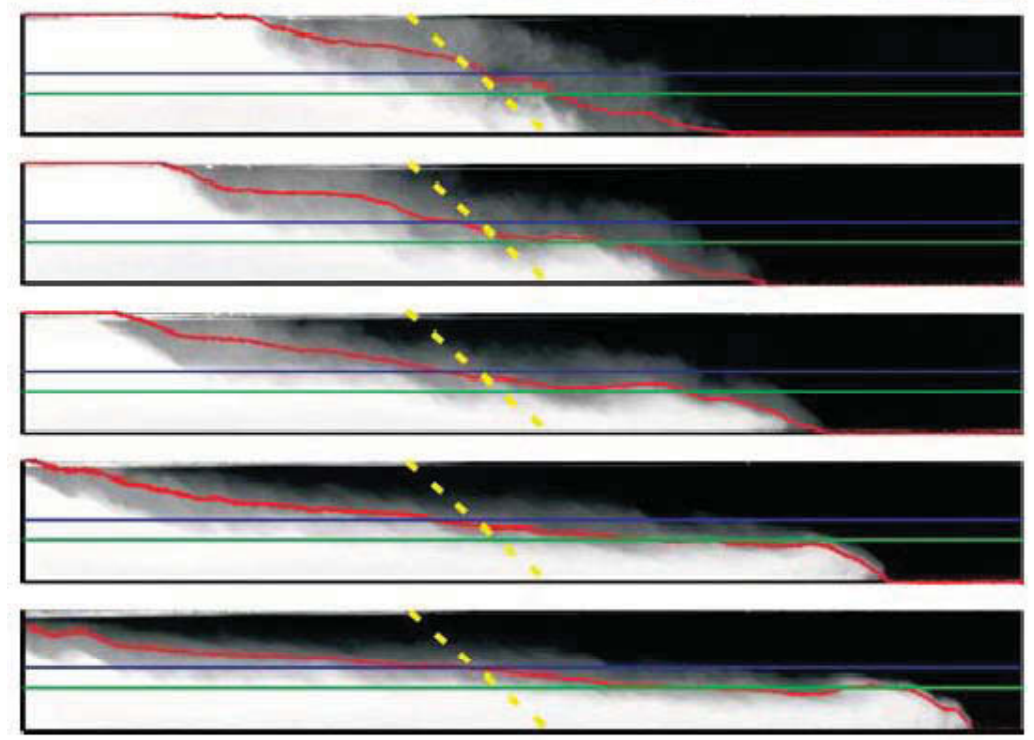

Figure 2-10. Full depth lock exchange experiment with the lock gate at an angle (Shin et al. (2004)).

Shin et al. (2004) also expanded Benjamin's model to the partial lock exchange cases. In their theory, they argued that energy and momentum can be transferred along the interface by internal waves. They showed that energy and momentum can be transferred towards the current front for partial depth locks less than about $0.76 \mathrm{H}$, but for deeper locks, the current travels faster than long interfacial waves. So, he suggested that for lower fractional depths, Benjamin's theory be modified to allow energy transfer. For partial depth cases (See Figure 2-11), based on the mass and momentum conservation, he obtained the expression

$$
\frac{U^{2}}{g H}=\left(\frac{\left(\rho_{2}-\rho_{1}\right) D(D-h)(H-h)}{2 h H\left(\rho_{2}(H-h)+\rho_{1} h\right)}\right) .
$$


By applying energy conservation assumption, they obtained the solution

$$
h=\frac{D}{2} \text {. }
$$

Substituting Eq. (2-17) into Eq. (2-16) gives

$$
\frac{U^{2}}{g H}=\left(\frac{\left(\rho_{2}-\rho_{1}\right) D(2 H-D)}{2 H\left(\rho_{2}(2 H-D)+\rho_{1} D\right)}\right) \text {. }
$$

(a)
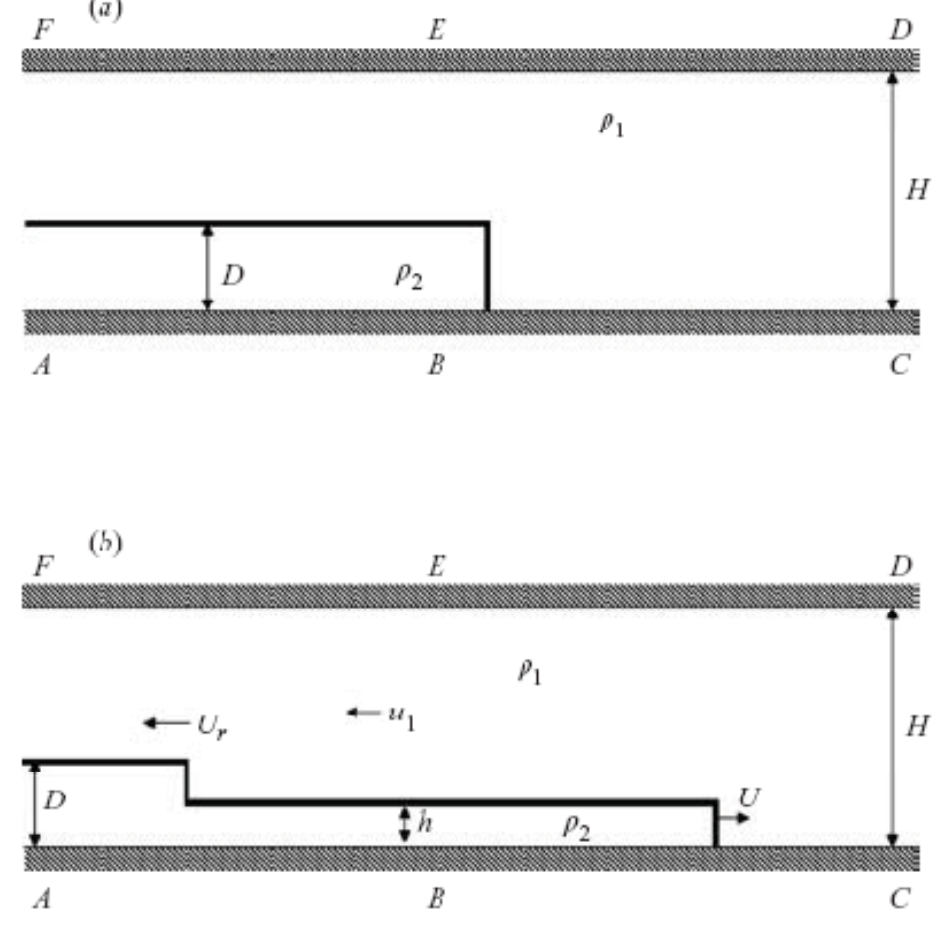

Figure 2-11. Schematic of a partial-depth lock exchange in a channel (a) before release and (b) after release (Shin et al. 2004).

The above energy-conservation solution was derived without using the Boussinesq approximation. Therefore, it is theoretically valid for any pair of densities; however, Shin et al. (2004) was not sure if it is valid for non-Boussinesq cases. For the Boussinesq cases, Eq. (2-18) can be rearranged as

$$
F_{H}=\frac{U}{\sqrt{g^{\prime} H}}=\frac{1}{2} \sqrt{\frac{D}{H}\left(2-\frac{D}{H}\right)} .
$$

In the limit of full depth case $(\mathrm{D}=\mathrm{H})$, Eq. (2-19) reduces to Benjamin's theory (Eq. (2-10)). Shin et al. (2004) compared their experimental data with the model, and showed very good agreement between them. They also argued that Benjamin's theory cannot apply for partial depth problems with $\mathrm{D}<0.76 \mathrm{H}$. For the gravity current in a deep ambient fluid, they suggested

$$
F_{h}=\frac{U}{\sqrt{g^{\prime} h}}=1 \text {. }
$$


Lowe et al. (2005) performed experiments and modeling for the entire density ratio range. They also repeated Keller and Chyou's (1991) theory, and derived another solution that involves only an expansion wave connecting the two gravity currents. To validate the models, they used their experimental data and Birman et al.'s (2005) computational solutions employing a combination of spectral and compact finitedifference methods. Their comparisons indicated that the theory without the bore gives the best agreement. They showed that the speeds of the current front were still constant for the non-Boussinesq cases, but the heavier current traveled faster than the light current (see Figure 2-12). The light non-Boussinesq current traveled at about the same speed as the Boussinesq current. The symmetry was also lost for the Boussinesq cases. But the depths of the leading parts of the two currents were close to the half depth of the fluid.

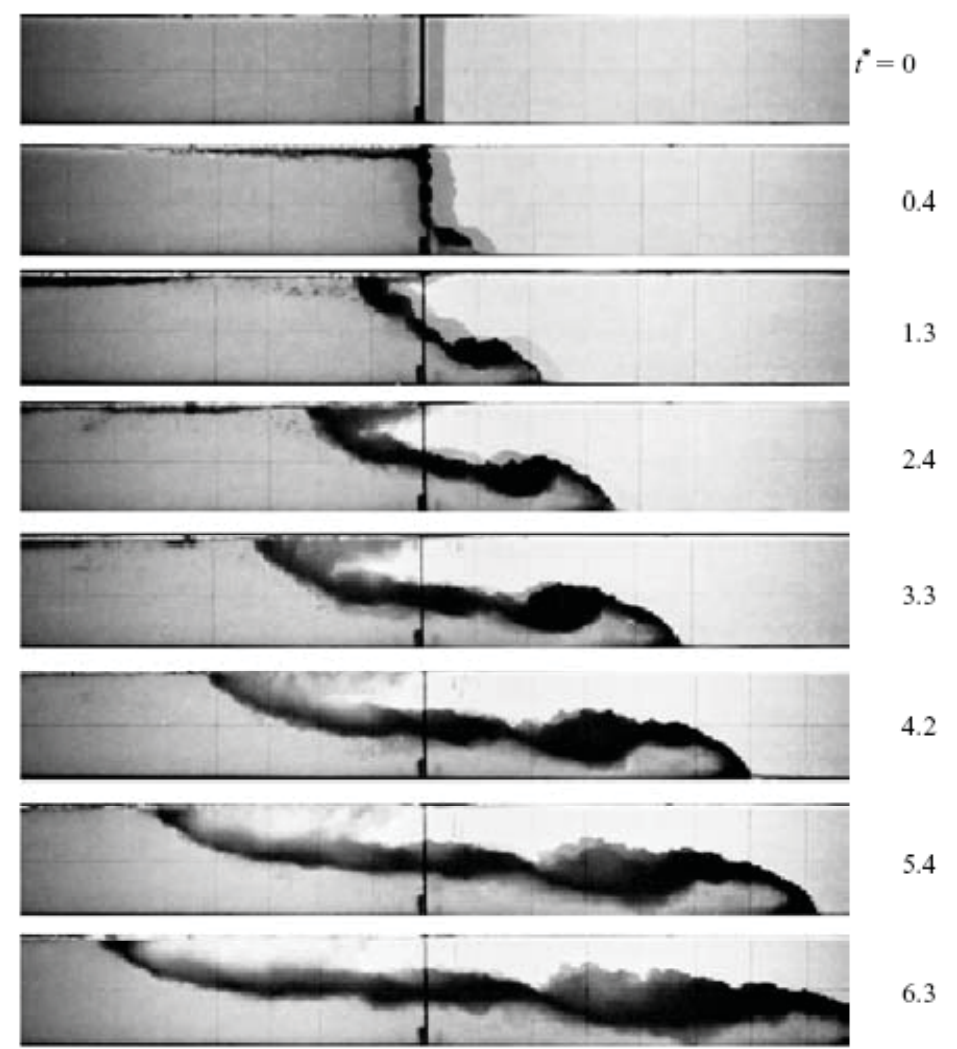

Figure 2-12. Lock exchange experiment for non-Boussinesq cases $(y=0.681)$.

Based on Keller and Chyou's (1991) observations and suggestions, there are two possible flow configurations for the non-Boussinesq lock-exchange flow (Lowe et al. (2005)). Figure 2-13 shows the schematics of the flow configurations. The flow configuration of Figure 2-13(a) occurs when $\gamma^{*}<\gamma<1$ and that of Figure 2-13(b) occurs when $0<\gamma<\gamma^{*}$. The speeds of the front are summarized below for each case (Lowe et al. 2005). Lowe et al. (2005) suggested the critical density ratio $\left(\gamma^{*}\right)$ has the value 0.281 . 
(a)

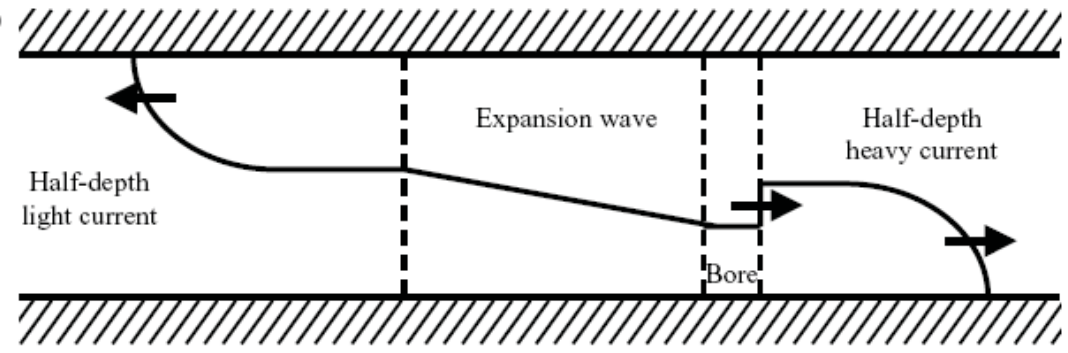

(b)

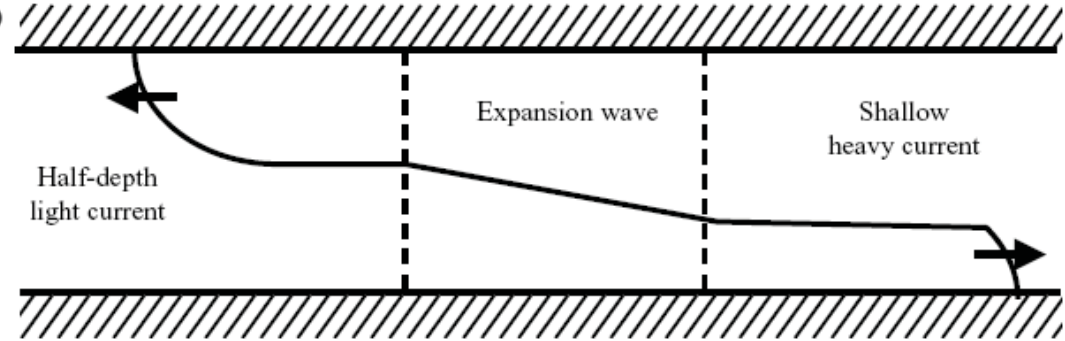

Figure 2-13. Schematic of two lock exchange configuration for non-Boussinesq flow.

\subsubsection{1 $\quad y^{*}<y<1$}

The speed and height of the left-propagating current are given by Benjamin's energy conserving current theory as

$U_{L}=\frac{1}{2} \sqrt{(1-\gamma) g H}$

$h_{L}=\frac{1}{2} H$.

Figure 2-14 shows the comparisons of the light current for various density ratios (Lowe et al. (2005)). In this figure, solid line is the value calculated by Eq. (2-21), and the points are experimental data obtained by previous investigations (Grobelauer et al. (1993), Keller and Chyou (1991), Birman et. al (2005)). The Reynolds numbers of the experiments varies from 10,000 to 100,000, so the results in Figure 14 show that the speed of the light current is independent of Re number and the model is in good agreement with the experimental data.

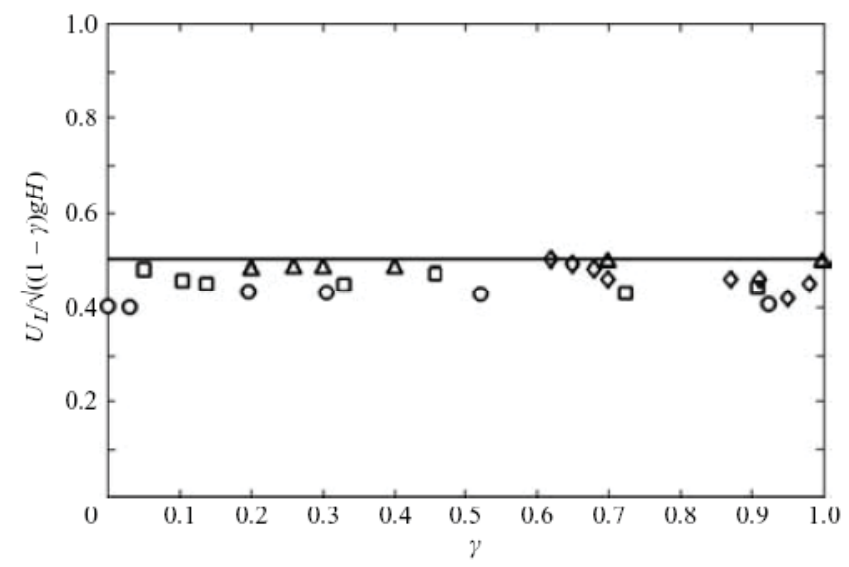

Figure 2-14. Speed of the light current for various density ratios (Lowe et al. (2005)). 


\subsubsection{2 $0<y<y^{*}$}

For the right-propagating current, the speed and the depth are expressed by

$U_{H}=\frac{1}{2} \sqrt{\frac{(1-\gamma)}{\gamma} g H}$

$h_{H}=\frac{1}{2} H$

The speed of the left-propagating current is the same as that expressed by Eq. (2-21). However, the heavy current has the different speeds calculated as

$U_{H}=\sqrt{(1-\gamma) g H}\left[\frac{1}{\gamma} \frac{h_{H}}{H}\left(2-\frac{h_{H}}{H}\right) \frac{1-h_{H} / H}{1+h_{H} / H}\right]^{1 / 2}$.

Figure 2-15 shows the comparisons of the speeds of the heavy currents for various density ratios. The theory in this figure shows good predictions for $\gamma>0.281$, but for less than that, the model over-predicts the experimental results a little bit. Figure 2-16 shows the comparisons of the theoretical front height and the experimental data (Lowe et al. 2005).

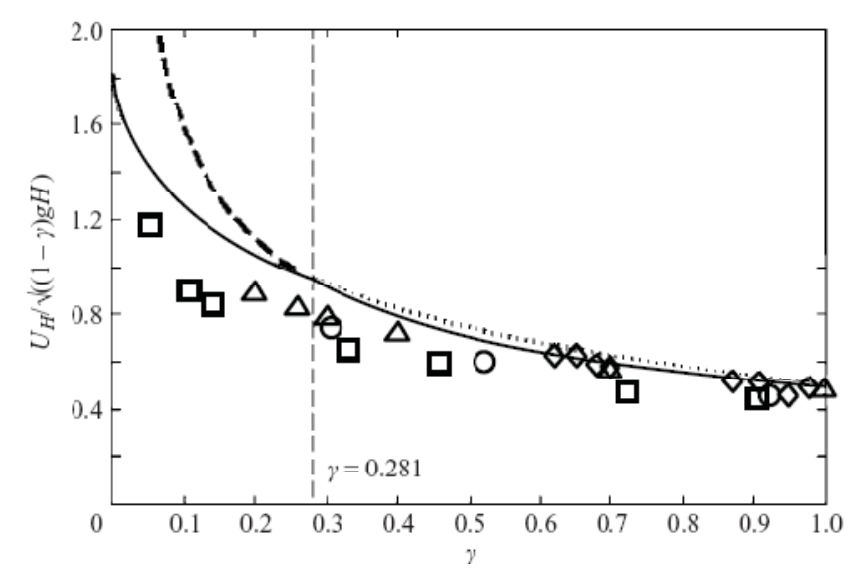

Figure 2-15. Speeds of the heavy currents (Lowe et al. 2005).

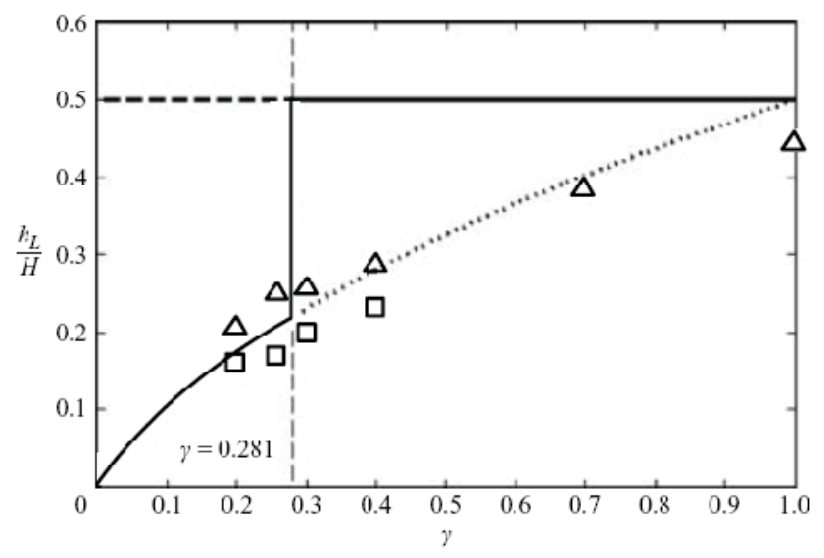

Figure 2-16. Flow depth of the heavy current (Lowe et al. 2005). 
Etienne et al. (2005) performed direct numerical simulation for the exchange flow of large density ratios as shown in Figure 2-17, and they compared the calculation results with the experimental data provided by Grobelbauer et al. (1993). Their simulation, using a dynamic mesh adaptation, covered the whole density ratio of the experiments and showed very good agreement with the experimental front velocities and Froude number variations.

(a)
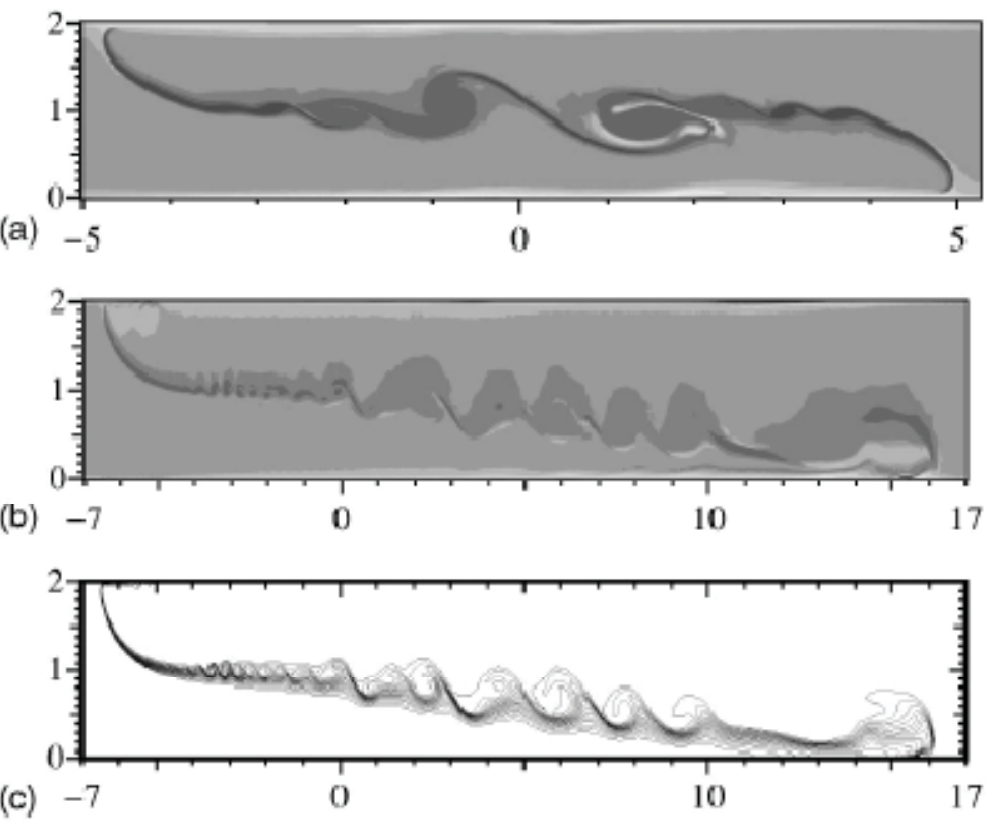

Figure 2-17. Nondimensional vorticity maps for the steady flow by DNS (Etienne et al. 2005).

Eugeny et al. (2007) argued that the solution by Shin et al. (2004) is valid only at sufficiently large values of the Reynolds number in which the viscous effect is not important. They carried out some experiments on the propagation speed of gravity currents at moderate values of gravity Reynolds numbers $(1,600<\operatorname{Re}<28,000)$, and developed a semiempirical model to predict the front propagation speed for various Re number ranges. Their model showed good agreement with the experimental results, but the applicability of such an approach to the general cases of decelerating flows with dissipation is still an open issue. They recommend

$$
\begin{aligned}
& \operatorname{Fr}(\alpha, \xi, \mathrm{Re})=\frac{U_{H}}{\left(g^{\prime} h\right)^{1 / 2}}=\left[\left(\frac{C \xi}{\operatorname{Re}} \varphi(\alpha)\right)^{2}+(1-\alpha)\right]^{1 / 2}-\frac{C \xi}{\operatorname{Re}} \varphi(\alpha) \\
& \varphi(\alpha)=\frac{1-\alpha}{\alpha^{5 / 2}}\left[1+\left(\frac{\alpha}{1-\alpha}\right)^{3}\right]
\end{aligned}
$$

where

$$
\begin{aligned}
& \alpha=h / H \\
& \operatorname{Re}=\left(\frac{g^{\prime} H^{3}}{v^{2}}\right)^{1 / 2}
\end{aligned}
$$




$$
\xi=L / H
$$

$C=2.4$ (recommended by Eugeny et al. (2007)).

\subsection{Analytical Estimations for VHTR Air-ingress Accident}

One of the main objectives of Task 1 is to identify what is the most important mechanism in the airingress process. Figure 2-18 shows the new air-ingress scenario proposed by Idaho National Laboratory (INL). According to this scenario, after the pipe break, the coolant (helium) in the vessel is rapidly blown into the reactor confinement with depressurization because the inside vessel pressure is much higher than that outside (Figure 2-18(a)). After depressurization, if the overall pressures between the inside and outside vessel are equilibrated, the outside air (or air/helium mixture) will move into the inside vessel forming stratified flow by density gradient (Figure 2-18(b)). This is referred to here as Stage 1 stratified flow. In Stage 1, the density gradient is generated first by molecular mass differences between helium coolant and air, and second by temperature gradient. After the helium/air mixture fills up the reactor bottom, Stage 2 stratified flow generally begins (See Figure 2-18(c)). In Stage 2, the density gradient flow is generated by the temperature gradient between reactor inside and outside, which drives cold air into the lower plenum where it expands by heating. Once the air is heated in the lower plenum, it (air or air/helium mixture) will have a buoyancy force to push up the helium coolant in the reactor core. The air will then slowly flow into the inside core. However, depending on the pipe size, heating conditions, and core designs, this process can be dominated by molecular diffusion because of insufficient buoyancy force. This situation is shown in the NACOK experiment explained in the following section. After the air fills up the reactor top, global natural circulation finally begins (See Figure 2-18(d)). Currently, there is no question that the dominant mechanism in the depressurization step is forced convection.
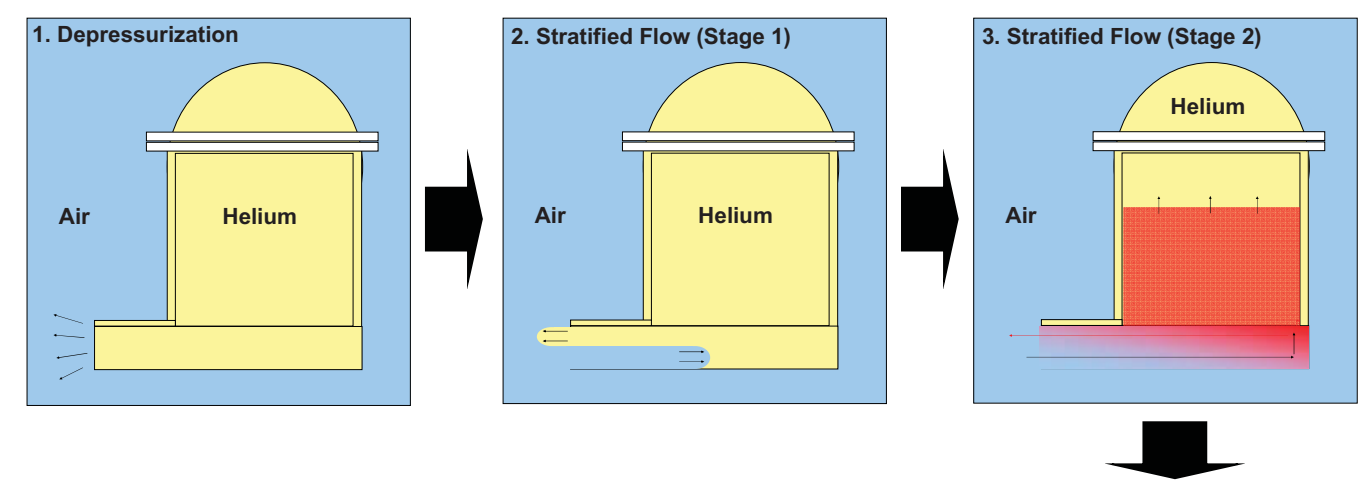

\section{Stratified Flow \\ Stage-1: Initially density gradient is driven by a factor of 26 and temperature.}

Stage-2: Air moves up into the reactor core and initiates the Onset of Natural Convection.

Figure 2-18. Air-ingress scenario (Idaho National Laboratory's [INL's] current understanding).

As previously mentioned in this report, there are two important physical mechanisms mainly affecting air-ingress process after depressurization: molecular diffusion and density gradient driven stratified flow. To compare the relative importance of these mechanisms, time scales were calculated for each at the 
different air-ingress steps. First, the relative time scales were estimated for molecular diffusion and density gradient driven stratified flow, then they were compared to each other. If the time scale is larger, it indicates that the mechanism is relatively slower and thus less important than the other. If the time scale is smaller, it indicates that the mechanism is faster and can be considered more important. The time scale comparisons thus provide a qualitative indication of which mechanism is more important. All the analytical calculations in this section are based on a simplified version of the $600 \mathrm{MW}$ gas turbine modular helium reactor (GTMHR) 'reference' design.

\subsubsection{Time scale Comparisons in Stage 1}

Figure 2-19 shows the schematics of Stage 1, which follows the depressurization process. In Stage 1 there are two air-ingress mechanisms: density gradient driven stratified flow and molecular diffusion. The density gradient driven stratified flow, known as gravity current, is driven by the density differences between air (reactor outside) and helium (reactor inside) in the VHTR. In this case, the heavy gas (air) intrudes into a light gas (helium) along with the hot-leg bottom. On the other hand, molecular diffusion is generated by the concentration gradient of air between the reactor inside and outside.

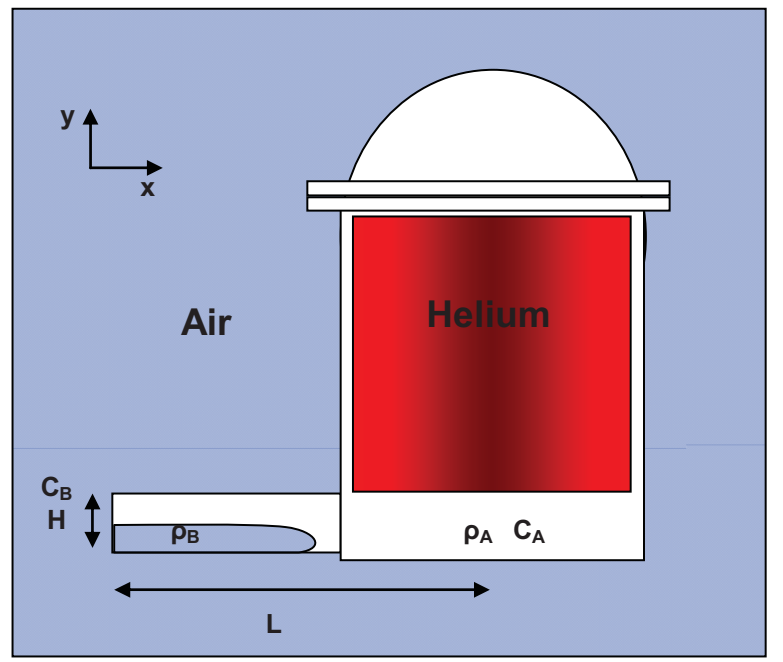

Figure 2-19. Schematics of Stage 1 (post-depressurization).

To compare the time scales of the stratified flow and the diffusion process, the species transport equation was adopted (Welty et al. 1984) as

$\frac{\partial C_{A}}{\partial t}=-v \cdot \nabla C_{A}+D_{A B} \nabla^{2} C_{A}$

where

$$
\begin{array}{ll}
C_{A} & =\text { gas concentration }\left(\mathrm{k}-\text { moles } / \mathrm{m}^{3}\right) \\
t & =\operatorname{time}(\mathrm{sec}) \\
v & =\operatorname{velocity}(\mathrm{m} / \mathrm{s}) \\
D_{A B} & =\text { diffusion coefficient }\left(\mathrm{m}^{2} / \mathrm{s}\right) .
\end{array}
$$


Since the y-directional flow is ignorable compared to x-direction in Figure 2-19, only x-directional flow were taken into consideration. Then, Eq. (2-32) is reduced to

$$
\frac{\partial C_{A}}{\partial t}=-U_{x} \frac{d C_{A}}{d x}+D_{A B} \frac{d^{2} C_{A}}{d x^{2}}
$$

Where $U_{x}=$ velocity in $\mathrm{x}$-direction $(\mathrm{m} / \mathrm{s})$.

In Eq. (2-33), the term in the left-hand side represents the concentration variations with time. The first term and the second term in the right-hand side represent contributions of convection and diffusion for species transport, respectively.

From Eq. (2-33), we can obtain the scaling formula

$$
\frac{C_{A}}{\Delta t} \sim U \cdot \frac{C_{A}}{L_{1}}+D_{A B} \frac{C_{A}}{L_{2}{ }^{2}}
$$

where

$$
\begin{aligned}
& \Delta t=\text { overall time scale ( } \mathrm{sec}) \\
& L_{1}=\text { length scale of convection (m) } \\
& L_{2}=\text { length scale of diffusion (m) }
\end{aligned}
$$

In this equation, the symbol, $(\sim)$ means that the left-hand side and the right-hand side have the same order of magnitude. Eq. then (2-34) reduces to

$$
\frac{1}{\Delta t} \sim \frac{1}{L_{1} / U_{x}}+\frac{1}{L_{2}^{2} / D_{A B}} .
$$

Therefore, the time scales for the convection (stratified in this case) and the diffusion can be defined by

$$
\Delta t_{g c} \sim L_{1} / U_{x},
$$

and

$$
\Delta t_{d} \sim L_{2}^{2} / D_{A B}
$$

\subsubsection{Estimation of Stratified Time Scale $\left(\Delta t_{g c}\right)$ in Stage 1}

In this section, the time scale for the stratified flow has been estimated in Stage 1. According to Eq. (3-36), the velocity $\left(U_{x}\right)$ and the length $\left(L_{l}\right)$ should be first determined in order to estimate the stratified flow time scale in Stage 1. The speed of the gravity current in the heavy side is generally determined by the following two equations as described in Section 2.2 (Lowe et al. 2005).

$$
\begin{aligned}
& \text { (i) } \gamma^{*}<\gamma<1 \\
& U=\frac{1}{2} \sqrt{\frac{(1-\gamma)}{\gamma} g H}
\end{aligned}
$$


where

$U=$ speed of heavy gravity current $(\mathrm{m} / \mathrm{s})$

$\gamma=$ density ratio $\left(\rho_{1} / \rho_{2}\right)$

$\gamma^{*}=$ critical density ratio $(=0.281$ (suggested by Lowe et al. (2005)))

$H=$ channel depth (m).

$g=$ gravity constant $\left(9.8 \mathrm{~m}^{2} / \mathrm{s}\right)$.

$\mathbf{0}<\gamma<\gamma^{*}$

$U=\sqrt{(1-\gamma) g H}\left[\frac{1}{\gamma} \frac{h}{H}\left(2-\frac{h}{H}\right) \frac{1-h / H}{1+h / H}\right]^{1 / 2}$

Where $h=$ current depth (m).

For the GTMHR reactor design, the maximum density ratio between inside (helium, $900^{\circ} \mathrm{C}, 1 \mathrm{~atm}$ ) and outside (air, $25^{\circ} \mathrm{C}, 1 \mathrm{~atm}$ ) of the vessel is estimated to be 0.036 in the air-ingress situation. It means that this flow is in the highly non-Boussinesq flow regime and will therefore follow Eq. (2-39) or Figures 2-20 and 2-21. The channel depth $(H)$ was determined to be $1.5 \mathrm{~m}$ based on the GTMHR cross duct design shown in Figure 2-22.

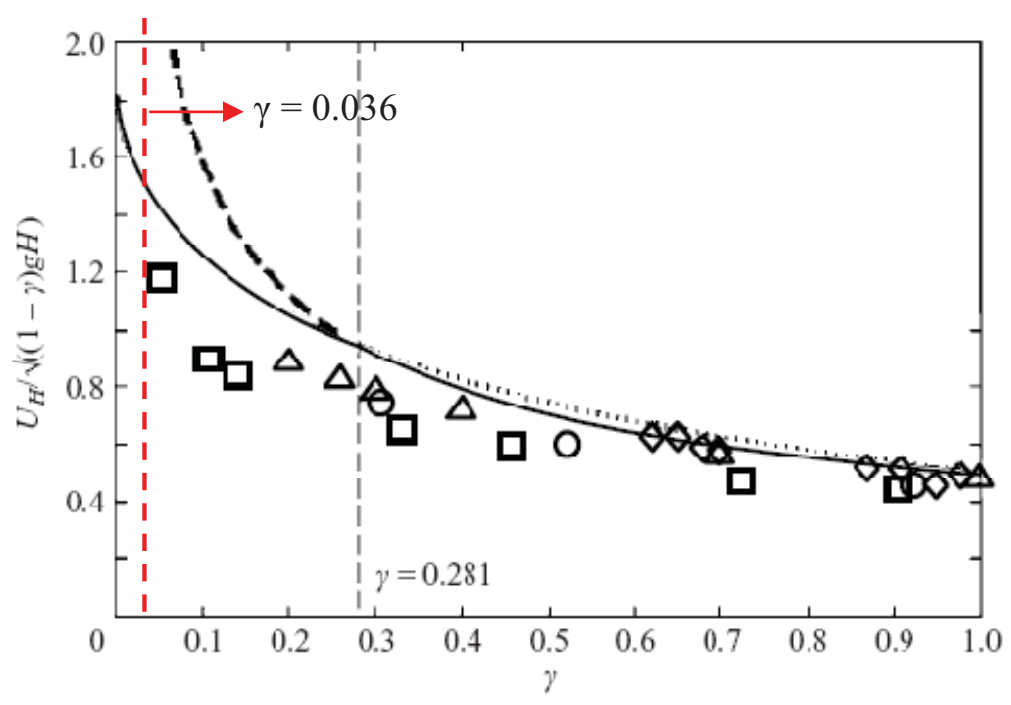

Figure 2-20. Speeds of the heavy currents (Lowe et al. 2005). 


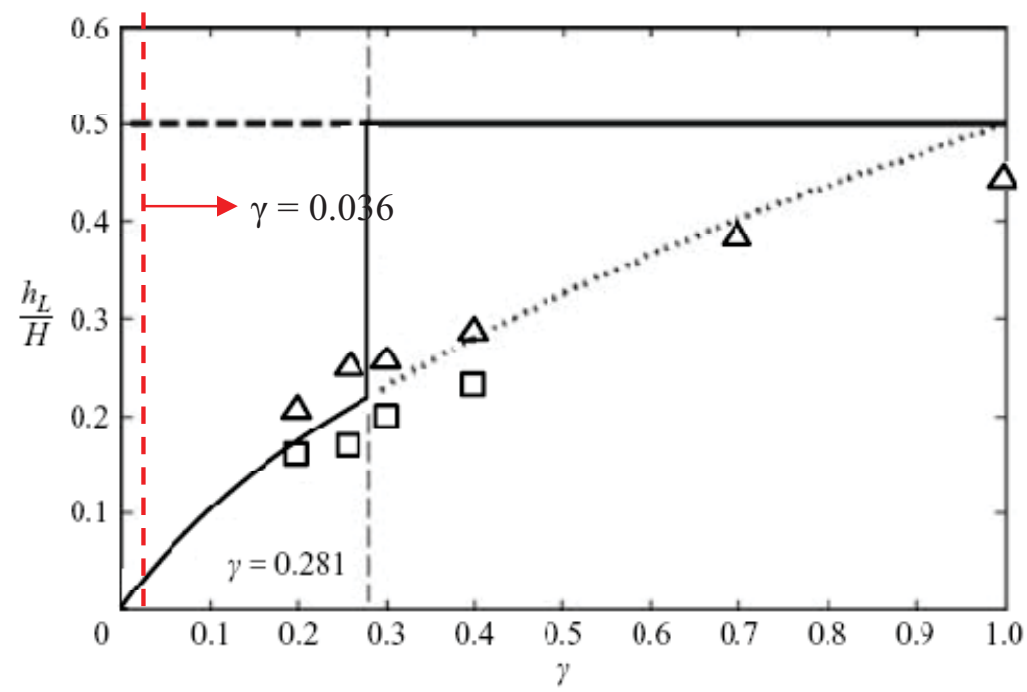

Figure 2-21. Flow depth of the heavy current (Lowe et al. 2005).

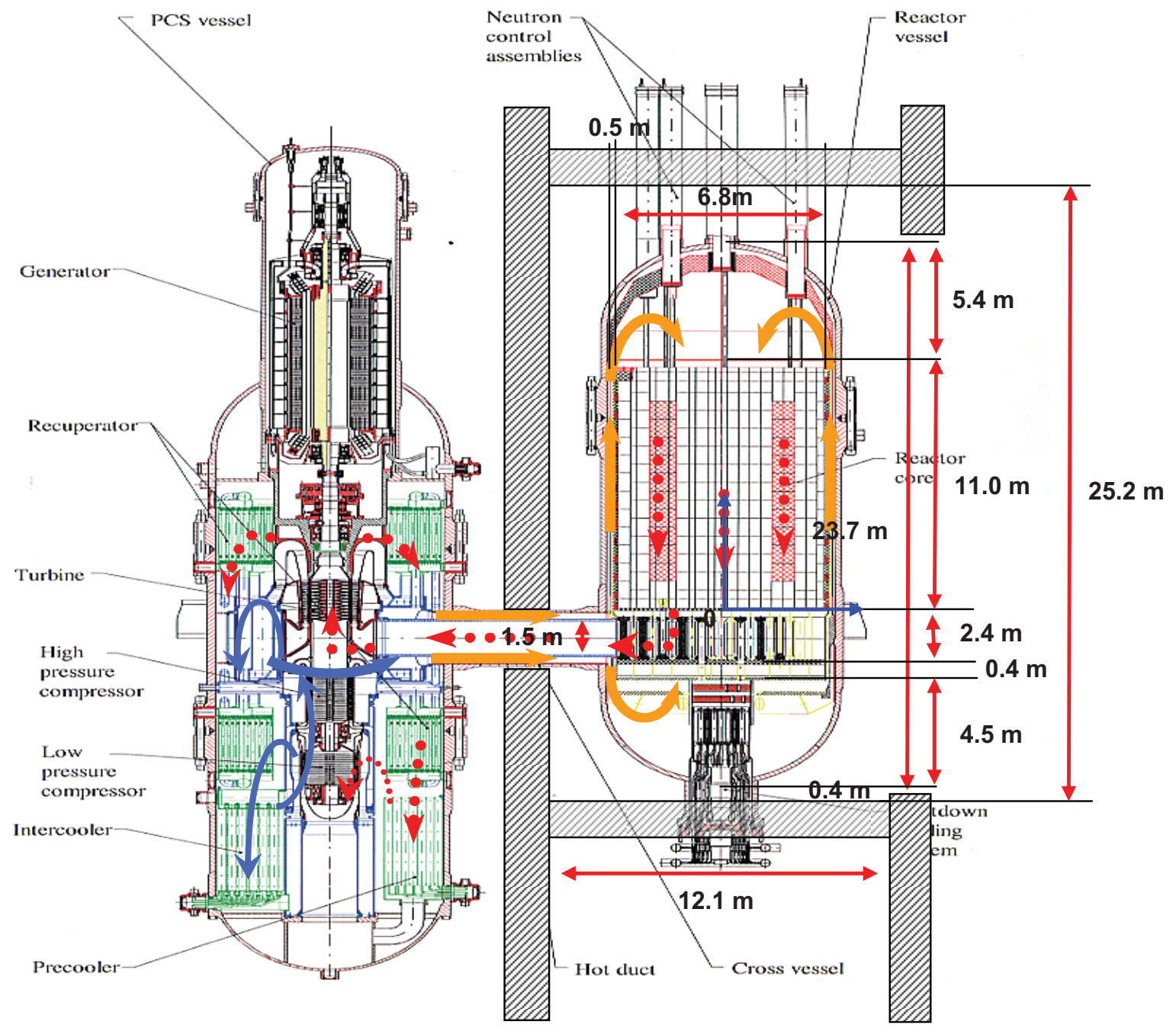

Figure 2-22. Schematics of 600 MWth GTMHR. 
The interpolation of the experimental data in Figures 2-20 and 2-21 were used rather than analytical model Eq. (2-39) to estimate the air current speed $(U)$ and depth $(h)$. The estimated current speed and depth in the GTMHR design are

$U=5.269 \mathrm{~m} / \mathrm{s}$

and

$h=0.06 m$.

The Reynolds number of this flow is estimated and the result is

$\operatorname{Re}=\frac{\rho U H}{\mu}=\frac{5.269 \mathrm{~kg} / \mathrm{m}^{3} \cdot 5.269 \mathrm{~m} / \mathrm{s} \cdot 1.5 \mathrm{~m}}{1.78 \times 10^{-5} \mathrm{~Pa} \cdot \mathrm{s}}=5.08 \times 10^{5}$.

The estimated Reynolds number $\left(5.08 \times 10^{5}\right)$ is quite a high value in the gravity current flow, and in these high Re numbers, the viscous effect is not generally important since the inertia force is much larger than the viscous force. The previous model assumptions (energy conservation and inviscid) are therefore valid (Eugeny et al. 2007) in this high Reynolds number regime $(\operatorname{Re}>10,000)$.

To estimate the stratified flow (convection) time scale, the length scale $\left(L_{l}\right)$ was determined to be a distance between the pipe break point and the center of the lower plenum. In this section, a minimum length scale of $3.4 \mathrm{~m}$, which is half of the lower plenum total length in the GTMHR reactor, has been arbitrarily determined to be the reference value. However, because the exact break point is flexible in the real situation, the sensitivity of length scales was taken into consideration in the parametric studies, wherein the length scale, $L_{1}$, was varied from $3.4 \mathrm{~m}$ to $8 \mathrm{~m}$. Examples of the time-scale calculation for the base case $\left(L_{1}=3.4 \mathrm{~m}\right)$ include:

- The length scale is

$$
L_{1}=3.4 m
$$

- The superficial velocity of the air-ingress can be calculated by

$$
U_{x}=\frac{U \cdot h}{H}=0.21 \mathrm{~m} / \mathrm{s}
$$

- Base on the above numbers, the calculated stratified flow time scale is

$$
\Delta t_{g c} \sim L_{1} / V=3.4 \mathrm{~m} / 0.21 \mathrm{~m} / \mathrm{s}=19.5 \mathrm{sec} .
$$

The physical meaning of this time scale is the duration for the air convective flow to fill one-half of the lower plenum in Stage 1.

\subsubsection{Estimation of Diffusion Time Scale $\left(\Delta t_{d}\right)$ in Stage 1}

To calculate the diffusion time scale, the diffusion coefficient $\left(D_{A B}\right)$ and length scale $\left(L_{2}\right)$ should be determined. The theoretical expression for the diffusion coefficient for gas pairs of nonpolar, nonreacting molecules (Welty et al. 1984) is

$$
D_{A B}=\frac{18.58 \cdot T^{3 / 2}\left[\frac{1}{M_{A}}+\frac{1}{M_{B}}\right]}{P \cdot \sigma_{A B}{ }^{2} \Omega_{D}}(\mathrm{~m} / \mathrm{s})
$$


where

$$
\begin{aligned}
T & =\text { temperature }(\mathrm{K}) \\
M & =\text { molecular weight }(\mathrm{kg} / \mathrm{kmol}) \\
P & =\text { absolute pressure }(\mathrm{atm}) \\
\sigma_{A B} & =\text { collision diameter (Lennard-Jones parameter) (Angstroms) } \\
\Omega_{D} & =\text { collision integral. }
\end{aligned}
$$

For air and helium molecules, the diffusion coefficient can be calculated as

$$
D_{\text {air-helium }}=7.92 \times 10^{-4} \mathrm{~m}^{2} / \mathrm{s}\left(\text { at } 900^{\circ} \mathrm{C}, 1 \mathrm{~atm}\right) \text {. }
$$

Since the diffusion length scale is not constant during the diffusion process, it is difficult to determine as a single number. For this reason, a different method was used to calculate the diffusion time scale. The main idea was to obtain the equivalent time scale, which has the same physical meaning as the convection time scale. The time scale of the diffusion is physically equivalent to that of the convection, when the average air concentration in the lower plenum becomes one-half of the external air concentration. The local air concentration $\left(C_{A i r}\right)$ in the lower plenum is mathematically expressed (Welty et al. 1984) as

$$
\frac{C_{A i r}(z, t)-C_{A i r, 0}}{C_{A i r, s}-C_{A i r, 0}}=1-\operatorname{erf}\left(\frac{z}{2 \sqrt{D_{\text {air-helium }} t}}\right)
$$

where

$$
\begin{aligned}
& z=\text { distance from air source }(\mathrm{m}) \\
& t=\text { time }(\mathrm{sec}) \\
& C_{A i r}(z, t)=C_{A, 0} \text { at } \mathrm{t}=0, \text { for all } \mathrm{z} \\
& C_{A i r}(z, t)=C_{A, s} \text { at } \mathrm{z}=0, \text { for all } \mathrm{t} \\
& C_{A i r}(z, t)=C_{A, 0} \text { as } \mathrm{z} \rightarrow \infty, \text { for all } \mathrm{t} .
\end{aligned}
$$

The definition of the error function in Eq. (2-48) is

$\operatorname{erf}(x)=\frac{2}{\pi} \int_{0}^{x} e^{-t^{2}} d t$

From Eq. (2-48), the average concentration in the lower plenum can be expressed as

$$
\left(\frac{C_{A i r}(z, t)-C_{A i r, 0}}{C_{A i r, s}-C_{A i r, 0}}\right)_{\text {average,LowerPlenum }}=\frac{1}{D_{L P}} \int_{\text {LowerPlemum }} 1-\operatorname{erf}\left(\frac{z}{2 \sqrt{D_{A B} t}}\right) \cdot d z
$$


where $D_{L P}=$ diameter of the lower plenum (m).

Therefore, the time scale of the diffusion can be calculated by solving

$$
\frac{1}{D_{L P}} \int_{\text {LowerPlenum }} 1-\operatorname{erf}\left(\frac{z}{2 \sqrt{D_{A B} t}}\right) \cdot d z=0.5 \text {. }
$$

In this work, the solution of Eq. (2-51) was calculated using MATLAB. The result of the diffusion time scale is

$$
t=\Delta t_{d}=1.29 \times 10^{4} \mathrm{sec} .
$$

\subsubsection{Comparisons of Time Scales}

Based on the results of Eq. (2-45) and Eq. (2-52), the convection time scale (=19.5 sec) is estimated to be about 785 times larger than the diffusion time scale $\left(=1.29 \times 10^{4} \mathrm{sec}\right)$, which means that the convection process is at least about 785 times faster than the diffusion process. If the overall time scale is calculated by using Eq. (2-35), it is

$\Delta t \sim 19.5 \mathrm{sec}$.

Figure 2-23 shows the relative time scales $\left(\Delta t_{d} / \Delta t_{g c}\right)$ as a function of the distance between pipe break location and lower plenum center. The relative time scale increases linearly with the length scale indicating that the stratified flow is the dominant mechanism in Stage 1, allowing the diffusion mechanism to be neglected.

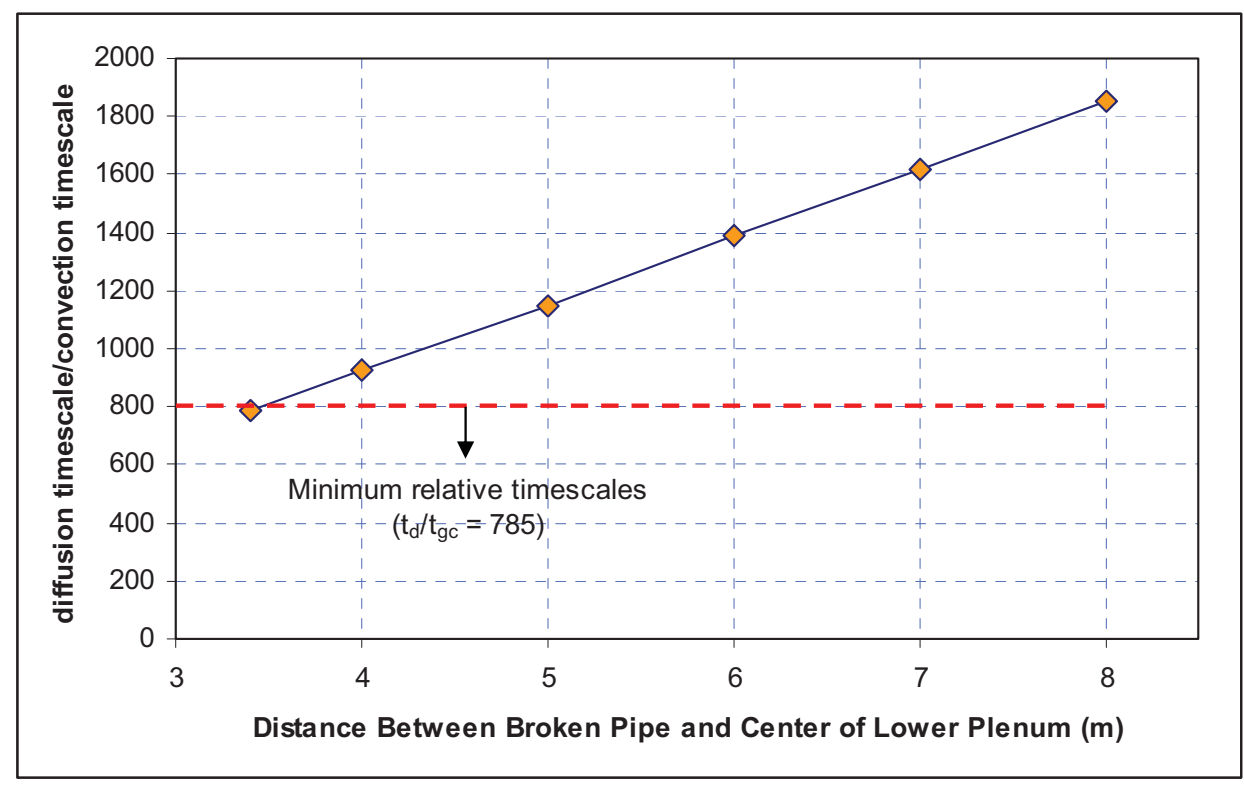

Figure 2-23. Correlation between length scale and relative time scales. 


\subsubsection{Time scale Comparisons in Stage 2}

After air flows into the reactor in Stage 1 by density gradient driven flow, the lower part of the core (lower plenum) is filled with air as shown in Figure 2-24. Once the air occupies the lower plenum, the main driving force which was generated by molecular mass differences (between air and helium) will disappear; however, temperature gradient between inside and outside reactors will still maintain density gradient even though the driving force is weaker than in Stage 1. It finally leads to another stratified flow or a local natural circulation flow. Since the temperature gradient is maintained during the whole airingress process, this stratified flow (Stage 2) will be continued until or even after the overall onset of natural circulation starts.

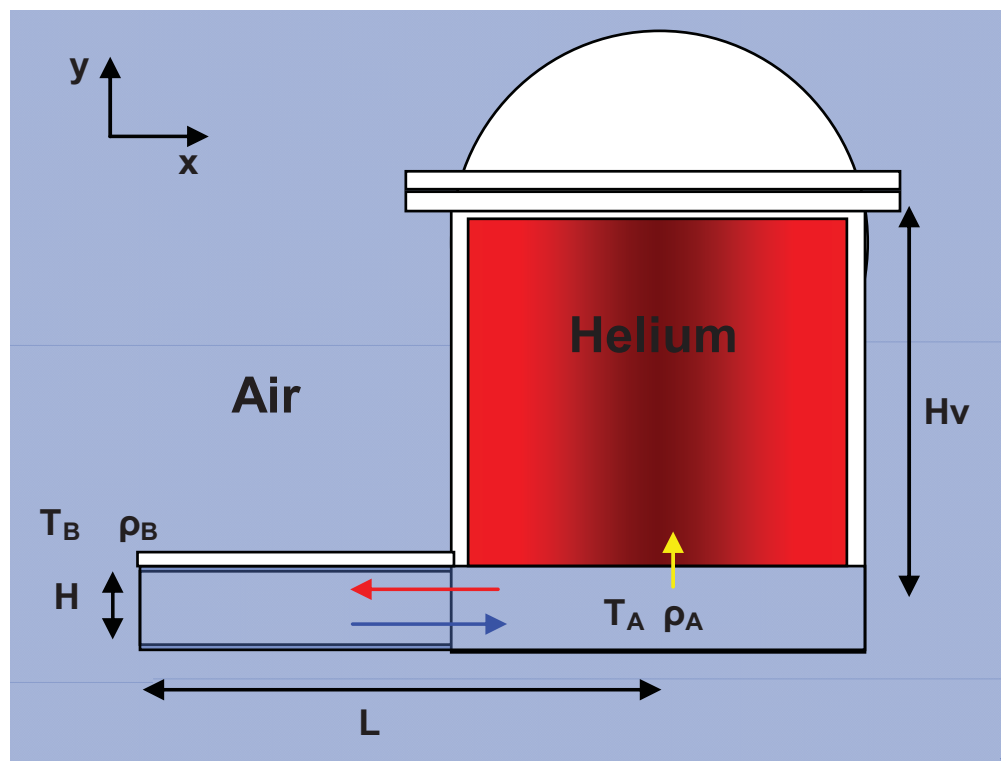

Figure 2-24. Schematics of Stage 2 air-ingress.

The majority of the inflow (blue arrow in Figure 2-24) in Stage 2 is expected to return to the reactor outside (red arrow) through the upper part of the channel. However, some portion of the flow can be moved into the reactor core (yellow arrow) slowly by a buoyancy force depending on the geometry and conditions. This buoyancy force is generated when the cold air moves into the lower plenum. If the cold air ingresses into the inside of the reactor, the air will interact with hot supporting structures. The air will be heated and expand, making it less dense than the unexpanded air, giving it a buoyancy force. If the buoyancy force is sufficient to overcome the static head in the core, the air will be able to move into the reactor core. If not, the air-ingress into the core will be controlled solely by molecular diffusion and turbulence mixing. Pressure build-up in the lower plenum was compared to the static head in the core to estimate the main air-ingress mechanism in Stage 2. It can be simply described as:

- Pressure Build-up (Buoyancy Force) > Hydrostatic Head : Convection Dominant

- Pressure Build-up (Buoyancy Force) < Hydrostatic Head : Molecular Diffusion Dominant.

The pressure build-up was estimated by energy conservation law and previous gravity current correlations described in Section 2.2. In this estimation, the friction loss and local heat transfer were not considered. The temperatures inside the reactor were assumed to be constant throughout the whole core. The temperatures inside and outside of the reactor were assumed to be $900^{\circ} \mathrm{C}$ and $25^{\circ} \mathrm{C}$, respectively. In this temperature condition, the density ratio is estimated to be 0.25 in non-Boussinesq flow regime. 
The total kinetic energy of the flow in the lower plenum is defined by

$K \cdot E_{\cdot 1}=\frac{1}{2} \rho_{A} \cdot u_{A}^{2}\left(J / m^{3}\right)$

where

$\rho_{A} \quad=$ density of the flow in the lower plenum $\left(\mathrm{kg} / \mathrm{m}^{3}\right)$

$u_{A}=$ velocity of the flow in the lower plenum $(\mathrm{m} / \mathrm{s})$.

In this derivation, it was assumed that the air has enough time to be heated up to the same temperature as the reactor inside. Therefore, the Kinetic energy of the air in the lower plenum can be derived by inserting Eq. (2-38) into Eq. (2-54) and the mass conservation law as

$K . E_{\cdot_{1}}=\frac{1}{2} \rho_{A} \cdot\left(\frac{1}{4} \cdot\left(\frac{1-\gamma}{\gamma}\right) \cdot g \cdot H\right) / \gamma^{2}$.

Therefore, Eq. (2-55) reduces to

$K . E_{\cdot_{1}}=\frac{\rho_{A} \cdot g \cdot H}{8} \cdot\left(\frac{1-\gamma}{\gamma^{3}}\right)$.

The Kinetic energy of the returning flow in the horizontal pipe can then be estimated by Eq. (2-21) as

$K \cdot E_{\cdot_{2}}=\frac{1}{2} \rho_{A} \cdot\left(\frac{1}{4} \cdot(1-\gamma) \cdot g \cdot H\right)$.

Therefore, Eq. (2-57) reduces to

$K \cdot E_{\cdot 2}=\frac{\rho_{A} \cdot g \cdot H}{8} \cdot(1-\gamma)$.

The pressure build-up can be estimated by energy conservation equation

$\Delta P=K \cdot E_{\cdot_{1}}-K \cdot E_{\cdot_{2}}=\frac{1}{8} \cdot\left(\rho_{A} \cdot g \cdot H \cdot(1-\gamma)\right) \cdot\left(\frac{1}{\gamma^{3}}-1\right)$.

In this estimation, effects of viscous dissipation and potential head were neglected.

The static head of the core can be calculated by the following hydraulic head equation.

Hydrostatic Head $=\left(\rho_{\text {core }}-\rho_{\text {riser }}\right) \cdot g \cdot H_{v}$

where

$$
\begin{aligned}
& \rho_{\text {core }}=\text { density of the coolant in the core }\left(\mathrm{kg} / \mathrm{m}^{3}\right) \\
& \rho_{\text {riser }}=\text { density of the coolant in the riser }\left(\mathrm{kg} / \mathrm{m}^{3}\right)
\end{aligned}
$$


Pressure build-up and static head were estimated for two different designs, one being the $600 \mathrm{MWth}$ GTMHR reactor and the other being the NACOK (an acronym of German words that stand for "Natural Convection in the Core with Corrosion") experiment (Schaaf et al. 1998)). The results are summarized in Table 2-1. In the GTMHR, the pipe diameter is $1.5 \mathrm{~m}$ and the core height is $11 \mathrm{~m}$. It has quite large pipe diameter but relatively shorter core height; however, the NACOK experiment has a $0.125 \mathrm{~m}$ pipe diameter and $7.334 \mathrm{~m}$ core height, which is a relatively large height compared to the pipe diameter. The pipe diameter is closely related to the pressure build-up as shown in Eq. (2-59). As the diameter increases, the pressure build-up increases proportionally. On the other hand, the core height is related to the static head (See Eq. (2-60)) where, if the core height is increased, the static head is also increased. According to the estimation in Table 2-1, the pressure build-up (24.18 Pa) in a GTMHR is larger than the average static head $(10.01 \mathrm{~Pa})$, indicating that the air will move into the core in Stage 2 for the GTMHR design. On the other hand, the pressure build-up $(1.101 \mathrm{~Pa})$ in the NACOK experiment is much smaller than the average static head $(9.6 \mathrm{~Pa})$, indicating that the air won't be able to move into the NACOK 'core'. The process therefore appears to be controlled by molecular diffusion in the NACOK experiment, even though there is a small stratified flow generated by the temperature gradient. This physical interpretation is consistent with previous NACOK experimental data.

Table 2-1. Comparisons of pressure build-up and static head for GTMHR and NACOK experiment.

\begin{tabular}{|lcl|}
\hline & GTMHR & NACOK \\
\hline Air Density Ratio $(\gamma)$ & 0.253 & 0.323 \\
Pipe Diameter (D) & $1.5 \mathrm{~m}$ & $0.125 \mathrm{~m}$ \\
Core Height (Hv) & $11 \mathrm{~m}$ & $7.334 \mathrm{~m}$ \\
Pressure Build-up (dP) & $24.18 \mathrm{~Pa}$ & $1.101 \mathrm{~Pa}$ \\
Static Head & $10.01 \mathrm{~Pa}$ & $9.6 \mathrm{~Pa}$ \\
\hline
\end{tabular}

The criteria of air movement into the core in Stage 2 was developed based on Eq. (2-59) and Eq. (2-60). According to the previous description, the criterion for the air movement into the core is

$\Delta P>$ Hydrostatic Head

Therefore, Eq. (2-61) can be rewritten as

$\frac{\Delta P}{\text { Hydrostatic Head }}>1$.

If Eq. (2-59) and (2-61) are inserted into Eq. (2-62), the result is

$\Pi_{2}=\frac{1}{8}\left(\frac{H}{H_{v}}\right) \cdot\left(\frac{\rho_{A}}{\rho_{\text {Core }}-\rho_{\text {Riser }}}\right)\left(\frac{(1-\gamma)\left(1-\gamma^{3}\right)}{\gamma^{3}}\right)>1$.

The physical meaning of nondimensional parameter, $\Pi_{2}$ in Eq. (2-63) is the ratio of the buoyancy force (pressure build-up) to the static head in Stage 2. If this value is larger than 1, the air will have enough buoyancy force to generate flow into the core. On the other hand, if the value is less than 1, the air will not have enough buoyancy force to generate flow into the core. The implication of this with respect to air-ingress mitigation is that decreasing the $\Pi_{2}$ value is beneficial in the mitigation of the air-ingress process. The following modifications are, in theory, shown to decrease the $\Pi_{2}$ value:

- Decreasing the diameter of the horizontal pipe $(H)$ 
- Increasing the height of the core $\left(H_{v}\right)$

- Increasing the $\rho_{\text {Core }}-\rho_{\text {Riser }}$ which can be achieved by increasing the riser temperature in the accident conditions or decreasing the core temperature in the accident conditions

- Increasing the density ratio $(\gamma)$, which can be achieved by increasing cavity temperature or decreasing lower plenum temperature.

\subsubsection{Calculation of Convection Time Scale $\left(\Delta t_{c}\right)$}

From the comparisons between buoyancy force and static head, it was found that the air will move into the reactor core by convective force. However, to estimate the relative importance of the mechanisms between molecular diffusion and the convective flow, the speed of the air movement into the core should be quantified. To estimate this velocity, three equations were set up for the following three flow paths (See Figure 2-25): Path-1: from core top to reactor outside; Path-2: from core bottom to core top; and Path-3: circulation flow in the lower plenum.

Path-1 (See Figure 2-25) can be expressed by the following Bernoulli's equation if the heat transfer effect in the riser is ignored:

$P_{1}+\frac{1}{2} \rho_{1} \cdot u_{1}^{2}=P_{2}+\frac{1}{2} \rho_{2} \cdot u_{2}^{2}+\rho_{A} \cdot g \cdot H_{v}$

Since heat is not transferred and there are very slight pressure differences between locations 1 and 2, the densities can be assumed to be almost the same between those two locations, given

$\rho_{1} \approx \rho_{2}$

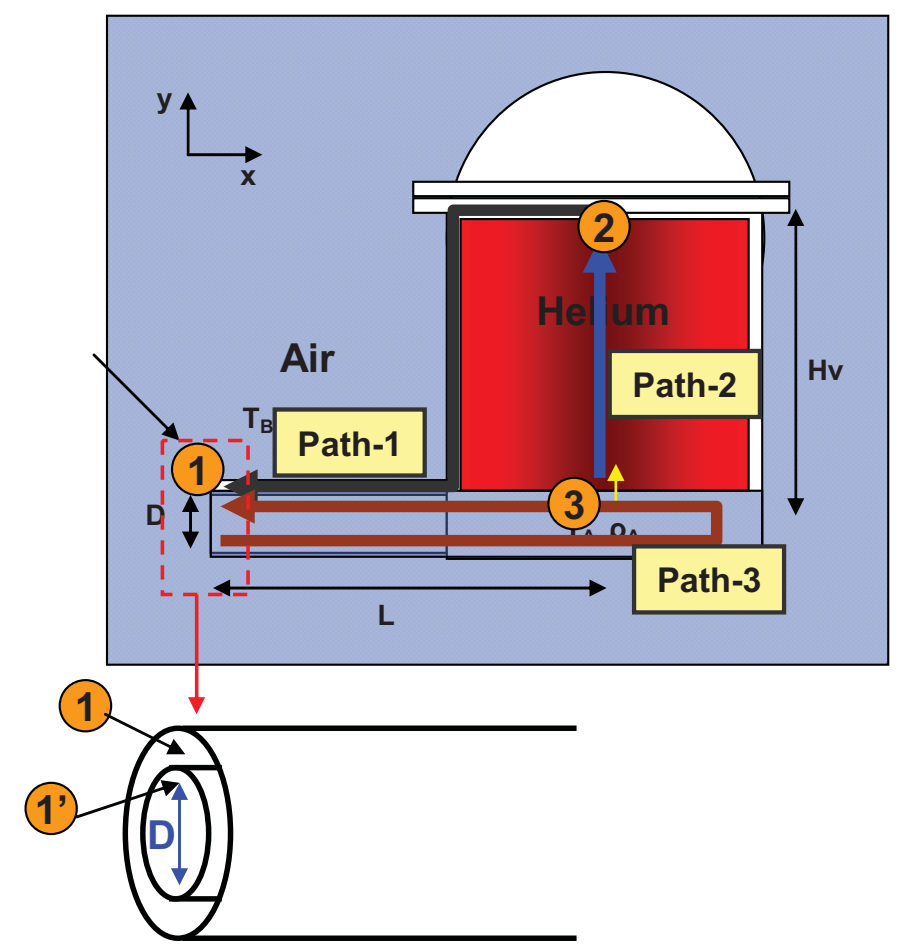

Figure 2-25. Schematics of the VHTR reactor for analytical modeling. 
Therefore, through mass conservation

$u_{1} A_{1}=u_{2} A_{2}$.

Since the friction effect cannot be ignored in the core, Path-2 (See Figure 2-25) can be expressed by

$P_{3}-P_{2}=32 \frac{\mu \cdot u_{\text {avg }} \cdot H_{v}}{D^{2}}+$ hydrostatic head

Since no heat transfer in the core region (uniform temperature) is assumed, the velocities in the core and location 2 (core top) may be considered to be the same as

$u_{\text {avg }} \approx u_{2}$.

Depending on the core density $\left(\rho_{\mathrm{c}}\right)$, which is an average density of the core fluids, the hydrostatic head can be expressed by

hydrostatic head $=\rho_{c} \cdot g \cdot H_{v}$

Path-3 was modeled very carefully. Figure 2-26 shows the assumed flow pattern in the lower plenum where there is a counter-current flow between upper and lower parts of the region and the lower flow changes its direction at the right side of the core. The contour plot in Figure 2-26 shows the flow angle in the lower plenum. Blue indicates a flow in the $\mathrm{x}$ direction, red indicates the negative $\mathrm{x}$ direction. This plot supports that the flow direction in our modeling is reasonably assumed.

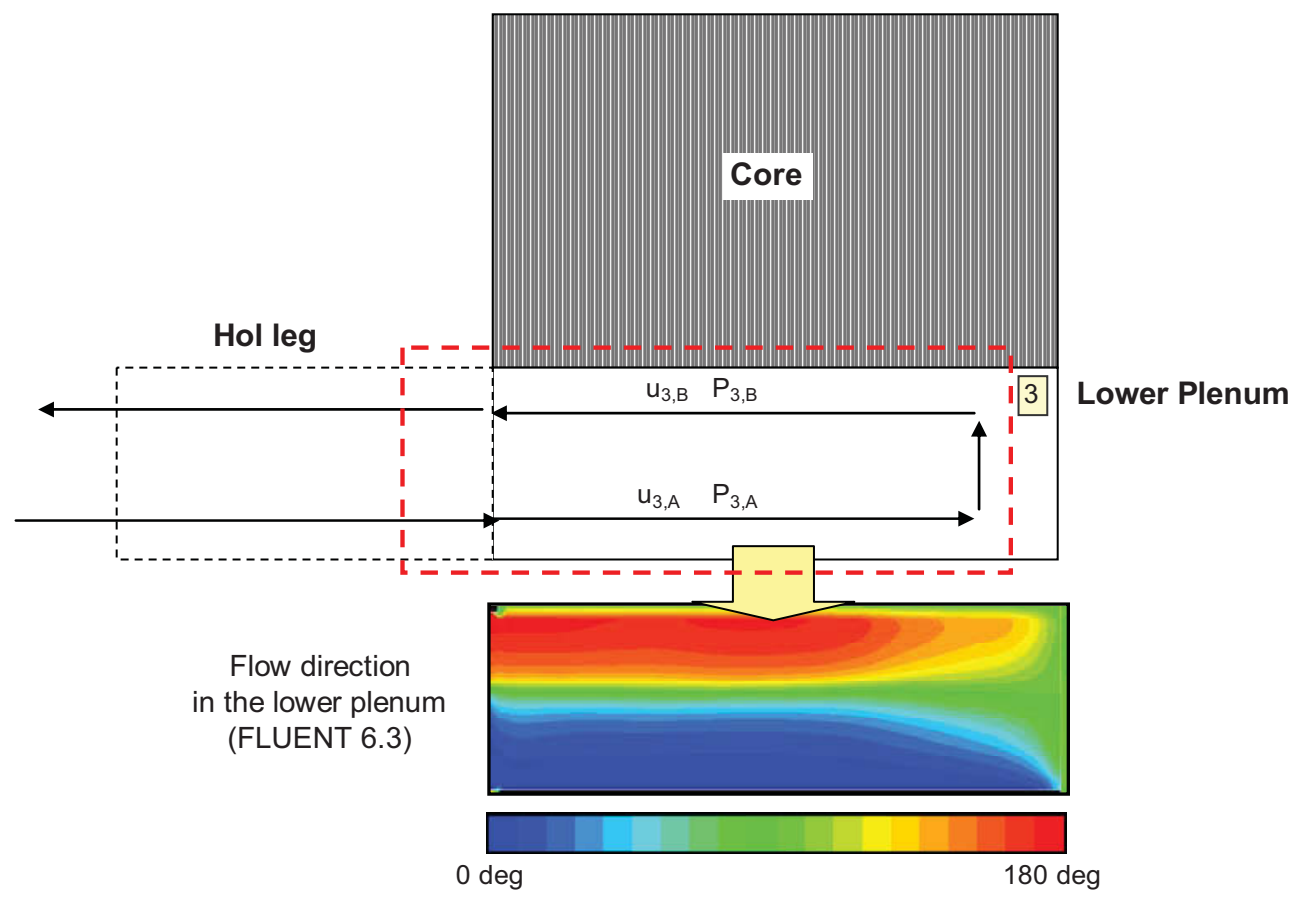

Figure 2-26. Circulation pattern in the location 3 (lower plenum). 
This modeling assumes that the kinetic energy difference between the lower and upper flows is converted to the pressure energy with negligible static head differences. The concept of this method is basically the same as that of pressure build-up modeling.

The derivation gives us

$$
P_{1}^{\prime}-P_{3}=P_{1}+\frac{1}{8} \rho_{3} \cdot g \cdot D \cdot(1-\gamma) \cdot\left(\frac{1}{\gamma^{3}}-1\right) \text {. }
$$

In this derivation, we ignored the hydrostatic head differences between location 1 and location 1' (See Figure 2-25). We can therefore assume that the static pressure in location 1 and location 1' will be the same. If the effect of the energy loss for turning the direction of the fluid (See Figure 2-26) is considered, Eq. (2-70) can be expressed as

$$
P_{1}-P_{3}=\frac{1}{8} \rho_{3} \cdot g \cdot H \cdot(1-\gamma) \cdot\left(\frac{1}{\gamma^{3}}-1\right) \cdot \eta \text {. }
$$

In this equation, the symbol $\eta$ represents the efficiency of energy conversion. From Eqs. (2-67), (2-69), and (2-71), we can obtain

$$
\begin{aligned}
P_{3}-P_{2} & =32 \frac{\mu \cdot u_{2} \cdot H_{v}}{D^{2}}+\rho_{c} \cdot g \cdot H_{v} \\
& =\frac{1}{8} \rho_{3} \cdot g \cdot H \cdot(1-\gamma) \cdot\left(\frac{1}{\gamma^{3}}-1\right) \cdot \eta-\frac{1}{2} \rho_{2} \cdot\left(\left(\frac{A_{2}}{A_{1}}\right)^{2}-1\right) \cdot u_{2}{ }^{2}+\rho_{A} \cdot g \cdot H_{v}
\end{aligned}
$$

Eq. (2-72) can be arranged as

$$
C_{1} \cdot u_{2}^{2}+C_{2} \cdot u_{2}+C_{3}=0
$$

where

$$
\begin{aligned}
& C_{1}=\frac{1}{2} \rho_{2} \cdot\left(\left(\frac{A_{2}}{A_{1}}\right)^{2}-1\right) \\
& C_{2}=32 \frac{\mu \cdot H_{v}}{D^{2}} \\
& C_{3}=\frac{1}{8} \rho_{3} \cdot g \cdot H \cdot(1-\gamma) \cdot\left(1-\frac{1}{\gamma^{3}}\right) \cdot \eta+\left(\rho_{c}-\rho_{A}\right) \cdot g \cdot H_{v}
\end{aligned}
$$

The core average density $\left(\rho_{\mathrm{c}}\right)$ in Eq. (2-76) can be defined as (See Figure 2-27)

$\rho_{c}=\left(\frac{H_{\text {air }}}{H_{v}}\right) \cdot \rho_{\text {air }}+\left(1-\left(\frac{H_{\text {air }}}{H_{v}}\right)\right) \cdot \rho_{\text {helium }}$

where 
$\rho_{\text {air }}=$ density of air (or heavy) gas

$\rho_{\text {helium }}=$ density of helium

$H_{\text {air }}=$ height of the air (or heavy gas) in the core

$H_{v} \quad=$ total core height.

Therefore, the speed of the flow in the core can be calculated by

$u_{2}=\frac{-C_{2}+\sqrt{C_{2}{ }^{2}-4 C_{1} \cdot C_{3}}}{2 C_{1}}$

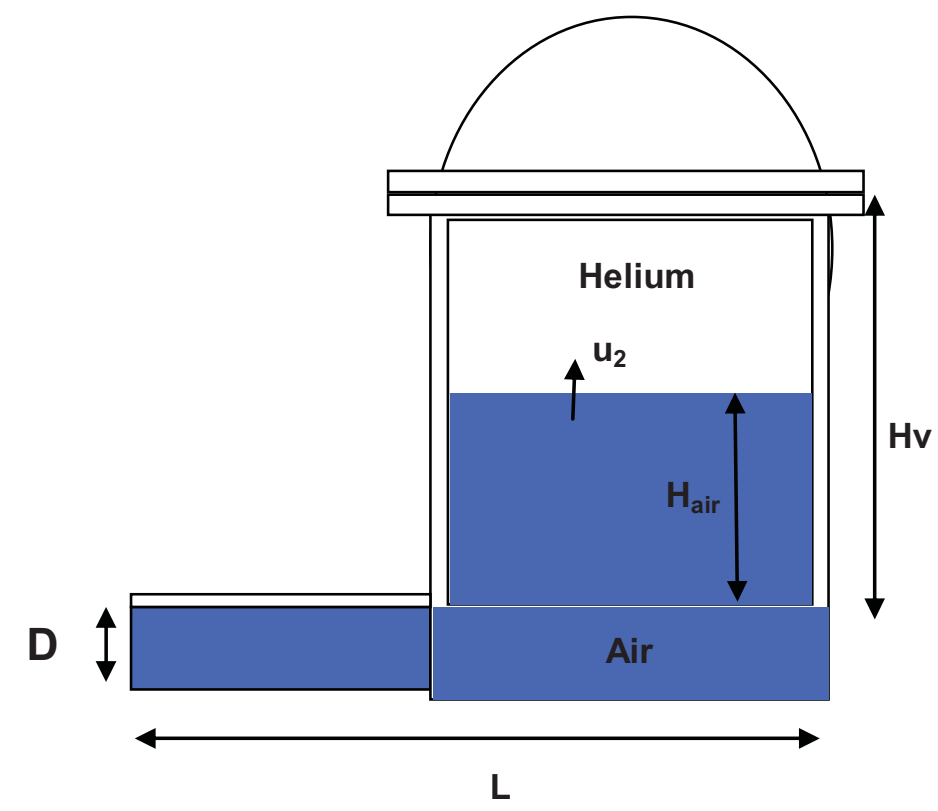

Figure 2-27. A sketch of the phenomena in Stage 2.

If Eq. (2-78) is applied to the $600 \mathrm{MWth}$ GTMHR core dimensions, the initial and average speeds of the core flow are calculated to be $0.487 \mathrm{~m} / \mathrm{s}$ and $0.26 \mathrm{~m} / \mathrm{s}$, respectively when the initial core is filled with only helium coolant and $A_{2} / A_{1}=1$. This speed is decreased with an increase of air height in the core. Figure 2-28 shows a sketch of the phenomena in Stage 2 and the velocity profile calculated by FLUENT 6.3 in the reactor core in the Stage 2 period of the stratified flow. Even though the temperature in the core is not uniform in the model (varying 500 to $\left.950{ }^{\circ} \mathrm{C}\right)$, the average velocity $(0.3 \mathrm{~m} / \mathrm{s})$ in this calculation is the same order as estimated by the simplified analytic solution $(\sim 0.26 \mathrm{~m} / \mathrm{s})$. Still, further validation work is required afterward.

Based on the estimated core flow velocity $(=0.26 \mathrm{~m} / \mathrm{s})$, the convection time scale by density gradient flow in Stage-2 was estimated as

$\Delta t_{c} \sim 42 \mathrm{sec}$ 


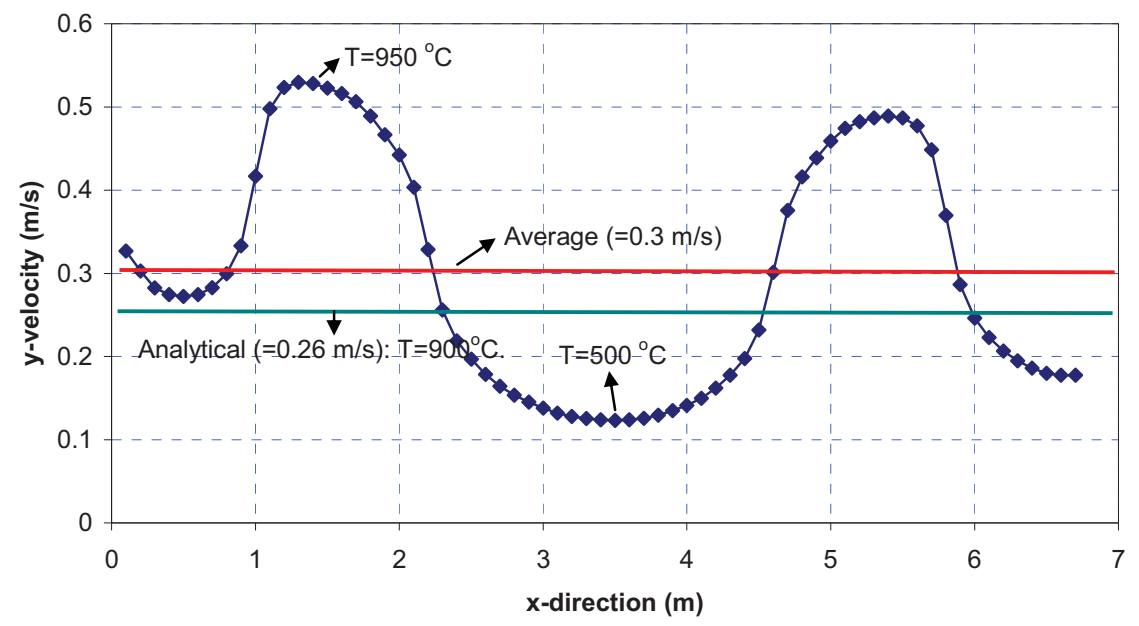

Figure 2-28. Calculated y-velocity profile in the reactor core during Stage 2 (FLUENT 6.3).

\subsubsection{Calculation of Diffusion Time Scale $\left(\Delta t_{d}\right)$}

To calculate the diffusion time scale, the diffusion coefficient $\left(D_{A B}\right)$ and length scale $\left(L_{2}\right)$ should be determined as done in the previous section. For air and helium molecules, the diffusion coefficient is

$D_{\text {air-helium }}=7.92 \times 10^{-4} \mathrm{~m}^{2} / \mathrm{s}\left(\right.$ at $\left.900^{\circ} \mathrm{C}, 1 \mathrm{~atm}\right)$.

The diffusion time scale in Stage 2 was calculated by the same method as carried out in Eqs. (2-50) and (2-51) in the previous section. The calculated time scale of the diffusion process to deliver the same amount of air into the core as the convection did during the convection time scale $(=42 \mathrm{sec})$ is

$\Delta t_{d} \sim 2.7 \times 10^{4} \mathrm{sec}\left(\right.$ at $\left.900^{\circ} \mathrm{C}, 1 \mathrm{~atm}\right)$.

\subsubsection{Comparisons of Time Scales}

Based on Eq. (2-79) and Eq. (2-88), we can estimate that the convection time scale is approximately 642-times larger than the diffusion time scale. It means that the convection process is about 642-times faster than the diffusion process. If calculated, the overall time scale is

$\Delta t \sim 42 \mathrm{sec}$.

This time scale is the same as the convection time scale, which means that Stage 2 is dominated by a stratified flow process. The diffusion effect may therefore be neglected. The results are summarized in Table 2-2.

Table 2-2. Comparisons of time scales for the GTMHR (Stage 2).

\begin{tabular}{|ll|}
\hline \multicolumn{2}{|c|}{ 600 MWth GTMHR } \\
\hline Channel Depth (D) (m) & 1.5 \\
Core Height (Hv) (m) & 11 \\
Average core flow velocity by buoyancy force (sec) & 0.26 \\
Convection Time scale (tc) (sec) (within $\left.\mathrm{H}_{\text {air }}\right)$ & 42 \\
Diffusion Time scale (td) $(\mathrm{sec})$ & $2.70 \mathrm{e} 4$ \\
$\mathrm{td} / \mathrm{tc}$ & 642 \\
\hline
\end{tabular}




\subsection{Discussions on Stage-2 and Early Onset of Natural Circulation}

This section discusses Stage-2 in the air-ingress accident scenario. As described and shown in Figure 2-18, INL proposed a new air ingress accident scenario consisting of the following four steps: depressurization, Stage-1 stratified flow, Stage-2 stratified flow, and onset of natural circulation (ONC).

- Depressurization: Depressurization step is followed by a pipe break. In this step, the coolant (helium) in the vessel is rapidly blown into the reactor confinement because of high pressure differences between the inside and outside vessel (Figure 2-18(1)). As the depressurization progresses, the pressure difference gets closer and is finally equilibrated.

- Stage-1 stratified flow: After the overall pressures between the inside and outside vessel are equilibrated during depressurization, the outside air (or air/helium mixture) moves into the inside vessel by the density gradient driven flow (Figure 2-18(2)). The density gradient is generated by different molecular mass and different temperature. In this step, air/helium mixture fills the reactor bottom.

- Stage-2 stratified flow: After the helium-air mixture fills up the reactor bottom, Stage 2 stratified flow begins (Figure 2-18(3)). In Stage 2, the density gradient flow is generated solely by the temperature gradient between the inside and outside of the reactor. The density gradient induced by the temperature gradient drives the cold air into the lower plenum where it is expanded by heating. Once the air is heated in the lower plenum, it (air or air-helium mixture) will have a buoyancy force that pushes it up into the reactor core or stay in the lower plenum.

- Onset of Natural Circulation: Once the reactor top is full, global natural circulation will begin (Figure 2-18(4)).

\subsubsection{Stage-2 Stratified Flow Issue}

After INL reported the new air-ingress accident scenario, including early ONC, there have been many discussions and disputes on potential nondiffusion mechanisms and early ONC caused by Stage- 2 stratified flow. These opinions are that Stage- 2 stratified flow is a very stable situation and therefore controlled by the slow molecular diffusion. Some researchers are also questioning if the CFD methods are valid for this problem based on the physical stability of two fluids. In the air-ingress accident, since the heavy air is placed under the light helium, they believe it is physically stable.

This prompted a more careful look into the Stage- 2 stratified flow phenomena to determine if this condition is physically-stable in real life situations. Figure 2-29 shows a schematic of the Stage-2 stratified flow. After Stage 1, the bottom of the reactor is filled with an air/helium mixture and the core with helium. Since the air/helium mixture density is higher than helium density, this situation is considered to be stable in the static condition. For example, if air tries to move up into the core, the hydrostatic head forces the air back to the original state. In order to move air to the riser and cold-leg, the air should have sufficient energy to lift the air to the top of the core. The graph on the right in Figure 2-29 illustrates the energy state of the Stage-2 stratified flow condition with an energy barrier for each side of the Stage 2 state. This energy barrier represents the maximum head to lift air up to the core top. Once the air is lifted to the top, it can be naturally flowed down through the riser by the gravity force, thereby initiating ONC. The stability of this system was determined by checking to see how big the energy barrier was and if the external perturbation could provide sufficient energy to overcome this. 


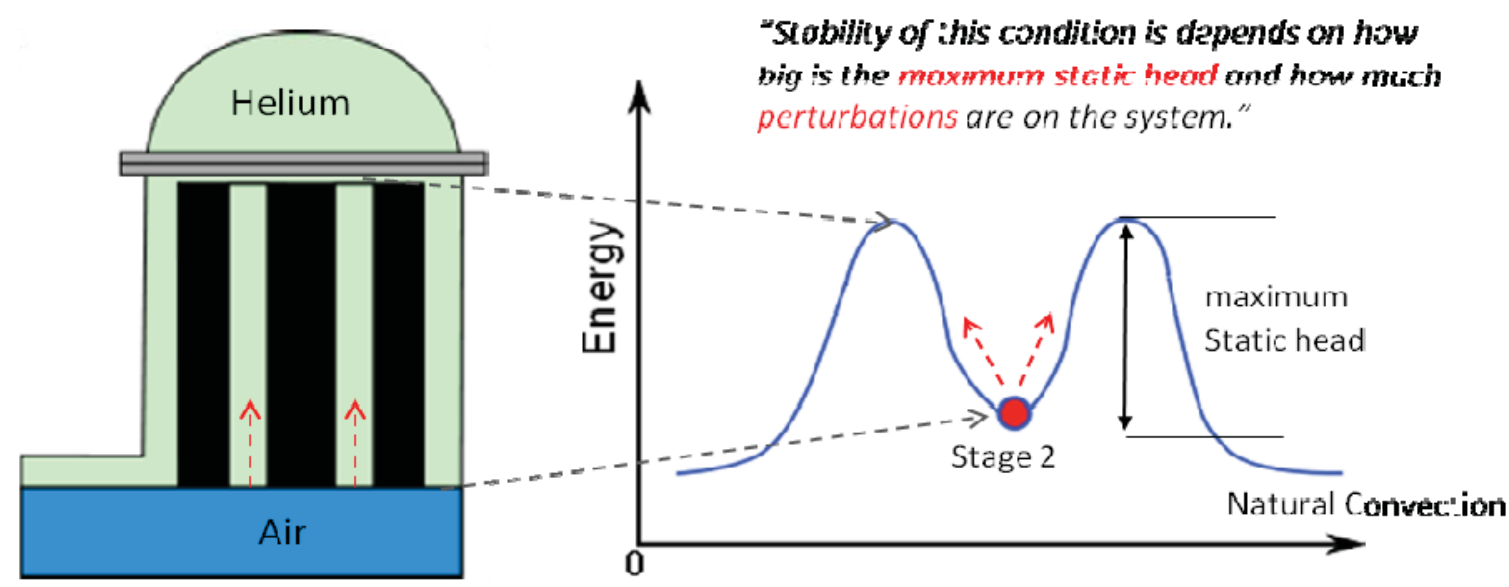

"Air should overcome hydrostatic head for the stage-2 to be controlleci ky convection."

Figure 2-29. Stability concept of Stage-2 in air-ingress accident.

\subsubsection{Estimation of Energy Barrier in Stage-2 Stratified Flow}

The energy barrier for ONC was calculated by an analytical method. Since the energy barrier is equivalent to the head required to lift air to the core top, the following static head equation was used:

$\Delta E=\left(\rho_{\text {mixture,core }}-\rho_{\text {helium,riser }}\right) \cdot g \cdot H$

where

$\Delta E \quad=$ energy barrier $\left(\mathrm{J} / \mathrm{m}^{3}\right)$

$\rho_{\text {mixture, } \text {,ore }}=$ air/helium mixture density in the core condition $\left(\mathrm{kg} / \mathrm{m}^{3}\right)$

$\rho_{\text {helium,riser }}=$ helium density in the riser condition $\left(\mathrm{kg} / \mathrm{m}^{3}\right)$

$g \quad=$ gravitational constant $\left(\mathrm{m} / \mathrm{s}^{2}\right)$

$H \quad=$ core height $(\mathrm{m})$.

This study selected the 600 MWth GTMHR as the reference design. The following basic assumptions were used:

- Core temperature: $900^{\circ} \mathrm{C}$

- Riser temperature: $400^{\circ} \mathrm{C}$

- Reactor pressure: $1 \mathrm{~atm}$

- Core height: $11.0 \mathrm{~m}$

- Air mole fraction of air/helium mixture: 0.5

- Hot leg diameter: $1.5 \mathrm{~m}$ 
Figure 2-30 shows the calculation results, which estimated the maximum static head to be $10.24 \mathrm{~Pa}$ (about $0.01 \%$ of the ambient pressure). This small static head indicates that the energy barrier is very small, a situation that might not be stable in the real reactor. From this number, the minimum compression ratio required to exceed this energy in the gas mixture was calculated. By the ideal gas law, the compression ratio $\left(d V / V_{0}\right)$ at the same temperature can be calculated by

$$
\frac{P_{1}}{P_{0}}=\frac{P_{0}+d P}{P_{0}}=\frac{V_{0}}{V_{1}}=\frac{V_{0}}{V_{0}+d V}
$$

where

$$
\begin{array}{ll}
P_{0} & =\text { initial pressure }(\mathrm{Pa}) \\
P_{1} & =\text { new pressure }(\mathrm{Pa}) \\
V_{0} & =\text { initial volume }\left(\mathrm{m}^{3}\right) \\
V_{1} & =\text { volume pressure }\left(\mathrm{m}^{3}\right) \\
d P & =\text { pressure change }(\mathrm{Pa}) . \\
d V & =\text { volume change }\left(\mathrm{m}^{3}\right) .
\end{array}
$$

\begin{tabular}{|c|c|c|}
\hline \multicolumn{3}{|c|}{ Core Side (Air+He, 50:50) } \\
\hline density (air+He, $900 \mathrm{C}, 1 \mathrm{~atm})$ & 0.166 & $\mathrm{~kg} / \mathrm{m} 3$ \\
\hline $\mathrm{g}$ & 9.8 & $\mathrm{~m} / \mathrm{s} 2$ \\
\hline h & 11 & m \\
\hline density ${ }^{*} g^{*} h$ & 17.8948 & $\mathrm{~Pa}$ \\
\hline \multicolumn{3}{|c|}{\begin{tabular}{|l|} 
Riser Side (He) \\
\end{tabular}} \\
\hline density (He, 400 C, 1atm) & 0.071 & $\mathrm{~kg} / \mathrm{m} 3$ \\
\hline$g$ & 9.8 & $\mathrm{~m} / \mathrm{s} 2$ \\
\hline h & 11 & m \\
\hline density $\mathrm{g}^{*} \mathrm{~h}$ & 7.6538 & $\mathrm{~Pa}$ \\
\hline \multicolumn{3}{|c|}{ Results } \\
\hline Static Head & 10.241 & $\mathrm{~Pa}$ \\
\hline Density (air, 25C, 1 atm) & 1.1452 & $\mathrm{~kg} / \mathrm{m} 3$ \\
\hline Equivalent Air Height & 0.9125044 & m \\
\hline Required Compression (dV/V) & $1.02 \mathrm{E}-04$ & \\
\hline Required Compression in LP & 0.154 & $\mathrm{~mm}$ \\
\hline
\end{tabular}

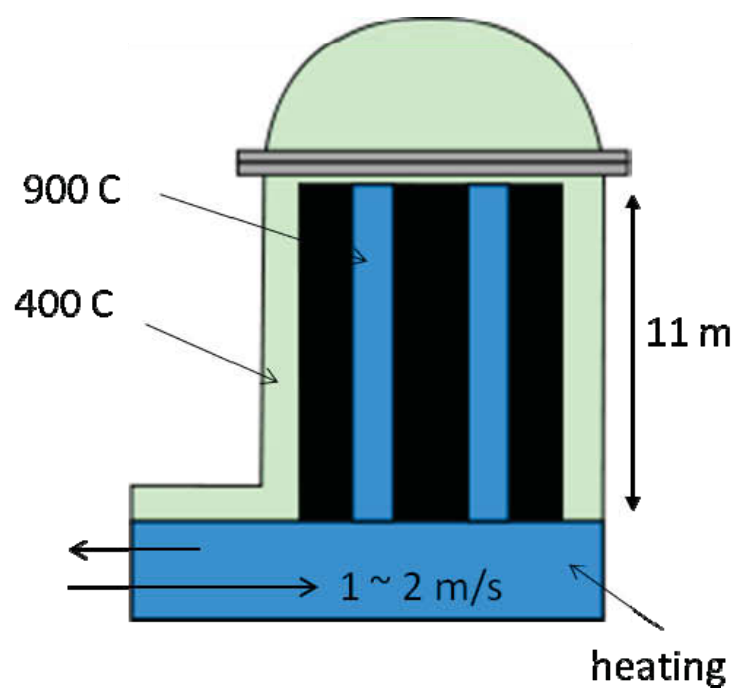

Figure 2-30. Estimated energy barrier for ONC.

The calculated compression rate required to overcome the energy barrier can be easily calculated from Equation (2-84). In this equation, $P_{0}, V_{0}$ is already given and $d P$ represents the energy barrier calculated by Equation (2-83). According to our analyses, about $0.01 \%$ of volume compression is required to overcome this energy barrier. It is equivalent to about $0.154 \mathrm{~mm}$ compression in the lower plenum volume. This volume change is actually very small, on a micrometer scale order, indicating that very slight gas compression in a microscale order can drive enough energy to immediately initiate ONC.

To determine if the Stage-2 stratified flow was stable, reactor flow conditions were scrutinized in greater detail. General lower plenum conditions in Stage-2 were as follows.

- Flow circulation speed: $1.5-3 \mathrm{~m} / \mathrm{s}$ 
- Flow heating from about $100-200$ to $600-900^{\circ} \mathrm{C}$. $\left(\mathrm{dT}=400\right.$ to $\left.800^{\circ} \mathrm{C}\right)$.

The air/helium mixture in Stage-2 is circulating in the lower plenum at about 1.5 to $2.0 \mathrm{~m} / \mathrm{s}$. At the same time, the flow is heated from 150 to $600-900^{\circ} \mathrm{C}$, meaning that the flow can be rapidly expanded inside the reactor. For the given temperature increase, the maximum possible volume change is estimated to be about two to three times that of the original flow volume. This volume expansion can increase the flow speed or the static pressure of the flow, indicating that only 0.002 to $0.005 \%$ volume changes can provide sufficient energy for ONC. Therefore, Stage- 2 is not always in a stable condition. Such stability will depend on the circulation speed, reactor inside temperature profile, and core height.

\subsection{Preliminary 2-D CFD Analysis for Density-Difference Driven Stratified Flow in DEGB Accident}

\subsubsection{Problem Description}

Preliminary calculations were performed using a commercial CFD code known as FLUENT 6.3 (2007) in order to estimate the air ingress that stems from density-gradient driven stratified flow. A short description of the underlying assumptions is given below. The FLUENT code has been used to model the hot duct and reactor vessel of the 600 MWth GTMHR (GA 1997) - a General Atomic, Inc. (GA) design with a prismatic core. Figure 2-31 shows the reactor configuration and simplified FLUENT model, specifying the overall size and dimensions. The total mesh number is 51,566. Figure 2-31 also shows a zoomed in picture of the core part of the meshes. In the real calculations, a much larger cavity and the real confinement size of the GTMHR was used based on the preconceptual design of GTMHR.

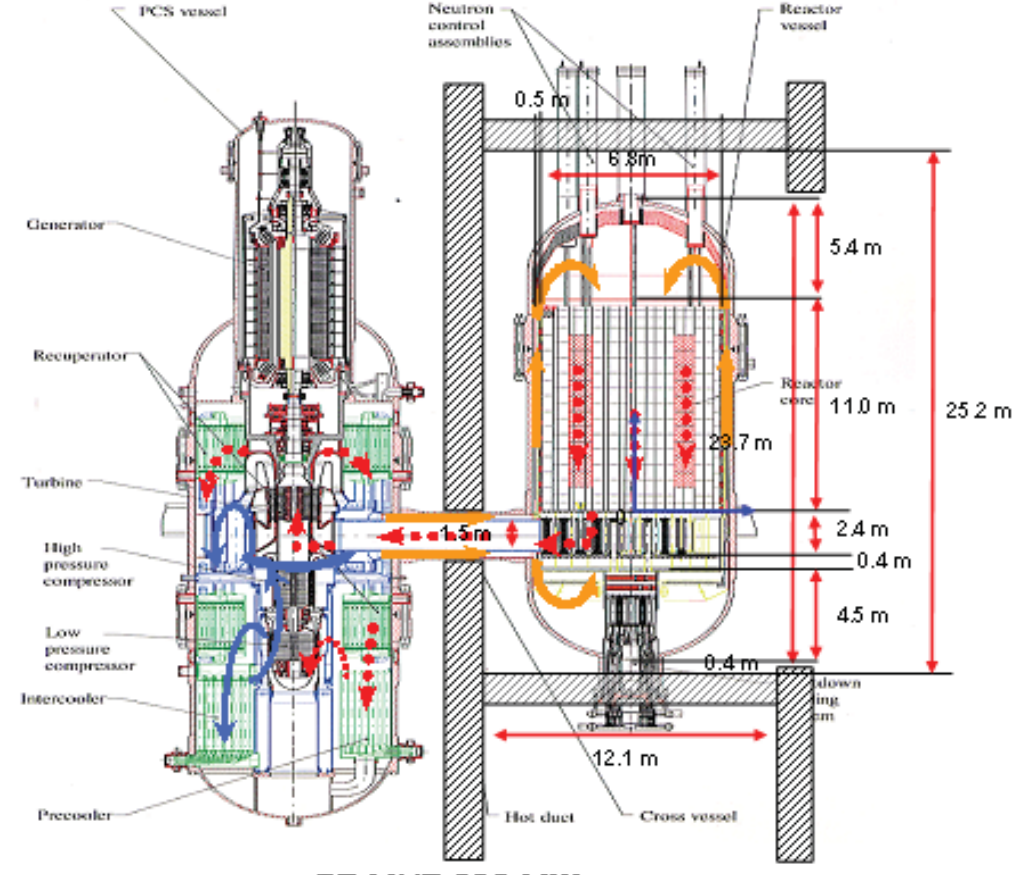

GT-MHR 600 MWt

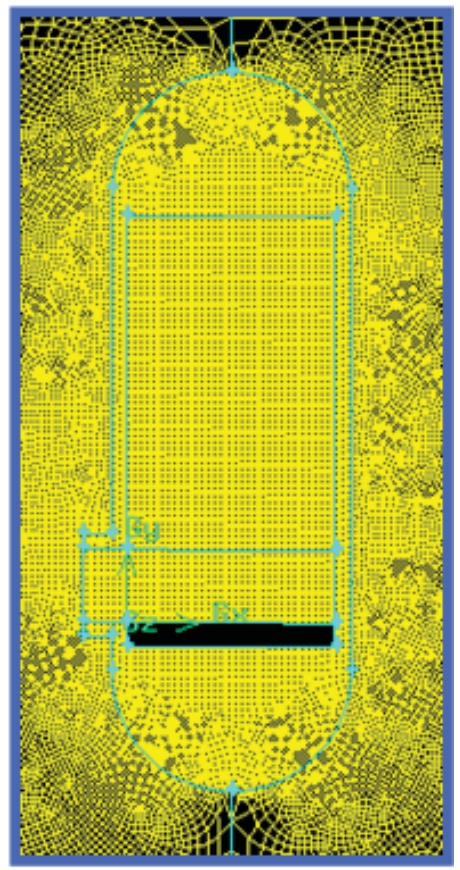

Figure 2-31. Reference reactor (GTMHR 600 MWth) and mesh of FLUENT 6.3. 
The FLUENT solver and model setup were as follows:

- Solver

- 2-D (hexagonal mesh)

- Segregated solver

- $\quad 1$ st order implicit

- Unsteady

- Noniterative time advancement

- Superficial velocity in porous formulation

- Energy equation solve

- Viscous model

- Realizable k-e model

- Species model

- Species transport equation

- Two gas species: Air/helium (diffusion coefficients were calculated by kinetic theory)

- Cavity size was determined by relative volume of vault-to-reactor (22 times larger than the reactor).

- Two different initial air-mole fractions in the cavity were considered: conservative and nonconservative

- Conservative: The cavity air mole fraction was assumed to be 1.00 .

- Nonconservative: Initial air mole fraction in the cavity was assumed to be 0.122 by simple analytic calculation of total air and helium inventories (See Table 2-3).

\subsubsection{Determination of Air Mole Fraction}

The density difference between the reactor cavity and the internal vessel is the main driving force in the stratified flow induction. It can therefore be easily understood that the stratified flow calculation will be highly affected by the air mole fractions. Table 2-3 summarizes the assumed sizes of the reference GTMHR system components. In this section, the volume of both cavity and containment were all considered in the total vault volume. Besides the vault size listed in Table 2-3, infinite vault size was also taken into consideration for conservative analyses.

Table 2-3. Assumed size of the GTMWR 600 MWth system components.

\begin{tabular}{|l|c|c|c|c|c|}
\hline & Confinement & Reactor & PCU & IHX & Vault \\
\hline Diameter $(\mathrm{m})$ & 29 & 7.6 & 7.8 & 3.8 & - \\
\hline Height $(\mathrm{m})$ & 43 & 24 & 30 & 14 & - \\
\hline Volume $\left(\mathrm{m}^{3}\right)$ & 28,388 & 1,088 & 1,433 & 159 & 25,708 \\
\hline Initial air mole fraction & 1 & 0 & 0 & 0 & - \\
\hline Initial pressure & $0.1 \mathrm{MPa}$ & $7 \mathrm{MPa}$ & $7 \mathrm{MPa}$ & $7 \mathrm{MPa}$ & - \\
\hline
\end{tabular}




\subsubsection{Determination of Core Initial Temperature}

The initial temperature distribution in the lower plenum, bottom reflector, and core were calculated by modeling the depressurization process using the GAMMA systems analysis code (Oh et al. 2006). Figure 2-32 shows the GAMMA nodalization for 600 MWth GTMHR. This simulation considered pipe break, depressurization, and the diffusion process. For simplicity, the density driven flow was not considered, because this calculation focuses on the only depressurization process in which the stratified flow does not yet occur.

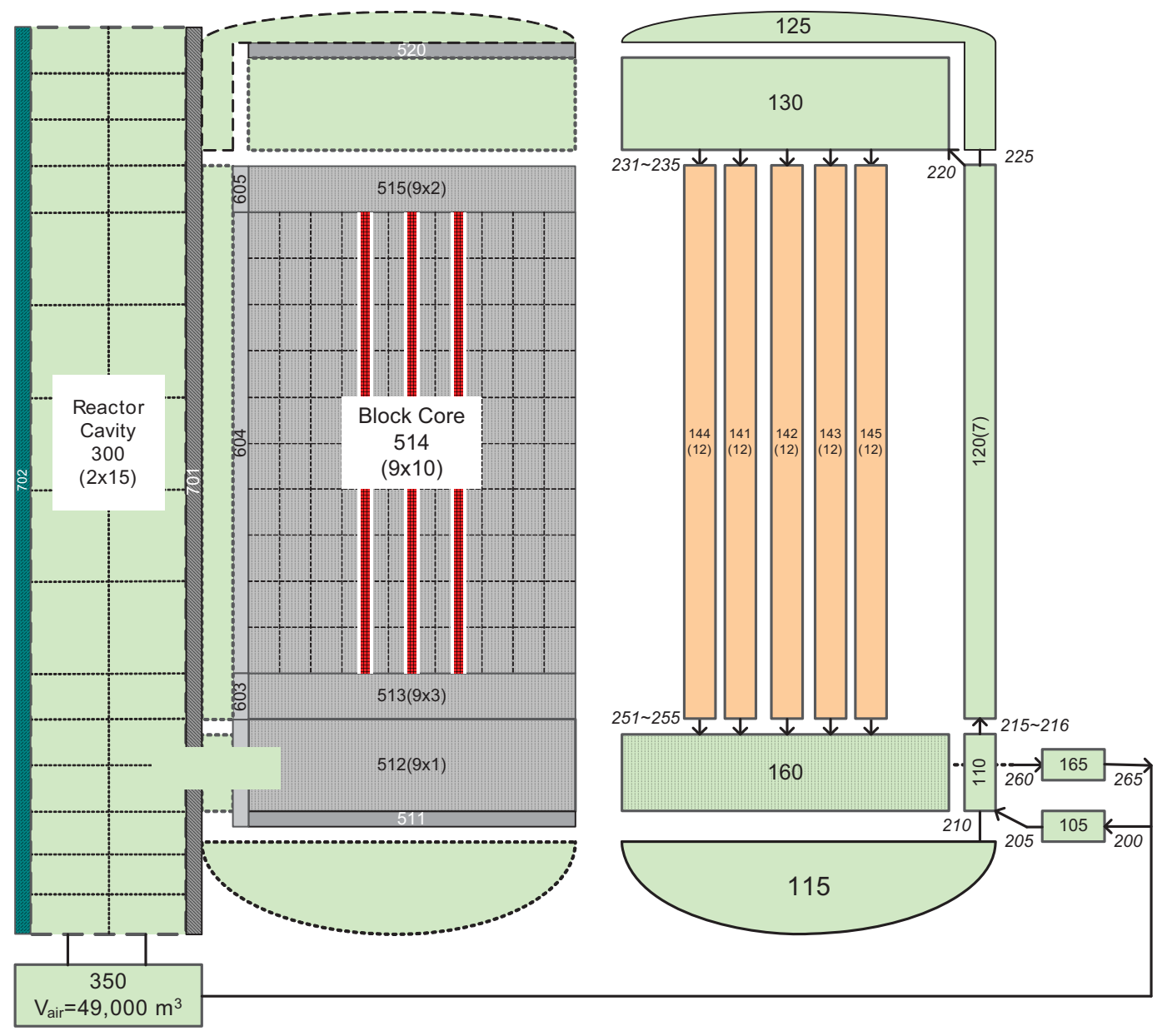

Figure 2-32. 600 MWth GTMHR GAMMA nodalization.

After depressurization analysis by GAMMA code, the core temperature distributions and air concentrations were implemented into the FLUENT input. Figure 2-33 shows the initial conditions for the stratified flow calculation for a nonconservative case. Initially, the reactor vessel was filled with helium.

The solid temperatures in this study were assumed to be constant during the CFD simulation. Figure 2-33 shows the GAMMA estimations on the solid temperatures during the initial 500 seconds following a postulated pipe break. The depressurization phase was completed by 200 seconds when the pressure and the flow between the inside and the outside reactors are equalized. In this calculation, the accident scenario transits to the molecular diffusion process in which the air diffuses into the reactor core slowly by 1-D pipe assumption. Figure 2-34 shows that the solid temperatures are not significantly changing with time for the given calculated time range, which is much larger than the CFD simulation period. Therefore, the constant solid temperature assumption is valid in this CFD analysis. 

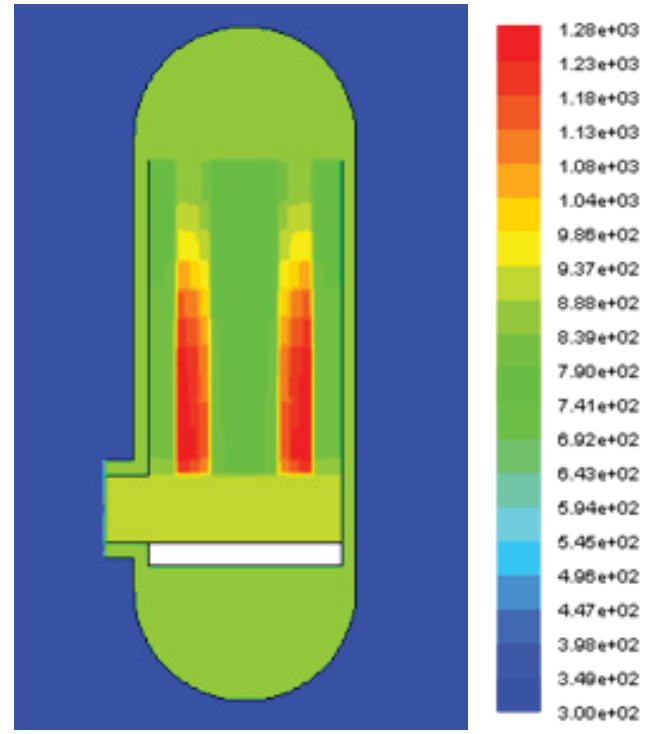

a. Temperature $(\mathrm{K})$

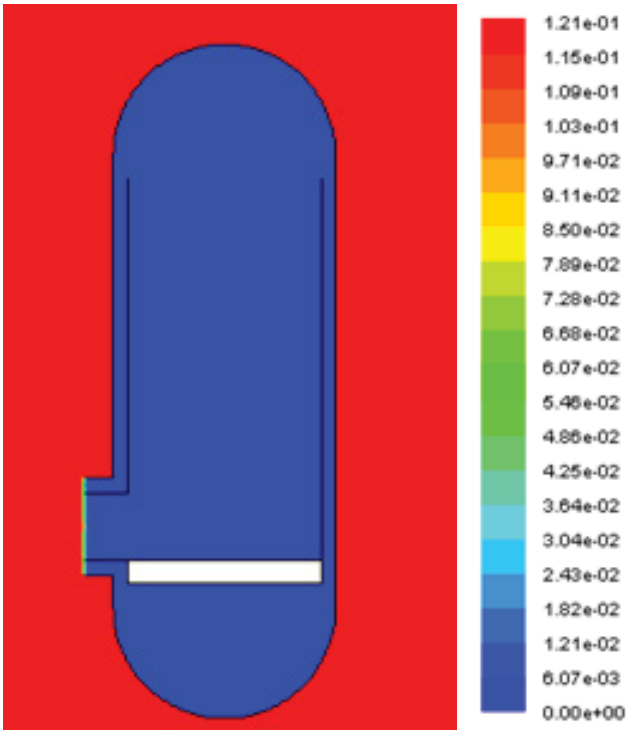

b. Air mole fraction (nonconservative)

Figure 2-33. Initial condition of temperature and air mole fraction for CFD analysis (non-conservative).

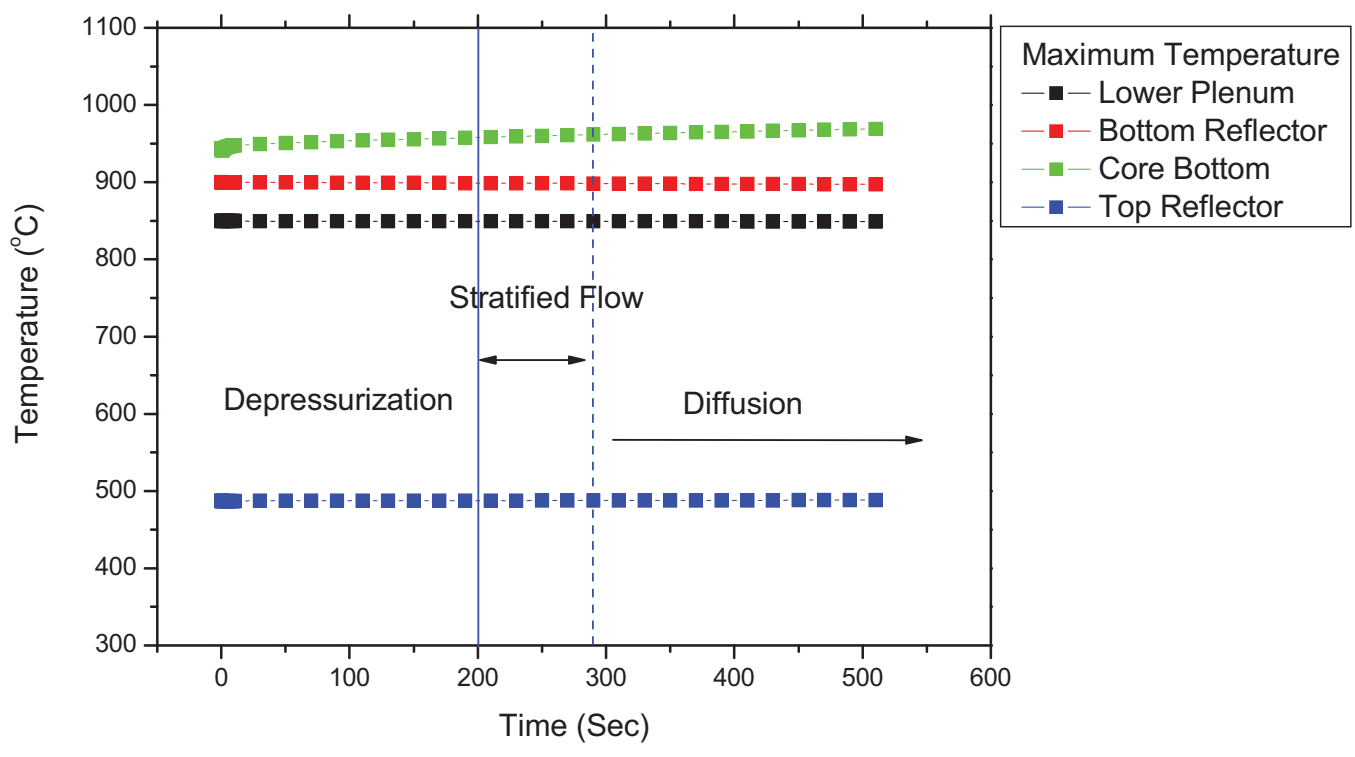

Figure 2-34. Time variations of the temperatures in the reactor core during an air-ingress accident.

\subsubsection{Determination of Porous Body Parameters}

The core and the lower plenum of the reactor were simplified to be porous bodies. In the FLUENT code, porous media are modeled by the addition of a momentum source term to the standard fluid equation. The source term is composed of two parts: a viscous loss term (Darcy, the first term), and an inertial loss term given as

$$
S_{i}=-\left(\sum_{j=1}^{3} D_{i j} \mu v_{j}+\sum_{j=1}^{3} C_{i j} \frac{1}{2} \rho v_{m a g} v_{j}\right)
$$


where $S_{i}$ is the source term for the $i$ th (x,y, or z) momentum equation, and $D$ and $C$ are prescribed matrices. This momentum sink contributes to the pressure gradient in the porous cell, creating a pressure drop that is proportional to the fluid velocity (or velocity squared) in the cell.

A homogeneous porous media equation is expressed in the formula

$$
S_{i}=-\left(\frac{\mu}{\alpha} v_{i}+C_{2} \frac{1}{2} \rho v_{m a g} v_{i}\right)
$$

where $\alpha$ is the permeability and $C_{2}$ is the inertial resistance factor, simply specifying $D$ and $C$ as diagonal matrices with $1 / \alpha$ and $C_{2}$, respectively, on the diagonals (and zero for the other elements).

FLUENT also allows the source term to be modeled as a power law of the velocity magnitude:

$$
S_{i}=-C_{0}|v|^{C_{1}}
$$

where $C_{0}$ and $C_{1}$ are user-defined empirical coefficients.

The two important parameters for defining porous media are porosity and permeability. The detailed processes used to determine these parameters for the reactor core and lower plenum are described below.

\subsubsection{Determination of Porosities}

The porosity is the volume fraction equal to the fluid volume over the total volume (where the total volume equals the fluid volume plus the structural volume) of the region in question. The porosity is used in the calculation of the heat transfer in the medium and in the time-derivative term in the scalar transport equations for unsteady flow. It also influences the calculation of the reaction source terms and body forces in the medium. These sources will be proportional to the fluid volume in the medium.

This study defined the porosities in two regions: the reactor core and the lower plenum. Figure 2-35 shows the single pattern element for the VHTR reactor core block. The porosity of the core zone in the FLUENT input can be determined by the equation

$$
\gamma_{\text {core }}=\frac{V_{\text {fluid }}}{V_{\text {total }}}=\frac{A_{\text {fluid }}}{A_{\text {total }}}=\frac{\frac{1}{8} \pi d^{2}}{\frac{\sqrt{3}}{4} p^{2}} .
$$

The porosity of the lower plenum can be determined from the geometry of Figure 2-36 and Figure 2-37. The porosity of the lower plenum in the FLUENT input can be determined by the following equations.

$$
\gamma_{\text {lowerplenum }}=\frac{V_{\text {fluid }}}{V_{\text {total }}}=\frac{A_{\text {fluid }}}{A_{\text {total }}}=\frac{\frac{\sqrt{3}}{4} p_{L P}{ }^{2}-\frac{1}{8} \pi d_{L P}{ }^{2}}{\frac{\sqrt{3}}{4} p_{L P}{ }^{2}}
$$

where $P_{L P}$ is the pitch of the lower plenum, and $d_{L P}$ is the diameter of the lower plenum.

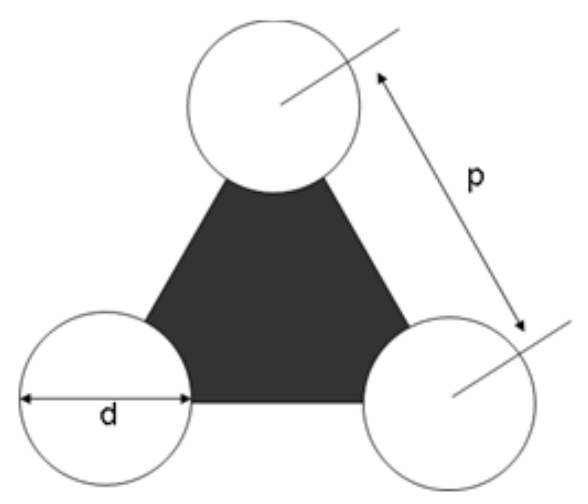

Figure 2-35. Core pattern $(d=1.58 \mathrm{~cm}, \mathrm{p}$ $=3.27 \mathrm{~cm}$ ). (Internal Design Review Presentation Material, General Atomics, San Diego, 1997) 


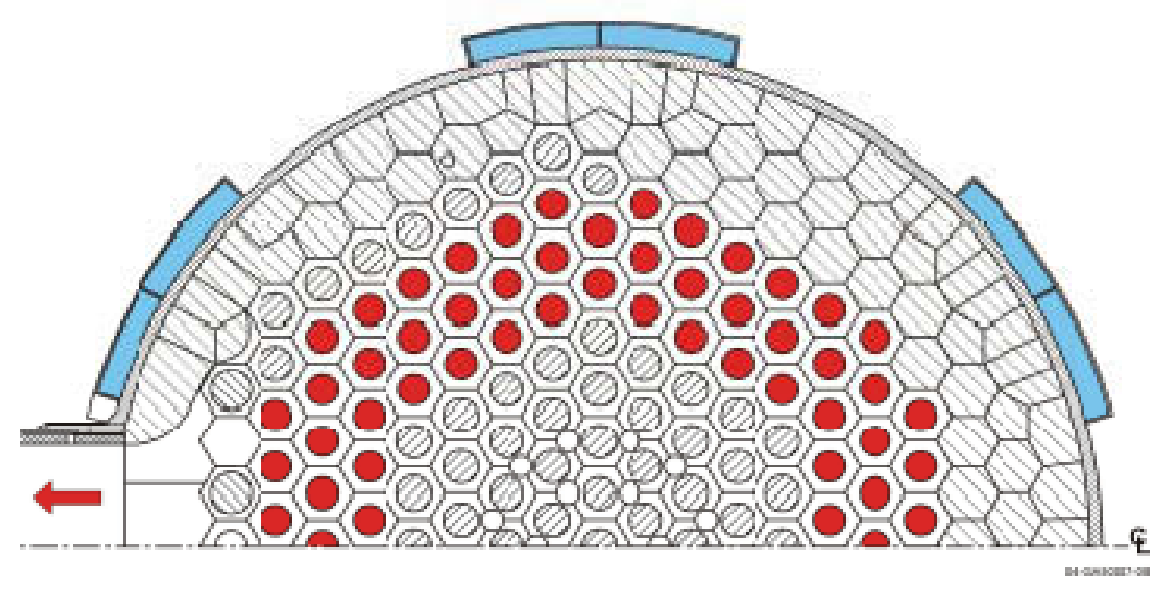

Figure 2-36. Typical geometry of lower plenum $\left(d_{L P}=0.212 \mathrm{~m}, P_{L P}=0.36 \mathrm{~m}\right)$. (The red circle represents rod.) (McEligot and McCreery 2004).
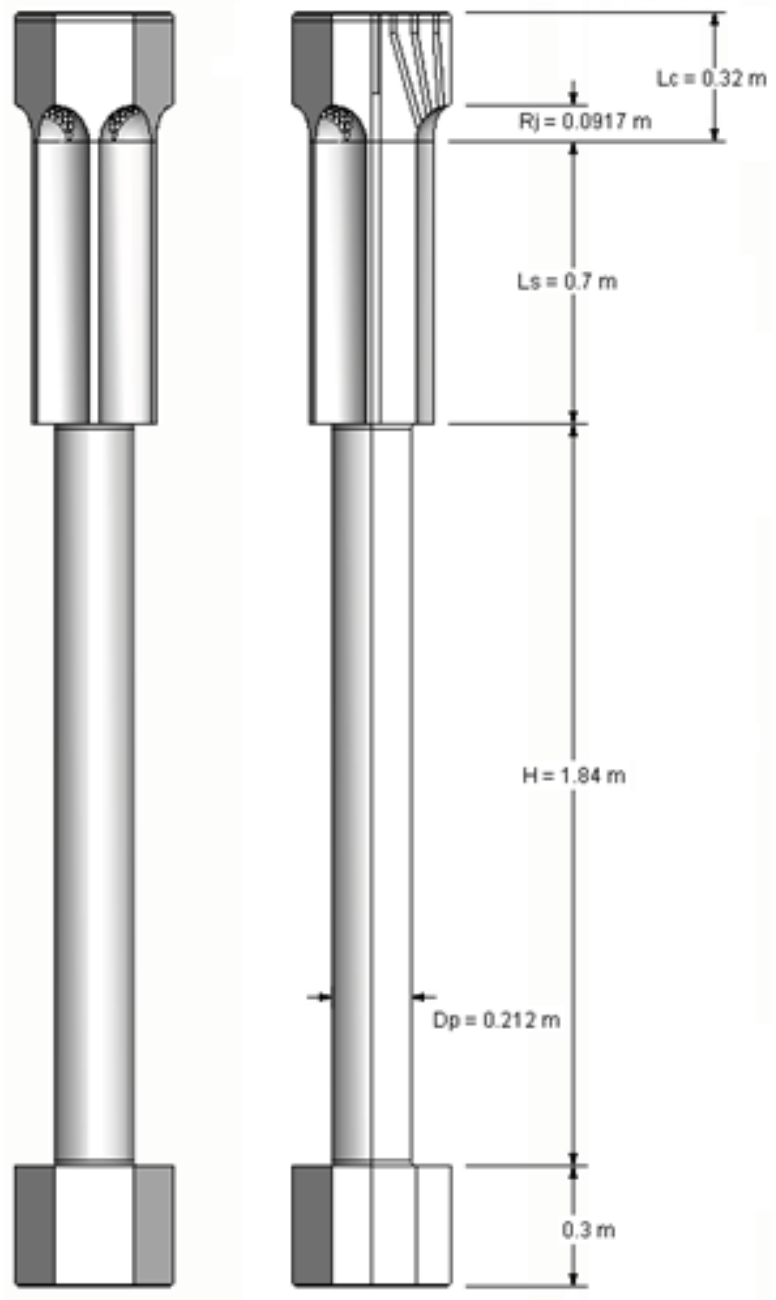

Figure 2-37. Detail view of lower plenum in 600 MWth GTMHR. 
From the above equations, the porosities of the reactor core and the lower plenum were calculated as follows:

- Core

- $\quad \gamma_{\text {core }}=0.21$

- Lower Plenum

- $\gamma_{\text {core }}=0.68$

\subsubsection{Permeability (Viscous and Inertial Resistance Coefficients)}

The porous media model incorporates an empirical determined flow resistance in a region of the model defined as porous. In essence, the porous media model is nothing more than an added momentum sink in the governing momentum equations.

In the reactor core, two permeabilities for different directions require definition: $\mathrm{x}$-direction (horizontal) and y-direction (vertical). Because there is no flow in the horizontal direction of the core, the permeability of the $\mathrm{x}$-direction in the core region is zero. The permeability of vertical direction was determined using the friction loss correlation. In this simulation, the flow rate of the core is governed by density-gradients and is very low. Hence, the flow field in the core for y-direction is assumed to be laminar flow (Lindburg 1997). 94):

In the laminar flow, the friction loss of the circular channel is expressed by Eqs. (2-90) through (2-

$$
\begin{aligned}
& \frac{\Delta P}{L}=f \frac{1}{2} \rho v^{2} \frac{1}{D} \\
& f=\frac{64}{\operatorname{Re}} \\
& \frac{\Delta P}{L}=\left(\frac{64}{\frac{\rho v D}{\mu}}\right) \frac{1}{2} \rho v^{2} \frac{1}{D}=\frac{32 \mu v}{D^{2}}
\end{aligned}
$$

Therefore,

$\frac{\Delta P}{L}=\frac{\mu}{\alpha} v=\frac{32 \mu v}{D^{2}}$

$\alpha=\frac{D^{2}}{32}$

The diameter of the channel in the core is $0.0158 \mathrm{~m}$, so the permeability of the y-direction is $7.8 \mathrm{e}-6 \mathrm{~m}^{2}$.

In the turbulent flow, the friction loss of the circular channel can be determined by the Moody diagram shown in Figure 2-38, which shows the friction loss data from laminar to turbulent region. 


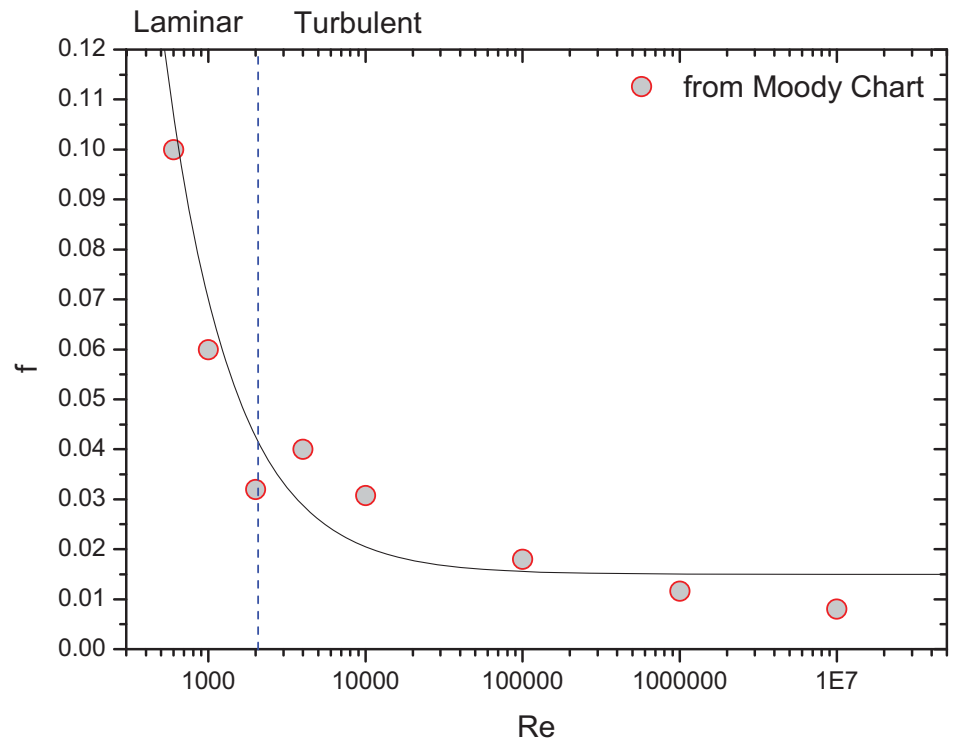

Figure 2-38. Friction factor correlation.

Based on the above graph, the friction factor equation can be correlated as

$$
f=0.015+\frac{55}{\mathrm{Re}}
$$

and the pressure drop equation as

$$
\Delta P=f\left(\frac{1}{2} \rho u^{2}\right)\left(\frac{L}{D}\right) .
$$

Inserting Equation (2-95) into Equation (2-96) derives

$$
\begin{aligned}
& \Delta P=\left(0.015+\frac{55}{\mathrm{Re}}\right)\left(\frac{1}{2} \rho u^{2}\right)\left(\frac{L}{D}\right) . \\
& \Delta P=\left(0.015+\frac{55}{(\rho u D / \mu)}\right)\left(\frac{1}{2} \rho u^{2}\right)\left(\frac{L}{D}\right) . \\
& \frac{\Delta P}{L}=\frac{55}{2 D^{2}} \mu u+\frac{0.015}{D}\left(\frac{1}{2} \rho u^{2}\right) .
\end{aligned}
$$

Therefore, permeability and $\mathrm{C}_{2}$ is

$$
\alpha=\frac{2}{55} D^{2}
$$

and

$$
C_{2}=\frac{0.015}{D}
$$


Because the diameter of the channel in the core is $0.0158 \mathrm{~m}$, the permeability and $\mathrm{C}_{2}$ can be determined as

$\alpha=9.07782 \times 10^{-6} \mathrm{~m}^{2}$

and

$C_{2}=0.949 m^{-1}$

Simulations involving highly anisotropic porous media like this may, over time, pose convergence trouble. This issue is addressed by limiting the anisotropy of the porous media coefficients to 2 or 3 orders-of-magnitude. Even if the medium's resistance in one direction is infinite, the resistance does not need to be set in that direction to be greater than 1,000 times the resistance in the primary flow direction (FLUENT user's guide [2007]). The permeability of the x-direction was therefore defined to be $7.8 \mathrm{e}-9 \mathrm{~m}^{2}$, even though it has zero permeability.

The permeability of the lower plenum y-direction was determined in the same manner to the core, but the flow in the lower plenum is not the circular channel flow. The hydraulic diameter was therefore used to determine the permeability. The hydraulic diameter of the lower plenum can be calculated as

$D_{h}=\frac{4 \text { Area }}{\text { Perimeter }}=0.46 \mathrm{~m}$ (for lower plenum)

Then, the permeability of the lower plenum y-direction can be calculated. The estimated permeability and $\mathrm{C}_{2}$ are calculated by Equation (2-100) and (2-101) as

$$
\begin{aligned}
& \alpha=0.00769 \mathrm{~m}^{2} \\
& C_{2}=0.0326 \mathrm{~m}^{-1}
\end{aligned}
$$

The permeability of the lower plenum in x-direction is different. In this region, the cross flow through the tube array is dominant. Figure 2-39 shows the flow and tube configuration. Figure 2-40 shows some experimental data and correlations considering this type of flow.

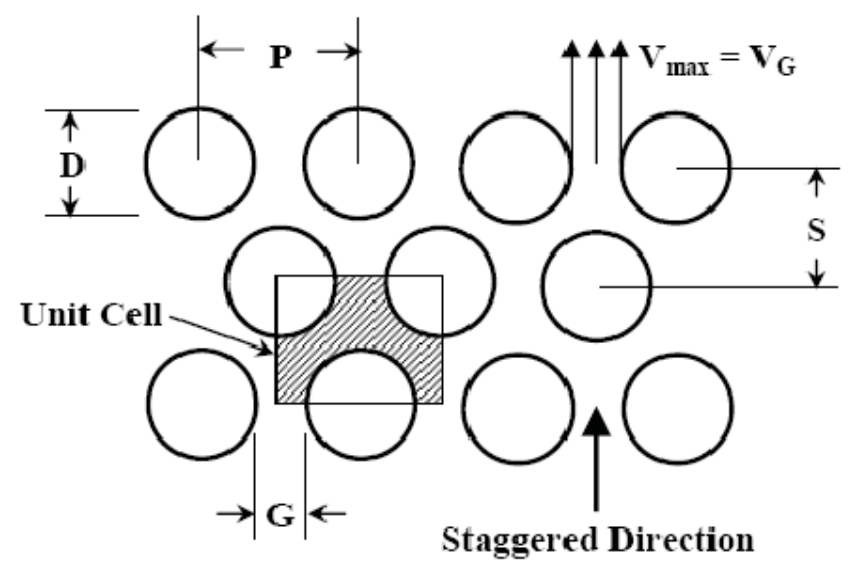

Figure 2-39. Equally spaced triangular tube array (Vassallo and Symolon 2007). 


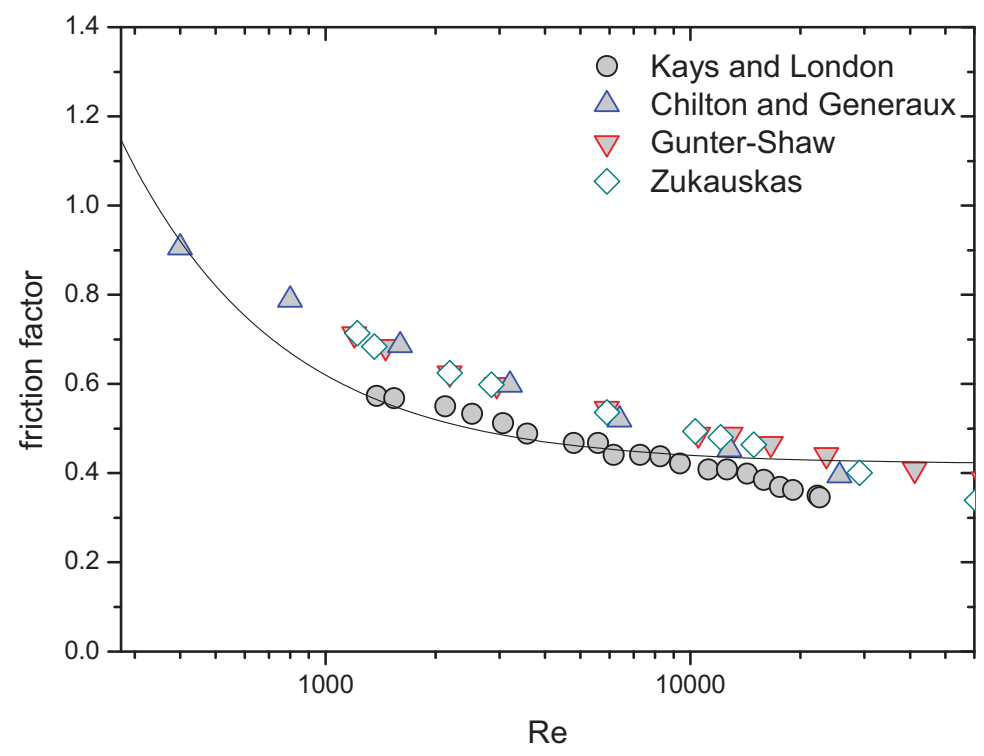

Figure 2-40. Cross-flow friction factor data for staggered tube arrays (Vassallo and Symolon 2007).

Table 2-4 shows the friction factor correlations for the cross flow through the tube array summarized by Kosar (2005).

Permeability and inertia resistance for staggered tube arrays was determined by correlating the friction factor in Figure 2-40 as

$$
f=0.42+\frac{200}{\operatorname{Re}}
$$

Finally, the following correlation can be derived as

$$
\frac{\Delta P}{L}=\frac{100}{D_{h}{ }^{2}} \mu u+\frac{0.42}{D_{h}}\left(\frac{1}{2} \rho u^{2}\right) .
$$

Permeability and $\mathrm{C}_{2}$ can therefore be determined by Equation (2-100) and hydraulic diameter $\left(D_{h}=\right.$ $0.46 \mathrm{~m})$ as

$$
\alpha=0.002116 \mathrm{~m}^{2}
$$

and

$$
C_{2}=0.913 m^{-1}
$$


Table 2-4. Correlation for the friction factor and their mean absolute errors. (Kosar et al. 2005)

\begin{tabular}{|c|c|c|c|c|c|c|}
\hline Correlation & Reference & Fluid & $\begin{array}{c}\text { Reynolds } \\
\text { Number }\end{array}$ & H/D & Configuration & Friction Factor \\
\hline 1 & $\begin{array}{l}\text { Chilton and } \\
\text { Generaux (1933) }\end{array}$ & Air & $\begin{array}{l}\text { Laminar } \\
\text { Region }\end{array}$ & $\begin{array}{l}\text { Long } \\
\text { Tubes }\end{array}$ & $\begin{array}{l}\text { Circular } \\
\text { Staggered }\end{array}$ & $f=\frac{106}{\operatorname{Re}}$ \\
\hline 2 & $\begin{array}{l}\text { Chilton and } \\
\text { Generaux (1933) }\end{array}$ & Air & $\begin{array}{l}\text { Turbulent } \\
\text { Region }\end{array}$ & $\begin{array}{l}\text { Long } \\
\text { Tubes }\end{array}$ & $\begin{array}{l}\text { Circular } \\
\text { Staggered } \\
\text { Inline }\end{array}$ & $\begin{aligned} f & =\frac{C}{\operatorname{Re}^{0.2}} \\
\mathrm{C} & =3 \text { for in-line tube configuration } \\
\mathrm{C} & =1.32 \text { for staggered tube configuration }\end{aligned}$ \\
\hline 3 & Jacob (1983) & Air & $\begin{array}{l}\text { Turbulent } \\
\text { Region }\end{array}$ & $\begin{array}{l}\text { Long } \\
\text { Tubes }\end{array}$ & $\begin{array}{l}\text { Circular } \\
\text { Staggered } \\
\text { Inline }\end{array}$ & $\begin{array}{l}\text { For inline configuration } \\
f=\frac{1}{\operatorname{Re}^{0.35}}\left(0.176+\frac{0.32\left(S_{L} / D\right)}{\left(S_{T} / D-1\right)^{0.43+\left(1.13 D / S_{L}\right)}}\right) \\
\text { For staggered configuration } \\
f=\frac{1}{\operatorname{Re}^{0.16}}\left(1+\frac{0.47}{\left(S_{T} / D-1\right)^{1.08}}\right)\end{array}$ \\
\hline 4 & $\begin{array}{l}\text { Gunter and Shaw } \\
\text { (1945) }\end{array}$ & Air & $\begin{array}{l}\text { Laminar } \\
\text { Region }\end{array}$ & $\begin{array}{l}\text { Long } \\
\text { Tubes }\end{array}$ & $\begin{array}{l}\text { Circular } \\
\text { In-line } \\
\text { Staggered }\end{array}$ & $\begin{array}{l}f=\frac{180}{\operatorname{Re}}\left(\frac{4 S_{T} S_{L}}{\pi D^{2}}-1\right)^{0.4}\left(\frac{D b_{G}}{S_{T}}\right)^{0.6} \\
b_{G}=\frac{S_{L}}{D} \text { (for inline tube configuration) } \\
b_{G}=\frac{S_{d}}{D} \text { (for staggered tube configuration) }\end{array}$ \\
\hline 5 & $\begin{array}{l}\text { Gunter and Shaw } \\
\text { (1945) }\end{array}$ & Air & $\begin{array}{l}\text { Turbulent } \\
\text { Region }\end{array}$ & $\begin{array}{l}\text { Long } \\
\text { Tubes }\end{array}$ & $\begin{array}{l}\text { Circular } \\
\text { In-line } \\
\text { Staggered }\end{array}$ & $\begin{array}{l}f=\frac{1.92}{\operatorname{Re}^{0.145}}\left(\frac{4 S_{T} S_{L}}{\pi D^{2}}-1\right)^{0.4}\left(\frac{D b_{G}}{S_{T}}\right)^{0.6} \\
b_{G} \text { is defined in the previous row }\end{array}$ \\
\hline 6 & $\begin{array}{l}\text { Bergelin et al. } \\
(1950)\end{array}$ & $\begin{array}{l}\text { Mobile } \\
\text { Oil }\end{array}$ & $\begin{array}{l}\text { Laminar } \\
\text { Region }\end{array}$ & $\begin{array}{l}\text { Long } \\
\text { Tubes }\end{array}$ & $\begin{array}{l}\text { Circular } \\
\text { In-line } \\
\text { Staggered }\end{array}$ & $\begin{array}{l}f=\frac{280}{\operatorname{Re}}\left(\frac{1}{a_{B}}\right)^{1.6} \\
\text { where } \\
a_{B}=\frac{S_{T}}{D} \\
\frac{S_{L}}{D} \geq \sqrt{2 \frac{S_{T}}{D}+1} \text { (Inline configuration) } \\
\text { or } \\
a_{B}=\frac{S_{T}}{D} \\
\frac{S_{L}}{D}<\sqrt{2 \frac{S_{T}}{D}+1} \text { (Staggered configuration) }\end{array}$ \\
\hline 7 & Damerow (1972) & Air & $\begin{array}{l}\text { Turbulent } \\
\text { Region }\end{array}$ & $\begin{array}{l}\text { Short } \\
\text { Tubes } \\
2<\mathrm{H} / \mathrm{D}<4\end{array}$ & Airfoils & $f=\left[8.24\left(S_{T} / D\right)^{-1.1}\right] \operatorname{Re}_{D}^{-0.16}$ \\
\hline 8 & Kast (1974) & Air & $\begin{array}{l}\text { Laminar and } \\
\text { Turbulent }\end{array}$ & $\begin{array}{l}\text { Long } \\
\text { Tubes }\end{array}$ & $\begin{array}{l}\text { Circular In-line } \\
\text { Staggered }\end{array}$ & $f=\frac{128}{\operatorname{Re}}+\frac{4}{\operatorname{Re}^{0.16}}$ \\
\hline 9 & $\begin{array}{l}\text { Metzger et al. } \\
(1982)\end{array}$ & Air & $\begin{array}{l}1,000- \\
100,000\end{array}$ & $\begin{array}{l}\text { Short } \\
\text { tubes } \\
\mathrm{H} / \mathrm{D}=1\end{array}$ & $\begin{array}{l}\text { Rectangular } \\
\text { Staggered Short } \\
\text { Tubes } \\
\mathrm{H} / \mathrm{D}=1, \mathrm{P}_{\mathrm{T}}=2.5 \\
\mathrm{P}_{\mathrm{L}}=1-5\end{array}$ & $\begin{array}{l}\text { For } 1,000<\operatorname{Re}<10,000 \\
f=1.268 \mathrm{Re}^{-0.132} \\
\text { For } 10,000<\operatorname{Re}<100,000 \\
f=7.04 \mathrm{Re}^{-0.318}\end{array}$ \\
\hline 10 & $\begin{array}{l}\text { Taborek } \\
\text { (1983) }\end{array}$ & $\begin{array}{l}\text { All } \\
\text { Fluids }\end{array}$ & All Re & $\begin{array}{l}\text { Long } \\
\text { Tubes }\end{array}$ & $\begin{array}{l}\text { Circular Both inline } \\
\text { and staggered }\end{array}$ & $\begin{array}{l}f=4 b_{1}\left(\frac{1.33}{S_{T} / D}\right)^{b}(\mathrm{Re})^{b 2} \\
b=\frac{b_{3}}{1+0.14(\mathrm{Re})^{b 4}}\end{array}$ \\
\hline
\end{tabular}




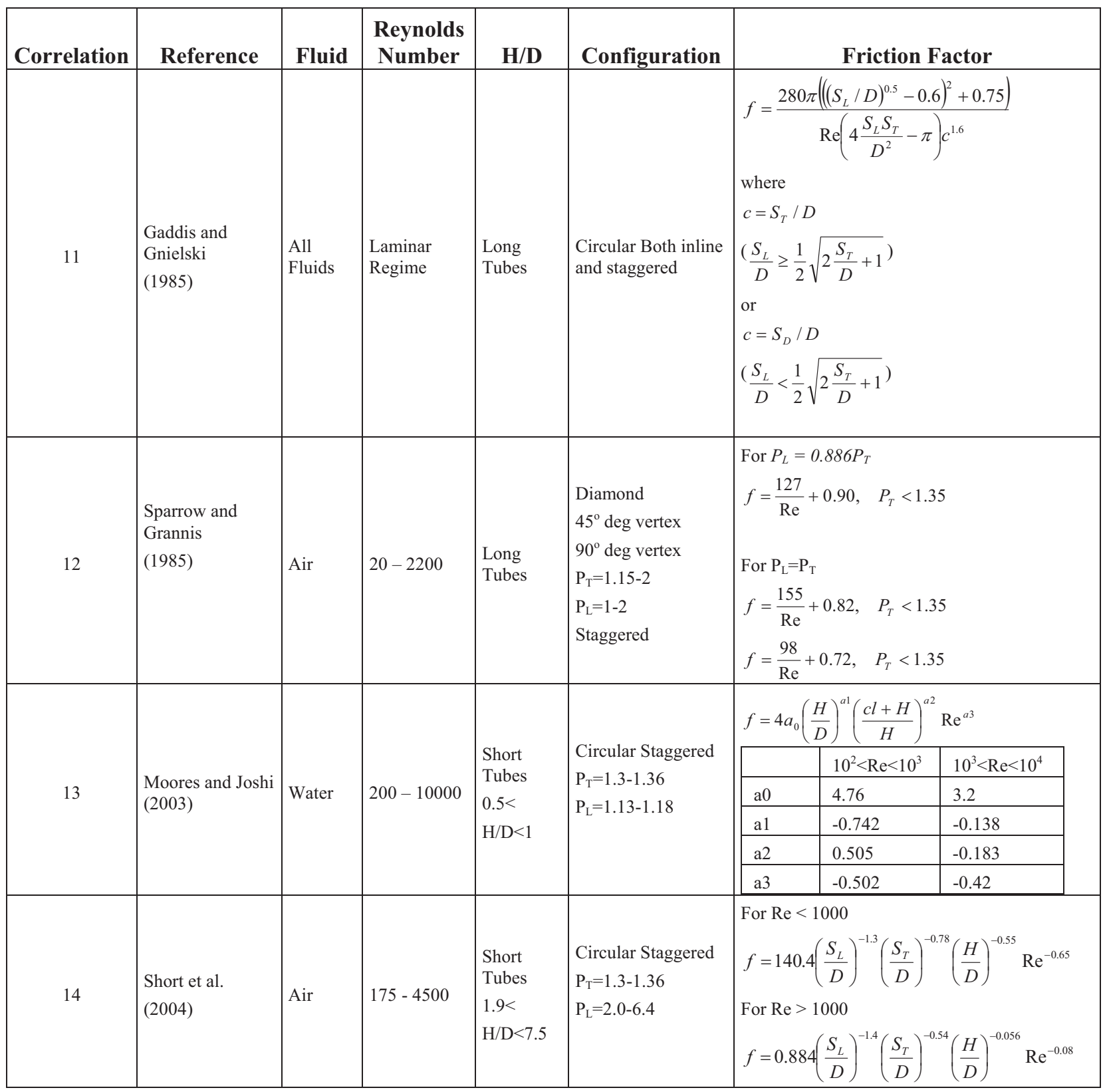

\subsubsection{Simulation Results}

Figure 2-41 is a snapshot of the FLUENT simulation in which the natural convection was initiated about 2-3 minutes after the stratified flow started. And, after 4 minutes, the whole reactor core and vessel was filled with air. It is much quicker than the previous predictions ( $150 \mathrm{hrs})$ using the diffusion driven flow assumption (Takeda 1997, Kadak 2005, Oh et al. 2006). 


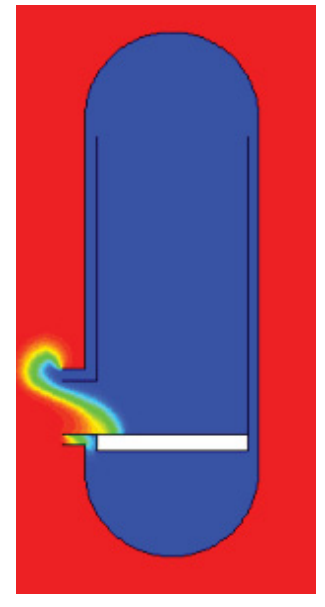

(a) $1.0 \mathrm{sec}$

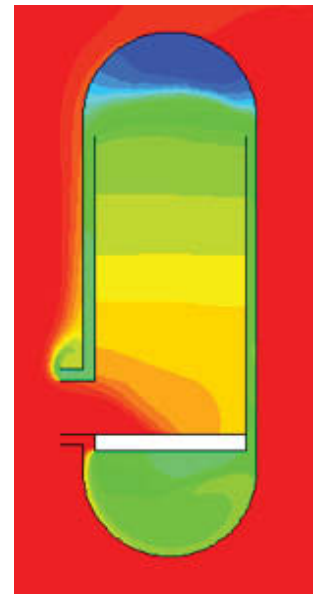

(f) $32.0 \mathrm{sec}$

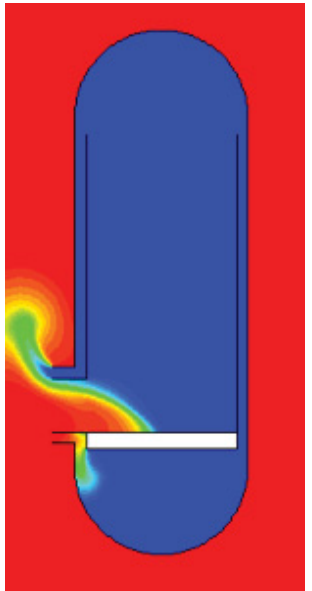

(b) $2.0 \mathrm{sec}$

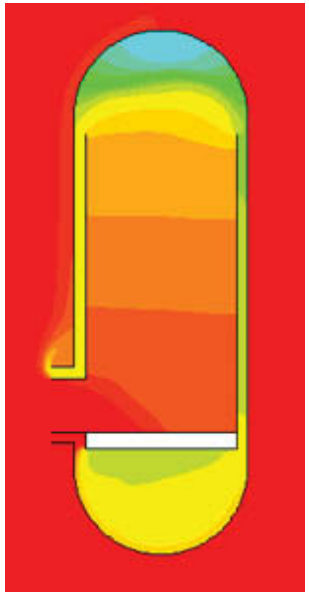

(g) $64.0 \mathrm{sec}$

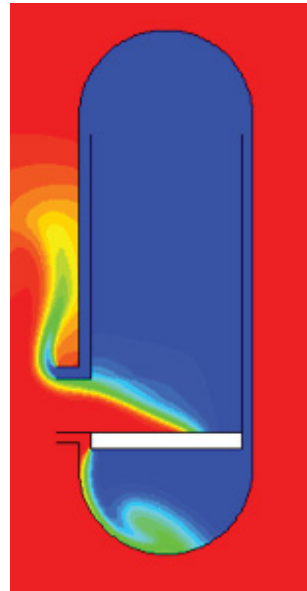

(c) $4.0 \mathrm{sec}$

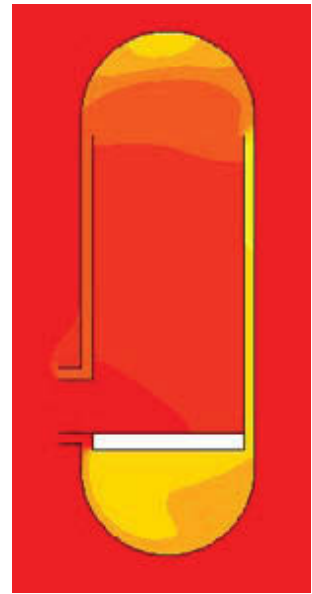

(h) $128.0 \mathrm{sec}$

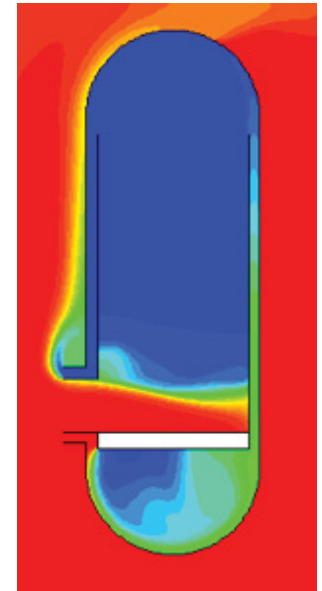

(d) $8.0 \mathrm{sec}$

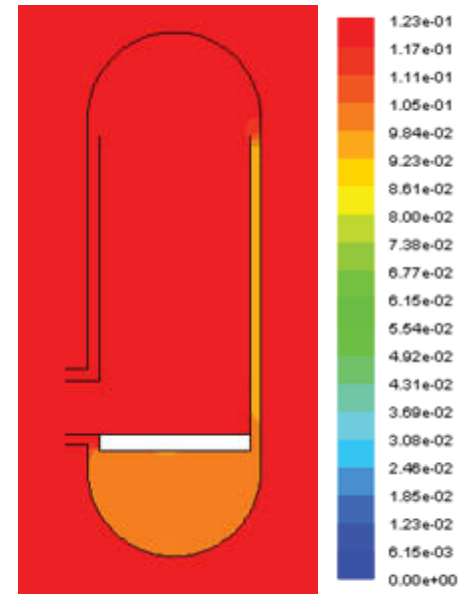

(i) $256.0 \mathrm{sec}$

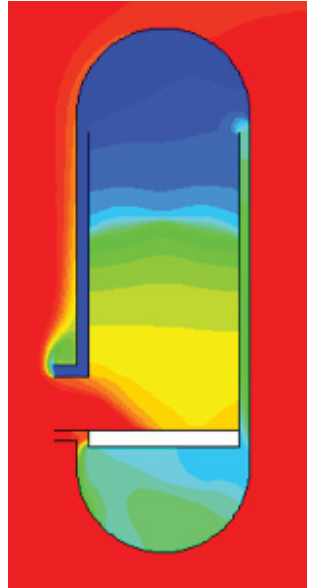

(e) $16.0 \mathrm{sec}$

Figure 2-41. CFD analysis results (air mole fraction) of stratified flow in VHTR (nonconservative).

\subsection{Preliminary 3-D CFD Analyses for DEGB Air-ingress Accident}

A 3-D CFD analysis with CFX-12 was performed for the air ingress accident of the $600 \mathrm{MWth}$ GTMHR reactor under the assumption of a DEGB in order to understand the air ingress behavior in detail and estimate the ONC time. According to previous research, FLUENT 2-D results show that the ONC time is about 200 seconds, which differs greatly from the 1-D GAMMA results of about 150 hours (Oh et al. 2008). The FLUENT 2-D analysis used a simplified porous model with a friction factor correlation and an approximated thermal equilibrium model to simulate the hydraulic resistance because of a friction and form loss and the heat transfer between the air and the solid structure in the lower plenum and the core block. The 3-D CFD analysis with the real grid model, especially for the lower plenum, was introduced to verify the 2-D FLUENT results.

The air ingress phenomenon is usually driven by the stratified flow and the pressure build-up in the lower plenum during air heat up and reduced inertia in the recirculation pattern. Air ingress may also be interrupted by the hydraulic resistance that takes place when the air passes a complicated geometry in the reactor. It can therefore be expected that an exactly simulated grid model for the complicated geometry of the lower plenum and core block can accurately predict the propagation of the air ingress inside the 
reactor. A grid interface function that connects two nonconformal meshes was used to complete the 3-D grid model because of the complicated nature of combining the consecutive mesh generation for the lower plenum, core blocks, and coolant riser within a single model.

The grid interface implemented in the CFX-12 (ANSYS 2009) is superior to that of other CFD codes (Kang 2006); however, the 3-D DEGB analysis by CFX-12 cannot simulate the helium blow-down phase with a decay heat generation in the core blocks. This is because CFX-12 has trouble obtaining fully converged solutions for the large pressure difference between the reactor and the confinement in the blow-down phase, and there is presently no implemented model for decay heat generation. The CFD calculations were therefore made at the pressure equalization between the confinement and the reactor vessel following the high pressure helium blow down to the confinement. The 3-D CFX analysis should therefore be carefully used to only predict the air ingress behavior because of the density driven stratified flow, buoyant flow by heat transfer, and hydraulic flow interrupted by complicated geometry. If the 3-D CFX analysis is able to predict the physical characteristics of an air ingress accident, the 3-D CFX analysis may also be used to find a mitigation method for the air ingress accident.

\subsubsection{3-D Grid Model}

In order to calculate the air inflow from the confinement into the reactor vessel through the broken pipes, a half symmetric grid model, shown Figure 2-42(a), simulating the confinement and the reactor vessel internal was generated based on the design data of the 600 MWth GTMHR (Oh et al. 2008). The inner and outer reflectors were also modeled to simulate the solid heat structure and the flow path formed from the core block upper region to the coolant riser upper region in the air ingress accident. A hexahedral mesh was separately generated by ICEM-CFD software (ANSYS 2008) for all regions in the reactor and confinement except the lower plenum, and then all separated models were connected by using the grid interface function of CFX-12. The lower plenum grid model was initially generated by using GAMBIT with hexahedral, tetrahedral, and pyramidal meshes (Johnson 2008). It was transformed to the grid model for CFX-12 by ICEM-CFD.

All meshes were densely distributed in a fluid region of the grid model, except the confinement, to prevent numerical diffusion and assure a low courant number (Eq. (2-111)). About two millions mesh cells were generated for all the core blocks to predict the air ingress more accurately because the expected flow regime in the core blocks is a buoyant flow because of heat transfer between the core block walls and the air. The 2-mm bypass gaps between the core blocks were neglected to avoid the large number of cells required to resolve a 2-mm gap. The expected CFD results with the bypass gap are not expected to differ greatly from those without the bypass gap. In the confinement, a coarse mesh distribution was used, except around the broken pipes and the reactor vessel wall, because locally precise CFD results are not necessary for the regions far from the broken pipes and the reactor vessel walls. Thirty CFX parallel licenses are being used to compute the air ingress phenomena in the HTGR reactor and the confinement with a total of 8.5 million meshes.

Courant Number $=\mathrm{V} \Delta \mathrm{t} / \Delta \mathrm{x}$

where

$$
\begin{aligned}
\mathrm{V} & =\text { Fluid velocity }(\mathrm{m} / \mathrm{s}) \\
\Delta \mathrm{t} & =\text { Time step }(\mathrm{sec}) \\
\Delta \mathrm{x} & =\text { Mesh length }(\mathrm{m}) .
\end{aligned}
$$



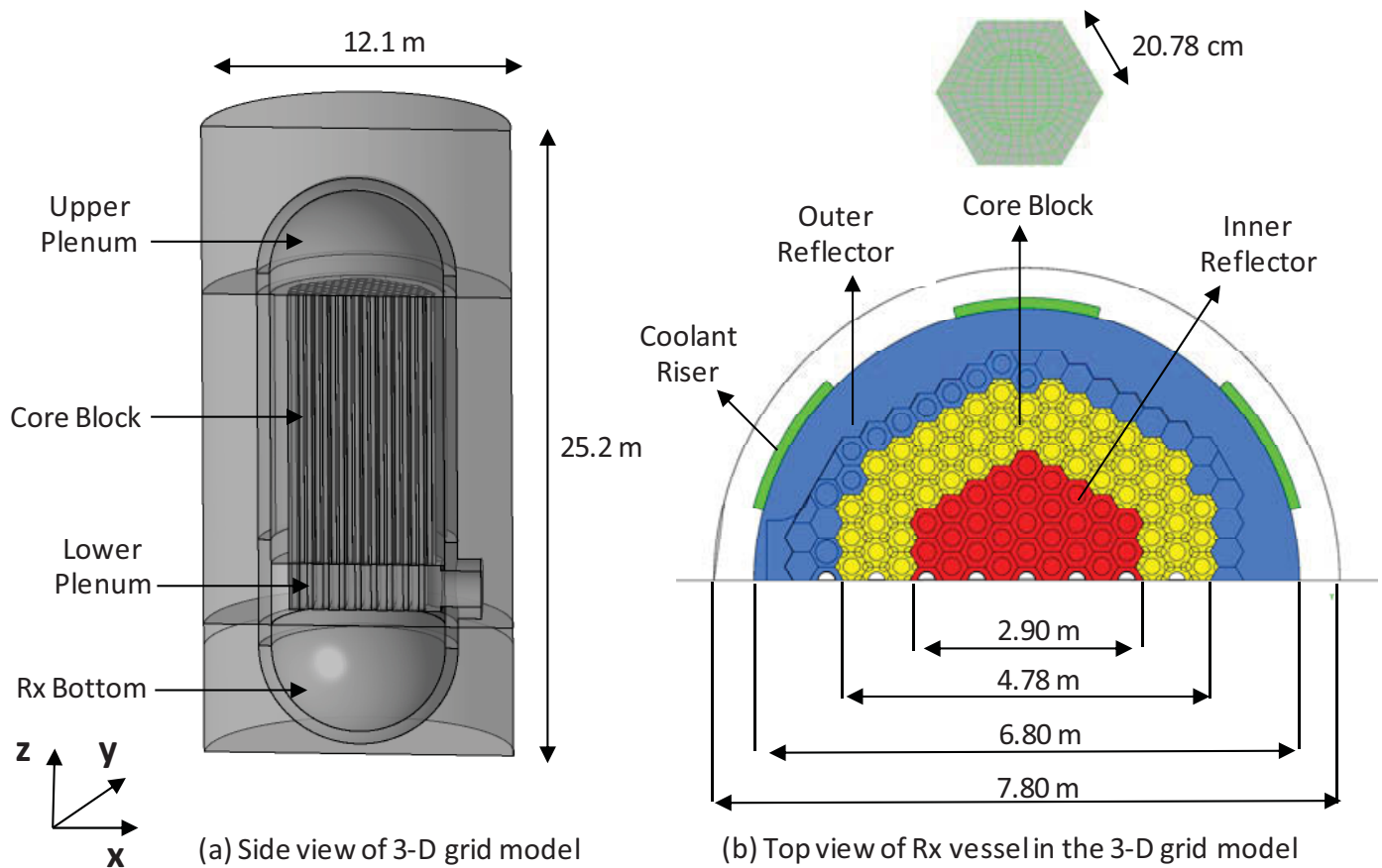

(1)

(b) Top view of Rx vessel in the 3-D grid model

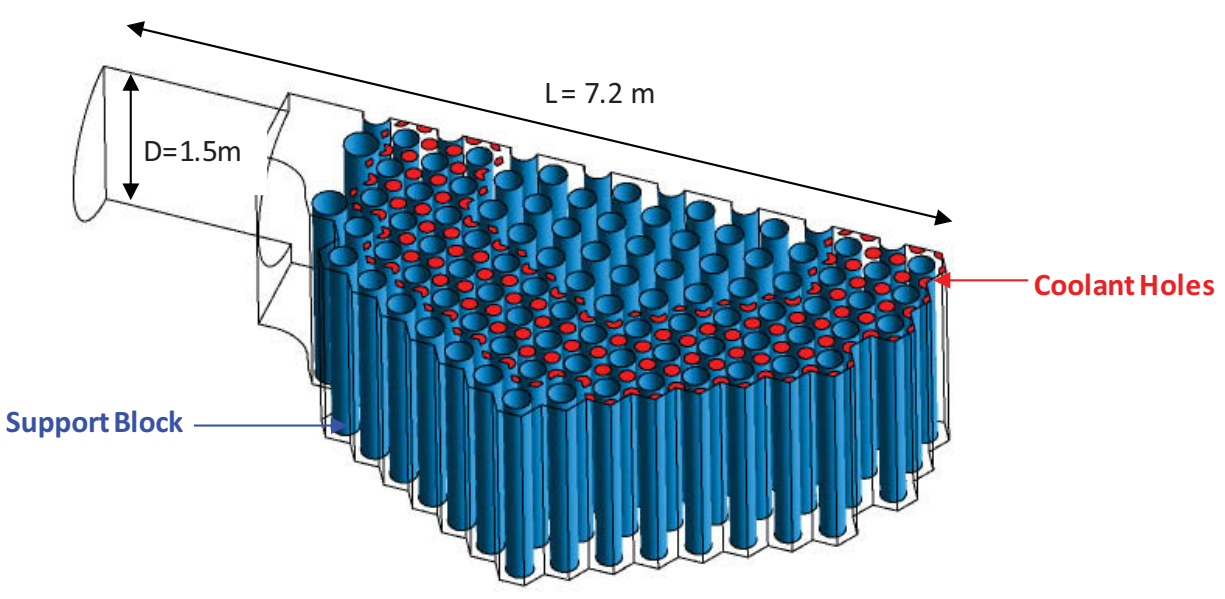

(c) Lower plenum model

\section{Figure 2-42. 3-D grid model for the DEGB analysis.}

The shutdown cooling system located in the reactor bottom region and several guide tubes in the upper plenum were neglected in the grid model because the anticipated advantages of those models are not essential in predicting the air ingress from the confinement into the core blocks and the coolant riser. The detailed information of the mesh distribution and the geometry are shown in Table 2-5. 
Table 2-5. Number of mesh and volume data for each region in the 3-D grid model.

\begin{tabular}{|c|c|c|}
\hline \multicolumn{2}{|r|}{ Reactor Internal } & Confinement \\
\hline Core Blocks & $\begin{array}{l}\text { Volume : } 60.35 \mathrm{~m}^{3} \text { (Volume Porosity : } 0.185 \text { ) } \\
\text { Height : } 10.82 \mathrm{~m} \\
\text { Hexahedral mesh : 2,248,560 }\end{array}$ & \multirow{6}{*}{$\begin{array}{l}\text { Volume : } 961.05 \mathrm{~m}^{3} \\
\text { Hexahedral mesh }: 621,183 \\
\text { Fluid volume ration the } \\
\text { confinement to the reactor } \\
\text { internal : } 3.81\end{array}$} \\
\hline Lower Plenum & $\begin{array}{l}\text { Volume }: 15.29 \mathrm{~m}^{3} \\
\text { Height }: 1.84 \mathrm{~m} \\
\text { Hexahedral mesh }: 677,917 \\
\text { Tetra mesh }: 25,940 \\
\text { Pyramids mesh }: 1,103 \\
\end{array}$ & \\
\hline Upper Plenum & $\begin{array}{l}\text { Volume : } 66.27 \mathrm{~m}^{3} \\
\text { Radius : } 3.4 \mathrm{~m} \\
\text { Hexahedral mesh : } 712,023 \\
\end{array}$ & \\
\hline Coolant Riser & $\begin{array}{l}\text { Volume : } 6.98 \mathrm{~m}^{3}\left(2.328 \mathrm{~m}^{3} \times 3\right) \\
\text { Height : } 9.87 \mathrm{~m} \\
\text { Hexahedral mesh : } 287,820\left(2.328 \mathrm{~m}^{3} \times 3\right)\end{array}$ & \\
\hline Rx Bottom & $\begin{array}{l}\text { Volume : } 82.33 \mathrm{~m}^{3} \\
\text { Hexahedral mesh }: 651,963\end{array}$ & \\
\hline \multirow[t]{2}{*}{ Reflector and Solid Regions } & $\begin{array}{l}\text { Volume : } 204.58 \mathrm{~m}^{3} \\
\text { Hexahedral mesh : 3,075,831 }\end{array}$ & \\
\hline & Total meshes number : $8,517,835$ & \\
\hline
\end{tabular}

\subsubsection{Initial, Boundary, Porous Media Conditions and Properties}

This 3-D DEGB CFX analysis assumed that the helium discharge from the reactor into the confinement through the broken pipes has already completed and global pressure equilibrium between the confinement side and the inside of the reactor. All initial conditions of the concentration, temperature, and pressure were computed using the GAMMA code and those values were used in CFD calculations as initial conditions. This was done because a large computation time would be necessary to get a well converged solution for the helium blow-down phase.

The initial conditions shown in Figure 2-43 for the air mass fraction, temperature, and confinement and reactor pressure, including the inner and outer reflectors, were given according to the GAMMA results and hand calculation results for the blow-down phase (Oh et al. 2008). The air mass fraction of 0.5 for the confinement was simply calculated by considering the pressure and volume difference between the confinement and the reactor with the ideal gas law during the blow-down phase. The initial pressure distribution along an elevation was automatically calculated by CFX-12 with a gravitational direction and density value.

Based on the GAMMA results, a constant temperature condition shown in Figure 2-44 for the wall boundary condition was applied along the core block walls, surface of the core support block, and surface of the reactor vessel. In the core wall temperature condition, the temperature of the core upper region shown in Figure 2-44 A is lower than that of the core lower region shown in Figure 2-44 B because the helium passes from the upper region into the lower region at the normal operation. The constant wall temperature conditions may be verified because the solid structure temperature is not changed, at least for several minutes. The symmetric condition is also applied on the 180 -degree cut plane of the grid model. 


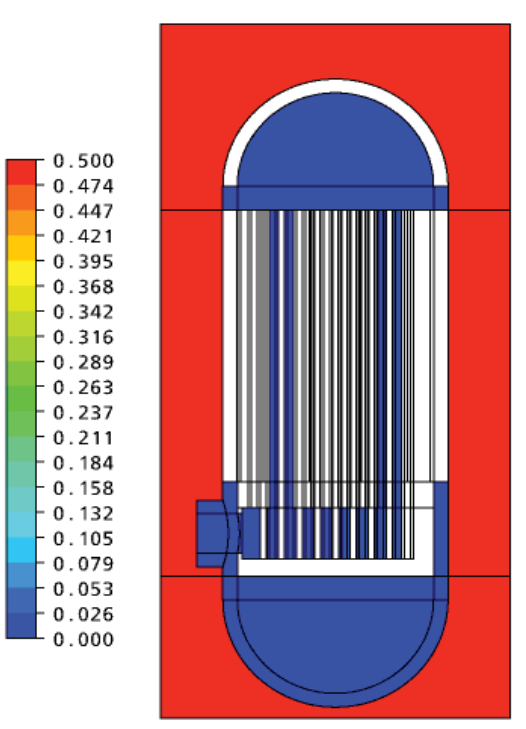

(a) Air mass Fraction

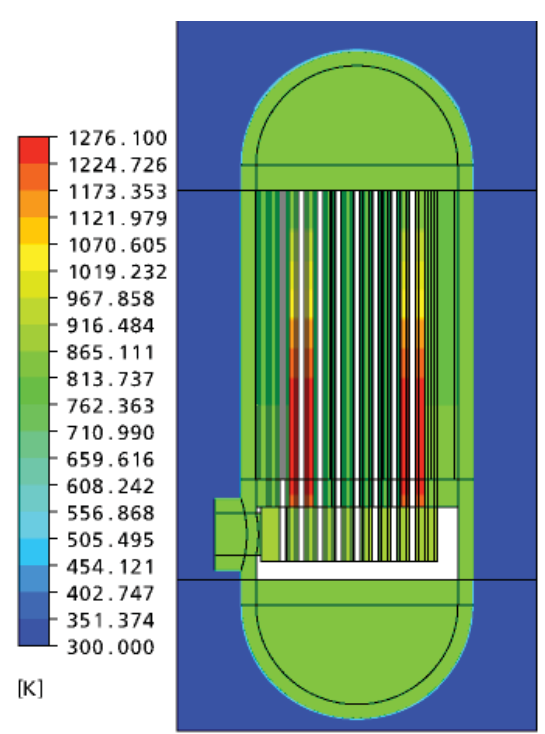

(b) Temperature (K)

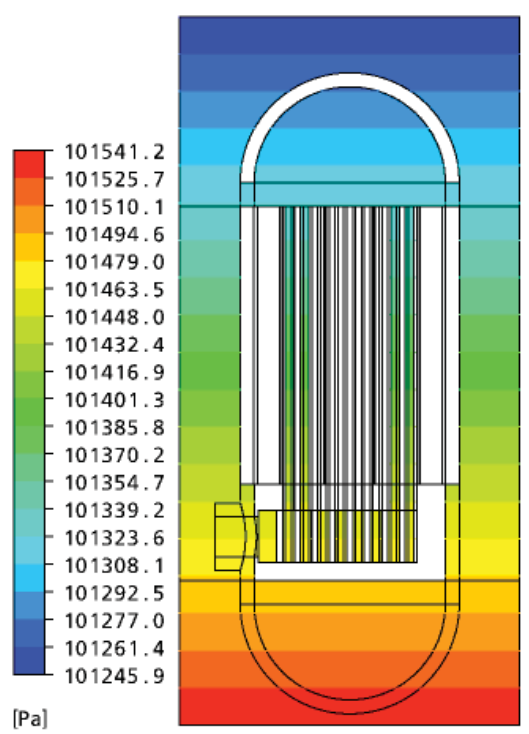

(c) Pressure $(\mathrm{Pa})$

(Contours are plotted on the plane of $y=0.01 \mathrm{~m}$. Symmetry plane is $\mathrm{y}=0.0 \mathrm{~m}$ )

Figure 2-43. Initial air mass fraction, temperature, and pressure conditions for 3-D CFX analysis.

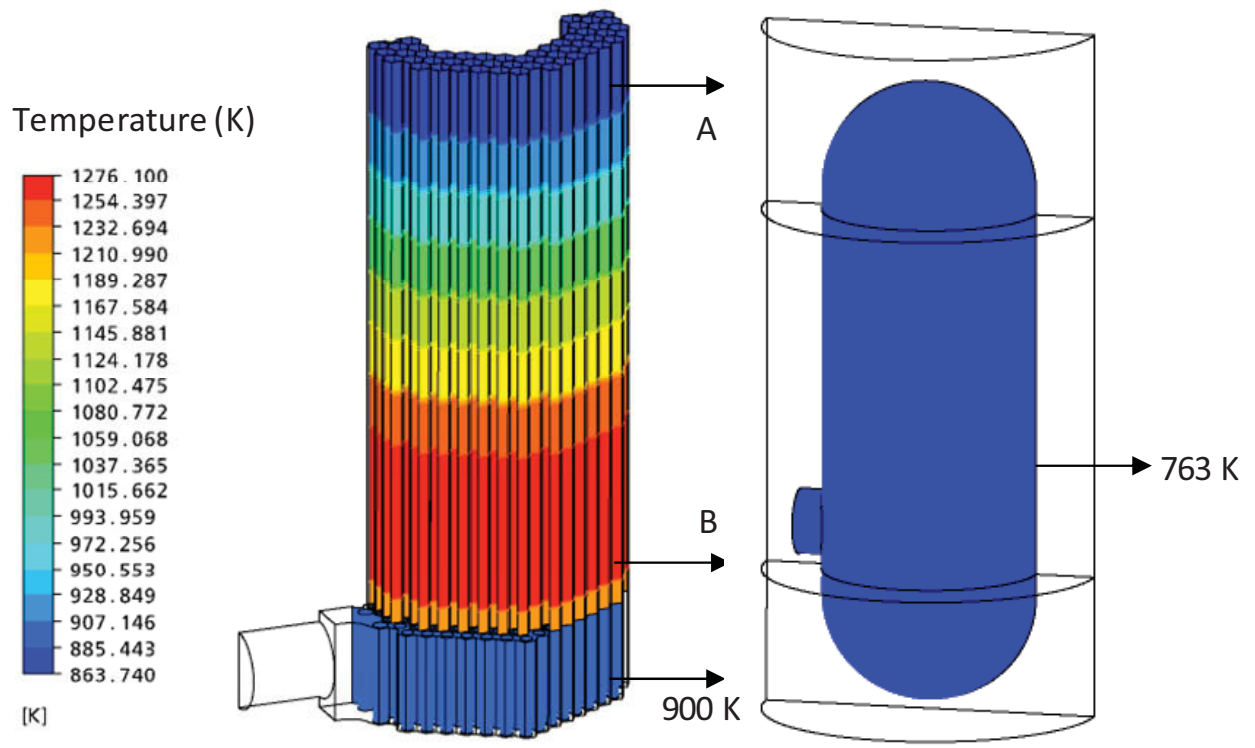

Figure 2-44. Wall temperature conditions for the core blocks, support blocks, and reactor vessel.

A porous media condition was applied to the core blocks to simulate a pressure drop through the core blocks when helium or air flows along the core blocks. This was done to simulate 108 coolant channels with diameters of 12.7 and $15.8 \mathrm{~mm}$ per core block (Oh et al. 2008). The porous media condition was given in terms of permeability $\left(\mathrm{K}_{\text {perm }}\right)$, a loss coefficient $\left(\mathrm{K}_{\text {loss }}\right)$, and volume porosity (Eq. (2-112)). The velocity $\left(\mathrm{V}_{\mathrm{i}}\right)$ used in the Eq. (2-112) is a true velocity that can be obtained by dividing the superficial velocity with the volume porosity (Eq. (2-113)). The true velocity concept of the porous media model may be important in the air ingress accident. The calculated turbulent viscosity based on the true velocity gradient can have an effect on the diffusion term of the species transport equation. 
$-\frac{\partial p}{\partial x_{i}}=\frac{\mu}{K_{\text {perm }}} V_{i}+K_{\text {loss }} \frac{\rho}{2}|V| V_{i}$

True Velocity $=$ Superficial Velocity/Volume Porosity

Experimental data are needed to give the accurate porous conditions simulating the core pressure drop under the air ingress accident because no other test data is available. Thus, conceptual design data regarding the core pressure drop (GA 1996) at a normal operation condition were introduced to generate the porous condition values. A theoretically obtained porous condition should also be verified by the comparison of the calculated pressure drop values and the conceptual design data before applying it to the air ingress accident analysis. A steady-state calculation was performed using normal GTMHR operating conditions (GA 1996) to show the pressure drop of the core blocks and the reactor vessel from the cold duct to the hot duct. The calculated pressure distribution is shown in Figure 2-45 and the comparison results of the core pressure drop and reactor pressure drop between the conceptual design data and CFD results (Table 2-6) show good agreement (within 10\%). It was therefore judged that these porous conditions may be used for the air ingress accident analysis.

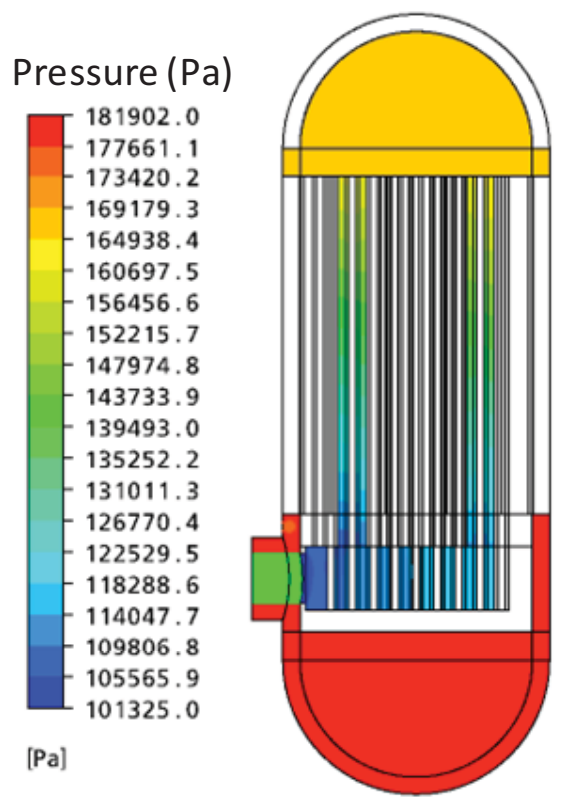

Figure 2-45. Pressure distribution results with the porous conditions under the normal operation conditions.

Table 2-6. Pressure drop results using the porous media conditions.

Porous Conditions:

- Volume porosity : 0.185

- Permeability : $9.706 \times 10^{-4} \mathrm{~m}^{2}$

- Resistance loss coefficient : $1.367 \mathrm{~m}^{-1}$

600 MWth GTMHR Normal Operation Conditions (GA 2006):

- He mass flow rate: $320 \mathrm{~kg} / \mathrm{s}$

- He average temperature through the core block: $743.65 \mathrm{~K}$

\begin{tabular}{|l|c|c|}
\hline & Conceptual Design Data & CFD Results \\
\hline Pressure drop of Rx vessel (Cold Duct to Hot Duct) & $71 \mathrm{kPa}$ & $78.8 \mathrm{kPa}$ \\
\hline Pressure drop of active core & $51 \mathrm{kPa}$ & $50.9 \mathrm{kPa}$ \\
\hline
\end{tabular}


The properties of the air and helium, such as thermal conductivity, molecular viscosity, and specific heat used for the 3-D CFX analysis, were cited from those of the FLUENT 2-D analysis, except for helium specific heat (ANSYS 2009). The National Aeronautics and Space Administration (NASA) format correlations in Table 2-7 were used for the helium specific heat property in the 3-D CFX analysis. The binary molecular diffusivity shown in Figure 2-46 was thus calculated by kinetic theory. The air and helium density was obtained by the ideal gas law. The graphite properties for thermal conductivity and specific heat for the inner and outer reflectors were quoted from the FLUENT 2-D analysis.

\section{Table 2-7. NASA format correlation for specific heat of helium.}

$-\mathrm{Cp} / \mathrm{R}=\mathrm{a} 1+\mathrm{a} 2 \mathrm{~T}+\mathrm{a} 3 \mathrm{~T}^{2}+\mathrm{a} 4 \mathrm{~T}^{3}+\mathrm{a} 5 \mathrm{~T}^{4}$

- $\mathrm{R}=2077[\mathrm{~J} / \mathrm{kg} \mathrm{K}]$ for helium

- Lower temperature $=300[\mathrm{~K}]$, Midpoint temperature $=1000[\mathrm{~K}]$, Upper temperature $=5000[\mathrm{~K}]$

- Lower interval coefficient:

$$
\begin{aligned}
& \mathrm{a} 1=0.02500000 \mathrm{E}+02[], \mathrm{a} 2=0.0 \mathrm{E}+00\left[\mathrm{~K}^{-1}\right], \mathrm{a} 3=0.0 \mathrm{E}+00\left[\mathrm{~K}^{-2}\right], \mathrm{a} 4=0.0 \mathrm{E}+00\left[\mathrm{~K}^{-3}\right], \\
& \mathrm{a} 5=0.0 \mathrm{E}+00\left[\mathrm{~K}^{-4}\right], \mathrm{a} 6=-0.07453750 \mathrm{E}+04[\mathrm{~K}], \mathrm{a} 7=0.09153488 \mathrm{E}+01[]
\end{aligned}
$$

- Upper interval coefficient:

$$
\begin{aligned}
& \mathrm{a} 1=0.02500000 \mathrm{E}+02[], \mathrm{a} 2=0.0 \mathrm{E}+00\left[\mathrm{~K}^{-1}\right], \mathrm{a} 3=0.0 \mathrm{E}+00\left[\mathrm{~K}^{-2}\right], \mathrm{a} 4=0.0 \mathrm{E}+00\left[\mathrm{~K}^{-3}\right], \\
& \mathrm{a} 5=0.0 \mathrm{E}+00\left[\mathrm{~K}^{-4}\right], \mathrm{a} 6=-0.07453750 \mathrm{E}+04[\mathrm{~K}], \mathrm{a} 7=0.09153489 \mathrm{E}+01[]
\end{aligned}
$$

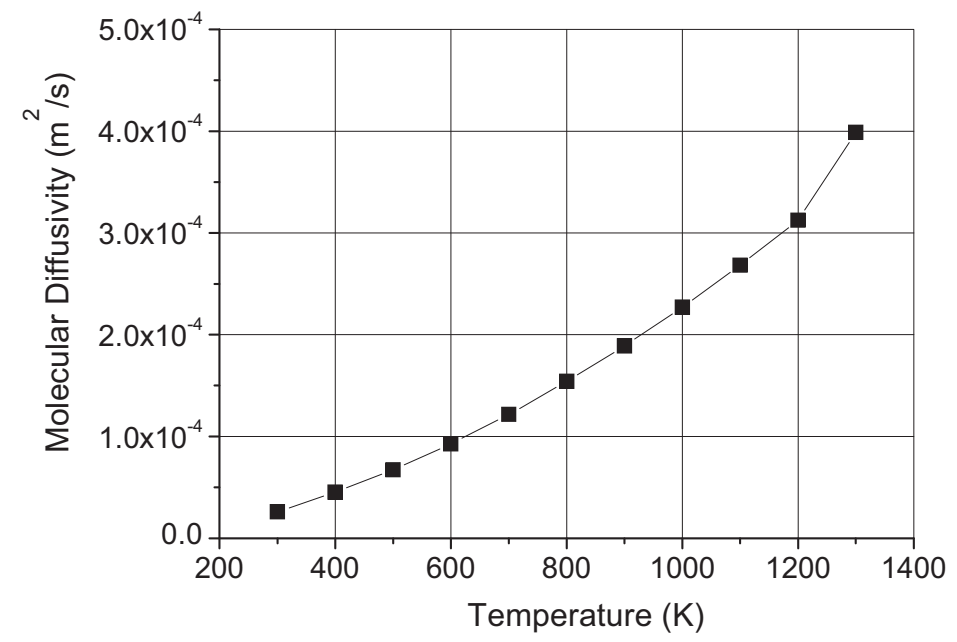

Figure 2-46. Binary diffusion coefficient between air and helium.

\subsubsection{Flow Field models and Numerical Models for the 3-D CFX Analysis}

The air ingress accident under the DEGB was treated as a convective flow, compressible flow, turbulent flow, species flow, buoyant flow, and transient flow. The governing equations (Eq. (2-114)$(2-120))$ used in this study are the continuity, Navier-Stokes, energy, and the species transport equations with a coupled solver algorithm (ANSYS 2009). Turbulent flow was modeled by the standard k- $\varepsilon$ turbulent model with the scalable wall function, and the buoyancy flow was modeled by the density difference (see Eq. (2-115)) (ANSYS, 2009). The governing equations used for the porous media are changed to Eq. $(2-121)$ by adding the volume porosity $(\lambda)$ and area porosity tensor $(K)$ into the general governing equations as follows: 


$$
\begin{aligned}
& \frac{\partial \rho}{\partial t}+\nabla \bullet(\rho \vec{V})=0 \\
& \frac{\partial(\rho \vec{V})}{\partial t}+\nabla \bullet(\rho \vec{V} \otimes \vec{V})=-\nabla p+\nabla \bullet\left[\mu_{e f f}\left(\nabla \vec{V}+(\nabla \vec{V})^{T}\right)\right]+\left(\rho-\rho_{r e f}\right) \vec{g} \\
& \frac{\partial\left(\rho h_{t o t}\right)}{\partial t}-\frac{\partial p}{\partial t}+\nabla \bullet\left(\rho \vec{V} h_{t o t}\right)=\nabla \bullet\left(\lambda \nabla T+\frac{\mu_{t}}{\operatorname{Pr}_{t}} \nabla h\right)+\nabla \bullet(\vec{V} \bullet \tau) \\
& \frac{\partial(\rho k)}{\partial t}+\nabla \bullet(\rho \vec{V} k)=\nabla \bullet\left[\left(\mu+\frac{\mu_{t}}{\sigma_{k}}\right) \nabla k\right]+P_{k}-\rho \varepsilon \\
& \frac{\partial(\rho \varepsilon)}{\partial t}+\nabla \bullet(\rho \vec{V} \varepsilon)=\nabla \bullet\left[\left(\mu+\frac{\mu_{t}}{\sigma_{\varepsilon}}\right) \nabla \varepsilon\right]+\frac{\varepsilon}{k}\left(C_{\varepsilon 1} P_{k}-C_{\varepsilon 2} \rho \varepsilon\right) \\
& \mu_{\text {eff }}=\mu+C_{\mu} \rho \frac{k^{2}}{\varepsilon} \\
& \frac{\partial(\rho \phi)}{\partial t}+\nabla \bullet(\rho \vec{V} \phi)=\nabla \bullet\left(D_{A B}+\frac{\mu_{t}}{S c_{t}}\right) \nabla \phi \\
& \frac{\partial(\gamma \rho \phi)}{\partial t}+\nabla \bullet(\rho K \cdot \vec{V} \phi)-\nabla \bullet(\Gamma K \cdot \nabla \phi)=r S
\end{aligned}
$$

where

$$
\begin{array}{lll}
\vec{V} & =\text { Velocity vector }(\mathrm{m} / \mathrm{s}) \\
\vec{g} & =\text { Gravitation vector }\left(\mathrm{m} / \mathrm{s}^{2}\right) \\
h_{t o t} & =\text { Total enthalpy }(\mathrm{J} / \mathrm{kg}) \\
D_{A B} & =\text { Binary diffusion coefficient }\left(\mathrm{m}^{2} / \mathrm{s}\right) \\
k & =\text { Turbulent kinetic energy }\left(\mathrm{m}^{2} / \mathrm{s}^{2}\right) \\
\varepsilon & =\text { Turbulent dissipation } \mathrm{rate}\left(\mathrm{m}^{2} / \mathrm{s}^{3}\right) \\
\lambda & =\text { Thermal conductivity }\left(\mathrm{W} / \mathrm{m} \mathrm{K}^{\mathrm{K}}\right) \\
\mu_{\text {eff }} & =\text { Effective viscosity }(\mathrm{Pa} \mathrm{sec}) \\
\phi & =\text { Variable } \\
\Gamma & =\text { Diffusion coefficient. }
\end{array}
$$


The transient calculation for a total time of 80 seconds with a time step of $0.001-0.005$ seconds was performed to carefully simulate the buoyant flow behavior because of the heat transfer from the solid structures into the air and helium. As a calculation method, about 3-10 iterations were performed per the time step until the mass, enthalpy, and velocity residual of the air reached a value below $1.0 \times 10^{-4}$. The RMS Courant number was maintained below 2.5. The numerical models used for the 3-D CFX analysis are summarized as:

- Pressure-velocity coupling

- Linear equation solver: Algebraic Multigrid

- Convection scheme: Upwind $1^{\mathrm{st}}: \phi_{i p}=\phi_{u p}$

- Transient scheme: Backward Euler $1^{\mathrm{st}}: \frac{\partial}{\partial t} \int_{v} \rho \phi d V=V\left(\frac{\rho \phi-\rho^{o} \phi^{o}}{\Delta t}\right)$

- $\quad$ Reynolds analogy: $\mathrm{Pr}_{\mathrm{t}}=0.9, \mathrm{Sc}_{\mathrm{t}}=0.9$

- 30 CPU parallel computations.

\subsubsection{Discussion on the CFD Analysis Results}

The 3-D CFD results of the air ingress accident are shown in Figures 2-47-2-49. The air mass fraction contours according to time (see Figure 2-47) show the air inflow pattern from the confinement side into the reactor internal side. Figure 2-47 shows the air entering into the hot and cold duct as soon as the CFD calculation starts. This is because the static head of the confinement side is slightly larger than that of the reactor internal side at the same elevation as much as the density difference between the air and the helium (see Figure 2-48(a) and (b)). Figure 2-48(a) shows the normalized pressure from the confinement ( $6.05 \mathrm{~m}$ from the center of the lower plenum) to the inlet point to the lower plenum $(3.5 \mathrm{~m}$ from the center of the lower plenum) while $\mathrm{z}=6.7 \mathrm{~m}$ represents the midpoint of the broken pipe height. The vertical line in Figure 2-48(a) is the pipe breach point and the curved line represents the curvature of the inlet pipe to the reactor vessel. Figure 2-48(b) shows a sudden density change at the breach point. Figure 2-48(c) shows the recirculation flow pattern at the breach point. Gravitational force directs the air inflow downward (see Figure 2-48(c)). Finally, instability may be developed on the interface between the air and helium when the air flows into the helium by Rayleigh-Taylor instability (Lowe et al. 2005).

As seen in Figure 2-47, the air arrives on the right end of the lower plenum at about 6 seconds and, after filling up the lower plenum and being heated by the support block, starts up into the core blocks right side at about 10 seconds. It takes approximately 50 seconds for the air in the lower part of the core block to move upward to the upper part by the buoyancy force generated by the density variation because of the heat transfer from the core block wall into the air. The air then arrives at the top of the coolant riser about 70 seconds after filling up the volume of the upper plenum near the core upper region (see Figure 2-47 and Figure 2-49; see A). The air that fills the upper plenum flows up through the reactor core, shown as two blank boxes in Figure 2-44. The air then moves downward along the coolant riser at about 80 seconds (see Figure 2-49 A), and is also located at the lower part of the coolant riser (Figure 2-49 B).

It is believed that this air came from the confinement through the cold duct after filling up the reactor bottom region by gravitational force as shown in Figure 2-49 C. From the air mass fraction contours, it can be expected that the air located on the upper region of the coolant riser can sufficiently reach the lower region of the coolant riser just 100-200 seconds after mixing with the air in the lower region. 

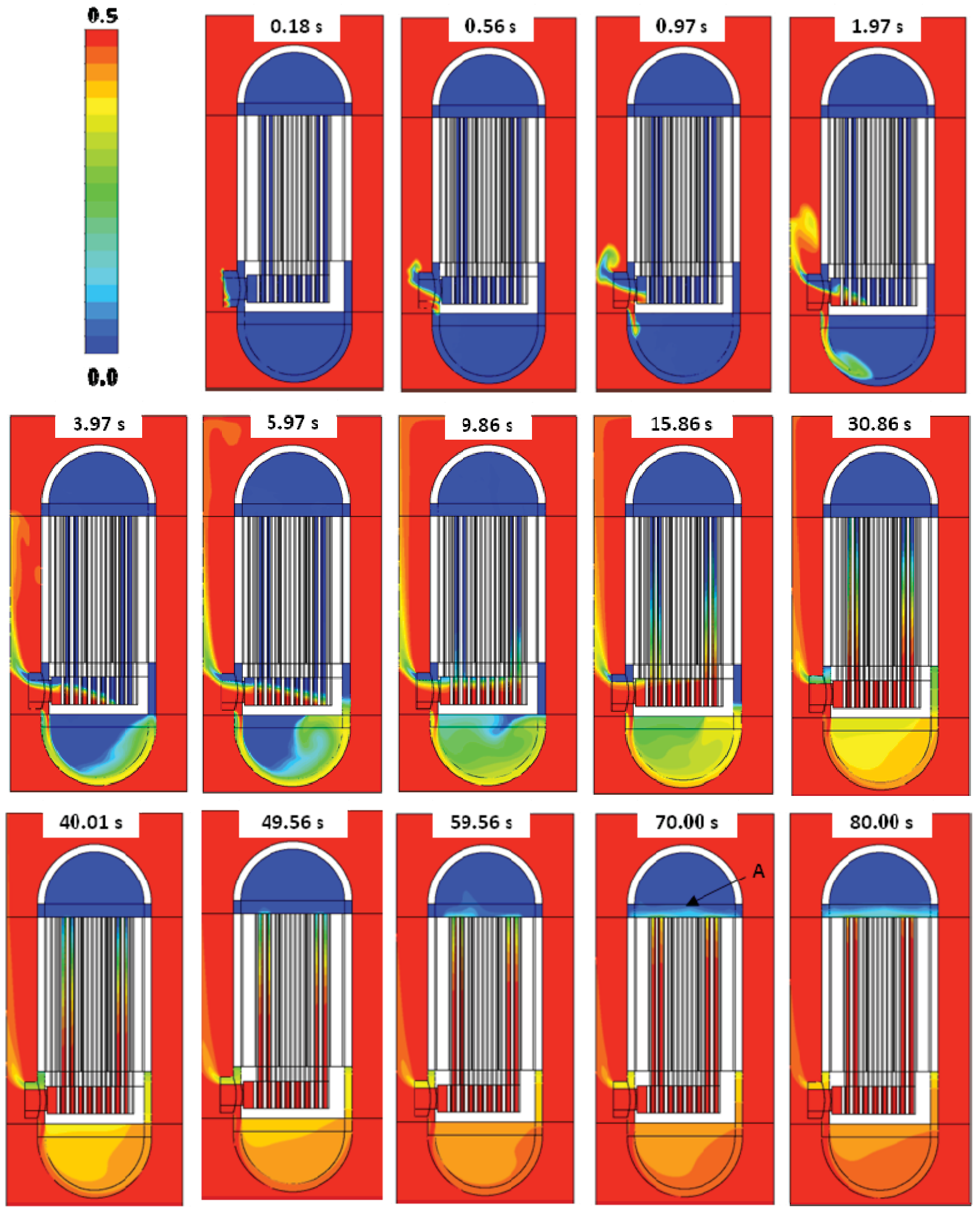

(Contours are plotted on the plane of $y=0.01 \mathrm{~m}$. Symmetry plane is $\mathrm{y}=0.0 \mathrm{~m}$ )

Figure 2-47. Variation of the air mass fraction according to time. 

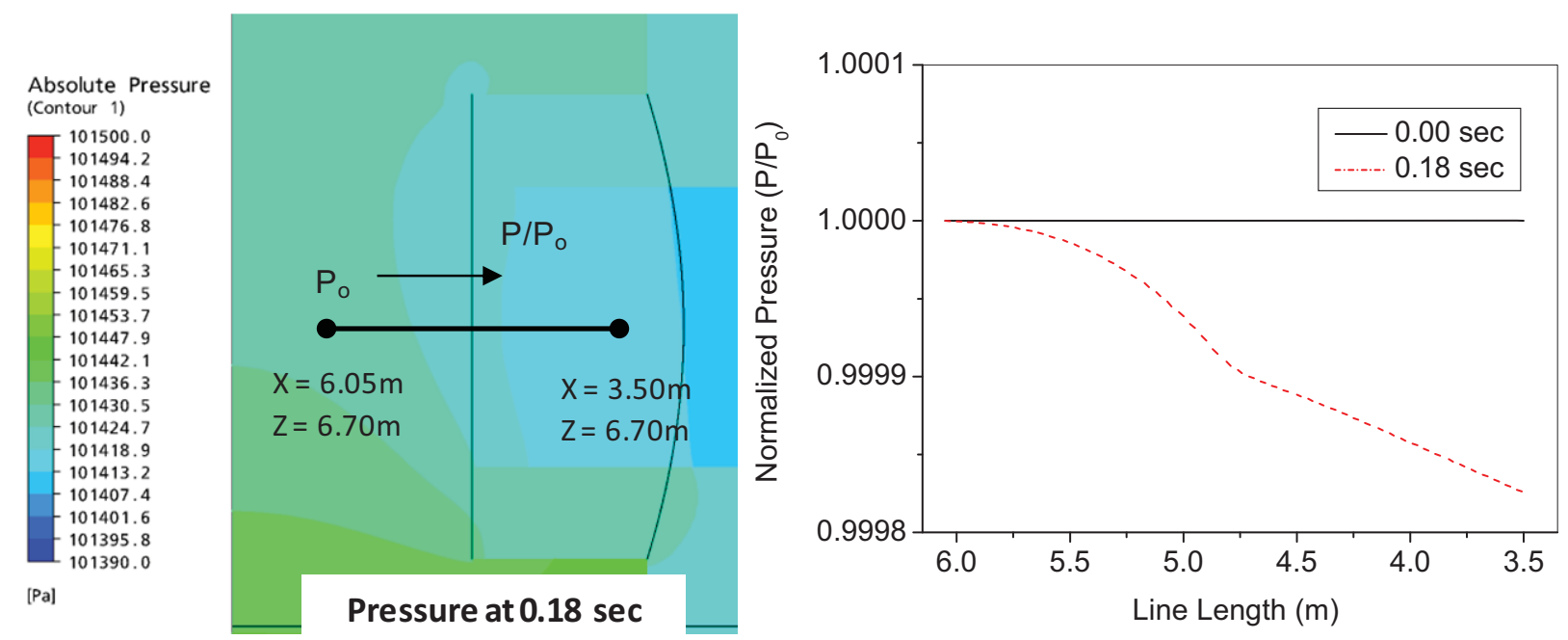

(a) Pressure contours and normalized pressure distribution along the line between $x=6.05 \mathrm{~m}$ and $\mathrm{x}=3.50 \mathrm{~m}$
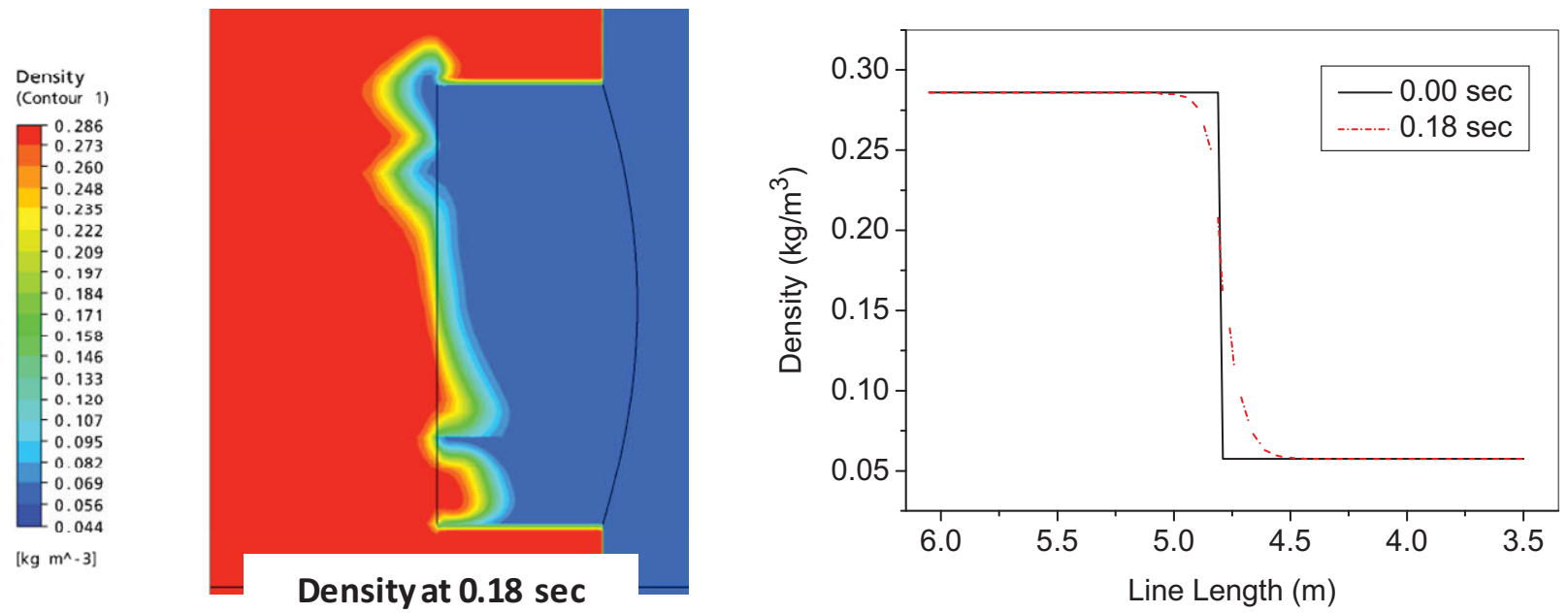

(b) Density contours and normalized density distribution along the line between $\mathrm{x}=6.05 \mathrm{~m}$ and $\mathrm{x}=3.50 \mathrm{~m}$.

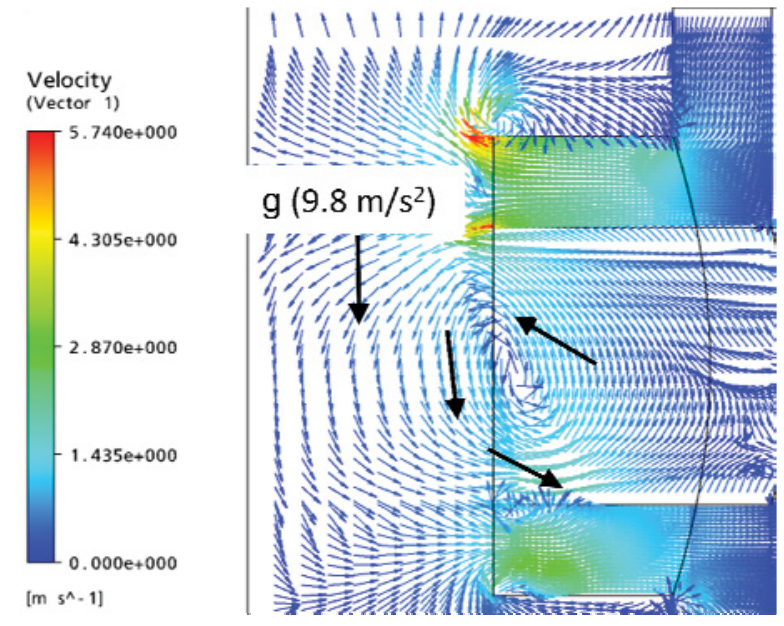

(c) Velocity profile on the plane of $\mathrm{y}=0.01 \mathrm{~m}$ at 0.18 seconds

(Contours are plotted on the plane of $y=0.01 \mathrm{~m}$. Symmetry plane is $\mathrm{y}=0.0 \mathrm{~m}$ )

Figure 2-48. Velocity profile, density, and pressure distribution at 0.0 seconds and 0.18 seconds. 


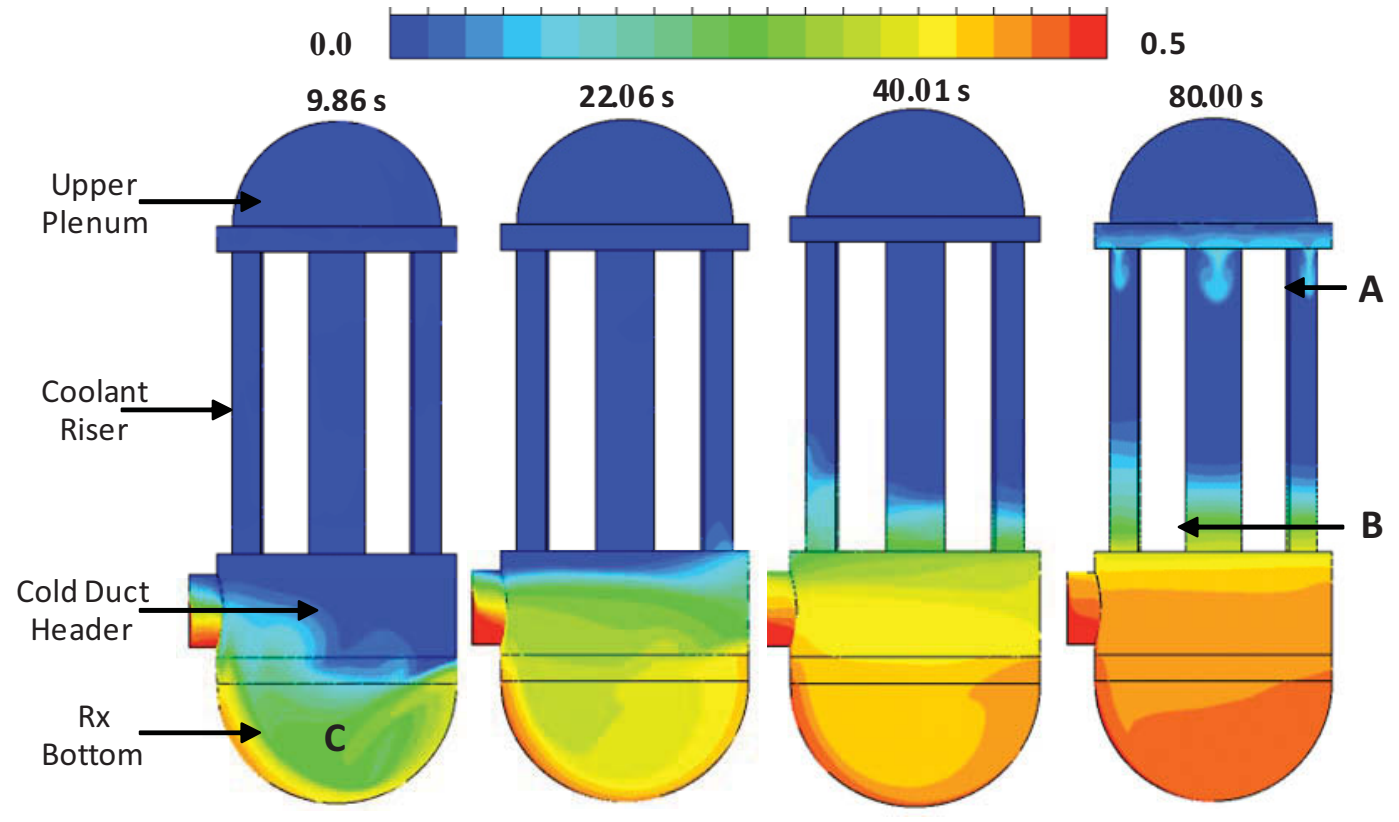

Figure 2-49. Air mass fraction of upper plenum, coolant riser, cold duct header, and reactor bottom.

Figure 2-50 shows the air mass fraction distribution on the hot and cold duct surface from a front view point. The air flows into the cold duct header through the lower region of the broken cold duct at the same time the helium counter-currently discharges through the upper region of the cold duct during the whole period. As time passes, the helium (blue color) in the helium discharge cold duct area steadily decreases. Figure 2-51(a) and (b) show air mass fractions and velocity vectors in the lower plenum at 5.96 seconds. As can be seen, a portion of air velocity vector moves to the reactor core. When the flow is recirculated at the end of the plenum wall, it loses the momentum, resulting in pressure build-up, which makes the air move upward, if the hydrostatic force is less than the pressure build-up.

The rate at which the helium area decreases is proportional to the helium inventory volume in the reactor vessel and the velocity of the air inflow. In the hot duct side, the same situation of the countercurrent flow driven by the density occurs just as on the cold duct side. The helium discharge through the upper region of the hot duct (see Figure 2-51(c)) continues until about 20 seconds. These different time scales for the discharge of helium through the cold and hot duct can be certified in terms of the volume averaged air mass fraction of the lower plenum, the reactor bottom, and the cold duct header as shown in Figure 2-52. The filling of the lower plenum with air is completed by about 20 seconds, whereas those of the reactor bottom and the cold duct are not completed until 80 seconds because the air through the cold duct moves downward and fills up the reactor bottom first. 


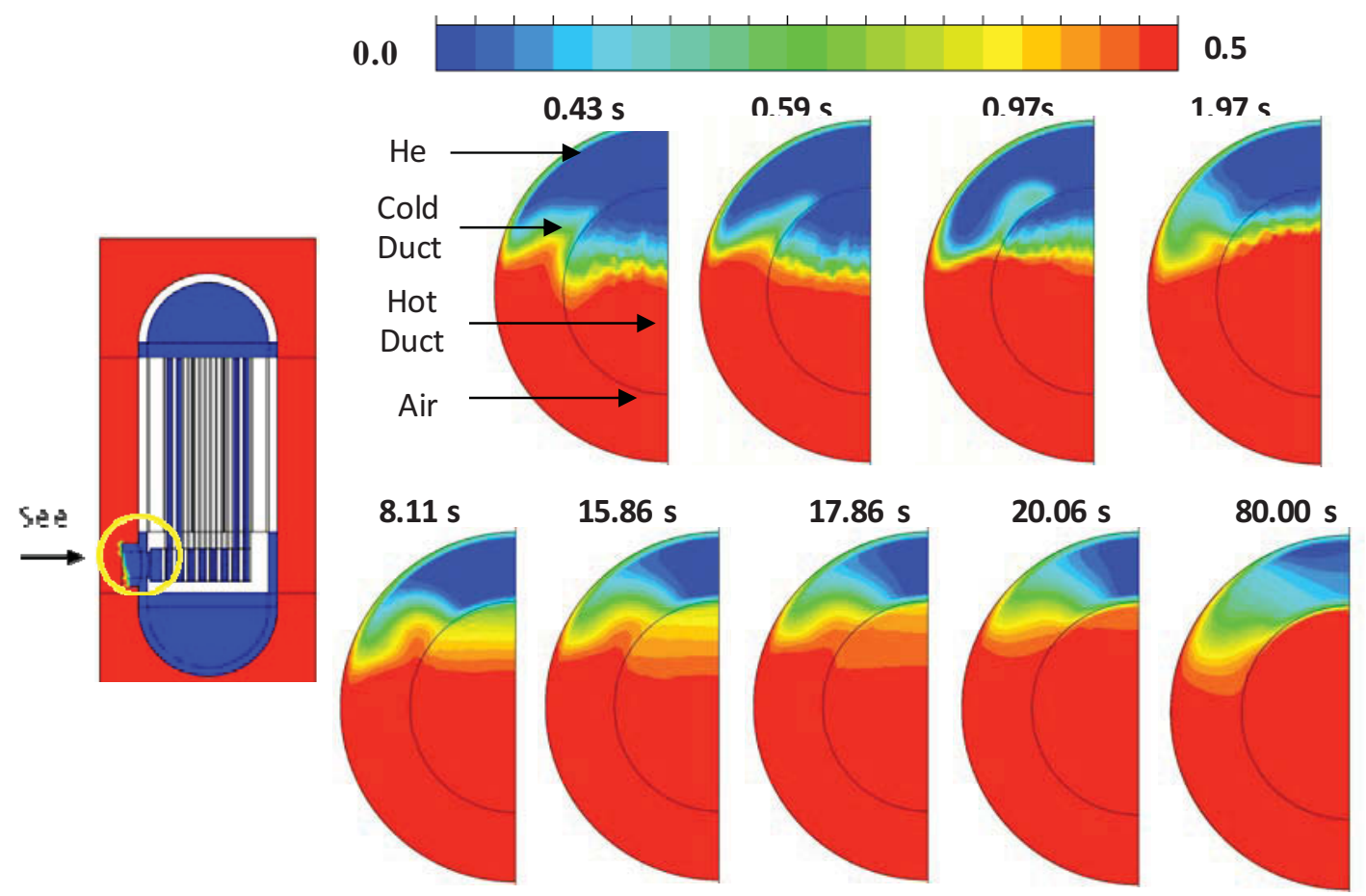

Figure 2-50. Air mass fraction of the hot and cold duct (front view)

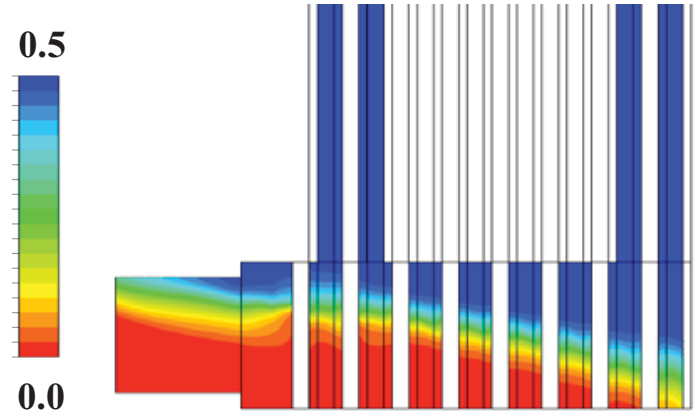

(a) Air mass fraction on plane of $y=0.01 \mathrm{~m}$

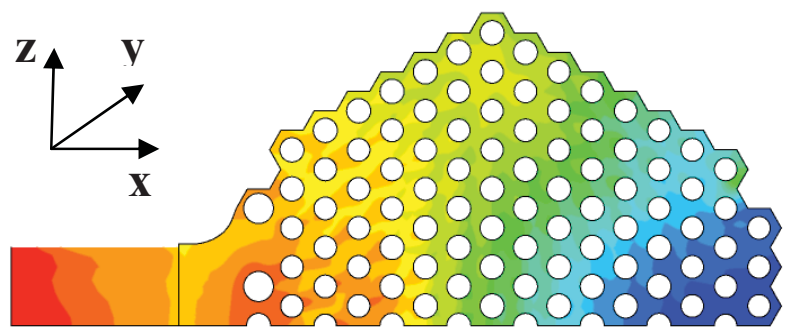

(b) Air mass fraction on plane of $\mathrm{z}=1 \mathrm{~m}$ from bottom of the lower plenum

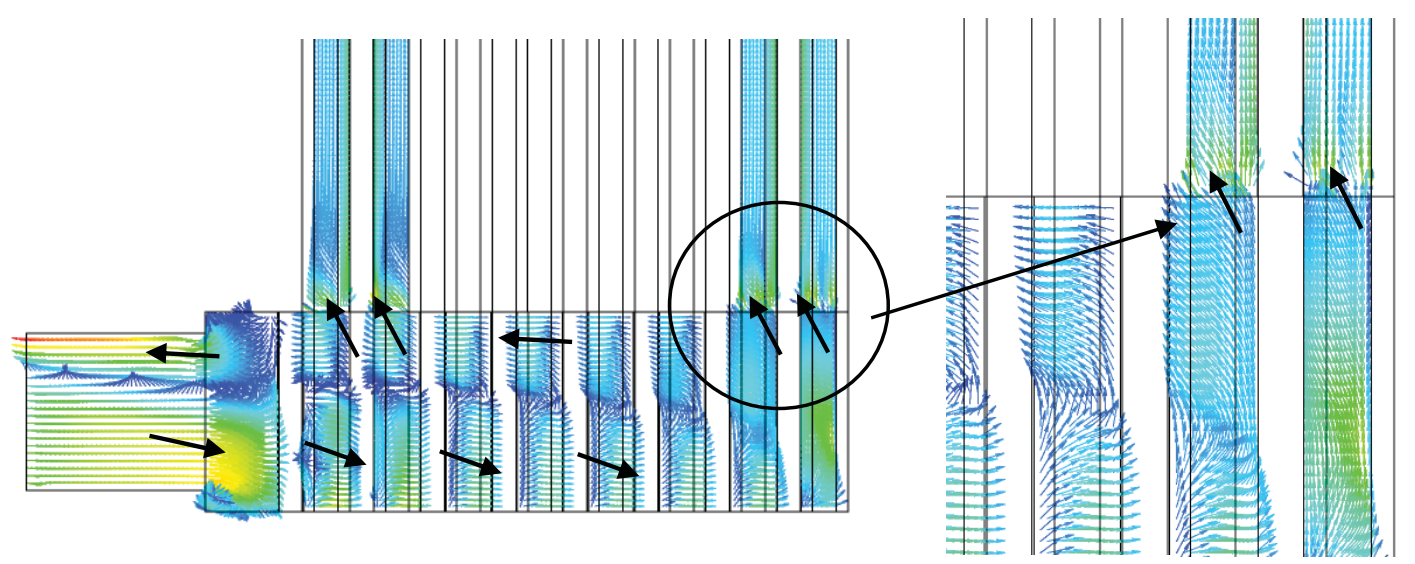

(c) Velocity profile on plane of $y=0.01 \mathrm{~m}$

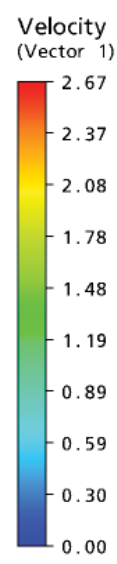

$\left[\mathrm{m} s^{\wedge}-1\right]$

Figure 2-51. Air mass fraction and velocity profile in the lower plenum at 5.96 seconds. 


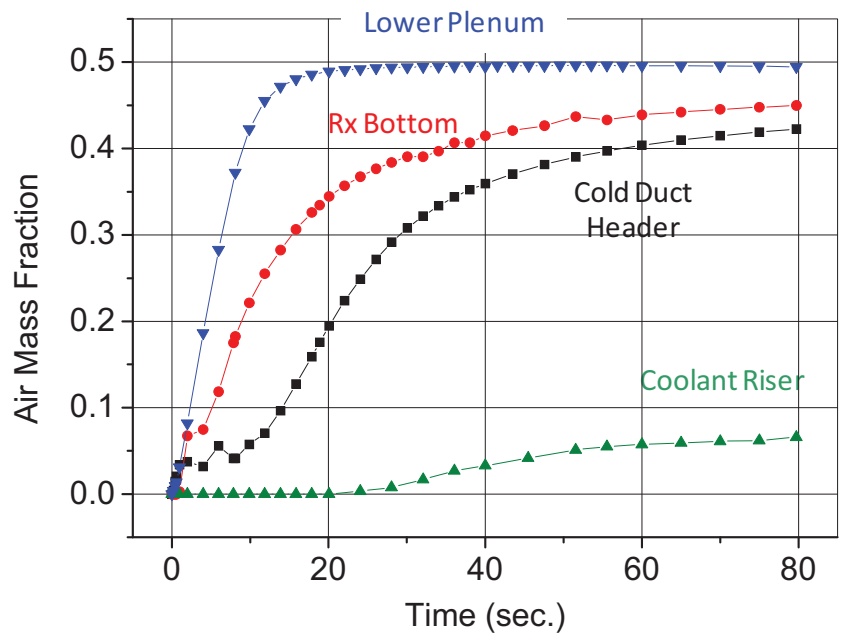

Figure 2-52. Volume averaged air mass fraction of the lower plenum, the reactor bottom, the cold duct header and the coolant riser (maximum value of the air mass fraction is 0.5 ).

The complete time of the helium discharge is very short when considering the lower plenum volume of $15.29 \mathrm{~m}^{3}$ and the helium discharge velocity of about $1.0-2.67 \mathrm{~m} / \mathrm{s}$ (see Figure 2-52). This situation may be caused from the helium located in the lower plenum at early stages that moves upward into the core blocks by the effect of the natural circulation along the core blocks. The development of the helium natural circulation along the core block because of the initial temperature difference may be confirmed in terms of the volume averaged velocity of the core block (see Figure 2-53). The velocity value shown in Figure 2-53 rapidly increases to about $1.1 \mathrm{~m} / \mathrm{s}$ for 3.0-7.0 seconds, and then decreases to about $0.2 \mathrm{~m} / \mathrm{s}$ at about 30.0 seconds. This natural circulation at an early stage may entrain the helium located in the lower plenum, and accelerate the helium circulation from the upper plenum region into the coolant riser.

Figure 2-54 shows the air mass fraction variation of the lower plenum, core, and core lower region according to time. An interested phenomenon is that the air mass fraction of the core starts to increase from about 10 seconds, even though about $80 \%$ of the lower plenum volume was already filled with air in the first 10 seconds. This may be caused by the discharging helium stream along the lower plenum upper region, thus preventing air penetration into the core blocks, or the air buoyancy force developed by the heat transfer from the support blocks being weak compared to the momentum of the helium discharging flow. However, we can know from the volume averaged temperature variation results of the lower plenum and the cold duct header (see Figure 2-55) that the starting time of the air flowing into the core block is closely related to the lower plenum temperature variation. The temperature graph of the lower plenum starts to increase at about 11 seconds from its continuous decreasing trend (see Figure 2-55 A), whereas the temperature of the cold duct header steadily decreased to the end of the CFD calculation because it did not have the heat structures of the support block in the lower plenum. The temperature increase from the decreasing trend can also be confirmed by the temperature contours at the plane of $\mathrm{z}=6.7 \mathrm{~m}$ in the lower plenum as shown in Figure 2-56(g). This may mean that the air heating time by the support block is an essential period for the air to have the buoyancy force because the buoyancy force can be developed by the density difference between a local value and an averaged value. It can therefore be expected that the starting time of the air flowing into the core block may be delayed if the air temperature of the lower plenum is maintained at a lower value. 


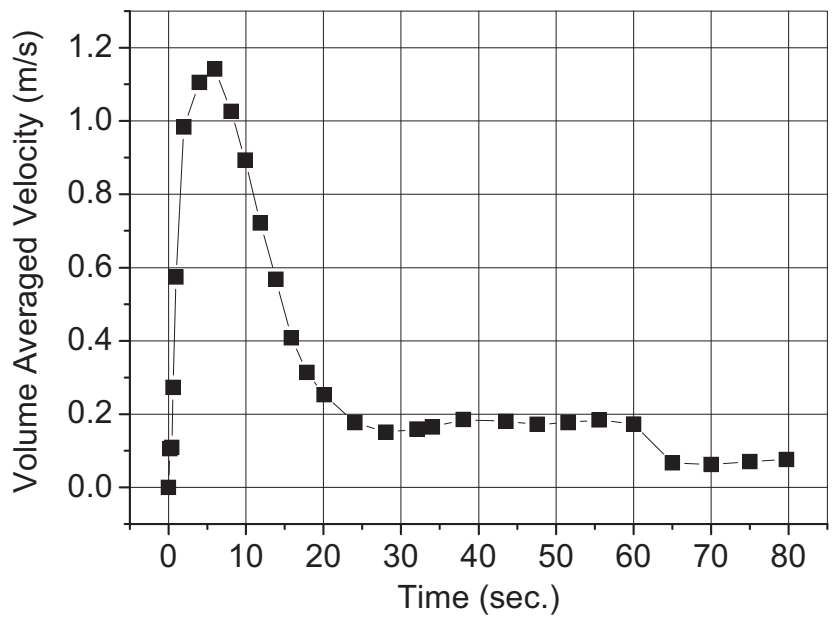

Figure 2-53. Volume averaged velocity of the core blocks.

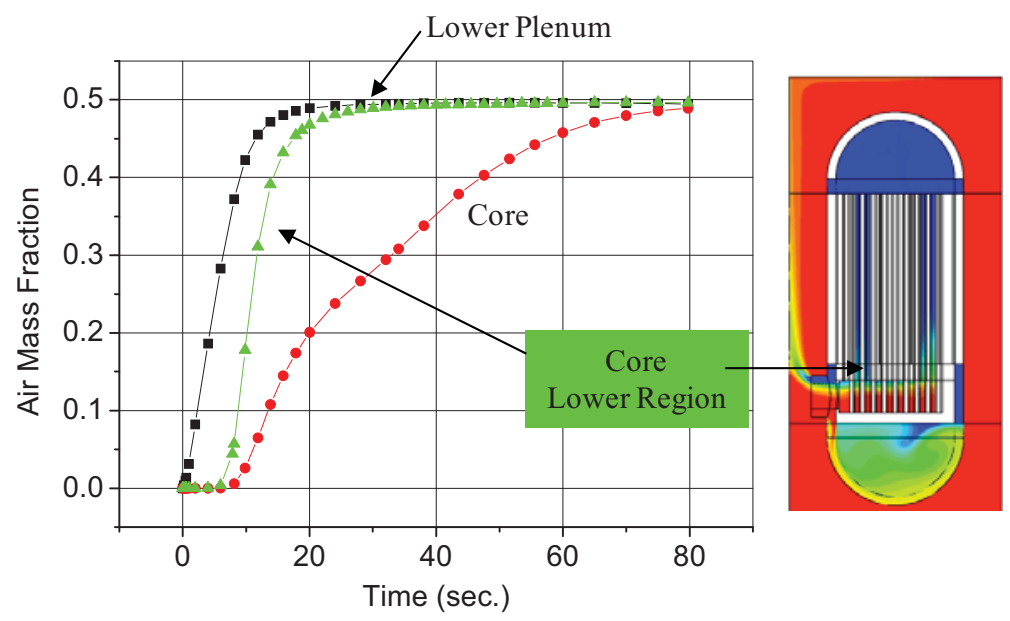

Figure 2-54. Volume and area averaged air mass fraction of the lower plenum, core blocks, and core inlet (maximum value of the air mass fraction is 0.5 ).

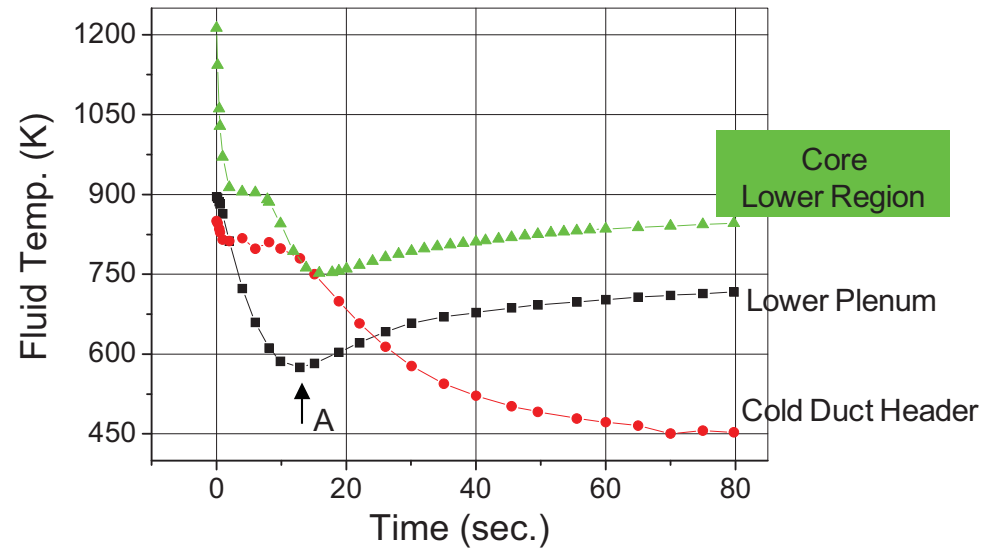

Figure 2-55. Volume and area averaged air temperature of the lower plenum, core blocks, and core inlet. 


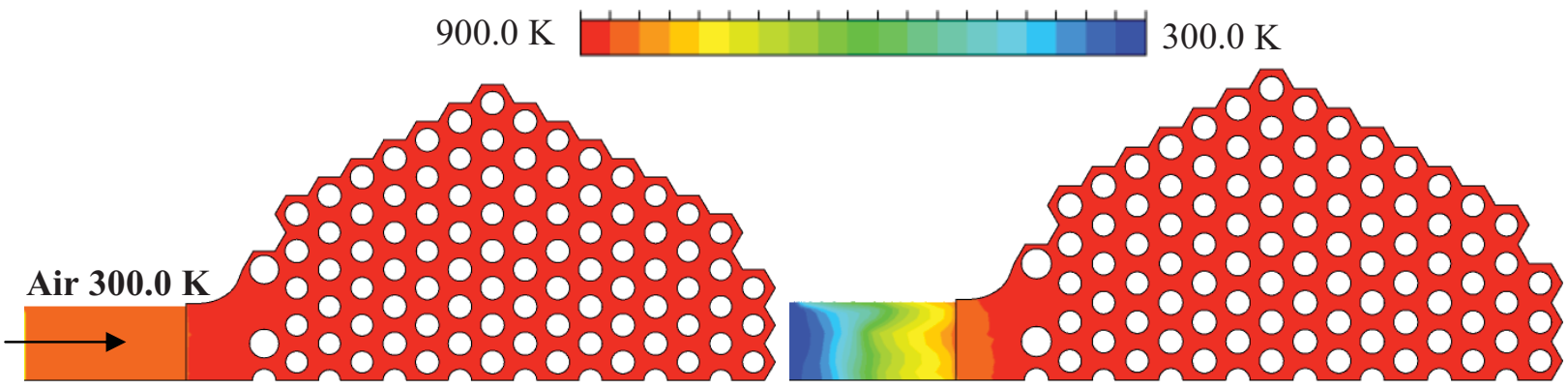

(a) $0.0 \mathrm{sec}\left(\mathrm{T}_{\text {avg }}=893.35 \mathrm{~K}, \mathrm{AMF}_{\text {avg }}=0.0\right)$

(b) $0.97 \mathrm{sec}\left(\mathrm{T}_{\mathrm{avg}}=855.54 \mathrm{~K}, \mathrm{AMF}_{\mathrm{avg}}=0.038\right)$
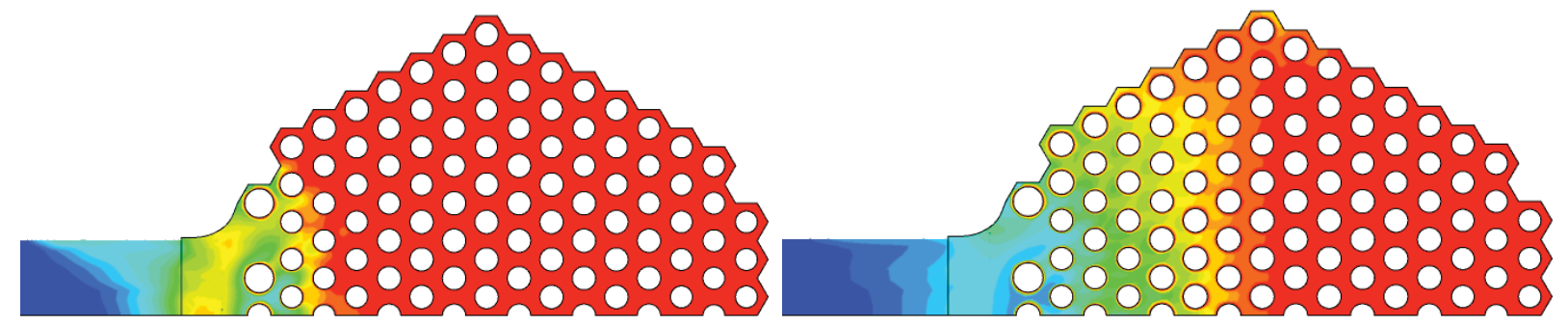

(c) $1.97 \mathrm{sec}\left(\mathrm{T}_{\mathrm{avg}}=805.27 \mathrm{~K}, \mathrm{AMF}_{\mathrm{avg}}=0.089\right)$

(d) $3.97 \mathrm{sec}\left(\mathrm{T}_{\mathrm{avg}}=717.56 \mathrm{~K}, \mathrm{AMF}_{\mathrm{avg}}=0.193\right)$
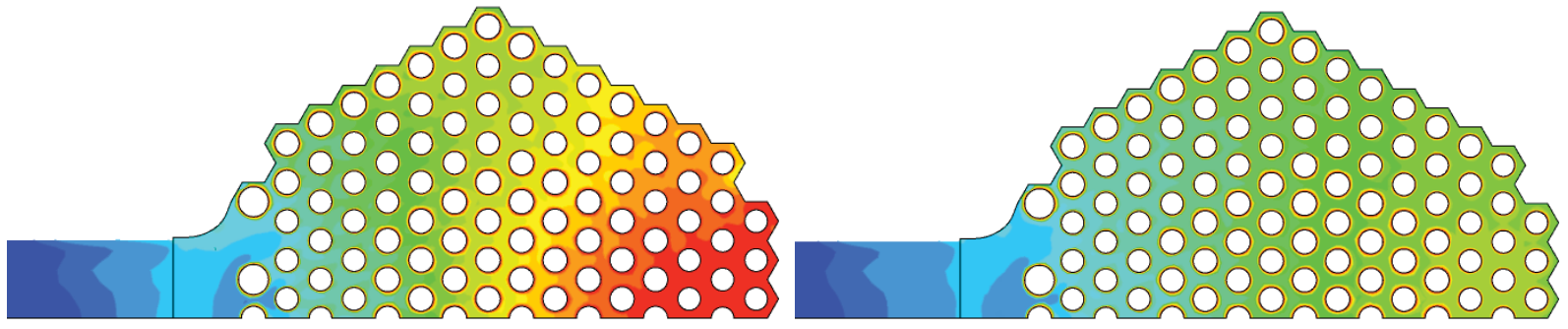

(e) $5.97 \mathrm{sec}\left(\mathrm{T}_{\text {avg }}=629.68 \mathrm{~K}, \mathrm{AMF}_{\mathrm{avg}}=0.335\right)$

(f) $9.86 \mathrm{sec}\left(\mathrm{T}_{\text {avg }}=535.51 \mathrm{~K}, \mathrm{AMF}_{\mathrm{avg}}=0.472\right)$
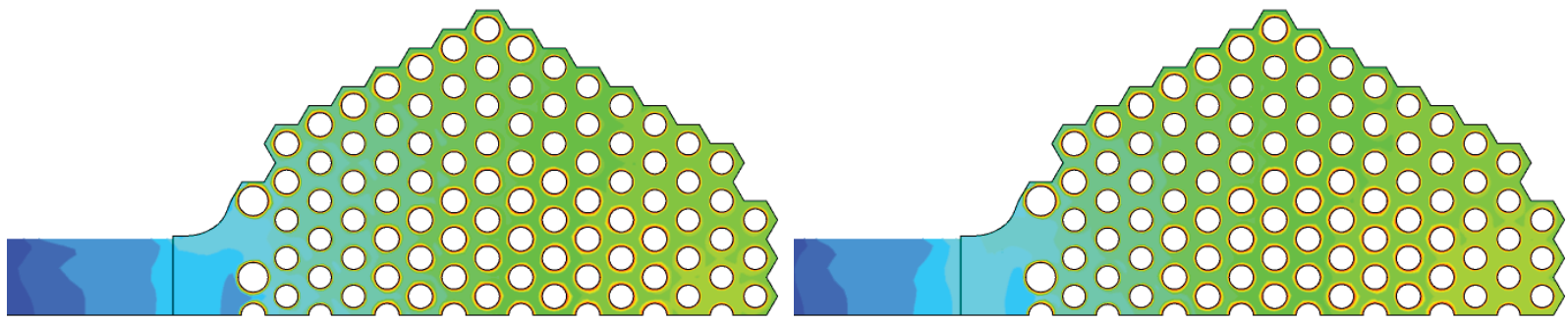

(g) $12.86 \mathrm{sec}\left(\mathrm{T}_{\text {avg }}=541.79 \mathrm{~K}, \mathrm{AMF}_{\text {avg }}=0.483\right)$

(h) $15.11 \mathrm{sec}\left(\mathrm{T}_{\mathrm{avg}}=557.85 \mathrm{~K}, \mathrm{AMF}_{\mathrm{avg}}=0.487\right)$

Figure 2-56. Temperature distribution on the plane of $z=6.7 \mathrm{~m}$ in the lower plenum (LP bottom: $z=7.624 \mathrm{~m}$, LP top: $z=7.624 \mathrm{~m}$, temperature and air mass fraction are averaged over the area of the plane at $z=6.7 \mathrm{~m}$ )

\subsubsection{Conclusions and Further Works}

The 3-D CFX results of the 3-D DEGB analysis by CFX-12 show that air can actively ingress the reactor vessel because the air inflow momentum generated by the stratified flow and the buoyant flow because of the heat transfer from the solid structures inside the reactor vessel sufficiently overcome the hydraulic resistance when the air passes the lower plenum and core blocks. This confirms that the previous FLUENT 2-D results with the porous media model are reasonable. The expected ONC time estimated by 3 -D CFX analysis is approximately 100 seconds, which is $50 \%$ of that of FLUENT 2-D analysis results. 
To confirm the starting time of the air flowing into the core blocks, a supplemental CFD calculation should be performed by changing the support block temperature. To reduce the uncertainty of the 3-D CFX results, several sensitivity calculations should be conducted by changing the numerical model for the convection term, the turbulent model, and the reference density value for the buoyant flow. The effect of the reference density value in the buoyant flow should also be carefully examined because the buoyant flow is a main driving force in the air ingress accident and its model is simply calculated by the density difference value based on the reference density value and the gravitational vector.

The qualitative results of the 3-D CFX analysis may not be changed because a lot of the heat structures definitely existed in the lower plenum and the density driven counter-current flow of air and helium is already verified by these experiments.

\subsection{2-D Preliminary Analyses on the Effect of Chemical Reaction in DEGB Air-ingress Accident}

Large amounts of graphite materials are used in VHTR cores, reflectors, and supporting structures. These graphite materials are very reactive to oxygen. The two main reactions between graphite and oxygen are

$\mathrm{C}+\mathrm{O}_{2} \rightarrow \mathrm{CO}_{2}$

and

$\mathrm{C}+\frac{1}{2} \mathrm{O}_{2} \rightarrow \mathrm{CO}$

The $\mathrm{R}-1$ reaction is dominant in low temperatures $\left(<750^{\circ} \mathrm{C}\right)$. The $\mathrm{R}-2$ reaction is dominant in high temperatures as shown in Figure 2-57. In the low temperature, the reaction is controlled by reaction kinetics and in the high temperature it is controlled by mass transfer rate (See Figure 2-58). This work implemented the following equations into the FLUENT code by a user defined function.

Overall Graphite Oxidation Rate (Oh et al. 2006).

$$
\frac{1}{R_{g}}=\frac{1}{R_{k}}+\frac{1}{R_{m}}
$$

where

$R_{g}=$ Overall graphite oxidation rate $(\mathrm{kg} / \mathrm{s})$

$R_{k}=$ Graphite oxidation rate estimated by Arrhenius model $(\mathrm{kg} / \mathrm{s})$

$R_{m} \quad=$ Graphite oxidation rate estimated by mass transfer $(\mathrm{kg} / \mathrm{s})$. 


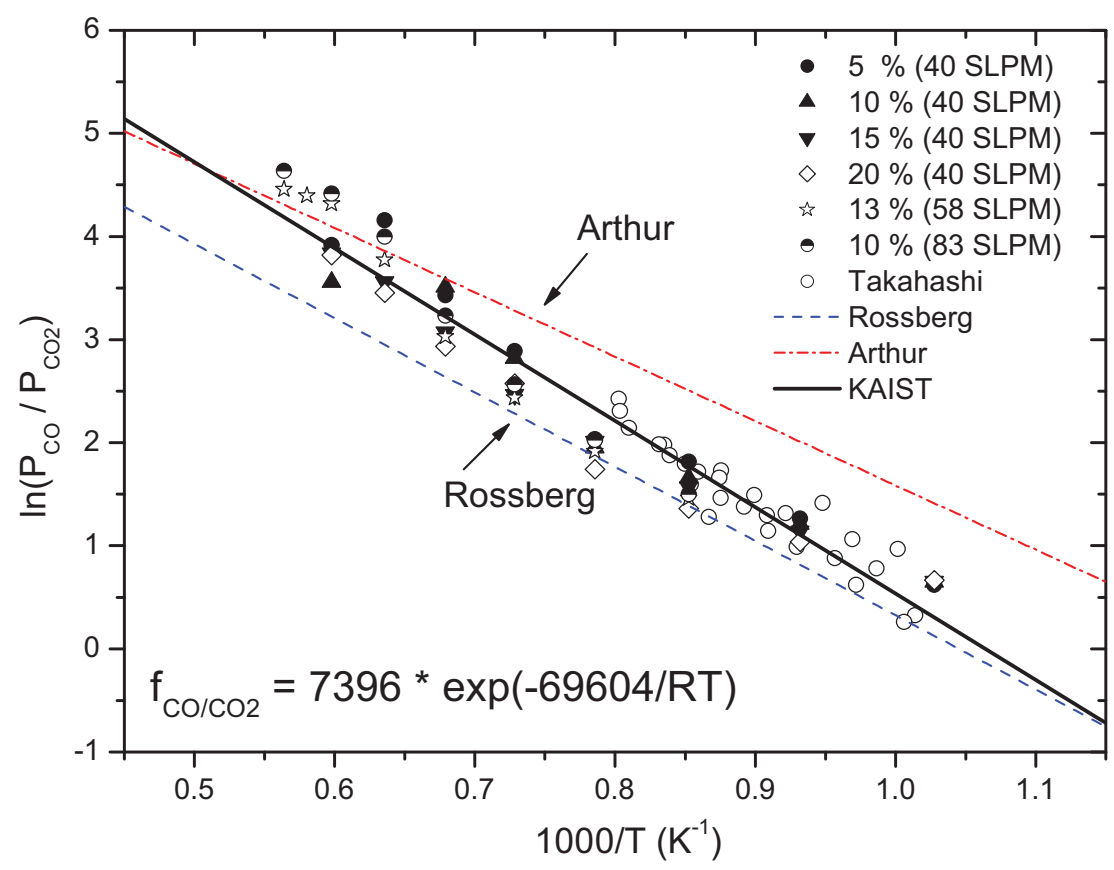

Figure 2-57. Ratio of $\mathrm{CO} / \mathrm{CO}_{2}$ with temperature (Oh et al. 2006).

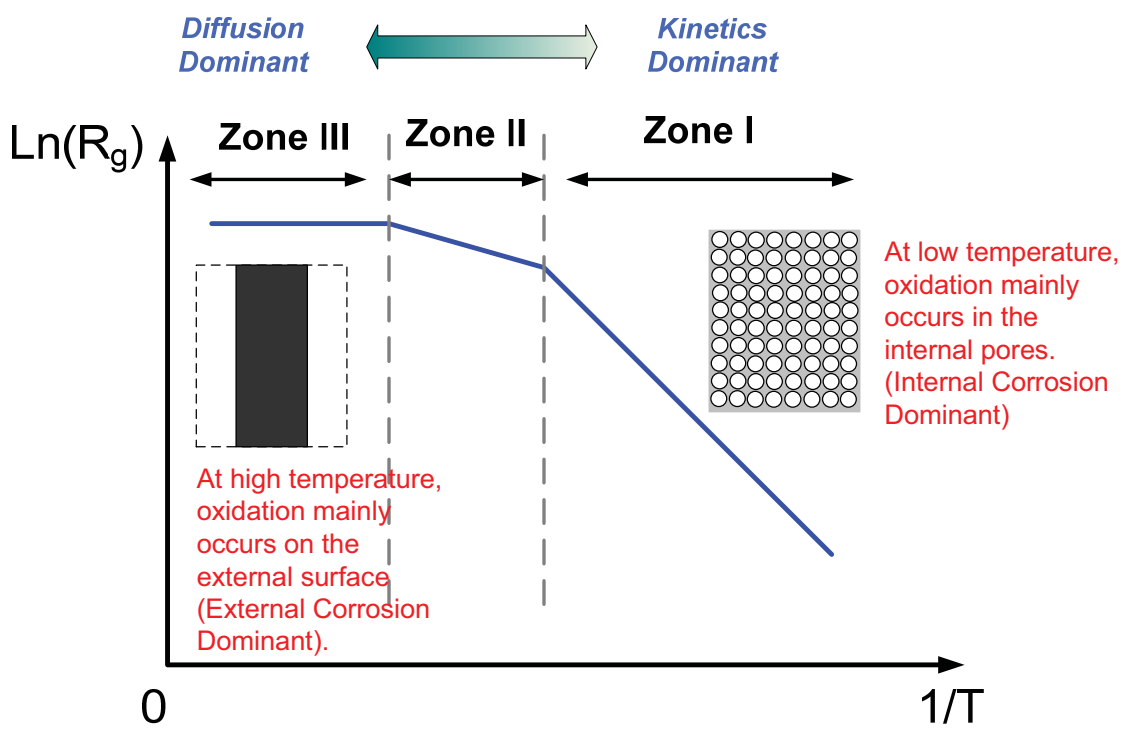

Figure 2-58. Effect of temperature on the graphite oxidation rates and oxidation regimes.

Kinetics Effect (Arrhenius model) (Oh et al. 2006)

$$
R_{k}=2552000 \cdot \exp \left(-\frac{218,000}{R \cdot T}\right) \cdot p_{O 2}^{0.75} \cdot \theta_{0} \cdot V
$$

where

$$
R=\text { Gas constant }(8.315 \mathrm{~kJ} / \mathrm{kmol} \mathrm{K})
$$


$T=$ Graphite surface temperature $(\mathrm{K})$

$P_{O 2}=$ Oxygen partial pressure $(\mathrm{Pa})$

$\theta_{0}=$ Graphite initial surface density $\left(\mathrm{m}^{2} / \mathrm{m}^{3}\right)$

$V \quad=\quad$ Apparent volume of the graphite structure.

\section{Mass Transfer}

$$
R_{m}=2 M_{c} \cdot K_{m}\left(C_{O_{2}, \infty}-C_{O_{2}, 0}\right) \cdot A
$$

where

$$
\begin{aligned}
& M_{c}=\text { Molecular mass of carbon, } \mathrm{C}(12 \mathrm{~kg} / \mathrm{kmol}) \\
& K_{m}=\text { Mass transfer coefficient of oxygen }(\mathrm{m} / \mathrm{s}) \\
& C_{O_{2}, \infty}=\text { Oxygen concentration in the bulk flow }\left(\mathrm{kmol} / \mathrm{m}^{3}\right) \\
& C_{\mathrm{O}_{2}, 0}=\text { Oxygen concentration at the graphite surface }\left(\mathrm{kmol} / \mathrm{m}^{3}\right) .
\end{aligned}
$$

CO/CO 2 Ratio (Oh et al. 2006)

$$
f_{\mathrm{CO} / \mathrm{CO}_{3}}=7,396 \exp \left(-\frac{69,604}{R \cdot T}\right) \text {. }
$$

The effect of burn-off in the FLUENT model was neglected because the time scale in the FLUENT simulation was several minutes in which the effect of burn-off on the reaction rate was negligible. For consideration of the chemical reaction, six gas species in total were taken into consideration in the species conservation model. The chemical species include $\mathrm{O}_{2}, \mathrm{~N}_{2}, \mathrm{CO}, \mathrm{CO}_{2}, \mathrm{H}_{2} \mathrm{O}$, and He. The model therefore solved five more equations than the previous FLUENT model, which did not consider chemical reactions. In this analysis, the computational speed significantly slowed down, even in the 2-D simplified geometries. The following summarizes the options and the models adopted in this calculation:

- 2-D

- Unsteady

- Segregated solver

- $2^{\text {nd }}$ order accuracy in time

- Noniterative time advancement

- Absolute velocity formulation

- Cell based gradient

- Physical velocity (in the porous media formulation)

- Laminar viscous model

- Energy equation solving

- Species transport equation solving:

- Six gas species: $\mathrm{O}_{2}, \mathrm{~N}_{2}, \mathrm{CO}, \mathrm{CO}_{2}, \mathrm{H}_{2} \mathrm{O}, \mathrm{He}$

- Multicomponent diffusion model 
- No consideration for thermal diffusion

- Pressure-velocity coupling method: PISO

- Pressure discretization method: PRESTO!

- Momentum: $2^{\text {nd }}$ order upwind

- Species: $2^{\text {nd }}$ order upwind.

The GTMHR was selected to be the reference reactor and DEGB was assumed. The geometry and mesh are the same as that used in the preliminary 2-D analyses in FY 2008. Initial air mass fraction in the confinement was assumed to be 0.5 . The results of the FLUENT simulation are briefly summarized in this section. Figure 2-59 shows the species concentration profiles in the reactor 13 seconds after depressurization, at which time the air has already filled the lower plenum and started to move into the reactor core by buoyancy force. The oxygen in the core is consumed by chemical reactions, resulting in $\mathrm{CO}$ and $\mathrm{CO}_{2}$ species being generated. This result also shows that the chemical reactions are mainly generated at the core bottom region, while little chemical reaction is generated in the lower plenum. This is because the temperature in the lower plenum is relatively much lower than it is in the core. The generated $\mathrm{CO}$ and $\mathrm{CO}_{2}$ gases move up through the core.
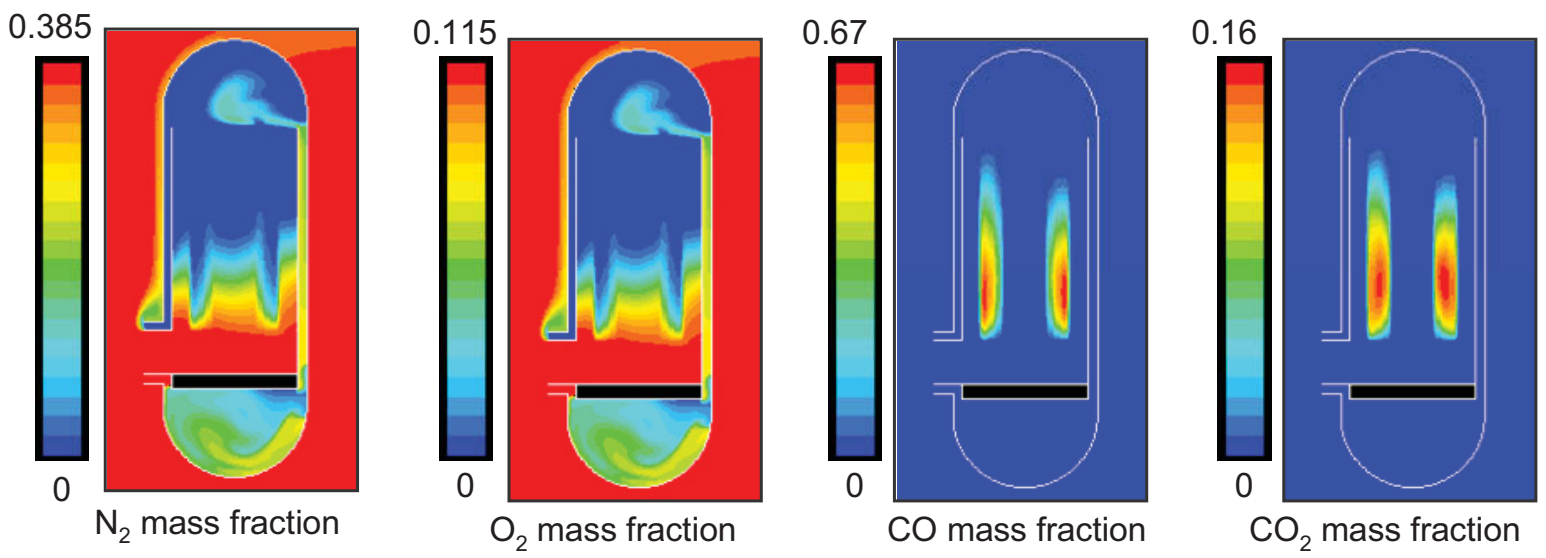

Figure 2-59. Species concentration profiles in the VHTR at 13 seconds.

Figure 2-60 shows the comparisons of the FLUENT calculations both with and without chemical reactions; helium concentrations are plotted in this figure for comparison. The chemical reaction accelerated the air-ingress process by moving more gases to the top. This occurred because the temperature in the reactor core is higher than $900^{\circ} \mathrm{C}$. CO generation (reaction $\mathrm{R}-1$ ) is therefore the dominant reaction in which one oxygen molecule is consumed and two $\mathrm{CO}$ molecules are generated. Therefore, a single gas species is generated by this reaction. Once CO is generated in the core, it will increase the pressure in the channel under the same temperature conditions. Graphite oxidation is an exothermic reaction, which increases the temperature at the reaction spot and generates more buoyancy force in the gases.

This preliminary calculation qualitatively indicates that the chemical reaction will accelerate the airingress process. However, for getting quantitative results, a detailed 3-D model is required as described in the previous section. The chemical reaction models and the user defined functions developed in this section can be directly implemented into the detailed 3-D CFD model. And, this detailed model will provide a more realistic and meaningful result. The detailed 3-D calculation for air-ingress with chemical reactions is currently planned. 


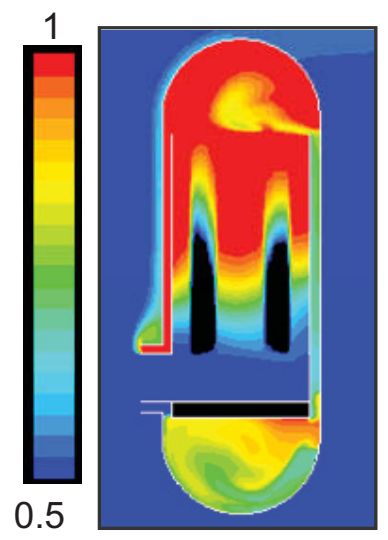

(a) With reaction model

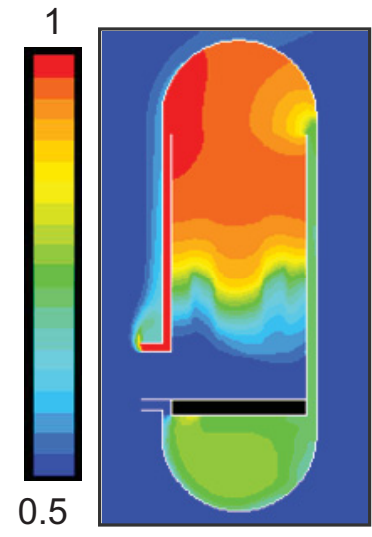

(b) Without reaction model

Figure 2-60. Comparisons of with and without chemical reaction models.

\subsection{Preliminary 2-D CFD Analyses on the Small Break Air-ingress Accident}

The air-ingress caused by a small pipe break is an important issue because it has a higher probability of occurrence than the DEGB. The purpose of this simulation is to (1) determine what mechanism dominates the initiation of air ingress, whether by molecular diffusion or density-gradient induced flow and to (2) find the timing of the natural convection that fills the reactor with air. It is strongly believed that the air ingress mechanism is dependent on the geometry and conditions of the break. This calculation pursues an interest in the air ingress mechanism, the flow path, and the timing of natural convection.

A design basis event (DBE-10) for the failure of a release valve that is less than 10 inch $^{2}$ was investigated. Air ingress analysis of a small pipe break $\left(82 \mathrm{~cm}^{2}\right)$ on the top of the steam generator was assumed (See Figure 2-61). This event has a higher probability of occurrence than other accident scenarios related to the depressurization LOCA and is considered by GA to be a design basis event.

This analysis is also based on a single failure of the check valve, which can happen when the corrosion materials and graphite particles are stuck into the hinge of the check valve. A 2-D FLUENT model was developed based on the gas turbine modular helium reactor, GTMHR design. The simulation continues, and some preliminary results are presented in this section. 


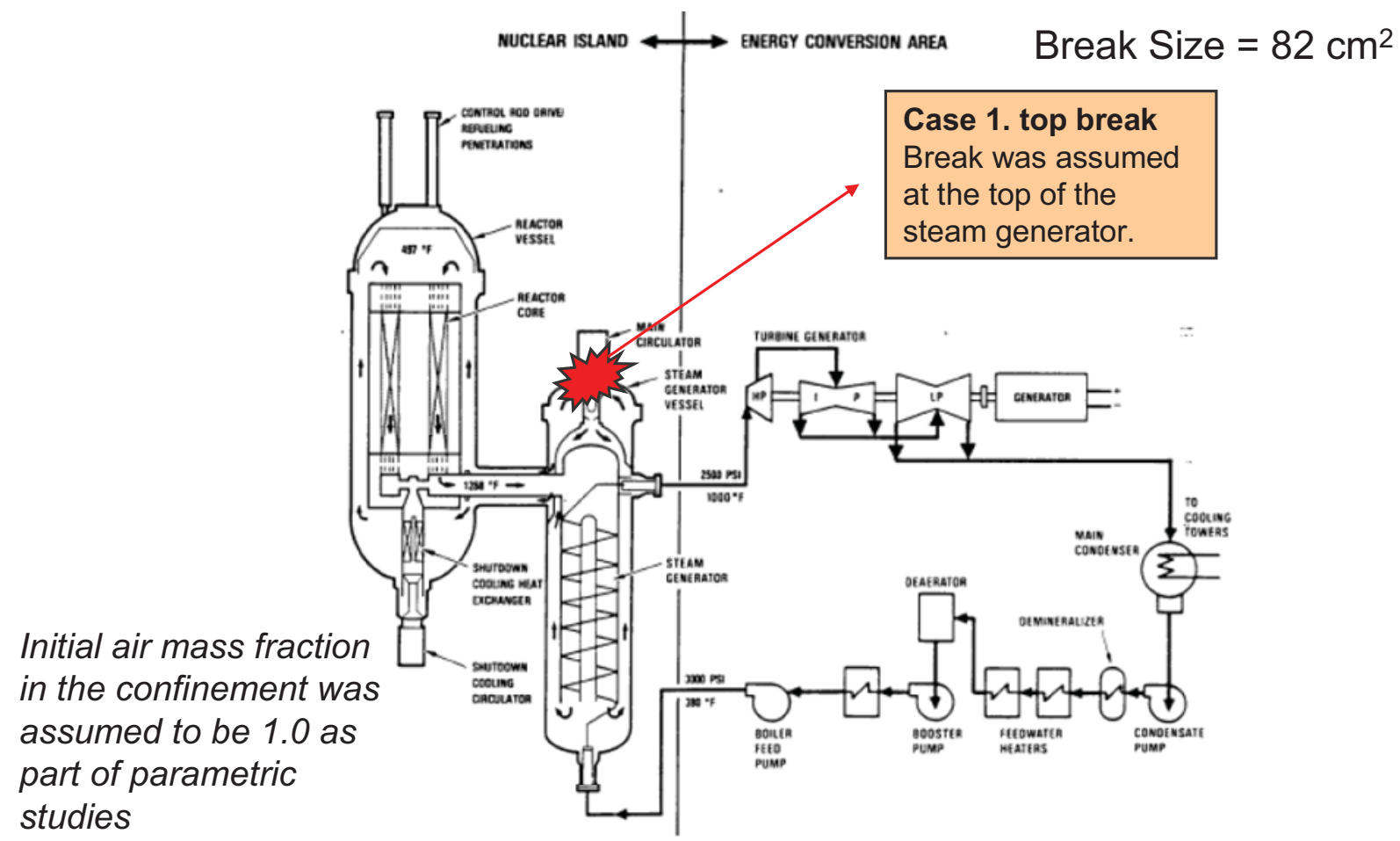

Figure 2-61. VHTR partial break schematic.

FLUENT (Version 6.3) was used with a simplified (2-D) model of the GTMHR into a 2-D geometry that includes GTMHR confinement, a reactor pressure vessel, and a steam generator. The break was assumed to occur at the top of the steam generator, which mimics a small break of the relief valve failure. Initial pressures at the break location were assumed to be the same between reactor inside and outside. The initial temperature distribution was obtained by GAMMA code calculation for depressurization. The options and the models adopted in our analyses are summarized as:

- 2-D

- Unsteady

- Segregated solver

- $2^{\text {nd }}$ order accuracy in time and space

- Noniterative time advancement

- Absolute velocity formulation

- Cell based gradient

- Physical velocity (in the porous media formulation)

- Laminar viscous model

- Energy equation solving

- Species transport equation solving:

- Two gas species: Air/Helium

- Multicomponent diffusion model (same as binary diffusion model in this case)

- No consideration for thermal diffusion 
- Pressure-velocity coupling method: PISO

- Pressure discretization method: PRESTO!

- Momentum: $2^{\text {nd }}$ order upwind

- Species: $2^{\text {nd }}$ order upwind.

Figure 2-62 shows the fluent simulation result $(10 \mathrm{sec})$ of the partial break simulation. In this simulation, it was visualized that the air-flow entered into the steam generator by density gradient. The flow, however, was not quite stratified because of the conflict between inflows (air) and outflows (helium). Figure 2-63 shows the velocity profile in the break location; the $\mathrm{x}$-axis represents horizontal locations, and the y-axis represents y-directional velocity. A positive velocity indicates upward flow (helium outflow) and a negative velocity indicates downward flow (air inflow). According to the FLUENT calculation, the average air inflow velocity is about $0.2 \mathrm{~m} / \mathrm{s}$ while helium outflow is about $0.6 \mathrm{~m} / \mathrm{s}$. This velocity gets slower with time, owing to the decrease of density gradient by mixing.

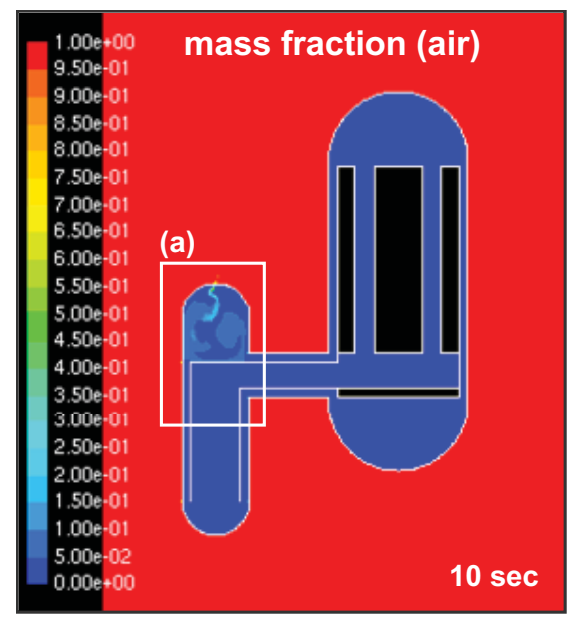

(a) air mass fraction

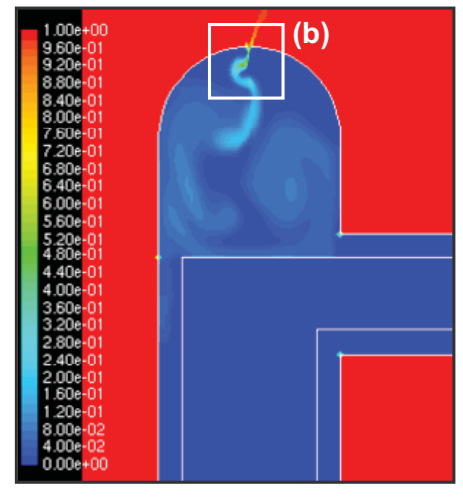

(b) y-velocity

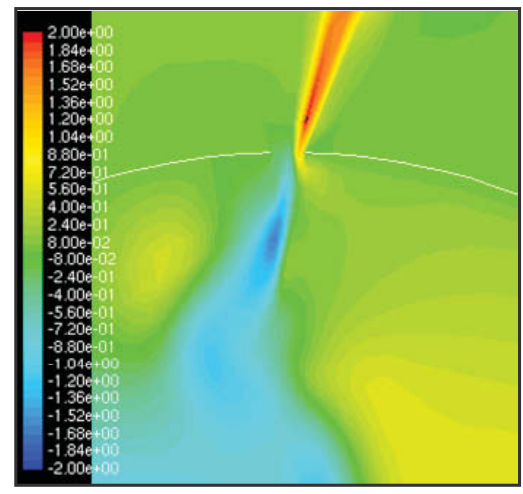

Figure 2-62. Fluent simulation of partial break situation (20 sec).

(a) y-velocity

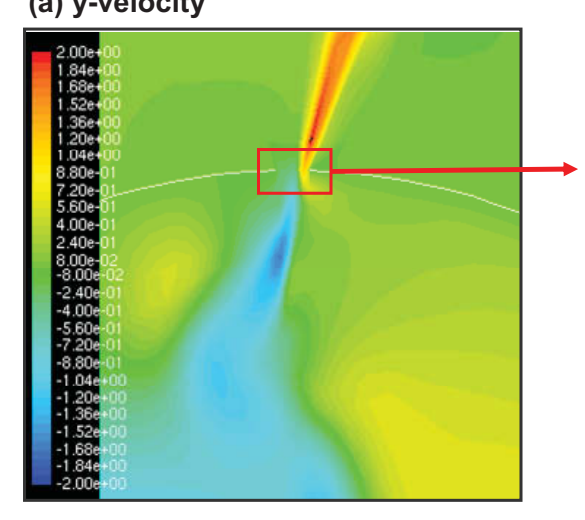

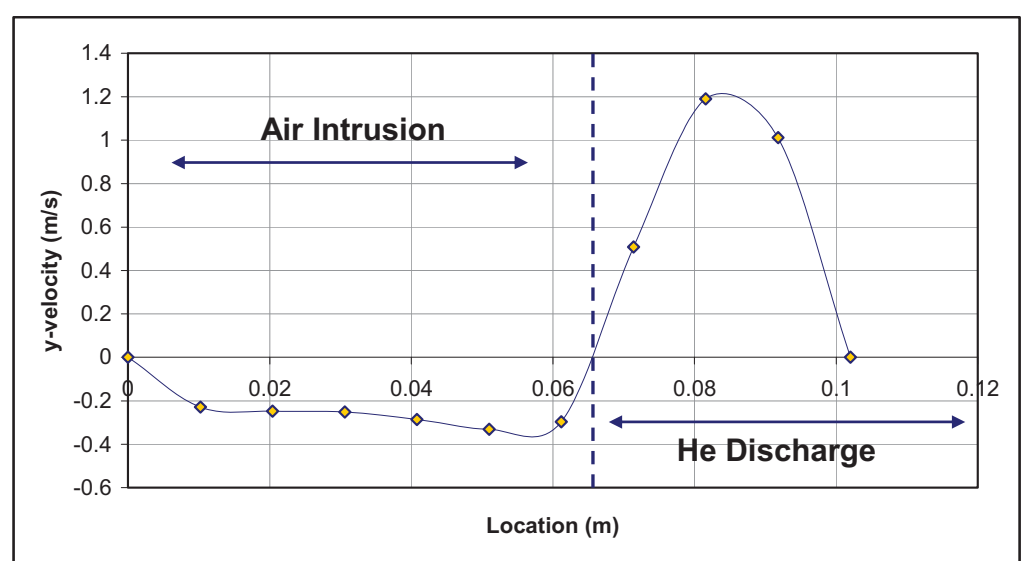

Flow velocity at the break location

Figure 2-63. Velocity profile at the break location (10 sec). 
Figure 2-64 shows the air mass fractions in the VHTR calculated by FLUENT code. According to this figure, the air (1) enters the steam generator through the broken section located at the top of the steam generator; (2) moves to the steam generator bottom by gravity force $(30 \mathrm{sec})$; (3) moves through the steam generator core and hot-leg, and finally (4) moves into the lower plenum and core (100 sec). Once the air goes into the reactor core, it is heated and easily moved up to the top $(200 \mathrm{sec})$. The air ultimately goes back to the steam generator through the cold-leg, which is the outer channel of the horizontal coaxial duct $(200 \mathrm{sec} \sim)$. This FLUENT simulation shows that the air-ingress process in the partial break situation is still very fast and highly dominated by density gradient flow rather than molecular diffusion.
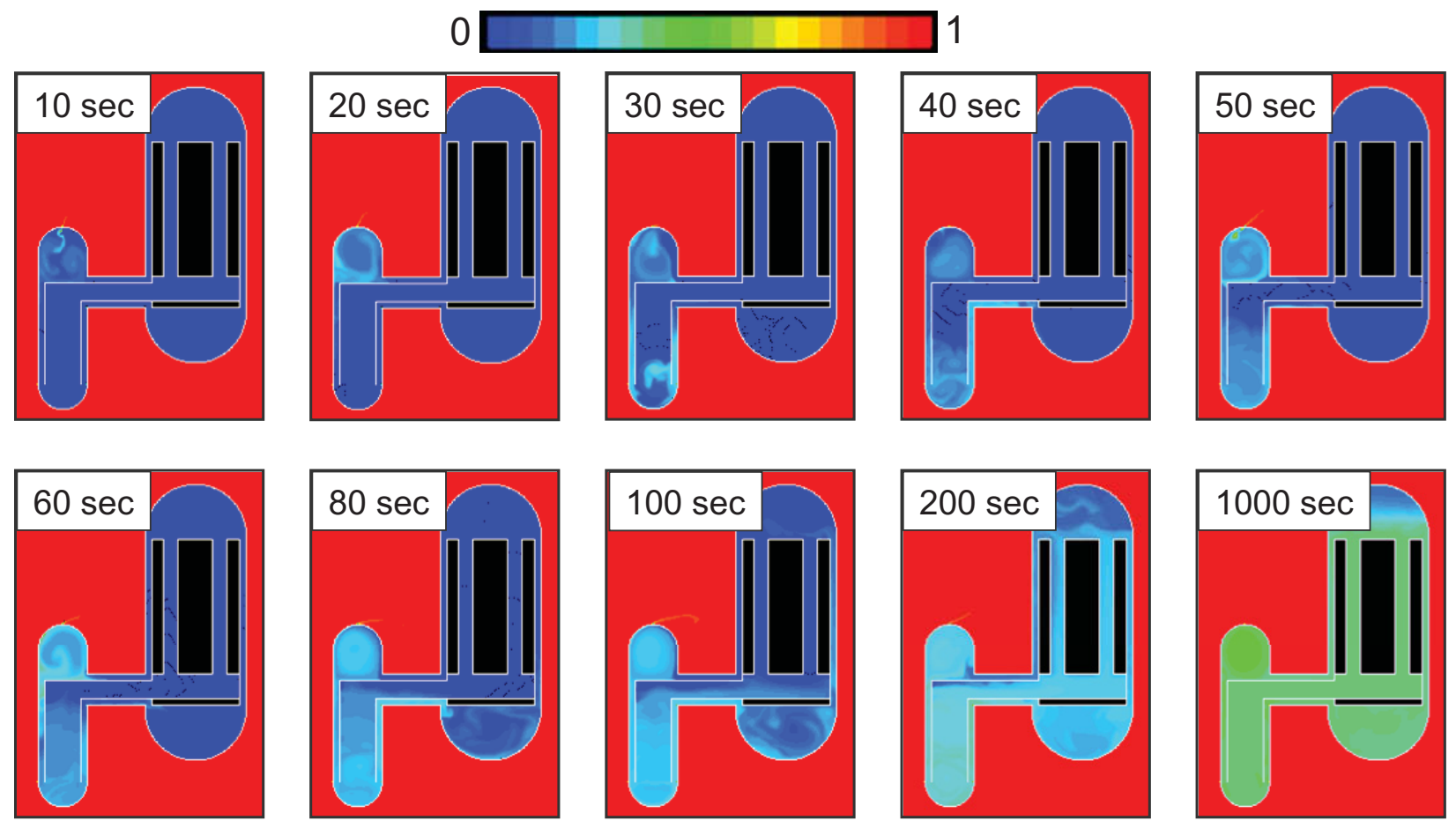

Figure 2-64. Air-mass fractions in the VHTR in the partial break situation (FLUENT results).

Figure 2-65 shows averaged air mass fractions in the reactor lower plenum and core. For the initial 200 seconds, air moves from the broken part to the lower plenum. From 200 to 500 seconds, the air mass fractions in the core and the lower plenum rapidly increased. After 500 seconds, the air mass fractions slowly increase with a global natural circulation. According to this result, after the global natural circulation starts, the air ingress speed decreases with time. This is because the density difference between the inside and outside of the reactor at the broken part also decreases. It is also possible that the global convective motion is preventing the air from coming in through the break at a significant rate.

The wall thickness effect in the FLUENT model in Figure 2-65 was ignored because considering the small wall will make the model size unnecessarily huge. In this section, the effect of the wall thickness is estimated by using two different FLUENT models as shown in Figure 2-66. These FLUENT models basically consist of two tanks in vertical arrangement. Between the two tanks, there is a small hole with $10 \mathrm{~cm}$. The upper tank is initially filled with air and the lower tank with helium. One model has the wall with $10 \mathrm{~cm}$ thickness, the other does not. The pressure and the temperature in the models were assumed to be $1.0 \mathrm{~atm}$ and $25^{\circ} \mathrm{C}$, respectively. 


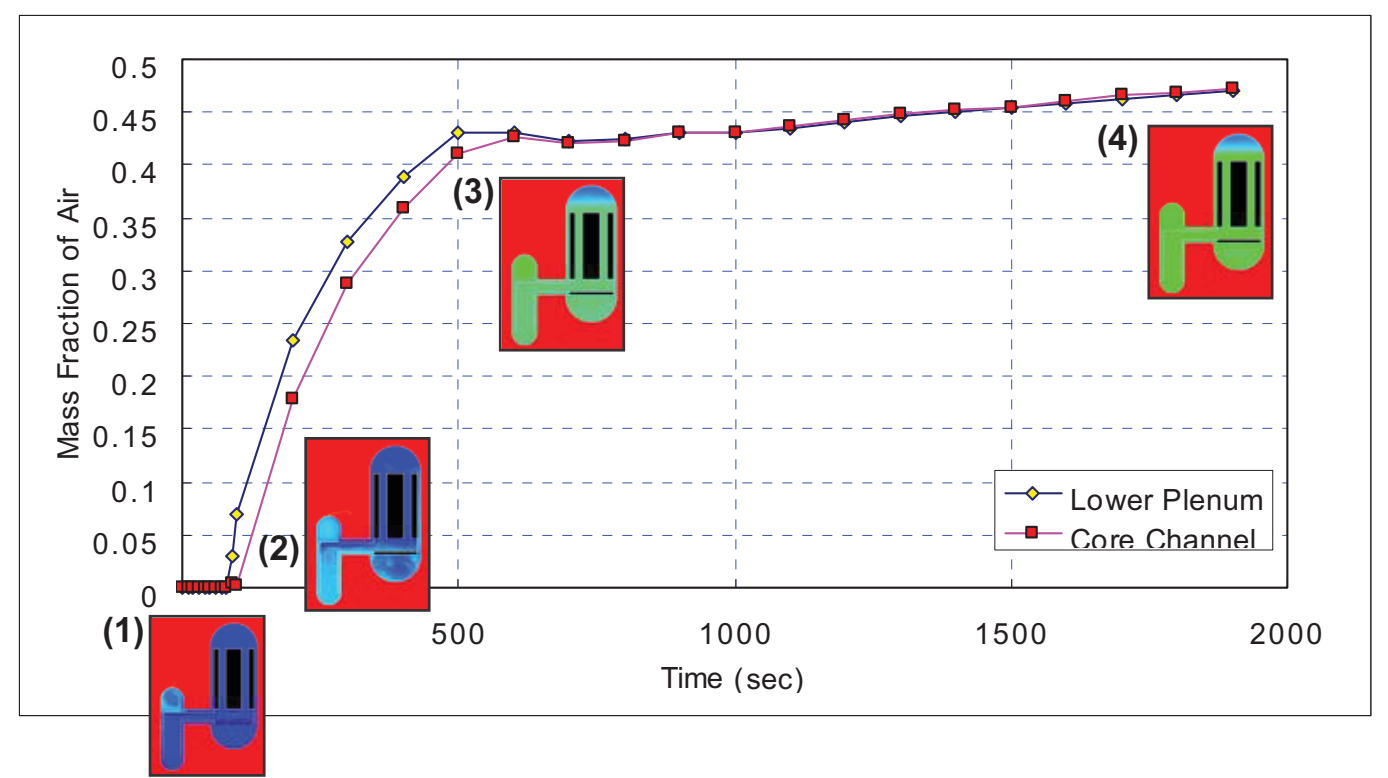

Figure 2-65. Average air mass fraction in the core and the lower plenum.

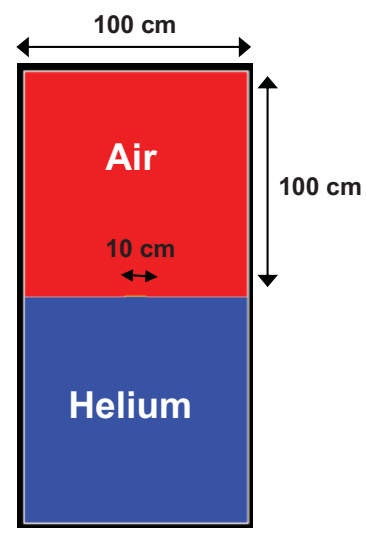

no wall

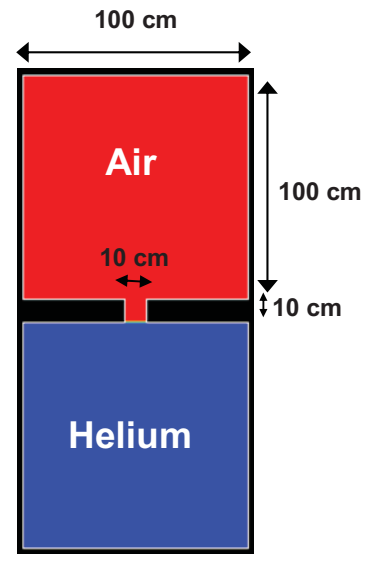

with wall

Figure 2-66. FLUENT models for wall thickness effect.

Figure 2-67 shows the results of the FLUENT calculations by plotting the averaged air mass fractions in the lower tank during 10 second simulations. This plot shows three different data sets: no-wall, withwall, and diffusion. The diffusion case was calculated by deactivating the gravity force in the FLUENT model. As shown, the averaged mass fractions of the with-wall case showed a similar trend to those of the no-wall case, even though specific flow distributions and patterns are not exactly the same. The diffusion case showed very different results from the other two cases. According to the estimation, the air-ingress speeds in the density gradient flows (no-wall and with-wall cases) were much faster than the diffusion case, even in the small hole. This indicates that even in the partial break situation, the density gradient effect is much more dominant than molecular diffusion.

Some preliminary CFD analyses have been performed to fully understand the partial break accidents before the detailed experimental design. One of the most important aspects in the partial break situation is that the flow characteristics are highly dependent on the break angle. Figure 2-68 shows the scheme of the general partial break situation. The cylinder is initially filled with helium (light fluid) while outside the cylinder is initially filled with air (heavy fluid.) 


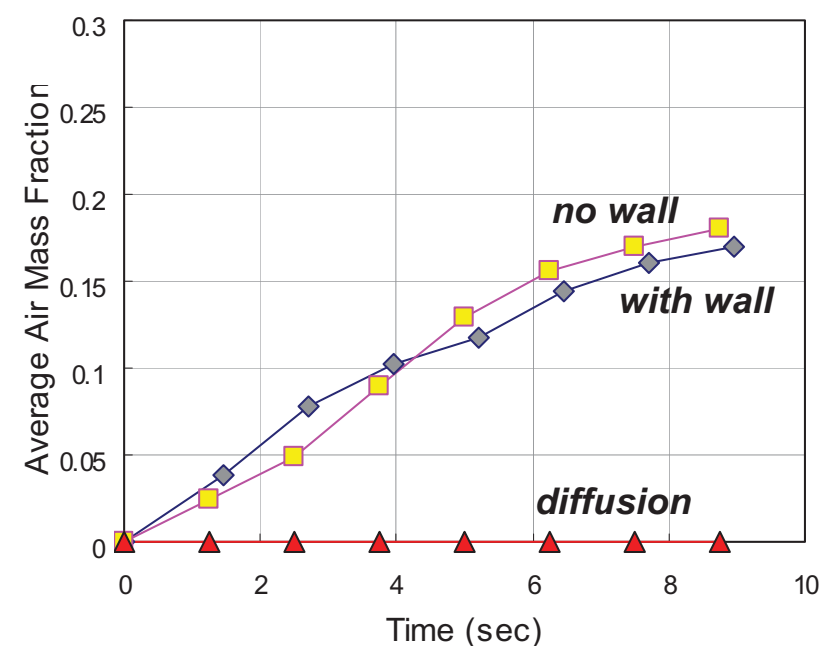

Figure 2-67. Effect of wall thickness in the partial break.

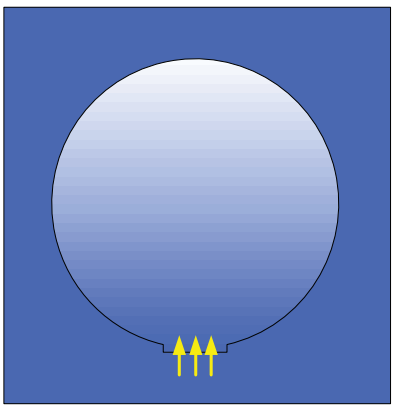

(a) Diffusion

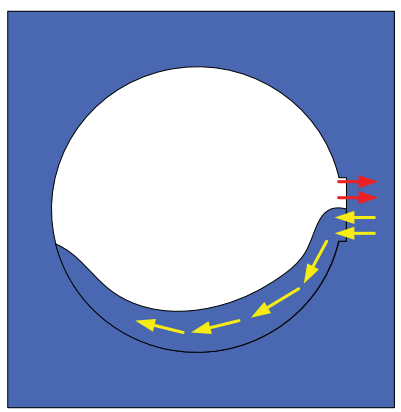

(b) Stratified Flow

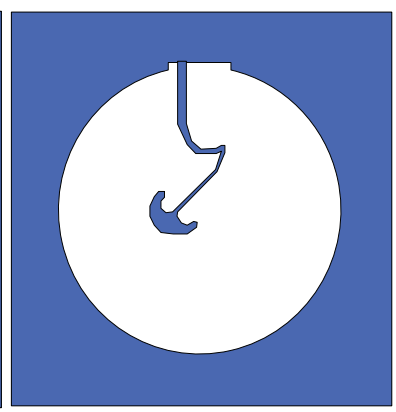

(c) Intermittent Flow

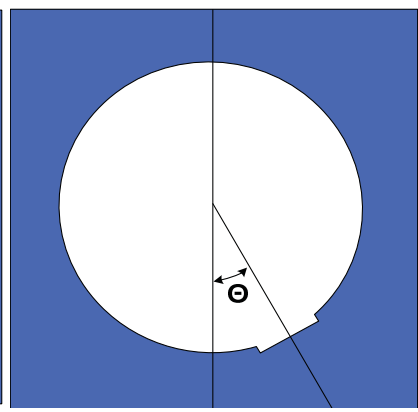

Angle of the hole

Figure 2-68. Three different regimes created depending on the break angle of the hole.

The first regime in Figure 2-68 is molecular diffusion. In the case where the break is at the bottom of the pipe, gravitational force keeps the air from mixing with the helium through either of the second two regimes. For this reason, only diffusion governs the exchange in this case. The second regime is gravitydriven flow. Heavier fluid goes into the lower part of the hole as a counter current flow that is stratified with the lighter fluid exiting in the upper portion. The last regime is an unstable gravity-driven flow for an angle of $180 \mathrm{deg}$. The helium exit flow is counter currently chocked with the air inlet flow. The air flow rate is not constant and will be much smaller than in the second regime.

The objective of the CFD calculation is to observe these three different regimes, depending on the angle. These analyses adopted a simple 2-D geometry, which consists of two cylinders with annular arrangements. The zone of interest is the inner cylinder because the mesh is more precise there than in the outer cylinder. The diameter of the inner cylinder was assumed to be $2.4 \mathrm{~m}$, which is the cold leg diameter in 600 MWth GTMHR design. The diameter of the outer cylinder was arbitrarily determined to be $7.2 \mathrm{~m}$. The break size was assumed to be $0.76 \mathrm{~m}$, which represents $10 \%$. To model the presence of two different species, the multiphase model in FLUENT was adopted, and a standard k- $\varepsilon$ turbulent model was selected for a preliminary study. Second order accuracy in time and space was considered to minimize numerical diffusion. Initially, the inner cylinder was filled with helium (light fluid) and the outer cylinder with air. Seven cases were made for the analyses: $0,45,90,112.5,135,157.5$ and 180 degrees. 
Figure 2-69 shows the simulation results for break angle $=0 \mathrm{deg}$, which represents the first regime (diffusion). The diffusion regime is very slow as predicted and no noticeable changes occur in our time scale of 10 seconds.

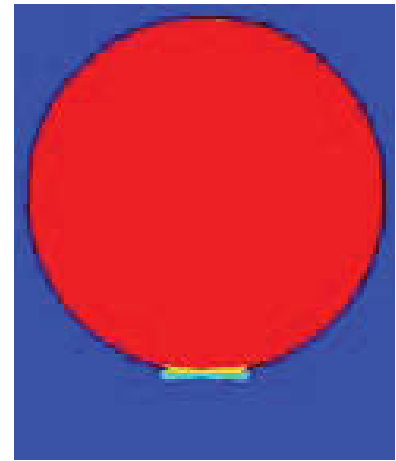

$\mathrm{t}=0$ seconds

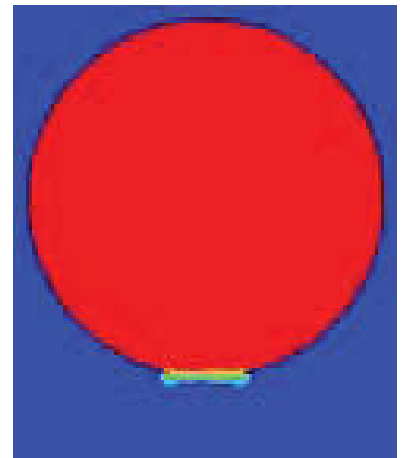

$\mathrm{t}=5 \mathrm{~seconds}$

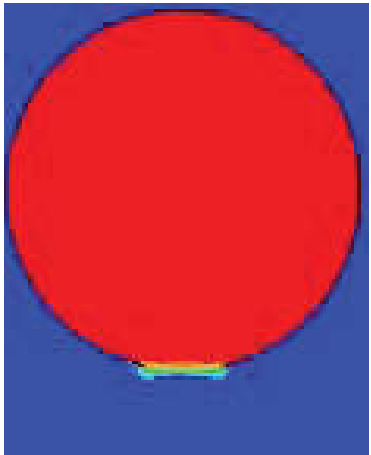

$\mathrm{t}=10$ seconds

Figure 2-69. Diffusion regime (break angle $=0 \mathrm{deg}$ ).

Figure 2-70 shows the simulation results for break angle $=112.5$ degrees, which represents the second regime (stratified flow). At the beginning, air enters the cylinder because of the density difference. Gravity forces the flow to follow the bottom part of the inner cylinder. It seems it doesn't lose so much energy, as it climbs at the altitude of the hole on the other side of the wall. Its momentum then forces it to spiral and mix with helium. After 6 seconds, a boundary begins to stabilize at the height of the hole. Wave motion can be seen on the interface. On the other hand, helium, which is lighter than the air, rises in the outer cylinder. A bubble of gas is created 1 second after the beginning and then mushrooms after 2 seconds. Next, the exiting plume straightens out.

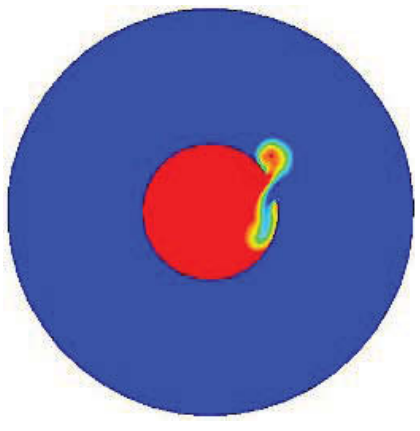

$\mathrm{t}=1 \mathrm{sec}$

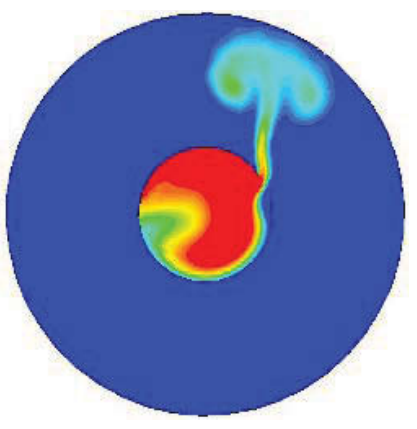

$\mathrm{t}=3 \mathrm{sec}$

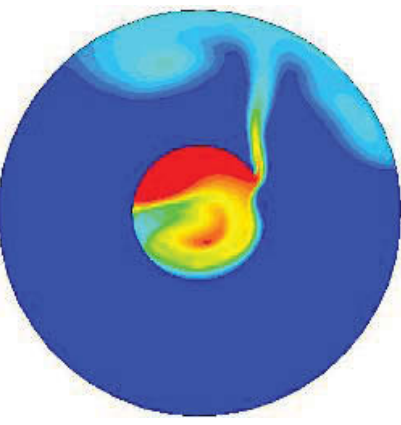

$\mathrm{t}=5 \mathrm{sec}$

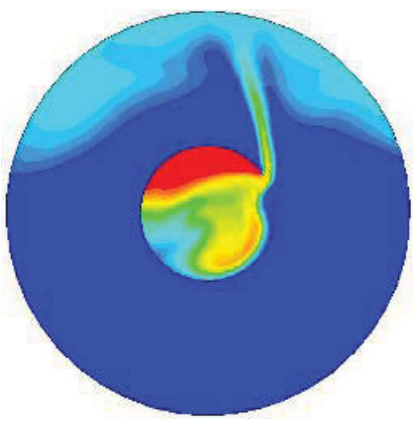

$\mathrm{t}=7 \mathrm{sec}$

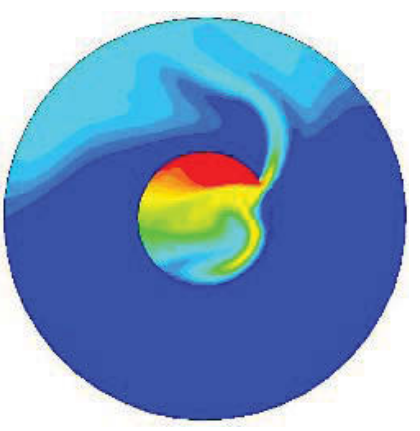

$\mathrm{t}=9 \mathrm{sec}$

Figure 2-70. Stratified flow regime (break angle $=112.5 \mathrm{deg}$ ). 
Figure 2-71 shows the simulation results for break angle $=180$, which represents the intermittent flow regime. The 180-degree case is totally different from the previous one. Air cannot flow directly along the bottom of a helium flow because of the orientation of the hole. The initial interface is unstable and the interface will roll-up. This helium bubble created within the outer tank is mirrored by an air bubble within the inner volume. The process of this exchange creates a pulsed flow across the break.

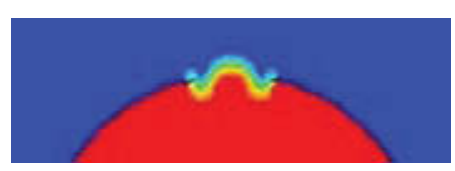

$\mathrm{t}=1 \mathrm{sec}$

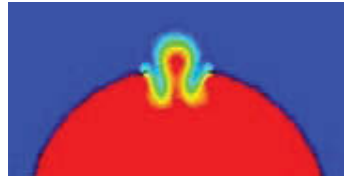

$\mathrm{t}=1.5 \mathrm{sec}$

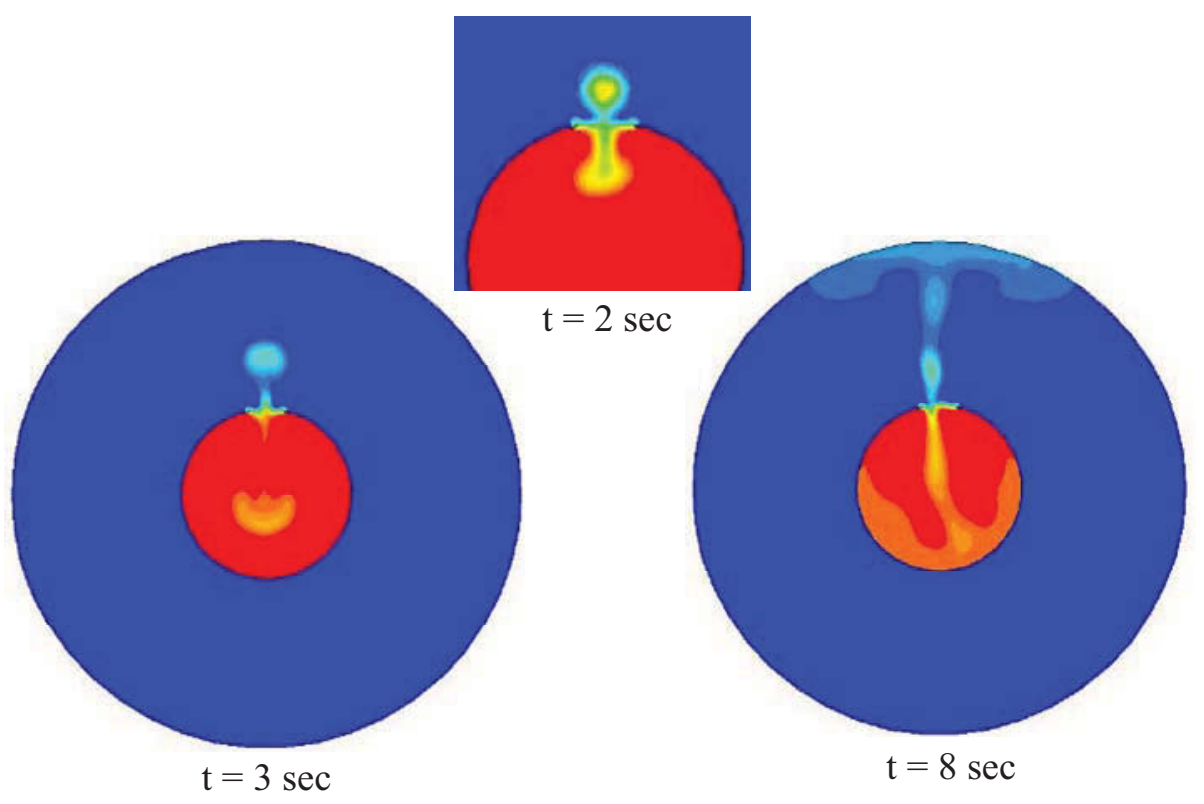

Figure 2-71. Intermittent flow regime (break angle $=180 \mathrm{deg}$ ).

Figure 2-72 shows the relations between break angles and maximum air flow rates. Air flow initially increases with break angle and peaks around 125 degrees. The flow rate then rapidly drops with angle, owing to the flow conflict between inflows and outflows as seen in the intermittent flow regime. When the break angles reach $180 \mathrm{deg}$, the flow rate is decreased to $25 \%$ of the maximum flow rate. 


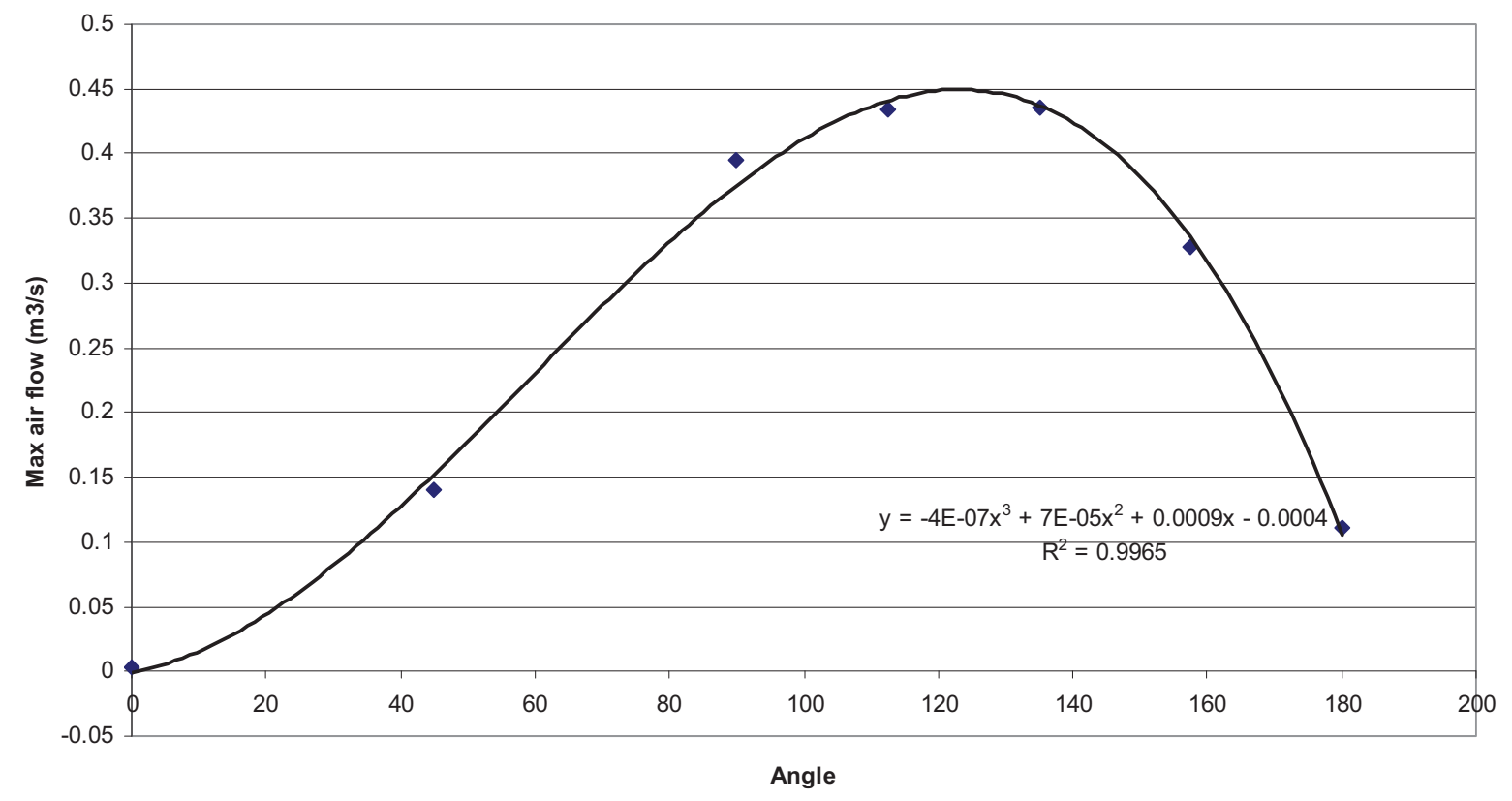

Figure 2-72. Break angle vs. maximum volumetric flow rate.

\subsection{Analyses on Natural Circulation Patterns in VHTR Air-ingress Accident}

This section focuses on the natural convection flow after ONC because the natural circulation flow significantly affects graphite oxidation and corrosion behaviors during the air-ingress accident. In the previous air-ingress studies, natural circulation flow was assumed to follow the following single flow path shown in Figure 2-73:

Cavity $\rightarrow$ hot-leg $\rightarrow$ lower plenum $\rightarrow$ core $\rightarrow$ upper plenum $\rightarrow$ riser $\rightarrow$ cold-leg $\rightarrow$ cavity.

From this assumption, the air-ingress speed has been estimated to be about 0.1 to $0.2 \mathrm{~m} / \mathrm{s}$, which is very slow. However, in the VHTR air-ingress accident, the reactor inside temperatures are generally much higher than the reactor outside by about 300 to $600^{\circ} \mathrm{C}$, making the outside gas density about 2 to 3 times larger than that of the inside, which might cause local density gradient driven countercurrent flow along the broken hot-leg, which is much more complicated than the simple 1-D flow path. Therefore, it is important to validate the previous 1-D assumptions for better analyses of the air-ingress accident in the future.

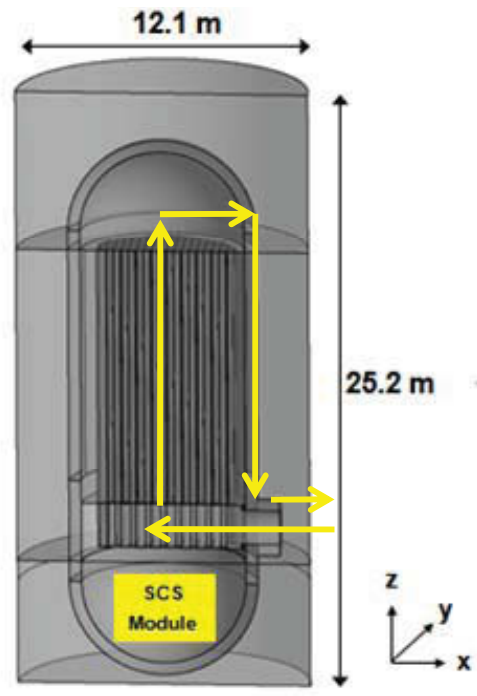

Figure 2-73. Previous natural circulation pattern assumed in 1-D simulation. 


\subsubsection{CFD Modeling and Basic Assumptions}

CFD analyses were performed in this study for characterizing natural circulation flow in the airingress accident. Figure 2-74 shows the FLUENT grid model developed for 3-D simulation. This model is based on the basic dimensions and geometries of the 600 MWth GTMHR (Oh et al. 2008). It was modeled as a half symmetric geometry for reducing grid numbers. The core, upper plenum, coolant riser, and bottom reflector were hexagonally meshed by ICEM-CFD software (ANSYS 2008), but the lower plenum was meshed by GAMBIT with mixed hexahedral, tetrahedral, and pyramidal meshes (Johnson 2008). Each grid model was separately imported into the FLUENT 6.3 and merged by grid interface. Table 2-8 summarizes the grid information of this FLUENT model.

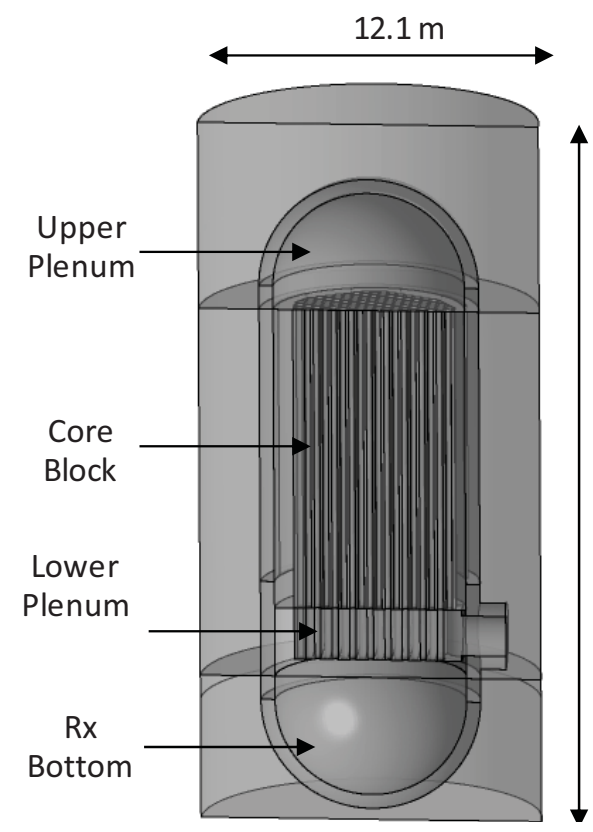

(a) Side view of 3-D grid model

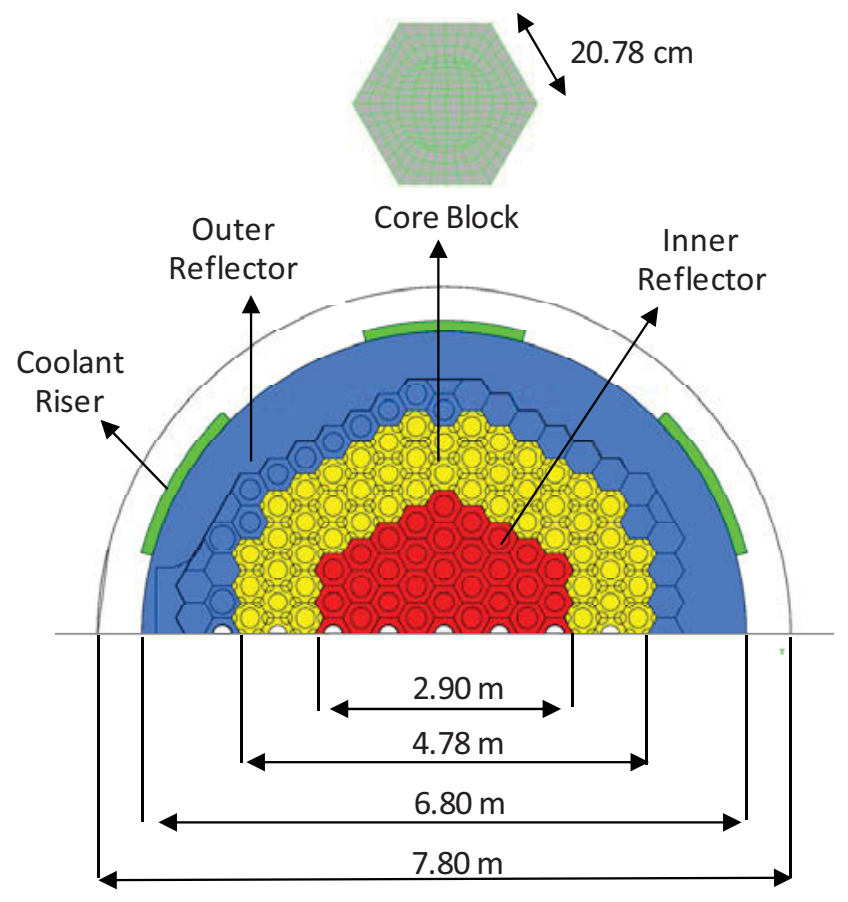

(b) Top view of Rx vessel in the 3-D grid model

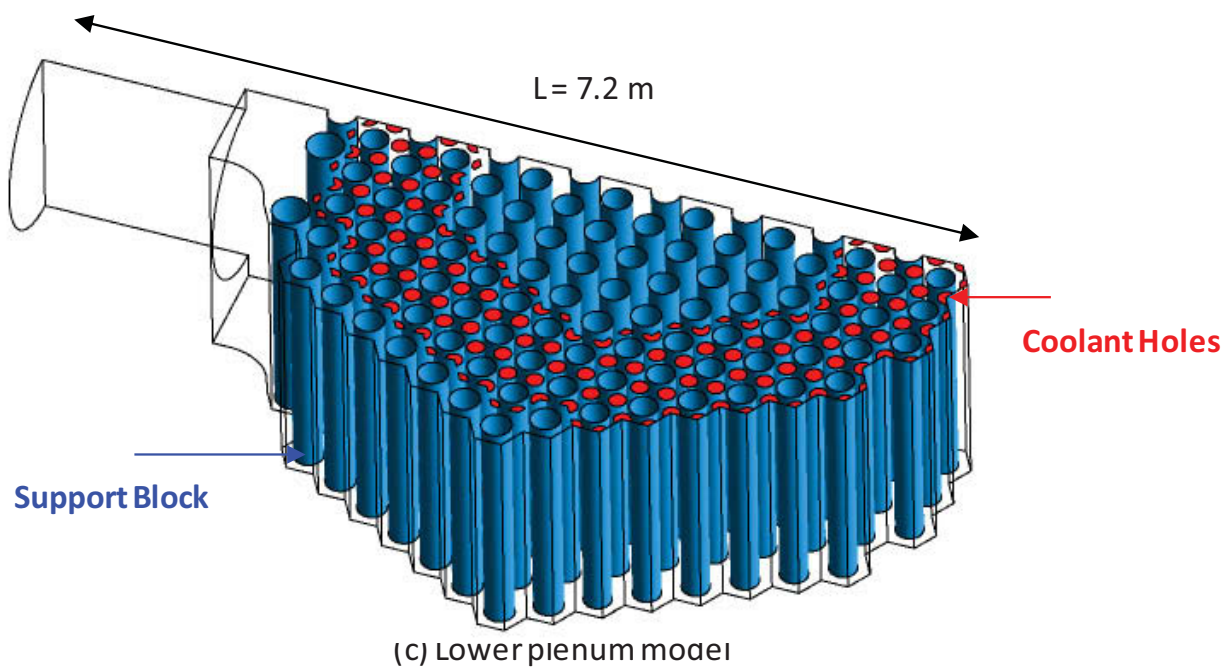

(c) Lower pienum moaeı

Figure 2-74. 3-D grid model for VHTR air-ingress accident. 
Table 2-8. Number of mesh and volume data for each region in the 3-D grid model.

\begin{tabular}{|c|c|c|}
\hline \multicolumn{2}{|r|}{ Reactor Internal } & Confinement \\
\hline Core Blocks & $\begin{array}{l}\text { Volume: } 60.35 \mathrm{~m}^{3} \text { (Volume Porosity: } 0.185 \text { ) } \\
\text { Height: } 10.82 \mathrm{~m} \\
\text { Hexahedral mesh: } 2,248,560\end{array}$ & \multirow{5}{*}{$\begin{array}{l}\text { Volume: } 961.05 \mathrm{~m}^{3} \\
\text { Hexahedral mesh: } 621,183 \\
\text { Fluid volume ration the } \\
\text { confinement to the reactor } \\
\text { internal: } 3.81\end{array}$} \\
\hline Lower Plenum & $\begin{array}{l}\text { Volume: } 15.29 \mathrm{~m}^{3} \\
\text { Height: } 1.84 \mathrm{~m} \\
\text { Hexahedral mesh: } \quad 677,917 \\
\text { Tetra mesh: } 25,940 \\
\text { Pyramids mesh: } 1,103\end{array}$ & \\
\hline Upper Plenum & $\begin{array}{l}\text { Volume: } 66.27 \mathrm{~m}^{3} \\
\text { Radius: } 3.4 \mathrm{~m} \\
\text { Hexahedral mesh: } \quad 712,023\end{array}$ & \\
\hline Coolant Riser & $\begin{array}{l}\text { Volume: } 6.98 \mathrm{~m}^{3}\left(2.328 \mathrm{~m}^{3} \times 3\right) \\
\text { Height: } 9.87 \mathrm{~m} \\
\text { Hexahedral mesh: } \quad 287,820\left(2.328 \mathrm{~m}^{3} \times 3\right)\end{array}$ & \\
\hline Rx Bottom & $\begin{array}{l}\text { Volume: } 82.33 \mathrm{~m}^{3} \\
\text { Hexahedral mesh: } \quad 651,963\end{array}$ & \\
\hline & Total meshes $=8,517,835$ & \\
\hline
\end{tabular}

Figure 2-75 shows the geometry and mesh of the model near the lower plenum where the meshes are densely distributed throughout the whole geometry. The core region of this model was simplified to be a porous body having 0.2 volume porosity. The porous media parameters related to the flow resistance were estimated using the circular channel friction data. The $2 \mathrm{~mm}$ bypass gaps in the core blocks were neglected to avoid computational limit.

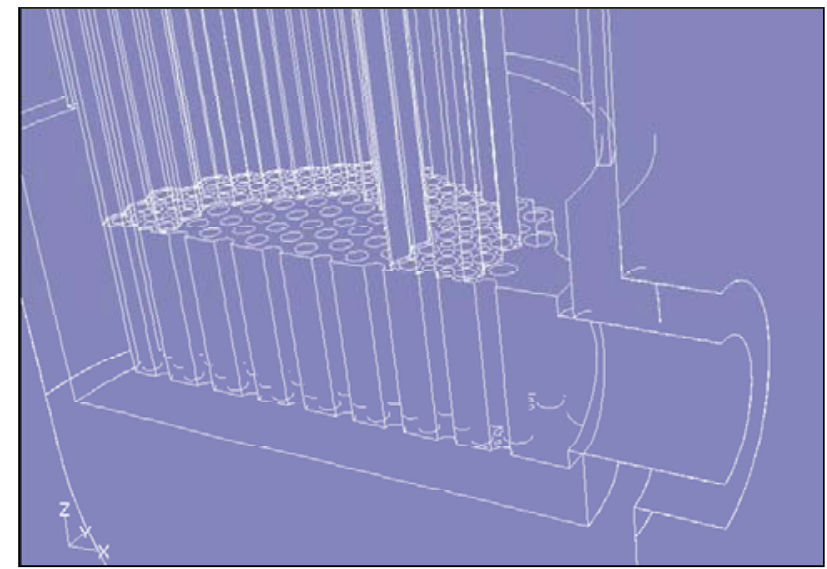

(a) Geometry

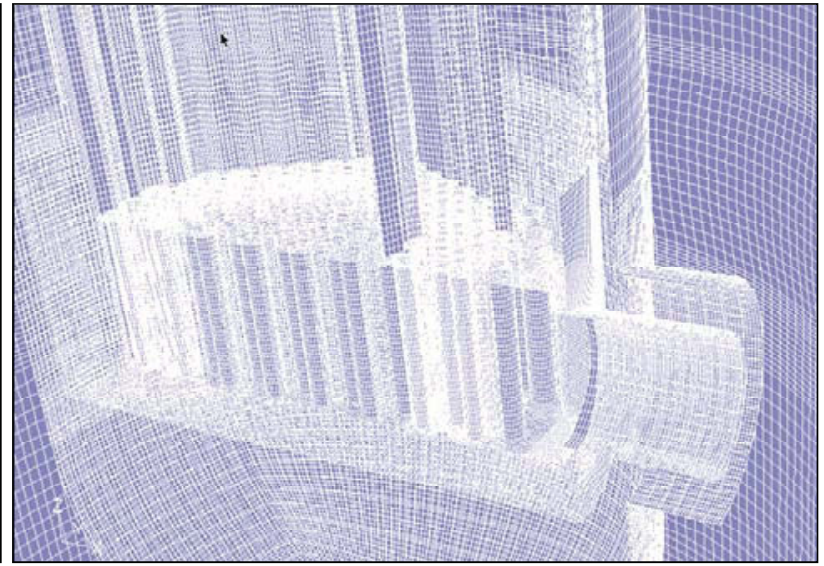

(b) Mesh

Figure 2-75. Geometry and mesh of the 3-D grid at the lower part of the reactor. 
Table 2-9 summarizes the FLUENT model setups. FLUENT Version 6.3.26 was used in this study and a general pressure based solver was selected with 20 parallel nodes. The steady-state option was used to reduce simulation time. According to the previous investigations, temperature variations with time in the post $\mathrm{ONC}$ is very slow because of the large heat capacity of the reactor solid structures. Figure 2-76 shows the temperature variations in the reactor during the air-ingress accident calculated by $\mathrm{Oh}$ et al. (2008). As shown in this figure, the temperatures in the reactor solid structures, including reactor core, lower plenum, upper plenum, and lower plenum, are not visibly changing in the given time ranges $(\sim 500 \mathrm{sec})$. Therefore, the steady-state option is considered as a good assumption in this study.

The basic governing equations solved here are a continuity equation, three momentum conservation equations, an energy conservation equation, and a species conservation equation. The reference turbulence model was selected as the Realizable k-e model based on two equations. We also performed parametric study for various boundary conditions including initial air mole fractions, vessel wall temperatures, cavity wall temperatures, and lower plenum temperatures. Three more viscous models were also considered in this parametric study in order to consider the effect of turbulence models on results. Additional turbulence models are the laminar model, standard k-w model, and Reynolds Stress Model (RSM). Fluid density was estimated by the incompressible ideal gas law. Since the Mach number is very low and the pressure variations throughout the model are negligible, this option is quite valid here. The other mixture properties, including heat capacity $\left(C_{p}\right)$, thermal conductivity $(k)$, and viscosity were calculated by mixing laws based on the mass weighted functions. A multicomponent diffusion model was selected for calculating gas diffusion. Chemical reactions, including graphite oxidation and CO combustion were not considered in this modeling because of complexities. It will be considered in the future studies.

Table 2-9. Reference FLUENT model setup.

\begin{tabular}{|ll|}
\hline \multicolumn{1}{|c|}{ Parameters } & \multicolumn{1}{c|}{ Settings } \\
\hline Code Version & FLUENT 6.3.26 \\
Solver Type & Pressure Based Solver \\
Dimension & 3-D \\
Steady/Unsteady & Steady \\
Number of CPUs & 20 \\
Velocity Formulation & Absolute \\
Gradient Option & Node Based \\
Porous Formulation & Physical Velocity \\
Viscous Model & Realizable k-e \\
PressureVelocity Coupling & SIMPLE \\
Air Mass Fraction & 0.5 \\
Energy Equation Solve & Yes \\
Species Equation Solve & Yes \\
Density & Incompressible Ideal Gas Law \\
Heat Capacity & Mixing Law \\
Thermal Conductivity & Mass Weighted Mixing Law \\
Viscosity & Mass Weighted Mixing Law \\
Diffusion & Multi-component \\
\hline
\end{tabular}




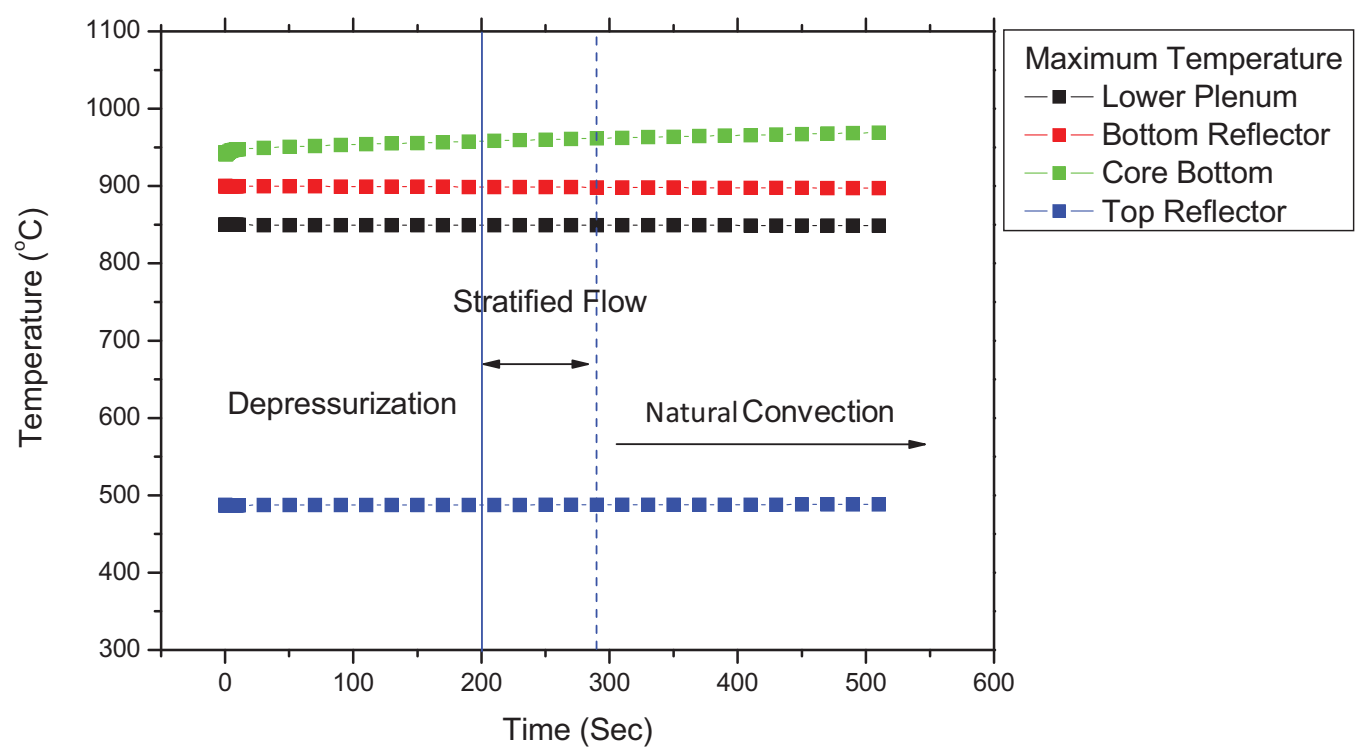

Figure 2-76. Temperature variations with time during air-ingress accident (Oh et al. 2008).

\subsubsection{Boundary Conditions}

Figure 2-77 shows the reference boundary conditions. The core temperature was determined by the GAMMA code simulation results (Oh et al. 2008, 2009). The reactor and the cavity side wall temperatures were assumed to be 763 and $473 \mathrm{~K}$ for the base case. The lower plenum temperature was assumed to be $1145 \mathrm{~K}$ at the wall. Cavity top and bottom walls were assumed to be adiabatic. These boundary conditions were changed in the parametric studies as listed in Table 2-10.

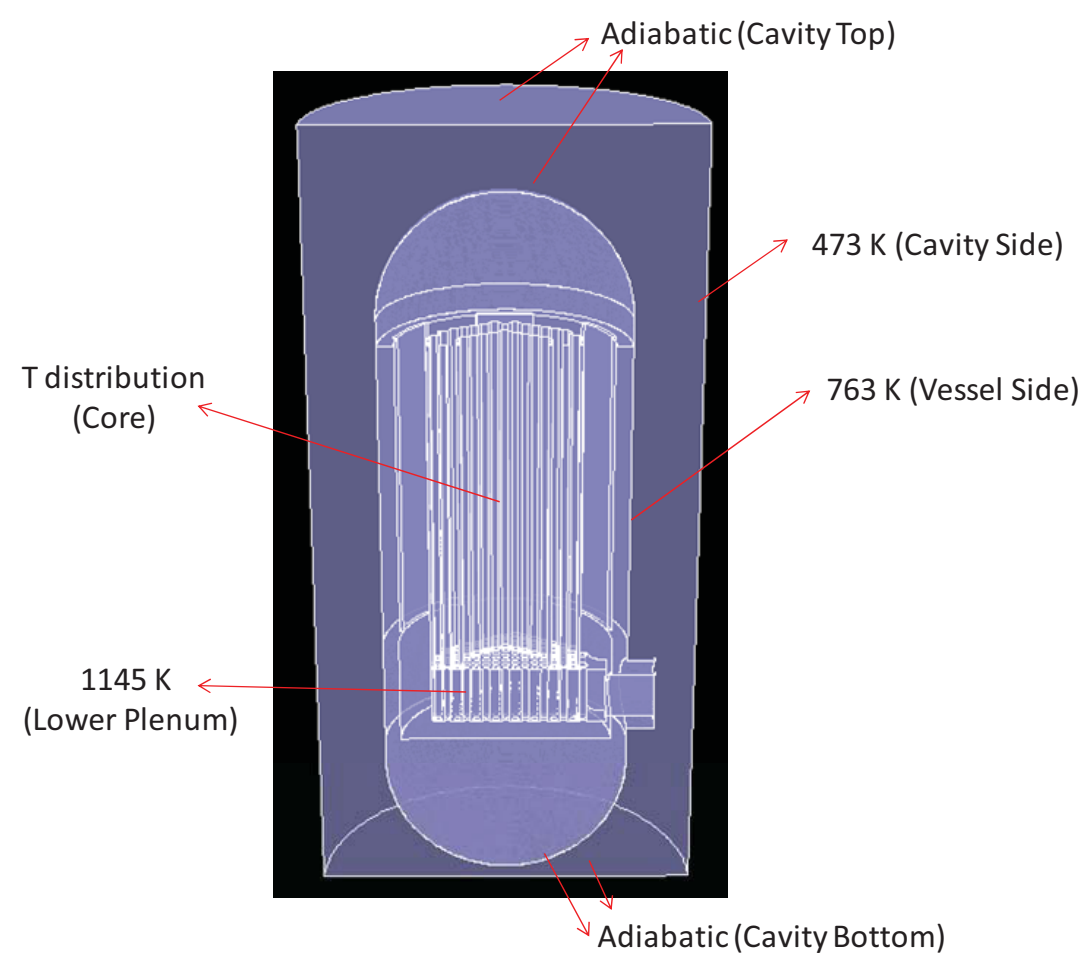

Figure 2-77. Reference boundary conditions for the CFD simulation. 
Table 2-10. Simulation cases and conditions.

\begin{tabular}{|llcccc|}
\hline \multicolumn{1}{c}{ Case } & \multicolumn{1}{c}{$\begin{array}{c}\text { Turbulence } \\
\text { Model }\end{array}$} & $\begin{array}{c}\text { Cavity Side } \\
\text { Wall T (K) }\end{array}$ & $\begin{array}{c}\text { Vessel Side } \\
\text { Wall T (K) }\end{array}$ & $\begin{array}{c}\text { Lower Plenum } \\
\text { Wall T (K) }\end{array}$ & $\begin{array}{c}\text { Air Mass } \\
\text { Fraction }\end{array}$ \\
\hline 1 (base) & Realizable k-e & 473 & 763 & 1145 & 0.5 \\
3 & Lam & 473 & 763 & 1145 & 0.5 \\
4 & k-w & 473 & 763 & 1145 & 0.5 \\
5 & RSM & 473 & 763 & 1145 & 0.5 \\
6 & Realizable k-e & 473 & 763 & 945 & 0.5 \\
7 & Realizable k-e & 473 & 763 & 1045 & 0.5 \\
8 & Realizable k-e & 373 & 763 & 1145 & 0.5 \\
9 & Realizable k-e & 573 & 763 & 1145 & 0.5 \\
10 & Realizable k-e & 473 & 763 & 1145 & 0.1 \\
11 & Realizable k-e & 473 & 763 & 1145 & 0.9 \\
12 & Realizable k-e & 473 & 663 & 1145 & 0.5 \\
\hline
\end{tabular}

\subsubsection{Results and Discussions}

This section summarizes the CFD simulation results with some discussions. Figures 2-78 through 2-82 show the base case results (See Figure 2-77 and Table 2-10). In the base case, the realizable k-e model was selected to be a turbulence model. The cavity and the vessel wall temperatures were assumed to be $473 \mathrm{~K}$ and $763 \mathrm{~K}$, respectively. The lower plenum temperature was set as $1145 \mathrm{~K}$ from the GAMMA code results. The core temperature profile was also obtained from the GAMMA code results. The initial air mass fraction in the cavity was assumed to be 0.5 , which was calculated from the GAMMA code assuming a complete mixing of helium and air in the cavity.

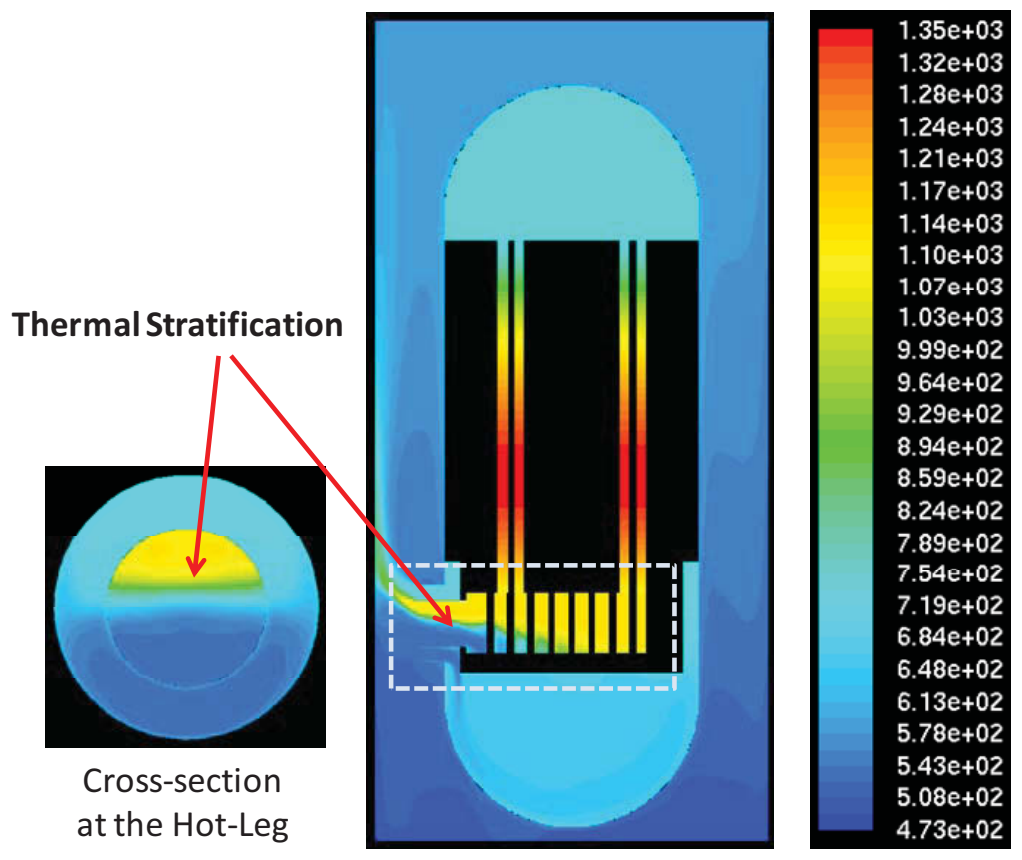

Figure 2-78. Temperature (K) contour plot (base case). 


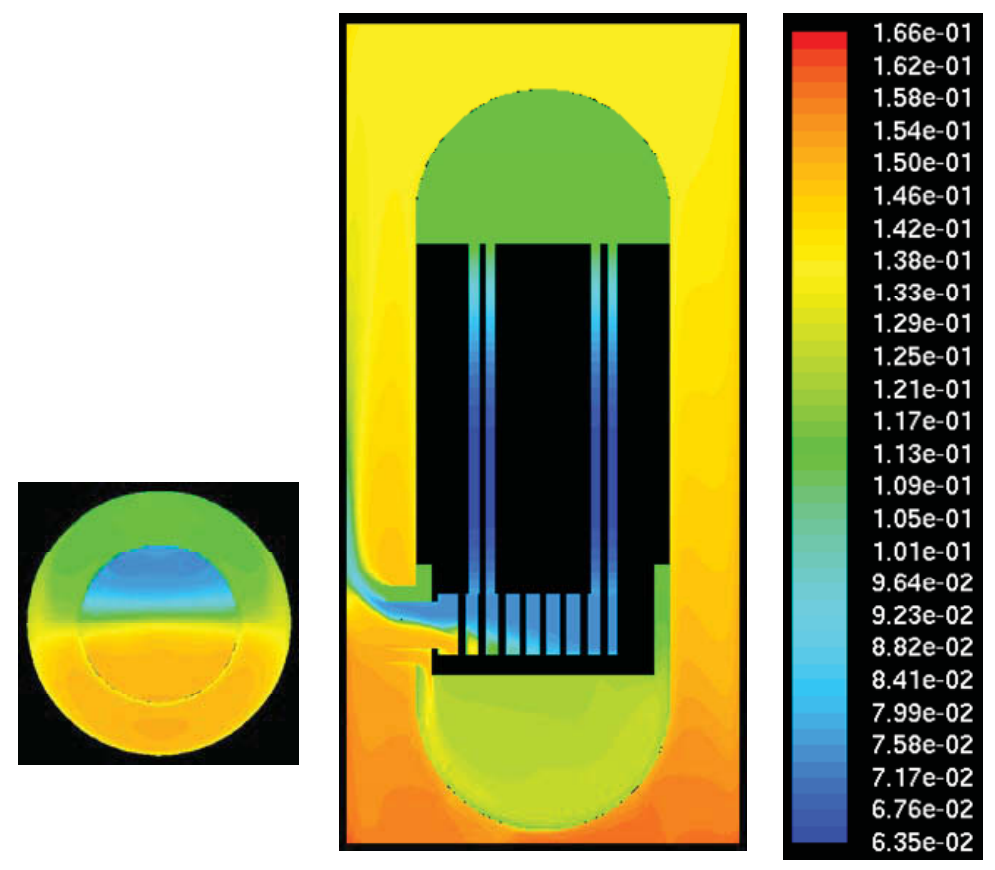

Figure 2-79. Density $\left(\mathrm{kg} / \mathrm{m}^{3}\right)$ contour plot (base case).

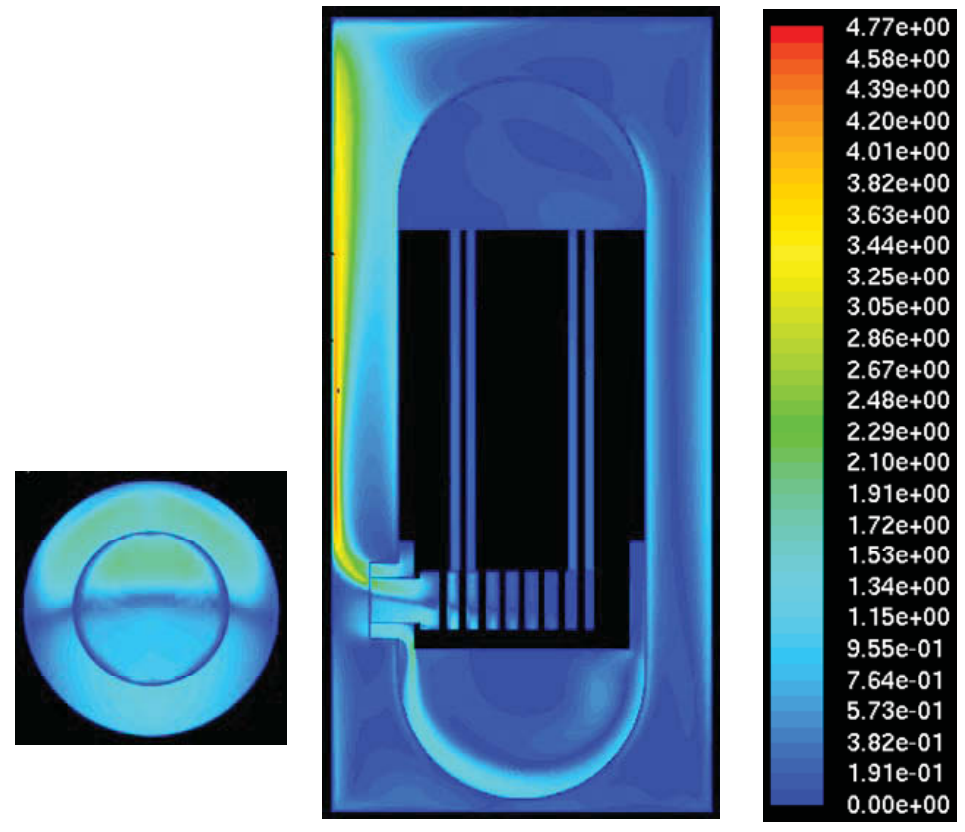

Figure 2-80. Velocity (m/s) contour plot (base case). 


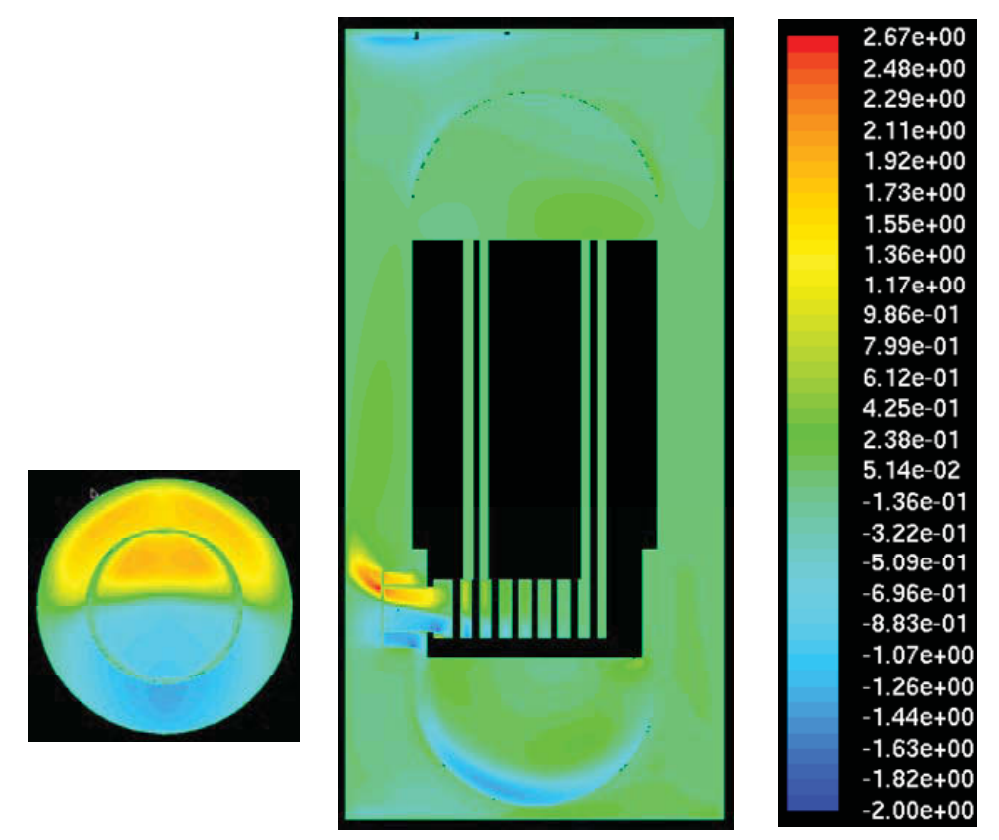

Figure 2-81. X-velocity (m/s) contour plot (base case).

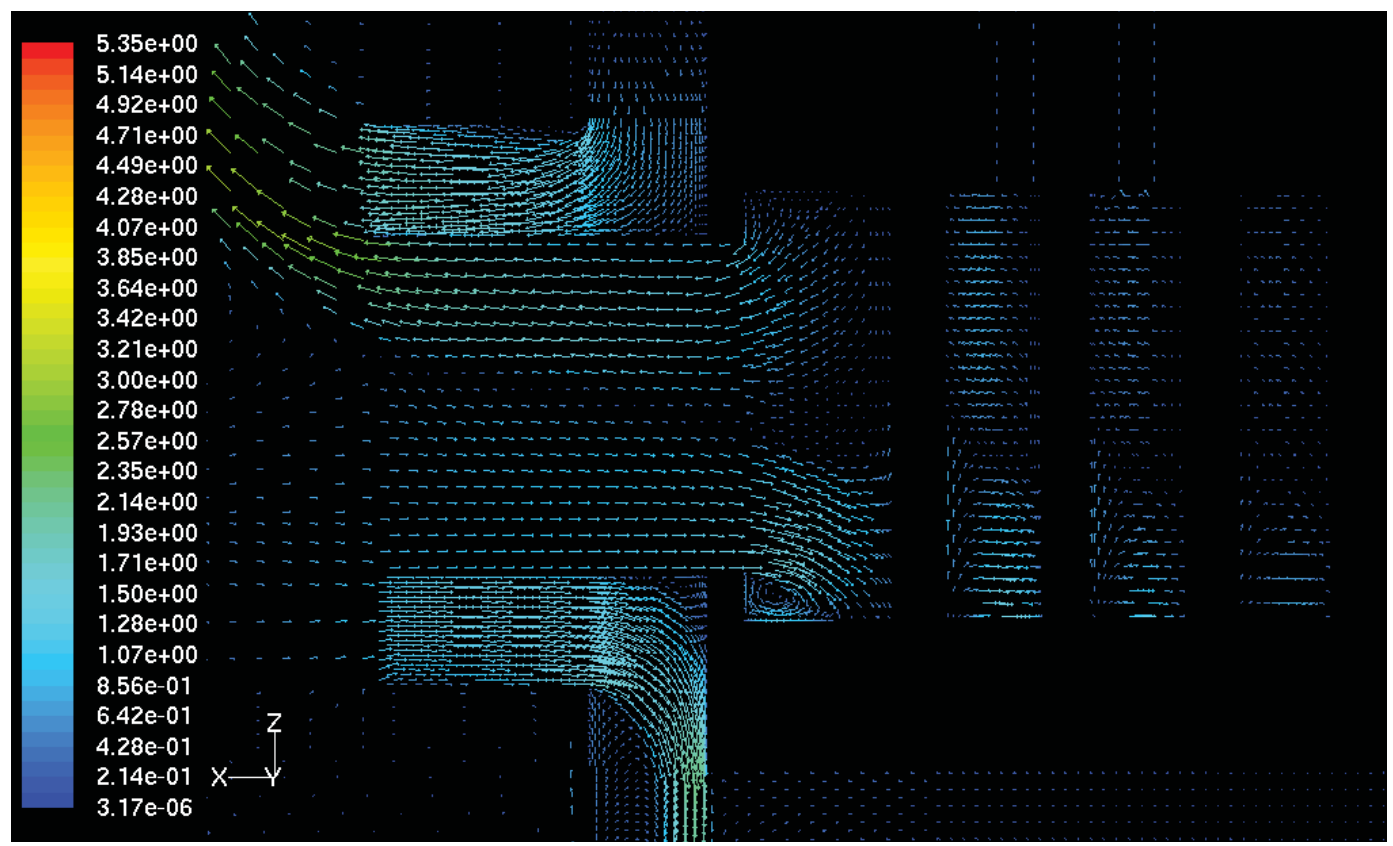

Figure 2-82. Velocity vector at the broken pipe (base case).

Figure 2-78 shows the contour plot of the temperature at the reactor cross-section. It is notable that the thermal stratification is shown at the hot-leg and the lower plenum. In this figure, the left bottom is occupied with cold fluids $(\sim 540 \mathrm{~K})$, and the right top with hot fluids $(\sim 1100 \mathrm{~K})$. This temperature gradient provides significant density gradient. Since the gas density is inversely proportional to the temperature, the maximum density is about two to three times larger than the minimum density in our calculations. Figure 2-79 shows the density distributions at the reactor cross-section. As shown in this figure, a clear density change exists between hot and cold fluids coupled with temperature. This thermal stratification is originated from the temperature difference between the inside and outside of the reactor vessel. During 
the air-ingress accident, the inside temperature is maintained high because of core decay heat and resident heat of the support structures. On the other hand, the outside temperature is maintained relatively low because of the cavity cooling system.

The thermal stratification is important because it is a good evidence that the stratified flow was driven by temperature gradient with recirculation flow patterns in the hot-leg and the lower plenum. The basic principle of this phenomenon is exactly the same as the density gradient driven stratified flow between air and helium shown after the depressurization phase. The only difference is that the density gradient of this case is originated from nonuniform temperature distributions in the reactor. Figures 2-80 and 2-81 show the contour plots for the absolute and x-directional velocities, respectively. These figures show the counter current flow in the hot-leg and the lower plenum. The blue color in Figure 2-80 represents the incoming flow while the red color represents the out-going flow. The vector plot in Figure 2-82 more clearly shows that a strong recirculation flow exists at the broken hot-leg. It also shows the cold fluids coming into the reactor through the lower part, and the hot fluids going out through the upper part. This recirculation pattern in the post $\mathrm{ONC}$ has never been reported or considered in the previous air-ingress studies.

The recirculation flow in the lower plenum is very important because this flow pattern may result in different accident consequences from those predicted in the previous studies. Figure 2-83 shows the comparisons of natural circulation patterns between the previous 1-D assumption and the multi-D simulation estimated by this study. The biggest difference between these two cases is the recirculation flow in the hot-leg and the lower plenum. In the 1-D simulation, the natural circulation pattern consists of a single flow path, so we cannot see any recirculation flows. However, the multi-D simulation shows strong recirculation flow patterns. According to the current study, it is anticipated that the recirculation flow will cause more graphite oxidation on the supporting graphite structure in the lower plenum. The following paragraphs discuss this more quantitatively.

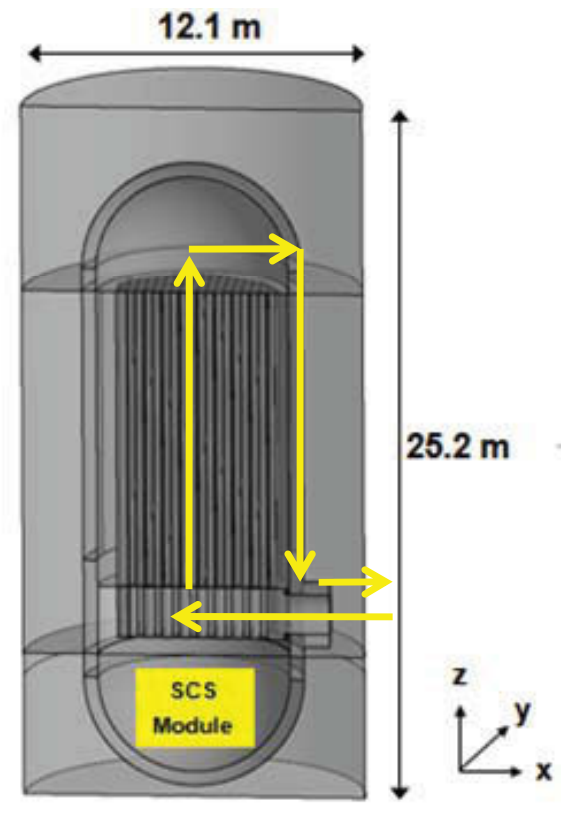

(a) 1-D assumption

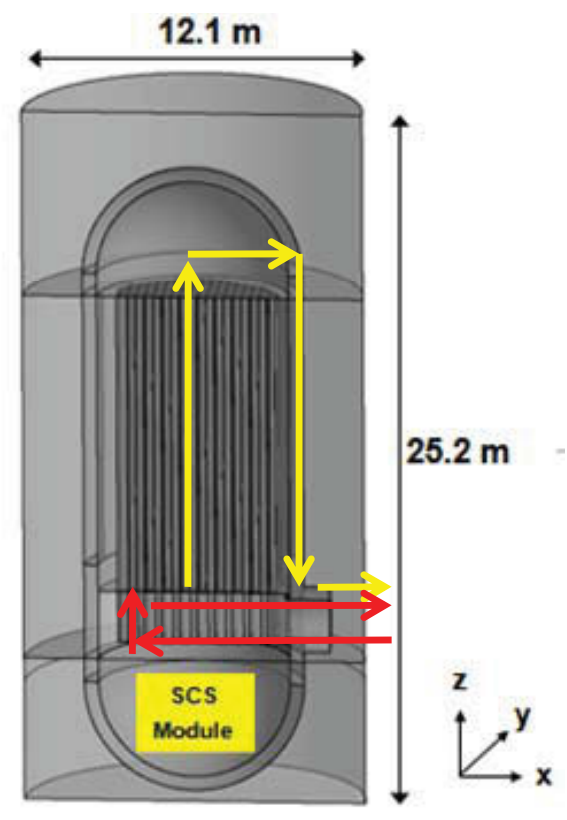

(b) Multi-D simulation

Figure 2-83. Comparisons of natural circulation flow patterns between 1-D assumption and multi-D simulation. 
Figure 2-84 plots the $\mathrm{x}$-velocity profile in the z-direction at the broken hot-leg. In this figure, the lefthand side is the hot-leg bottom and the right-hand side is the hot-leg top. The positive $(+)$ velocity represents the inward flow and the negative velocity (-) represents the outward flow. This figure clearly shows the counter current flow throughout the pipe. The ingress speed of the cold fluid in this figure ranges approximately from a very small velocity to $1 \mathrm{~m} / \mathrm{s}$. The maximum speed is $1.08 \mathrm{~m} / \mathrm{s}$, and the average speed is about $0.74 \mathrm{~m} / \mathrm{s}$. The superficial velocity, which is averaged by the whole pipe crosssectional area, is $0.46 \mathrm{~m} / \mathrm{s}$. Table $2-11$ summarizes the estimated air-ingress speeds for various boundary conditions and turbulence models. 1-D simulation results are also listed in the first row for comparisons. The 1-D simulation was conducted by the GAMMA code (Oh et al. 2008). According to their calculations, the air-ingress speeds in the 1-D simulation range between 0.03 and $0.05 \mathrm{~m} / \mathrm{s}$. The air-ingress speeds from 3-D CFD simulations are about an order of magnitude faster than that from 1-D GAMMA simulation, meaning that graphite oxidation will be generated in the reactor about 10 times more than 1-D simulation. It also indicates that the recirculation flow will significantly accelerate the air-ingress process. For the natural circulation flow in the core, both 1-D and 3-D simulations predict the same order of magnitude air-ingress speeds, which ranged from approximately 0.1 to $0.2 \mathrm{~m} / \mathrm{s}$.

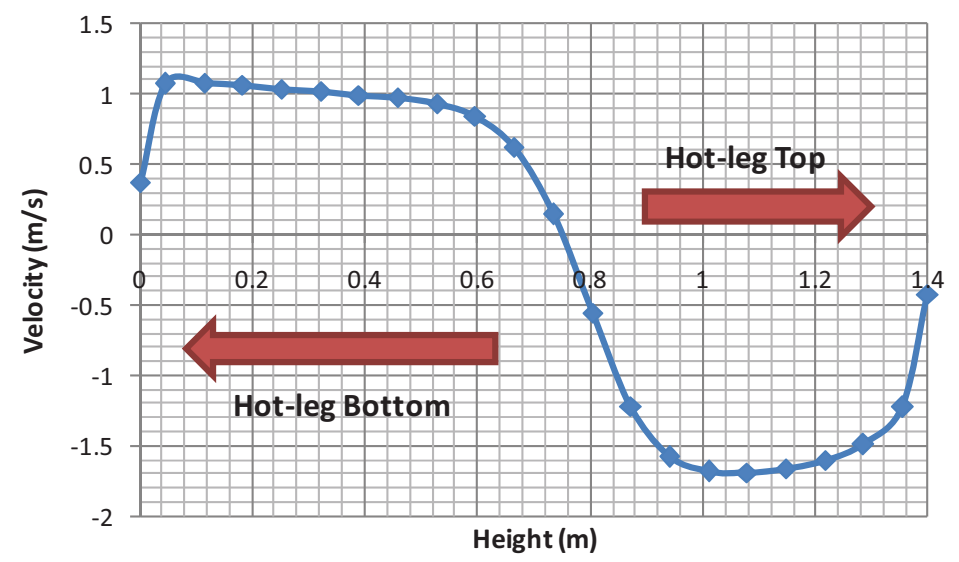

Figure 2-84. x-velocity profile at the broken hot-leg (base case).

Table 2-11. Estimated air-ingress speeds at the broken hot-leg.

\begin{tabular}{|l|c|c|c|}
\hline & $\begin{array}{c}\text { Average Speed } \\
(\mathbf{m} / \mathbf{s})\end{array}$ & $\begin{array}{c}\text { Maximum Speed } \\
(\mathbf{m} / \mathbf{s})\end{array}$ & $\begin{array}{c}\text { Superficial Speed } \\
(\mathbf{m} / \mathbf{s})\end{array}$ \\
\hline 1-D Modeling (GAMMA code) & $0.03-0.05$ & $0.03-0.05$ & $0.03-0.05$ \\
\hline Case 1 (base) & 0.74 & 1.08 & 0.46 \\
\hline Case 2 & 0.80 & 1.00 & 0.48 \\
\hline Case 3 & 0.73 & 1.19 & 0.45 \\
\hline Case 4 & 0.73 & 0.98 & 0.45 \\
\hline Case 5 & 0.7 & 1.01 & 0.43 \\
\hline Case 6 & 0.73 & 1.07 & 0.45 \\
\hline Case 7 & 0.74 & 1.04 & 0.46 \\
\hline Case 8 & 0.75 & 1.05 & 0.47 \\
\hline Case 9 & 0.69 & 1.08 & 0.44 \\
\hline Case 10 & 0.88 & 1.07 & 0.51 \\
\hline Case 11 & 0.76 & 1.04 & 0.47 \\
\hline Case 12 & 0.71 & 1.07 & 0.45 \\
\hline
\end{tabular}


Table 2-11 also shows that the boundary conditions do not highly affect the recirculation speed calculation results. In the parametric study, the temperatures were changed within $\pm 100 \mathrm{~K}$ from the base case. Therefore, the maximum temperature difference is $200 \mathrm{~K}$ for each boundary. The average velocities for all the cases range between 0.7 to $0.75 \mathrm{~m} / \mathrm{s}$. The biggest output changes are observed when the air mass fractions change. Increasing the air mass fraction clearly increases the air-ingress speed. It is because the driving force is more increased for the larger densities at the same temperature gradient. This table also shows that the effect of turbulence models is very small on the air-ingress speed estimation. However, relatively large differences were observed between the laminar and the turbulence models. According to the calculations, the laminar model predicts faster air-ingress speed because the turbulence mixing effect diminishes the temperature and density gradient in the flow. Reduced temperature and density gradients generate less driving force for the recirculation.

\subsubsection{Summary of CFD Natural Circulation Analyses}

Natural circulation patterns in the air-ingress accident were investigated by CFD methods. The 600 MWth GTMHR was selected to be the reference design and modeled by a half symmetric 3-D geometry. The 180-degree half symmetric model was absolutely necessary because of the unique characteristics of the GTMHR cross-duct. The grid model was divided into six regions (lower plenum, core, reactor top, reactor bottom, riser, and cavity) and merged by a grid interface function. In this model, the core was simply assumed to be a porous body with 0.2 volume porosity, assuming the pebble core. The parameters related to the permeability were estimated based on the circular channel friction data. The $2 \mathrm{~mm}$ bypass gaps in the core blocks were neglected. The 3-D CFD results were finally compared with the 1-D simulation results using the GAMMA code. Overall, the results indicate that the 1-D air-ingress modeling may significantly distort the real air-ingress process and consequences. The notable results are as follows:

- 1-D and 3-D simulation show a very different flow pattern.

- In the 1-D simulation, the natural circulation path consists of a single route from hot-leg to cold-leg.

- In the 3-D simulation, the natural circulation consists of two flow paths: (1) recirculation flow in the lower plenum, and (2) normal natural circulation through the core.

- The recirculation speed predicted in the lower plenum using 3-D CFD simulations is much faster than the natural circulation speed calculated using 1-D GAMMA simulations.

- The estimated air-ingress speeds by the $3-\mathrm{D}$ simulations are about $0.46 \mathrm{~m} / \mathrm{s}$, while the $1-\mathrm{D}$ simulation estimates the speed to be only 0.02 to $0.03 \mathrm{~m} / \mathrm{s}$. The 3-D simulation predicts an order of magnitude faster air-ingress speed into the reactor than the 1-D simulation.

- Faster air-ingress speed will generate more graphite oxidation in the real situation than predicted by the previous GAMMA 1-D simulations.

- The effect of turbulence models is not large in this study. However, there are some differences between the laminar and the turbulence models. The laminar option provides the most conservative results because of no turbulence mixing term, which diminishes density gradient. 


\section{TASK 2: EXPERIMENTAL STUDY ON THE STRATIFIED FLOW 3.1 Introduction}

Air-ingress is potentially the most serious accident in a VHTR. This type of accident occurs when a pipe breaks inside the reactor, allowing the air surrounding the reactor to rapidly ingress into the reactor vessel. This type of accident will result in serious problems such as chemical reactions between oxygen and the core graphite structures, which will subsequently heat up the reactor core even more, damaging the structural integrity and releasing toxic gases such as $\mathrm{CO}$ and $\mathrm{CO}_{2}$.

Many computational and theoretical works have been carried out to understand what would happen in an actual air-ingress accident. However, validation data are required to support this air-ingress theory and the computational results. Table 3-1 shows the previous and current experimental data used to validate computer codes for air-ingress analyses. This table lists 14 different experimental sets covering diffusion, natural convection, radiation, chemical reaction, and porous media models in the code; however, no good data are presently available validating stratified flow.

Table 3-1. Previous and current validation data for air-ingress analyses.

\begin{tabular}{|c|c|c|c|c|c|c|c|}
\hline & Test Facility & D & NC & $\mathbf{R}$ & C & $\mathbf{P}$ & etc \\
\hline 1 & Pipe Network, NWU & & & & & & $\mathrm{O}$ \\
\hline 2 & Blowdown, NWU & $\mathrm{O}$ & & & & & $\mathrm{O}$ \\
\hline 3 & Buncan \& Toor's Experiment & $\mathrm{O}$ & & & & & \\
\hline 4 & Inverse U-tube single/multiple channel test & 0 & 0 & & & & \\
\hline 5 & Ogawa's circular tube test & & & & $\mathrm{O}$ & & \\
\hline 6 & Takahashi's annular tube test & & & & 0 & & \\
\hline 7 & VENTURApebble bed test & & & & $\mathrm{O}$ & $\mathrm{O}$ & \\
\hline 8 & Inverse U-tube air ingress experiment & 0 & $\mathrm{O}$ & & $\mathrm{O}$ & & \\
\hline 9 & HTTR simulated air ingress experiment & $\mathrm{O}$ & $\mathrm{O}$ & & 0 & & $\mathrm{O}$ \\
\hline 10 & Vertical slot experiment & $\mathrm{O}$ & $\mathrm{O}$ & & & & \\
\hline 11 & NACOK natural convection test & & $\mathrm{O}$ & & & $\mathrm{O}$ & \\
\hline 12 & SANA-1 afterheat removal test & & & & & $\mathrm{O}$ & \\
\hline 13 & HTTR RCCS mockup test & & $\mathrm{O}$ & $\mathrm{O}$ & & & \\
\hline 14 & SNU RCCS test & & 0 & 0 & & & \\
\hline
\end{tabular}
D: Diffusion NC: Natural Convection P: Porous Media

\section{R: Radiation C: Chemical Reaction}

A number of sets of experiments were planned to understand stratified flow phenomena and validate the computer codes with physical models. This experimental study covers a separate effort to couple effects related to the stratified flow.

\subsection{Isothermal Stratified Flow Experiment}

The current section describes separate-effect experiments for understanding stratified flow phenomena in the air-ingress accident and for generating data for validation of computer codes, including CFD codes or system analysis codes. This experiment is aimed at the phenomena that occur for a doubleended-guillotine break (DEGB) scenario. Although the DEGB scenario is recognized as a beyond design basis accident (BDBA) scenario, stratified flow air ingress experiments are being conducted to study this 
experimental break configuration because: (a) it is a limiting event in that it is the largest break size and traditional safety research performed by the U.S. Nuclear Regulatory Commission has always focused on limiting events, and (b) the geometry is considerably simpler than the more likely small leak scenarios.

Density gradient driven flow is a new issue in the VHTR safety analysis field. The detailed mechanisms for the whole scenario have not yet been fully understood or validated up to the level of satisfaction for the safety analyses. This section summarizes the objective of this experiment.

\subsubsection{Scope and Objectives}

The two main objectives in the stratified flow experiment are to understand density gradient driven flow phenomena in the broken circular pipe and to provide data for the validation of computer codes such as CFD or system analysis codes.

To meet the first objective, the following characteristics of the flow phenomena typical to the VHTR DEGB scenario were investigated in the experiment (See Figure 3-1):

- Characteristics of gravity current in the horizontal pipe

- Characteristics of gravity current at the expansion point from the pipe to the vessel.

Previous research has been conducted for the gravity current flow in the civil engineering application. This gravity current flow is very similar to the air-ingress situation, but has some differences, in particular, for the channel cross-section shapes. Figure 3-2(a) shows the channel shape in previous gravity current studies. These previous experiments were carried out by using rectangular channels. In the rectangular channel, the channel height is not changed along the horizontal axis. It indicates that the current speed will be consistent along the axis, and the flow regime in each horizontal location will be the same. This configuration visualizes 2-D flow, so the experimental results were well matched to the previous theoretical models derived in the 2-D axis. In the air-ingress condition, which consists of a circular channel, the channel height is changed along the horizontal axis (see Figure 3-2(b)). At the center, the height is large, and at the side, the height is very small. Therefore, at the center, the current speed will vary from the speed near the sides. This might provide different flow regimes for different locations

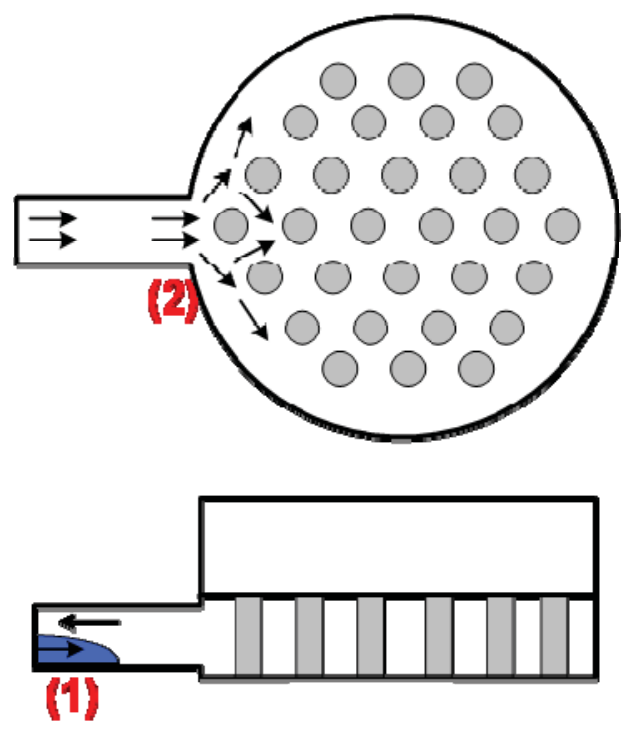

(1) Stratiled Flew in the horizental Pipe

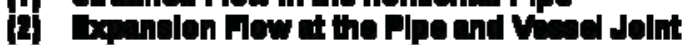

Figure 3-1. Flows of interest in the isothermal fullbreak experiment. in the circular pipe. The circular pipe appears to show some 3-D effect on the gravity current flow. It is therefore necessary to see the differences of the stratified flow between a rectangular channel and a circular pipe. It is therefore not clear whether the previous 2-D models are still valid for the circular geometry. 


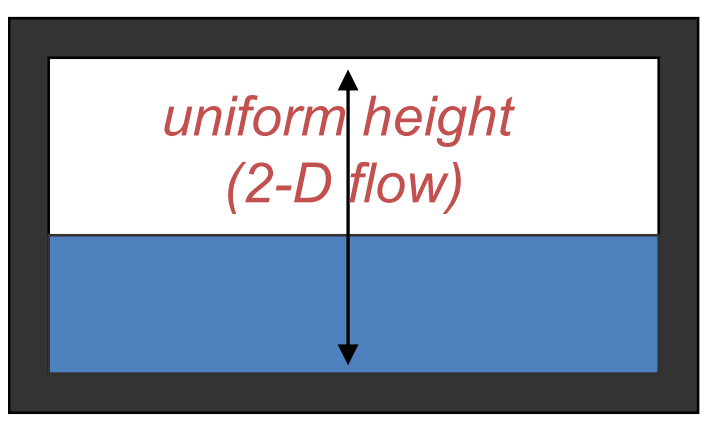

(a) Previous gravity current studies

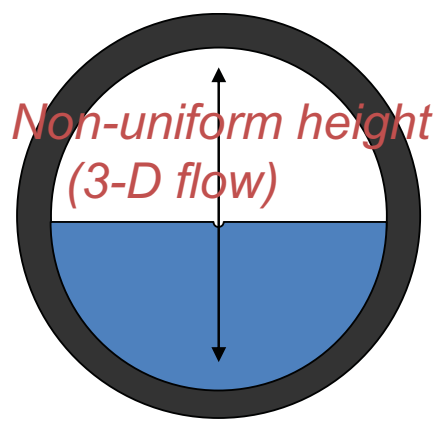

(b) VHTR air-ingress situation

Figure 3-2. Comparisons between previous gravity current studies and VHTR air-ingress condition.

\subsubsection{Experimental Setup}

Air-ingress experiments of a gas-cooled reactor were conducted using a scaled-down test apparatus based on the General Atomics GTMHR design. The DEGB was considered the worst case of the air ingress accidents. A pipe break accident scenario that mimics the DEGB was demonstrated by opening a pneumatic knife gate valve. The pressure and temperature were measured by transducers on each cylinder for pressure and by thermocouples for temperature measurements. Flow visualization was recorded by a video camera. The operating conditions were ambient pressure and temperature.

The experiments mimicked air ingress into a typical NGNP gas-cooled reactor lower plenum as a countercurrent air and helium flow when the inlet pipe break occurs in the hot duct. A liquid system with water, salt water, or water-sucrose was used. The liquid-liquid system data was used for validating the computational fluid dynamic models.

The DEGB isothermal experiment consists of two tanks and a horizontal pipe that connects the two tanks, as shown in Figure 3-3. Along the horizontal pipe is a sliding gate valve (Dezurik Knife Gate Valve, KCB, 8, F1, S2, TDP-EPDM*CY-PC8CS,4VG63) installed to separate the tanks. Initially, both tanks were filled with different fluids having different densities, and the valve was closed. Once the predetermined initial conditions were achieved, the experiments were started by quickly opening the valve. After the valve is open, a counter-current stratified flow formed in the test-section where the heavy fluid intrudes into the light fluid at the bottom, and the light fluid intrudes into the heavy fluids at the top. A port at the bottom of the two tanks was linked to a differential pressure transmitter to be used for setting up the initial pressure equilibrium and stabilization. Each tank also had a pressure transducer for independent pressure monitoring. The tanks and the pipe were made of transparent acrylic for optical measurements and flow visualization. The detailed test section designs and blueprints are enclosed in the Appendix A.

Figure 3-4 shows the experimental setup, which consists of two tanks, two horizontal pipes, and a gate valve for separation of two tanks. The horizontal pipe diameter and length were designed to be $0.2 \mathrm{~m}$ and $1.0 \mathrm{~m}$, respectively. The tank diameter was $0.9 \mathrm{~m}$, and the height was $1.0 \mathrm{~m}$. Initially, the gate valve was closed, and water and brine filled the two tanks, respectively. The height of the brine and the water at the initial condition were about one-third of the full tank height $(=0.3 \mathrm{~m})$. After achieving a predetermined condition, the experiment was started by opening the gate valve with 80 psi of compressed air. The valve was pneumatically controlled by a solenoid valve. The gate opening is the same size as the horizontal pipe to avoid flow distortion when liquid flows through the gate valve. 


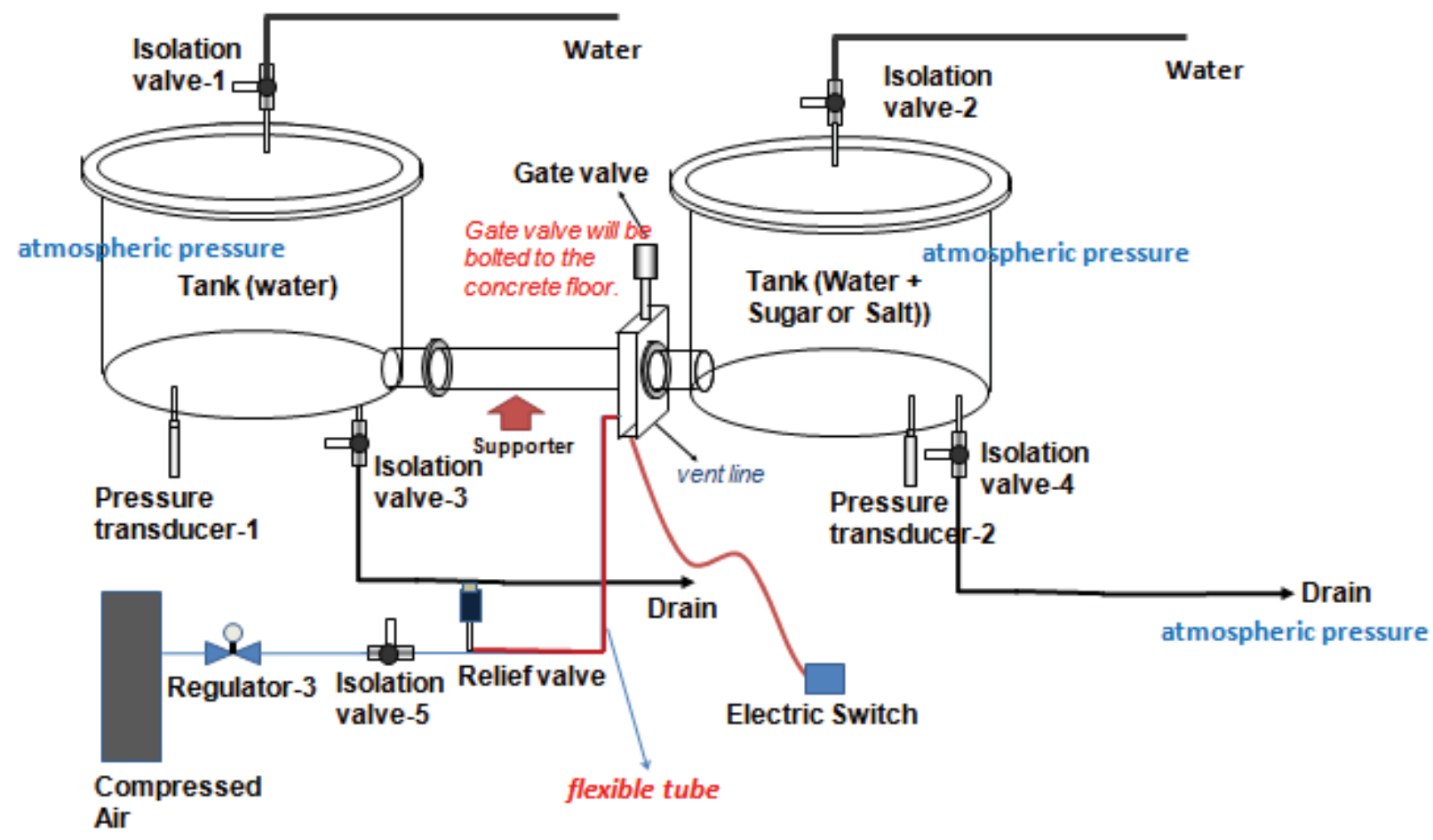

Figure 3-3. Schematics of isothermal DEGB experiment.
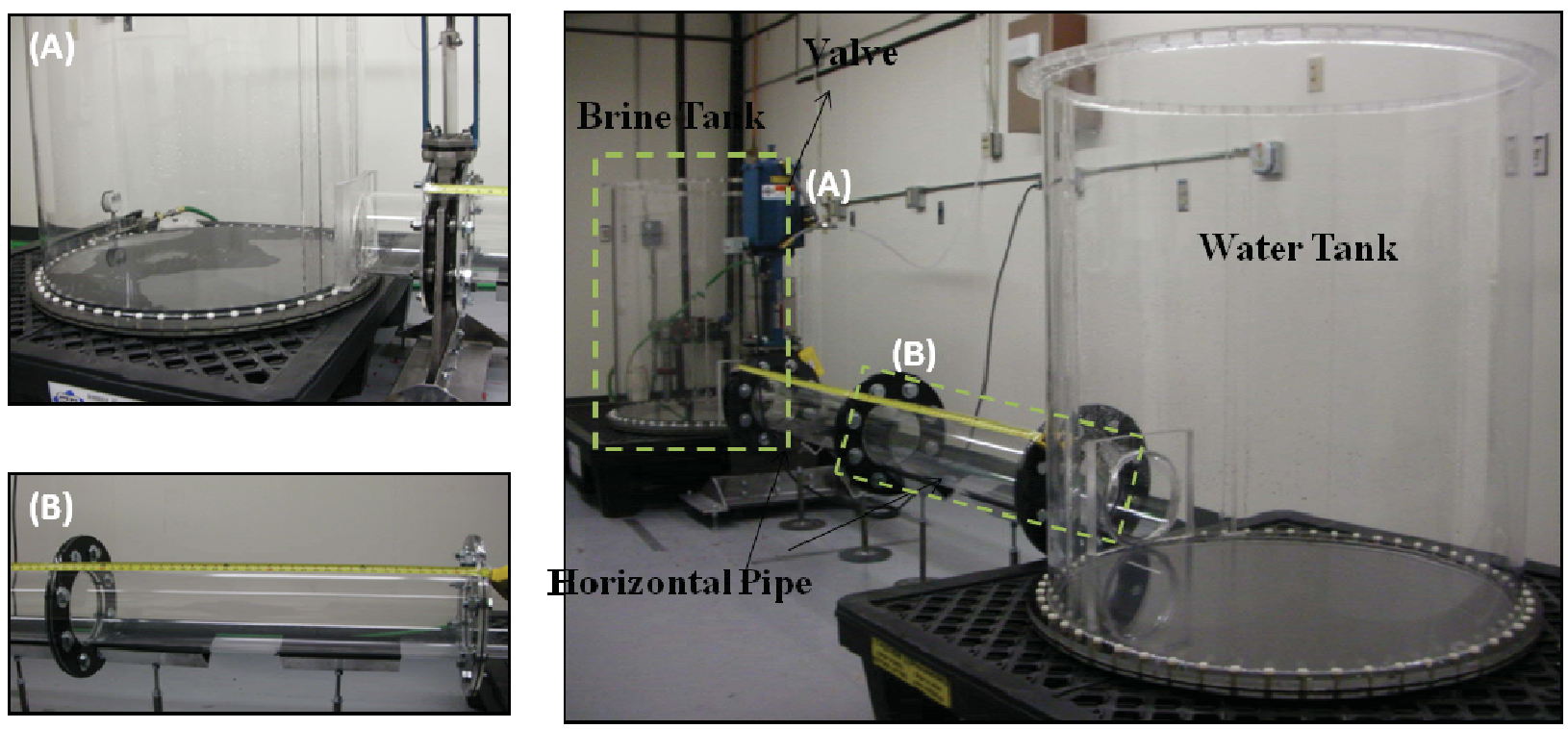

Figure 3-4. Isothermal stratified flow experimental setup for brine-water.

Figure 3-5shows the gate valve installed in the test section. This gate valve is actuated by compressed air. The compressed air is controlled by a solenoid valve and an electrical switch. The valve is vertically oriented and mounted on the concrete floor. Figure 3-6 shows the overall setup of the test facility. The detailed valve-mount design and stress analysis results are described in Appendix B. 

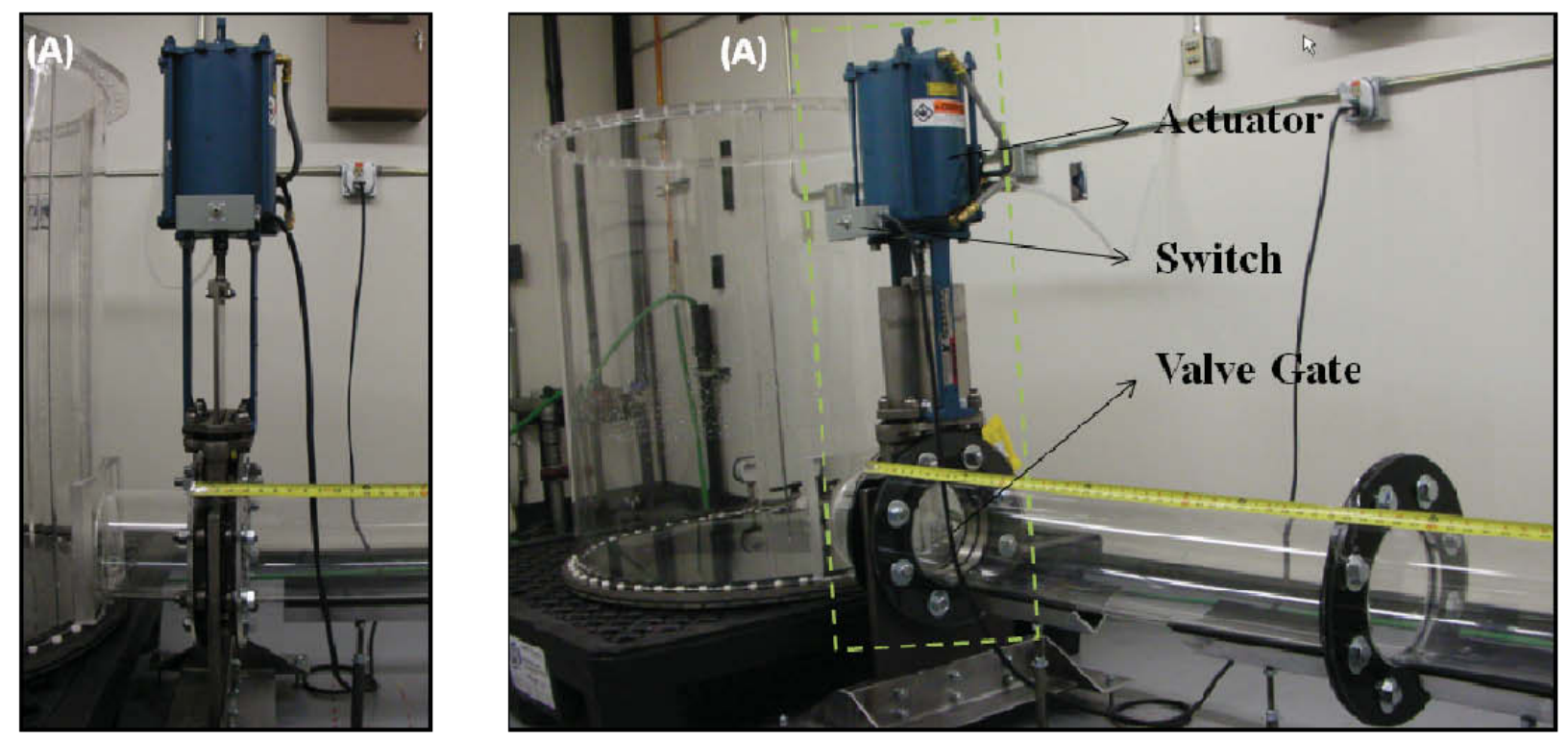

Figure 3-5 Gate valve installation.
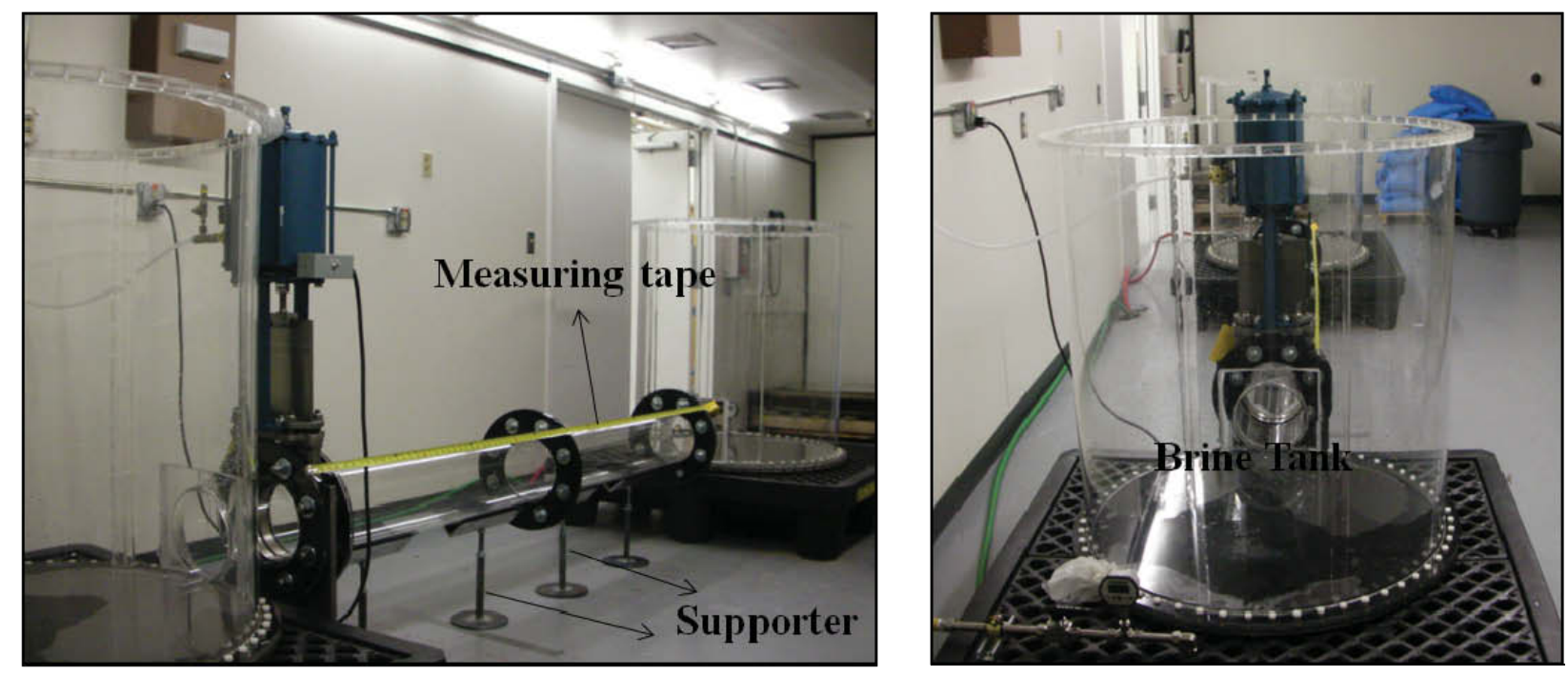

Figure 3-6. Overall setup of isothermal experiment.

The experimental test matrix is given in Table 3-2. The experiment used brine and sucrose as heavy fluids and water and air as light fluids. The density ratios are changed between 0.98 and 0.866 , except for the air/water experiment. The total number of test cases is 9 , including the air and water experiment. This experiment covers Reynolds numbers from $2.3 \times 10^{4}$ to $3.79 \times 10^{5}$. The main flow is therefore in the turbulent flow regime, which is the same as predicted in the real air-ingress accident. In this regime, the gravity current or density gradient stratified flow is not affected by the viscous effect.

Before starting the experiment, the fluid's densities (specific gravity) were measured by a hydrometer. Later, the brine and sucrose were separately sampled for validating measurement. Figure 3-7 shows the brine and sucrose samples taken in a small bottle. In each sample, fluid types and measuring date were written on the bottle surface. 
Table 3-2. Experimental conditions and case summary.

\begin{tabular}{|c|c|c|c|c|c|}
\hline & Heavy Fluid & Light Fluid & $\begin{array}{l}\text { Heavy Fluid } \\
\text { Density }\left(\mathrm{kg} / \mathrm{m}^{3}\right)\end{array}$ & $\begin{array}{c}\text { Light Fluid } \\
\text { Density }\left(\mathrm{kg} / \mathrm{m}^{3}\right)\end{array}$ & Density Ratio \\
\hline Case 1 & Sugar & Water & 1020 & 1000 & 0.980 \\
\hline Case 2 & Sugar & Water & 1025 & 1003 & 0.979 \\
\hline Case 3 & Salt & Water & 1045 & 1002 & 0.959 \\
\hline Case 4 & Sugar & Water & 1061 & 1002 & 0.944 \\
\hline Case 5 & Sugar & Water & 1080 & 1002 & 0.928 \\
\hline Case 6 & Sugar & Water & 1100 & 1000 & 0.909 \\
\hline Case 7 & Salt & Water & 1130 & 1002.5 & 0.887 \\
\hline Case 8 & Sugar & Water & 1155 & 1000 & 0.866 \\
\hline Case 9 & Water & Air & 1000 & 1.2 & 0.0012 \\
\hline
\end{tabular}

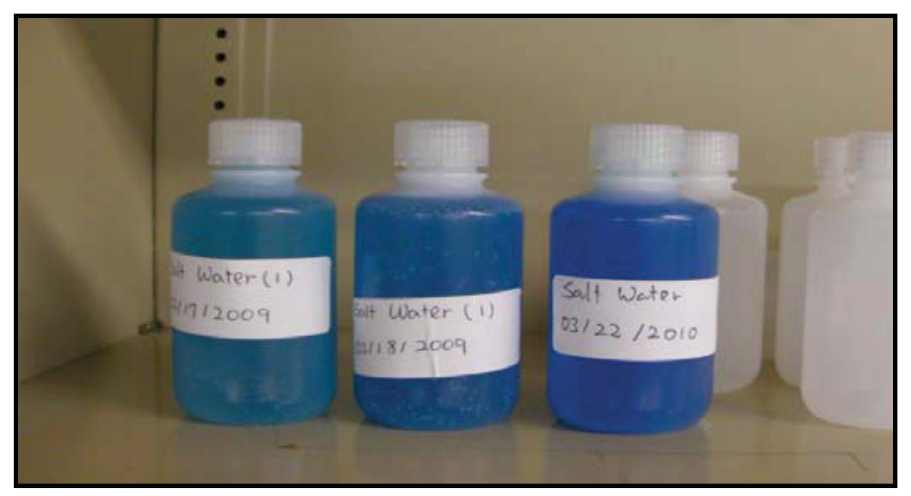

Figure 3-7. Brine and Sucrose Samples.

\subsubsection{Experimental Procedure}

The isothermal experimental procedure is as follows:

1. The knife gate valve, isolation Valves 3 and 4, are closed as shown in Figure 3-3 above.

2. Water is added to each tank through a water feed line.

3. Salt (or sugar) and a $100 \mathrm{ppm}$ concentration of dye (indigo type blue dye, certified by International to the American National Standards Institute/National Science Foundation Standard 60 for use in drinking water) are added to a tank, the brine is stirred for mixing, and a $10 \mathrm{~mL}$ brine sample is taken for a density measurement.

4. Isolation Valve 5 is opened, and the pneumatic knife gate valve is opened by turning the switch on.

5. The flow pattern is observed in the horizontal pipe.

6. The flow is recorded using a video camera.

7. After the experiment, the power source to the knife gate valve is deenergized.

8. Isolation Valves 3 and 4 are opened to discharge water to sewage (the sewage drain is located in the floor of Room E). 


\subsubsection{Measurement}

This experiment measured two parameters for characterizing stratified flow-current velocity and current depth - using two digital video cameras (30 frames per second) were used. One camera, installed on the top of the horizontal pipe, measured the time when the current front passes through premarked positions. Since the marking positions are known, the current speed can be calculated by measuring time. To measure the current depth, the other camera was installed at the side of the horizontal pipe. The analysis was conducted by image-processing software. The measured data are compared with the CFD simulations and the theoretical models.

\subsubsection{Experimental Results and Discussions}

Figure 3-8 shows the heavy-fluid current propagation through the horizontal pipe for Case 8 (watersucrose, density ratio $=0.866$ ). In this case, the heavy-fluid was sucrose, and the light-fluid was water. The density ratio was 0.866 , which means that the sucrose is about $13.4 \%$ heavier than the water. As shown in Figure 3-8, the current rapidly propagates through the pipe, occupying about one-half of the pipe diameter. This result is consistent with the previous observations reported for the lock exchange flow in the Boussinesq flow regimes. In Case 8 , the heavy current travelling speed was estimated at $\sim 0.26 \mathrm{~m} / \mathrm{s}$.
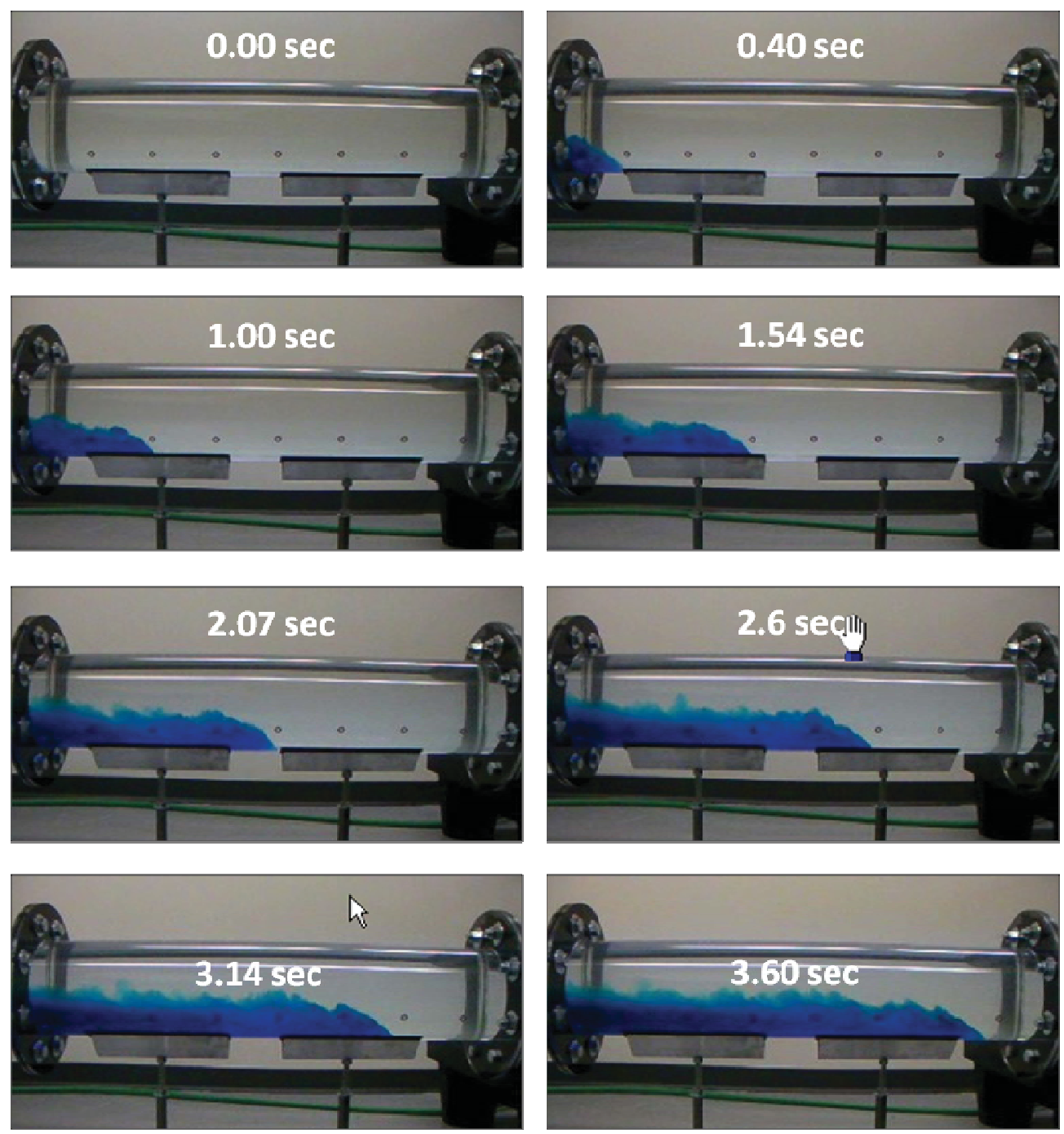

Figure 3-8. Progress of gravity current and stratified flow (water-sucrose, density ratio $=0.866$ (Case 8)). 
The experimental results are summarized in Table 3-3 and Figure 3-9. The first column in Table 3-3 represents the traveling distance, which was determined by measuring marking spots. The columns from the second to the end show the traveling time when the current arrived at the marking spots. Figure 3-9 plots these data. As shown, the traveling distance has a linear relationship with the traveling time. It means that the heavy current speed is constant throughout the axial direction of the horizontal pipe. This is because of the high Reynolds numbers of the gravity current. For high Reynolds numbers, inertia force dominates viscous effects, so the flow can be considered as an inviscid flow, indicating that the frictional loss can be ignored. The current speed can be calculated by an inverse of the slope of each dataset. As shown in the figure, the current speed is significantly affected by density ratios. The lowest density ratio is 0.866 and the largest is 0.98 for the liquid-liquid experiment. The velocity difference between these two cases are about a factor of $2.5\left(\mathrm{~V}_{\mathrm{r}=0.866}=0.254 \mathrm{~m} / \mathrm{s}\right.$, and $\left.\mathrm{V}_{\mathrm{r}=0.98}=0.101 \mathrm{~m} / \mathrm{s}\right)$. If the density ratio is small (the density differences are large), the current speed is fast because of the larger density gradient. The current speed $(\mathrm{V}=1.69 \mathrm{~m} / \mathrm{s})$ for the air-water experiment was estimated to be even an order faster than for the liquid-liquid experiment.

Table 3-3. Experimental results (traveling distance $[\mathrm{x}]$ vs. time).

\begin{tabular}{|l|c|c|c|c|c|c|c|c|c|}
\hline $\mathbf{x}(\mathbf{m})$ & $\begin{array}{c}\text { Case 1 } \\
\text { time (s) }\end{array}$ & $\begin{array}{c}\text { Case 2 } \\
\text { time (s) }\end{array}$ & $\begin{array}{c}\text { Case 3 } \\
\text { time (s) }\end{array}$ & $\begin{array}{c}\text { Case 4 } \\
\text { time (s) }\end{array}$ & $\begin{array}{c}\text { Case 5 } \\
\text { time (s) }\end{array}$ & $\begin{array}{c}\text { Case 6 } \\
\text { time (s) }\end{array}$ & $\begin{array}{c}\text { Case 7 } \\
\text { time (s) }\end{array}$ & $\begin{array}{c}\text { Case 8 } \\
\text { time (s) }\end{array}$ & $\begin{array}{c}\text { Case 9 } \\
\text { time (s) }\end{array}$ \\
\hline 0 & 0 & 0 & 0 & 0 & 0 & 0 & 0 & 0 & 0 \\
\hline 0.127 & 1.54 & 1.13 & 0.67 & 0.73 & 0.53 & 0.47 & 0.5 & 0.4 & - \\
\hline 0.254 & 3.2 & 2.3 & 1.6 & 1.47 & 1.26 & 1 & 1.2 & 1 & - \\
\hline 0.381 & 4.54 & 3.6 & 2.54 & 2.2 & 2.06 & 1.54 & 1.8 & 1.54 & - \\
\hline 0.508 & 5.6 & 4.9 & 3.6 & 2.93 & 2.66 & 2.07 & 2.47 & 2.07 & - \\
\hline 0.635 & 6.74 & 5.8 & 4.87 & 3.8 & 3.33 & 2.6 & 3.07 & 2.6 & - \\
\hline 0.762 & 8 & 7 & 5.94 & 4.6 & 4 & 3.14 & 3.6 & 3.14 & - \\
\hline 0.889 & 9.14 & 8 & 6.94 & 5.4 & 4.66 & 3.74 & 4.14 & 3.6 & - \\
\hline 1.016 & 10.07 & 8.87 & 7.67 & 6.13 & 5.13 & 4.14 & 4.54 & 4 & 0.6 \\
\hline
\end{tabular}

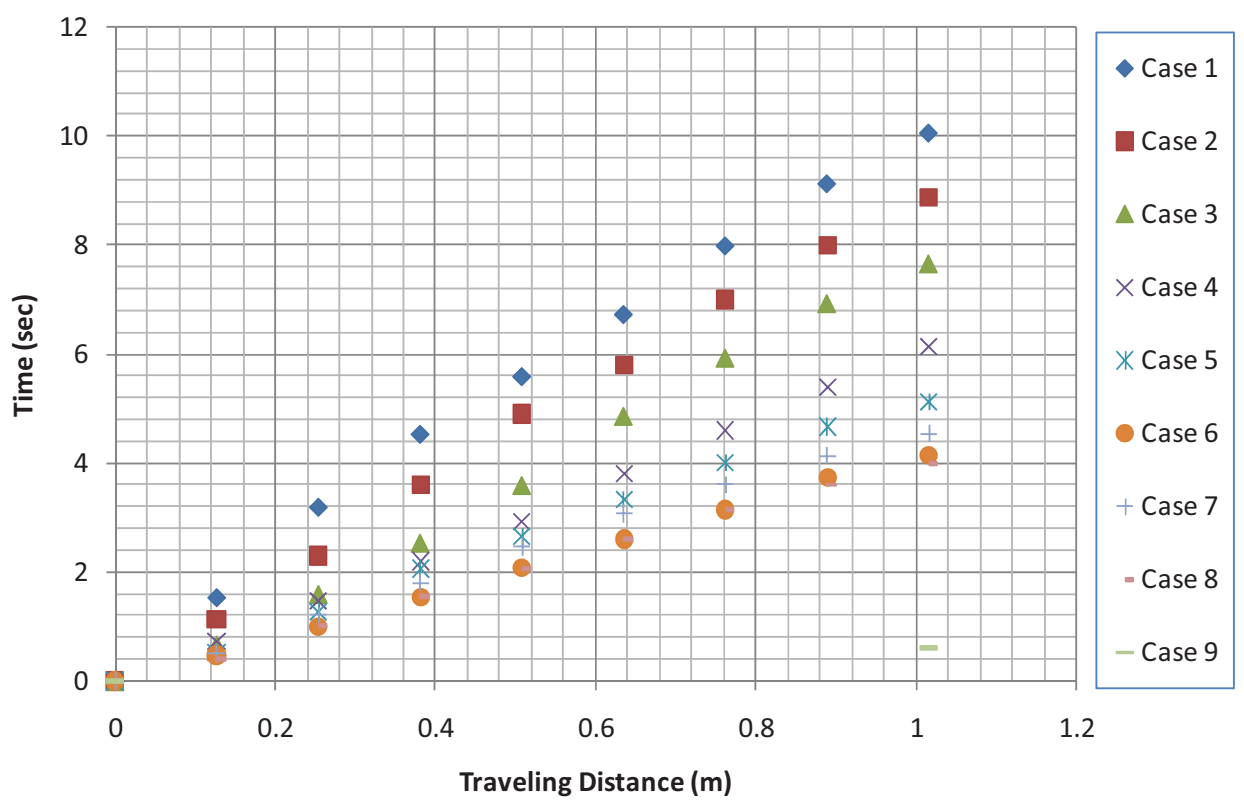

Figure 3-9. Experimental results (traveling distance vs. time). 
Table 3-4 summarizes the estimated current speeds between the experiments and theoretical estimations. The heavy current speeds in this experiment range between 0.101 and $1.69 \mathrm{~m} / \mathrm{s}$. As shown in the table, the theoretical estimation is in good agreement with the experimental data (within $10 \%$ error) for all the cases. The comparisons between the experiment and the theoretical models are discussed in more detail in the next section.

Table 3-4. Comparisons of current speeds between experiment and Benjamin's theoretical model.

\begin{tabular}{|l|l|l|l|l|l|l|l|l|l|}
\hline Velocity $(\mathrm{m} / \mathrm{s})$ & Case 1 & \multicolumn{1}{|c|}{ Case 2 } & Case 3 & \multicolumn{1}{|c|}{ Case 4 } & Case 5 & \multicolumn{1}{|c|}{ Case 6 } & \multicolumn{1}{c|}{ Case 7 } & \multicolumn{1}{c|}{ Case 8 } & \multicolumn{1}{c|}{ Case 9 } \\
\hline Experiment & 0.1009 & 0.1155 & 0.1375 & 0.1657 & 0.1814 & 0.2241 & 0.2381 & 0.254 & 1.69 \\
\hline Theory & 0.1028 & 0.1145 & 0.146 & 0.1705 & 0.191 & 0.2127 & 0.237 & 0.26 & 1.68 \\
\hline Error $(\%)$ & 1.88 & 0.86 & 6.18 & 2.89 & 5.29 & 5.08 & 0.46 & 2.36 & 0.59 \\
\hline
\end{tabular}

\subsection{Validation of CFD Code for Stratified Flow (Water/Brine(Sucrose))}

CFD methods for a gravity current flow were validated by analyzing CFD and comparing the results with experimental data. Details of this validation are described in this section.

\subsubsection{Preliminary CFD Result and Validation Study with Experiment}

\subsubsection{CFD Specification and Fluid Properties}

FLUENT 6.3.26 (ANSYS 2008) was used to understand stratified flow in small density ratio cases (from Case 1 to Case 8 in the isothermal experiment). Figure 3-10 indicates the overall geometry of the present CFD model of isothermal experiment simulations.

$<$ Overall geometry of CFD model $>$

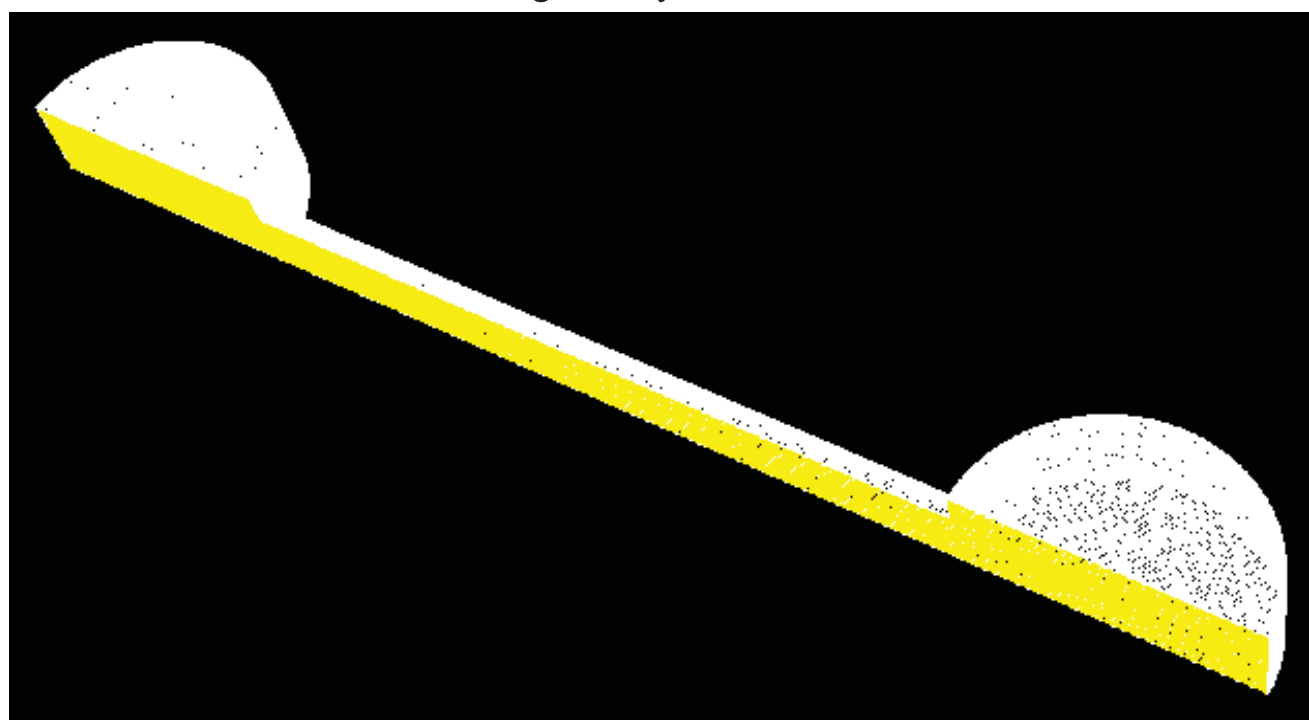

$<$ Side view of model $>$

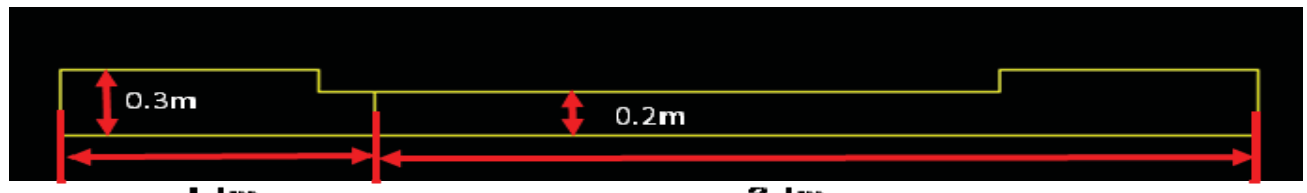

I. Im

3.1m

Figure 3-10. Overall geometry with normal mesh and side view from symmetry surface. 
A 3-D model was developed to simulate the air-ingress phenomena using the INL experimental setup. The overall geometry of the CFD model used for the experiment consisted of three basic parts as shown in Figure 3-10: a heavy fluid brine or sucrose tank (upper left), a light fluid water or air tank (lower right), and a connecting pipe (going between the two tanks). Three different types of polyhedral meshes (coarse, normal, and fine) were generated and used for the grid sensitivity and convergence study. The first 3-D model developed used a coarse mesh with appropriate grid sizes. Rediscretization with half of coarse mesh grid spacing (normal mesh) and finer meshes were then used as part of Richardson extrapolation method.

The FLUENT specification and model used in this simulation are listed as follows:

- Solver:

- Solver: pressure based

- Formulation: implicit

- Space: 3-D double precision

- Time: unsteady

- Velocity formulation: absolute

- Gradient I option: green-gauss cell based

- Unsteady formulation: $2^{\text {nd }}$-order implicit

- Pressure-velocity coupling: PISO.

- Discretization:

- Pressure: PRESTO!

- Momentum: $2^{\text {nd }}$-order upwind

- Turbulent kinetic energy: $2^{\text {nd }}$-order upwind

- Turbulent dissipation rate: $2^{\text {nd }}$-order upwind

- Species: $2^{\text {nd }}$-order upwind

- Energy: $2^{\text {nd }}$-order upwind.

- Viscous Model:

- Turbulence model: realizable k-e

- Wall function: standard wall function.

- Energy equation.

- $\quad$ Species transport model:

- Mixture material: Mixture-template

- 2 species: water and brine.

- $\quad$ Species transport model:

- Mixture material: Mixture-template.

Figure 3-11 shows the initial condition. The left side is filled with brine water that is slightly heavier than pure water density (red: heavy fluid), and right side is filled with pure water (blue: light fluid). Therefore, as transient simulation starts, the heavy fluid intrudes into the light fluid by driving force of density-driven current, which is also defined as stratified flow. Initial conditions for temperature and pressure of both fluids were set as $300 \mathrm{~K}$ and $1 \mathrm{~atm}$. identically. 


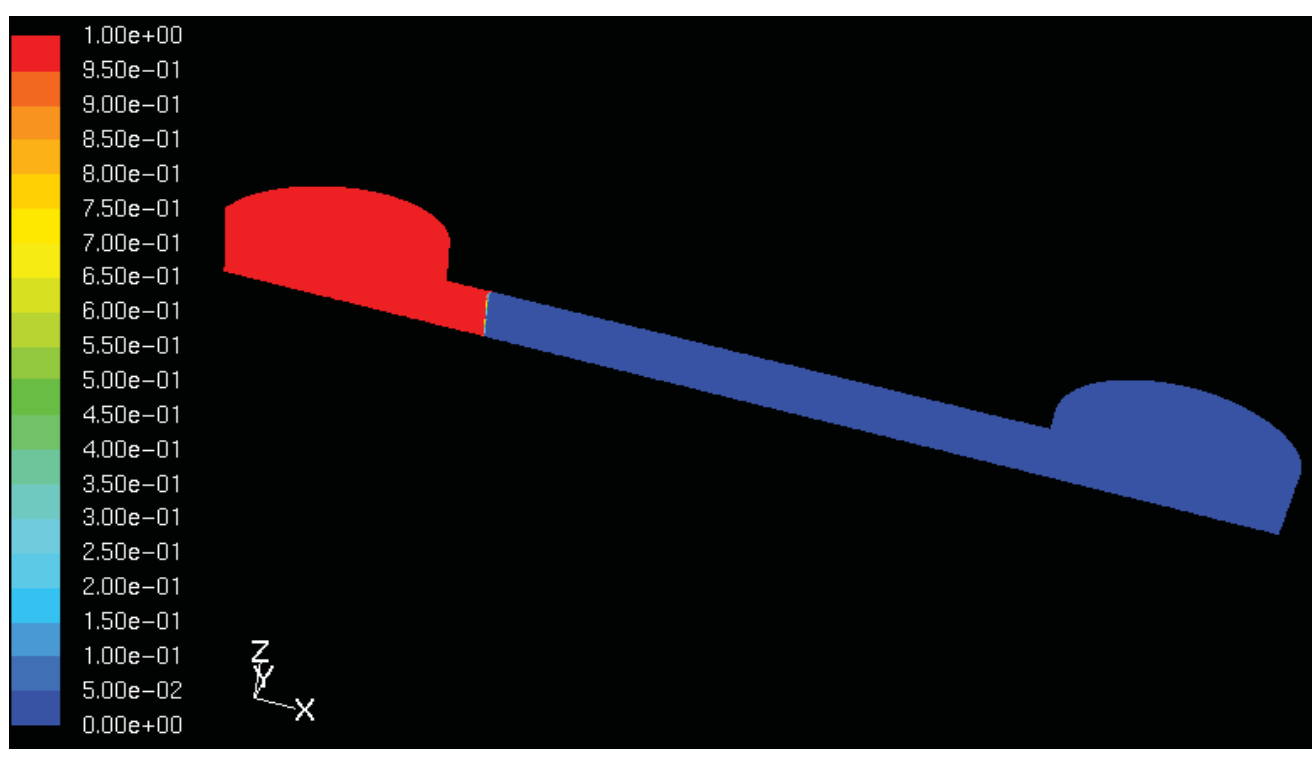

Figure 3-11. Initial Brine-water mass fraction (Red: brine, Blue: water)

Figure 3-12 shows how brine (heavy fluid) intrudes into the water in the lower zone while water (relatively light fluid) moves forward into the brine zone by expressing contour of brine mass fraction at different times (transient simulation result). Three types of mesh (coarse, normal, and fine) were implemented for predicting appropriate front speed in this study. Those CFD simulation results observe quite constant increments of front-head position by marching time. It is basically considered that fluid intrusion speed is almost constant, which means good agreement with previous theoretical models (Benjamin's Theory, invicid flow assumption). Brine water intrusion is demonstrated in 3-D simulation results by contouring of the brine mass fraction at $\mathrm{t}=1.1,3.1$, and 5.1 sec. Constant front speed and constant fluid height were also observed in $3-\mathrm{D}$ view.

The fluid conditions of isothermal experiments conducted at INL varied. Four fluid properties are necessary in fluent simulation to define fluid behavior and solve the mass, momentum, energy, and species transport equation: density, dynamic viscosity, specific heat, and thermal conductivity. The heavy fluids used in the experiment were made from mixing sugar or salt into pure water, so the density and viscosity of the heavy fluids in each test varied based on the amount of sugar or salt added. Densities for each test case were measured, and viscosities for each test were also calculated by the correlation between density and viscosity provided from the National Institute for Standards and Technology (NIST) (see Figure 3-13) fluid properties database. Those updated fluid properties (density and viscosity) for brine were implemented into fluent to capture better physical phenomena of stratified flow intrusion. 

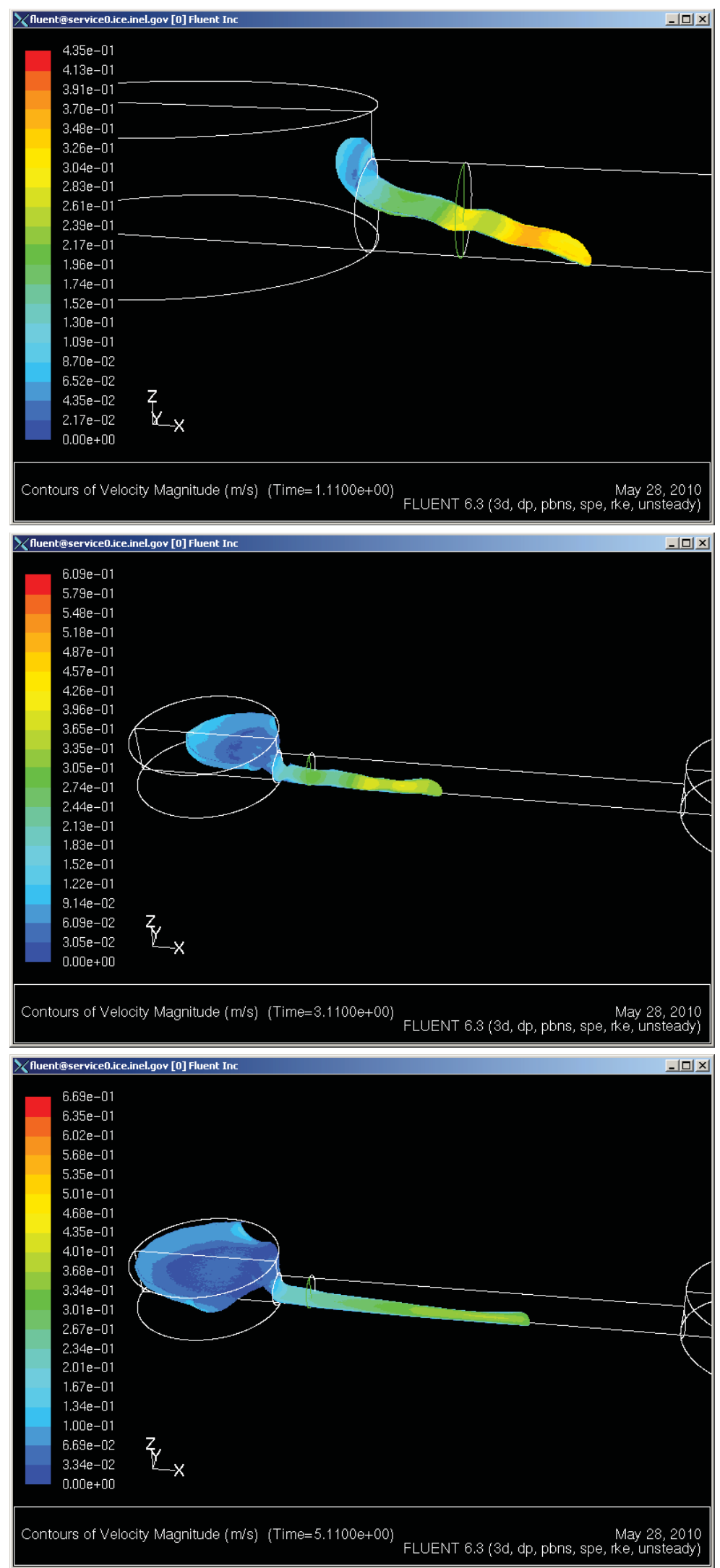

Figure 3-12. Visualization of brine water intrusion from tilted top view at marching time frame. 


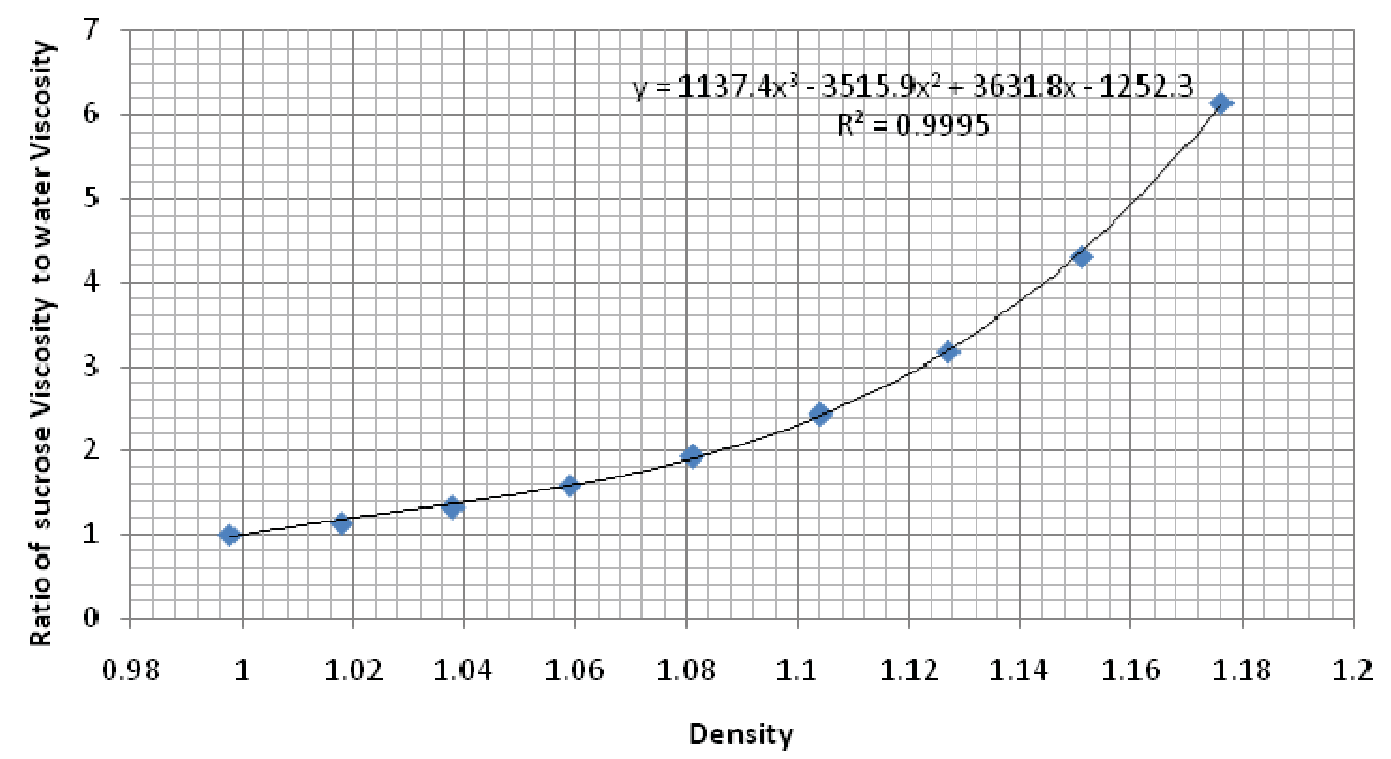

Figure 3-13. The correlation of density and ratio of sucrose viscosity to water viscosity.

\subsubsection{Grid Refinement Study}

As mentioned in the previous section, the grid refinement study was conducted for CFD validations of the spatial dimension and better prediction of interest variable (front-head speed). The calculation method for front-head speeds in different mesh types (coarse, normal, and fine) is defined with the same method used in the experimental section. Figures 3-14 through 3-16 show how brine water intrudes into pure water in three mesh-type simulations. As shown in the figures, more blur interface line is observed in coarse meshes, and more sharpness and distinctive interface lines are shown in finer meshes. This sharpness affects front-head position determination and gives accuracy on front-head speed calculation. An early propagation in Figure 3-16 shows wavy motions, which are captured for initial perturbation in finer meshes. The proper mesh quality provides a more reliable CFD calculation as well as validation. However, the finer mesh requires more computing time. In this section, eight experiment measurement data (from Case 1 to Case 8) are compared with the CFD calculation using the asymptotic approach. It is expected the computing cost will be very high. A solution that avoids this problem is discussed in the next section.

The locations of front heads were determined in this experiment by observing a video clip taken downstream of the current flow, front head, and some billows. So, the front-head positions in simulations were also calculated by the brine water mass fraction at the bottom of pipe channel. Figure 3-17 shows that the mass fraction of brine ranges from 0 to 1 . The front head speed was estimated using the distance from one position to others in the travel timing, which is superimposed on the front head ( 0.5 brine mass fraction in Figure 3-17). 

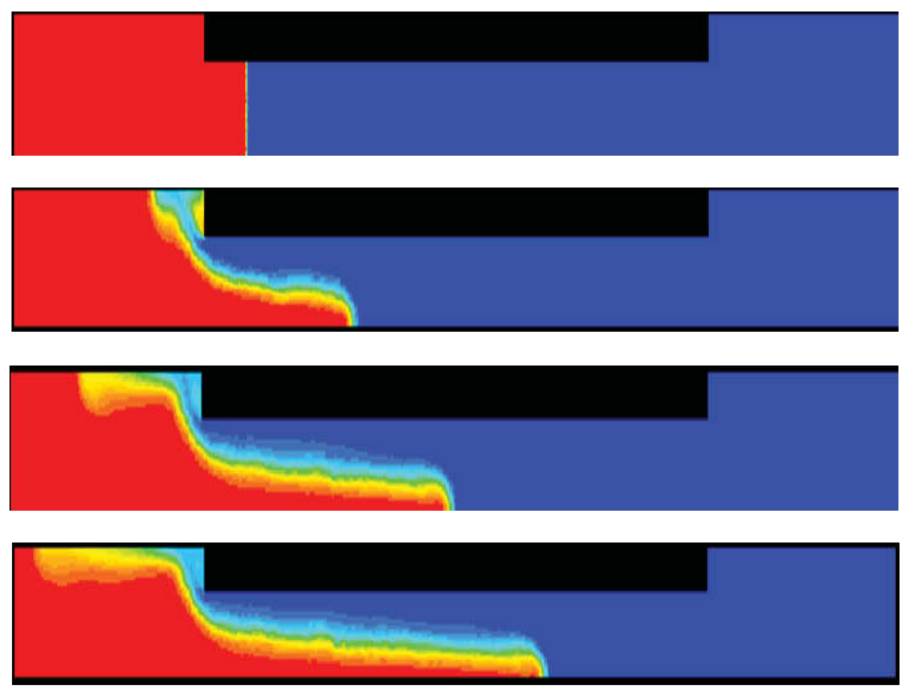

Figure 3-14. Brine intrusion simulation with coarse mesh.
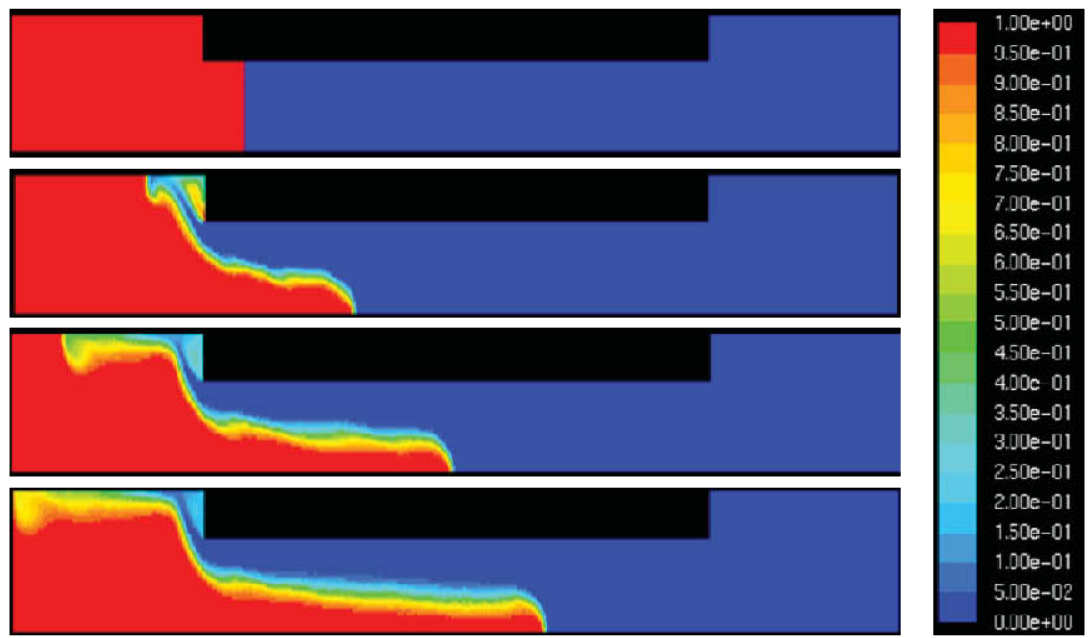

Figure 3-15. Brine intrusion simulation with normal mesh.
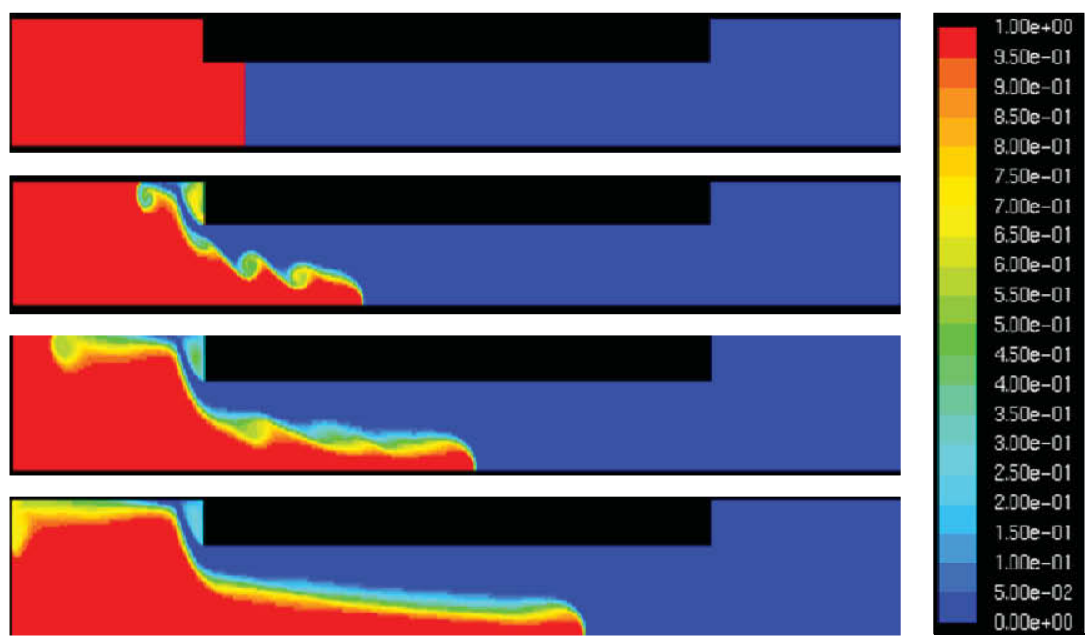

Figure 3-16. Brine intrusion simulation with fine mesh. 


\section{Brine mass fraction at the bottom of pipe (Normal mesh)}

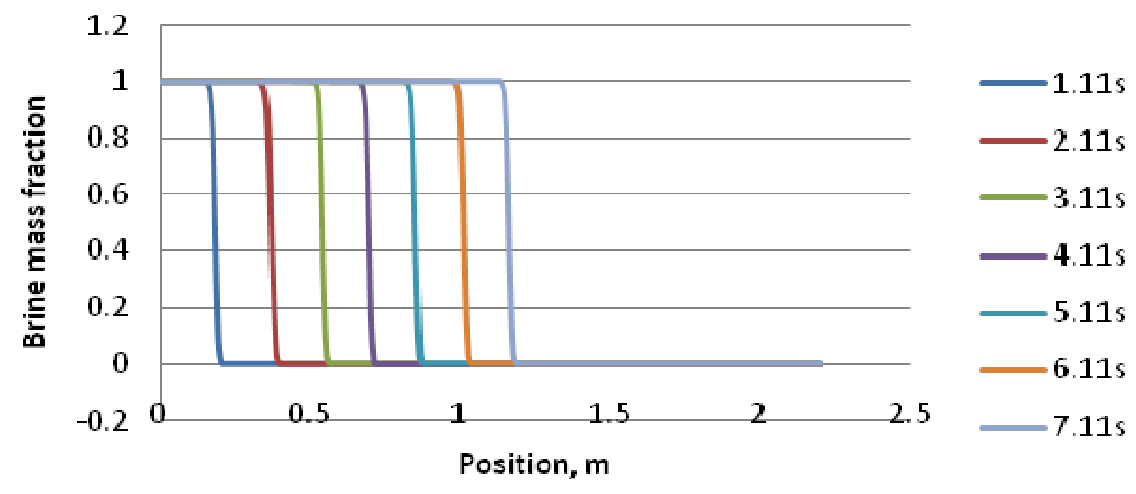

\section{Brine mass fraction at the bottom of pipe (coarse mesh)}

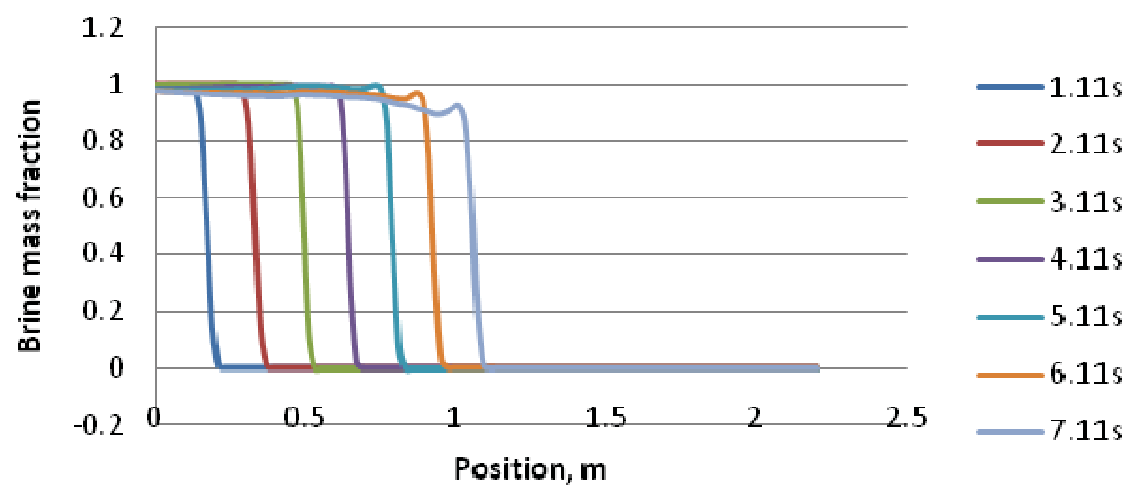

Figure 3-17. Brine mass fraction at the bottom as time marches until 7 seconds (normal, coarse mesh)

Normal mesh was found to have a more distinctive interface between the brine and water compared to the coarse mesh. Based on Figure 3-17, using normal mesh, the edges of the brine or head of the intrusion fluid is well defined, making it easy to determine half of the brine mass fraction because of this sharp interface. The accumulated numerical diffusion in normal mesh is considered smaller than the accumulated numerical diffusion in coarse mesh, which leads to better sharpening of the interface at normal mesh. It is also observed that more blurriness and less sharpening are found at the edge interface in the small density-difference case because the density-driven driving force is degraded by smaller density differences. Figure 3-18 compares the calculated intrusion-front speeds for three different mesh qualities. As shown, the calculated intrusion-front speed varies by mesh types. In order to have different mesh types, a coarse mesh is first defined with appropriate grid size, rediscretization is performed with half of the coarse mesh gird spacing (normal mesh), and fine mesh is generated out of normal by conducting rediscretization. The ratio of total cell number of normal (fine) mesh to that of coarse (normal) mesh is not exactly eight because the CFD model is a full 3-D unstructured grid. It is, therefore, more appropriate to use normalized grid spacing for a refinement study in the unstructured grid case. 


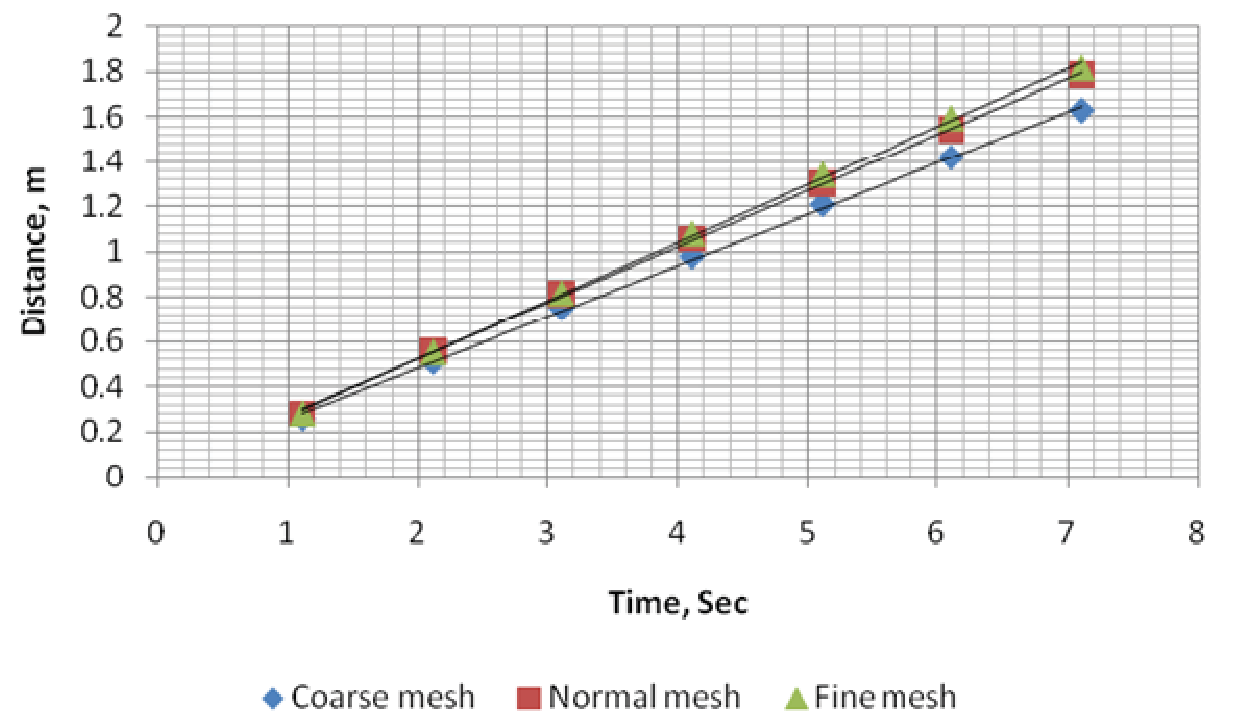

Figure 3-18. Front speed calculation in different mesh (coarse, normal, and fine).

With grid triplet (set of fine mesh, normal mesh, and coarse mesh) solutions, the unknown variables A, B, and p ought to be obtained from simple algebra as shown in Figure 3-19 where $\mathrm{p}$ is the order of convergence in the Richardson extrapolation curve and A is the predicted value of CFD simulation result at asymptotic zero spacing grid. With constant $p$ value, two solutions from normal grid and coarse grid allow the A value (Asymptotic front heat speed) to be calculated as:

$p=\frac{\ln \left(\frac{y_{3}-y_{2}}{y_{2}-y_{1}}\right)}{\ln \frac{x_{2}}{x_{1}}}$

$y=A+B x^{p}, p,\left(x_{2}, y_{2}\right),\left(x_{3}, y_{3}\right)$ are known

$\ln (y-A)=\ln (B)+p \ln (x)$

$\left.\ln \left(\frac{y_{2}-A}{y_{3}-A}\right)=\ln \left(\frac{x_{2}}{x_{3}}\right)^{p} 3-4\right)$

$A=\frac{0.5^{p} \times y_{3}-y_{2}}{0.5^{p}-1}, \frac{x_{2}}{x_{3}}=0.5$

where $p=$ order of convergence, $y_{1}=$ front heat speed in coarse grid, $y_{2}=$ front head speed in normal grid, $y_{3}=$ front head speed in coarse grid, and $x=$ normalized grid spacing. If $p$ is greater than unity, it converges while $p$ values less than unity make the solution diverge.

The uncertainty of CFD prediction is also performed based on the Grid Convergence Index (GCI). GCI-23 is calculated instead of GCI-12 because the normal and coarse mesh solutions are utilized for the Richardson extrapolation method.

Table 3-5 summarizes front-head speed in a different grid type and normalized spacing. Each solution is properly converged with $10^{-4}$ of convergence criteria (continuity, momentums, energy, and species). It is seen that front-head speed increases as the mesh gets finer and as normalized spacing is reduced. The objective of this CFD calculation is to validate the experiment using the front-head speed calculation. It is therefore necessary to achieve the best prediction with less numerical error either in a spatial or temporal dimension. The Richardson extrapolation method is a well known method to validate the CFD result and give asymptotic value at zero spacing (Roche 1998). 


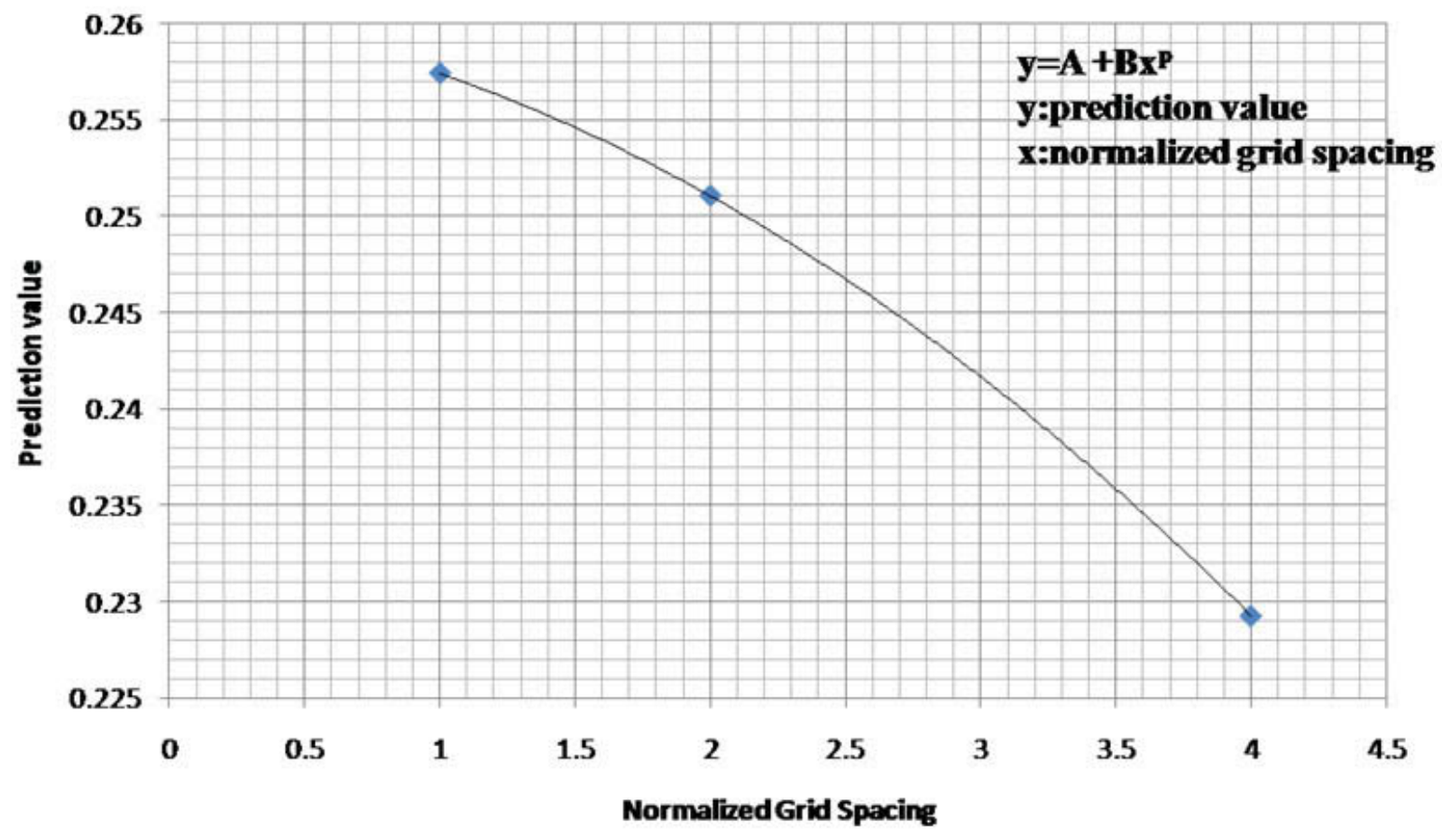

Figure 3-19. Correlation of grid spacing and prediction value in grid convergence study.

Table 3-5.Front head speed calculation at different normalized spacing

\begin{tabular}{|lcc|}
\hline \multicolumn{1}{r}{ Grid type } & Normalized Spacing & Front Head Speed \\
\hline Fine & 1 & $0.257 \mathrm{~m} / \mathrm{s}$ \\
Normal & 2 & $0.251 \mathrm{~m} / \mathrm{s}$ \\
Coarse & 4 & $0.229 \mathrm{~m} / \mathrm{s}$ \\
\hline
\end{tabular}

The order of convergence from Equations. 3-1 to 3-5 above is calculated as

$p=\frac{\ln \left(\frac{0.229-0.251}{0.251-0.257}\right)}{\ln 2}=1.78$

Applying the Richardson extrapolation with two meshes (normal, fine), the asymptotic front head speed at zero grid spacing is calculated as

asymptotic front head speed $=0.257+\frac{(0.257-0.251)}{2^{1.78}-1}=0.260 \frac{\mathrm{m}}{\mathrm{s}}$

The GCI provides an error band on each grid type. The factor of safety is recommended to be Fs $=1.25$ for comparisons over three grids. The GCI for a fine grid (GCI-12) and normal grid (GCI-23) is

$G C I 12=\frac{1.25\left(\frac{0.257-0.251}{0.257}\right)}{\left(2^{1.78}-1\right)} * 100=1.26 \%$

Same approach applies for GCI-23 as

GCI23 $=\frac{1.25\left(\frac{0.251-0.229}{0.229}\right)}{\left(2^{1.78}-1\right)} * 100=4.45 \%$ 
Checking that the solutions were in the asymptotic range of convergence, is accomplished by

$\frac{4.45}{2^{1.78} * 1.26}=1.025$

which is approximately 1.0 , indicating that the solution is well within the asymptotic range of convergence. Figure 3-20 shows the estimated front head speed $(\mathrm{m} / \mathrm{s})$ at the zero spacing with the course, normal, and fine mesh results.

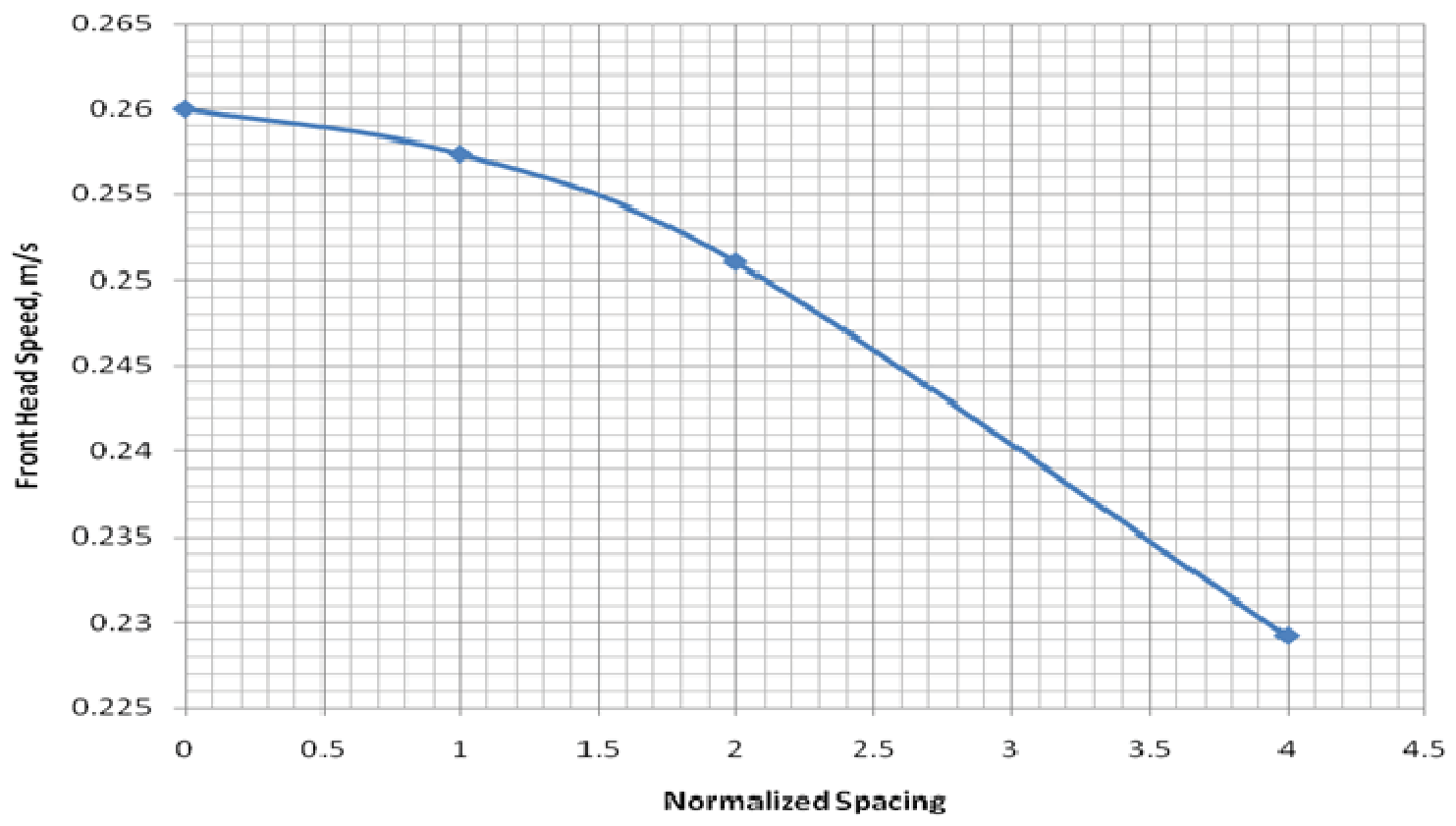

Figure 3-20. Simulated front head speed and calculated front head speed at zero grid spacing with order of convergence, $p=1.71$ (Case 8).

Based on the CFD calculation, the front-head speed in density-driven brine-water stratified flow is estimated to be $\mathrm{V}=0.26 \mathrm{~m} / \mathrm{s}$, with an error band of $1.26 \%$ for GCI- 12 and $4.45 \%$ for GCI-23. In the actual measured front-head speed of the experiment conducted for this case (Case 8), the identical condition with CFD was $0.254 \mathrm{~m} / \mathrm{s}$. Therefore, the CFD result with the asymptotic approach (Richardson extrapolation) is quite reliable for validating experiment data.

Validating the CFD front-head speed prediction by comparing FLUENT calculation with INL isothermal experimental data (as described in the previous section), INL conducted eight sets of isothermal stratified flow tests for liquid-to-liquid having different fluid densities. Heavy liquid density varies from 1.020 to $1.155 \mathrm{~kg} / \mathrm{m}^{3}$. One significant driving force of stratified flow is density difference (different densities of brine water with pure water generate different current speeds). Figure 3-9 and Table 3-4 summarize the current speeds of heavy fluids.

The previous subsection showed three sets of FLUENT calculations with coarse, normal, and fine meshes to determine asymptotic front-head speed. Although these three sets of different grid size models provide a good way to calculate asymptotic front-head speed, it requires tremendous computing time to validate each case, especially the fine mesh model, which consumes more than 72 hours of computing time for 20 seconds of transient simulation. Computing time versus accuracy is a key issue in validating CFDs. However, in this experiment, the overall flow behavior was consistent with different fluid properties, which provided good accuracy with reduced computing time. It was assumed that the order of convergence value, $p$ (see Equation 2-1) is fully independent with each experimental test case because 
fluid properties have a very small range of variance. In addition, an INL isothermal test is being conducted with a very small density-different case calculated as $\sim 1, \gamma=$ density ratio of the low density to the heavy density, which induces relatively slow stratified flow current in the channel.

Figure 3-21 compares front-head speed prediction by CFD calculation with the asymptotic approach and experiment measurement in which the CFD prediction qualitatively indicates good agreement with experiment data. In order to validate the procedure between code prediction and experiment data, however, it is necessary to take an uncertainty concept into consideration for both the CFD code and experiment.

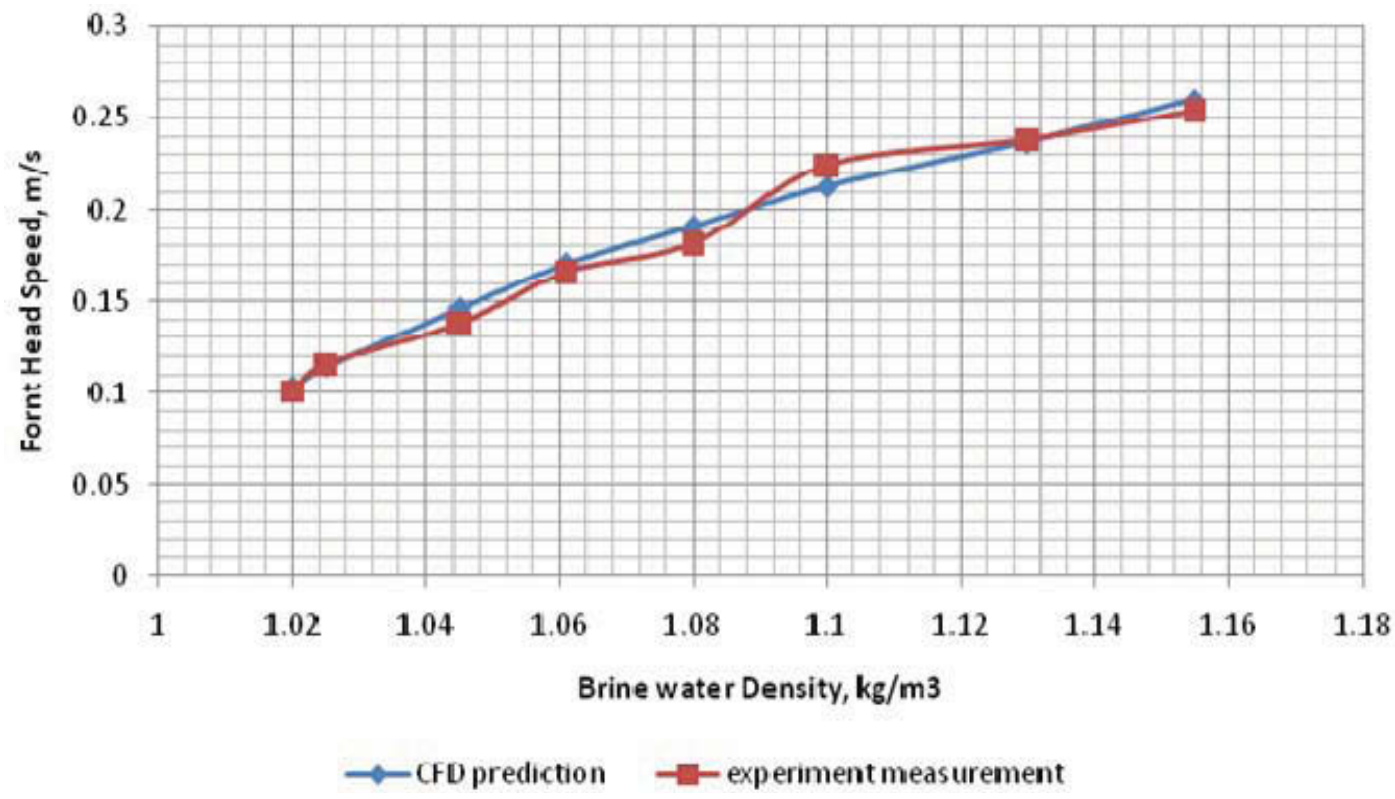

Figure 3-21. Comparison of CFD result and experiment measurement of front head speed.

The linearized approximation uncertainty method (Kline and McClintonck 1953) was used to evaluate the uncertainty of front head velocity with two measured quantities $(\mathrm{x}, \mathrm{t})$ as shown in the following mathematical procedure:

$\mathrm{V}=\frac{\mathrm{x}}{\mathrm{t}}$

$(\mathrm{dV})^{2} \approx\left[\frac{\partial}{\partial \mathrm{x}}\left(\frac{\mathrm{x}}{\mathrm{t}}\right)\right]^{2}(\mathrm{dx})^{2}+\left[\frac{\partial}{\partial \mathrm{t}}\left(\frac{\mathrm{x}}{\mathrm{t}}\right)\right]^{2}(\mathrm{dt})^{2}$

$d V=\sqrt{\left[\frac{\partial}{\partial x}\left(\frac{x}{t}\right)\right]^{2}(d x)^{2}+\left[\frac{\partial}{\partial t}\left(\frac{x}{t}\right)\right]^{2}(d t)^{2}}=\sqrt{\left[\left(\frac{1}{t}\right)\right]^{2}(d x)^{2}+\left[\left(\frac{-x}{t}\right)\right]^{2}(d t)^{2}}$

This method allows for calculating the uncertainty of measured front-head speed from both local and global points of view. Usually, local uncertainty from two local data points is relatively smaller than global uncertainty (with starting-point data and ending-point data). In this case, uncertainty in the distance between the start and end region is larger than that of the local region. Both cases of uncertainty were therefore calculated to obtain the mean value of experiment uncertainty for the front head speed. The uncertainties of front-head speed were also predicted for the CFD code using the asymptotic approach of the Richardson extrapolation method and GCI uncertainty error band. The uncertainties of front-head speed in the experiment and CFD code are tabulated in Table 3-6. 
Table 3-6. Uncertainty of front head speeds from experiments and CFD code calculations.

\begin{tabular}{|l|c|c|c|c|c|c|c|c|}
\hline & Case 1 & Case 2 & Case 3 & Case 4 & Case 5 & Case 6 & Case 7 & Case 8 \\
\hline $\begin{array}{l}\text { Uncertainty of } \\
\text { Experiment (\%) }\end{array}$ & 10.47 & 10.69 & 10.95 & 9.02 & 10.77 & 10.91 & 10.52 & 13.51 \\
\hline $\begin{array}{l}\text { Uncertainty of CFD } \\
\text { results (\%) }\end{array}$ & 11.08 & 7.34 & 5.42 & 5.07 & 4.10 & 4.03 & 3.44 & 4.45 \\
\hline
\end{tabular}

The uncertainty of CFD results calculated using Equation 2-8 in the lower brine-water density cases $\left(1.02 \mathrm{~kg} / \mathrm{m}^{3}\right)$ indicate around $11 \%$, compared to the other cases. It is related to how the order of convergence in the whole validation procedure is selected. The order of convergence value is obtained from Case 8 . Density of brine water and pure water are $1.155 \mathrm{~kg} / \mathrm{m}^{3}$ and $1.001 \mathrm{~kg} / \mathrm{m}^{3}$, respectively. The order of convergence value, $\mathrm{p}=1.78$, was determined from three sets of solutions with different mesh sizes. Using the $\mathrm{p}$ value as a general order of convergence in the validation procedure, only two mesh cases (normal and coarse) are used to calculate asymptotic front-head speed. This saves a considerable amount of computing time with reliable accuracies (uncertainty of 3 to $5 \%$ for most of cases) for eight cases of other validation procedures. The uncertainties of CFD results for most of the cases are also shown in Table 3-6.

When computation code is validated with experimental data, it is often tempting to directly compare computational results with experimental data. This comparison, however, can only give a qualitative validation. In the same manner, it can be stated that INL isothermal experimental results are well validated against Fluent CFD results as shown in Figure 3-21. More advanced validations were achieved by using the asymptotic Richardson extrapolation and GCI. Figure 3-22 shows that the CFD code agrees with experiment measurement in stratified flow and also that CFD uncertainty bands agree with experimental uncertainty bands. CFD code is therefore validated for the isothermal experiment in both a qualitative as shown in Figure 3-21 and a quantitative way as shown in Figure 3-22.
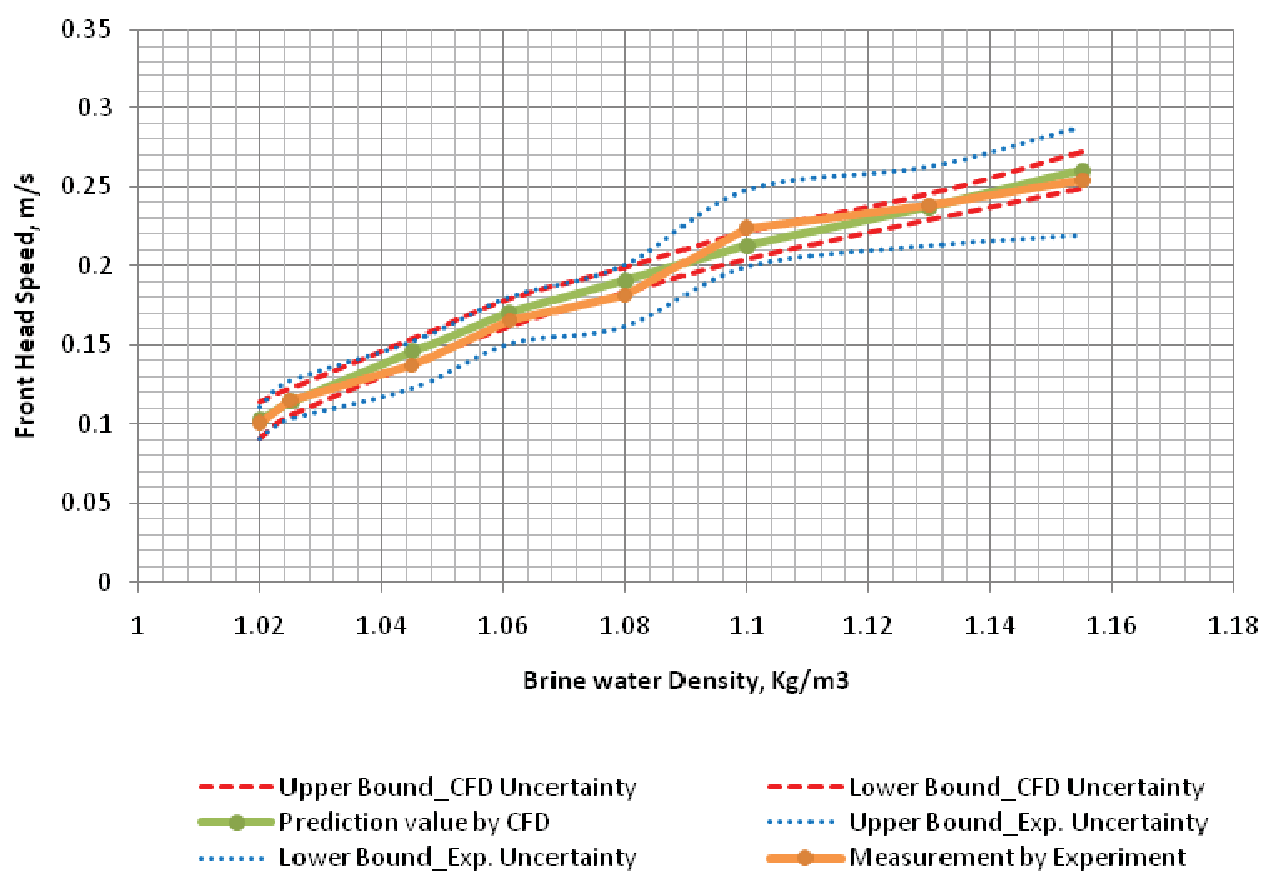

$$
\begin{aligned}
& \ldots-- \text { Lower Bound_CFD Uncertainty } \\
& \ldots . . . \text { Upper Bound_Exp. Uncertainty } \\
& \text { Measurement by Experiment }
\end{aligned}
$$

Figure 3-22. Plot of experimental data with uncertainty band and CFD prediction value with code uncertainty band comparison between experiment measurement and previous model (Simpson model (1979) and Benjamin model (1968)) 
A density-driven stratified flow has drawn considerable attention in many fields. A number of researchers have investigated how to predict the current speed or height of flow under different conditions using proper assumptions. A comparison study was conducted wherein two models, the Simpson model (1979) and the Benjamin model (1968), were reviewed to check the feasibility of the reference theoretical model for this experiment validation. Both models aim to predict current speeds in various conditions. A considerable difference in the two models is whether one takes internal flow (Benjamin model) or external flow (Simpson model) into account. The Benjamin model is considered more feasible in physically capturing the INL isothermal experiment because the experimental condition is designed to investigate internal (in pipe) stratified flow behavior. The Simpson model, which is a function of g'(reduced gravity), d1(height of current flow), and D (total height of channel), predicts front-head speed as follows:

$U_{\text {Simpson }}=f\left(g^{\prime}, d_{1}, D\right)$

$U=1.2 \times\left(g^{\prime} d_{1}\right)$, when $\frac{d_{1}}{D} \ll 0.075$

$U=0.5 \times\left(\frac{d_{1}}{D}\right) \times\left(g^{\prime} d_{1}\right)$, when $0.075<\frac{d_{1}}{D} \leq 0.5$

$U=0.396 \times\left(g^{\prime} d_{1}\right)^{\frac{1}{2}}, \frac{d_{1}}{D}=0.5($ Simpson model $)$

Complying with the Simpson model, INL experimental data are thus plotted as

$U=0.65 \sim 0.75 \times\left(g^{\prime} d_{1}\right)^{\frac{1}{2}}, \frac{d_{1}}{D}=0.5(I N L$ experiment $)$

It was then found that INL experimental front-head velocities are approximately 1.6 to 1.87 times larger than those of the Simpson model. However, expressing INL experimental data along with the Simpson model form shows very good agreement with the inviscid model (red dash line), which represents the Benjamin model (see Figure 3-23). The Benjamin model uses internal flow assumption to predict front head speed as follows:

$U_{\text {Benjamin }}=f\left(g^{\prime}, D\right)$

$U=0.5 \times\left(g^{\prime} D\right)^{\frac{1}{2}}$, when $\frac{d_{1}}{D}=0.5($ Benjamin model $)$

$U=0.46 \sim 0.55 \times\left(g^{\prime} D\right)^{\frac{1}{2}}$, when $\frac{d_{1}}{D}=0.5$ (INL experiment $)$

The INL experimental data agree quite well with the Benjamin model; there is a $10 \%$ deviation. Current INL experiment conditions are physically well-matched with Benjamin model assumptions and conditions. It therefore makes sense to use the Benjamin model to check INL experiment data. 


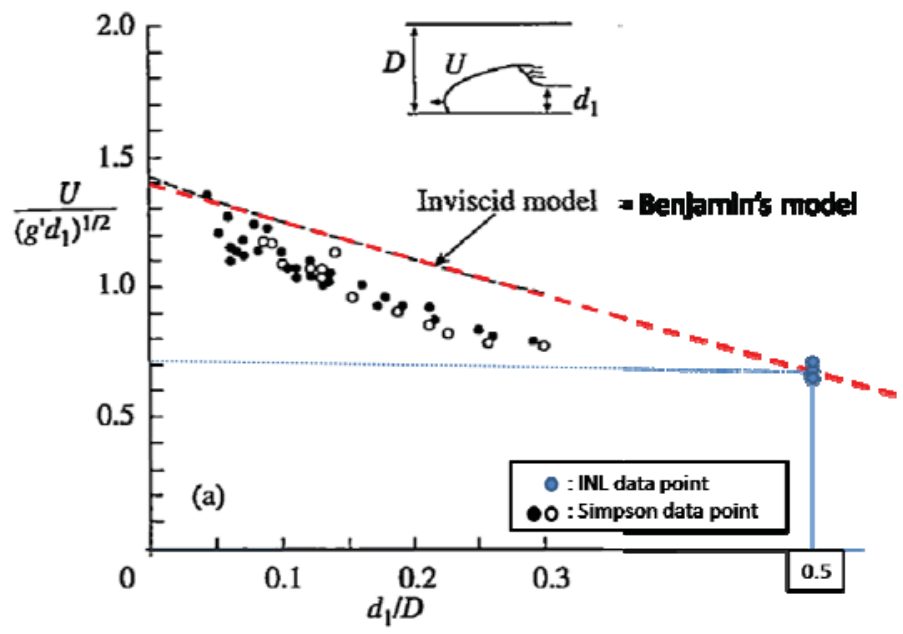

Figure 3-23. Measured front head speed U, scaled with as a function of of INL experimental data and Britter and Simpson's data (1979).

\subsection{Validations on the CFD code for Air/Helium Stratified Flow}

\subsubsection{Experiment Description}

In this section, the CFD results were compared to the experimental results obtained by Grobelbauer et al. (1993) in ETH Zurich as a part of the CFD validation study. Their experiments were based on both a series of lock-exchange flows with gases of different densities in a closed channel of a square crosssection. They focused on the quantitative measurement of front velocities of the gravity current flows. The experiment results covered the full range of gas intrusions, heavy as well as light, for the gravity current flows in the lock-exchange situations.

Figure 3-24 shows the experimental setup. A closed channel of cross-section $0.3 \times 0.3 \mathrm{~m}^{2}$ and total length of either 3.8 or $4.5 \mathrm{~m}$ is divided into chambers of unequal size separated by a quick-operating gate. The chamber lengths are 3.8 and $0.8 \mathrm{~m}$ (or $1.5 \mathrm{~m}$ ) respectively. The chambers were filled with gases of different densities, and to this end they were equipped with valves at the end walls. A gas heavier than air was supplied through the low valve and air let out through the top valve; vice versa for gases lighter than air. The concentration of gas in the chambers was monitored during the filling process. Prior to a test it would be above $95 \%$ for the large chamber and above $97 \%$ for the smaller chamber. The temperature was monitored. Prior to a test the temperature difference relative to the ambient was always less than $2{ }^{\circ} \mathrm{C}$.

According to Grobelbauer et al. (1993), the experimental scatter is mainly contributed by the manually opened gate. However, the time required to open the gate was very short compared to characteristic flow times. The gate velocity was reported to be 3 to $4 \mathrm{~m} / \mathrm{s}$, while flow velocity was reported to be 0.2 to $1.8 \mathrm{~m} / \mathrm{s}$. The velocity of the front was measured by using seven hot-wire probes placed along the floor (for heavy gas) or along the ceiling (for lighter gas). These probes were used as trip wires to give the signal of the arrival time of the current front. Figure 3-25 shows the propagation velocities of the fronts of heavy gas and light gas. The experiment was conducted with combinations of five different gases: air, argon, carbon dioxide, Freon 22, and helium, producing density ratios ranging from 0.046 to 0.9. Seven different gas combinations were taken into consideration (See Table 3-7). Each gas combination was tested in two configurations: first with the heavy gas in the smaller chamber and with the light gas in the larger, and then vice versa. 


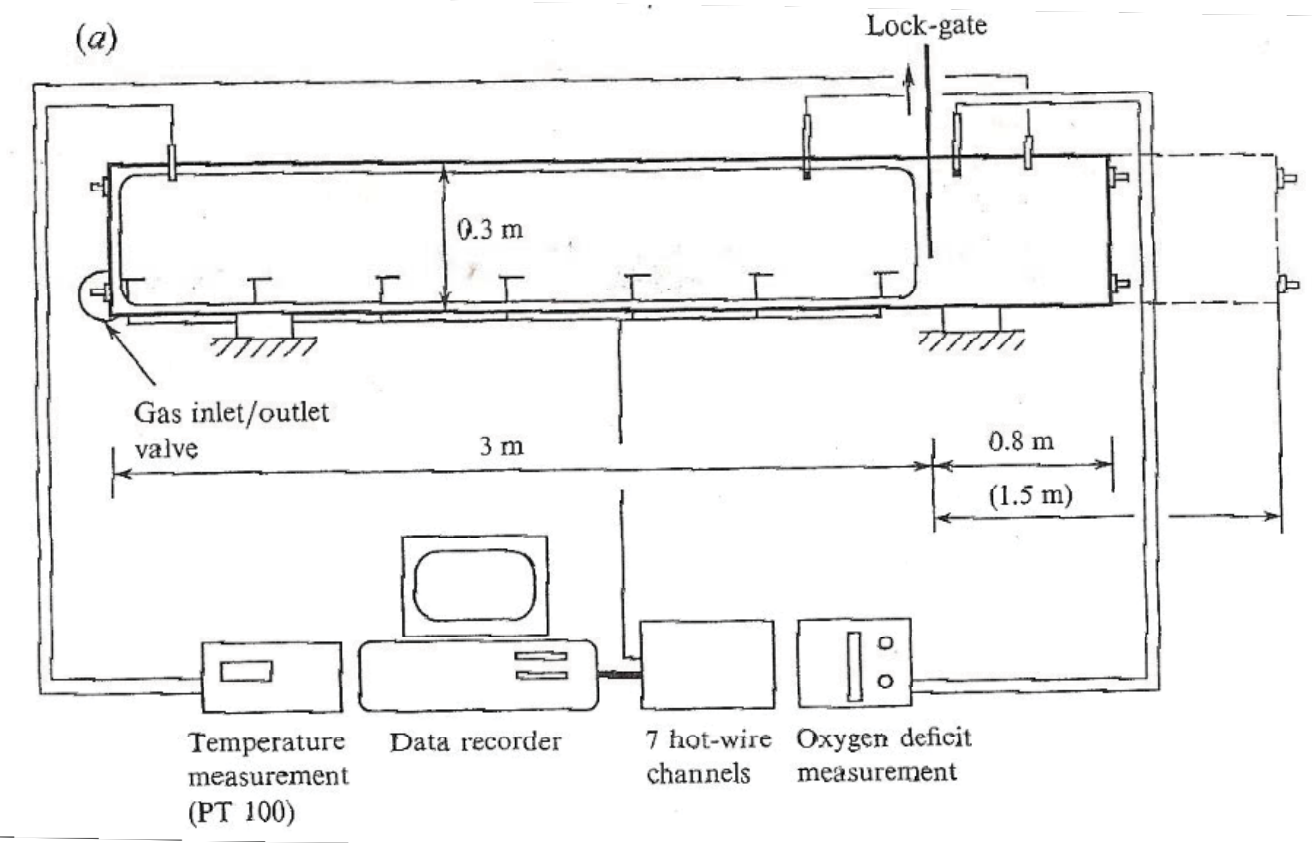

Figure 3-24. Experimental arrangement (Grobelbauer et al. 1993).

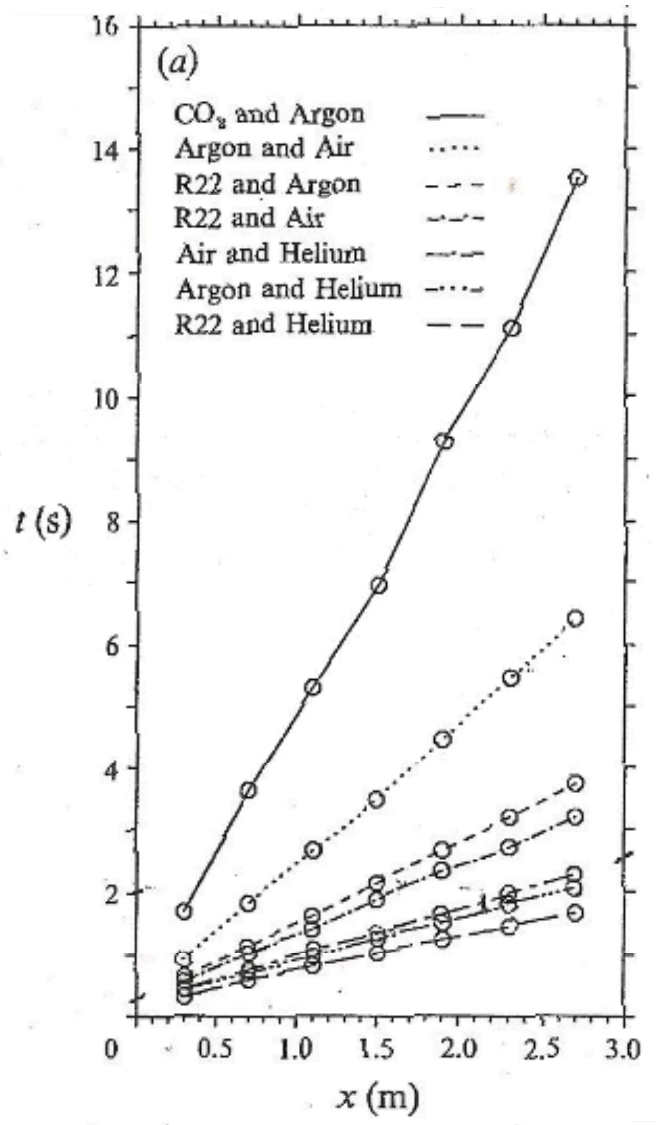

(a) Dense gas

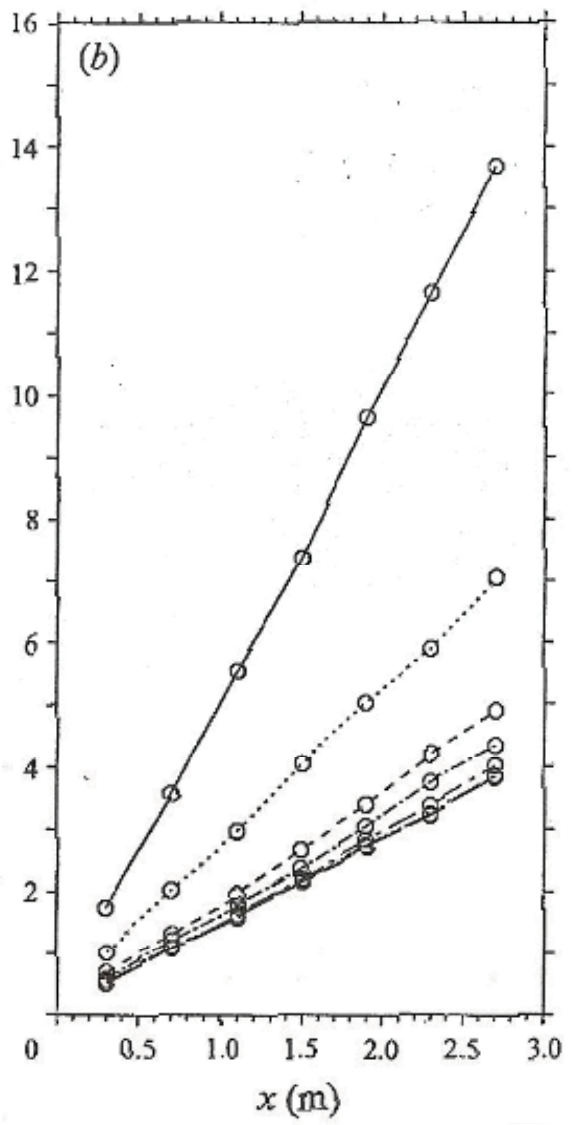

(b) Light gas

Figure 3-25. Propagation velocities of dense gas and light gases (Grobelbauser et al. 1993). 
Table 3-7. Gas combinations used for Grobelbauser et al. (1993)'s experiment.

\begin{tabular}{|l|c|}
\hline \multicolumn{1}{|c|}{ Gases } & Density Ratio $\left(\boldsymbol{\rho}_{\mathbf{L}} / \boldsymbol{\rho}_{\mathbf{H}}\right)$ \\
\hline $\mathrm{CO}_{2} /$ argon & 0.90 \\
\hline Argon/air & 0.72 \\
\hline R22/argon & 0.46 \\
\hline R22/air & 0.33 \\
\hline Air/helium & 0.14 \\
\hline Argon/helium & 0.1 \\
\hline R22/helium & 0.046 \\
\hline
\end{tabular}

The test-section was made of transparent material to allow visualization. The current depths were another interest in the experiment besides the current speed. Some initial visualization trials were made using smoke from commercial smoke pellets. These pellets, however, generated smoke by burning and the heat released changed the density distribution. A second trial has been performed by the method based on the evaporation of oil from a vertical wire. By this method, they obtained nice photos that visualize that the flow is not symmetric and that the light-gas front is less blunt and appear more stable than the heavy-gas fronts. However, they could not obtain the good quantitative data for the current depth.

\subsubsection{Numerical Simulations and Comparisons}

FLUENT 6.3, a general purpose CFD code, was used for simulating the experiments. Figure 3-26 shows the geometry and mesh of the FLUENT model. Since the experiment by Grobelbauer et al. (1993) is based on the simple lock-exchange flows in the rectangular channel, the 2-D assumption is quite valid here. The model consists of two boxes of different sizes. The left one is $0.3 \mathrm{~m}$ high and $3.0 \mathrm{~m}$ long. The right one is $0.3 \mathrm{~m}$ high and $0.8 \mathrm{~m}$ long. The mesh type is hexagonal, and three different sizes of mesh were considered in the grid sensitivity study: coarse, normal, and fine. The mesh sizes are $0.04 \mathrm{~m}$ (coarse), $0.02 \mathrm{~m}$ (normal), and $0.01 \mathrm{~m}$ (fine) for each grid.

(a) Geometry

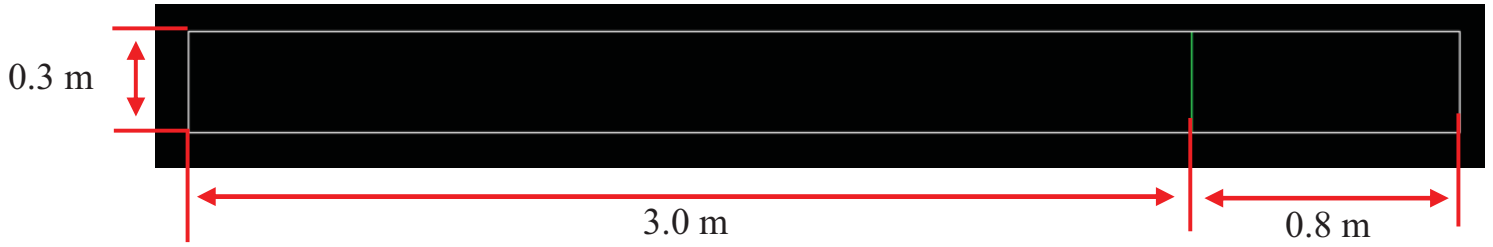

(b) Mesh (Fine)

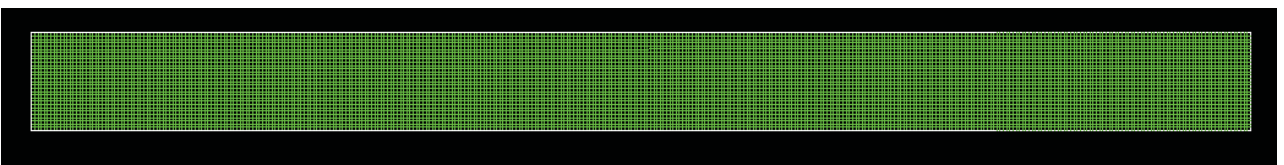

Figure 3-26. Geometry and FLUENT mesh.

The following summarizes the FLUENT options and models used for the base calculations.

- Solver:

- Solver: Pressure Based

- Formulation: Implicit

- 2-D double precision 
- Unsteady

- Velocity Formulation: Absolute

- Gradient Option: Green-Gauss cell based

- Pressure-Velocity Coupling: Simple

- Pressure: Standard

- Momentum: $2^{\text {nd }}$ Order

- Turbulence Kinetic Energy: $2^{\text {nd }}$ Order

- Turbulence Dissipation Rate: $2^{\text {nd }}$ Order

- Species: $2^{\text {nd }}$ Order

- Energy: $2^{\text {nd }}$ Order

- Viscous Model:

- Turbulence model: k-e realizable

- Wall function: standard wall function

- Energy equation

- $\quad$ Species transport model:

- 2 species: Air and Helium

- Property ModelsDensity: Incompressible ideal gas

- Heat capacity: mixing law

- Thermal conductivity: mixing law

- Viscosity: ideal gas mixing law.

Figure 3-27 shows the initial simulation condition. The left hand side was filled with helium and the right hand side with air. Therefore, this simulation demonstrates the heavy fluid intrusion into the light fluid. Initial temperature was set as $300 \mathrm{~K}$ and pressure at $1 \mathrm{~atm}$.

\section{Air Mass Fraction}

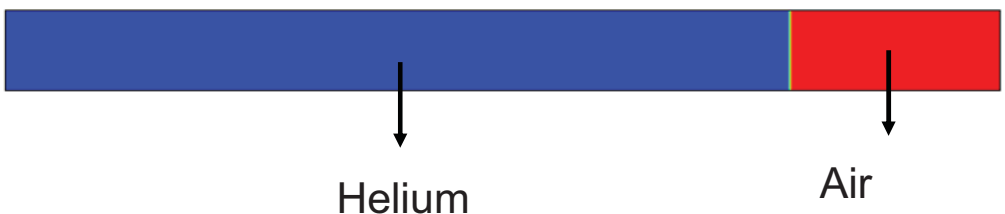

Figure 3-27. Initial air mass fractions. 
Figures 3-28, 3-29, and 3-30 show the contour plots of air mass fractions at different times for fine, normal, and coarse meshes, respectively. These figures clearly show that air rapidly intrudes into the helium side. According to these figures, the front speed of the air looks constant along with time. They show that air travels almost the same distance in the same time intervals. It strongly supports the invicid flow assumptions used in the previous theoretical derivations.

\section{$0.5 \mathrm{sec}$}

\section{$1.0 \mathrm{sec}$}

\section{$1.5 \mathrm{sec}$}

\section{$2.0 \mathrm{sec}$}

\section{$2.5 \sec$}

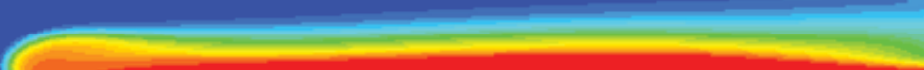

Figure 3-28. FLUENT simulation (air mass fraction (fine mesh)).

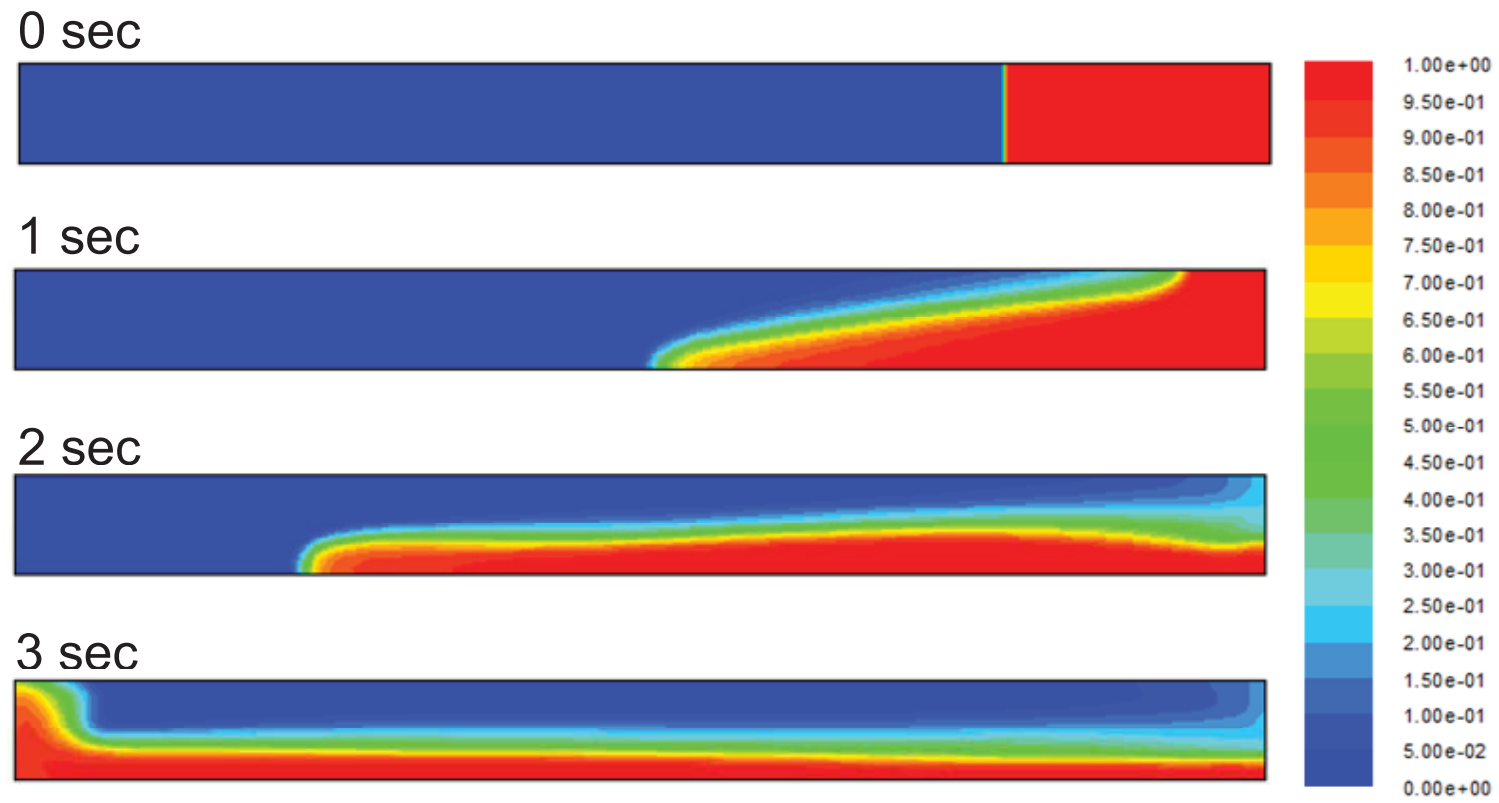

Figure 3-29. FLUENT simulation (air mass fraction (normal mesh)). 


\section{$0 \mathrm{sec}$}

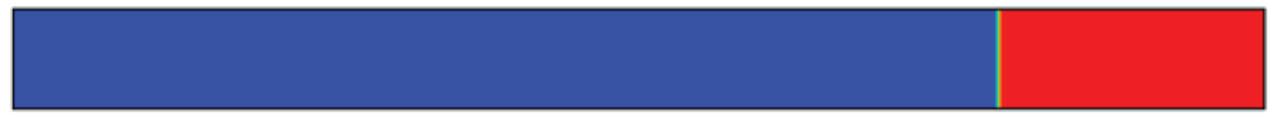

$1 \mathrm{sec}$

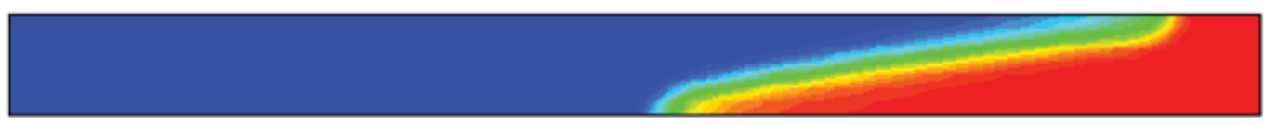

\section{$2 \mathrm{sec}$}

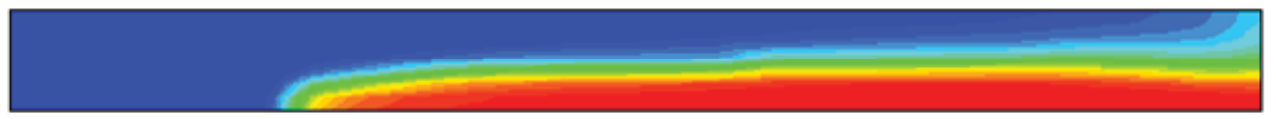

\section{$3 \mathrm{sec}$}

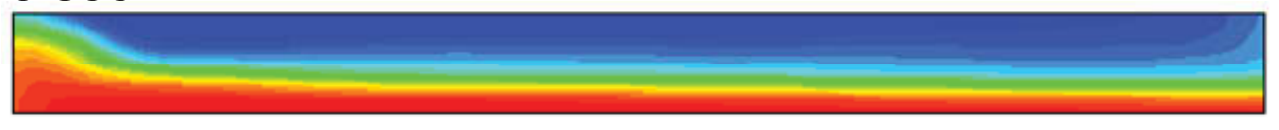

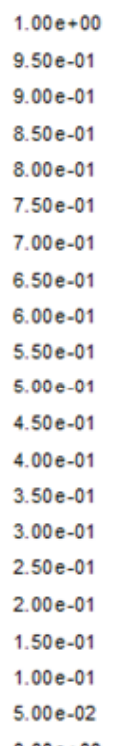

$0.00 e+00$

Figure 3-30. FLUENT simulation (air mass fraction [coarse mesh]).

A grid sensitivity study was performed for our simulation. Since the front speed is the main comparison parameter in the current validation, we first defined the current speed in the simulation with the same method used in the experiment. In the experiment, the locations of the current were determined by hot-wires installed at the bottom of the test-section. So, the current locations in the simulations were also determined by the air concentrations at the bottom plane of the bottom. Figure 3-31 shows the air mass fractions at the bottom plane and includes five different curves for different times: 0.5, 1.0, 1.5, 2.0, and 2.5 seconds. The current locations were determined to be the intersections between air mass fraction curves and x-axis.

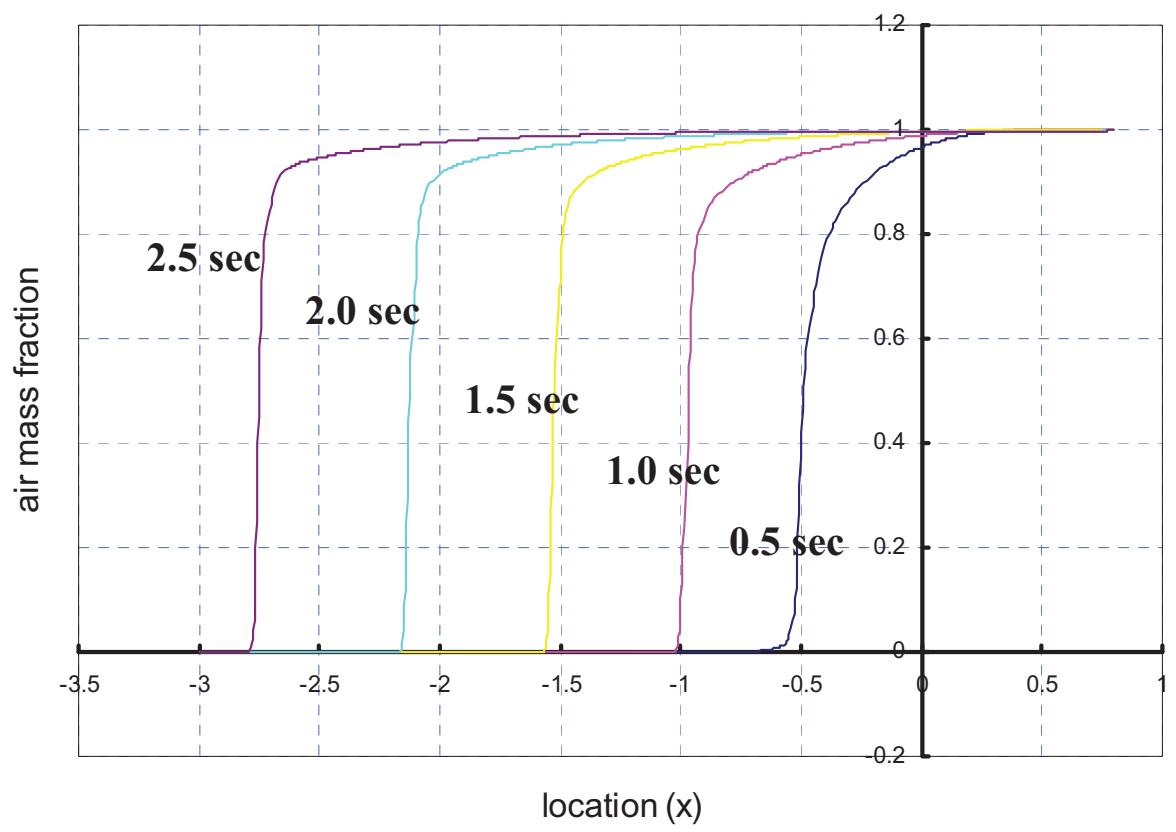

Figure 3-31. Air mass fraction at the bottom plate (current locations). 
Based on Figure 3-31, the propagation distances were obtained for different times. Figure 3-32 compares the calculated propagation distances for three different quality meshes, showing that the calculated propagation distances are quite close to each other, even though the quality of the meshes differs. Especially, if we look at the data at $\mathrm{t}=2.5$ seconds, three different models predict almost the same propagation distances (within $1 \%$ ).

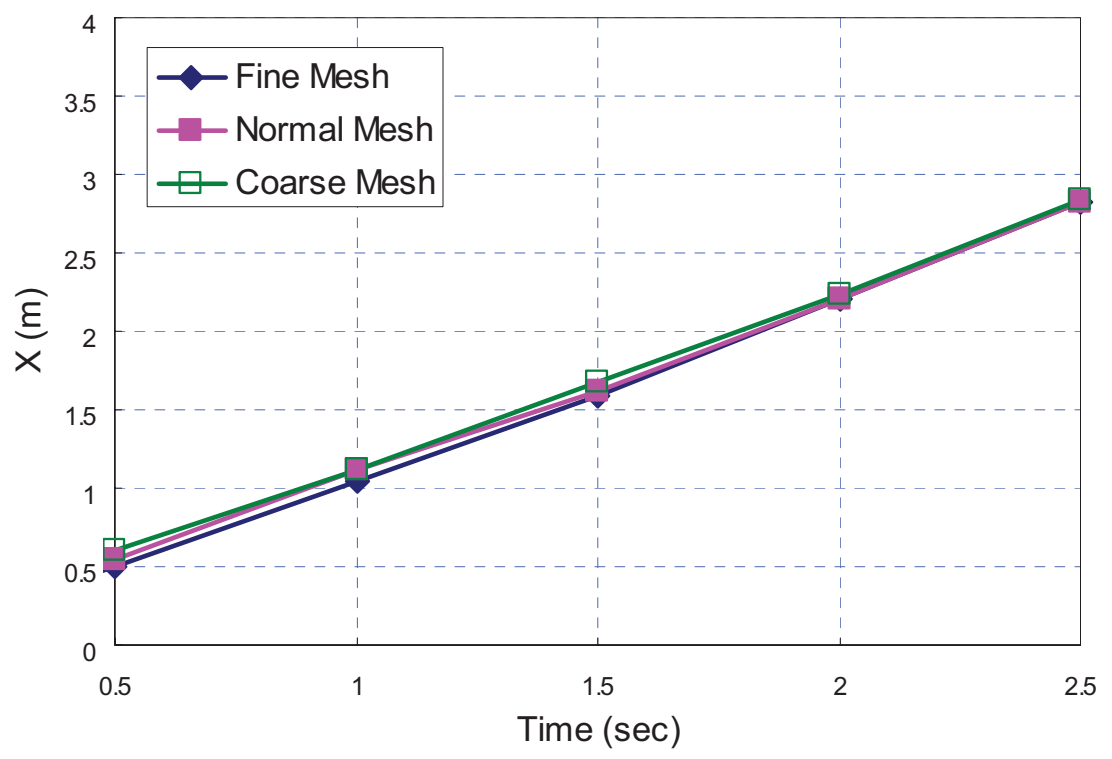

Figure 3-32. Comparisons between different mesh sizes.

A method based on the Richardson extrapolation (Roache et al. 1998) was used to quantitatively estimate the grid convergence. The objective of this CFD analyses was to determine the current front speed along with the channel. Table 3-8 indicates the grid information and the calculated front speeds by CFD calculations. Each solution was properly converged with respect to iterations. The column indicated by spacing is the spacing normalized by the spacing of the finest grid.

Table 3-8. Grid information and front speed.

\begin{tabular}{|l|c|c|}
\hline \multicolumn{1}{|c|}{ Grid } & Normalized Grid Spacing & Front Speed (m/s) \\
\hline 1 Fine & 1 & 1.19 \\
\hline 2 Normal & 2 & 1.18 \\
\hline 3 Coarse & 4 & 1.16 \\
\hline
\end{tabular}

From the above information, the order of convergence is calculated as

$p=\ln ((1.16-1.18) /(1.18-01.19)) / \ln (2)=1$.

We can apply Richardson extrapolation using the two finest grids to obtain an estimate of the value of the front speed at zero grid spacing as

$V_{h}=1.19+(1.19-1.18) /\left(2^{1}-1\right)=1.2 \mathrm{~m} / \mathrm{s}$.

Figure 3-33 plotted the simulated front speeds and the estimated front speed at zero grid spacing. The grid convergence index (GCI) for the fine grid solution was computed using a factor of safety of $\mathrm{F} s=1.25$. The GCI for grids 1 and 2 is 


$$
\left.G C I_{12}=1.25 \times(1.19-1.18) / 1.19\right) /\left(2^{1}-1\right) \times 100=1.050 \%
$$

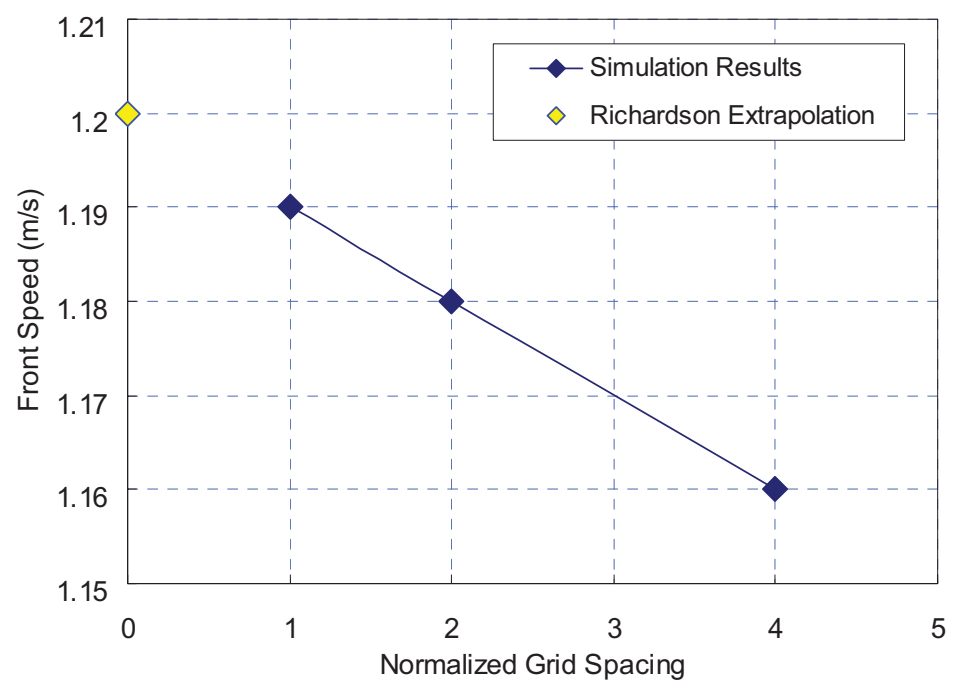

Figure 3-33. Simulated front speeds and estimated front speed at zero grid spacing.

and the GCI for grid 2 and 3 is

$$
\left.G C I_{23}=1.25 \times(1.18-1.16) / 1.18\right) /\left(2^{1}-1\right) \times 100=2.119 \%
$$

The formula used to check that the solutions were in the asymptotic range of convergence is

$$
2.119 /\left(2^{1} \times 1.050\right)=1.0085
$$

which is approximately 1.0, indicating that the solutions are well within the asymptotic range of convergence.

Based on the calculation in Eqs. (3-22) through (3-26), we can say that the front speed is estimated to be 1.2 within an error of $1.050 \%$. The front speed estimated in the dine mesh is only deviated from this value with an error of $0.84 \%$, indicating that the simulated results by fine mesh are quite reliable.

More calculations were carried out to look at the turbulence model effect on the results. Figures 3-34, 3-35, and 3-36 show the contour plots on the air mass fractions for k-w, standard k-e, and RSM, respectively. All the simulations have been performed by using fine mesh grid and the same model setup as the base calculation except for turbulence models. The three figures show that the overall qualitative flow behaviors are not dependent on the different turbulence model. Figure 3-37 shows the comparisons between the CFD simulation results and the experimental data. The compared parameter is the front location of the heavy current (air). This figure shows that the realizable k-e and RSM models are well predicting the front locations and that the data in the beginning shows more deviations than those in the later. It is because in the actual experiment, the opening gate is not instantaneous, and the gate opening process disturbs the flow field. After 1 second, the experimental data and the CFD results show quite good agreement. Table 3-9 summarizes the comparisons of the front speed between the experiment and the CFD simulations. To estimate the front speeds correctly, only the data after 1.5 seconds were used by ignoring initial disturbed data. In the experiment, the air current speed was estimated to be $1.25 \mathrm{~m} / \mathrm{s}$. The calculated CFD results are $0.92 \mathrm{~m} / \mathrm{s}$ (standard k-e), $1.19 \mathrm{~m} / \mathrm{s}$ (realizable $\mathrm{k}-\mathrm{e}), 1.12 \mathrm{~m} / \mathrm{s}(\mathrm{k}-\mathrm{w})$, and $1.20 \mathrm{~m} / \mathrm{s}$ 
(RSM). As shown in the table, the realizable k-e and RSM models show good predictions of front speeds. The errors of the k-e model and the RSM model are 5.04\% and 4.2\%, respectively.

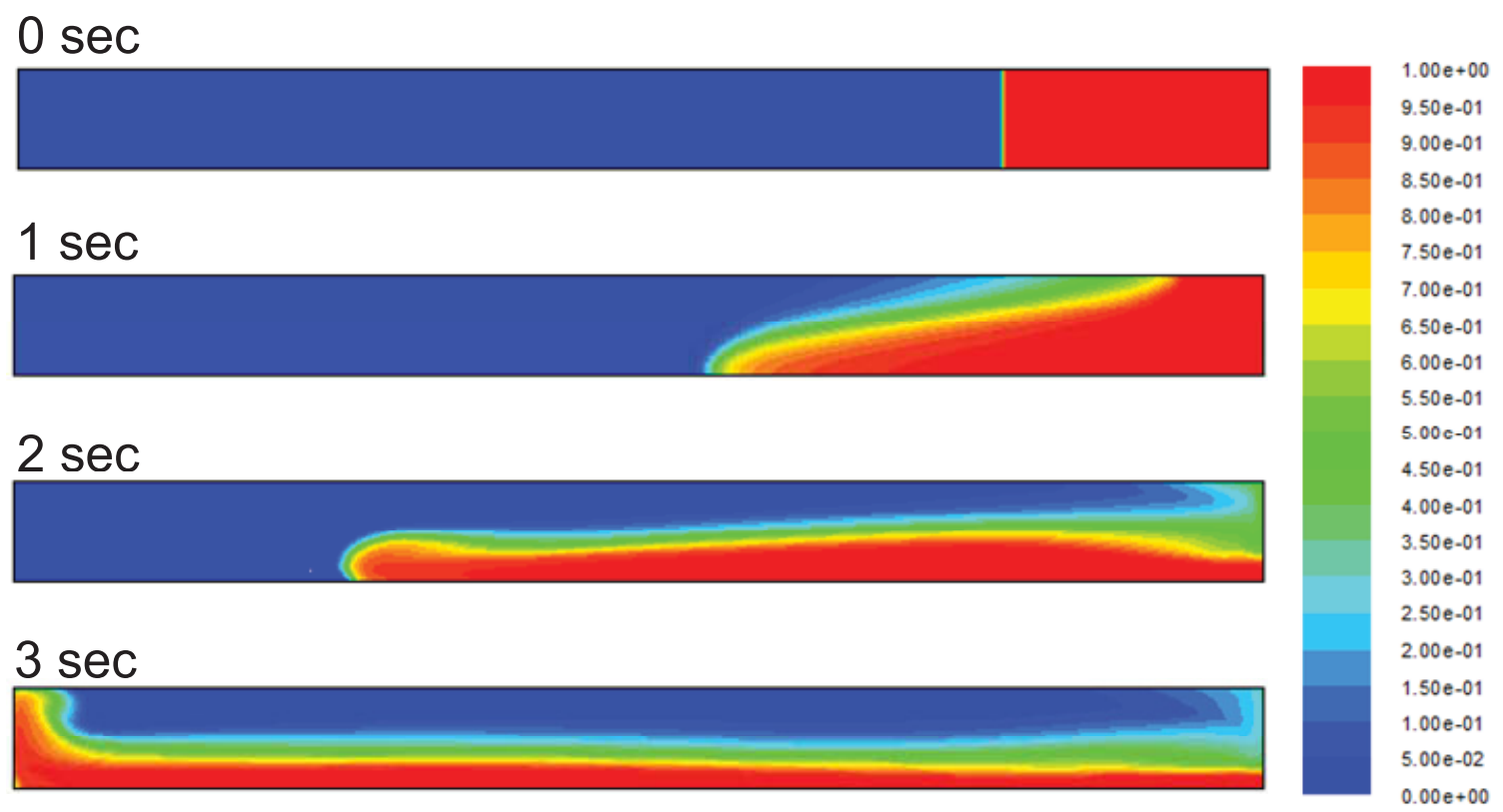

Figure 3-34. FLUENT simulation (air mass fraction, k-w model, fine mesh).

\section{$0 \mathrm{sec}$}

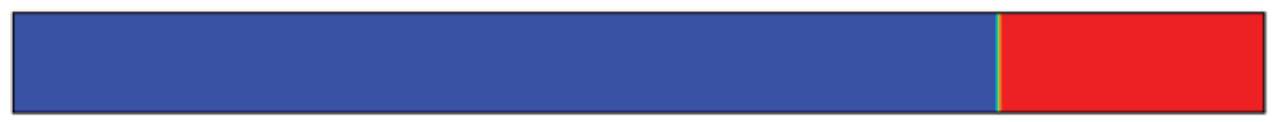

\section{$1 \mathrm{sec}$}

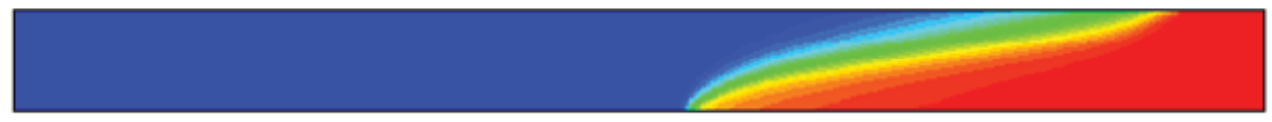

\section{$2 \mathrm{sec}$}

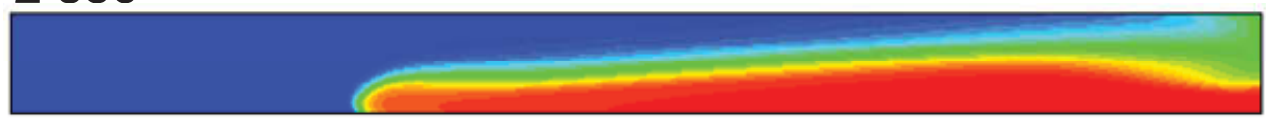

\section{$3 \mathrm{sec}$}

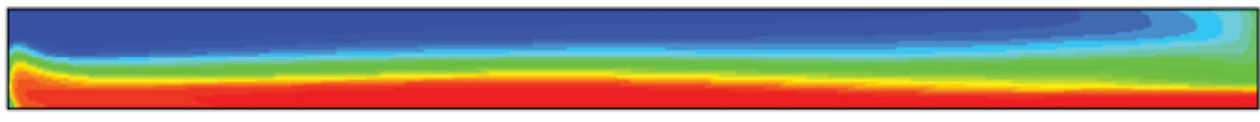

Figure 3-35. FLUENT simulation (air mass fraction, standard k-e model, fine mesh). 
$0 \mathrm{sec}$

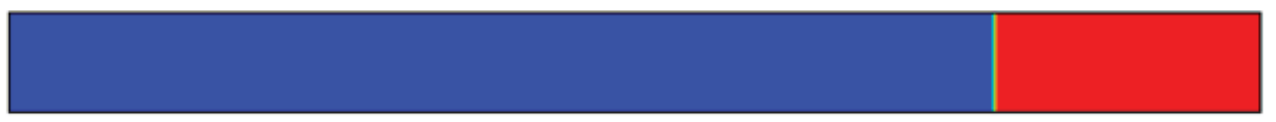

\section{$1 \mathrm{sec}$}

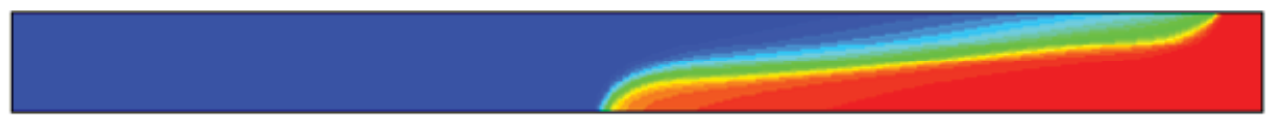

\section{$2 \mathrm{sec}$}

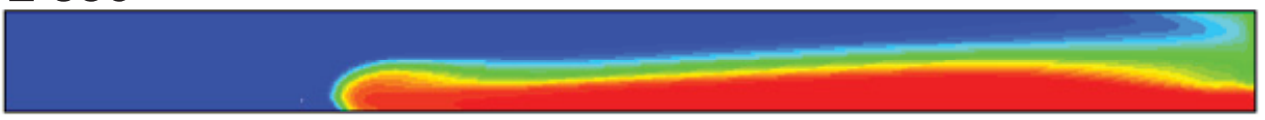

\section{$3 \mathrm{sec}$}

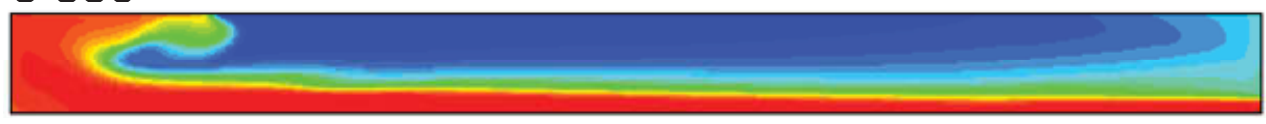

Figure 3-36. FLUENT simulation (air mass fraction, RSM model, fine mesh).

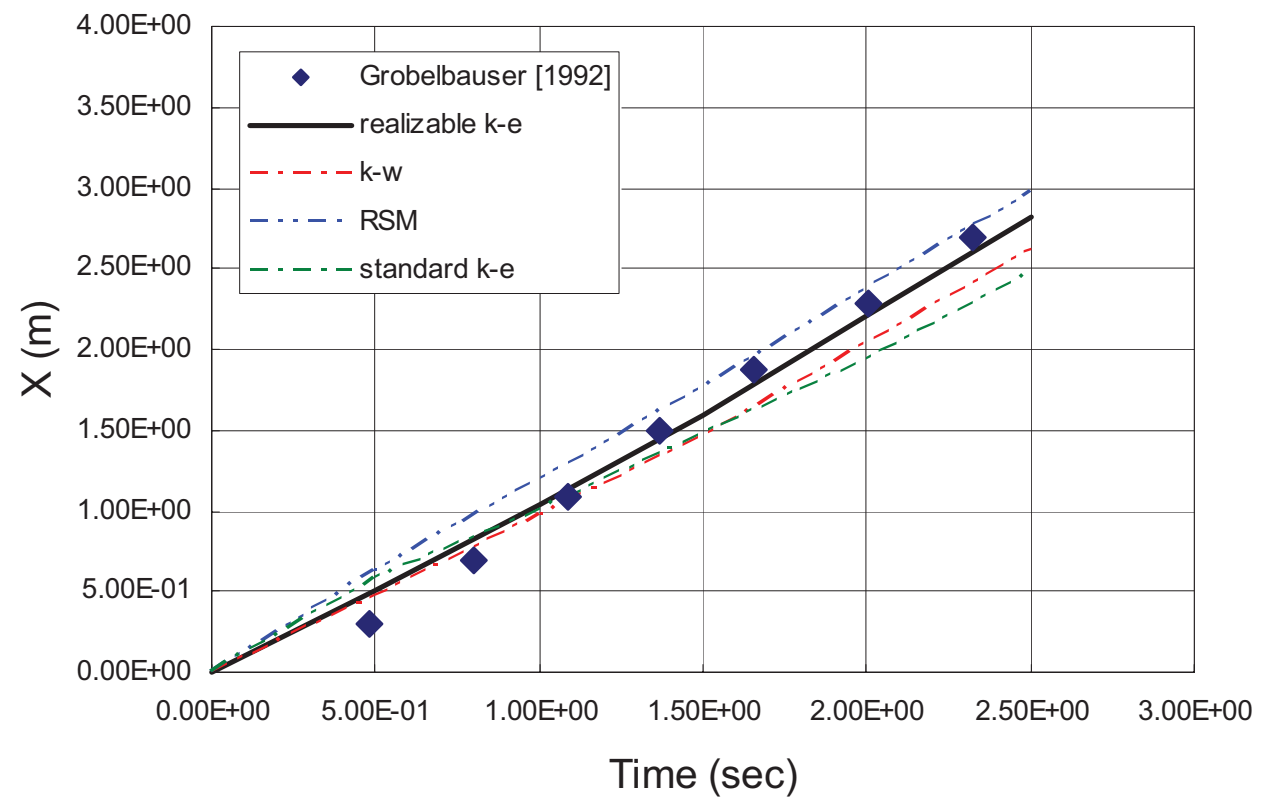

Figure 3-37. Comparisons between CFD results and experimental data (front location).

Table 3-9. Comparisons between CFD results and experimental data (current speed).

\begin{tabular}{|l|c|c|}
\hline & Current Speed (m/s) & Error (\%) \\
\hline Experimental Data (Grobelbauer et al. 1993) & 1.25 & - \\
\hline Standard k-e model & 0.97 & 28.9 \\
\hline Realizable k-e model & 1.19 & 5.04 \\
\hline k-w model & 1.12 & 11.6 \\
\hline RSM model & 1.20 & 4.2 \\
\hline
\end{tabular}


In the above validation, heavy gas intrusion (air) into light gas (helium) has been taken into consideration, and the calculation results showed very good agreement with the experimental data. In the following section, the opposite case has been considered when the light gas (helium) is intruding into heavy gas (air). All the basic simulation setups are chosen to be the same as those used in the above simulation except for the initial air mass fractions. Figure 3-38 shows the initial air mass fractions assumed in the simulation. In this simulation, the left-hand side is initially filled with air while the righthand side is filled with helium. The realizable k-e model has been selected to be the reference turbulence model. The fine mesh has been used for calculations.

Figure 3-39 shows the calculated contour plots on air mass fraction for different times. The red color represents air and the blue color represents helium. Helium is smoothly intruded into the air side with almost constant speed occupying half of the channel height. It looks like the light current (helium) is showing perfect energy conserving flow satisfying Benjamin's theory. This simulation result is also consistent with the previous experimental observations by Lowe et al. (2005).

To determine current locations, the helium mass fractions on the upper plate have been plotted for different times (see Figure 3-40). The intersections between helium mass fraction curves and x-axis were chosen as the current locations.

Figure 3-41 shows the comparisons on the current locations (helium) between experiment (Grobelbauer et al. 1993) and FLUENT simulations. The calculated results showed very good agreement with the experimental data. Table 3-10 summarizes the estimated current speeds. The current speed obtained by experimental data to be $0.68 \mathrm{~m} / \mathrm{s}$ and that of simulation $0.72 \mathrm{~m} / \mathrm{s}$. This result indicates that the deviation of the simulation results is only $5.56 \%$ from the experimental data.

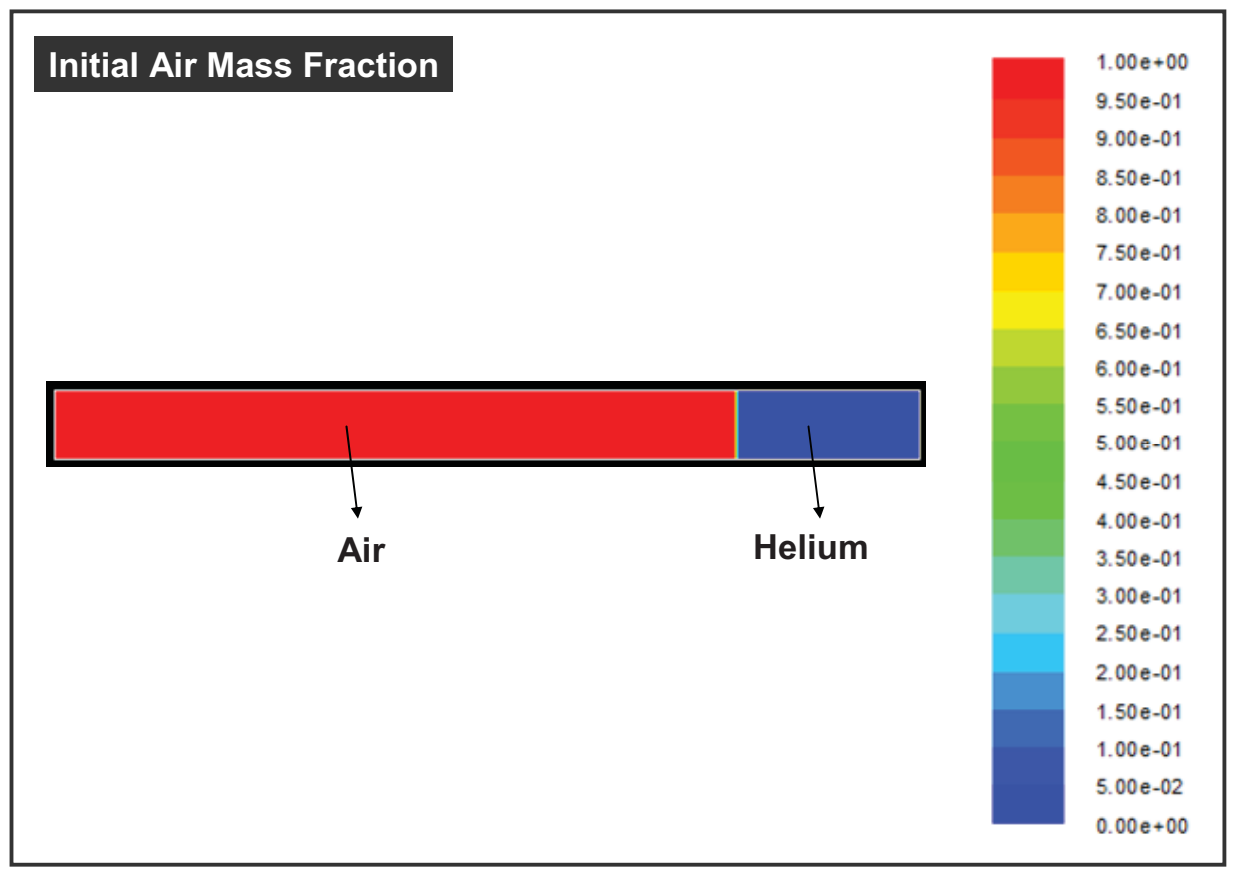

Figure 3-38. Initial air mass fraction for light gas intrusion (helium). 


\section{Sec}

\section{$0.5 \mathrm{Sec}$}

\section{$1.0 \mathrm{Sec}$}

\section{$1.5 \mathrm{Sec}$}

\section{$2.0 \mathrm{Sec}$}

\section{$2.5 \mathrm{Sec}$}

3.0 Sec

\section{$3.5 \mathrm{Sec}$}

\section{$4.0 \mathrm{Sec}$}

Figure 3-39. FLUENT simulation for light gas intrusion (air mass fraction, realizable k-e model, fine mesh).

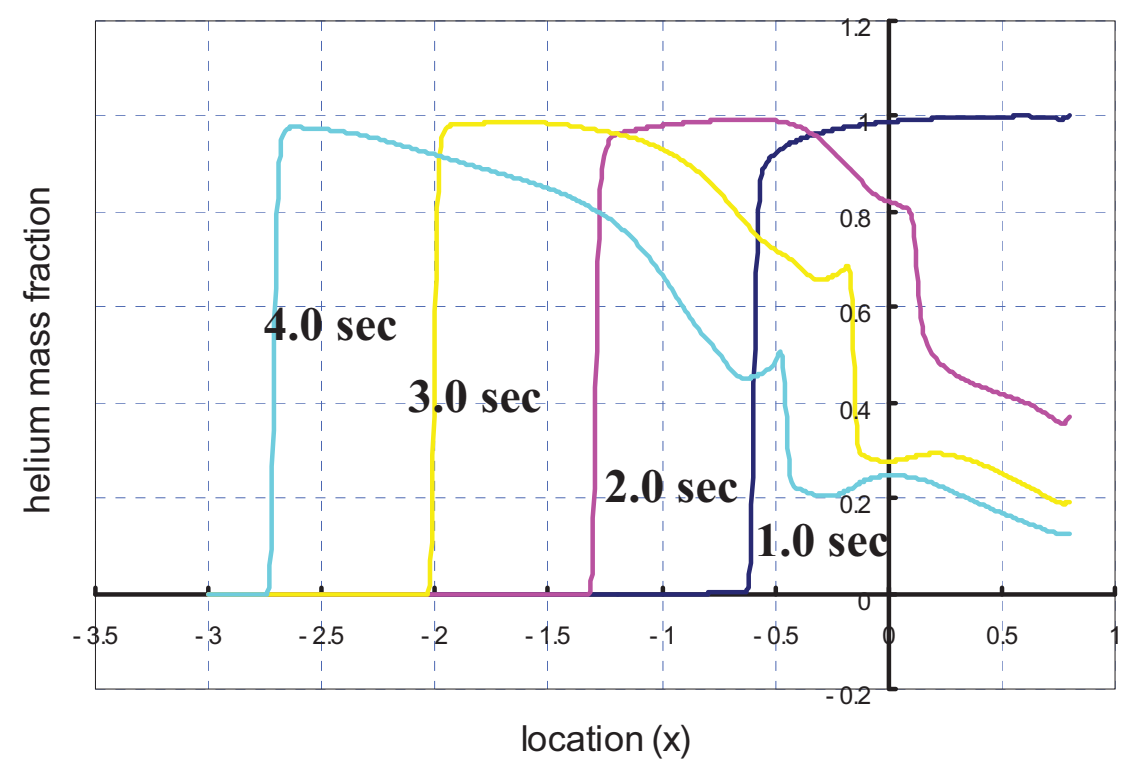

Figure 3-40. Helium mass fraction at the upper plane for light gas intrusion. 


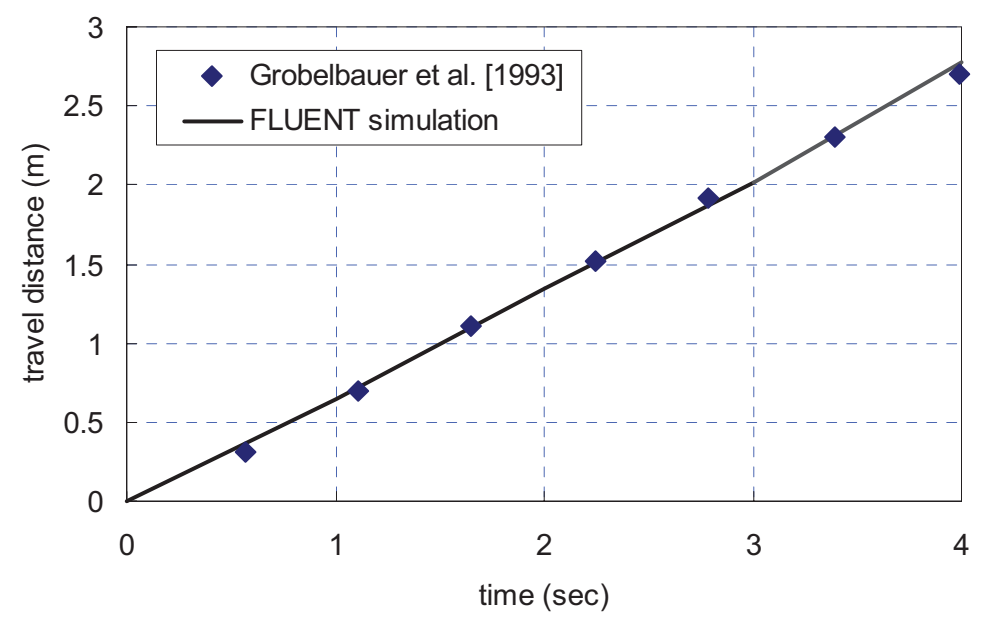

Figure 3-41. Comparisons between CFD results and experimental data (light gas intrusion).

Table 3-10. Comparisons between CFD results and experimental data (light gas intrusion).

\begin{tabular}{|l|c|c|}
\hline & Current Speed (m/s) & Error (\%) \\
\hline Experimental Data (Grobelbauer et al. 1992) & $0.68 \mathrm{~m} / \mathrm{s}$ & - \\
\hline FLUENT (Realizable k-e model) & $0.72 \mathrm{~m} / \mathrm{s}$ & $5.56 \%$ \\
\hline
\end{tabular}




\section{TASK 3: ADVANCED GRAPHITE OXIDATION STUDY}

\subsection{Introduction}

Graphite oxidation in an air-ingress accident is an important issue for VHTR safety because of its potential problems such as core heat-up, graphite strength degradation, and release of some toxic gases such as $\mathrm{CO}$ and $\mathrm{CO}_{2}$. The oxidation process of graphite is affected by various factors, including temperature, pressure, oxygen concentration, types of graphite, graphite shape and size, flow distribution, etc. The effects of these factors have been documented by a number of previous investigations (Kim and NO 2006, Fuller et al. 1997, Moorman 1984, Ogawa 1993, Contescu 2008, etc.), and good models have been developed for estimating the graphite oxidation process in an air-ingress accident.

One of the main issues regarding graphite oxidation is the mechanical degradation of the graphite. For analyzing this problem, it is important to understand the relationship between the oxidation degree and the strength degradation. In addition, the change of oxidation rate by graphite oxidation degree (burn-off: ratio of the oxidized graphite density to the original density) should be quantified because graphite strength degradation is followed by graphite density decrease, which highly affects oxidation rates and patterns. Since the density change is proportional to the internal pore surface area, these parameters should be quantified in advance. Regarding those issues, the following tasks were performed:

1. Experiment on the fracture of oxidized graphite and validate the previous correlations.

2. Experiment on the change of oxidation rate using graphite density and data collection.

3. Measure the BET surface area of the graphite.

The data for Task 3 was measured and provided by Contescu (2008) in Oak Ridge National Laboratory (ORNL) so that data given in this report is not based on the INL experiment. Tasks 1 and 2 were performed at INL for H451 (Great Lakes Carbon Corporation), IG-110 (Toyo Tanso Co., Ltd), and NBG-18 (SGL Group) graphite. The reason for the use of those graphite materials is because their chemical and mechanical characteristics are well identified by the previous investigations, and therefore it was convenient for us to access the published data, and to apply and validate our new methodologies.

\subsection{Experiment on the Graphite Oxidation}

\subsubsection{Introduction}

As mentioned, the effect of oxidation on the strength of graphite is an important issue for safety analysis of the air-ingress accident in VHTRs because strength degradation by oxidation leads to potential core collapse problems. Although the relationship between strength and oxidation has been studied by a number of various investigators (Eto and Growcock 1981, Wichner 1976, Engle 1977, etc.), in most cases, the majority of the data has been obtained only under $10 \%$ graphite burn-off. This means that the correlations are only valid within very limited burn-off ranges and the use of those correlations for the higher burn-off may not be valid yet. Actually, the potential graphite structure collapse will occur at high burn-off range (higher than 50\%). It is therefore very important to confirm the validity of the previous correlations for use at the wider ranges. This experiment attempted to obtain the graphite strength data at high burn-off ranges. To achieve this goal, an experimental method and procedure were newly developed. The degree of burn-off in which the graphite loses its mechanical strength has been measured and was finally used for validation and improvement of the previous correlations.

\subsubsection{Background}

The literature survey revealed that it was technically very difficult to apply the standard methods of the mechanical strength measurement to highly oxidized graphite. Consequently, most of the data were 
obtained under 10\% burn-off in the previous investigations (Eto and Growcock 1981, Wichner 1976, Engle 1977, etc.). Figure 4-1 shows some experimental data for IG-11 graphite measured by Eto and Growcock (1983) wherein all the data ranges within 7\% burn-off. Eto and Growcock (1983) measured the strength of the three types of oxidized graphite (IG-11, PGX and H451), and recommended the following equation form.

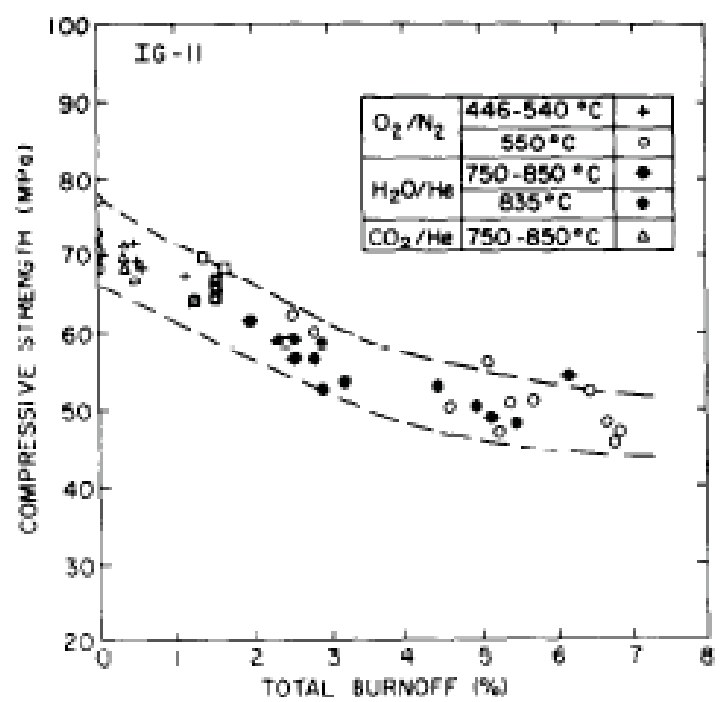

Figure 4-1. Experimental data of compressive strength with burn-off (Eto and Growcock 1983).

$S / S_{0}=\alpha \cdot \rho^{\prime \beta}+(1-\alpha) \cdot \rho^{\prime \delta}$

where

$$
\begin{aligned}
& S \quad=\text { Compressive Strength }(\mathrm{Pa}) \\
& S_{0} \quad=\text { Initial compressive strength }(\mathrm{Pa}) \\
& \rho^{\prime} \quad=\text { Ratio of the density between oxidized graphite and the original. }
\end{aligned}
$$

In their research, the graphite was oxidized up to a certain level of burn-off and then the sample was tested by a standard method and testing machine. Table 4-1 summarizes the parameters in Equation (4-1) for graphite PGX, H451, and IG-11.

Table 4-1. Parameters in Equation (4-1) for various graphite materials (Eto and Growcock 1983).

\begin{tabular}{|l|l|c|c|c|}
\hline \multicolumn{1}{|c|}{ Graphite } & \multicolumn{1}{c|}{ Mode } & $\boldsymbol{\alpha}$ & $\boldsymbol{\beta}$ & $\boldsymbol{\gamma}$ \\
\hline PGX & Compression & 0.94 & 9.4 & 194 \\
\hline PGX & Tension & 0.86 & 10 & 100 \\
\hline H451 & Compression & 0.79 & 3.5 & 71.5 \\
\hline H451 & Tension & 0.62 & 3.5 & 30 \\
\hline IG-11 & Compression & 0.83 & 4.0 & 40 \\
\hline
\end{tabular}

This equation assumes that the graphite's strength will approach zero as the density decreases and finally become zero at $100 \%$ burn-off. This assumption is conceptually reasonable, but some experimental observations show that the graphite losses its mechanical strength at a certain burn-off level 
lower than $100 \%$ because the corrosion breaks all the connections between inside pore structures in very high burn-off.

Because of the technical difficulties associated with the direct measurement of highly oxidized graphite strength, a new method is proposed. Figure 4-2 shows the basic idea of this method, which measures the degree of burn-off at which the graphite loses its strength by a very small load. The load is very small compared to the original material strength so that the stress can be assumed to be almost zero. Figure 4-2 depicts this data point as a red circle.

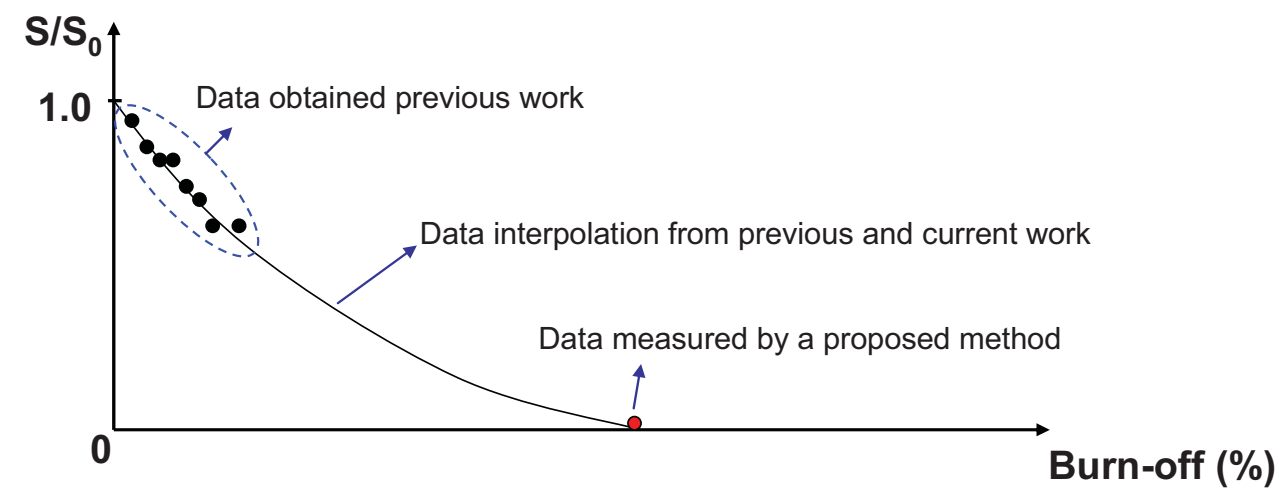

Figure 4-2. Basic idea on the prediction of graphite compressive strength.

The method used to measure this data is simple as described in detail in the next section. Simply putting the graphite with a small load on its top is oxidized at constant temperature and flow rate conditions. If it is weaken by the load, the weakened burn-off will be detected through a sudden drop in the graphite mass signal. Once the data is obtained, the strength can be interpolated between the previous and the new data points. The smooth change of the graphite mechanical strength was assumed in the interpolated region.

\subsubsection{Experimental Facility and Setup}

The experiments have been carried out on the graphite oxidation test station at INL, which is built for evaluation of the American Society for Testing and Materials (ASTM) standard test method (Contescu 2008) for graphite. The ASTM test standard was developed for determining and rating the oxidation resistance of nuclear grade graphite. A protocol containing instructions for setting up a test station was developed by ORNL and distributed to several laboratories for independent evaluation of the protocol's robustness in terms of repeatability. Basically the same experimental setup as the ASTM protocol was maintained for this work, but it was slightly adjusted for this purpose.

The schematic of the oxidation test station included as Figure 4-3 shows the graphite sample suspended below a balance inside the Inconel tube, which is surrounded by the furnace. The Inconel tube is connected to nitrogen and air supplied from the bottom and desiccated to eliminate oxidation from moisture. The nitrogen is used during this process to avoid oxidation when the furnace is heating up. When the gas temperature is stabilized at target value it is switched to air to start oxidation. The test can take from a couple of hours to a few days, so the data is gathered automatically using LabVIEW (National Instruments) until the desired burn-off is achieved as shown in Figure 4-4. 


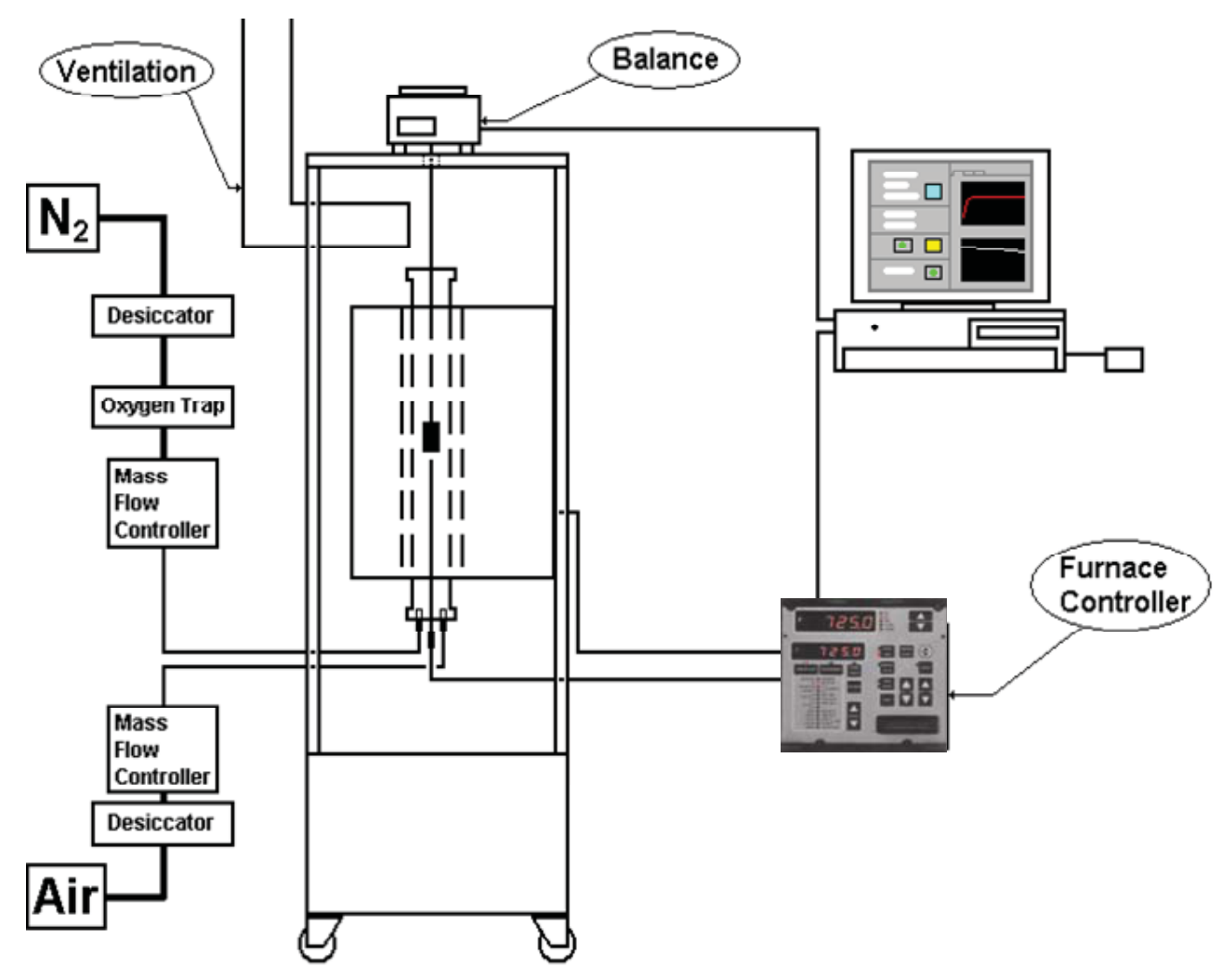

Figure 4-3. Schematic of the graphite oxidation test station setup.

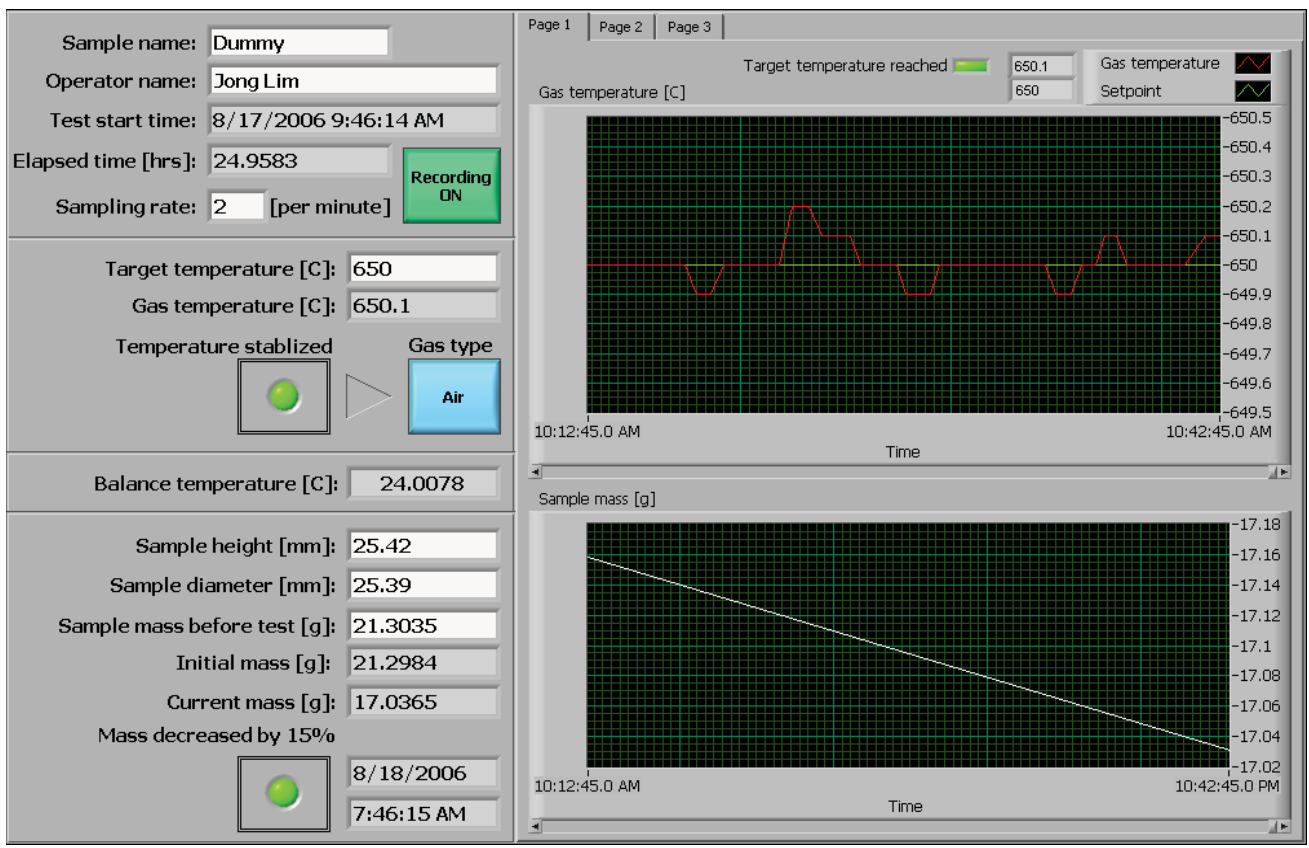

Figure 4-4. LabVIEW program user interface.

The LabVIEW program retrieves the sample mass and temperature at a constant sampling rate and records it in a data file with useful introductory information as shown in Figure 4-5. File names are automatically generated to include the sample name, target temperature, test start date, and time. 


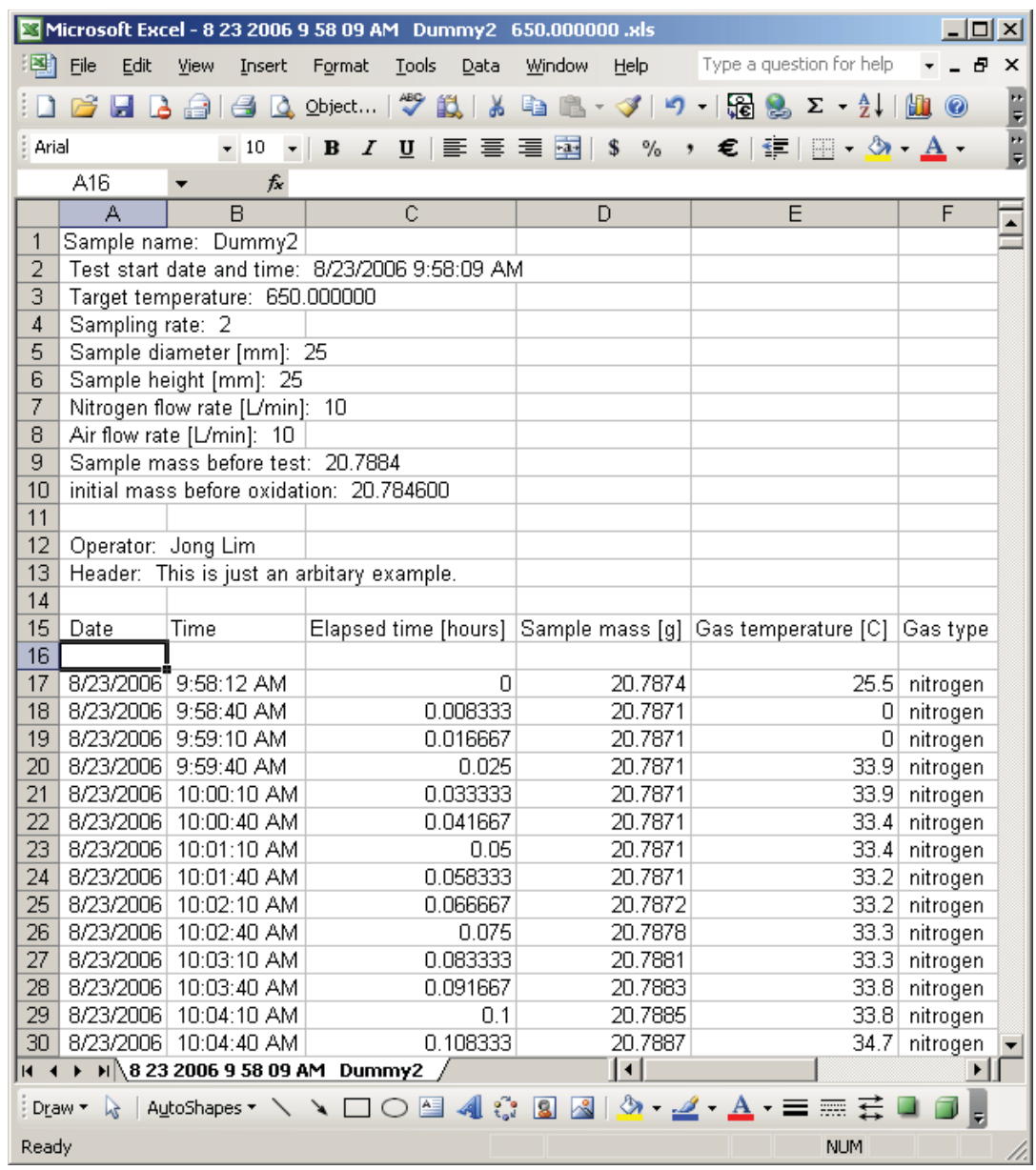

Figure 4-5. Data file example.

Figure 4-6 shows the schematic of the sample holder that was set up for the experiment. It was installed at the center of the electrical furnace. The sample holder is made of titanium and has a rectangular cage shape with a loading material and graphite sample inside. A loading material is made of titanium and initially placed on the top of the graphite sample.

The graphite sample is a cylinder with a hole at the center. Its dimensions are 1.0 inch outer diameter, 1.0 inch height, and 0.5 inch inner-hole diameter. The loading material has the cylindrical shape with a small tip at the bottom center to fit the loader to the sample. The size of the tip is made to be a little bit smaller than the hole to avoid thermal expansion problems. The experiment is performed at the low temperature $\left(650^{\circ} \mathrm{C}\right)$ during which the reaction kinetics dominate the graphite corrosion process. In this regime, the graphite corrosion mainly decreases the graphite density with degradation of mechanical strength, maintaining its original shape and size. If
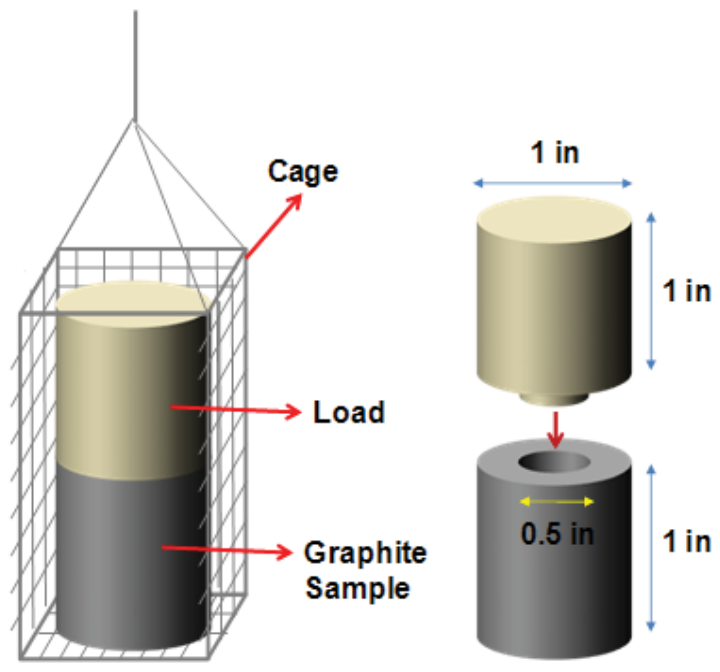

Figure 4-6. Schematic of the sample holder and loader setup. 
the graphite is fully corroded, the sample will lose its strength by the loading materials. The broken ashes will then fall down through the metal mesh at the bottom of the cage, sending a sudden decrease signal of graphite mass as detected by the balance connected to the cage and the sample.

\subsubsection{Results and Discussions}

\subsubsection{Graphite Oxidation Degree vs. Graphite Mechanical Strength}

Three samples were tested. Figures 4-7, 4-8, and 4-9 show the sample mass change with time measured for IG-110 graphite. The graph in these figures shows (a) the overall trend of the sample mass changes for the whole test time and (b) the sudden mass changes in detail at the sample fractured time. These figures show that this experiment provides very good repeatability. The sudden mass drops were observed at almost the same time, regardless of the different samples. The average burn-off for the fracture in IG-110 is $64.7 \%$. 


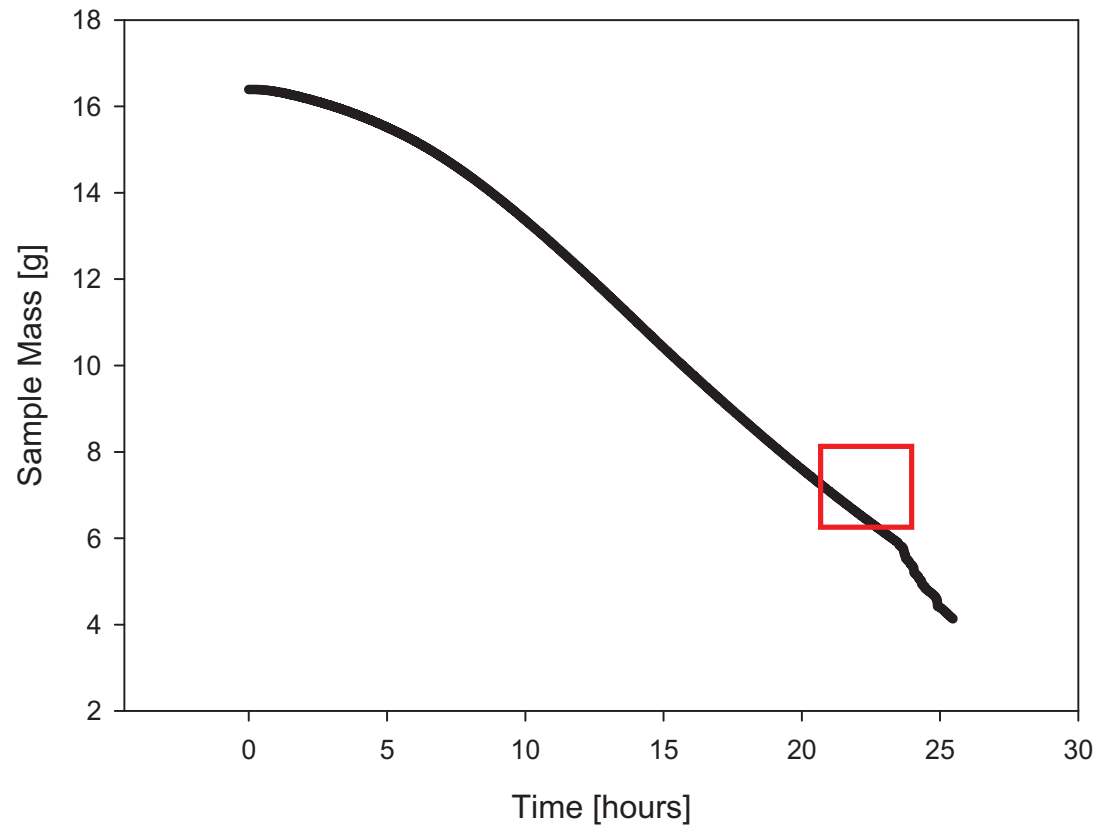

(a) Overall trend of the sample mass change.

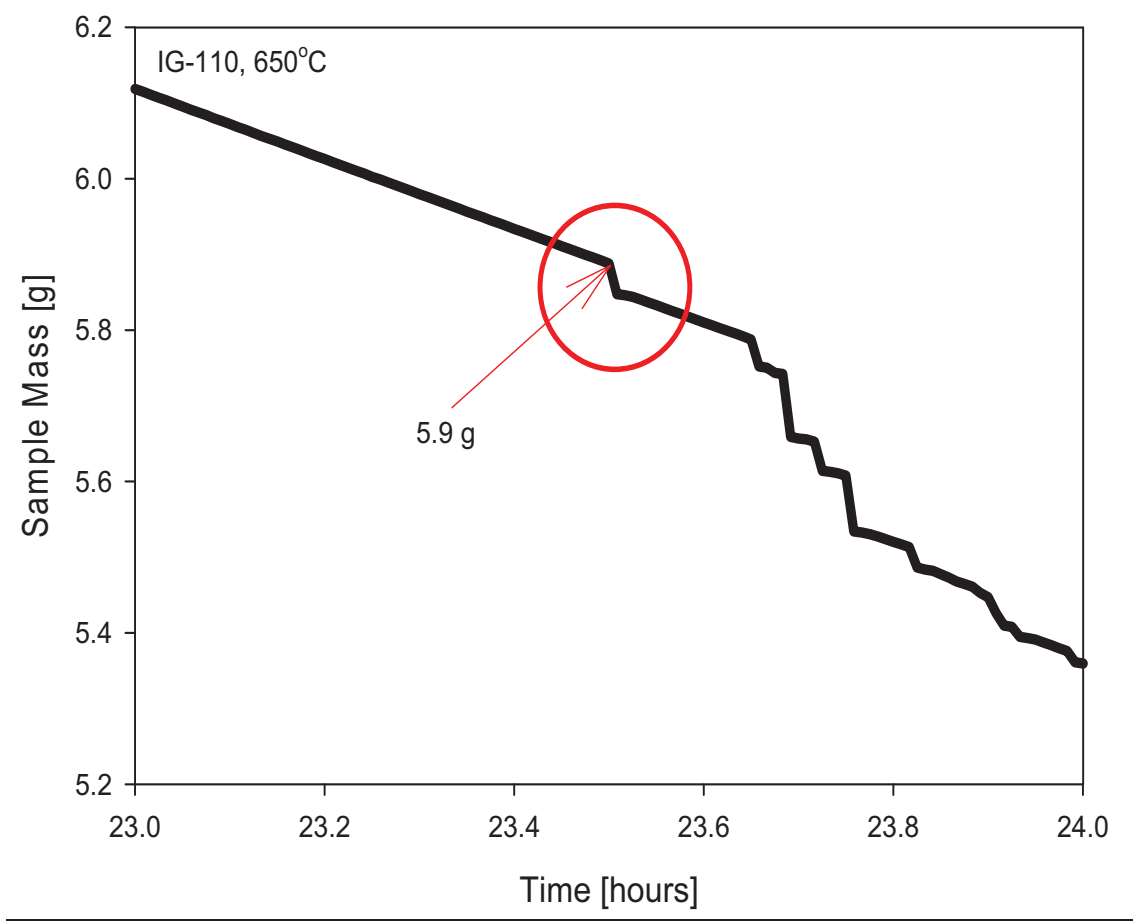

(b) Sudden drop of mass at the broken point.

Figure 4-7. Variations of sample mass with time (IG-110 Case $\left.1,650^{\circ} \mathrm{C}\right)$. 


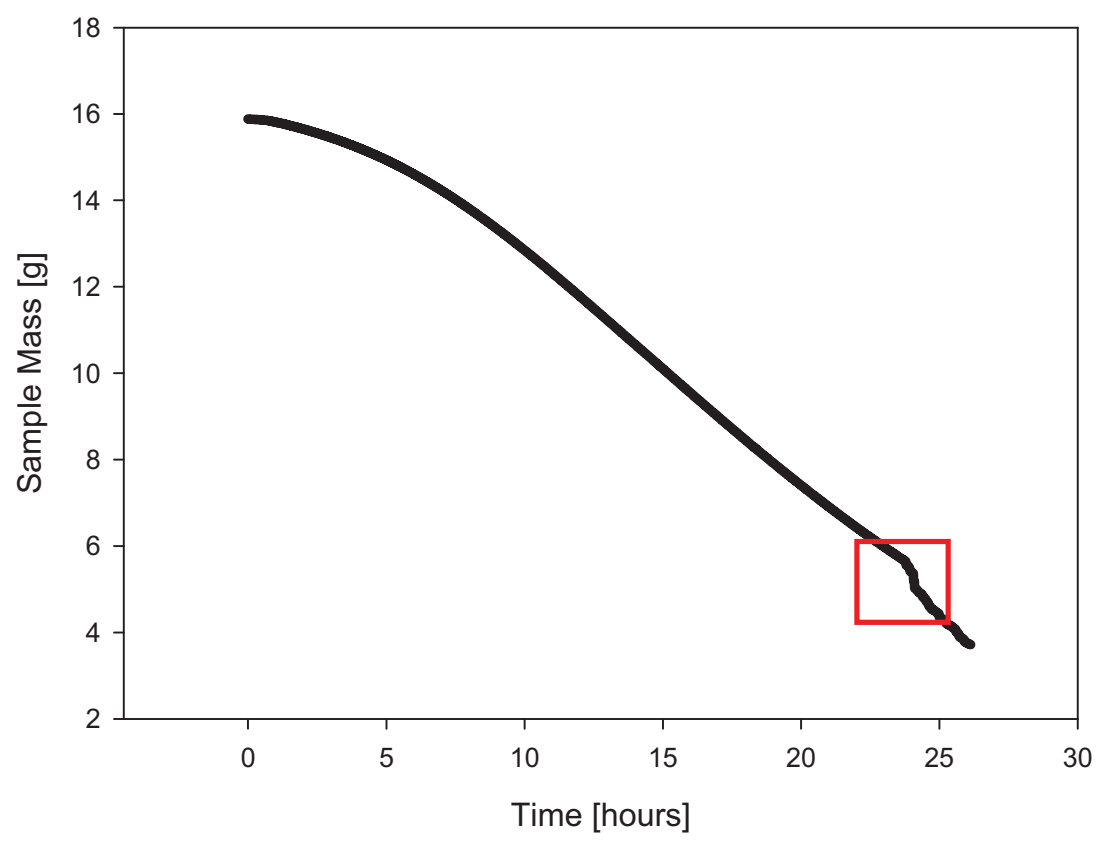

(a) Overall trend of the sample mass change.

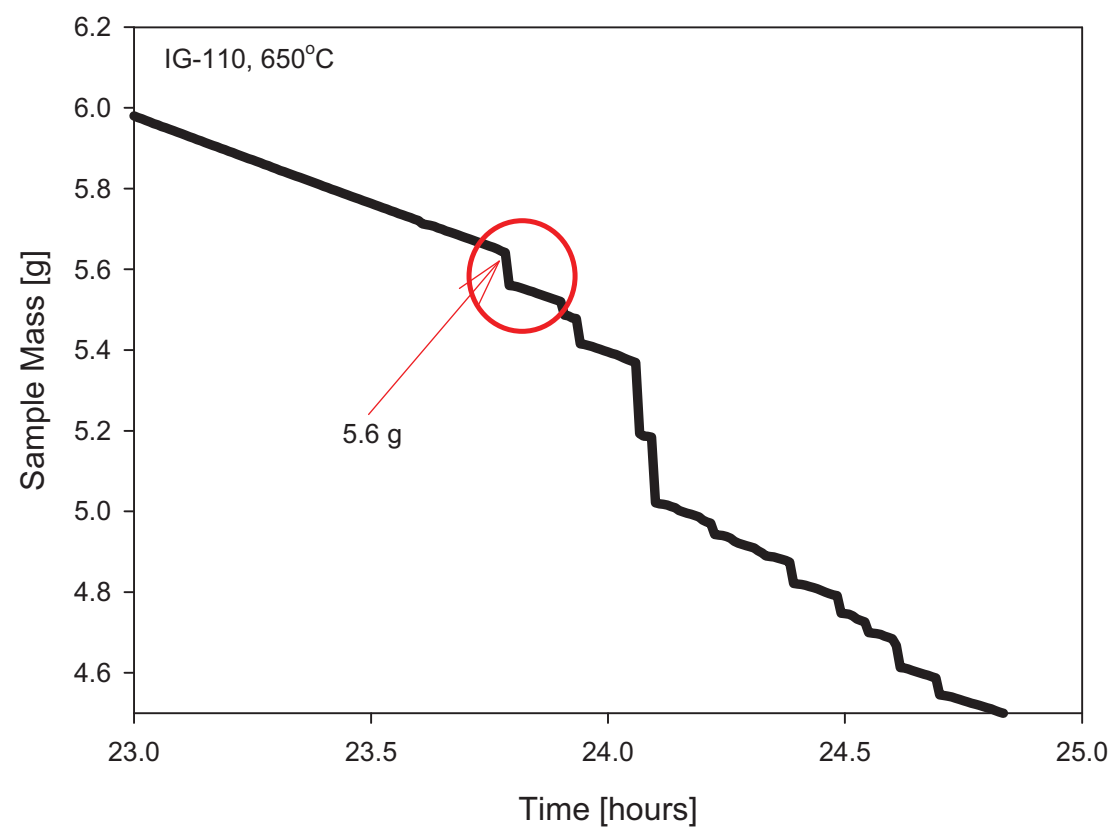

(b) Sudden drop of mass at the broken point.

Figure 4-8. Variations of sample mass with time (IG-110 Case $2,650^{\circ} \mathrm{C}$ ). 


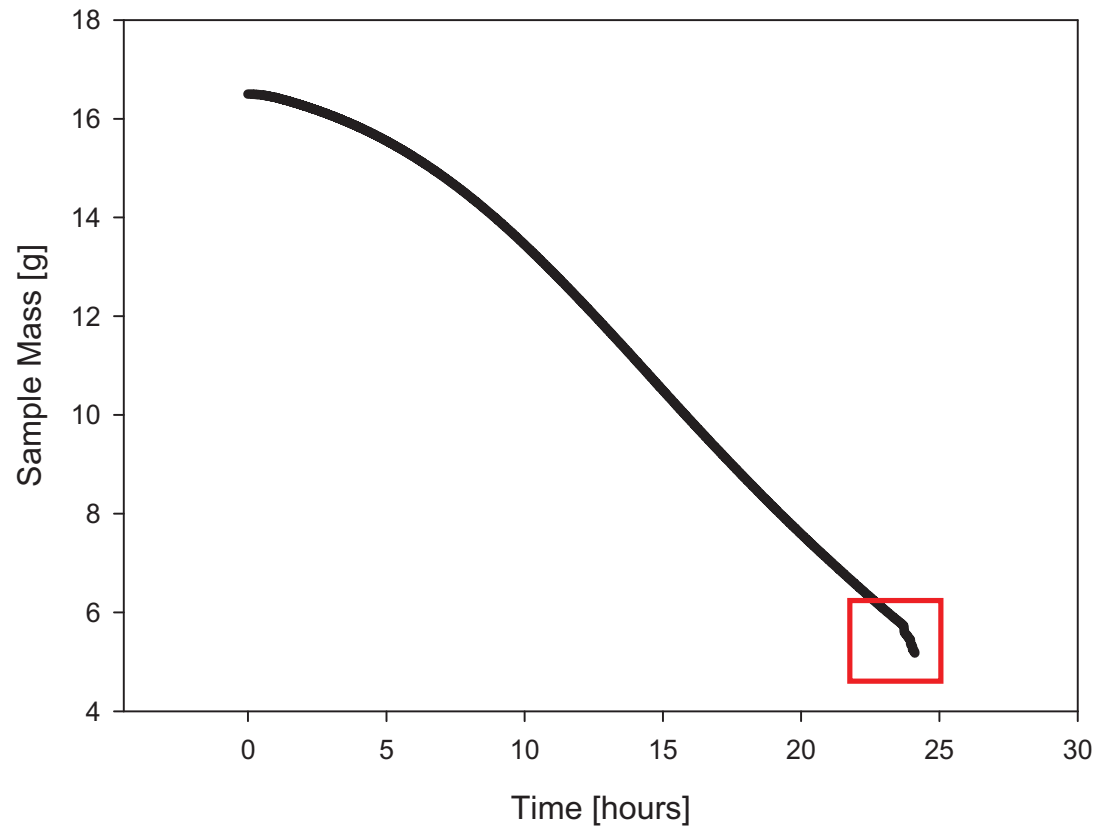

(a) Overall trend of the sample mass change.

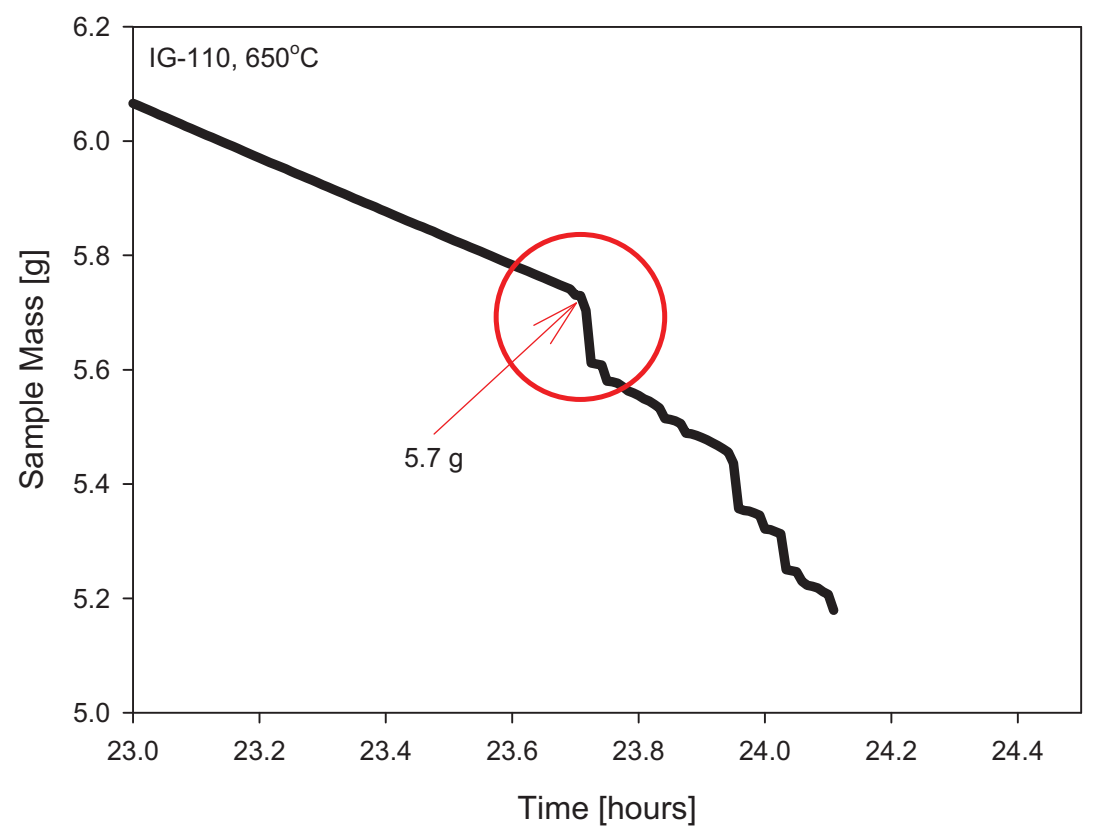

(b) Sudden drop of mass at the broken point.

Figure 4-9. Variations of sample mass with time (IG-110 Case $3,650^{\circ} \mathrm{C}$ ). 


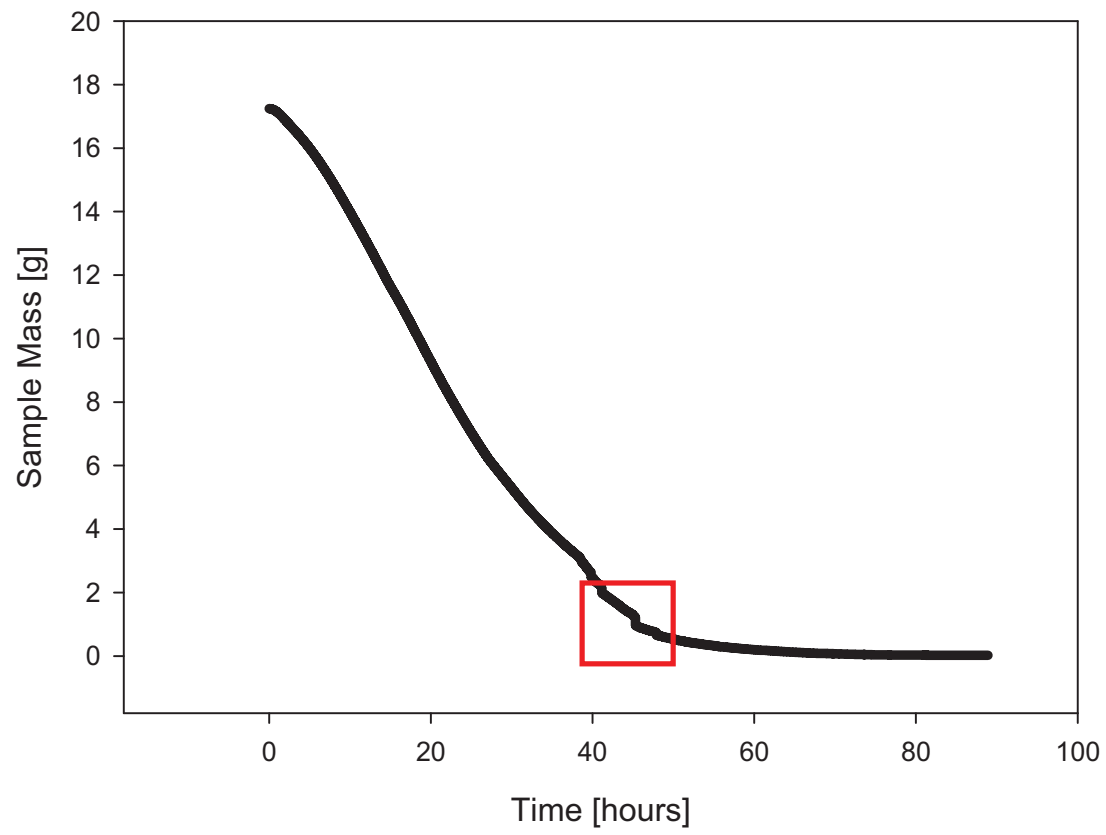

(a) Overall trend of the sample mass change.

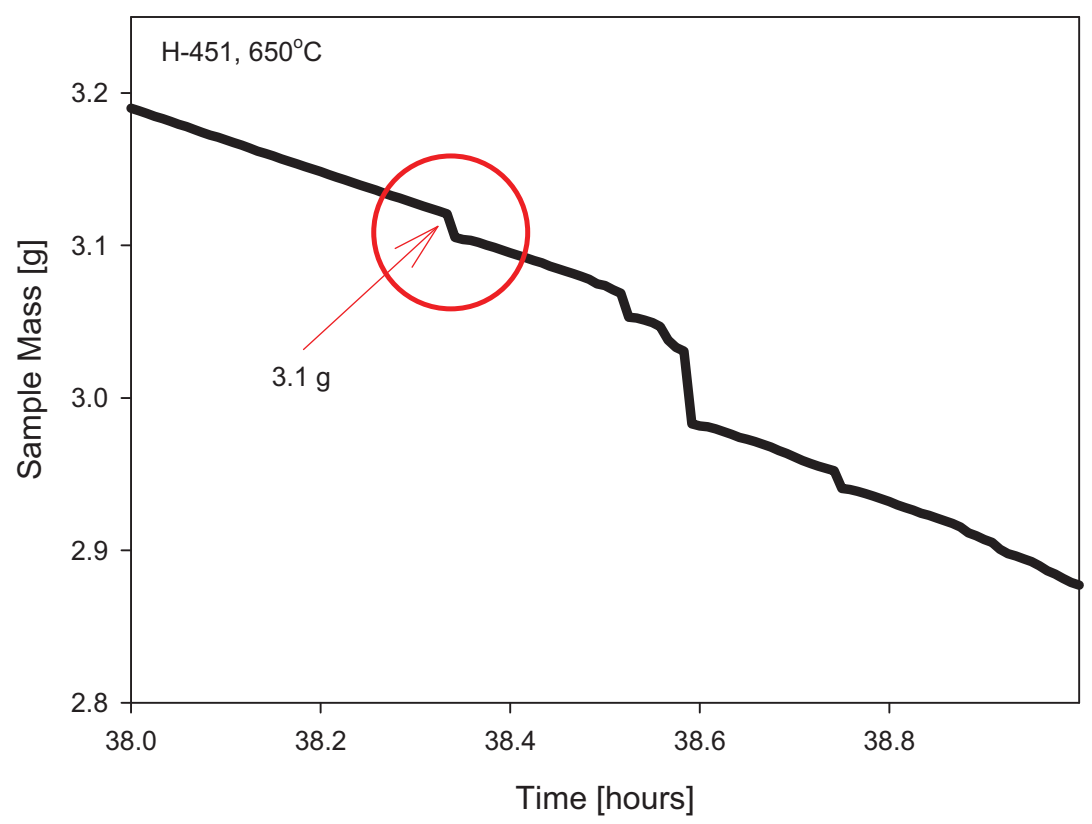

(b) Sudden drop of mass at the broken point.

Figure 4-10. Variations of sample mass with time (H451 Case $\left.1,650^{\circ} \mathrm{C}\right)$. 


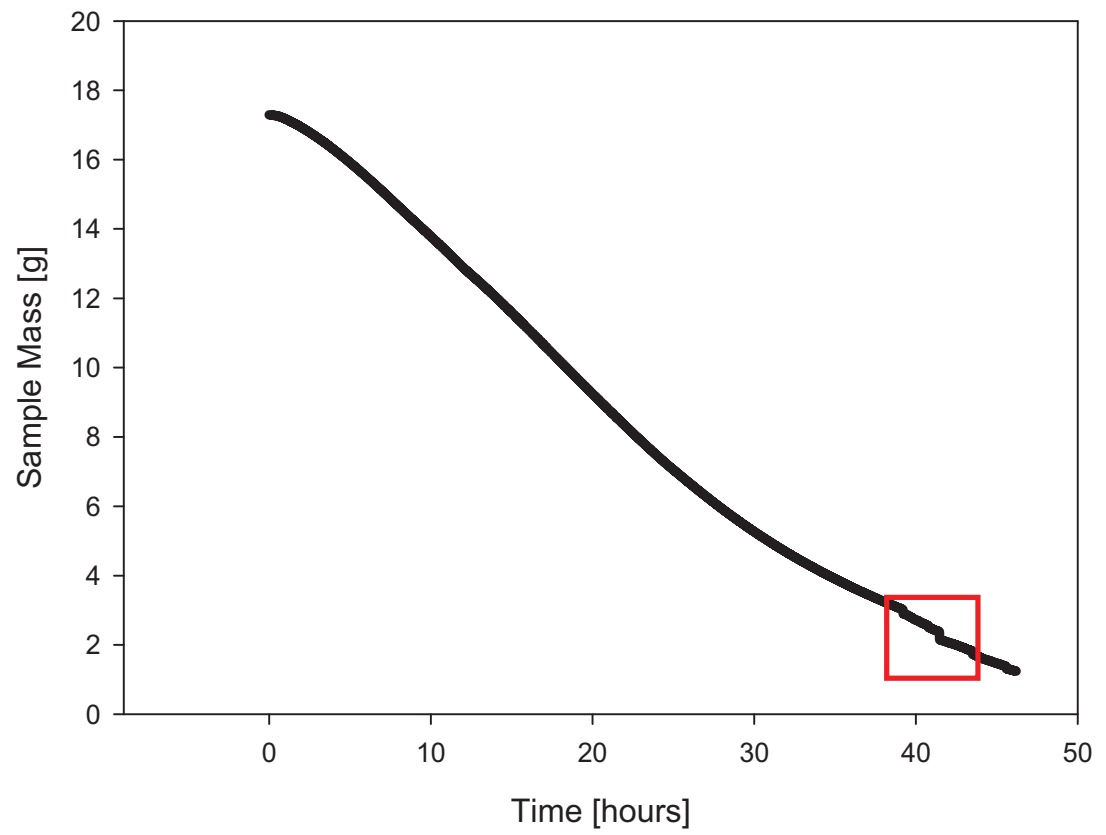

(a) Overall trend of the sample mass change.

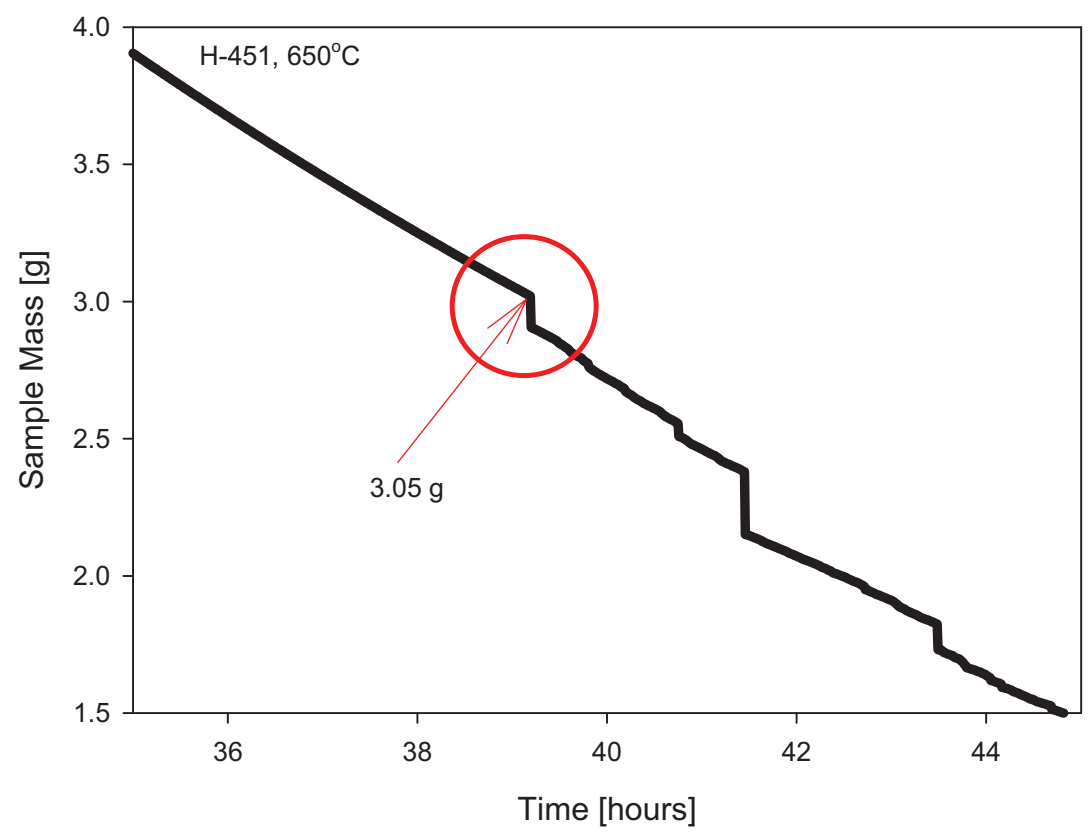

(b) Sudden drop of mass at the broken point.

Figure 4-11. Variations of sample mass with time ( $\mathrm{H} 451$ Case $2,650^{\circ} \mathrm{C}$ ). 


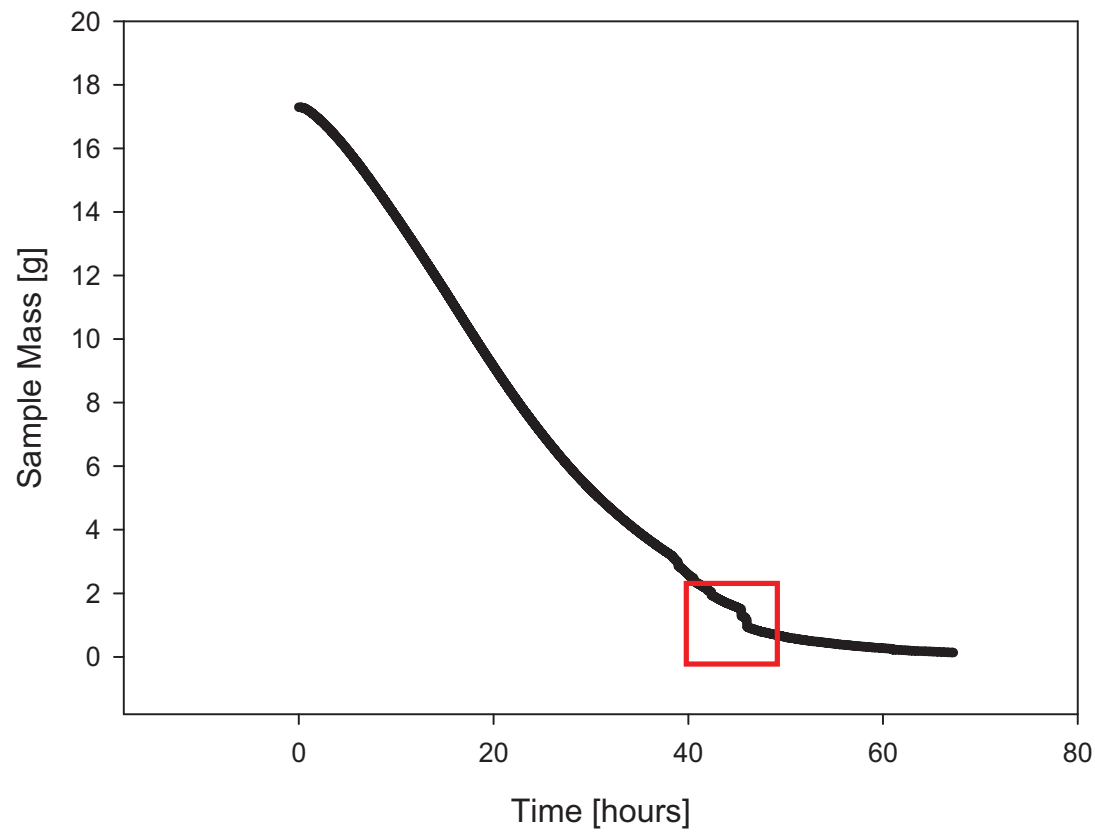

(a) Overall trend of the sample mass change.

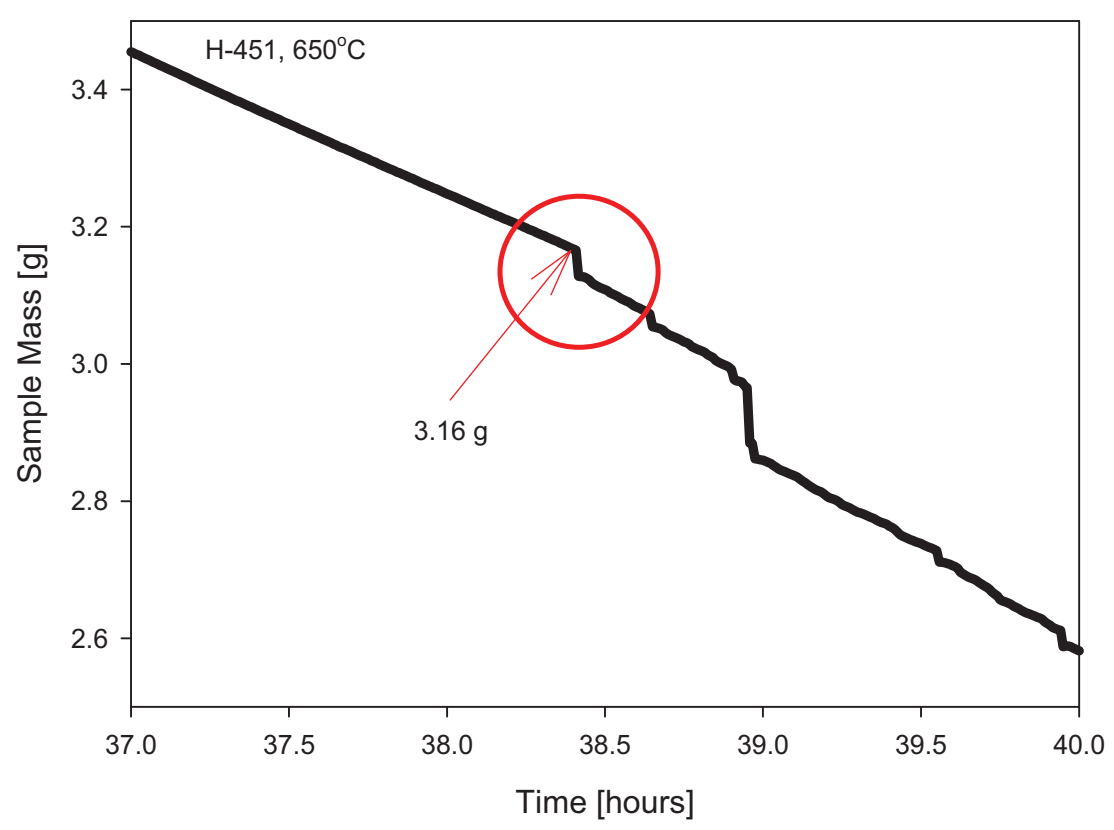

(b) Sudden drop of mass at the broken point.

Figure 4-12. Variations of sample mass with time ( $\mathrm{H} 451$ Case $\left.3,650^{\circ} \mathrm{C}\right)$. 


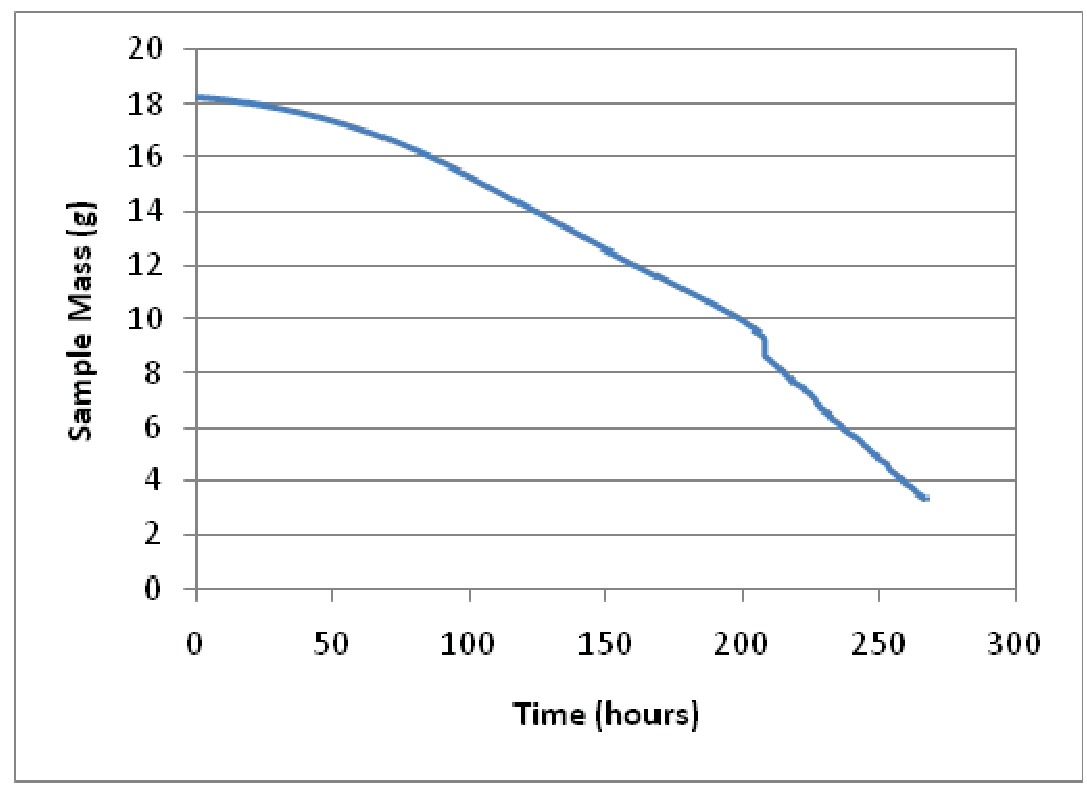

(a) Overall trend of the sample mass change.

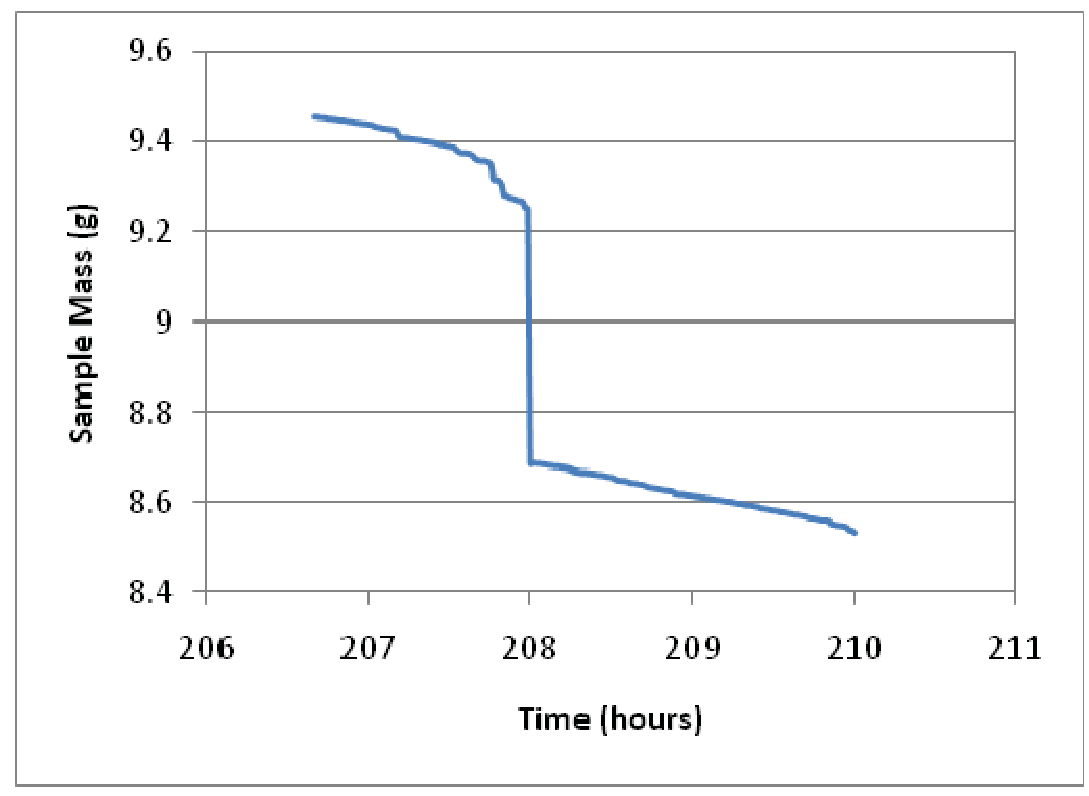

(b) Sudden drop of mass at the broken point.

Figure 4-13. Variations of sample mass with time (NBG-18, $650^{\circ} \mathrm{C}$ ).

Table 4-2. Summary of the experimental data.

\begin{tabular}{|l|l|l|l|l|l|l|l|l|}
\hline \multicolumn{3}{|c|}{ IG-110 } & \multicolumn{3}{c|}{ H451 } & \multicolumn{3}{c|}{ NBG-18 } \\
\hline $\begin{array}{c}\text { Initial } \\
\text { Mass [g] }\end{array}$ & $\begin{array}{c}\text { Fractured } \\
\text { Mass [g] }\end{array}$ & $\begin{array}{c}\text { Fractured } \\
\text { Burn-off }\end{array}$ & $\begin{array}{c}\text { Initial } \\
\text { Mass [g] }\end{array}$ & $\begin{array}{c}\text { Fractured } \\
\text { Mass [g] }\end{array}$ & $\begin{array}{c}\text { Fractured } \\
\text { Burn-off }\end{array}$ & $\begin{array}{c}\text { Initial } \\
\text { Mass [g] }\end{array}$ & $\begin{array}{c}\text { Fractured } \\
\text { Mass [g] }\end{array}$ & $\begin{array}{c}\text { Fractured } \\
\text { Burn-off }\end{array}$ \\
\hline 16.40 & 5.9 & 64.02 & 17.24 & 3.1 & 82.01 & 18.28 & 9.25 & 49.42 \\
\hline 15.88 & 5.6 & 64.74 & 17.29 & 3.05 & 82.36 & & & \\
\hline 16.50 & 5.7 & 65.45 & 17.3 & 3.16 & 81.73 & & & \\
\hline
\end{tabular}


Figure 4-14 depicts the plot of normal compressive strength versus burn-off for IG-110 graphite. The graph includes the data from Ishihara et al. (2004) and this work. Two correlations are plotted in the figure: one is Eto and Growcock's (1983) correlation for IG-11 graphite (See Table 4-1), the other is the correlation fitted by all the data including this work. Eto and Growcock's correlation shows quite good agreement with the experimental data for the whole range but a small over-prediction of the strength at high burn-off. According to Eto and Growcock's equation, the strength of the graphite at the $65 \%$ burnoff is predicted to be $870,000 \mathrm{~Pa}$, while the stress load at the bottom part of the VHTR graphite structure is $100,000 \mathrm{~Pa}$. The equation will thus estimate that the graphite structure is still secure for fracture, even though in the real situation, the support graphite loses its integrity at this point. Eto and Growcock's equation predicts that the fracture point of the graphite support structure will be $79.6 \%$ burn-off, which is $14.6 \%$ larger than the point at which the IG-110 graphite loses its mechanical strength. In the new conservative fitting, the following correlation shows very good agreement with the whole experimental data, and is recommended for the final IG-110 graphite:

$$
S / S_{0}=\left(\rho / \rho_{0}\right)^{6.5} \text {. }
$$

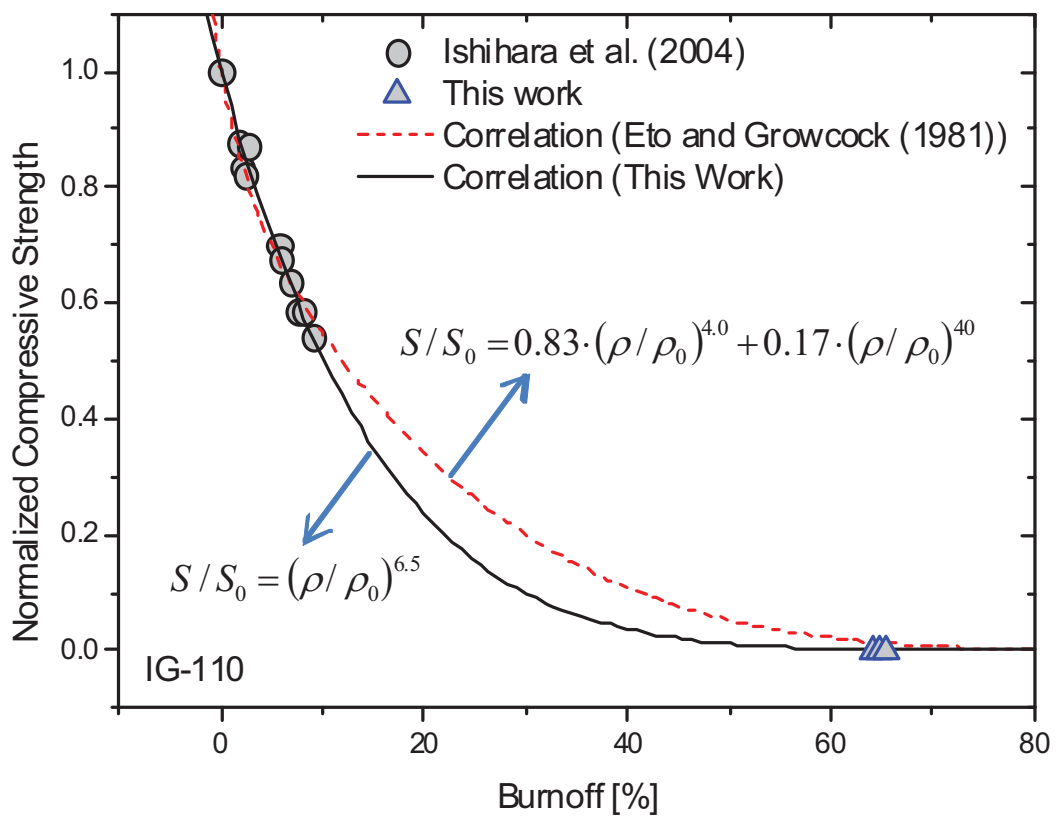

Figure 4-14. Normal compressive strength vs. burn-off (IG-110).

Figure 4-15 presents experimental data about the relationship between normal compressive strength and burn-off for H451 graphite. It also plots two correlations: one is developed by Eto and Growcock (1983) and the other is developed here by fitting all of the data, including this work. Accordingly, both correlations show very good agreement with the experimental data for the whole range. In particular, Eto and Growcock's correlation shows very good agreement at the low burn-off region, while the following correlation provides more conservative predictions:

$$
S / S_{0}=\left(\rho / \rho_{0}\right)^{6.25} \text {. }
$$




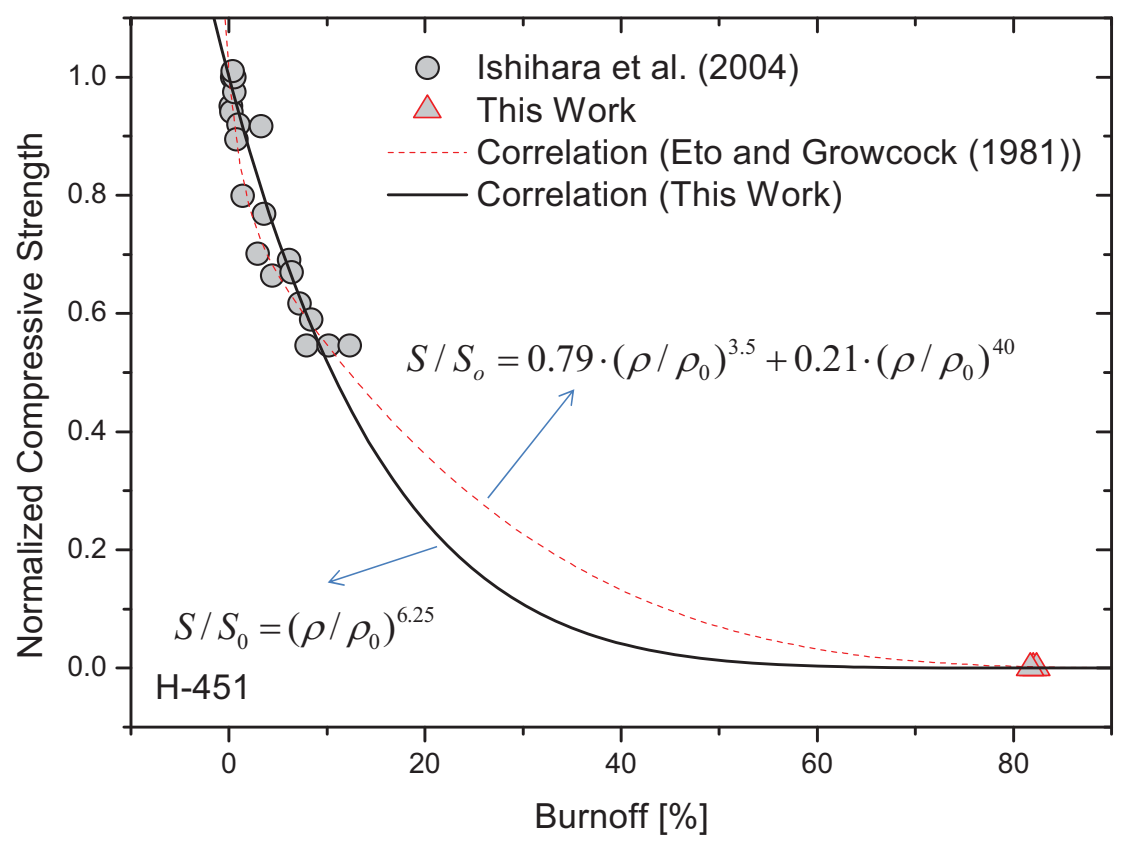

Figure 4-15. Normal compressive strength vs. burn-off (H451).

\subsubsection{Graphite Surface Area Density}

Graphite has lots of inside pores. The surface area of those pores is very important for predicting oxidation. This section evaluates the surface area densities for various types of graphite based on the BET-specific surface area data provided by Contescu (2008) at ORNL and other literature.

The BET method is used to measure specific surface area based on the BET theory, which is well known for the physical adsorption of gas molecules on a solid surface. This method is based on the following assumptions: (1) homogeneous surface equals energy surface, (2) only the uppermost molecules of a multilayered adsorbate are in dynamic equilibrium with the vapor, (3) the heat of adsorption of the second and higher layers equal the heat of condensation, (4) a molecule covered by another molecule cannot evaporate, (5) at saturation the number of layers becomes infinite, and (6) there is no lateral interaction between adsorbed molecules. Adsorption gases are generally nitrogen at low temperature $(77 \mathrm{~K})$, krypton at low temperature, water vapor, hydrocarbons of various types, and inert gases. For more information about this method refer to Bruauer et al. (1938) and Fagerlund (1973).

Figure 4-16 shows the plot of the BET surface area for various graphite materials (NBG18, NBG10, and PCEA) measured by Contescu (2008). Table 4-3 summarizes the collected data for the surface area density for graphite materials. 


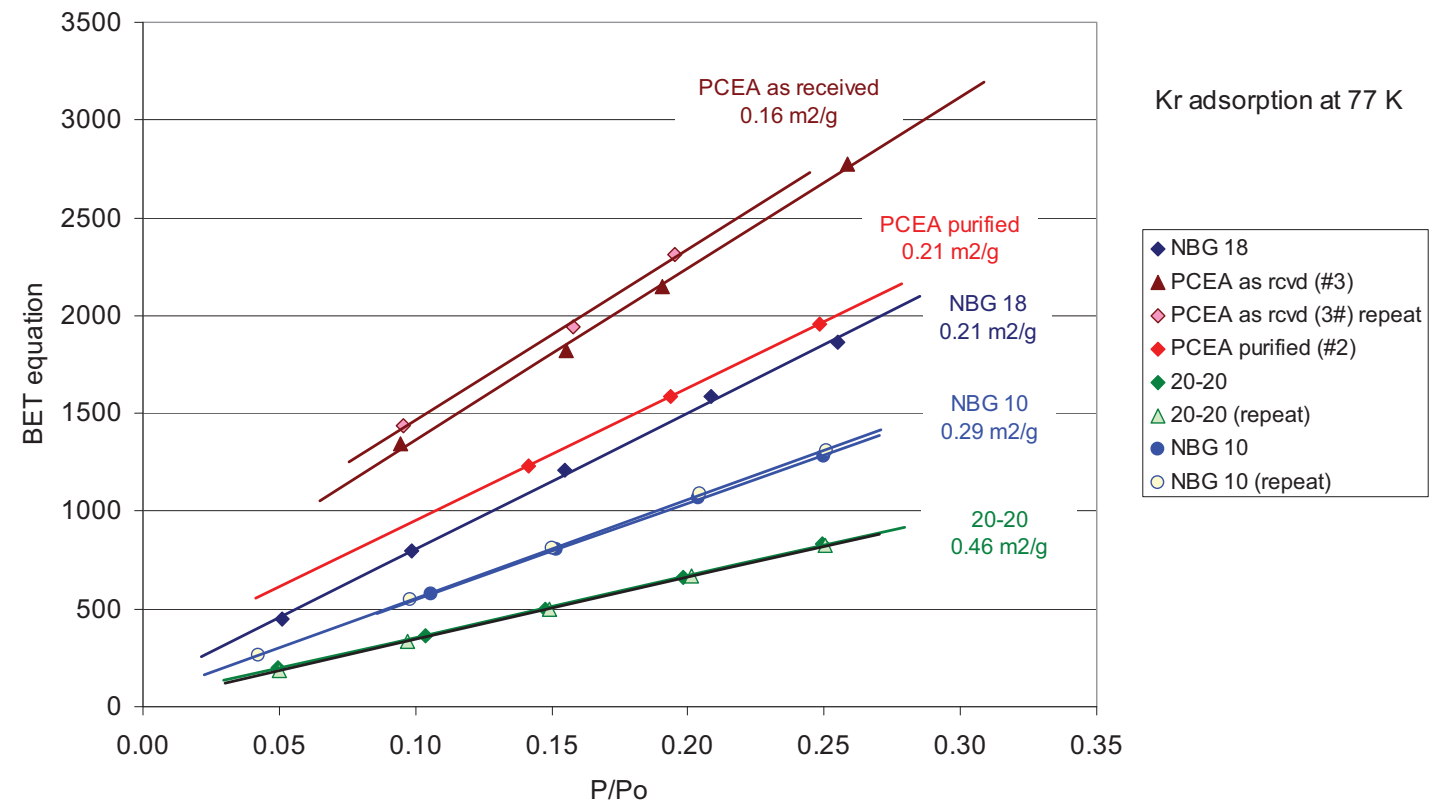

Figure 4-16. Measurement of BET surface area by Contescu (2008).

Table 4-3. Summary of surface area density for graphite.

\begin{tabular}{|l|c|c|c|}
\hline & $\begin{array}{c}\text { Density } \\
{\left[\mathbf{g} / \mathbf{m}^{\mathbf{3}}\right]}\end{array}$ & $\begin{array}{c}\text { Specific Surface Area } \\
{\left[\mathbf{m}^{\mathbf{2}} \mathbf{g}\right]}\end{array}$ & $\begin{array}{c}\text { Surface Area Density } \\
{\left[\mathbf{m}^{\mathbf{2}} / \mathbf{m}^{\mathbf{3}}\right]}\end{array}$ \\
\hline NBG-18 (Contescu 2008) & 1790 & 0.21 & 375.9 \\
\hline NGB-10 (Contescu 2008) & 1790 & 0.29 & 519.1 \\
\hline PCEA (Contescu 2008) & 1790 & 0.21 & 375.9 \\
\hline 20-20 (Contescu 2008) & 1790 & 0.46 & 823.4 \\
\hline IG-11 (Eto and Growcock 1981) & 1750 & 2.8 & 8900 \\
\hline IG-110 (Nakano et al. 1997) & 1780 & 0.5 & 1320 \\
\hline H451 (Pawelko et al. 2001) & 1760 & 0.75 & 1211 \\
\hline PGX (Eto and Growcock 1981) & 1730 & 0.7 & \\
\hline
\end{tabular}

\subsubsection{Graphite Oxidation Degree vs. Graphite Oxidation Rate}

The effect of burn-off is very important for predicting oxidation rate of graphite because the reaction rate is highly dependent on the degree of burn-off. Usually, the reaction rate increases with the increasing burn-off in the beginning (Velasquez et al. 1978). This is because of the increase of the reaction surface as the pores grow larger and the closed porosity opens. Then, the reaction rate decreases at high burn-off because the pores join together, thus decreasing the reaction surface area. The effect of burn-off is usually considered by an empirical factor, $F_{b}$, as

$r_{g}=k_{0} \exp \left(-\frac{E_{A}}{R \cdot T}\right) \cdot P_{O_{2}}^{n} \cdot F_{b}$

where

$$
r_{g} \quad=\text { reaction rate }
$$




$$
\begin{array}{ll}
k_{0} & =\text { pre-exponential factor } \\
E_{A} & =\text { activation energy } \\
R & =\text { gas constant } \\
T & =\text { temperature } \\
P_{O_{2}}^{n} & =\text { oxygen partial pressure } \\
n & =\text { order of reaction } \\
F_{b} & =\text { empirical factor. }
\end{array}
$$

The physical meaning of $F_{b}$ is the ratio of reacting surface area of oxidized graphite to that of original graphite. Therefore, $F_{b}$ equals to 1.0 for the original graphite and 0.0 for the completely burned graphite. The $F_{b}$ has the equation form

$$
F_{b}=A_{\text {oxidized }} / A_{\text {original }}
$$

where

$$
\begin{aligned}
& A_{\text {oxidized }}=\text { reacting surface area for the oxidized graphite } \\
& A_{\text {original }}=\text { reacting surface area for the original (unoxidized) graphite (burn-off }=0 \% \text { ). }
\end{aligned}
$$

As described above, the reacting surface area initially increases with the reaction because of the increased pore size. However, as the reaction proceeds, the reacting surface area decreases again because of the diminishes of the enlarged pores. Therefore, the $F_{b}$ value starts at 1.0, and then initially increases with oxidation. After a certain level of burn-off, it starts to decrease again, and finally drops to 0.0 at $100 \%$ burn-off.

In this report, the $F_{b}$ factor has been experimentally obtained as a function of burn-off for various forms of graphite: IG-110, H451, NBG-10, NBG-18, and V483T. The $F_{\mathrm{b}}$ factors of IG-110 and H451 were measured by the experimental setup used in Section 4.1.2, and for NBG-10, NBG-18, and V483T, the data published in Fuller and Okoh (1997), Moorman et al. (1999), and Hinssen et al. (2008) were used.

Figures 4-17 through 4-21 show the plots of $F_{b}$ versus burn-off. Figure 4-17 (a) shows the results for IG-110 graphite. This graph includes three datasets: Fuller and Okoh (1997), Kim et al. (2006), and (3) the experimental results obtained in this work. According to the figure, the data from Kim et al. (2006) and this work show very good agreement. However, the data from Fuller and Okoh (1997) shows some discrepancies from other data. The reason is not yet identified. In Kim et al.(2006) and the current experiments, the maximum oxidation rate occurs at about $35 \%$ burn-off, at which time the oxidation rate is about 6.4 times that of the initial oxidation rate. Figure 4-17 (b) shows the data for H451 graphite. In this material, the maximum oxidation rate occurred between 30 and 50\% burn-off, and is about 3.5 times larger than the initial rate. 


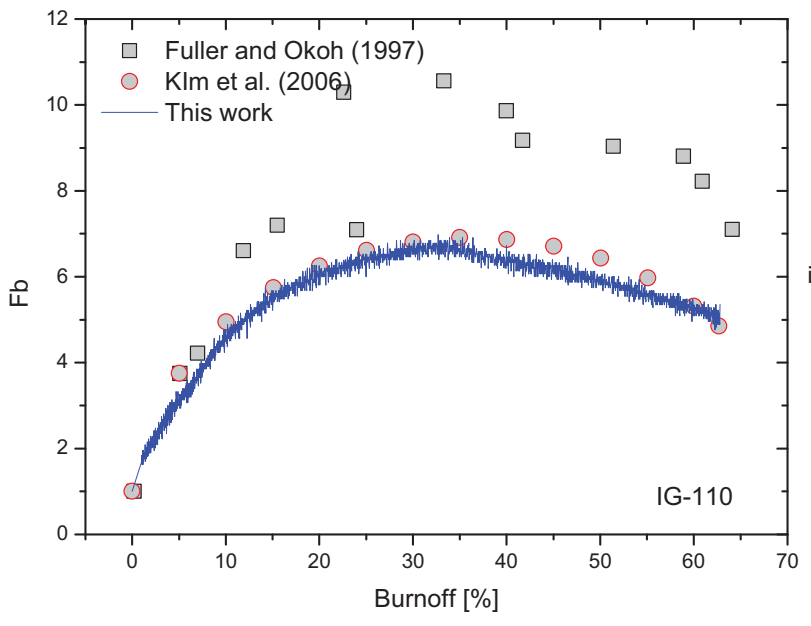

(a) IG-110 Graphite

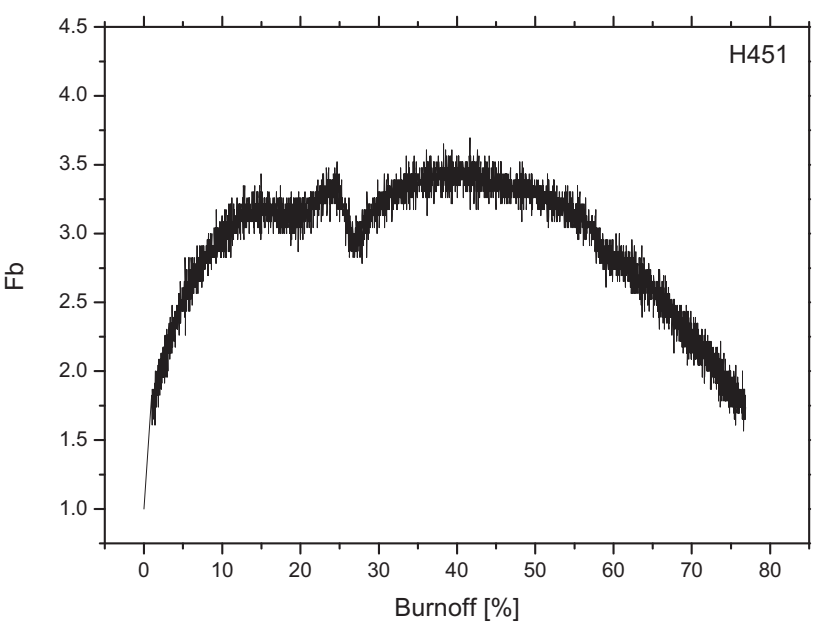

(b) H451 Graphite

Figure 4-17. Burn-off vs. $F_{b}$ (IG-110 and H451).

Figure 4-18 plots the surface area densities and the reaction rates for IG-110 and H451 graphite as a function of burn-off. The surface area densities of Figure 18(a) were obtained by multiplying the initial surface area density of the graphite in Table 4-3 by the $F_{b}$ value (See Equation 4-5). The reaction rates in the Figure 4-18(b) are the experimental data. Generally, the reaction rates are proportional to the reacting surface area. Therefore, the initial oxidation rate is smaller in IG-110 graphite $\left(890 \mathrm{~m}^{2} / \mathrm{m}^{3}\right)$ than in $\mathrm{H} 451$ graphite $\left(1,320 \mathrm{~m}^{2} / \mathrm{m}^{3}\right)$. However, as oxidation progresses, the surface area density of IG-110 increases more rapidly than that of H451. Finally, the oxidation rate of IG-110 becomes larger than that of H451 for more than $10 \%$ burn-off, meaning that only comparisons based on the surface area densities or reaction rates of the original graphite can lead to a misunderstanding about the graphite oxidation characteristics. For example, comparisons show that H451 graphite has better oxidation resistance than IG-110 in the long process, which is more important in the air-ingress accident.

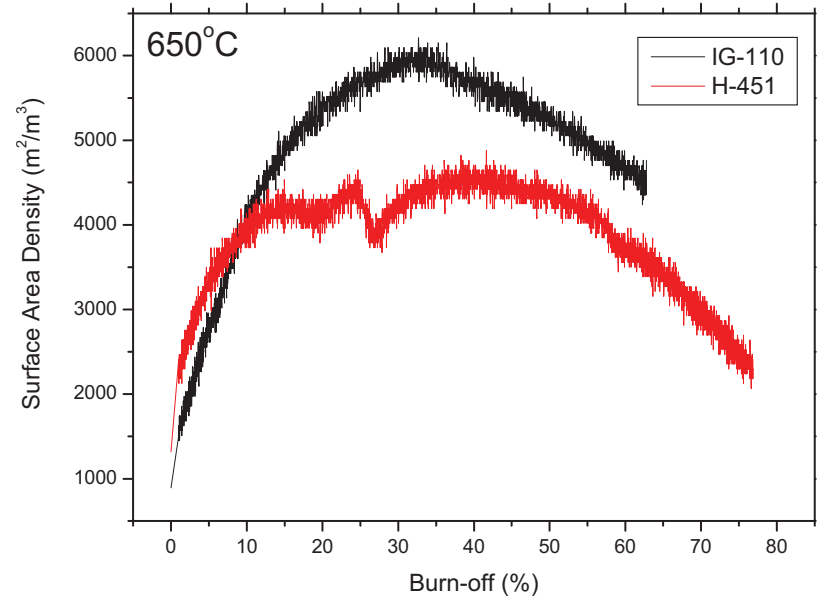

(a) Burn-off vs. Surface Area Density

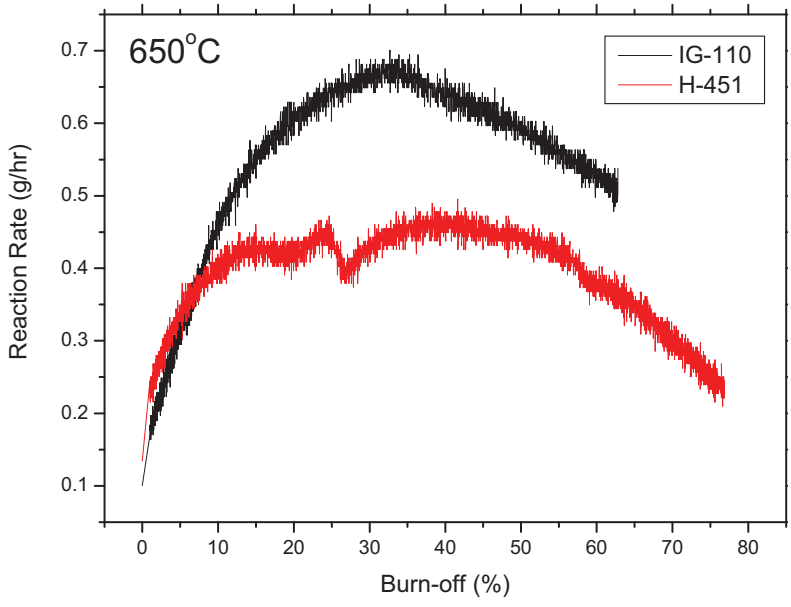

(b) Burn-off vs. Reaction Rate

Figure 4-18. Comparisons of surface area density and oxidation rate between IG-110 and H451. 
Figures 4-19 and 4-20 show the data for NBG-10 and NBG-18 manufactured by SGL group, respectively. These data are based on the experimental data reported by Hinssen et al. (2008). According to these figures, the maximum oxidation rate occurred at 30\% for both graphite types. Figure 4-21 shows the data for V484T, which is based on the data reported by Moorman et al. (1999). All of these graphs and data can be used in predicting the graphite oxidation rate at the specific burn-off in the air-ingress analysis of the VHTRs.

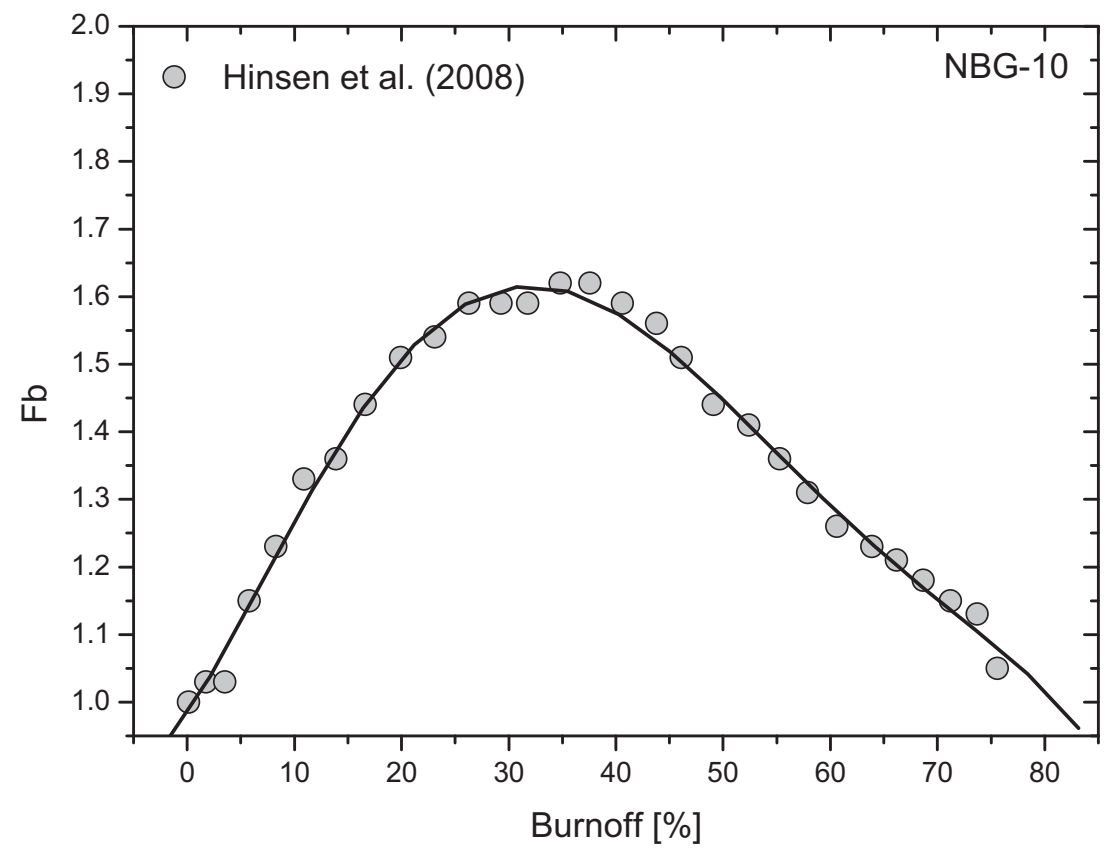

Figure 4-19. Burn-off vs. $F_{b}$ (NBG-10).

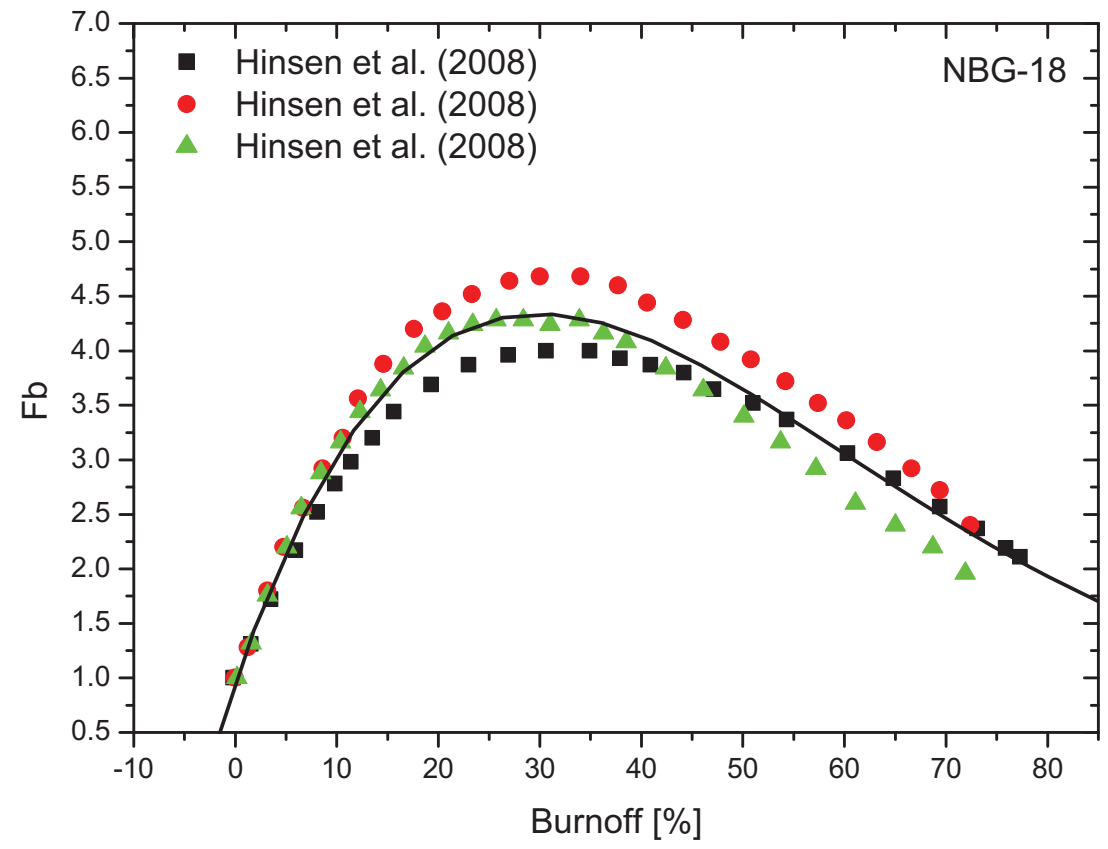

Figure 4-20. Burn-off vs. $F_{b}$ (NBG-18). 


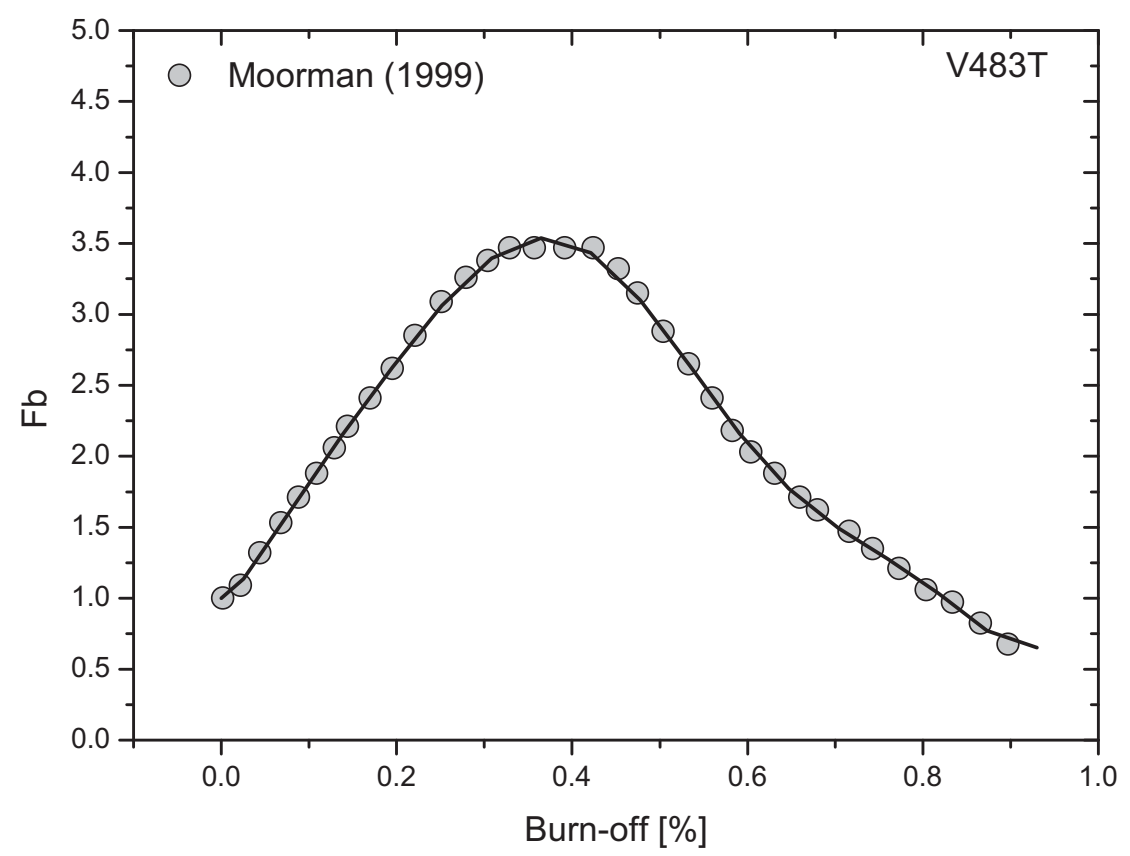

Figure 4-21. Burn-off vs. $F_{b}$ (V484T).

\subsection{Modeling of Graphite Oxidation and Fracture in Air-ingress}

Inherent safety features of the VHTR graphite core design could be compromised if the core supporting structures collapse and damage the fuel blocks, potentially leading to release of fission products. Because graphite does not easily oxidize and the amount of oxygen available in the reactor confinement structure is limited, such a fracture is likely a very low probability event, perhaps well beyond design basis. But because the consequences are severe, the event does warrant some study. Graphite oxidation will occur to some extent after any air-ingress accident. Unless mitigating action is taken, the graphite support structures may gradually erode over time altering their shapes and mechanical properties. To determine the time scale of the graphite support structure fracture, a computational stress analysis was performed with ABAQUS (Ver. 6.75) using the transient corrosion depth, temperature and graphite burn-off predicted by the GAMMA.

\subsubsection{Stress Analysis Strategy}

As shown in Figure 4-22, the core is made of several layers of graphite blocks. To finish the computational analysis in a reasonable amount of time, instead of modeling the entire core, only one vertical column of the support block and plenum directly below the fuel blocks - the parts subjected to most stress and oxidation damage - were analyzed (indicated by green arrows in Figure 4-22).

As shown in Figure 4-23(b), each block is usually surrounded by six adjacent blocks. However, the presence of adjacent blocks was ignored because there is approximately $2 \mathrm{~mm}$ clearance between the block surfaces (GA 1997). Because of the gap, they are not exerting force on each other, so the vertical columns of the blocks were assumed to be independent of each other. 


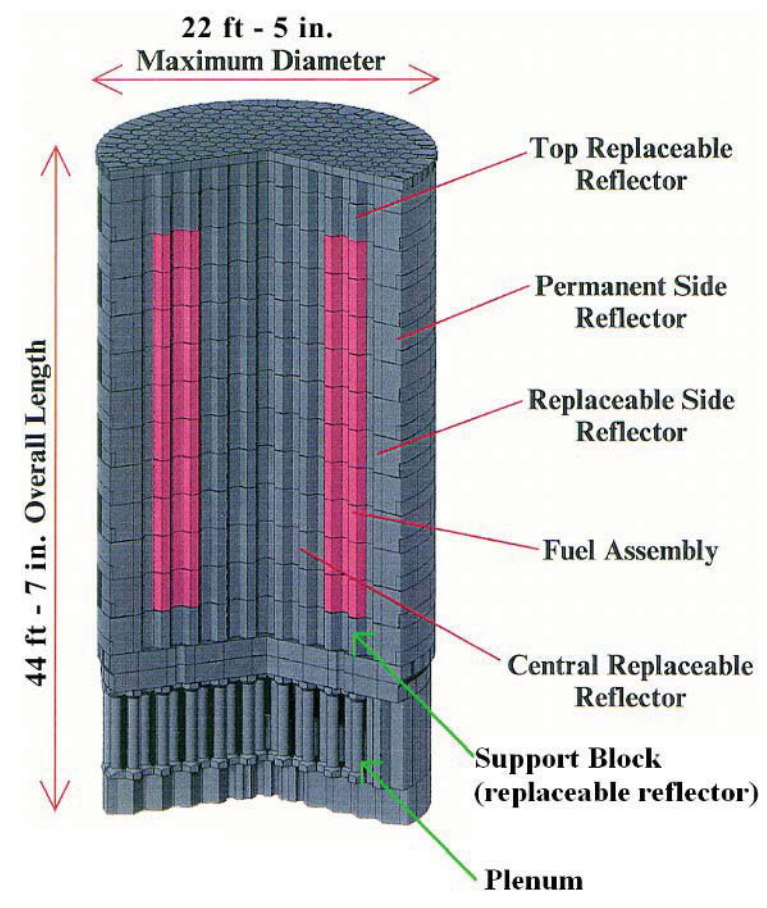

Figure 4-22. GTMHR core side view (Shenoy 2007).

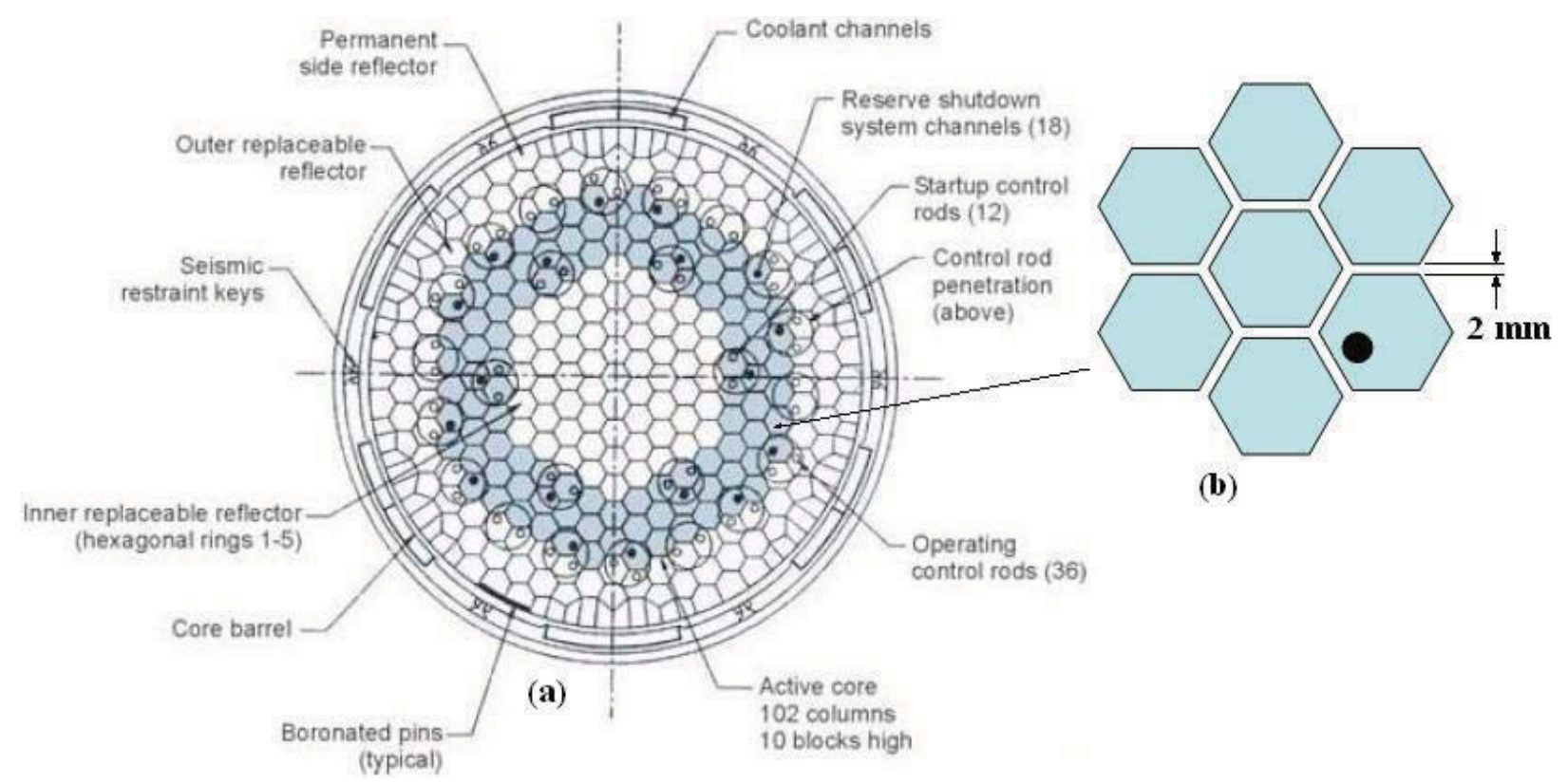

Figure 4-23. Top cutaway view of the core (Cocheme 2004).

Figure 4-24 shows the whole and cut-away views of the support block and the plenum that were analyzed. Oxidation damages the graphite structure by two different means: burn-off and corrosion. Burn-off refers to the oxidation of the graphite's internal body, causing reduction of density, leading to reduction of stiffness (young's modulus) and mechanical strength. Corrosion refers to oxidation taking place on the outer surfaces of the structure exposed to airflow. The corrosion decreases the cross-sectional area available to support the weight. As corrosion continues, the cross-sectional area decreases until the stress exceeds the mechanical strength of the graphite, leading to structural fractures. 


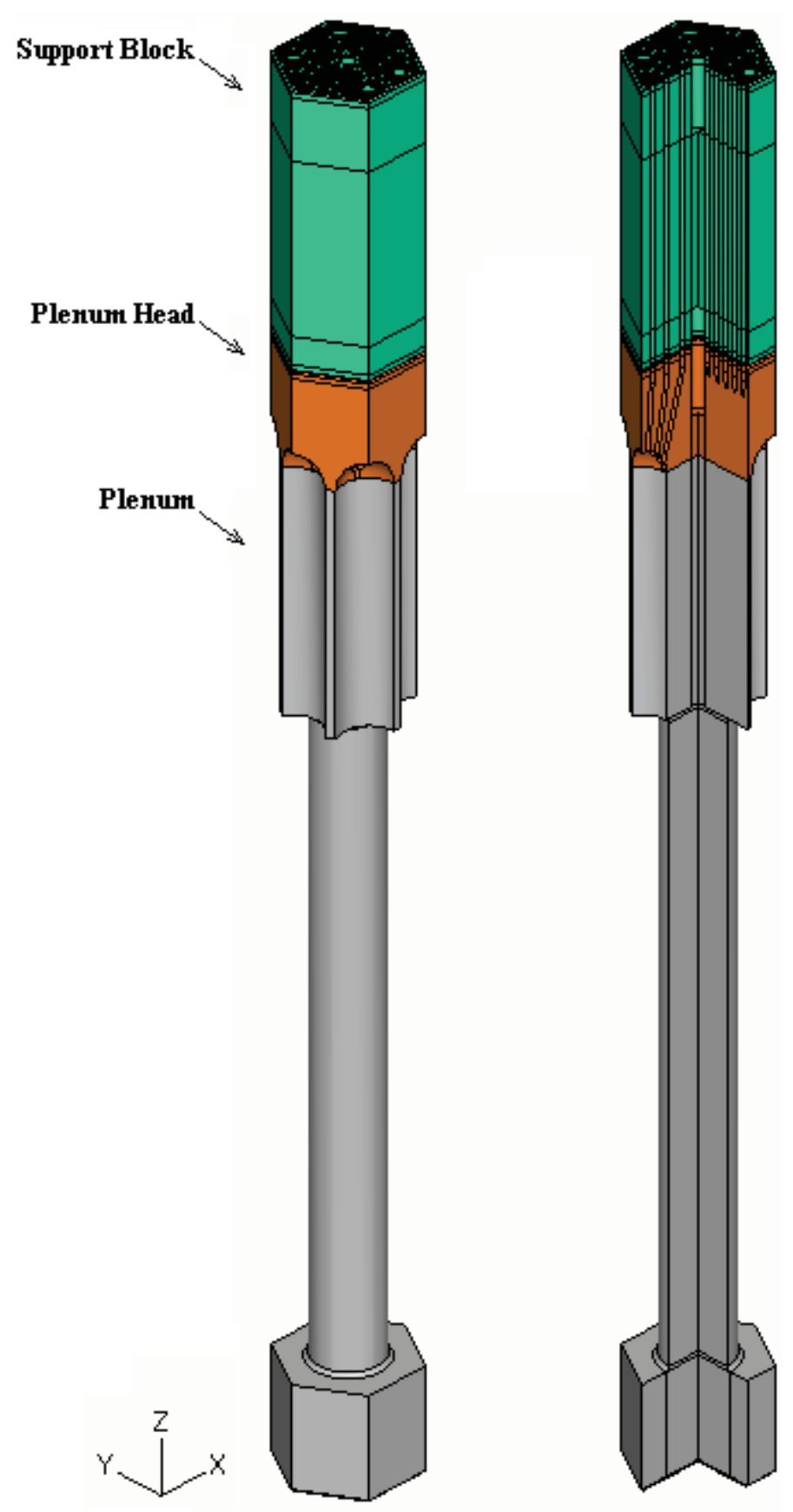

Figure 4-24. ABAQUS generated whole and cut view of the support block and plenum.

\subsubsection{Structural Dimensions}

\subsubsection{Support Block Dimensions}

The support block is modeled using the dimensions of General Atomics' GTMHR fuel block design shown in the Figure 4-25. The support block is almost identical to the fuel block, except the support block does not have fuel or LBP holes. Parts without exact dimensions given were approximated from the appearance of the drawing, indicated with an asterisk. 


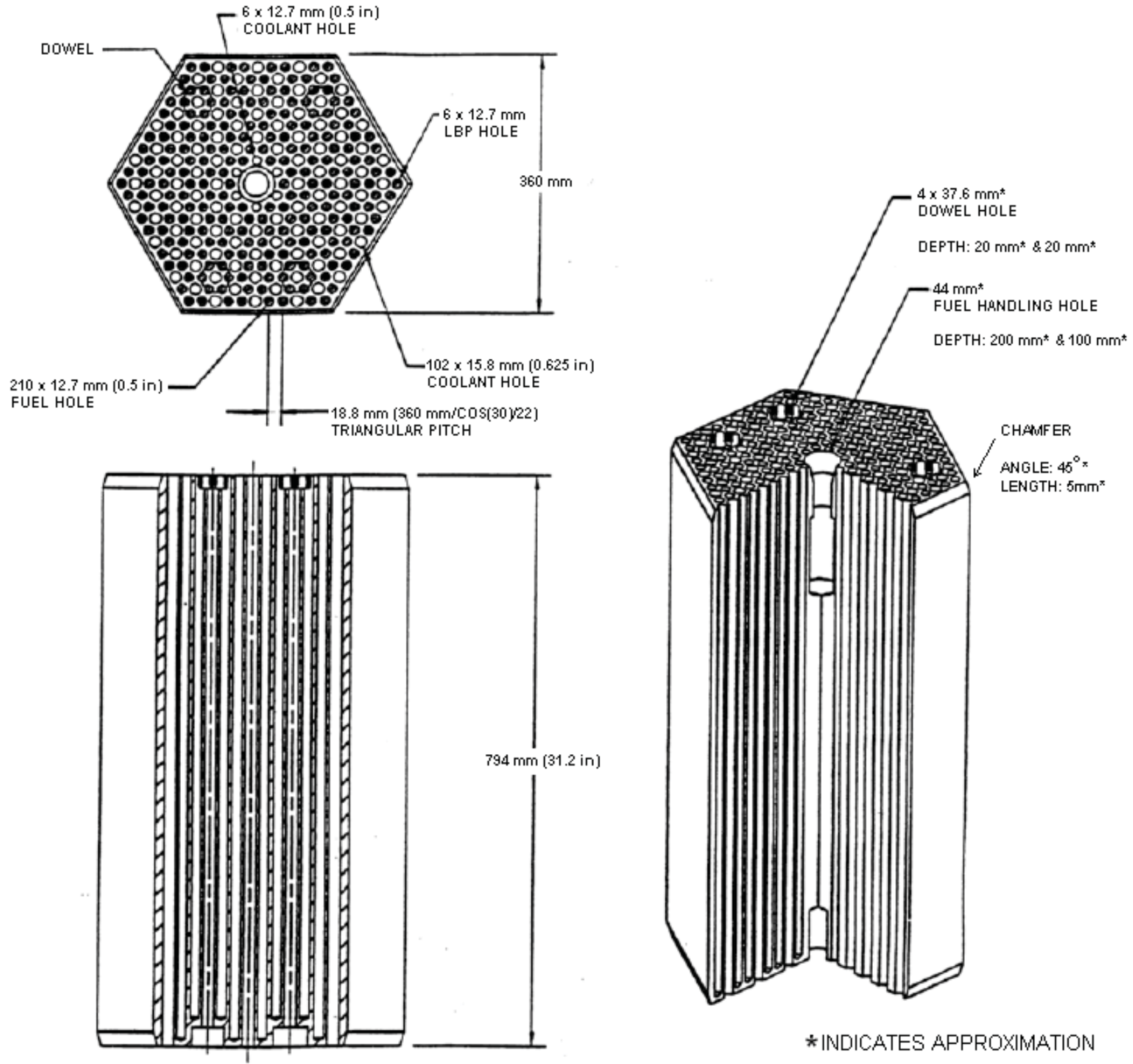

Figure 4-25. The dimensions of a General Atomics' GTMHR fuel block. (GA 1997, Cocheme 2004, GA 2003).

\subsubsection{Plenum Dimensions}

The dimensions used for modeling the plenum, shown in Figure 4-26(a), are based on the order of magnitude estimates for geometric ratios suitable for normal operation of a 600 MWth GTMHR (McCreery 2004). The information on how the coolant channels converge into the jet hole was not available; therefore, they were personally designed using rotational symmetry such that an equal number of channels converge into each jet holes as shown in the Figure 4-26(b). 


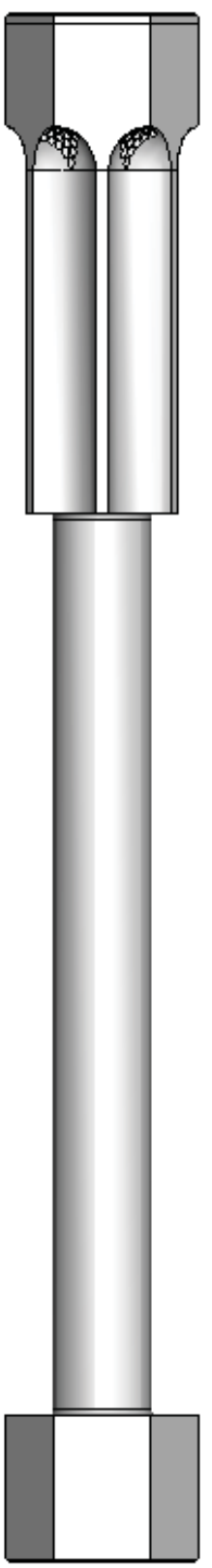

(a)

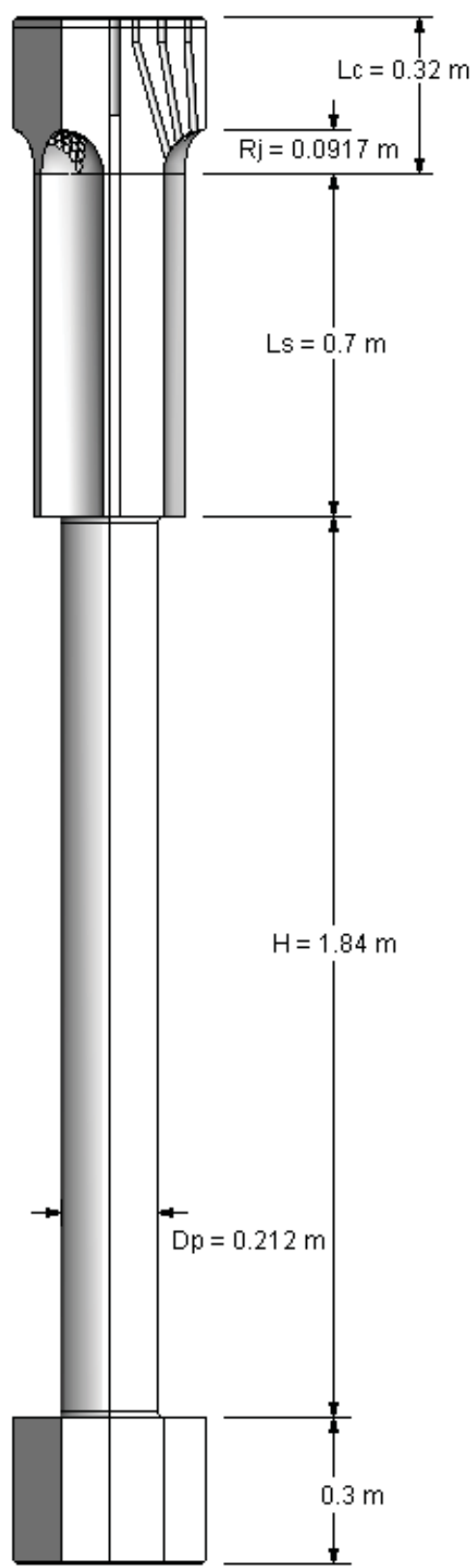

(a)

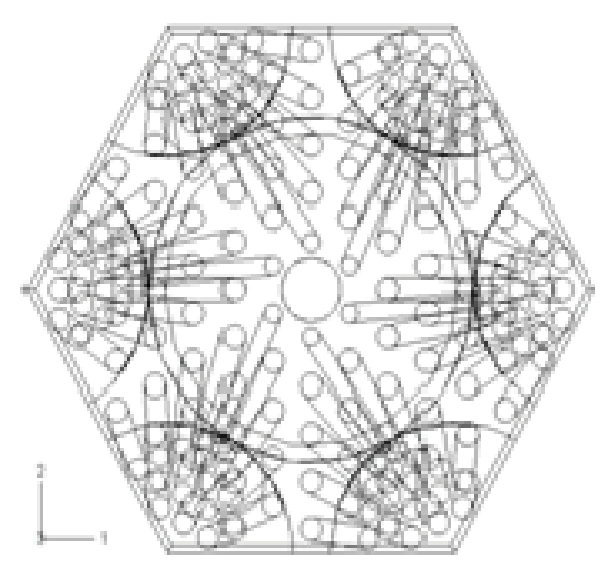

(b)

Figure 4-26. Plenum (a) side views (with dimensions), and (b) wireframe top view.

\subsubsection{Change of Temperature, Burn-off, and Corrosion Thickness}

The graphs in Figures 4-27, 4-28, and 4-29 show the corrosion depth, temperature, and burn-off as a function of time for sections of the support block and plenum. The corrosion and burn-off start almost immediately after the LOCA because the natural convection starts almost immediately, supplying oxygen to the core. The temperature and availability of oxygen play key roles in oxidation damage. The heat from an exothermic oxidation reaction causes the temperature to rise, which also increases the oxidation rate. The corrosion is highest on the lower plenum sections because air enters from the bottom and rises up. The corrosion decreases toward higher sections because of depleted oxygen, but from Section 6 and above, the temperature is significantly higher to offset this trend. 


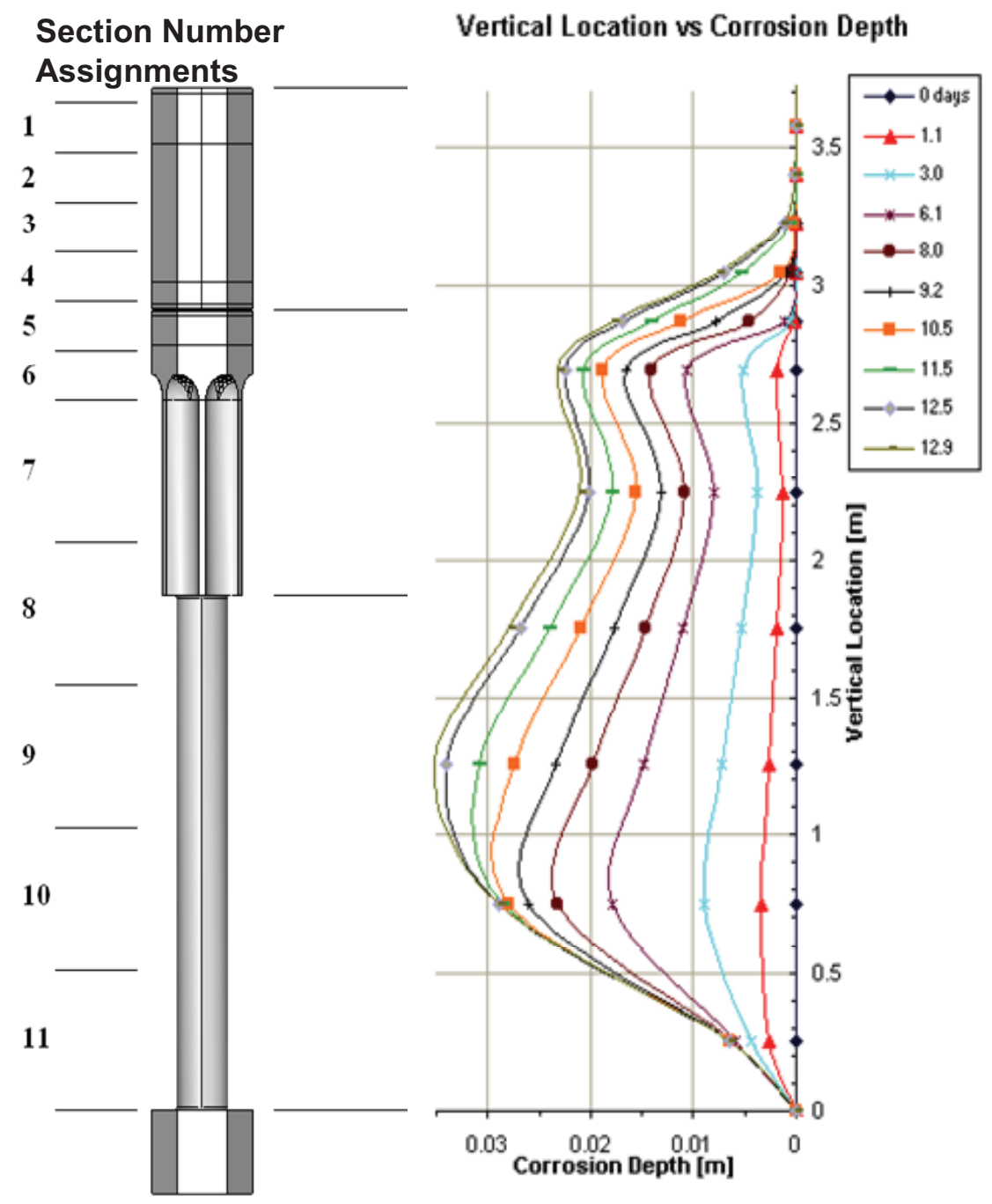

Figure 4-27. Corrosion depth and section assignments.

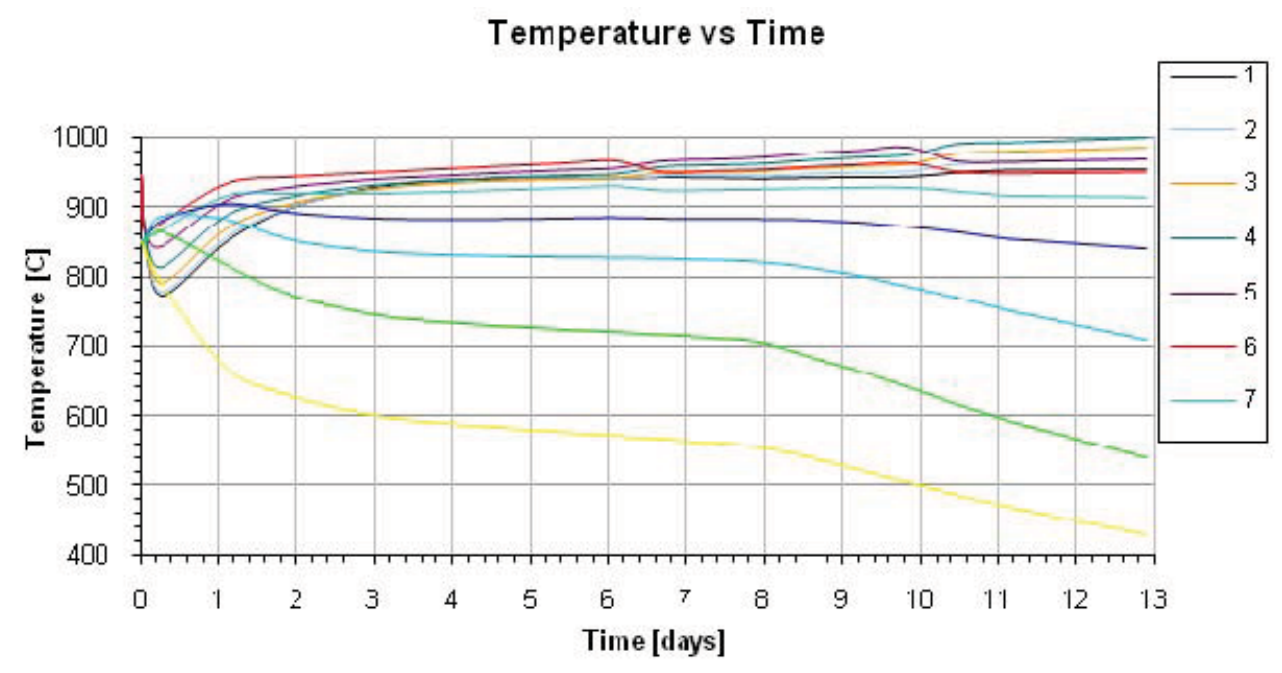

Figure 4-28. GAMMA result of temperature over time. 


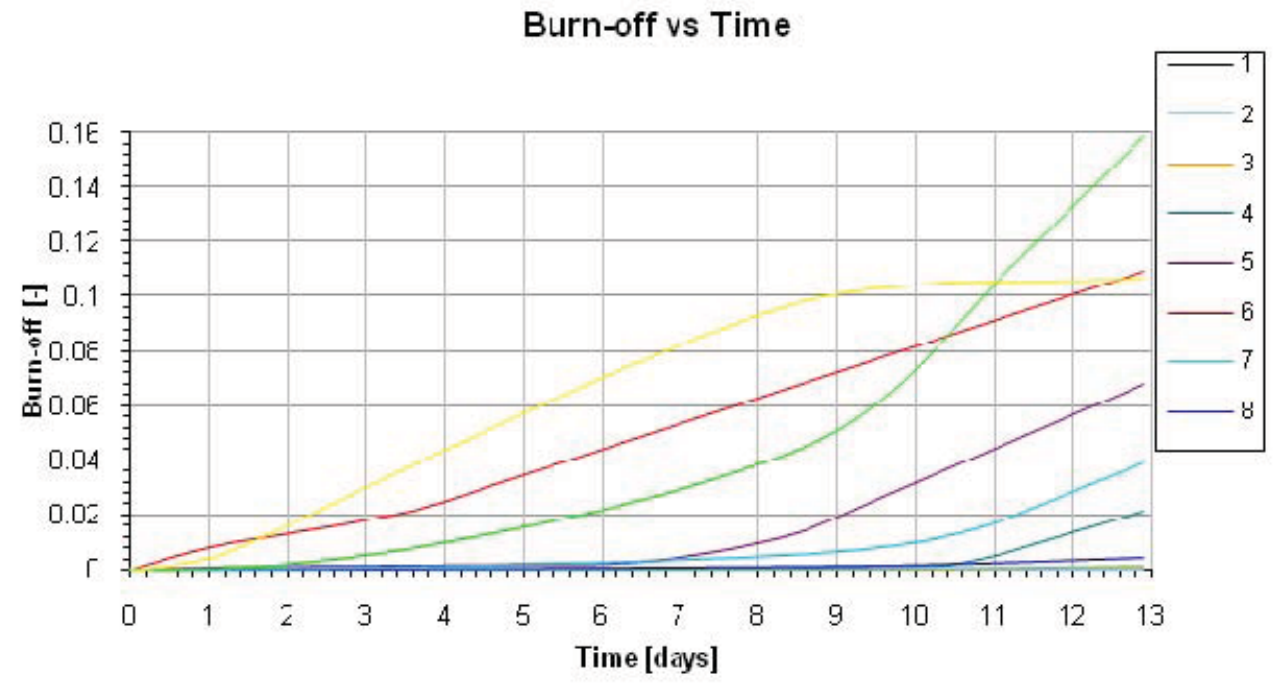

Figure 4-29. GAMMA result of burn-off over time.

Mechanical fracture first occurs in Section 6 and above because these parts have numerous coolant channels, and the cross sectional area to support the load decreases significantly with corrosion on the channel walls. Even though the corrosion and burn-off is highest in lower plenum sections, they are not enough to exceed mechanical strengths or cause buckling.

\subsubsection{Material Database}

Mechanical properties are important to the accuracy of the analysis, so a detailed material database was built to address the effects of temperature, burn-off, and irradiation.

\subsubsection{Properties in Standard Conditions}

The GTMHR's core was assumed to be made of IG-110, a high-strength, fine-grained graphite with isotropic mechanical properties (Ishihara et al. 2004). Thermo mechanical properties of the IG-110 in its normal state are shown in Table 4-4. Because the ultimate strengths exhibit statistical variations, the minimum compressive and tensile strengths were determined from statistical treatment of the strength data such that it can be said with $95 \%$ confidence that $99 \%$ of the graphite samples survive beyond the specified values (Ishihara et al. 2004).

Table 4-4. Basic thermo mechanical properties of IG-110 at standard conditions. (Ishihara et al. 2004, Burchell 1991).

\begin{tabular}{|l|l|}
\hline \multicolumn{1}{|c|}{ Density } & \multicolumn{1}{|c|}{$\mathbf{1 7 8 0} \mathbf{~ k g} / \mathbf{m}^{\mathbf{3}}$} \\
\hline Mean Young's Modulus & $7.9 \mathrm{GPa}$ \\
\hline Poisson Ratio & 0.14 \\
\hline Mean Compressive Strength & $76.8 \mathrm{MPa}$ \\
\hline Minimum Compressive Strength & $61.3 \mathrm{MPa}$ \\
\hline Mean Tensile Strength & $25.3 \mathrm{MPa}$ \\
\hline Minimum Tensile Strength & $19.4 \mathrm{MPa}$ \\
\hline Mean Thermal Expansion Coefficient $\left(293-673^{\circ} \mathrm{C}\right)$ & $4.06 \times 10^{-6} \mathrm{~K}^{-1}$ \\
\hline Thermal Conductivity $\left(600^{\circ} \mathrm{C}\right)$ & $80 \mathrm{~W} / \mathrm{m}-\mathrm{K}$ \\
\hline
\end{tabular}




\subsubsection{Tensile and Compressive Strength vs. Burn-off}

The experimental data for the change of tensile and compressive strength because of burn-off are shown in Figures 4-30(a) and 4-30(b). The relationship between the strengths and the burn-off is exponential. Also, the ratio of the instantaneous compressive strength to initial compressive strength, S/So, for both tensile and compressive strengths, show a virtually identical trend to that of function of burn-off.

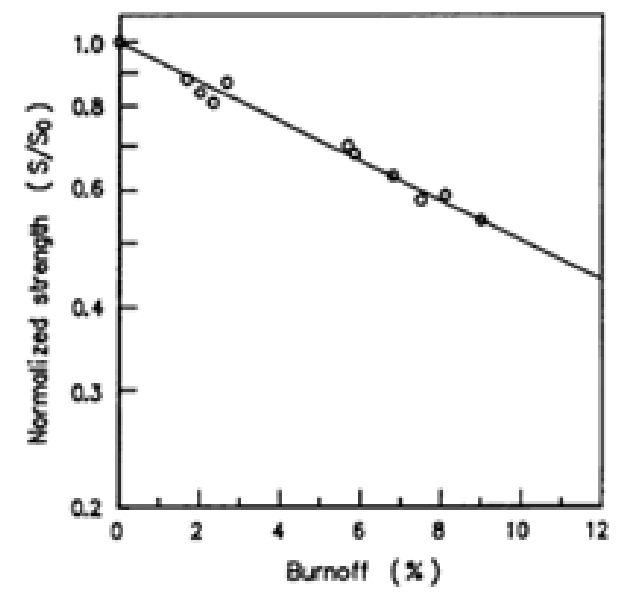

(b) Compressive strength

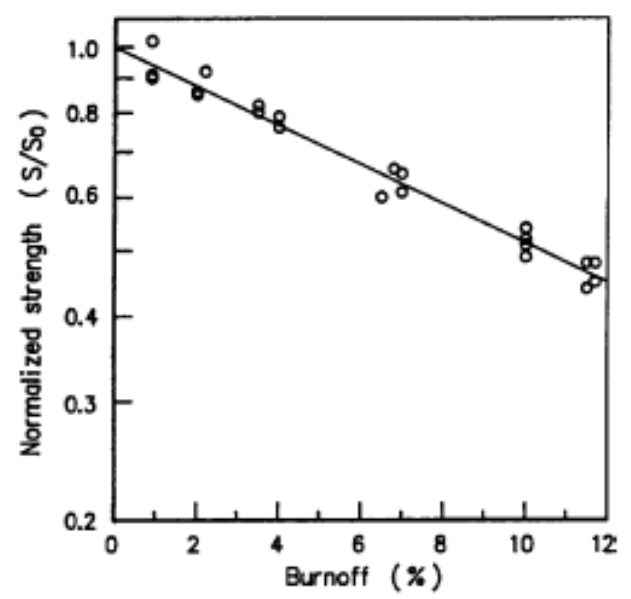

(c) Tensile strength

Figure 4-30. (a) Tensile strength as function of bun-off; (b) compressive strength as function of burn-off (Ishihara et al. 2004).

\subsubsection{Tensile and Compressive Strength vs. Temperature}

As shown in the Figure 4-31, the Young's modulus ratio appears to show square root relationship with the tensile strength. Considering that $\mathrm{S} / \mathrm{So}$ ratios for tensile and compressive strength behave almost exactly the same as function of burn-off, their behaviors with respect to the temperature change might be very similar with each other. Therefore, the S/So ratio for compressive strength was assumed to behave the same as the $\mathrm{S} / \mathrm{So}$ ratio for the tensile strength as function of temperature.

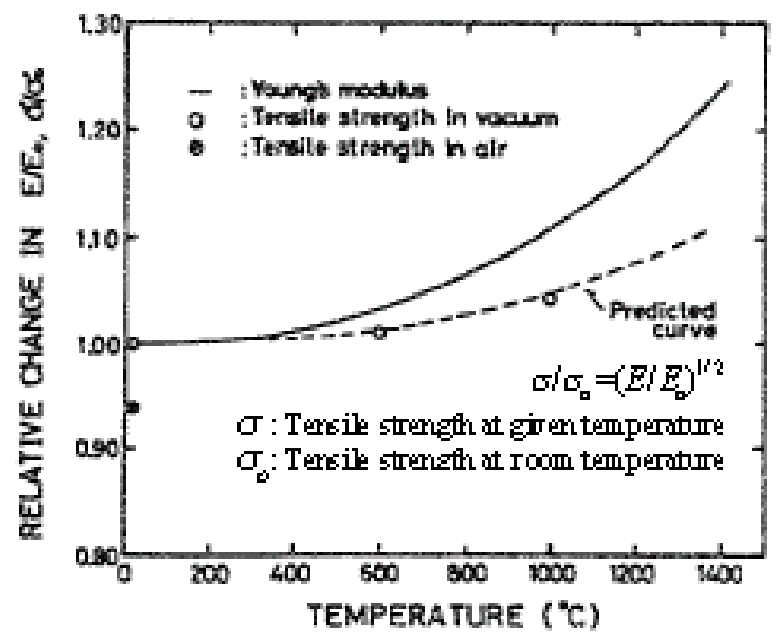

Figure 4-31. Tensile strength as function of temperature (Eto et al. 1986). 


\subsubsection{Young's Modulus vs. Temperature and Burn-off}

The experimental data and coalitions for the change of Young's modulus because of temperature and burn-off are shown in the Figure 4-32.

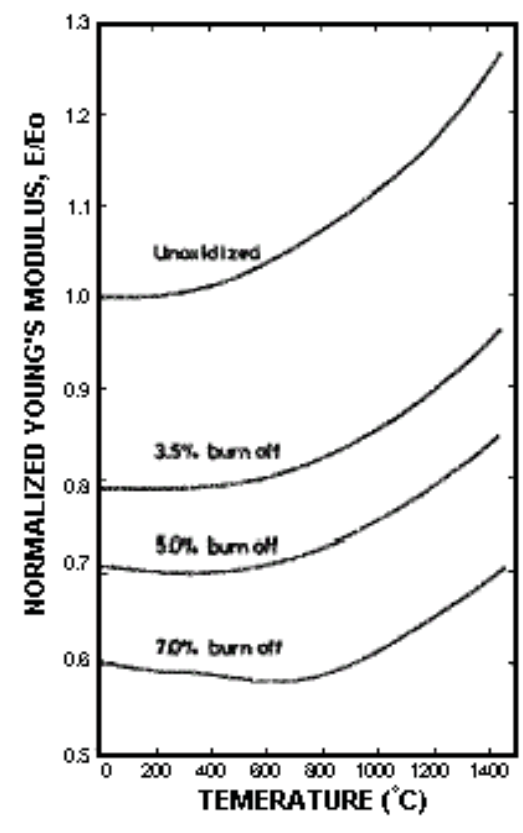

(a)

$$
\begin{aligned}
E / E_{o}= & \left(8.885 \times 10^{-12} \times T^{3}+1.275 \times 10^{-7} \times T^{2}-2.430 \times 10^{-5} \times T+0.999\right) \\
& \times\left(\rho / \rho_{0}\right)^{(0.00134 \times T+6.579)} \quad \text { Below } 800^{\circ} \mathrm{C} \\
E / E_{0}= & \left(8.885 \times 10^{-12} \times T^{3}+1.275 \times 10^{-7} \times T^{2}-2.430 \times 10^{-5} \times T+0.999\right) \\
& \times\left(\rho / \rho_{0}\right)^{(0.0000682 \times T+7.540)} \quad \text { Above } 800^{\circ} \mathrm{C}
\end{aligned}
$$

$E$ : Y oung s modulus at given temperature and bum-off

$E_{0}$ : Y oung's modulus at room temperature and no burn-off

$T:$ Temperature

$\rho$ : Density at given temper ature and burn- off

$\rho_{o}$ : Density at room temperature and no burn off

(b)

Figure 4-32. (a) The change in Young's modulus because of oxidation as a function of temperature (best fit of experiment data), and (b) the correlations for the Young's modulus as a function of temperature and burn off (Eto et al. 1986).

\subsubsection{Young's Modulus and Mechanical Strengths vs. Irradiation}

A change of mechanical properties from irradiation is expected to be negligible because of low neutron dose experienced by the support block and plenum. Ishihara et al. (2004) performed mechanical tests on IG-110 samples that were irradiated up to neutron fluence of $3.8 \times 10^{22} \mathrm{n} / \mathrm{cm}^{2}$ (neutron energy $>0.05 \mathrm{MeV}$ ) at a temperature of $600^{\circ} \mathrm{C}$, resulting in neutron dose of $\sim 25 \mathrm{dpa}$ (Burchell et al. 1996). Figures 4-33(a) and 4-33(b) show the change of Young's modulus and mechanical strength as a function of dpa.

As a reference example, the central replaceable reflectors indicated in the Figure 4-22 experience the neutron fluence of $6.7 \times 10^{20} \mathrm{n} / \mathrm{cm}^{2}$ per year (neutron energy $>0.1 \mathrm{MeV}$ ) with a neutron dose of $0.56 \mathrm{dpa}$ per year (Bratton et al. 2005). In contrast, the support block and plenum experience neutron fluences of $9.1 \times 10^{15} \mathrm{n} / \mathrm{cm}^{2}$ per year $[\mathrm{E}>0.1 \mathrm{MeV}]$ and $3.7 \times 10^{17} \mathrm{n} / \mathrm{cm}^{2}$ per year, respectively, with negligible neutron dose in dpa for both sections (Bratton et al. 2005). Using the central reflector case as the reference and taking the neutron fluence ratio, the neutron dose received by support block and plenum are $7.606 \times 10^{-6}$ and $3.1 \times 10^{-4}$ dpa per year, respectively. Considering that the projected plant design life is 60 years (Bratton et al. 2005), the total neutron dose received by the support block and plenum is $4.56 \times 10^{-4}$ and $1.85 \times 10^{-2} \mathrm{dpa}$, respectively. As is apparent on Figures 4-33(a) and 4-33(b), mechanical property changes because of irradiation at these dpa are probably negligible, therefore the effect of irradiation was ignored for the stress analysis. 

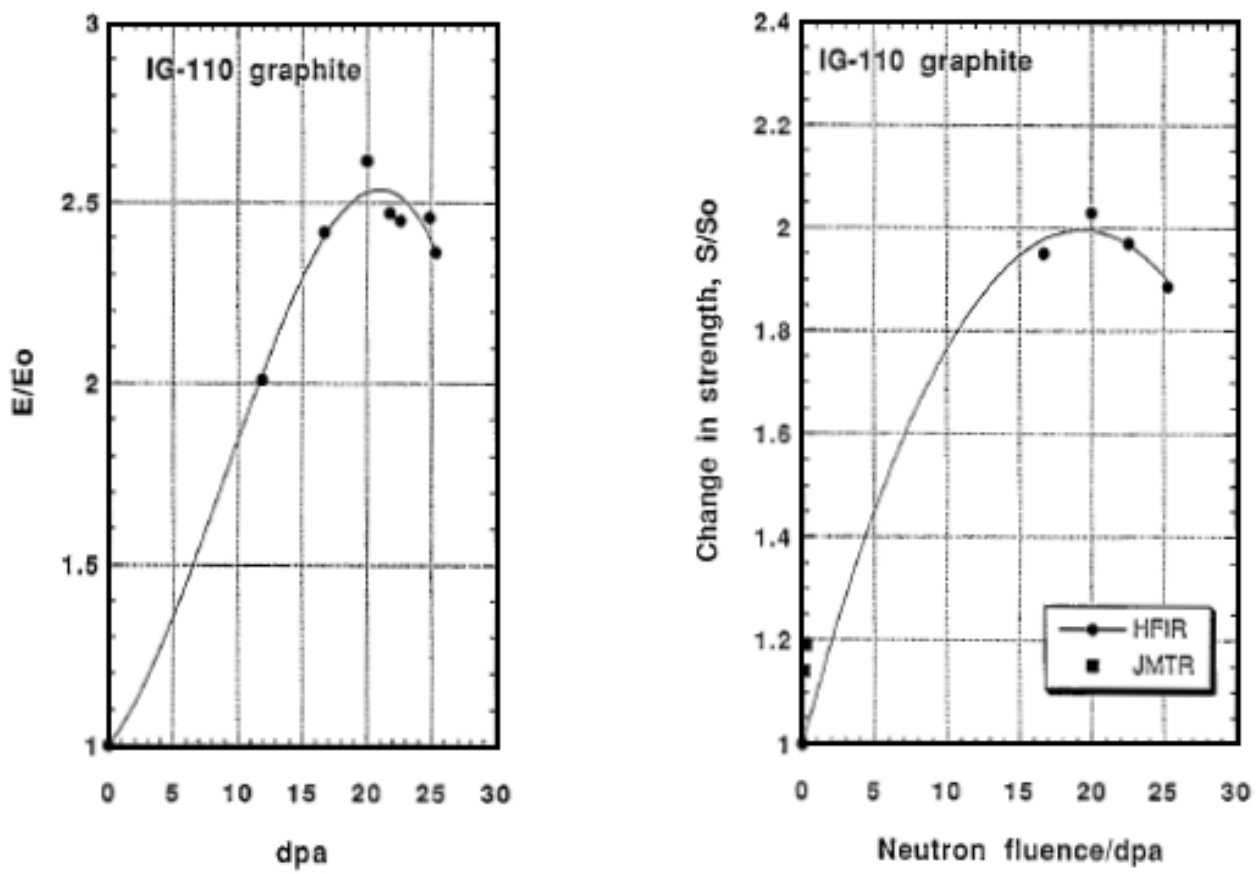

Figure 4-33. Young's modulus (a) and mechanical strength (b) as function of dpa caused by irradiation (Burchell et al. 1996).

Normal operating temperatures for support block and plenum are about $1000^{\circ} \mathrm{C}$, and $1000-1200^{\circ} \mathrm{C}$, respectively (Bratton et al. 2005). Because they experience irradiation at temperatures higher than the irradiation temperature in Ishihara et al.'s experiment, the Young's modulus and strength might behave a little differently than the data shown in Figure 4-33. However, considering that mechanical properties tend to recover back toward the original values at higher temperature because of the annealing effect (Burchell et al. 1996), it is most likely that the effect of irradiation on the Young's modulus and strength is less at the higher irradiation temperatures at which the support block and plenum normally operate.

\subsubsection{Treatment of Material Properties during Analysis}

\subsubsection{Young's Modulus vs. Strain}

The IG-110 is treated as an ideal brittle ceramic material for this application because it exhibits elastic behavior with a constant Young's modulus and does not undergo any plastic deformation before reaching the strength limit. As a relative example, Figure 4-34(a) shows the compressive stress-strain curve of UNS31803 steel, a ductile metal. As a ductile metal, its stress-strain curve is characterized by its ability to undergo plastic deformation up to a large strain value. It initially shows elastic behavior, but after about $700 \mathrm{MPa}$, starts to plastically deform until it eventually fails at the strain of 0.35 . Figure 4-34(b) shows the stress-strain curve for IG-110. Please note the scale of the strain, which is 10-times smaller than that in Figure 4-34(a). Unlike the steel, IG-110, being a brittle ceramic material, is not able to handle much strain before fracture and shows almost no plastic deformation. Plotting the IG-110 stress-strain curve on Figure 4-34(b) appears as almost a straight line. 

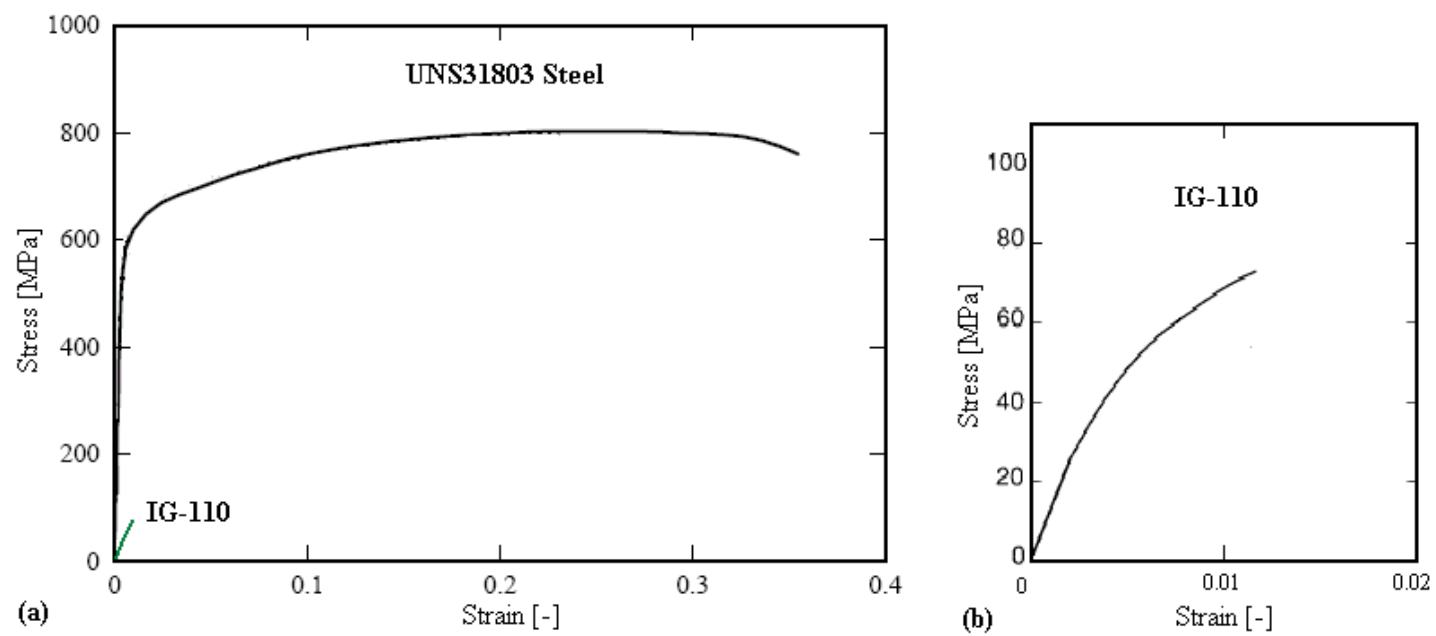

Figure 4-34. (a) Compressive stress-strain curve for UNS31803 Steel (Rasmussen 2001), and (b) Compressive stress-strain curve for IG-110 (Fuji et al. 1997).

\subsubsection{Effect of the Temperature, Burn-off, and Irradiation}

During the analysis, the young's modulus and density were estimated for the section number, the respective temperature and burn-off of the time point shown in Figures 4-27, 4-28, and 4-29, respectively, where the effect of irradiation was ignored since it is probably negligible.

\subsubsection{Fracture Criteria}

For this report, the principal stress fracture criterion is used as the fracture criteria. The fracture is assumed to occur if the minimum principal stress (maximum compressive stress) exceeds the ultimate compressive strength, or if the maximum principal stress (maximum tensile stress) exceeds the ultimate tensile strength.

\subsubsection{Initial Un-oxidized State Results}

\subsubsection{Load Stress}

As the first step, the support block and plenum in unoxidized condition was analyzed to understand general stress distribution. Figure 4-35 shows the maximum compressive stress distribution on the support block and plenum in unoxidized condition, analyzed using 1/6 cyclic symmetry. As shown in Figure 4-35, the maximum stress is concentrated on the root of the plenum, indicated in red color, which corresponds to $1 \mathrm{MPa}$, far below the mechanical strength limit of undamaged IG-110. Although not shown on the figure, the maximum tensile stress is $0.1 \mathrm{MPa}$, again far below the tensile strength limit. Because the corrosion and burn-off on the lower part of the plenum and the upper part of the support block is negligible, stress distribution on these parts would be about the same on other time points, and it is unnecessary to include them in the analysis. For this reason, only the parts between points L1 to L2, indicated in Figure 4-35, were analyzed to reduce the size of the model and computation time. 


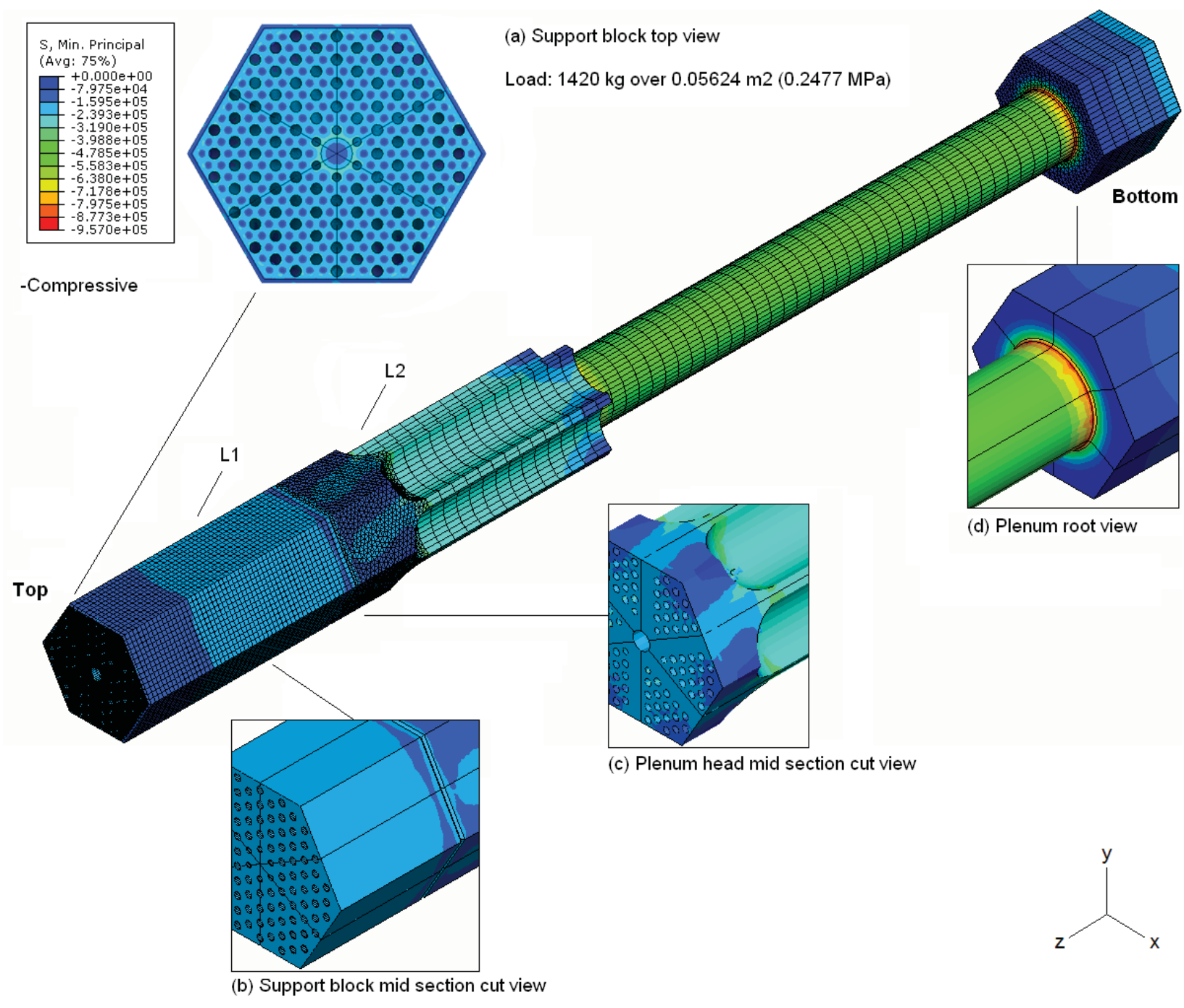

Figure 4-35. Compressive stress distribution on an unoxidized support block and plenum.

\subsubsection{Analytical Validation}

It is important to check the validity of the result obtained from finite element analysis by comparing it to the analytical estimate. For the middle of the support block and the middle of the plenum, analytical solutions to compressive stresses in z-axis direction (top to bottom) can be calculated by the simple stress relationship

$$
\sigma=\frac{m g}{A}
$$

where

$m=$ Total mass of objects being supported by the area $(\mathrm{kg})$

$g=$ The gravitational constant, $9.81\left(\mathrm{~m} / \mathrm{s}^{2}\right)$

$A=$ The cross sectional area perpendicular to the loading $\left(\mathrm{m}^{2}\right)$. 
The two locations were chosen because the stress distribution on the cross sectional areas at these points are redistributed to be very uniform, and the loading scheme is almost uniaxial in z-axis direction such that analytical solution using the above equation should yield the same result as Figure 4-36. As shown, there are nine different stress components in 3-D principal stress that are accounted for to determine the maximum tensile and compressive stresses at a given point.

However, when the loading scheme is simple, such that all of stress components except one normal stress component are negligible, the analytical solution using Equation (4-6) is approximately equal to the principal stress. At the middle of the support block and the middle of the plenum, all stress components except the normal stress in $\mathrm{z}-$ axis are negligible.

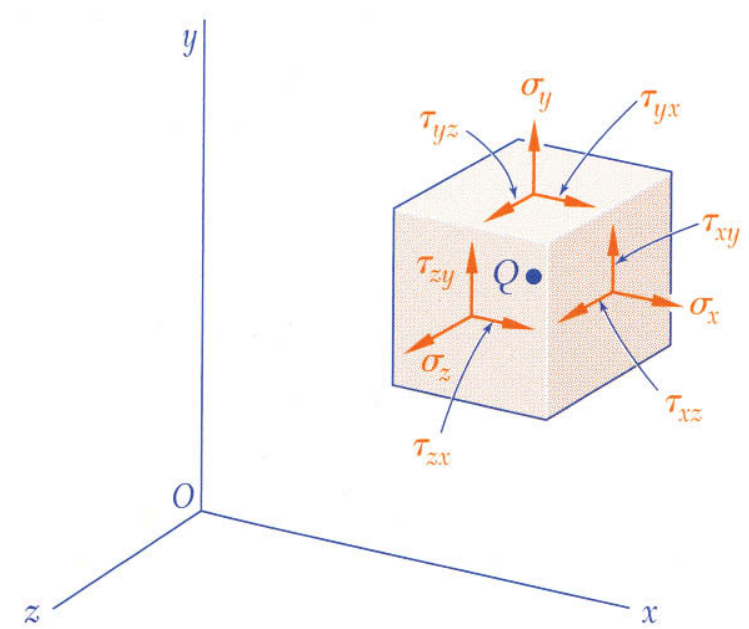

Figure 4-36. The principal stress components (Beer et al. 2006).

As shown in the Table 4-5, there is reasonable agreement between the result of the analytical calculation and the ABAQUS result, which suggests the finite element analysis was done correctly.

Table 4-5. Comparison of the analytical solution to the ABAQUS result.

\begin{tabular}{|c|c|c|c|c|}
\hline Location & $\begin{array}{l}\text { Supported } \\
\text { Mass } \\
(\text { kg) }\end{array}$ & $\begin{array}{c}\text { Cross- } \\
\text { Sectional Area } \\
\left(\mathrm{m}^{2}\right)\end{array}$ & $\begin{array}{c}\text { Compressive } \\
\text { Stress } \\
\text { (Pa) }\end{array}$ & $\begin{array}{c}\text { ABAQUS } \\
\text { Result } \\
(\mathbf{P a})\end{array}$ \\
\hline Middle of the support block & 1,500 & 0.09148 & $1.636 \mathrm{e} 5$ & $1.625 \mathrm{e} 5$ \\
\hline Middle of the plenum & 1,800 & 0.0353 & $5.002 \mathrm{e} 5$ & $4.915 \mathrm{e} 5$ \\
\hline
\end{tabular}

\subsubsection{Thermal Stress}

Thermal stress analysis was done for a small representative part of the support including three coolant channels, indicated in Figure 4-37(a). When the coolant channel's outer surface experiences an exothermic surface oxidation reaction, this is the most likely location for the thermal stress fracture. The temperature difference between the outer surface and the inner body is expected to be on the order of tens of degrees, therefore the coolant channel surface temperature of $1300^{\circ} \mathrm{C}$ and internal body temperature of $1250^{\circ} \mathrm{C}$ were assumed.

As apparent in the Figure 4-37(c) and 4-37(d), both the compressive and tensile stresses are below strength limits. Considering that the actual temperature difference between the coolant channel surface and the internal body is most likely much smaller than $50^{\circ} \mathrm{C}$, the thermal stresses are likely smaller and thus negligible. Combined loading and thermal stress analysis was done for the nonuniform oxidation case for which the effect of thermal stress was again negligible. 


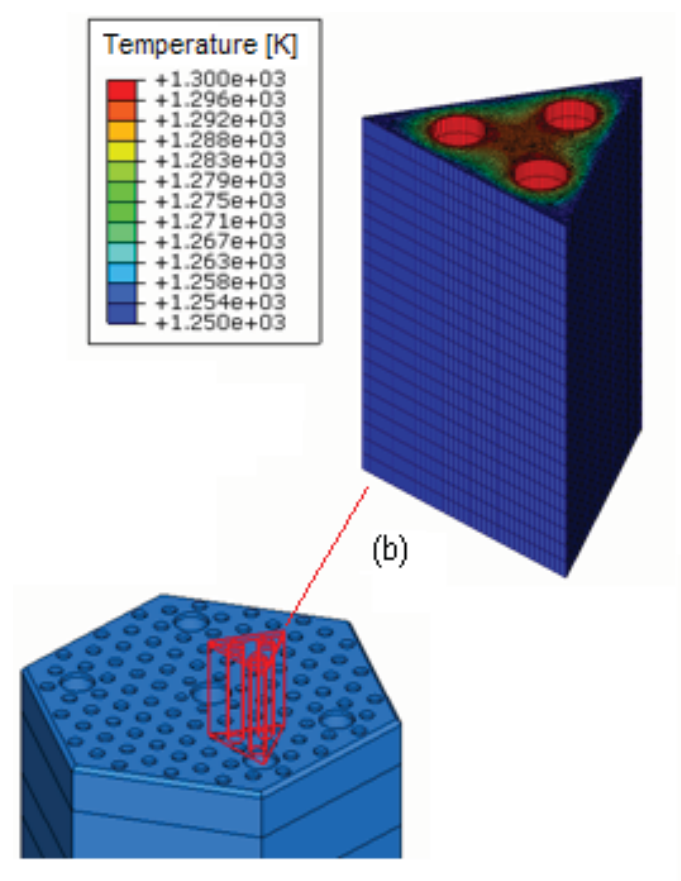

(a)

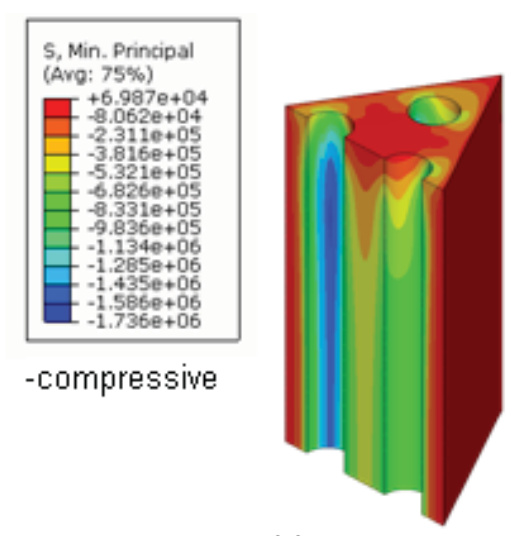

(c)

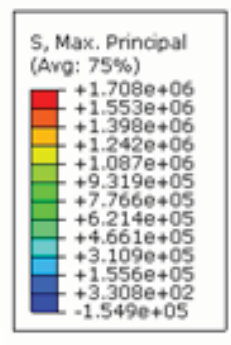

ttensile

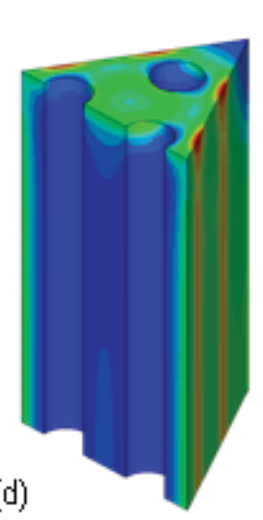

Figure 4-37. (a) Upper part of support block with triangular prism; (b) Mesh and temperature distribution; (c) Compressive stress; and (d) Tensile stress.

\subsubsection{Oxidized State Results}

\subsubsection{Oxidized State Model}

For oxidized states, simple modifications were made to the height of the plenum head section and support block to make them more structurally sound. Stress concentration would occur at the contact surface between the support block and plenum head (critical location in Figure 4-38), primarily because of the chamfer feature, which significantly reduces the size of cross sectional area available to support the load in heavily corroded states. The structure could last longer if the interacting surface is relocated to a higher place where oxidation damage is negligible. This is accomplished by adjusting the height of the plenum head and the support block by $25 \mathrm{~cm}$.

Among the dimensions used for the plenum, some of them are not yet set in stone and are open to modifications, such as the height of the plenum head and support block. The plenum head's height was increased by $0.25 \mathrm{~m}$, which consequently decreased the support block's height by $0.25 \mathrm{~m}$. From a manufacturing standpoint, there should be no difficulty in making these modifications. Most of the fuel and support blocks have straight channel holes drilled $0.8 \mathrm{~m}$ long. With the height modification, instead of drilling a $0.05 \mathrm{~m}$ long hole from the top of plenum head, it is now increased to $0.3 \mathrm{~m}$, which is still much shorter than $0.8 \mathrm{~m}$. With the height of the plenum increased by $0.3 \mathrm{~m}$, the chamfer and interacting surface are relocated away to a place where corrosion and burn-off are almost negligible. With the interacting surface and chamfer relocated, the cross-sectional area at the critical location is increased, which relieves the stress concentration. And because the critical location is now placed in the middle of a continuous body, it is more structurally sound. 


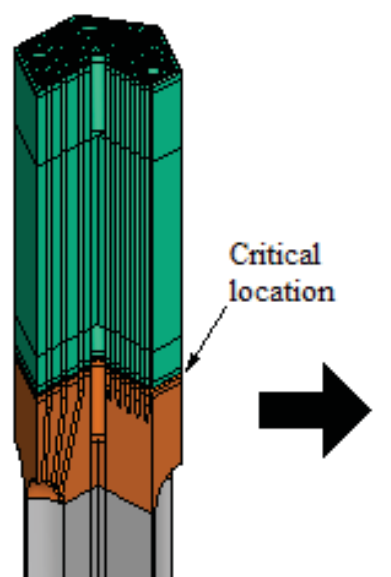

(a)

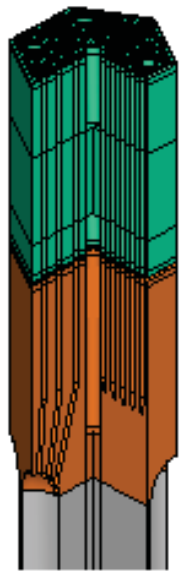

(b)

Figure 4-38. Modification of plenum head's height block by $25 \mathrm{~cm}$.

Figure 4-39 shows the 1/6 cyclic symmetry unit of the modified plenum head at particular times during transient. As corrosion progresses and the coolant channels collapse together, the plenum head eventually develops pillars, which are the remains of the thickest parts of the plenum head. Table 4-6 shows the material properties used for each day.
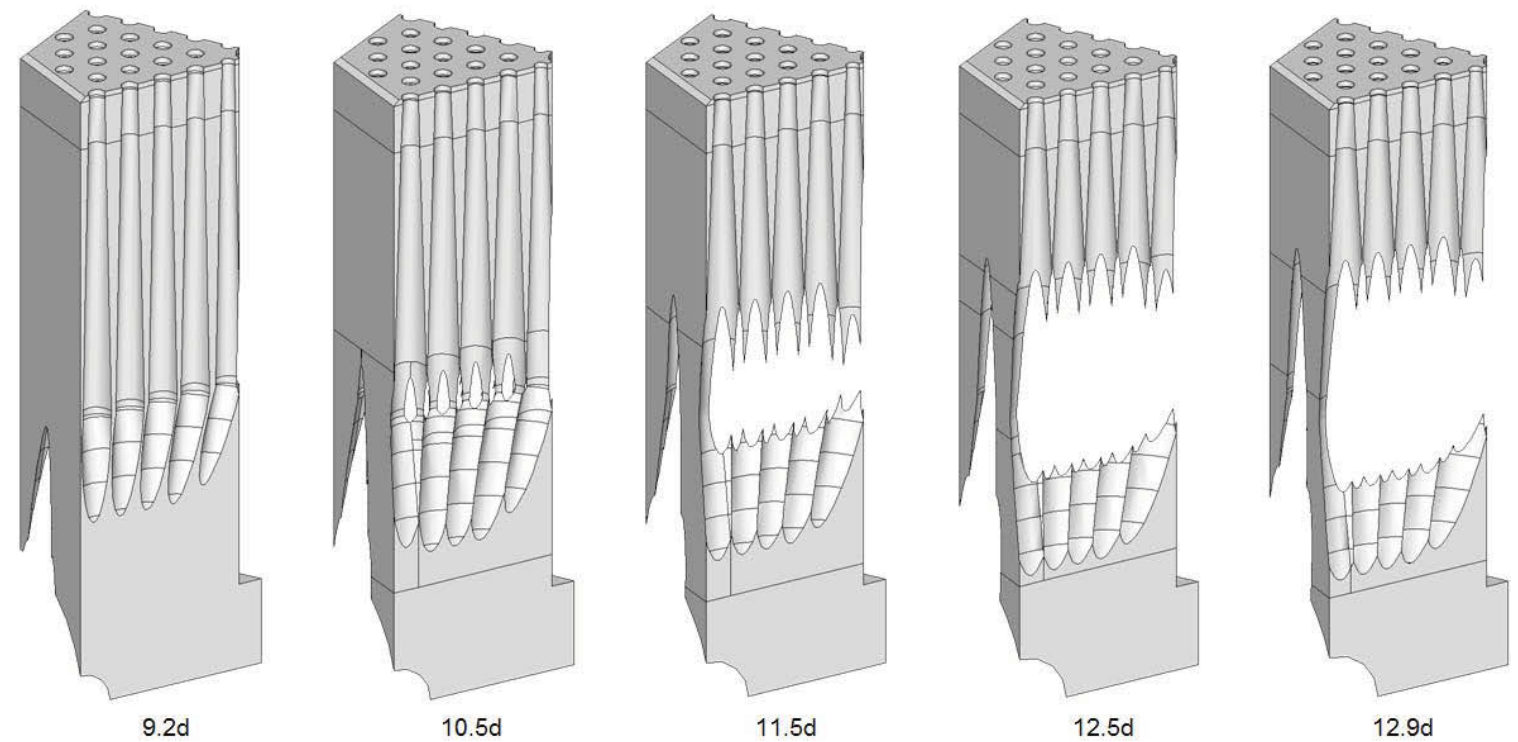

Figure 4-39. Assumed 1/6 cyclic symmetry units of the modified plenum head for each day.

Table 4-6. Material properties at each day.

\begin{tabular}{|c|c|c|c|c|c|c|}
\hline Day & $\begin{array}{c}\mathbf{T} \\
(\mathbf{K})\end{array}$ & $\begin{array}{c}\text { Burn-off } \\
(-\mathbf{-}\end{array}$ & $\begin{array}{c}\text { Density } \\
\left(\mathbf{k g} / \mathbf{m}^{\mathbf{3}}\right)\end{array}$ & $\begin{array}{c}\mathbf{E} \\
(\mathbf{G P a})\end{array}$ & $\begin{array}{c}\text { Compressive Strength } \\
(\mathbf{M P a})\end{array}$ & $\begin{array}{c}\text { Tensile Strength } \\
(\mathbf{M P a})\end{array}$ \\
\hline 9.2 & 962 & 0.0743 & 1,648 & 4.7403 & 36.8721 & 11.6692 \\
\hline 10.5 & 950 & 0.0861 & 1,627 & 4.2800 & 34.0125 & 10.7641 \\
\hline 11.5 & 950 & 0.0960 & 1,609 & 3.9292 & 31.7850 & 10.0592 \\
\hline 12.5 & 950 & 0.1050 & 1,593 & 3.6323 & 29.8870 & 9.4585 \\
\hline 12.9 & 950 & 0.1090 & 1,586 & 3.5068 & 29.0802 & 9.2032 \\
\hline
\end{tabular}




\subsubsection{Oxidized State Model}

Figures 4-40 and 4-41 show the maximum compressive and tensile stresses for two different locations, the edge and inside, indicated in Figure 4-42(b) and 4-42(c) for compressive stress and Figures 4-43(b) and 4-43(c) for tensile stress. From observing Figure 4-42(a) and 4-42(b), it can be seen that the bottom half of the pillar is slanted to the left, which creates a counter clockwise bending moment, causing compressive stress as shown in Figure 4-42(b) toward the left side and tensile stress as shown in Figure 4-42(b) toward the right side. In contrast, the top half of the pillar is straight, and because of the counter clockwise bending moment of the bottom half, the top half of the pillar is subjected to compressive stress on the right side and tensile stress on the left side. Local maximum near the edge occurs because the bending moment causes the greatest stress on the outer edge, which gets exacerbated by the decreasing cross-sectional area toward the edge because of the triangular shape. The stress is relieved toward the inner section where the cross-sectional area gets relatively larger.

Note that exceeding the strength limit on the edge does not necessarily lead to fracture, because, after edge portion crumbles, the stress get redistributed to the inner section, and the inner section's cross sectional area is most likely wide enough to handle the additional loading without much change in stress concentration. But, exceeding the mechanical strength of the inner section is a definite sign of fracture because fracture of the inner section results in significant loss of cross sectional area to handle the load.

The stress concentrations are increasing almost exponentially over time because they are inversely proportional to the cross sectional area, which is also decreasing over time because of corrosion. The compressive strength shown in Figure 4-40 and 4-41 is exceeded first on the edge after 11.5 days, and both mechanical strengths are exceeded on the inside after 12.9 days.

Maximum Compressive Stress

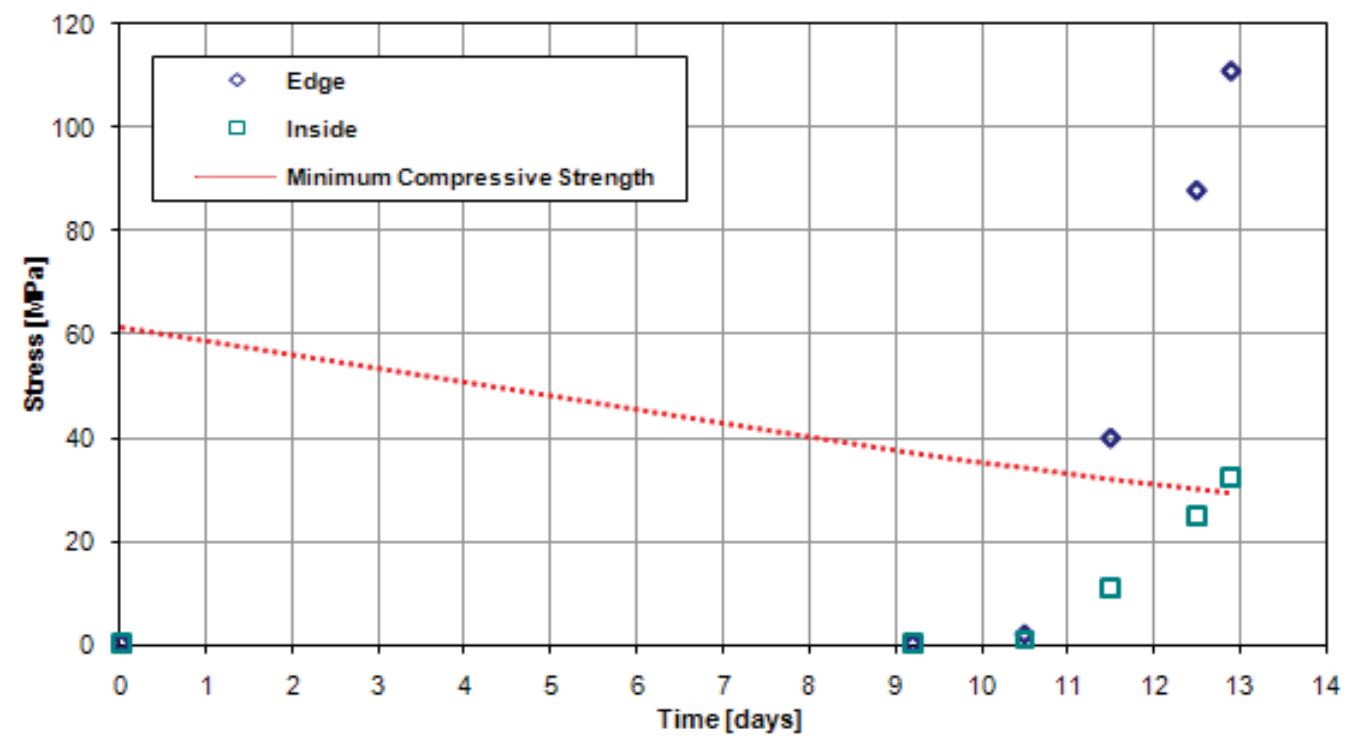

Figure 4-40. Maximum compressive stress over time. 


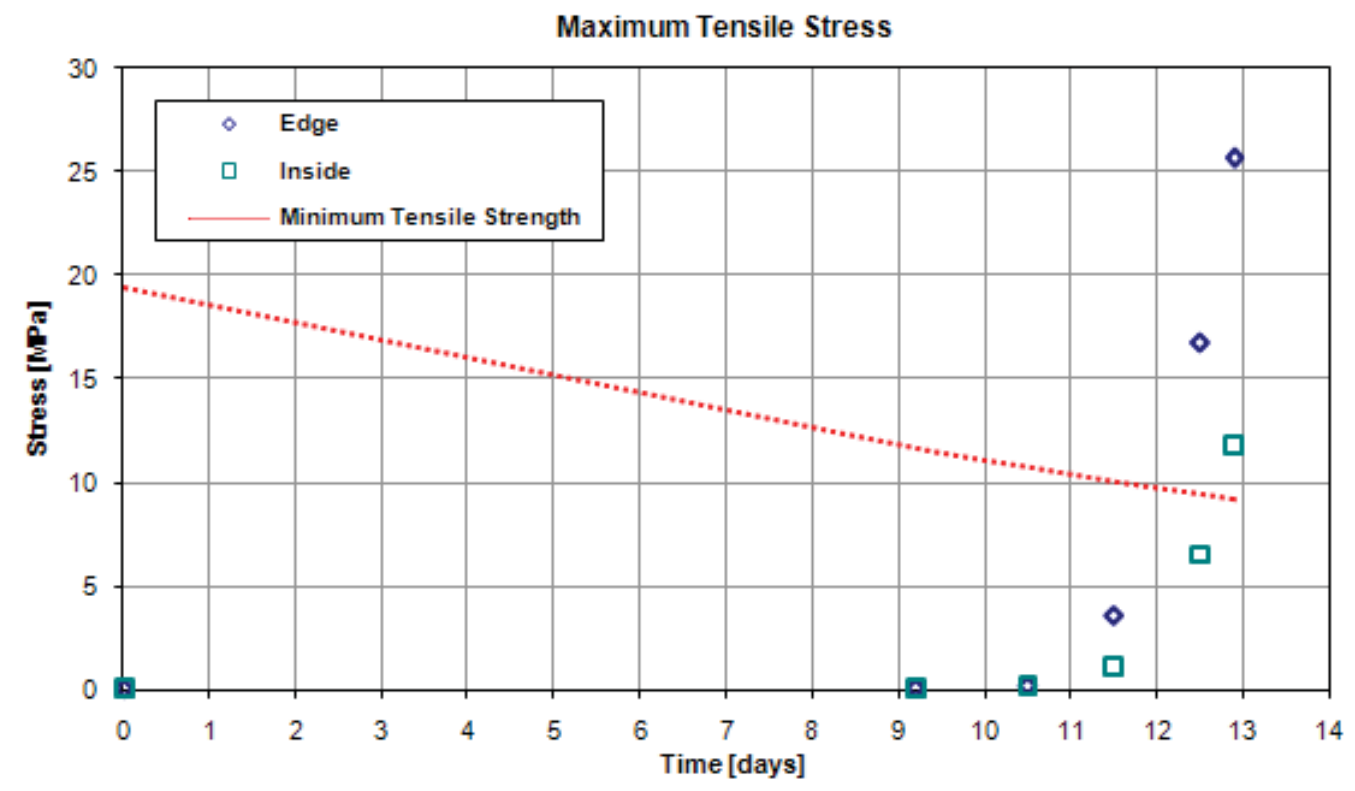

Figure 4-41. Maximum compressive tensile stress over time.

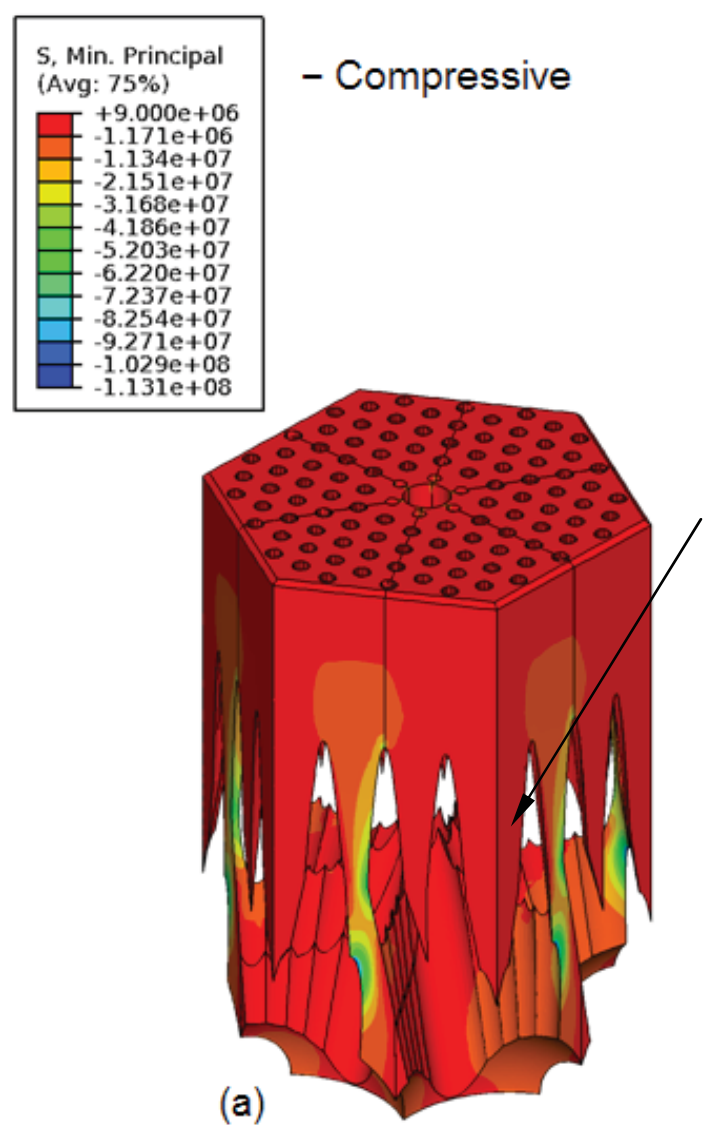

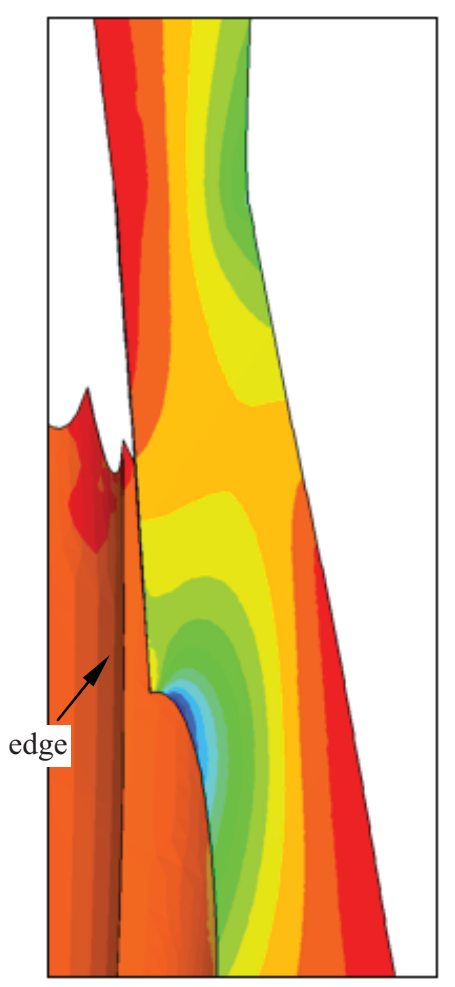

(b)

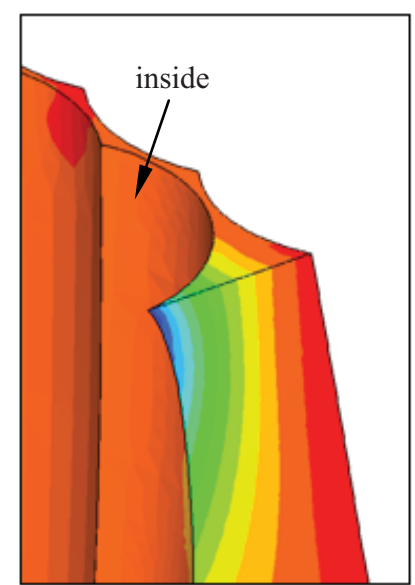

(c)

Figure 4-42. Compressive stress distribution of non-uniform corrosion model, 12.25 days after LOCA. 


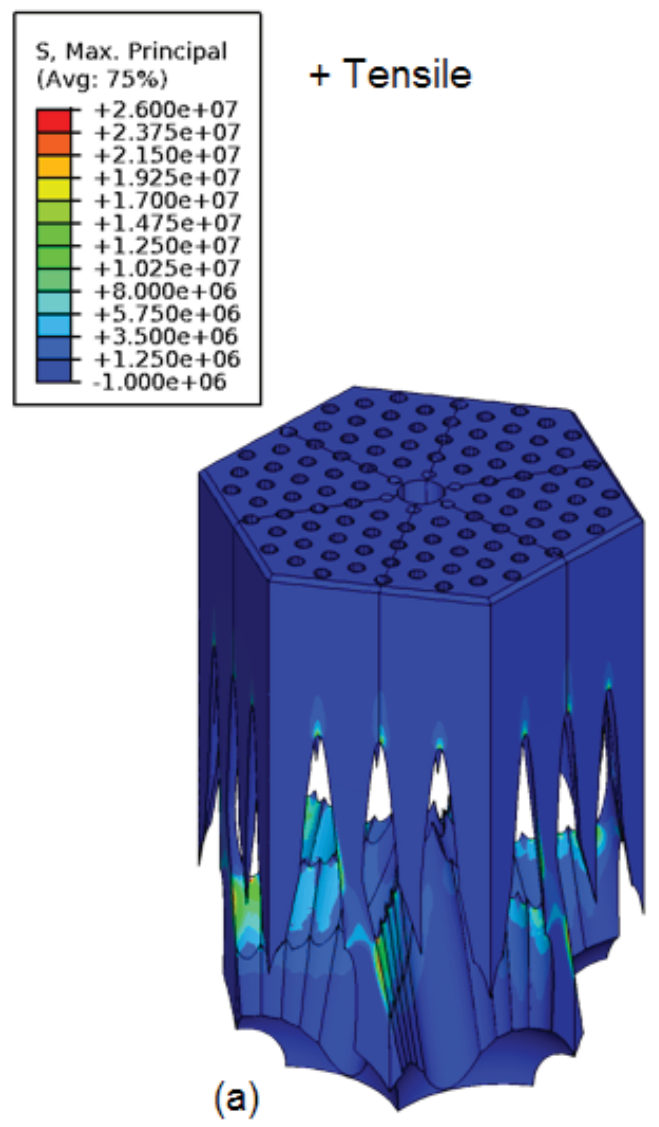

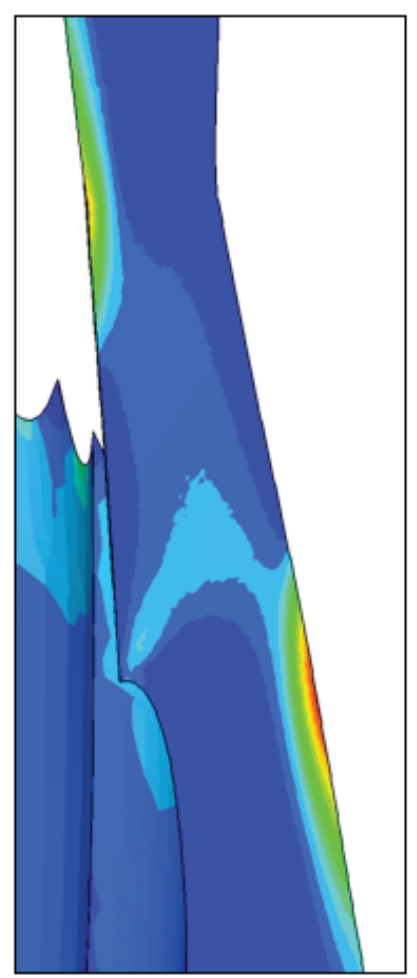

(b)

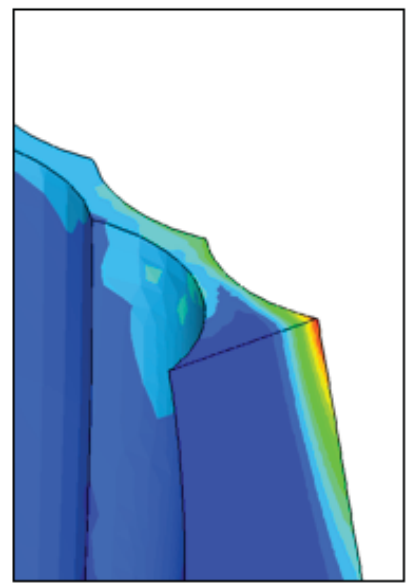

(c)

Figure 4-43. Tensile stress distribution of non-uniform corrosion model, 12.25 days after LOCA.

\subsection{Estimation of Conservative Burn-off Criteria for Graphite Structure}

Degradation of graphite structural integrity by oxidation is currently the subject of research in VHTR safety. The degradation is processed by two different mechanisms. Internal corrosion in the graphite pores lowers the graphite compressive strength with pore collapses, and the external corrosion leads to the stress concentration with overall size reduction. If left unabated, this degradation can result in the major fracture of core structures.

In this section, simple simulations were carried out to estimate how much graphite should be burned before it fails. So, this study is aimed at providing the maximum allowed burn-off limit in certain conditions and conservatively accounts for the additional thermal energy released in the oxidation reaction. There are some advantages in this approach. First, this method provides direct estimation of structural fracture in the VHTR core. The fracture criteria can be directly implemented in the code. Therefore, this method is much faster and convenient than the approach used in the previous section. Second, it provides much more conservative predictions than the previous section. If the calculation result is within our criteria, we can have some confidence for the graphite structures to be secure.

The modeling and simulation in this section is based on the 600 MWth GTMHR core design, but the same methodology can be applied to the other reactor types. Figure 4-44 shows the basic pattern in the GTMHR bottom reflector, which is the most vulnerable to oxidation damage from an air-ingress accident (Kim et al. 2008). In addition, the bottom reflector suffers from the highest mechanical stress on it. 


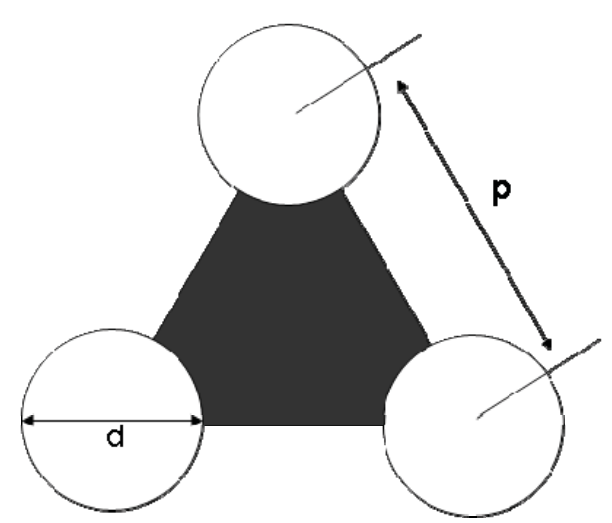

Figure 4-44. Unit block schematics.

Oxidation generally causes structural problems in two ways: the decrease of size will increase the stress concentration for unit surface area, and the increase of internal pore size will weaken the structure by decreasing its compressive strength. Eventually, if the stress exceeds the compressive strength, the structure can be considered fractured. Therefore, predicting graphite compressive strength and crosssectional surface area are the essential parts of estimating graphite fracture.

The changes of graphite compressive strength can be calculated by predicting the change of graphite density because density is the main variable of graphite's compressive strength. Changes in graphite cross-sectional areas can be calculated by predicting the corrosion depths of the flow channels. This study simply modeled the changes of the graphite density and cross-sectional area as detail below.

First, the oxidized mass of the outside (by external corrosion) and inside (by pore oxidation) can be respectively expressed as

$d M_{\text {sur }}=\rho \cdot(\pi r L) \cdot d r$

$d M_{\text {in }}=-A \cdot L \cdot d \rho$

where

$d M_{\text {sur }}=$ Oxidized mass change of graphite on the external surface $(\mathrm{kg})$

$d M_{i n}=$ Oxidized mass change of graphite in the inside of the pores $(\mathrm{kg})$

$\rho=$ Density of graphite $\left(\mathrm{kg} / \mathrm{m}^{3}\right)$

$r \quad=\quad$ Radius of the flow channel $(\mathrm{m})$

$L \quad=\quad$ Length of the channel (m)

$A=$ Cross-sectional area $\left(\mathrm{m}^{2}\right)$.

$d M_{\text {sur }}$ and $d M_{\text {in }}$ can be expressed by the total mass changes, $d M_{t o t}$ as

$d M_{\text {sur }}=f \cdot d M_{\text {tot }}$ 


$$
d M_{\text {in }}=(1-f) \cdot d M_{\text {tot }}
$$

where

$$
\begin{aligned}
& d M_{\text {tot }}=\text { Total oxidized mass change of graphite at a certain time }(\mathrm{kg}) \\
& f \quad=\text { Fraction of surface reaction to total reaction. }
\end{aligned}
$$

The fraction, $f$ in Eq. (4-9) and (4-10) is a very complicated function determined by oxidation and flow variables such as temperature, pressure, flow velocity, and oxygen concentration. Therefore,

Eq. (4-7) and (4-8) can be rewritten by

$$
\frac{d r}{d M_{t o t}}=\frac{f}{\rho \cdot(\pi r L)}
$$

and

$$
\frac{d \rho}{d M_{t o t}}=-\frac{(1-f)}{A \cdot L}
$$

The cross-sectional area $A$ in Eqs. (4-8) and (4-12), where the load is imposed on, can be expressed by

$$
A=\frac{\sqrt{3}}{4} p^{2}-\frac{1}{2} \pi r^{2} \text {. }
$$

The total graphite burn-off, $B_{t o t}$ can be defined as

$$
B_{t o t}=\frac{M_{t o t}}{\rho_{0} \cdot V_{0}}=\frac{M_{t o t}}{\rho_{0} \cdot A_{0} \cdot L}
$$

where

$$
\begin{aligned}
& M_{t o t}=\text { Total oxidized mass of graphite }(\mathrm{kg}) \\
& \rho_{0}=\text { Initial density of graphite }\left(\mathrm{kg} / \mathrm{m}^{3}\right) \\
& L \quad=\text { Length of the channel }(\mathrm{m}) \\
& A_{0} \quad=\text { Initial cross-sectional area }\left(\mathrm{m}^{2}\right)
\end{aligned}
$$

Therefore, the total oxidized mass is express by

$$
M_{\text {tot }}=\rho_{0} \cdot A_{0} \cdot L \cdot B_{\text {tot }} .
$$

If we normalized the density of the oxidized graphite at a certain time, it can be expressed by

$$
\rho^{\prime}=\frac{\rho}{\rho_{0}}
$$


Inserting Eqs. (4-13), (4-15), and (4-16) into Eqs. (4-11) and (4-12) derives the differential equations

$$
\frac{d A}{d B_{t o t}}=-\left(A_{0} f\right) \cdot\left(\frac{1}{\rho^{\prime}}\right)
$$

and

$$
\frac{d \rho^{\prime}}{d B_{t o t}}=-\left(A_{0}(1-f)\right) \cdot\left(\frac{1}{A}\right) .
$$

By solving those two differential equations, the changes of graphite cross-sectional area $(A)$ and normalized density $\left(\rho^{\prime}\right)$ can be estimated as a function of total graphite burn-off $\left(B_{t o t}\right)$. The stress on the structure can be estimated by the information of the cross-sectional area $(A)$, and the compressive stress can be estimated by the changes of the normalized densities $\left(\rho^{\prime}\right)$. The stress and the compressive strength can then be calculated as

$$
P=\frac{W_{0}}{A}
$$

where

$$
\begin{aligned}
& P=\text { Stress }(\mathrm{Pa}) \\
& W_{0}=\operatorname{Load} \text { on the structure }(\mathrm{N})
\end{aligned}
$$

and

$$
S=S_{0} \cdot \rho^{\prime \beta}
$$

where

$$
\begin{aligned}
& S=\text { Compressive Strength }(\mathrm{Pa}) \\
& S_{0}=\text { Initial compressive strength }(\mathrm{Pa}) \\
& \beta=\text { Constant depending on the graphite types. }
\end{aligned}
$$

The correlations between local density and compressive stress were obtained by Oh et al (2008). Figures 4-45 and 4-46 show the data for IG-110 and H451, respectively. According to the research, the constants, $\beta$ are 6.5 for IG-110, and 6.25 for $\mathrm{H} 451$ graphite. The initial compressive strength of those graphite materials are 70.5 and $52.7 \mathrm{MPa}$, respectively.

Criteria (a) represents that if the stress $(S)$ imposed on the structure exceeds the compressive strength $(P)$ of the oxidized graphite, it will fail. Criteria (b) represents that if the normalized density $(\rho$ ') exceeds a certain limit $\left(\rho^{\prime}\right.$ limit $)$, it will fail. The normalized density limit was obtained from the data measured by Oh et al. (2008). The burn-off level in which the graphite losses its mechanical strength was experimentally measured, which provided the data for IG-110 and H451 graphite. The normalized density limits are 0.35 and 0.18 for IG-110 and H451, respectively. Criteria (c) represents that if the adjacent two flow channels are collapsed, it will fail. 


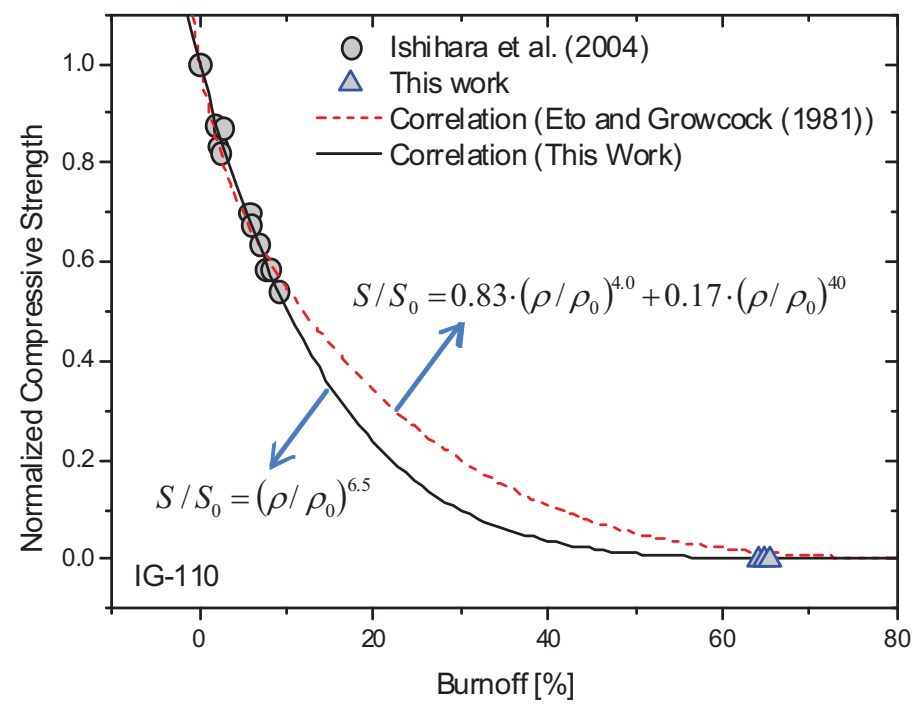

Figure 4-45. Relation between local burn-off and normalized compressive strength (IG-110).

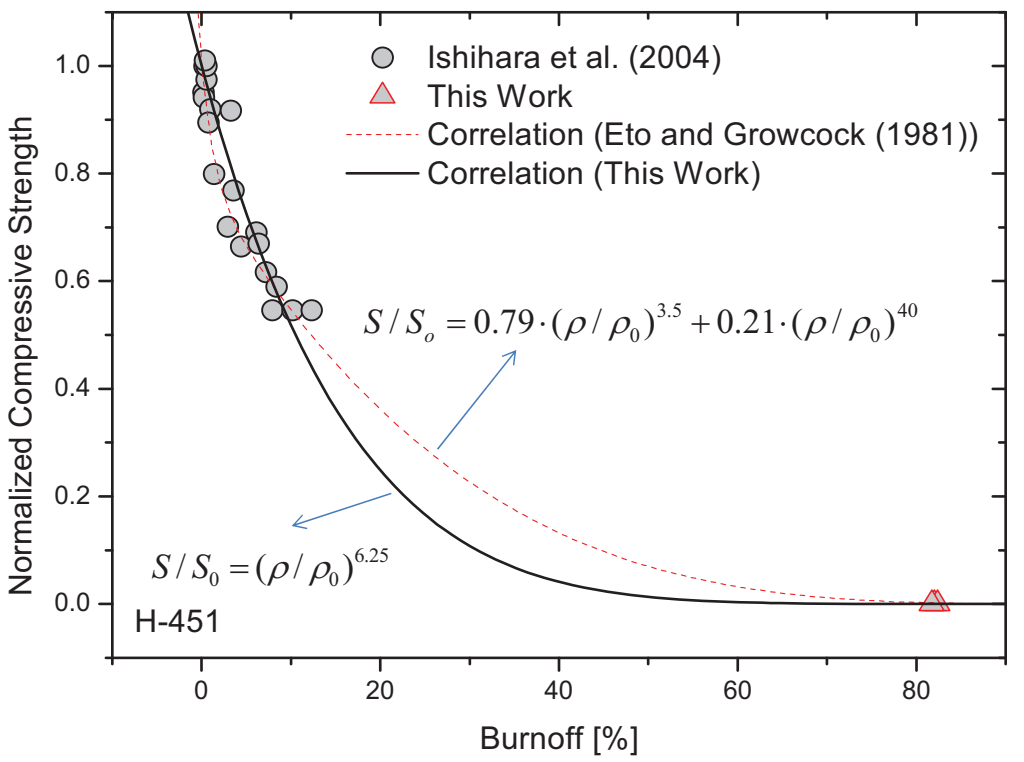

Figure 4-46. Relation between local burn-off and normalized compressive strength (H451).

To judge the fracture of the graphite structure, the following three criteria were used in this research:
a. $\quad \mathrm{S}>\mathrm{P}$
b. $\quad \rho^{\prime}<\rho^{\prime}$ limit
c. $\mathrm{p}<2$ r.

To simulate the fracture of the graphite structure, MATLAB SIMULINK was selected in this research. Figures 4-47, 4-48, and 4-49 show three different models developed: constant f, randomly sampled $\mathrm{f}$ (uniform distribution), and randomly sampled $\mathrm{f}$ (Gaussian distribution). The f-value represents the ratio of the external surface reaction to the total reaction. As mentioned, this value depends on various fluid and flow conditions, and therefore varies significantly during an air-ingress accident. This research 
randomly picked up the f value between 0 and 1 at every time-step because this value cannot be determined yet. Theoretically, randomly selected f value based on uniform distribution provides the largest uncertainty because the deviation from the mean value is the largest in this distribution. However, in the uniform distribution, the average f-value converges to 0.5 , which is the mean value of the probability distribution function. For this reason, the Gaussian distribution was also used with different mean values ranging from 0 to 1 . Statistically, the randomly selected f-value will cover all possible scenarios of air-ingress accidents. The constant $f$ value case, in which the $f$ value was maintained constant at every time-step, was used for the base data. Table 4-7 summarizes the simulation input parameters. In this simulation, the input parameters were determined based on the $600 \mathrm{MWth}$ GTMHR reactor.

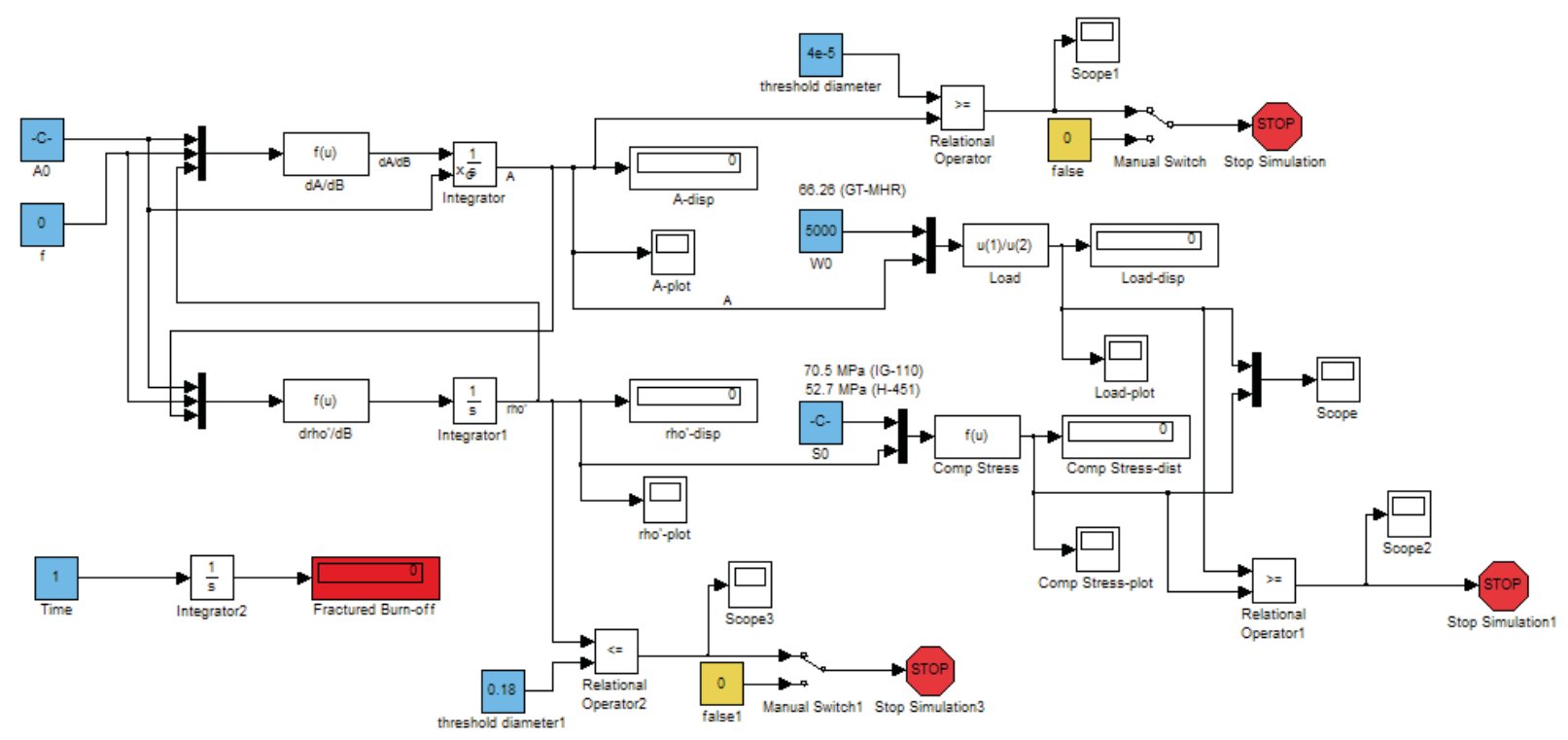

Figure 4-47. Modeling of graphite fracture by corrosion damage (constant f).

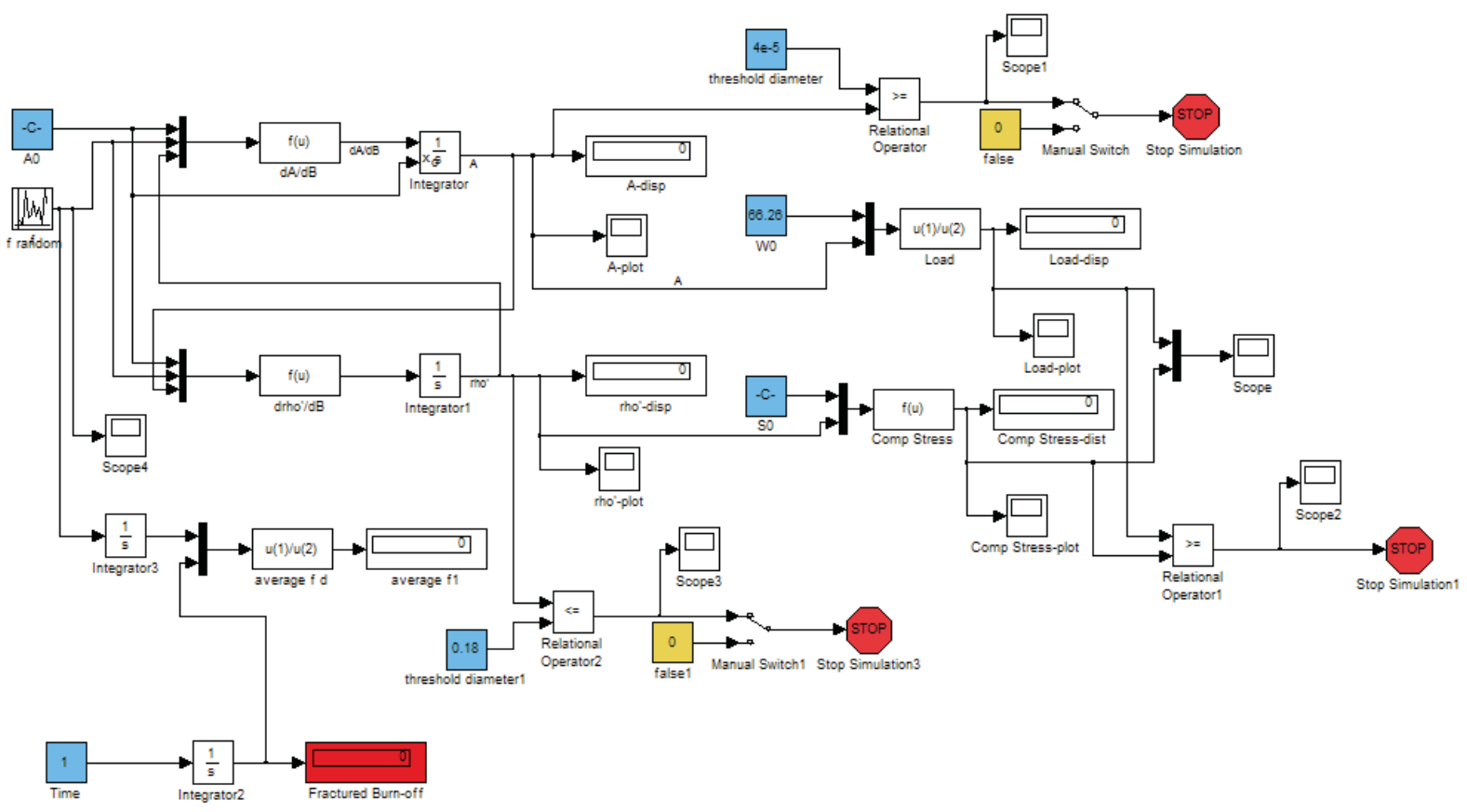

Figure 4-48. Modeling of graphite fracture by corrosion damage (randomly sampled $f$ [uniform]). 


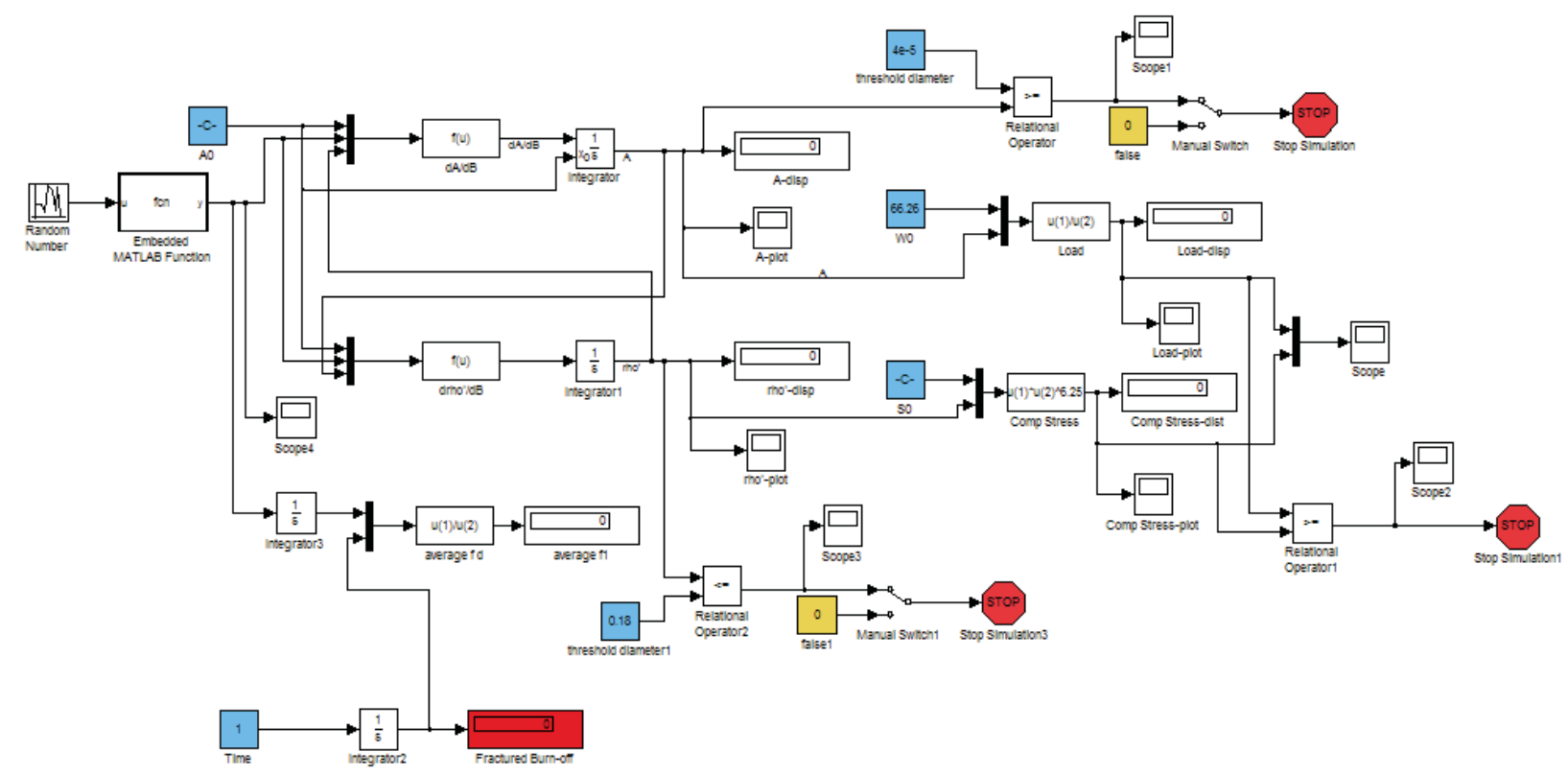

Figure 4-49. Modeling of graphite fracture by corrosion damage (randomly sampled f [Gaussian]).

Table 4-7. Input parameters for graphite oxidation and structural integrity.

\begin{tabular}{|l|l|}
\hline \multicolumn{1}{|c|}{ Parameters } & \multicolumn{1}{c|}{ Values } \\
\hline Initial cross-sectional area $\left(\mathrm{m}^{2}\right)$ & 0.00037 \\
\hline Load for a unit cell $(\mathrm{N})$ & 66.26 \\
\hline Initial compressive strength $(\mathrm{MPa})$ & $70.5(\mathrm{IG}-110), 52.7(\mathrm{H} 451)$ \\
\hline
\end{tabular}

Figure 4-50 shows the calculated data for f-values $(f)$ vs. total fractured burn-off $\left(B_{t o t}\right)$ based on the IG-110 graphite properties. In this calculation the maximum allowable burn-off $(0.6)$ was viewed at $\mathrm{f}$-value $=0$, meaning that the graphite becomes the most vulnerable to the oxidation damages when the whole reaction is dominated in the inside pores. As the f-value increases, the fractured burn-off also increases almost linearly. It indicates that the reduction of compressive strength by internal corrosion plays a significant role in the graphite fracture mechanism. The allowable burn-off was estimated to be 0.93 at $\mathrm{f}=0.8$. After $\mathrm{f}=0.8$, the fractured burn-off was estimated to decrease because of different fracture criteria (Criteria (c): flow channel collapsing). Thus, at large f values, the external surface reaction plays a main role in the fracture because at even very high burn-offs, the compressive strength of the graphite has not reached the maximum stress imposed on the structure.

Figures 4-50 and 4-51 show that the data obtained from random $\mathrm{f}$ values are in good agreement with those from constant $\mathrm{f}$ values, within a $4 \%$ maximum error (red line), so conservatively, the maximum allowable total burn-off can be determined from the lowest value of the lower red line. The estimated allowable burn-off is 0.58 with a $99 \%$ confidence level. It indicates that the graphite structure will not fail at lower than 0.58 in total burn-off. 


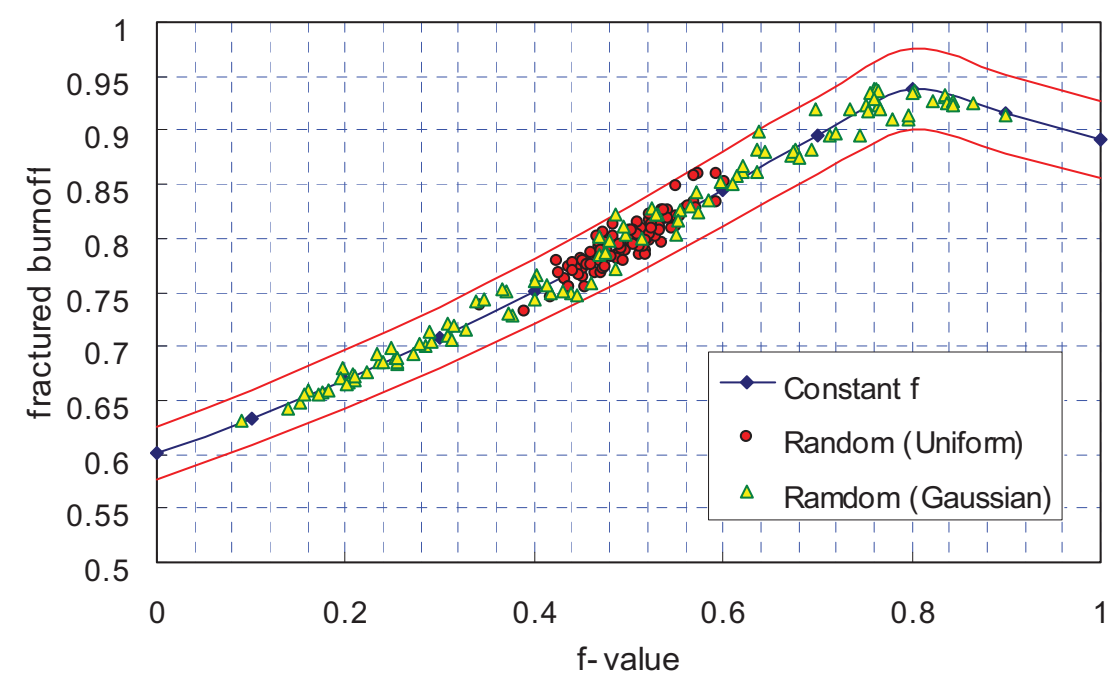

Figure 4-50. Relations between f-value ( $f$ ) vs. fractured total burn-off (IG-110).

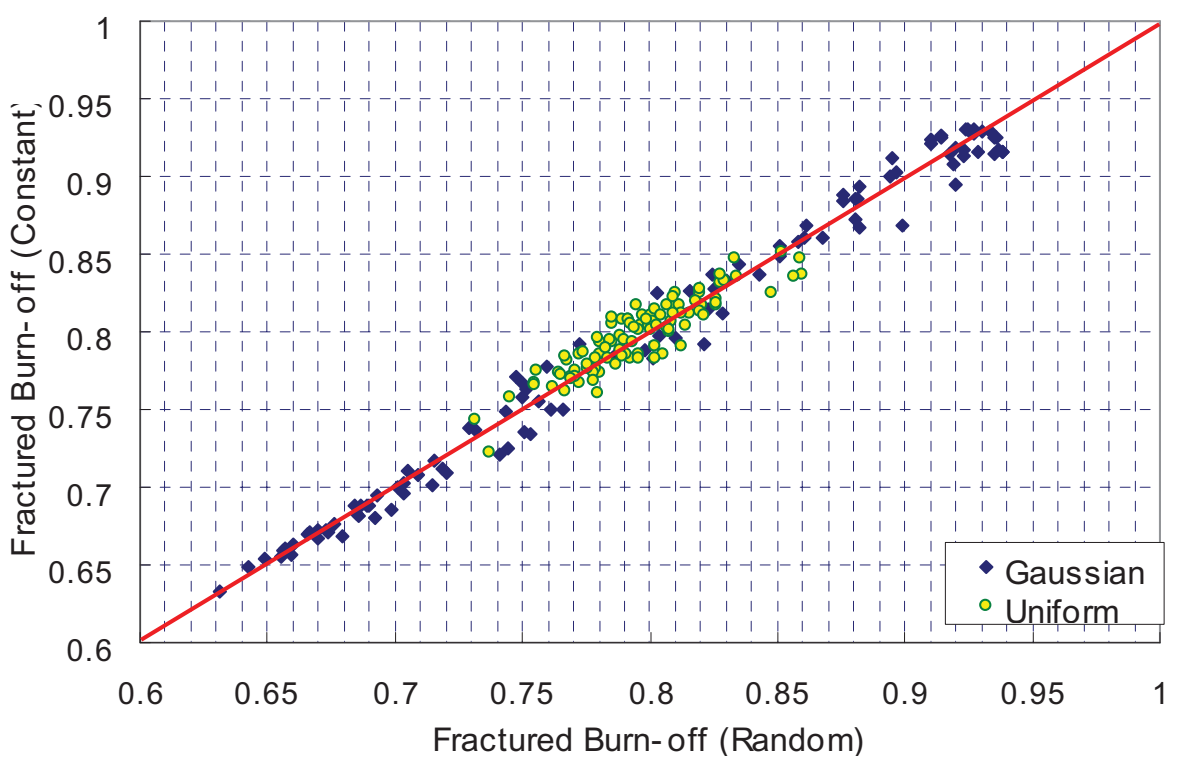

Figure 4-51. Fractured burn-off (Random f) vs. fractured burn-off (constant f) - IG-110.

Figures 4-52 and 4-53 show the calculated results for H451 graphite. The same methodologies used in Figures 4-50 and 4-51 were applied to these analyses. The results show that the minimum allowable total burn-off for this material is 0.57 , almost the same as that of the IG-110 graphite. 


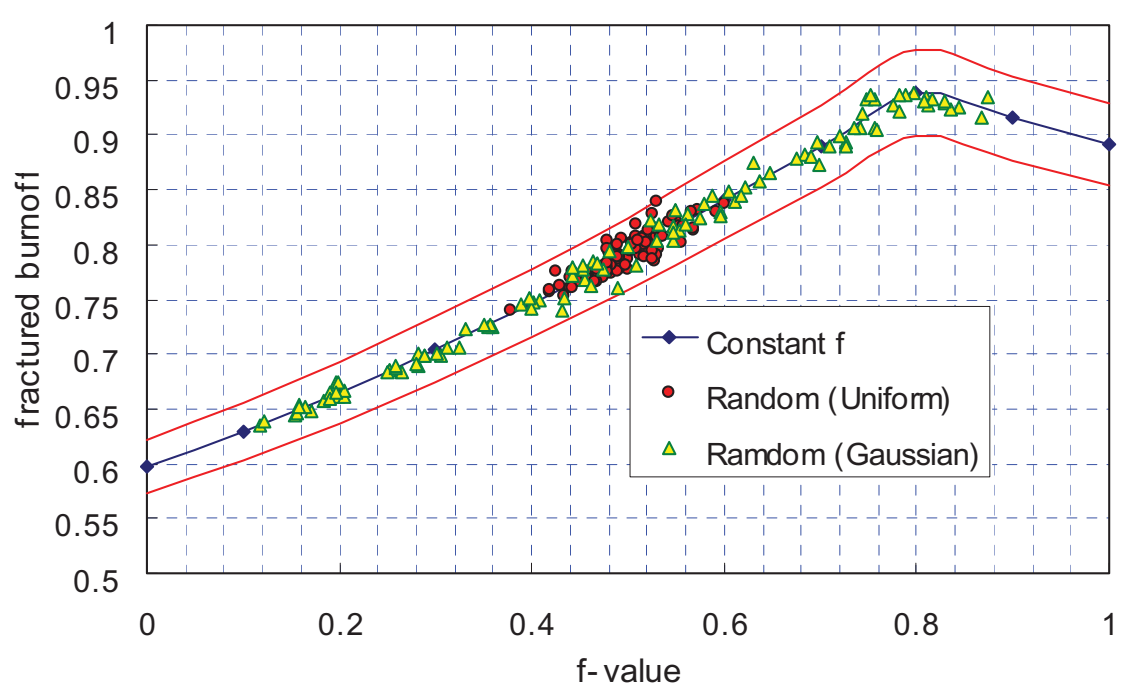

Figure 4-52. Relations between f-value ( $f$ ) vs. fractured total burn-off (H451).

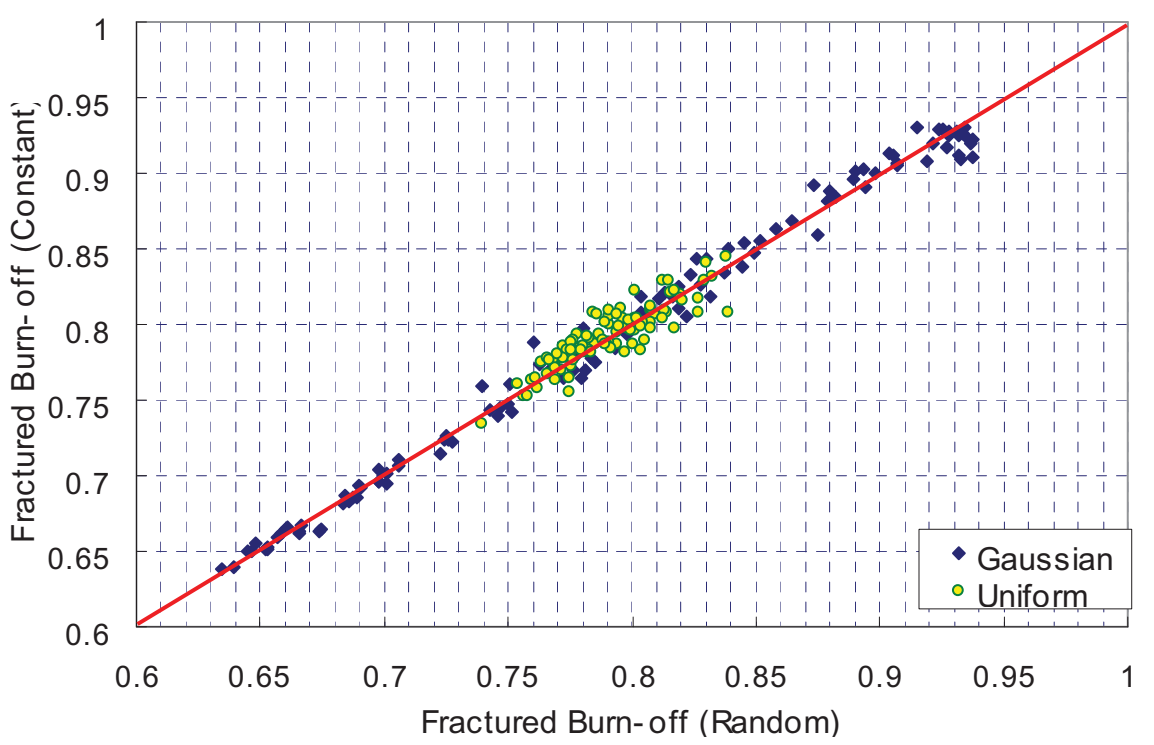

Figure 4-53. Fractured burn-off (random f) vs. fractured burn-off (constant $\mathrm{f}$ ) - H451.

\subsection{Implementation of Advanced Graphite Oxidation Model into GAMMA code and Analyses}

The original graphite oxidation models in the GAMMA code have been updated. In the updated models, the reaction kinetics, mass transfer, moisture, burn-off, and conservative fracture criteria have been considered. The following summarizes the models.

The overall graphite oxidation rate is affected by two mechanisms; reaction kinetics and mass transfer. At temperatures below about $700^{\circ} \mathrm{C}$, reaction kinetics dominates the oxidation rate. Therefore, the overall rate follows Arrhenius model very well at this range. However, at high temperature above about $950^{\circ} \mathrm{C}$, the oxidation rate is dominated by the mass transfer of oxygen molecules in the working fluids. In the intermediate temperature ranges, the oxidation rate is affected by both kinetics and mass transfer (See Figure 4-54). 


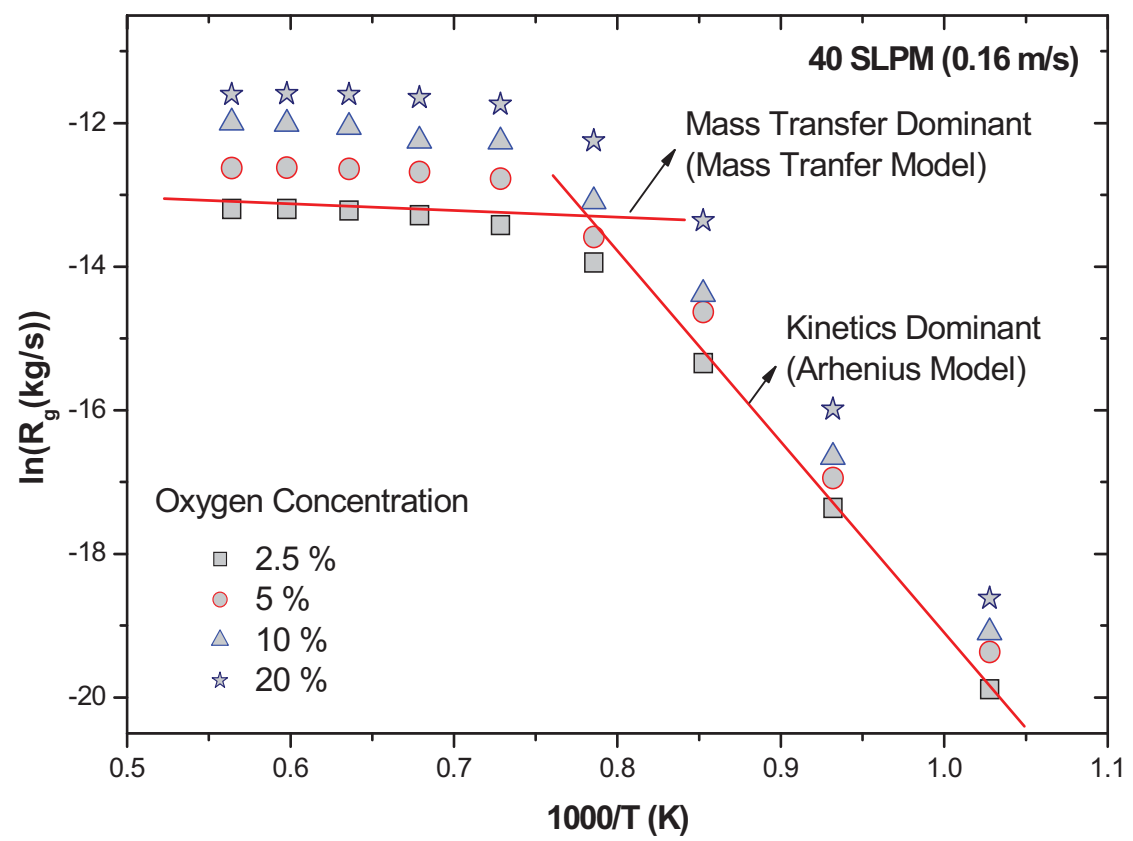

Figure 4-54. Overall graphite oxidation rate (Oh et al. 2006).

The overall graphite oxidation rate can be determined by (Oh et al. 2006)

$$
\frac{1}{R_{g}}=\frac{1}{R_{k}}+\frac{1}{R_{m}}
$$

where:

$R_{g} \quad=$ Overall graphite oxidation rate $(\mathrm{kg} / \mathrm{s})$

$R_{k} \quad=\quad$ Graphite oxidation rate estimated by Arrhenius model $(\mathrm{kg} / \mathrm{s})$

$R_{m} \quad=$ Graphite oxidation rate estimated by mass transfer $(\mathrm{kg} / \mathrm{s})$.

$R_{k}$ and $R_{m}$ in Eq. (4-21) can be determined by the following equations. The oxidation rate by kinetics effect can be calculated by the Arrhenius equation form (Oh et al. 2006)

$$
R_{k}=2552000 \cdot \exp \left(-\frac{218,000}{R \cdot T}\right) \cdot p_{O 2}^{0.75} \cdot M_{B}(B) \cdot \theta_{0} \cdot V
$$

where

$$
\begin{aligned}
& R=\text { Gas constant }(8.315 \mathrm{~kJ} / \mathrm{kmol} \mathrm{K}) \\
& T \quad=\text { Graphite surface temperature }(\mathrm{K}) \\
& P_{O 2}=\text { Oxygen partial pressure }(\mathrm{Pa}) \\
& M_{B}(B)=\text { Multiplication factor involved in the burn-off degree }
\end{aligned}
$$


$\theta_{0}=$ Graphite initial surface density $\left(\mathrm{m}^{2} / \mathrm{m}^{3}\right)$

$V \quad=\quad$ Apparent volume of the graphite structure.

The multiplication factor, $M_{B}(B)$ in Eq. (4-22) can be determined by the relationship between the burn-off and oxidation rate shown in Figure 4-55.

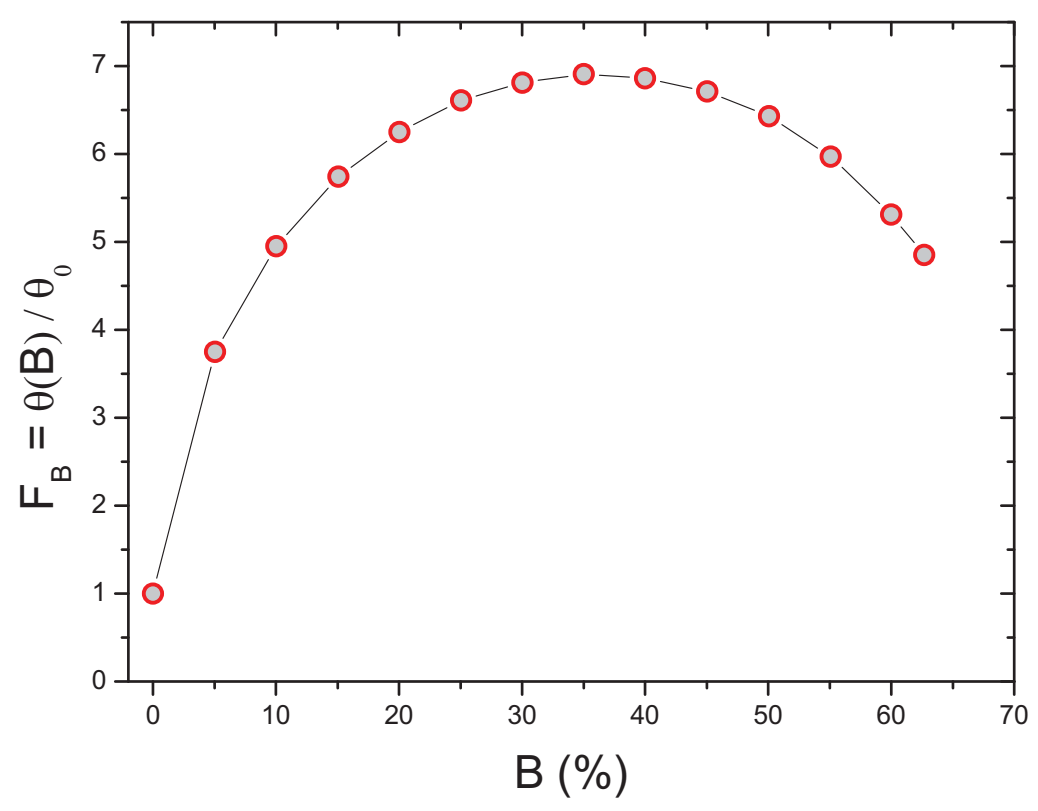

Figure 4-55. Relationship between burn-off (\%) and multiplication factor (Oh et al. 2008).

The burn-off $(B)$ in this figure is defined by

$$
B=\frac{\rho_{0}-\rho(t)}{\rho_{0}}
$$

where

$$
\begin{aligned}
& \rho_{0}=\text { Initial graphite density }\left(\mathrm{kg} / \mathrm{m}^{3} \mathrm{~s}\right) \\
& \rho(t)=\text { Graphite density at time, } \mathrm{t}\left(\mathrm{kg} / \mathrm{m}^{3} \mathrm{~s}\right) .
\end{aligned}
$$

Graphite oxidation rate by the mass transfer can be calculated by the general mass transfer model

$$
R_{m}=2 M_{c} \cdot K_{m}\left(C_{O_{2}, \infty}-C_{O_{2}, 0}\right) \cdot A
$$

where

$$
\begin{aligned}
& M_{c}=\text { Molecular mass of carbon, } \mathrm{C}(12 \mathrm{~kg} / \mathrm{kmol}) \\
& K_{m}=\text { Mass transfer coefficient of oxygen }(\mathrm{m} / \mathrm{s}) \\
& C_{O_{2}, \infty}=\text { Oxygen concentration in the bulk flow }\left(\mathrm{kmol} / \mathrm{m}^{3}\right)
\end{aligned}
$$




$$
C_{O_{2}, 0}=\text { Oxygen concentration at the graphite surface }\left(\mathrm{kmol} / \mathrm{m}^{3}\right) \text {. }
$$

In the mass transfer dominant condition in which the temperature is very high, the surface concentration of the oxygen, $C_{O 2,0}$ is assumed to be zero. The mass transfer coefficient, $K_{m}$ is a function of flow parameters; temperature, pressure, and velocities. When the effect of moisture is considered, the mass transfer rate is corrected as (Kim et al. 2008)

$$
R_{m, \text { moist }}=0.5 \cdot R_{m} \text {. }
$$

The rate of graphite oxidation is affected by an oxidation history following density and overall size changes. It is therefore necessary to track the density and the size of the graphite structure in calculation. The changes of graphite overall size and density can be estimated by (Kim et al. 2008)

$$
\frac{d \rho(t)}{d t}=-(1-f) \cdot R_{g} / V(t)
$$

and

$$
\frac{d z(t)}{d t}=\frac{1}{A(t)} \cdot \frac{f \cdot R_{g}}{\rho(t)}
$$

where

$f \quad=\quad$ Ratio of external reaction rate to total reaction rate

$V(t)=$ Apparent volume of graphite structure at time, $\mathrm{t}\left(\mathrm{m}^{3}\right)$

$z(t)=$ Corrosion depth of the graphite structure $(\mathrm{m})$

$A(t)=$ Apparent reacting surface area of graphite $\left(\mathrm{m}^{2}\right)$.

Eqs. (4-21) and (4-22) calculate the density changes and corrosion depth changes by oxidation, respectively. The volumes and surface areas are updated in each time-step based on the original geometries and the corrosion depth calculated. The ratio of external reaction to total reaction can be calculated by (Kim et al. 2008)

$$
f=\frac{1 / R_{m}}{1 / R_{k}+1 / R_{m}} .
$$

As mentioned in previous sections, one of the important issues in VHTR safety is fracture of the graphite structure by oxidation damages during the air-ingress accident. The oxidation damages generally degrade the structural integrity of graphite in two ways. Internal oxidation in the graphite inside pores degrades the graphite's compressive strength, which is a resistive force under the compressive force. External oxidation in the graphite structure outside concentrates the stress on the smaller region by reducing the cross-sectional surface area where the compressive force is imposed. In Section 4.3, the minimum allowable total burn-off for IG-110 and H451 was estimated to be 0.58 and 0.57 , respectively.

The total burn-off can be calculated and updated in the computer codes by

$$
\frac{d M_{g}(t)}{d t}=-R_{g}
$$


and

$$
B_{t}(t)=\frac{\rho_{0} V_{0}-M_{g}(t)}{\rho_{0} V_{0}}
$$

where

$$
\begin{aligned}
& M_{g}=\text { Graphite mass in the unit cell }(\mathrm{kg}) \\
& B_{t}=\text { Total graphite burn-off. }
\end{aligned}
$$

All the graphite oxidation models were implemented into the GAMMA system analyses code. Figure 4-56 shows the flowchart of advanced graphite oxidation models in the upgraded GAMMA code.

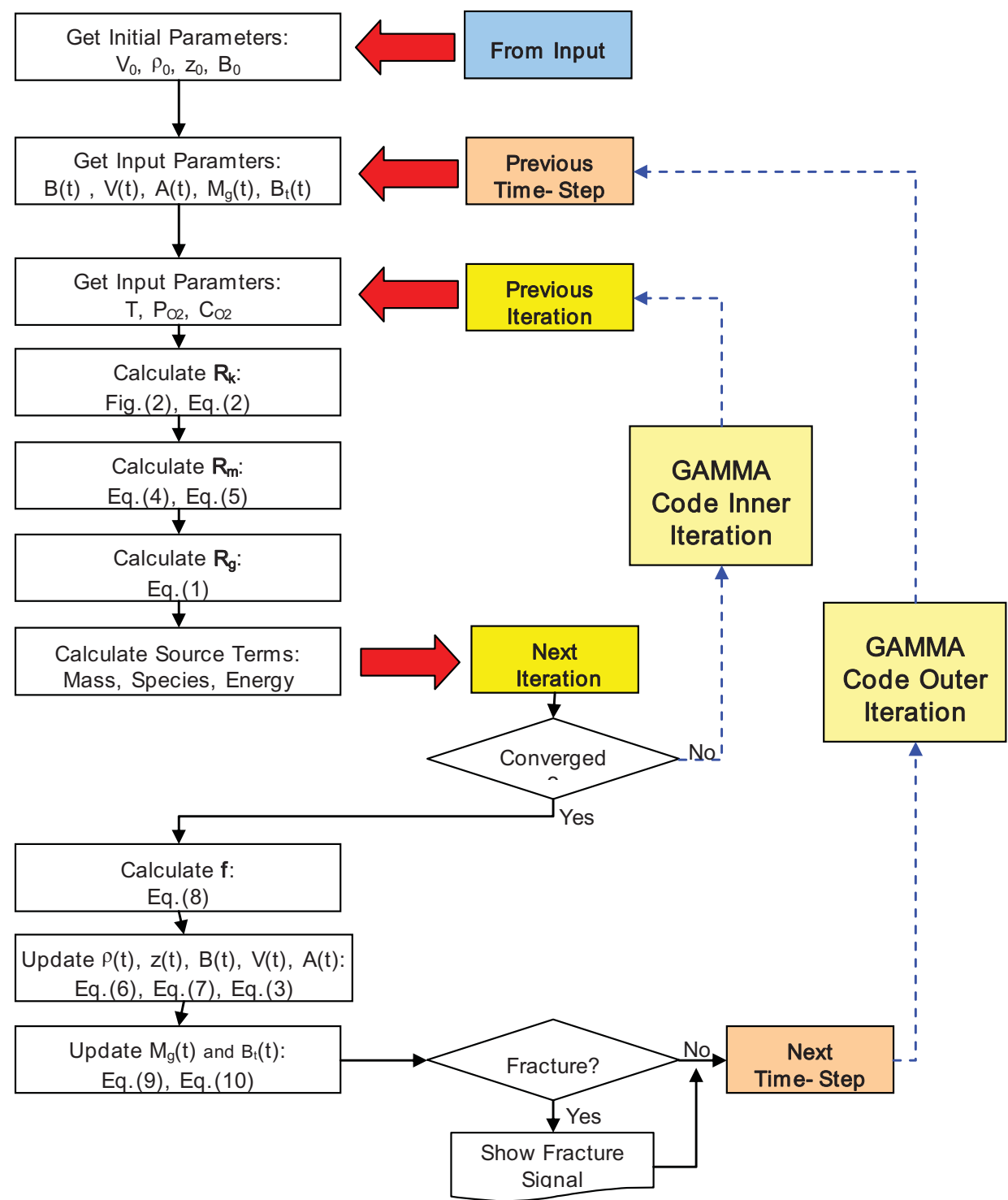

Figure 4-56. Flowchart of the advanced oxidation model. 
The air-ingress analyses in this section were performed by the GAMMA code with the upgraded graphite oxidation model. The initial conditions of air-ingress after onset-natural-circulation were estimated by the FLUENT code. For this calculation, FLUENT code output data such as quasi-steady state value of air distribution, temperature, and flow rate were implemented as initial GAMMA code values.

Figure 4-57 shows the code input nodalization where 2-D geometry models are used for the reactor cavity to consider the heat removal by natural convection flow, and for the solid structures including the core and reflector blocks to consider multidimensional heat conduction. Heat transport in the prismatic core is greatly complicated by the combined effect of solid conduction in the fuel, the graphite matrix and gas, and contact conduction and radiation in the fuel and fuel block gaps. In this simulation, the coolant channel and the fuel compact were separately treated by 1-D fluid equations and 2-D heat conduction equation from the graphite matrix, respectively. A porous media approach was applied to the reactor core, reflector, and plenum regions. The radiation heat exchanges were considered in every cavity and plenum. The air-cooling reactor cavity cooling system was modeled using the 1-D pipe network for the air flow loop and the 3-D tube model for the cooling tubes. Following the accident, since a reactor trips immediately, the core power is determined directly from the decay heat curves.

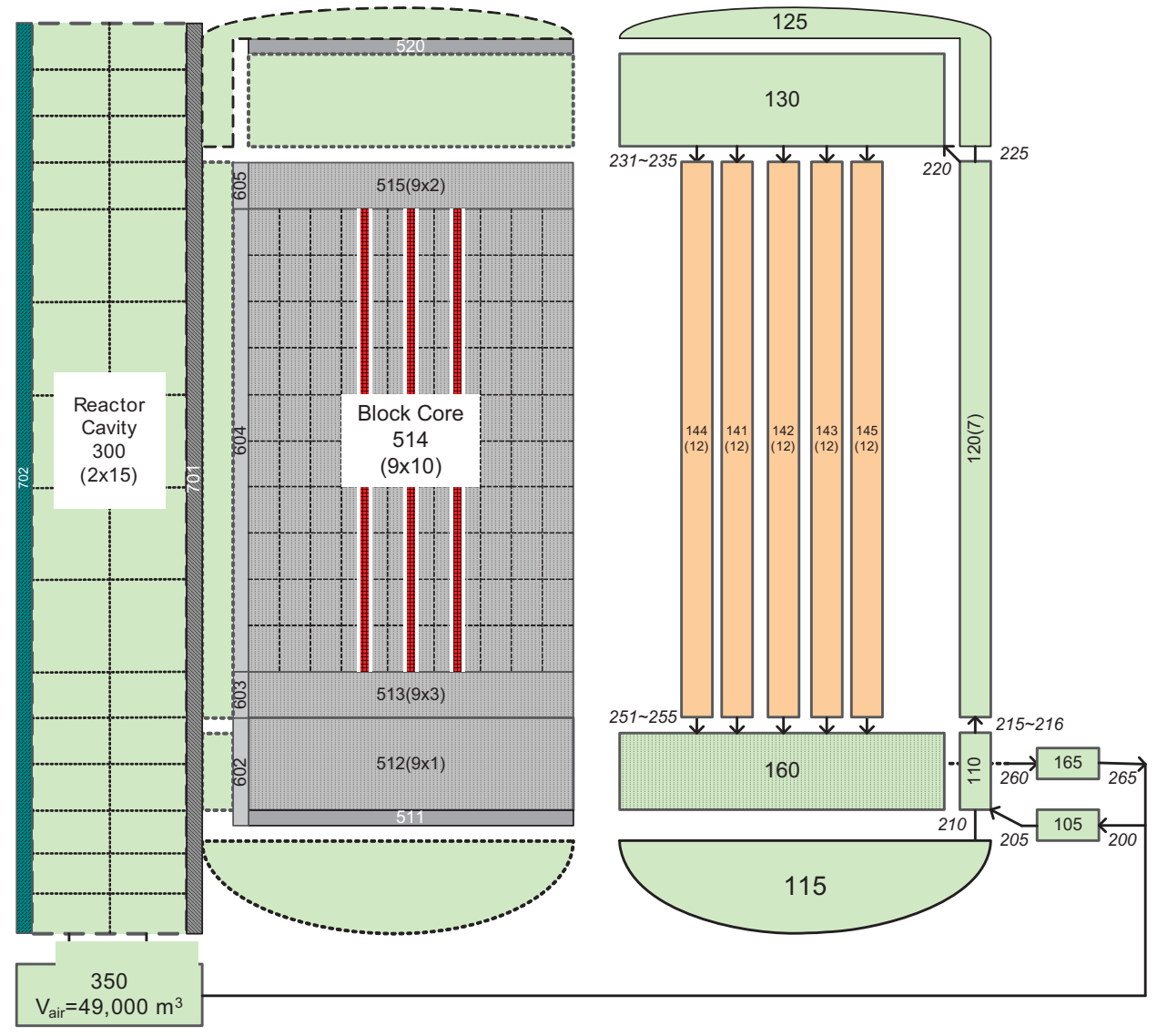

Figure 4-57. 600 MWth GTMHR code nodalization (GAMMA).

A summary of the modeling follows:

- $\quad$ 1-D (previous calculation), 2-D (current) flow modeling, 2-D axi-symmetric solid modeling

- $\quad$ Six gas species $\left(\mathrm{He}, \mathrm{O}_{2}, \mathrm{~N}_{2}, \mathrm{CO}_{2}, \mathrm{CO}, \mathrm{H}_{2} \mathrm{O}\right)$

- Irradiation/radiosity method at the cavity and plenums 
- Porous media in the core and plenum

- Natural convection in the cavity

- Infinite air inventory in the cavity.

Figure 4-58 shows the results of the core maximum temperature with time where the core maximum temperature does not exceed $1600^{\circ} \mathrm{C}$, the temperature limit in the VHTR core for fuel security. This result is consistent with that obtained in FY 2008.

Figure 4-59 shows the total burn-off variations in the bottom reflector, in which the graphite is the most seriously damaged. In this analysis, the graphite was assumed to be IG-110, isotropic grade graphite produced by Toyo Tanso Co., Ltd. The red line in each figure shows the minimum allowable burn-off, which is 0.58 for IG-110. Figure 4-59 also shows that the total burn-off goes beyond the fracture criteria after 88 hours, indicating that the graphite supporting structure risks fracture at about 88 hours after depressurization. Figure 4-60 shows the variations of graphite total burn-off in the lower plenum with time. According to this calculation, the lower plenum was estimated to start having a risk of fracture at about 112 hours after depressurization.

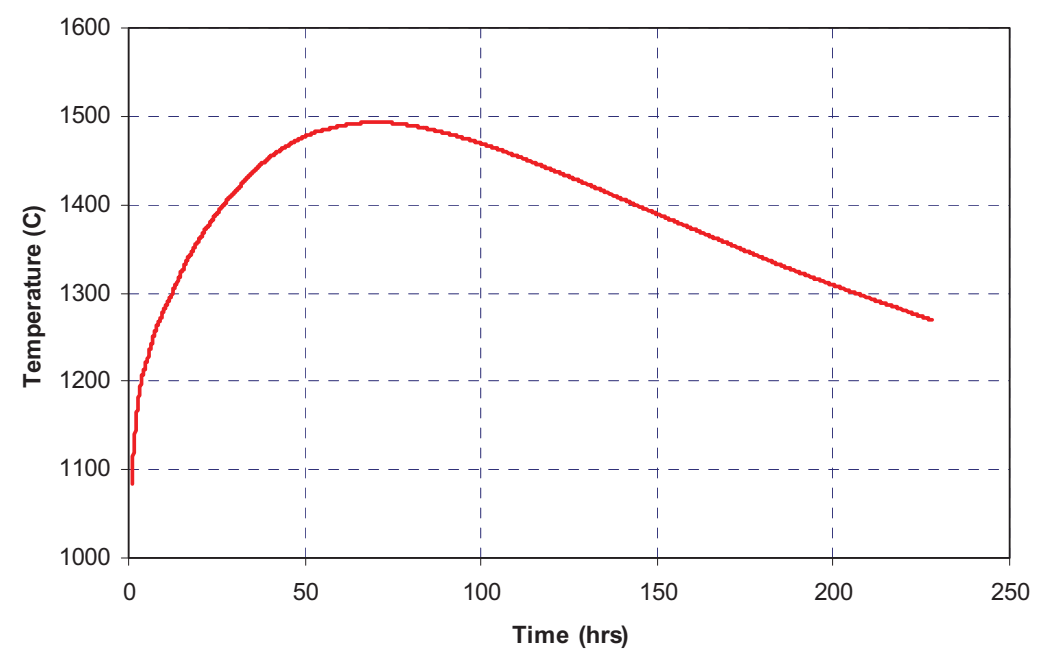

Figure 4-58. Time vs. core maximum temperature.

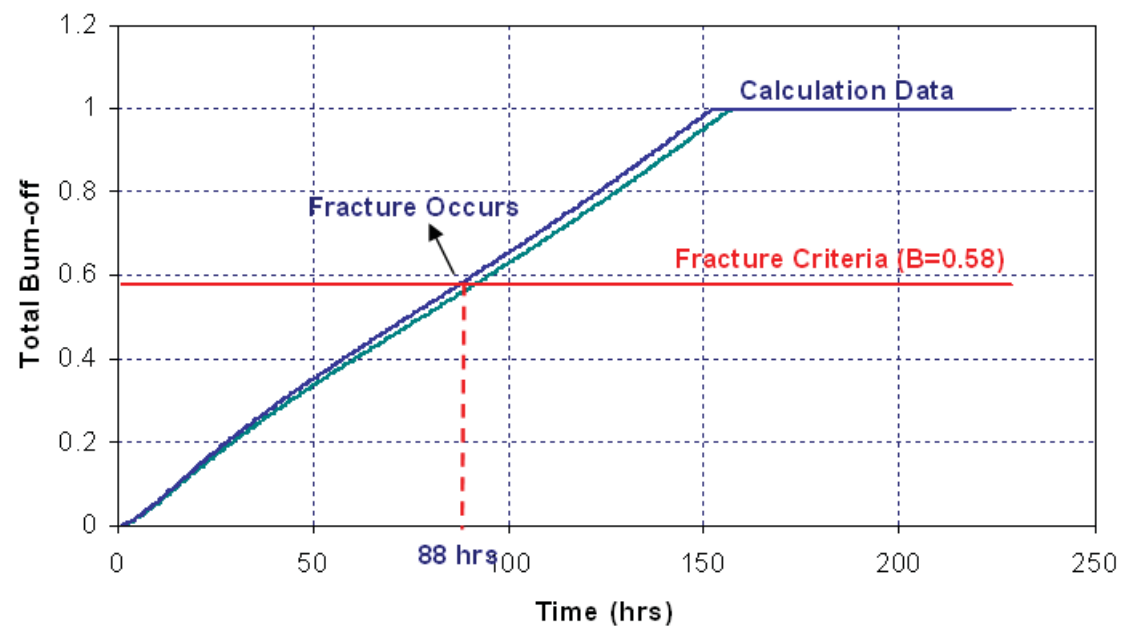

Figure 4-59. Time vs. total burn-off (bottom reflector - IG-110). 


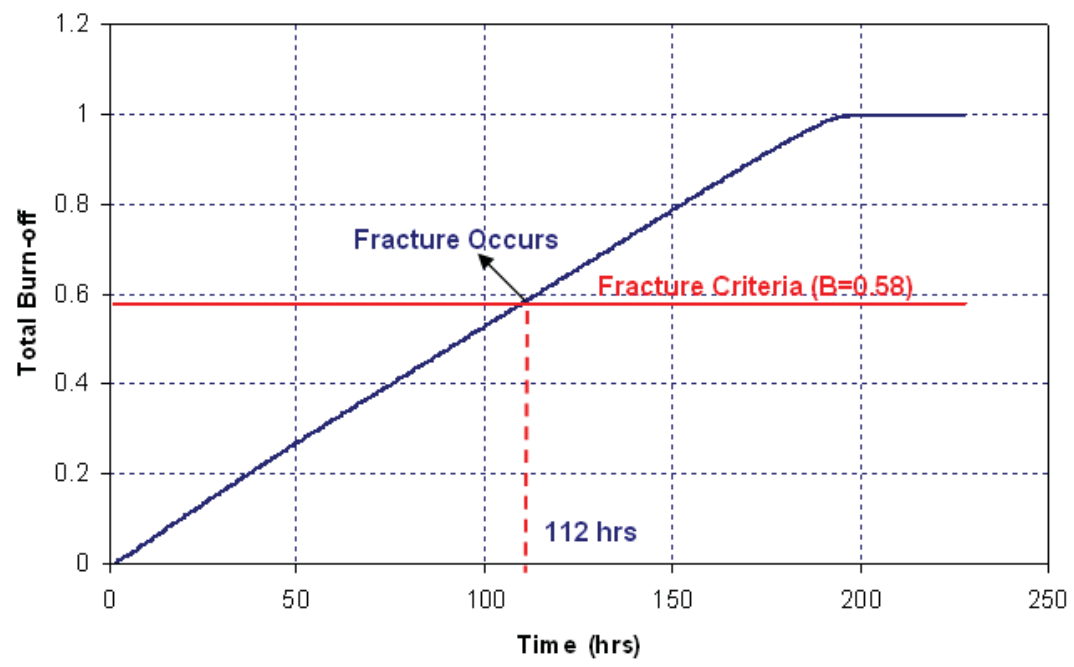

Figure 4-60. Time vs. total burn-off (lower plenum - IG-110).

Figure 4-61 shows the comparisons of fracture between IG-110 and H-451 graphite. According to this figure, IG-110 and H-451 are estimated to have failed at 88 hours and 105 hours, respectively, indicating that the H-451 graphite is a little bit more secure than the IG-110 graphite. The main reason is that $\mathrm{H}-451$ is less oxidizable than IG-110, especially in the higher burn-offs.

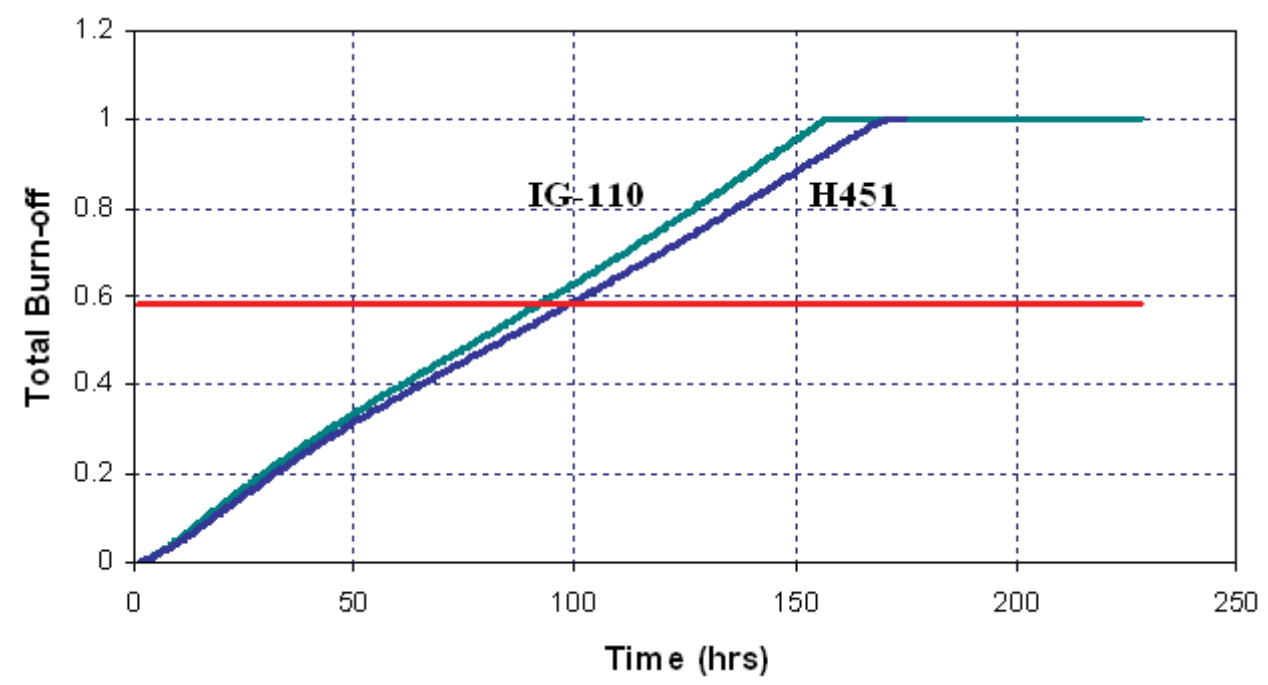

Figure 4-61. Comparisons of IG-110 and H-451 for fracture at the bottom reflector. 


\section{TASK 4: AIR INGRESS MITIGATION STUDY}

A LOCA, which can cause depressurized conduction cool down, is considered a critical event for a VHTR. Following helium depressurization, it is anticipated that unless countermeasures are taken, air will enter the core through the break leading to oxidation of the in-core graphite structure. Thus, without mitigation features, a LOCA will lead to an air ingress event, which may lead to exothermic chemical reactions of graphite with oxygen. Under extreme circumstances, a loss of core structural integrity may occur along with excessive release of radiological inventory. The rate of graphite oxidation and the likelihood of extensive structural damage can be assessed with a combination of analytical investigation, simulations of simplified core models, and experimental validations.

This task studied air ingress mitigation concepts. Some important factors affecting an air-ingress accident were first determined using a root-cause diagram. Overall air-ingress mechanisms and their relationships are also studied here, followed by an explanation of some previously recommended airingress mitigation concepts. Based on recent findings, the validity of those methods are reevaluated and discussed in more detail. Some possible air-ingress mitigation methods are also introduced and explained and some of them are validated by computational methods. Finally, two air-ingress mitigation methods are recommended as potential candidates.

\subsection{Previous Air-ingress Mitigation ideas and Discussions}

Some air-ingress mitigation concepts are recommended by some investigators, the helium injection method currently being one of the most well-known ideas. This concept is based on the counter-diffusion process first recommended by Yan et al (2008). The idea is to inject helium into the top of the VHTR vessel through an orifice located downstream of the helium storage tank. CFD simulations were used to validate this method. After reviewing their work for this project, it was concluded that it may not work if a DEGB occurs in the horizontal pipe. The reasons for this conclusion are summarized in this section.

An axi-symmetricity was assumed using the Gas Turbine High Temperature Reactor 300 (GTHTR300) design. As shown in Figure 5-1, the GTHTR300 has a cross duct with a $2 \mathrm{~m}$ diameter. When an axi-symmetric assumption was made in Yan's calculation, the inlet pipe surface area did not represent the actual cross duct and was therefore simulated based on a thin slit geometry instead of a $2 \mathrm{~m}$ diameter inlet pipe size.

The momentum created by the top helium injection was calculated using the following equation and the supersonic velocity helium through the orifice flow area of $0.011 \mathrm{~mm}^{2}$.

$\dot{m}=A \cdot P_{o} \cdot \sqrt{k \cdot \frac{1}{R \cdot T} \cdot\left[\frac{2}{k+1}\right]^{\frac{k+1}{k-1}}}$

where

$\dot{m}=$ mass flow in $\mathrm{kg} / \mathrm{hr}$

$\mathrm{A}=$ orifice cross sectional area

$P_{o}=$ helium storage tank pressure

$\mathrm{k}=$ ratio of heat capacity at constant pressure and volume. 


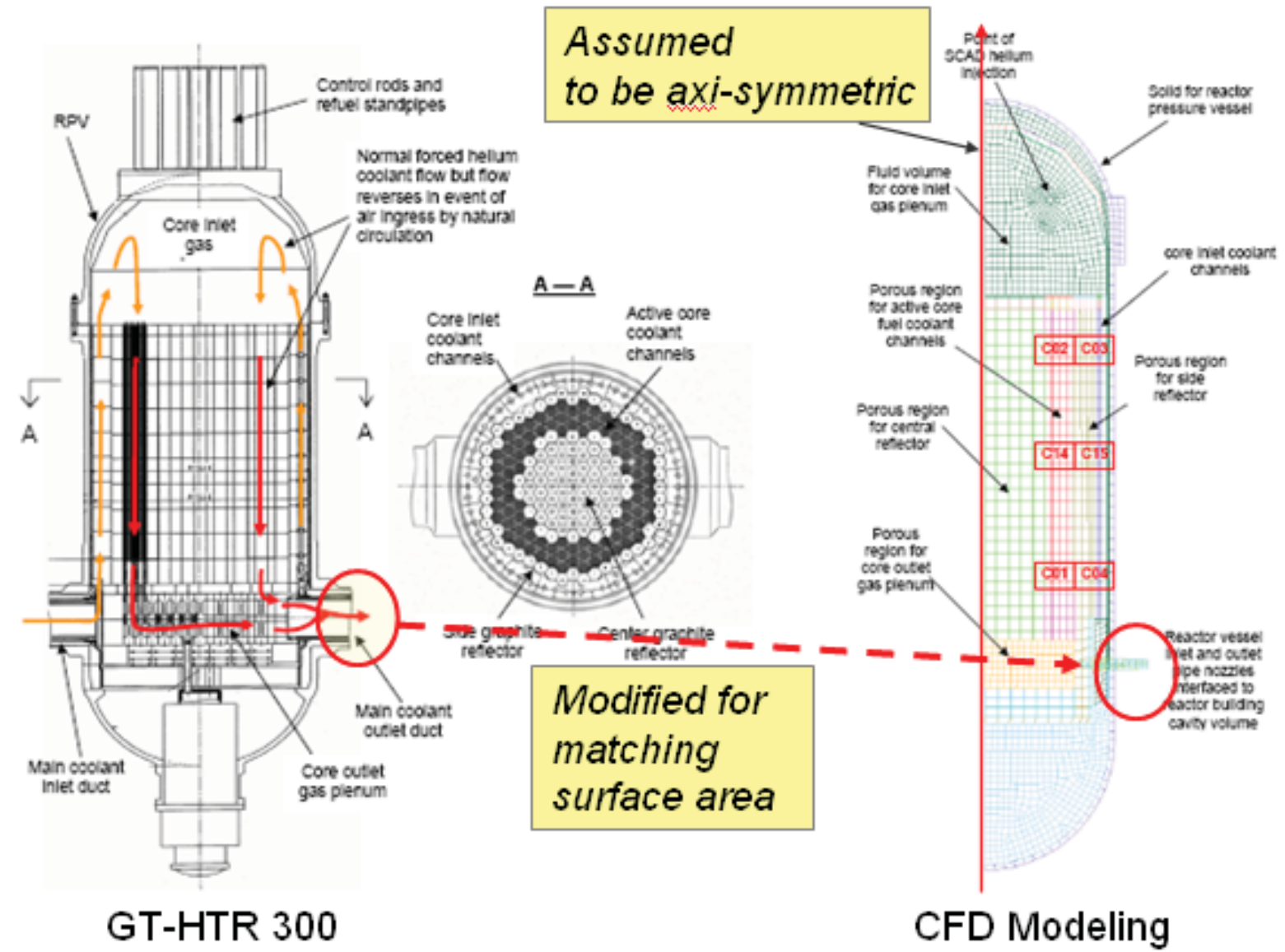

Figure 5-1. GTHTR300 geometry and CFD model (Yan et al. 2008).

The mass flow was converted to $\mathrm{kg} / \mathrm{sec}$ and multiplied by the sound velocity of helium, which equals to $1,134 \mathrm{~m} / \mathrm{s}$ at the ambient condition.

The momentum created by the helium top injection is 0.062 Newton, which is compared with $155 \mathrm{~N}$ - the buoyancy forces created in the lower plenum of the VHTR. Therefore, the buoyancy force is much larger than that of the top injected flow by a factor of 2,500. It is therefore not feasible to prevent air flow to the reactor by injecting helium in the top.

\subsection{Overview of Air Ingress Accident and General Mitigation Concepts}

Before discussing air-ingress mitigation methods, it is helpful to look at the overall picture of the airingress accident. Figure 5-2 is a top to bottom root-cause diagram that shows the overall sequence of the air-ingress accident. Figure 5-2 indicates that graphite structure weakening is now the most serious concern in the air-ingress accident because it can lead to the fission product release and eventually to potential core weakening. Graphite structural weakening can be generated by two main causes associated with graphite oxidation: (1) degradation of graphite structures, and (2) increases in local load (stress). The graphite structure degradation is mainly caused by internal oxidation reaction in the graphite pores. The oxidation between oxygen and graphite attacks internal graphite pore structures by weakening mechanical strength. This internal oxidation occurs at rather low temperatures where the oxidation rate is slow and is controlled by reaction kinetics regime. When the internal corrosion is dominant, only graphite density is decreased without external shape and size changes. Generally, the kinetics-controlled regime is the major 


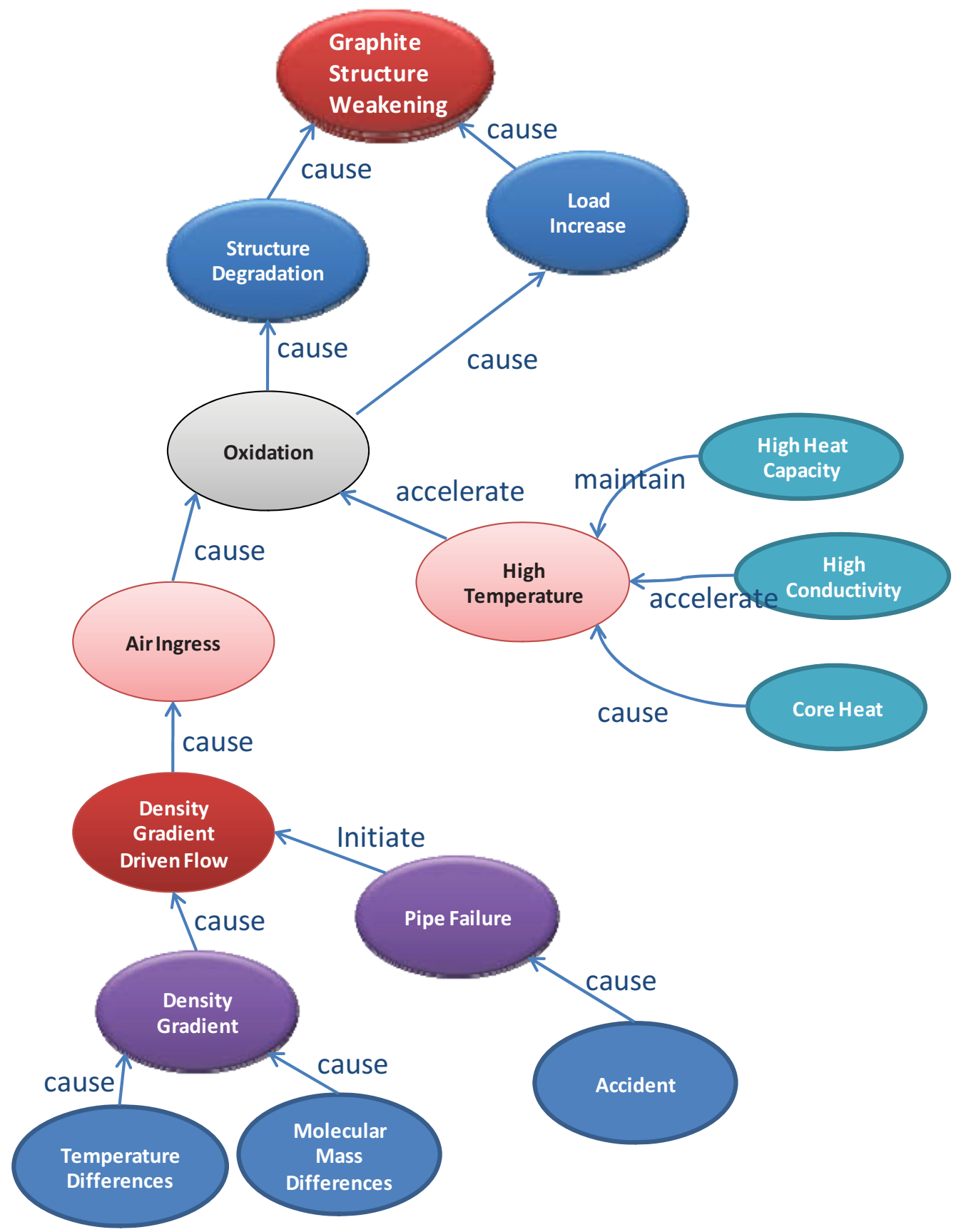

Figure 5-2. Root causes of Air-Ingress Accident.

oxidation mechanism at lower than about $650^{\circ} \mathrm{C}$. On the other hand, the local stress increase is caused by external corrosion, which occurs when the oxidation rate is very fast and the process is controlled by the mass transfer of oxygen in the flow (diffusion-controlled regime). The external corrosion changes the graphite's external shape by decreasing the local supporting area. The decrease of the local supporting area concentrates the load and stress on this region. Generally, graphite oxidation is dominated by the external corrosion at higher than $950^{\circ} \mathrm{C}$. At intermediate temperatures between 650 and $950^{\circ} \mathrm{C}$, both internal and external corrosions are mixed together. 
As shown in the middle of Figure 5-2, weakening of the graphite structure is caused by (1) air ingress, and (2) high temperature. The root cause of these conditions originates from the temperature and molecular mass differences between the inside and outside of the reactor vessel. Since the oxygen in the ingressed air is the main reactant, graphite oxidation does not occur without air-ingress. The oxidation rates and structural fracture are greatly affected by how fast the air ingresses. According to the Arrhenius model, the high temperature in the reactor inside significantly accelerates the graphite oxidation because the oxidation reaction exponentially increases with temperature.

The air-ingress speed is reportedly dependent on two physical mechanisms: molecular diffusion, and density gradient driven flow. However, recent studies for the DEBG showed that molecular diffusion is negligible compared to density gradient flow, since the molecular diffusion process is too slow. For this reason, molecular diffusion is removed from the diagram in Figure 5-2. Density gradient flow is generated in the VHTR air-ingress accident by density gradient from either molecular mass differences or temperature gradients between the inside and outside of the reactor. The initial density gradient flow is generated by molecular mass differences between helium (inside) and air (outside). However, after the air fills the bottom of the reactor vessel, the temperature gradient is the main driving force of the density gradient flow. According to this study, the density gradient flow driven by the temperature gradient is even maintained after the onset natural circulation (ONC) by accelerating air-ingress into the lower plenum one order of magnitude higher than the global natural circulation through the core.

Some basic concepts for air-ingress mitigation can be derived based on the above results. If the main causes of the root-cause diagram shown in Figure 5-2 are prevented or mitigated, the air-ingress consequences can be mitigated. The basic air-ingress mitigations are to prevent:

1. Fracture (under structural degradation or load increase conditions)

2. Structural Degradation (under oxidation environment)

3. Load Increase (under oxidation environment)

4. Oxidation (under air-ingress and high temperature conditions)

5. Air Ingress (under density gradient existing conditions)

6. Density Gradient Driven Flow (under density gradient existing conditions)

7. Density Gradient (under temperature and molecular mass difference existing conditions)

8. Temperature Gradient (between inside and outside of the reactor)

9. Molecular Mass Difference (between inside and outside of the reactor)

10. High Temperature (in the reactor inside).

The following sections will introduce some methods to satisfy above concepts and show some validation results. Again, the root causes of graphite damages originated from the temperature difference and mass difference between the confinement and reactor vessel following the initiation of air ingress. CFD models were developed to determine the side helium injection effect on the air mass fraction in the core and the lower plenum. The rationale for the injection is two-fold: the injection will reduce temperature in the lower plenum and dilute the concentration of air mixture.

\subsection{Air-ingress Mitigation Methods}

This section introduces some air-ingress mitigation methods based on the basic mitigation concepts. The ideas discussed here are still in the conceptual state, so technical readiness or effectiveness is not discussed here in detail. Economical issues are also not yet in this scope, but will be covered in future studies. 


\subsubsection{Prevention (or Mitigation) of Graphite Fracture}

If the graphite structure fracture can be prevented in the reactor, the weakening and the following fission product release can be protected. The following describes some of the conceptual ideas for preventing graphite structure fracture in air-ingress accidents conditions.

The main concept for preventing core weakening is illustrated in Figure 5-3. In the original design, each supporter sustains the bottom reflector and core blocks separately so if a supporter fails, the bottom reflector and core blocks supported by the supporter also fall down, even though the neighboring supporters are secure. According to our analyses, the graphite oxidations in the supporters (lower plenum) are not uniform and highly concentrated on some local blocks. Our idea is to locate a supporting structure that is not reactive to the oxygen as shown in the new design in Figure 5-3. In the new design, even though some supporters may fail because of severe corrosion, the neighboring structure can sustain the core securely. The supporting structure can be either a single structure or multiple structures having the same concept. The candidate material of the supporting structure can be graphite with oxidation resistant coating, ceramic materials, or some high temperature metals such as titanium. Further investigations are required in the future.

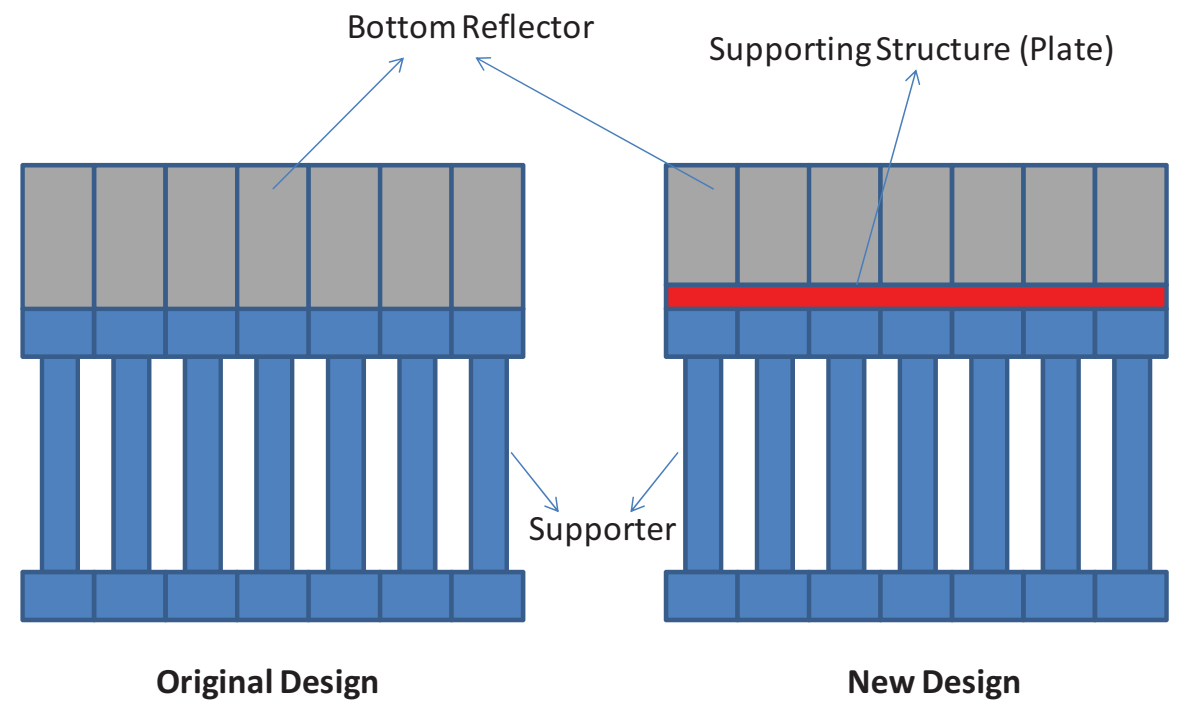

Figure 5-3. Concept of using supporting structure for preventing core weakening.

\subsubsection{Prevention (or Mitigation) of Structural Degradation}

If the structural degradation of the support structure can be prevented, the structure weakening and the following fission product release can be protected. Since the structural degradation is closely related to the graphite oxidation process, the ideas of this part are incorporated in the Section 4.

\subsubsection{Prevention (or Mitigation) of Load Increase}

If the local load increase of the support structure can be prevented, the structure weakening and resulting release of fission products can be prevented. According to the previous investigations, the graphite oxidation during the air-ingress accident is highly concentrated on upper part of the lower plenum and the lower part of the bottom reflector. Therefore, if corrosion is prevented in these areas, fracture of the support structure can be mitigated. Corrosion in these areas can be reduced by applying oxidation-resistant coatings on the flow channels. Silicon carbide ( $\mathrm{SiC}$ ) is one of the candidate coating materials. 


\subsubsection{Prevention (or Mitigation) of Oxidation}

Since graphite oxidation is the main cause of all related air-ingress accident problems, preventing oxidation in the reactor is a very effective way to mitigate air-ingress. Some of the possible conceptual ideas for mitigating oxidation are described below.

\subsubsection{Injection of Helium Into the Lower Plenum}

The first proposed method for mitigating oxidation is to inject helium into the lower plenum (See Figure 5-4). The main idea of this method is to inject light helium gas into the lower plenum from the lower plenum side and separate air inflow into two layers. Since the helium gas is much lighter than the air gas, the upper part of the air flow will be replaced by the helium gas, which will then move into the core channels instead of air, thus protecting the core from oxidation damage. Injecting helium into the lower plenum vessel wall of a prismatic-type reactor could also mitigate air ingress and minimize graphite oxidation by diluting the oxygen concentration and reducing the buoyancy force by lowering fluid temperature in the lower plenum. To validate this method, a CFD simulation was performed. The simulation results showed that the helium replaces the air flow into the core and significantly reduces the air concentration in the core and bottom reflector, potentially leading to significant oxidation damages without the helium injection. According to the simulation results, even small helium flow was sufficient toblock air moving up to the core, mitigating the air-ingress successfully. This method is discussed in detail with validation calculations in Section 5-5.

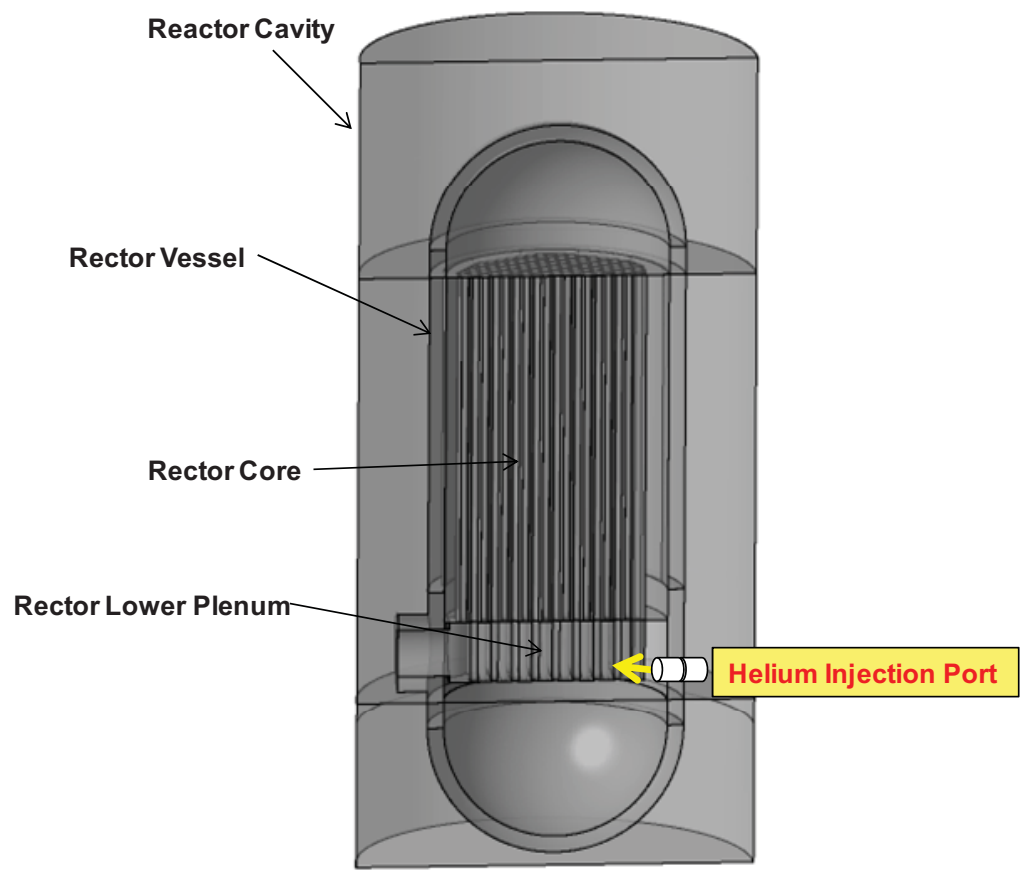

Figure 5-4. Air-ingress mitigation method at the lower plenum.

\subsubsection{Injection of or Absorbent of Oxygen or Substance Reactive to Oxygen}

Since graphite oxidation is the reaction between oxygen and graphite, removing oxygen from the air can effectively mitigate oxidation related problems. One way to accomplish this is to inject oxygen absorbent or a substance reactive to oxygen into the reactor during the air-ingress accident. Graphite (carbon) powder can be a good candidate since it easily attaches onto the support structures and reacts with oxygen. 


\subsubsection{Protective Coating on the Support Structures}

The oxidation resistant coating can mitigate the graphite oxidation by preventing oxygen from coming into contact with support structures. Silicon carbide (SiC) is one of the candidate materials for this option.

\subsubsection{Reactor Enclosure with Opening and Relief at the Bottom}

A reactor enclosure with openings and reliefs at the bottom are recommended to reduce air content. This concept is illustrated in the conceptual drawing in Figure 5-5 (the scale and layout does not represent an actual VHTR system). The basic idea behind this method is to enclose the reactor as shown in the figure. This enclosure is not the pressure boundary. Openings located at the bottom of the enclosure, are equipped with a relief valve so the air inside of the enclosure can be discharged through this opening during the depressurization stage. Once the pressure between the inside and outside is balanced, the flow will stop, leaving only a small amount of air inside the enclosure. Once the pressure between the inside and outside enclosure is balanced, air ingress into the enclosure can be controlled by molecular diffusion process because the opening is located at the bottom. Even though the density gradient flow is generated in the reactor, the air supply into the enclosure is limited by the slow diffusion process. This concept can change the air-ingress process from the density gradient driven process to the molecular diffusion process. In the confinement, the relief line is recommended to be downward as shown in the figure in order to limit air-ingress into the confinement by diffusion in case the relief valve closing fails.

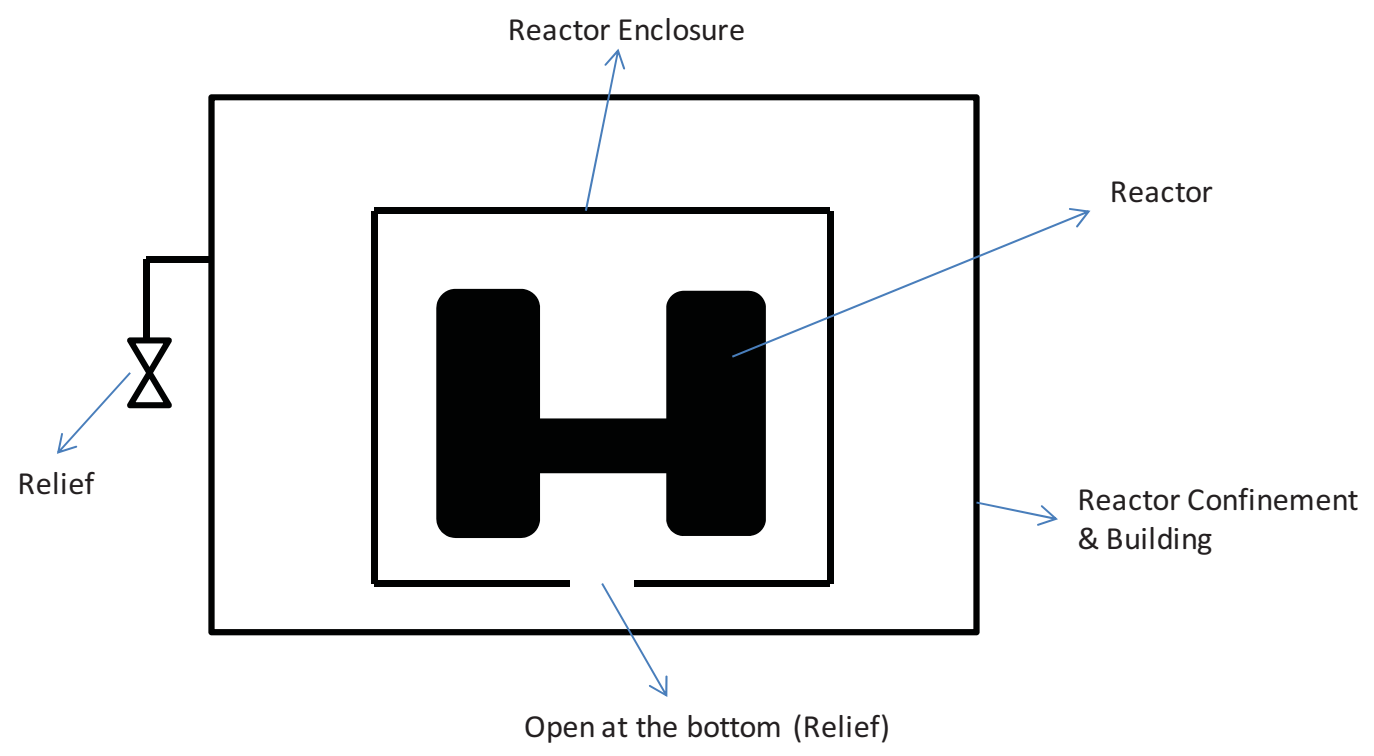

Figure 5-5. Concept of reactor enclosure with opening at the bottom.

\subsubsection{Prevention (or Mitigation) of Air Ingress}

Prevention of air-ingress into the reactor system can mitigate air-ingress consequences by reducing graphite oxidation. One of the methods using an enclosure was already described in the previous section. Another method to prevent air ingress is to install Fluidic diodes in the riser, which control the flow directions. In the normal operation, the flow direction in the riser is upward, but in the accident condition, it is downward. Therefore, if the Fluidic diode is set to allow only upward directional flow in the riser, the global natural circulation will be prohibited by preventing air flow into the core. Since the riser has the lowest temperature in the reactor, installing a Fluidic diode in the riser is quite realistic. The Fluidic diode will work effectively for the DEGB and the small break. 


\subsubsection{Prevention (or Mitigation) of Density Gradient Driven Flow}

According to FY 2009 studies, the Stage-2 stratified flow is very important in the whole air-ingress process. In this stage, the air-ingress is governed by a density gradient caused by a temperature gradient between the inside and outside of the reactor vessel. This mechanism is quite similar to the thermal stratification phenomena.

The current issue in an air-ingress accident is graphite structural degradation by oxidation and corrosion. The graphite structures in the lower plenum and the core can be seriously damaged by active reactions with oxygen at high temperature. Therefore, the rate of air-ingress into the reactor is directly related to the degree of oxidation damage. The air-ingress rate is a very strong function of the air-ingress mechanisms. The air can be moved into the reactor either by a global natural circulation or by a recirculation flow between the lower plenum and the confinement. It is obvious that these two different mechanisms will provide different air-ingress rates, eventually leading to the different oxidation damage. Generally, the global natural circulation is considered more serious than just recirculation flow in the lower plenum.

The ratio between hydrostatic head and pressure buildup in Stage 2 determines early natural circulation. If the hydrostatic head is larger, the air-ingress mechanism is dominated by a recirculation flow. However, if the buoyancy force or pressure build-up is larger, the mechanism will be directly transited to the overall natural circulation. In FY 2009, a nondimensional parameter was defined by as

$$
\Pi_{2}=\frac{1}{8}\left(\frac{H}{H_{v}}\right) \cdot\left(\frac{\rho_{A}}{\rho_{\text {Core }}-\rho_{\text {Riser }}}\right)\left(\frac{(1-\gamma)\left(1-\gamma^{3}\right)}{\gamma^{3}}\right) .
$$

The physical meaning of the nondimensional parameter, $\Pi_{2}$ in Equation (5-1) is the ratio of the pressure build-up to the hydrostatic head in Stage 2. If this value is larger than 1, the air will have enough buoyancy force to generate flow into the core. If the value is less than 1 , the air will not have enough buoyancy force to generate flow into the core. The implication of this with respect to air-ingress mitigation is that decreasing the $\prod_{2}$ value is beneficial in the mitigation of the air-ingress process. In FY 2009, the following concepts were briefly mentioned for air-ingress mitigation:

- Decreasing the diameter of the horizontal pipe $(\mathrm{H})$

- Increasing the height of the core (Hv)

- Increasing the $P_{\text {Core }}-P_{\text {Riser }}$, which can be achieved by increasing the riser temperature or decreasing the core temperature in accident conditions

- Increasing the density ratio $(\gamma)$, which can be achieved by increasing cavity temperature or decreasing lower plenum temperature.

A sensitivity study was performed to understand and determine the better mitigation method. The basic idea is as follows. If the nondimensional parameter is large, the air-ingress mechanism is governed by recirculation flow (thermal stratification). If the nondimensional parameter is small, the air-ingress mechanism is governed by global natural circulation. Therefore, decreasing the nondimensional parameter indicates the better method to mitigate air-ingress.

The sensitivity study took the following eight variables into account; Table 5-1 also summarizes these input variables and their reference conditions:

- $\quad$ Pipe diameter $(\mathrm{H})$

- Core Height $\left(\mathrm{H}_{\mathrm{v}}\right)$ 
Table 5-1. Input variables and reference values for the sensitivity analyses.

\begin{tabular}{|l|l|l|l|}
\multicolumn{1}{c}{ Parameters } & \multicolumn{2}{c|}{ Symbol } & \multicolumn{1}{c|}{ Unit } \\
\hline Pipe Diameter & $\mathrm{H}$ & $\mathrm{m}$ & 1.5 \\
\hline Core Height & $\mathrm{H}_{\mathrm{v}}$ & $\mathrm{m}$ & 11 \\
\hline Lower Plenum T & $\mathrm{T}_{\mathrm{L}}$ & ${ }^{\circ} \mathrm{C}$ & 600 \\
\hline Core T & $\mathrm{T}_{\mathrm{C}}$ & ${ }^{\circ} \mathrm{C}$ & 950 \\
\hline Riser T & $\mathrm{T}_{\mathrm{R}}$ & ${ }^{\circ} \mathrm{C}$ & 500 \\
\hline Confinement T & $\mathrm{T}_{0}$ & ${ }^{\circ} \mathrm{C}$ & 150 \\
\hline Confinement P & $\mathrm{P}_{0}$ & atm & 1 \\
\hline Confinement Air concentration & $\mathrm{mf}$ & $\#$ & 0.1 \\
\hline
\end{tabular}

- Lower Plenum Temperature $\left(\mathrm{T}_{\mathrm{L}}\right)$

- Core Temperature $\left(\mathrm{T}_{\mathrm{C}}\right)$

- $\quad$ Riser Temperature $\left(\mathrm{T}_{\mathrm{R}}\right)$

- Confinement Temperature $\left(\mathrm{T}_{0}\right)$

- Confinement Pressure $\left(\mathrm{P}_{0}\right)$

- Confinement Initial Air Mole Fraction $\left(\mathrm{m}_{\mathrm{f}}\right)$.

A local sensitivity study was performed based on the partial derivatives of each parameter. The derivatives were numerically calculated using an Excel spreadsheet. The input variations were decided to be $1 \%$. This small range is considered to maintain the linearity of the variables. Eventually, the derivatives have been normalized for comparisons by the reference values.

Table 5-2 summarizes the results of the sensitivity analyses. The interpretation of these results is as follows: If the number is high, it means that the input variable is sensitive to the nondimensional parameter (ratio of pressure buildup to hydrostatic head). If the sign of the number is negative, it is recommended to reduce the input variables for air-ingress mitigation; if the sign is positive, it is recommended to increase the input variables for mitigation.

Table 5-2. Results of the sensitivity analysis.

\begin{tabular}{|l|l|l|}
\hline \multicolumn{3}{|c|}{ Local Sensitivity } \\
\hline \hline Pipe Diameter & $\mathrm{H}$ & 1 \\
\hline Core Height & $\mathrm{H}_{\mathrm{V}}$ & -0.99 \\
\hline Lower Plenum T & $\mathrm{T}_{\mathrm{L}}$ & 2.25 \\
\hline Core T & $\mathrm{T}_{\mathrm{C}}$ & 12.74 \\
\hline Riser T & $\mathrm{T}_{\mathrm{R}}$ & -10.84 \\
\hline Confinement T & $\mathrm{T}_{0}$ & 1.66 \\
\hline Confinement P & $\mathrm{P}_{0}$ & 0 \\
\hline Confinement Air conc. & $\mathrm{mf}$ & -5.98 \\
\hline
\end{tabular}

The table shows that the most important and sensitive variables are the core and the riser temperatures. The sensitivity indices are 12.7 and -10.8 , respectively, indicating that decreasing core temperature and increasing riser temperature are highly preferred for air-ingress mitigation. Practically, the core temperature decrease is not easy because of large heat capacities in the core solid structures. Increasing 
riser temperature is easier, but will lead to an increased core temperature to compensate for that effect. Therefore, both variables do not look feasible for air-ingress mitigation in a practical sense.

Air mole fraction in the confinement is the next sensitive variable. Increase of air mole fraction will mitigate the air movement into the core. However, increase of the air mole fraction will lead to more oxygen ingress into the reactor, causing increased core oxidation.

The lower plenum and confinement temperatures are the next sensitive variable. Decreasing both parameters is preferred for air-ingress mitigation. The pipe diameter and the core height follow them with the same order of sensitivities. The confinement pressure has no effect on the nondimensional parameter.

The above results can be used for making some concepts to mitigate the air-ingress, especially in the Stage 2 .

\subsubsection{Prevention (or Mitigation) of Density Gradient}

Since the density gradient is closely related to the temperature gradient and the molecular mass differences, this part is incorporated in the Sections 6 and 7.

\subsubsection{Prevention (or Mitigation) of Molecular Mass Differences}

Decreasing molecular mass differences between the inside and outside of the reactor can initially mitigate Stage-1 stratified flow, but even if Stage-1 stratified flow is mitigated, Stage- 2 stratified flow, which is based on the temperature gradient, rapidly dominates the air-ingress process. Therefore, preventing molecular mass differences does not look efficient for air-ingress mitigation.

\subsubsection{Prevention (or Mitigation) of Temperature Gradient}

Decreasing the temperature gradient between the inside and outside of the reactor is very helpful in mitigating air-ingress by reducing recirculation flow in the lower plenum. To decrease the temperature gradient between the inside and outside of the reactor, the supporter length can be increased as shown in Figure 5-6. In the accident condition, the heat is coming from the core on the top of the supporter, so if the length of the supporter is increased, the temperature at the lower part of the supporter will decrease proportional to the length. This reduced supporter temperature will reduce the temperature gradient between the inside and outside of the reactor.

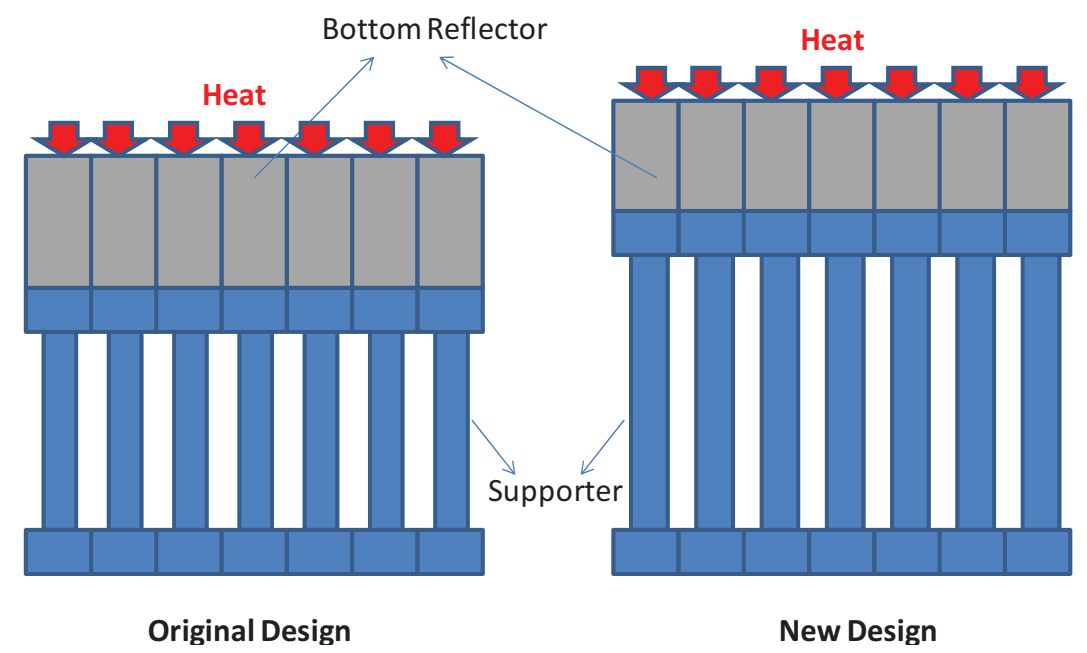

Figure 5-6. Concept of increasing supporter height. 


\subsubsection{Prevention (or Mitigation) of High Temperature Inside the Reactor}

Decreasing the temperature of the lower plenum is very helpful in mitigating support fracture because the graphite oxidation exponentially increases with the temperature. The method for increasing the supporter, which is described in the Figure 5-6, can also be used here. The decreased lower plenum temperature will reduce the risk of structural fracture.

\subsection{Validation of Air-ingress Mitigation Methods on Helium Injection and Reactor Enclosure}

Two air-ingress mitigation methods proposed in the Section 5.4 were validated by CFD simulations. The validation methods are:

- Air-ingress mitigation using lower plenum helium injection

- Air-ingress mitigation using the reactor enclosure concept.

The details of this validation are described in this section.

\subsubsection{Validation of Air-ingress Mitigation using Lower Plenum Helium Injection}

The previous section proposed helium injection into the lower plenum as one of the good air-ingress mitigation concepts. The main idea of this method is to change the density-gradient-flow-controlled airingress process to the molecular-diffusion controlled process. This study shows how effectively this method will work for air-ingress mitigation.

\subsubsection{Overview of Helium Injection Method for Air-ingress Mitigation}

Figure 5-7 shows the basic concept of the helium injection method for air-ingress mitigation. The main idea of this method is to inject light helium gas into the lower plenum from the lower plenum side and separate air inflow into two layers. Since the helium gas is much lighter than the air gas, the upper part of the air flow will be replaced by the helium gas. This helium gas will then move into the core channels instead of the air by buoyancy force, thus protecting the core from oxidation damage. An injection of helium into the lower plenum vessel wall for the prismatic type reactor could also mitigate air ingress and minimize graphite oxidation by diluting the oxygen concentration and reducing the buoyancy force by lowering fluid temperature in the lower plenum. Helium can be supplied from the helium storage tank that already exists in the VHTR system for maintaining the system pressure during operation as shown in Figure 5-8. By setting up the check valve at the entrance, a reverse flow from the system to the storage tank can be protected. Since the stored helium is generally separated into several tanks, helium injection can be securely accomplished. even if one or two valves fail.

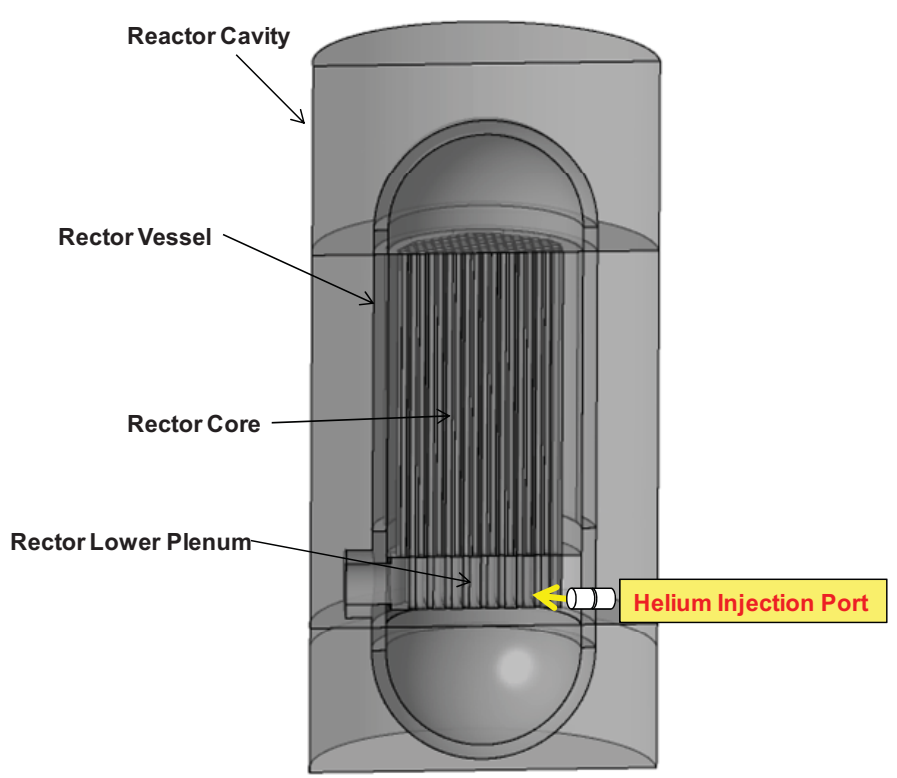

Figure 5-7. Air-ingress mitigation method by helium injection at the lower plenum. 


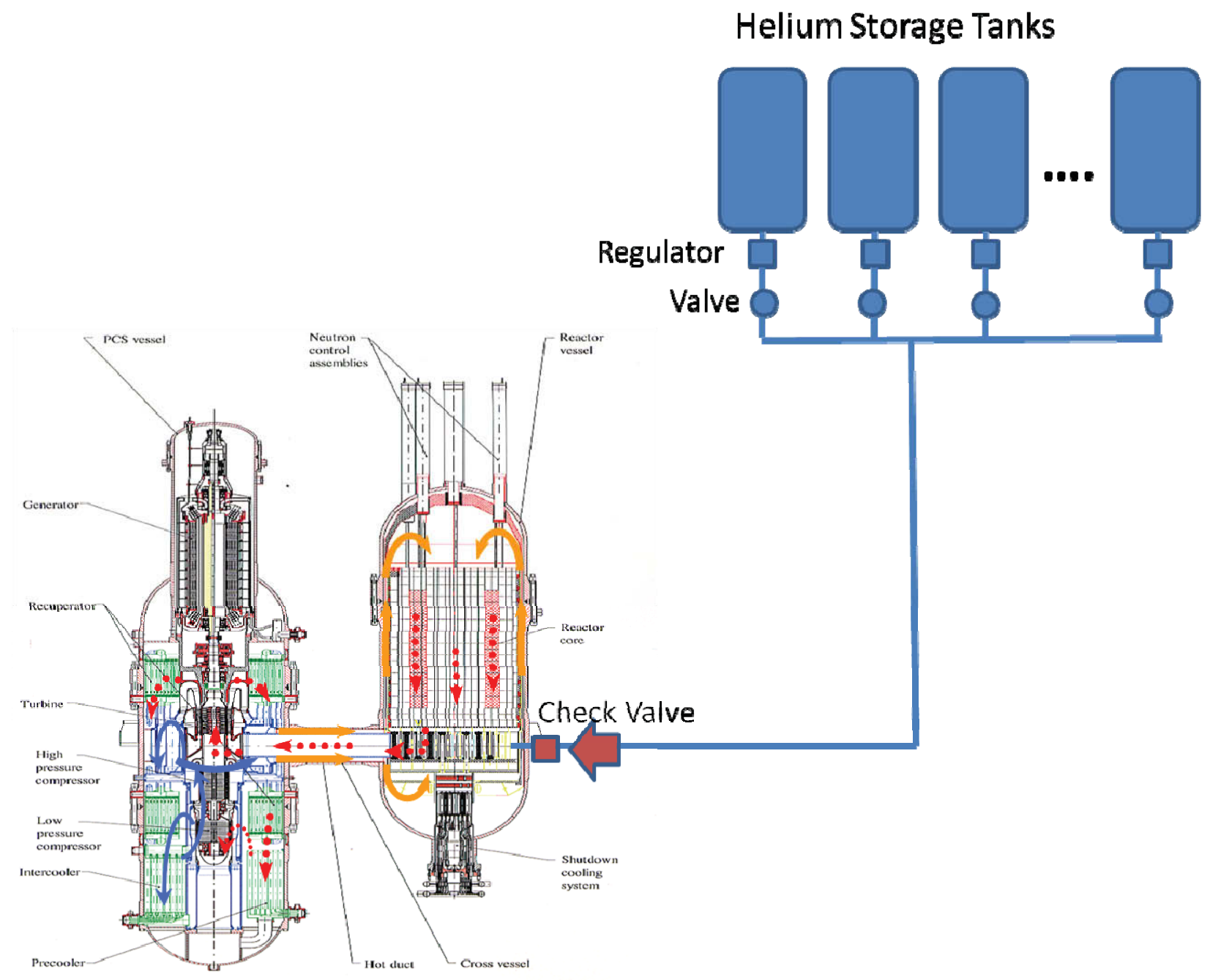

Figure 5-8. Concept of helium injection system.

\subsubsection{Modeling the Helium Injection Method using FLUENT Code}

This method was validated by performing a CFD simulation. The 600 MWth GTMHR was selected as a reference design and the FLUENT 6.3 code was used for simulation. The details are described below.

\section{Reference System}

Figure 5-9 shows a schematic of the 600 MWth GTMHR, which was selected as the reference design for analyses. The GTMHR is a nuclear fission power reactor design under development by GA. This helium cooled, graphite moderated reactor uses TRISO fuel compacts in a prismatic core design. Helium enters the prismatic core of this reactor at $490^{\circ} \mathrm{C}$ and a flow rate of $7 \mathrm{MPa}$ through the riser and exists at $850^{\circ} \mathrm{C}$ and a flow rate of $320 \mathrm{~kg} / \mathrm{s}$. Helium is heated to $850^{\circ} \mathrm{C}$ in the reactor. Hot helium at high pressure is fed into the turbine, which rotates the compressor and the generator where the helium expands. 


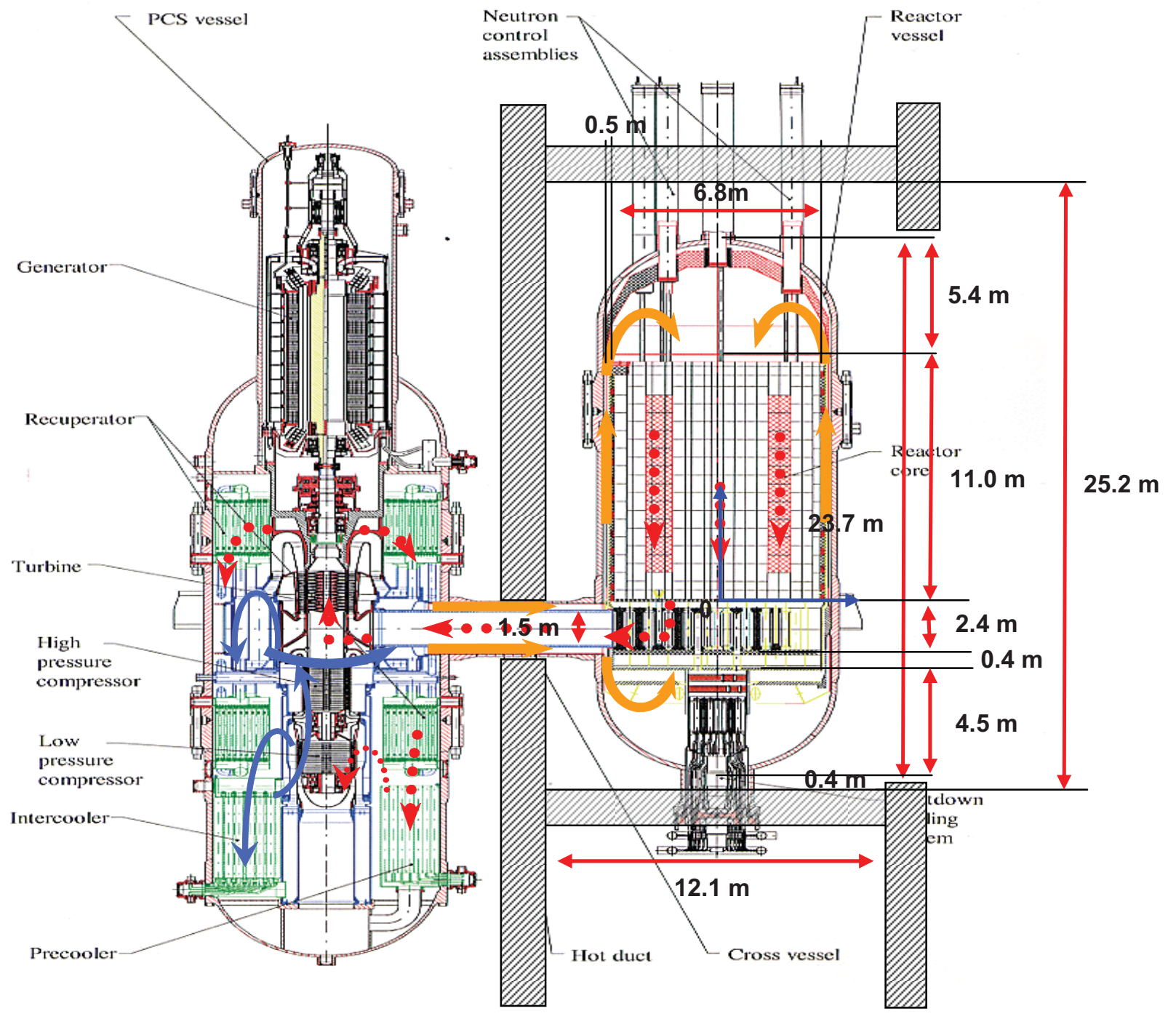

Figure 5-9. Geometry and size of 600 MWth GTMHR.

\section{2-D Grid Model and Model Setup}

Figure 5-10 shows the 2-D grid model for the CFD analyses. The grid model was developed by the GAMBIT mesh generation software. The geometry was simplified to be 2-D for saving computation time. The grid mode was divided into six regions: core, lower plenum, hot-leg, cold leg, vessel inside, and cavity. Among them, the core was assumed to be the porous media. The porous media parameters can be found in the INL report prepared by Oh et al. (2008). Figure 5-10 (b) shows more fine meshes around the vessel, which were created using GAMBIT with the aspect ratio of unity. The model was hexagonally meshed using the mapping scheme in the inside reactor and the pave scheme in the outside of the reactor. The total number of mesh was 55,590. The injection port was located at the side of the lower plenum, and the size was assumed to be $30 \mathrm{~cm}$ in diameter. About 10 meshes were located at this injection port. 


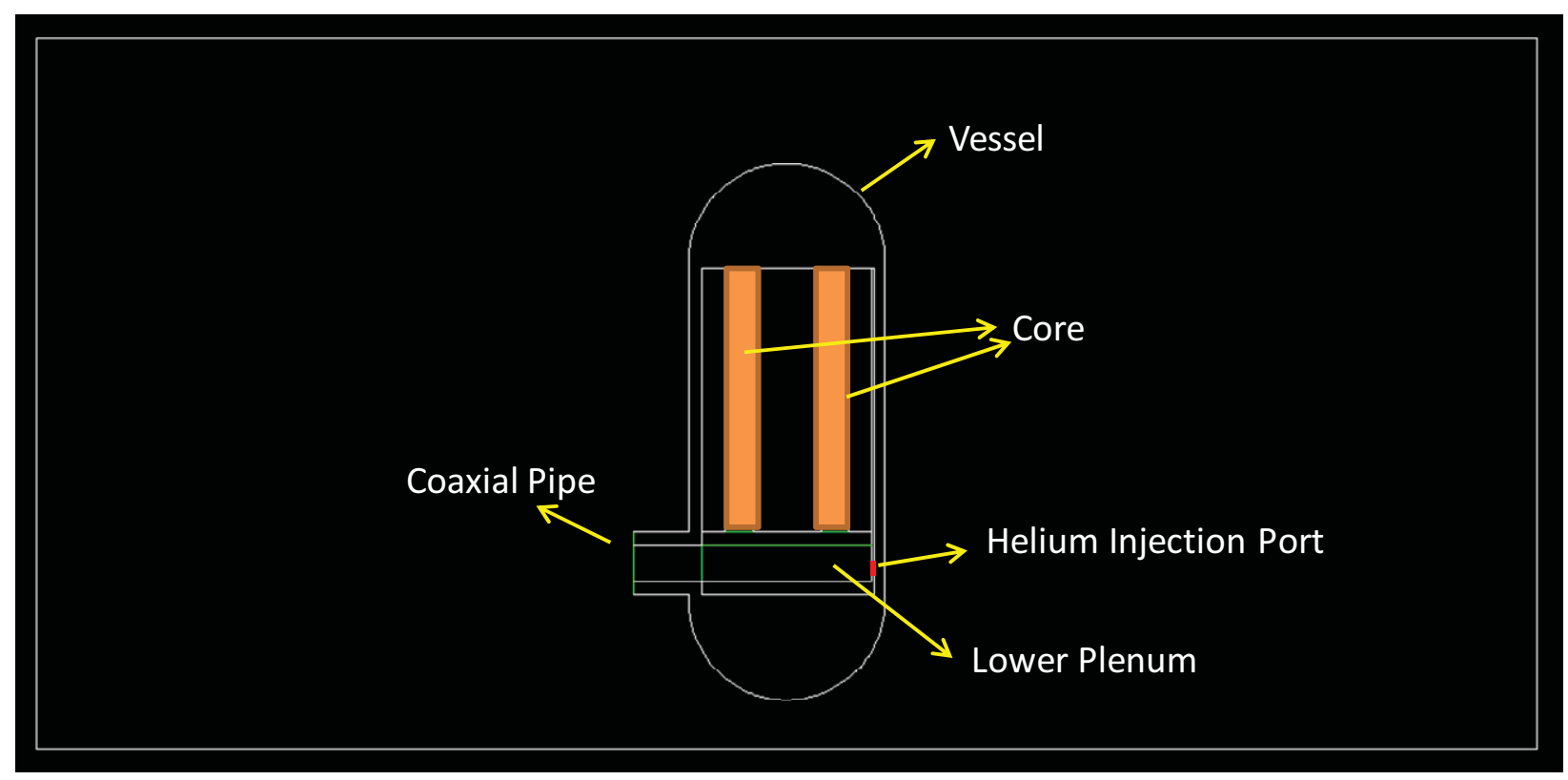

(a) Geometry summary.

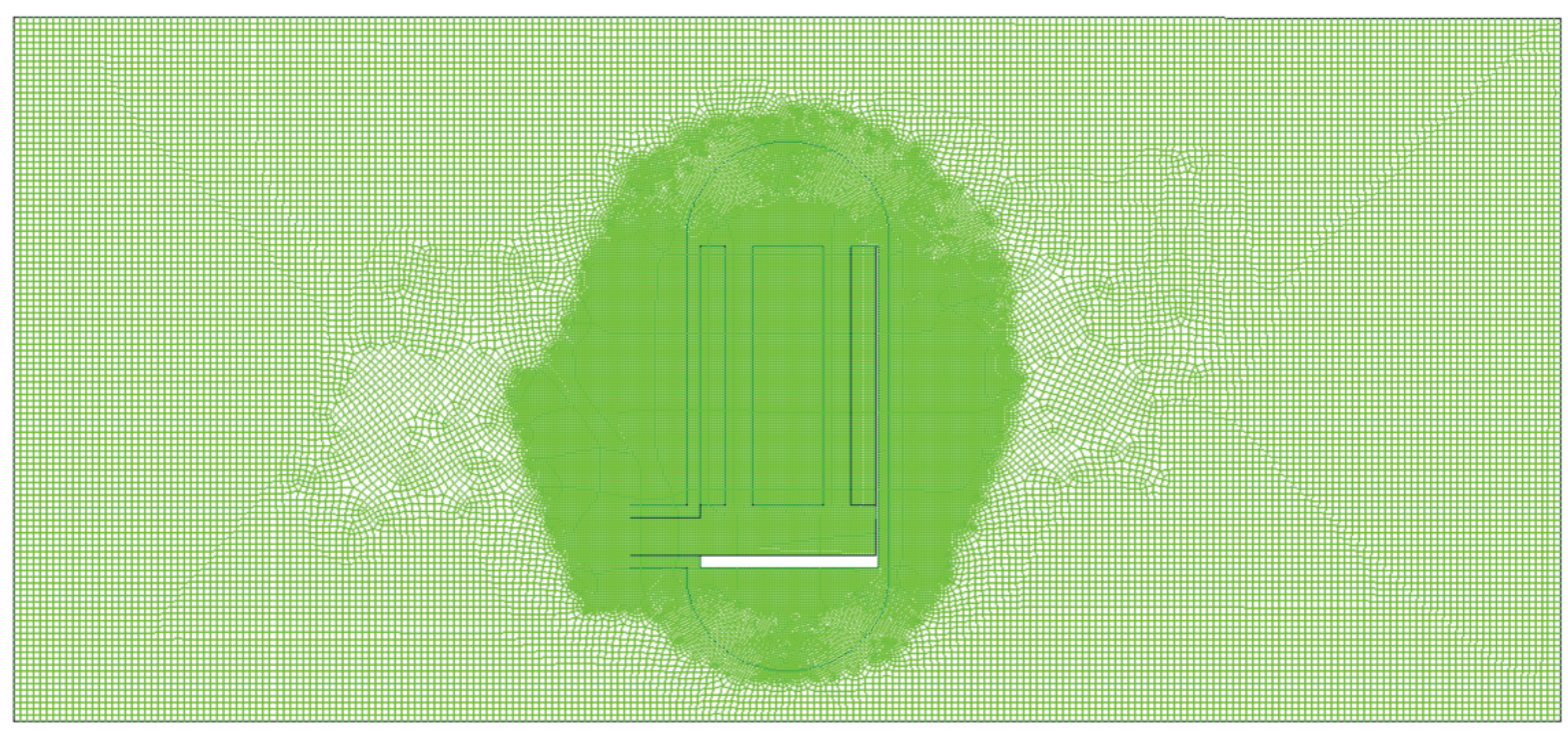

(b) Grid model (55,590 cells).

Figure 5-10. 2-D FLUENT model (geometry and grid model).

Table 5-3 summarizes the FLUENT solver setup. The FLUENT version used here was 6.3.26. The pressure based solver was selected as a solver type. The model was 2-D, and the time scheme was implicit. This simulation was unsteady and the starting point was after depressurization process was completed and the pressures were equilibrated. We used 20 parallel CPUs for simulations. The viscous model was selected to be a laminar model for conservative analyses. Energy and species conservation was also solved here. Two gas species were considered in the calculation; air and helium. Incompressible ideal gas law was used for density estimation, and mixing laws for other properties. A multicomponent diffusion model was used for molecular diffusions. 
Table 5-3. Reference FLUENT model setup.

\begin{tabular}{|ll|}
\hline \multicolumn{1}{|c|}{ Parameters } & \multicolumn{1}{c|}{ Settings } \\
\hline Code Version & FLUENT 6.3.26 \\
Solver Type & Pressure Based Solver \\
Time Scheme & Implicit \\
Dimension & 2-D \\
Steady/Unsteady & Unsteady \\
Number of CPUs & 20 \\
Velocity Formulation & Absolute \\
Gradient Option & Node Based \\
Porous Formulation & Physical Velocity \\
Viscous Model & Laminar \\
PressureVelocity Coupling & SIMPLE \\
Air Mass Fraction & 0.5 \\
Energy Equation Solve & Yes \\
Species Equation Solve & Yes \\
Density & Incompressible Ideal Gas Law \\
Heat Capacity & Mixing Law \\
Thermal Conductivity & Mass Weighted Mixing Law \\
Viscosity & Mass Weighted Mixing Law \\
Diffusion & Multi-component \\
\hline
\end{tabular}

\section{Initial and Boundary Conditions}

Figure 5-11 shows the initial air concentration and the temperature profile of the simulation. Initially, the air concentration in the inside of the vessel was assumed to be 0.0 , since the helium coolant occupies this region before air ingress. In the outside of the vessel, the initial air mass fraction of 0.5 was used, which was calculated from the GAMMA code after depressurization. The outside temperature of the reactor was assumed to be $300 \mathrm{~K}$, and the inside was determined based on the GAMMA code results for depressurization phase (Oh et al. 2009). The lower plenum temperature profile was assumed as uniform since the inside structures were not considered here because of the 2-D geometries. The riser temperature at the vessel side was assumed to be $775 \mathrm{~K}\left(500^{\circ} \mathrm{C}\right)$ and the cavity wall (or confinement wall) temperature was assumed to be $300 \mathrm{~K}$. The upper and the lower domes were assumed to be adiabatic. Coupled wall heat transfer options were selected for other walls to consider heat exchanges through the walls. The helium injection velocity was selected between 0.0 and $2.0 \mathrm{~m} / \mathrm{s}$ to see the effect of injection speed. 


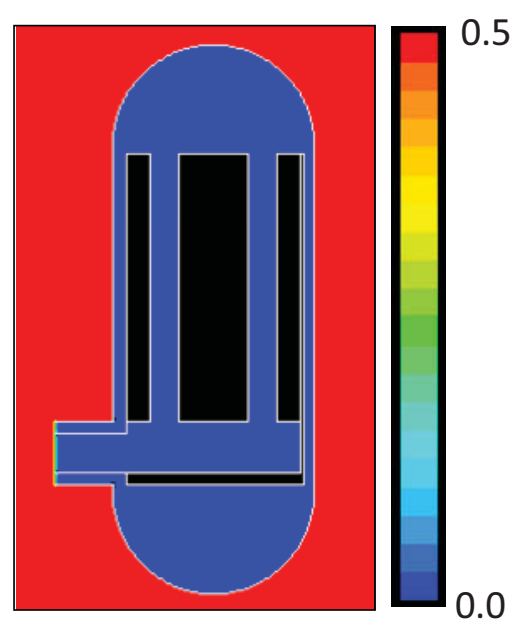

(a) Air mass fraction

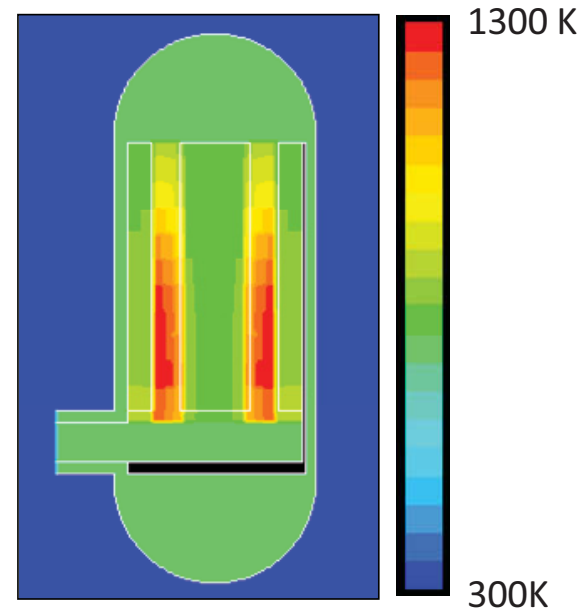

(b) temperature

Figure 5-11. Initial conditions (air mass fraction, temperature)

\subsubsection{Results and Discussions}

Figures 5-12 through 5-18 show the CFD results for the six cases with different injection velocities. The selected velocities are $0.0,0.05,0.1,0.5,1.0$, and $2.0 \mathrm{~m} / \mathrm{s}$, respectively. Figure $5-12$ shows the contour plot of the air mass fractions for the no helium injection case $\left(\mathrm{V}_{\text {inj }}=0.0 \mathrm{~m} / \mathrm{s}\right)$ wherein the air initially enters the reactor by density gradient flow and is driven into the core rapidly by buoyancy force generated in the lower plenum. This is the reference case to be compared with mitigation results.

Figure 5-13 shows the air mass fractions for $\mathrm{V}_{\text {inj }}=0.05 \mathrm{~m} / \mathrm{s}$ in which the core air concentration was slightly reduced but the effect was very small. When the helium is injected by $0.1 \mathrm{~m} / \mathrm{s}$ (See Figure $5-14$ ), the air concentration in the core and the upper part of the lower plenum was much more reduced. When the helium is injected to the lower plenum with the speed higher than $1.0 \mathrm{~m} / \mathrm{s}$, most of air in the core and the upper lower plenum was replaced by the injected helium flow, showing clear separation of air and helium. This effect is contributed by low helium density compared to that of air. Air flow was clearly separated from the helium, and returned back to the broken hot-leg by recirculation flow. The majority of the helium injected at the lower plenum moved into the core and was released out of the vessel through the cold-leg. Based on previous air-ingress researches, the upper part of the lower plenum and the lower part of the bottom reflector are known to be the most seriously corroded and damaged by graphite oxidation because of relatively high temperature and large air concentrations. Helium injection at the lower plenum is thus considered very effective in mitigating air-ingress consequences, since the injected helium successfully covers the seriously-damaged part. This indicates that the helium injection can protect not only the core but also the lower plenum and bottom reflectors from serious oxidation damage. Figure 5-18 shows the comparisons on the air distributions in the reactor for various injection speeds.

As shown above, the injection fluid and the mass flow rate are very important. Based on the $200 \mathrm{~m}^{3}$ helium storage tank with approximately $86 \mathrm{~atm}$ (assumption), a helium flow rate of $0.5 \mathrm{~m} / \mathrm{s}$ will last 6 days; the decay heat is so low after a 3-day delay by the injection that oxidation cannot be a serious problem. According to the previous investigations, the maximum temperatures of the bottom reflector and lower plenum drop down to about $725^{\circ} \mathrm{C}$ and $550^{\circ} \mathrm{C}$, respectively. For this idea, we propose using air velocity higher than $0.5 \mathrm{~m} / \mathrm{s}$ to achieve sufficiently low air concentration in the core. The helium can be supplied from the helium supply tanks and helium storage tanks already installed in the system to maintain the pressure in the system. The air-ingress delay time can be varied, depending on the helium storage tank size and injection velocity. 


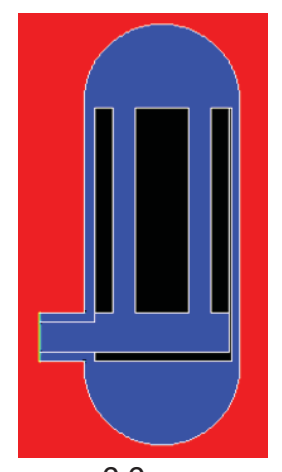

$0.0 \mathrm{sec}$

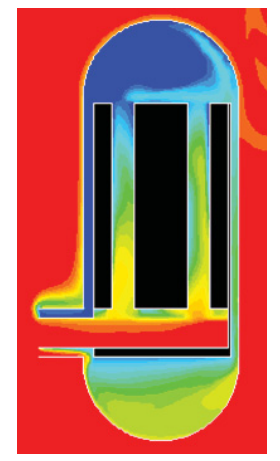

$20.0 \mathrm{sec}$

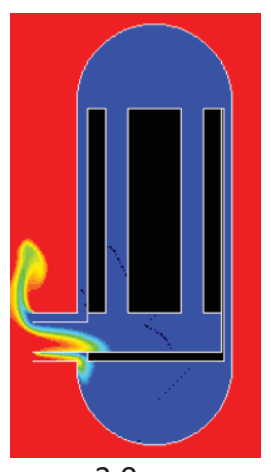

$2.0 \mathrm{sec}$

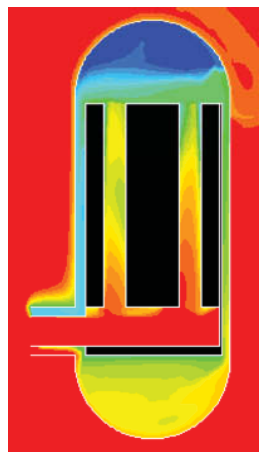

$40.0 \mathrm{sec}$

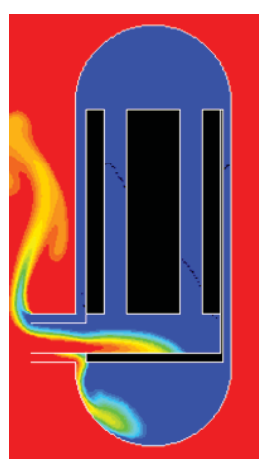

$4.0 \mathrm{sec}$

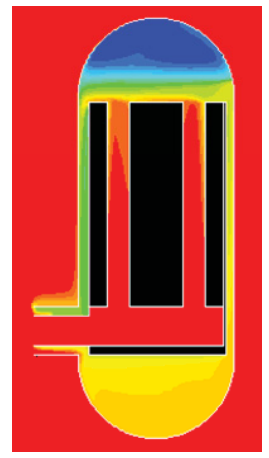

$80.0 \mathrm{sec}$

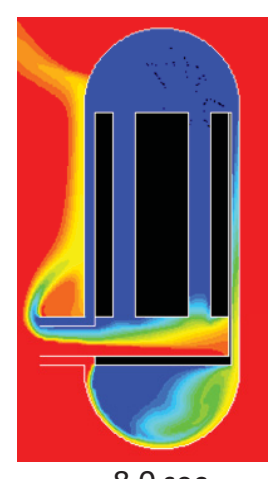

$8.0 \mathrm{sec}$
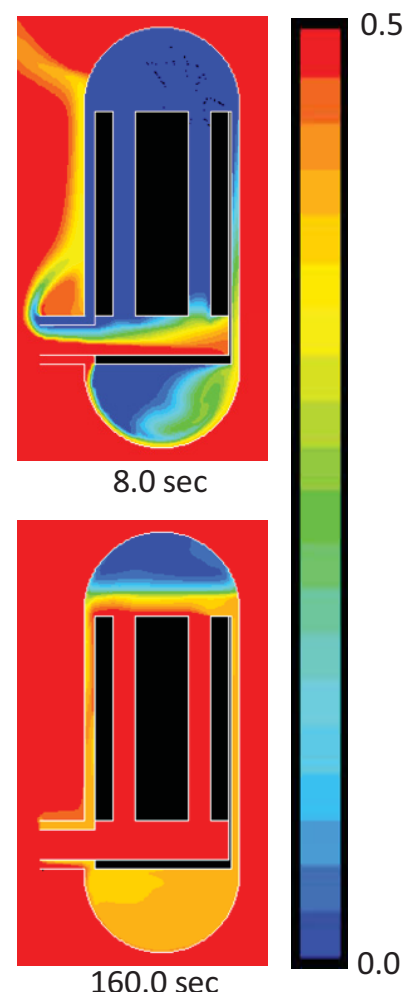

Figure 5-12. No injection $\left(V_{i n j}=0.0 \mathrm{~m} / \mathrm{s}\right)$.

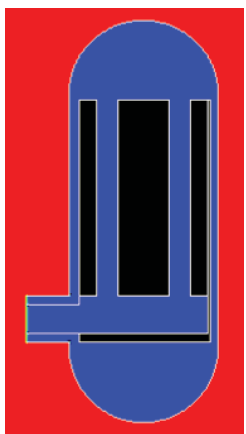

$0.0 \mathrm{sec}$

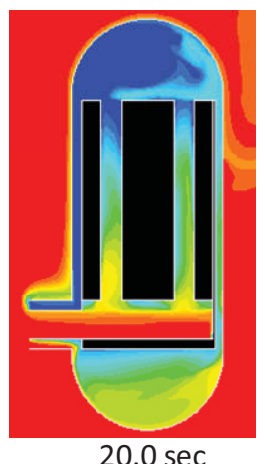

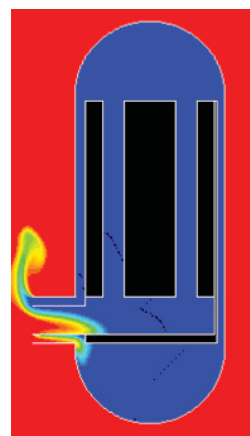

$2.0 \mathrm{sec}$

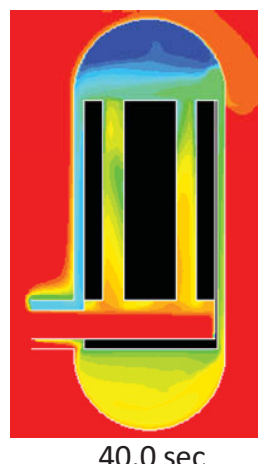

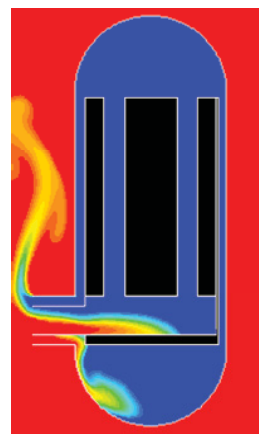

$4.0 \mathrm{sec}$

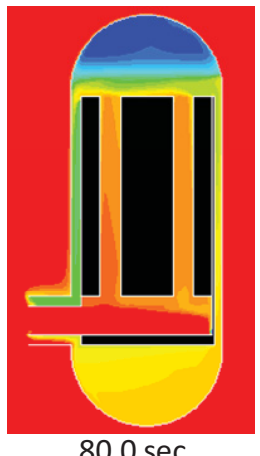

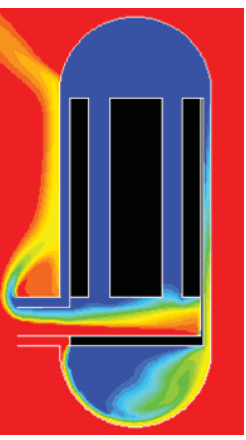

$8.0 \mathrm{sec}$

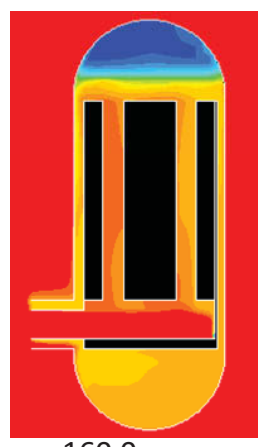

$160.0 \mathrm{sec}$

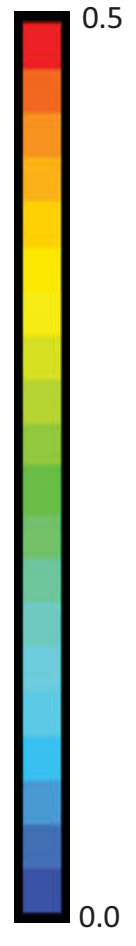

Figure 5-13. Helium injection $\left(V_{\mathrm{inj}}=0.05 \mathrm{~m} / \mathrm{s}\right)$. 

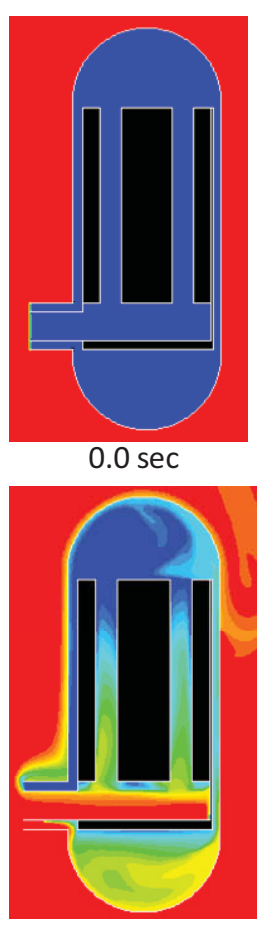

$20.0 \mathrm{sec}$
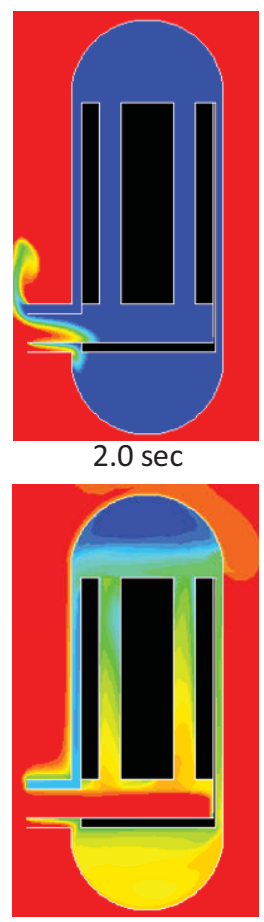

$40.0 \mathrm{sec}$
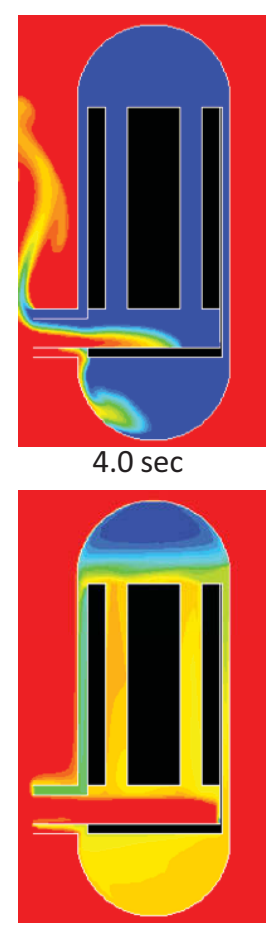

$80.0 \mathrm{sec}$
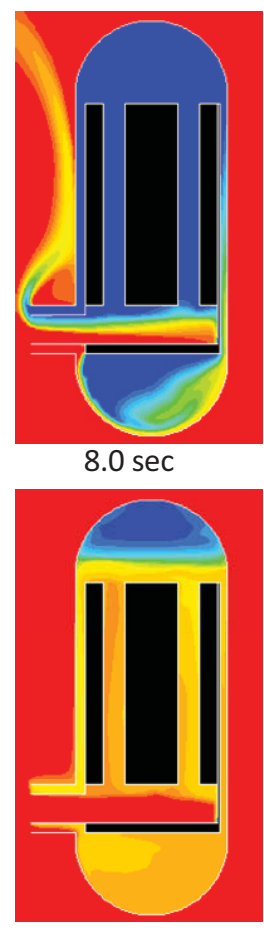

$160.0 \mathrm{sec}$

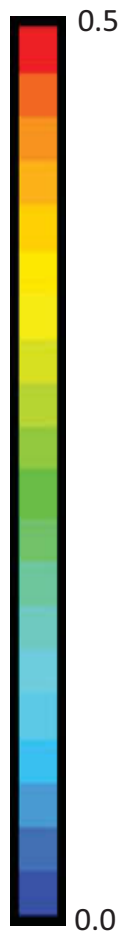

Figure 5-14. Helium injection $\left(V_{\mathrm{inj}}=0.1 \mathrm{~m} / \mathrm{s}\right)$.

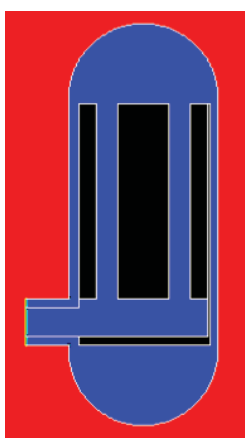

$0.0 \mathrm{sec}$

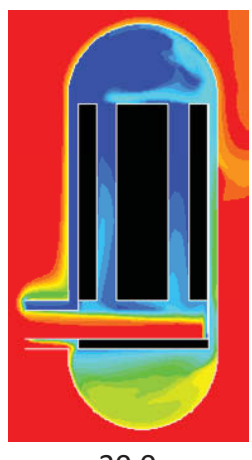

$20.0 \mathrm{sec}$

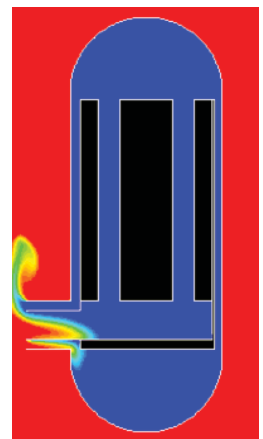

$2.0 \mathrm{sec}$

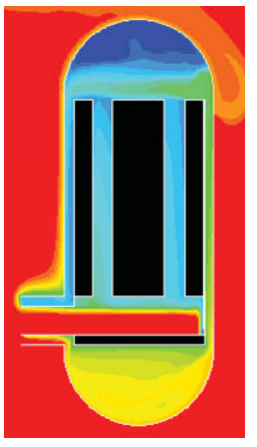

$40.0 \mathrm{sec}$

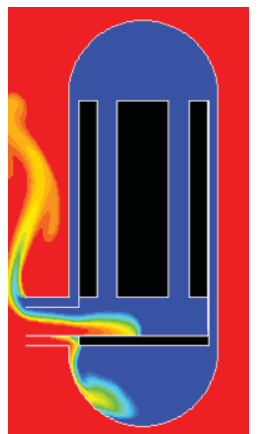

$4.0 \mathrm{sec}$

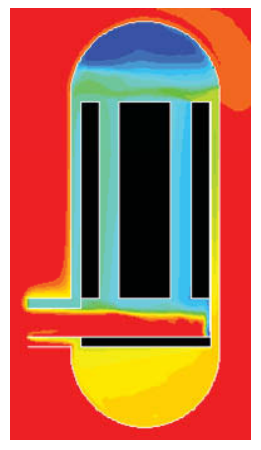

$80.0 \mathrm{sec}$

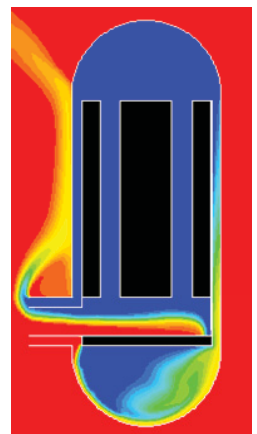

$8.0 \mathrm{sec}$

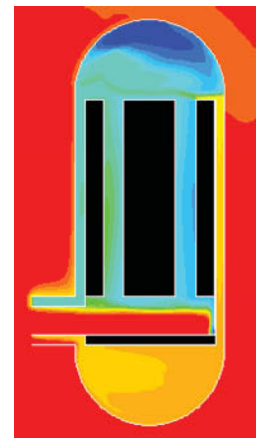

$160.0 \mathrm{sec}$

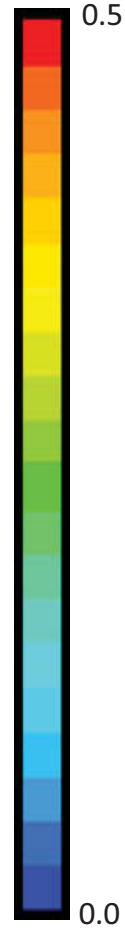

Figure 5-15. Helium injection $\left(V_{i n j}=0.5 \mathrm{~m} / \mathrm{s}\right)$. 

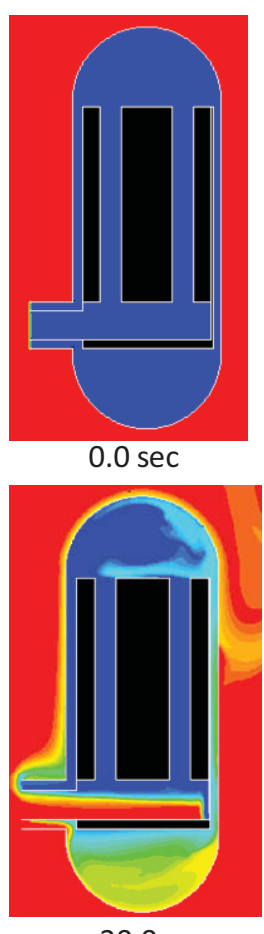

$20.0 \mathrm{sec}$
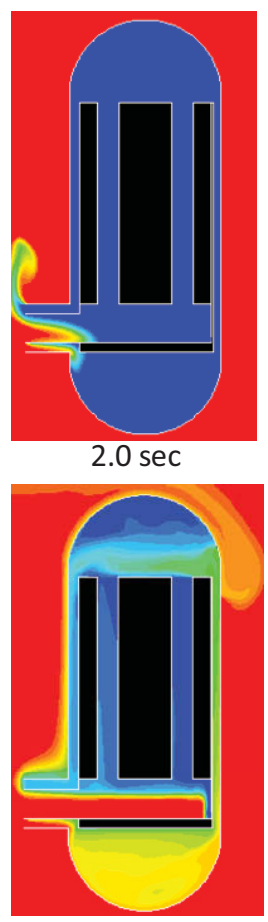

$40.0 \mathrm{sec}$
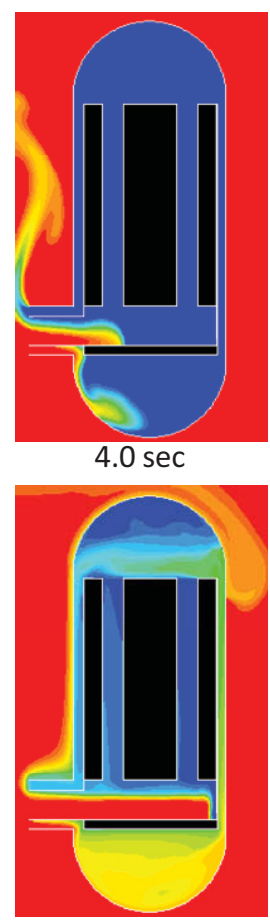

$80.0 \mathrm{sec}$
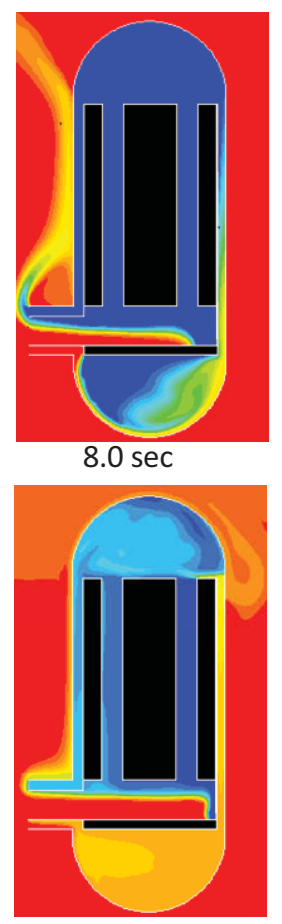

$160.0 \mathrm{sec}$

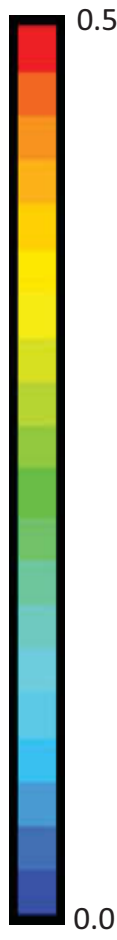

Figure 5-16. Helium injection $\left(V_{\mathrm{inj}}=1.0 \mathrm{~m} / \mathrm{s}\right)$.

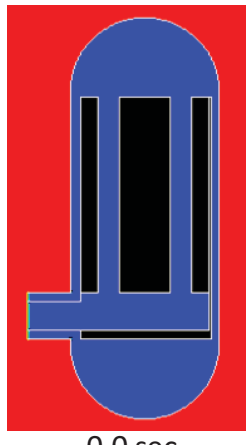

$0.0 \mathrm{sec}$

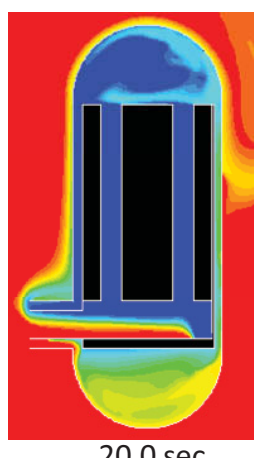

$20.0 \mathrm{sec}$

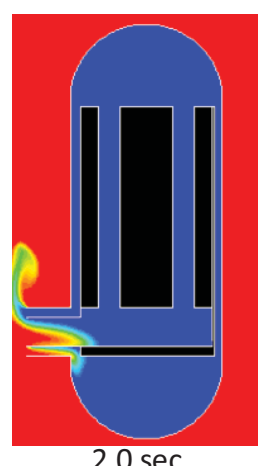

$2.0 \mathrm{sec}$

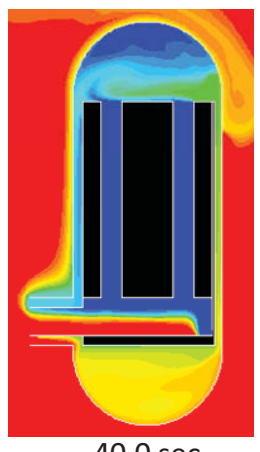

$40.0 \mathrm{sec}$

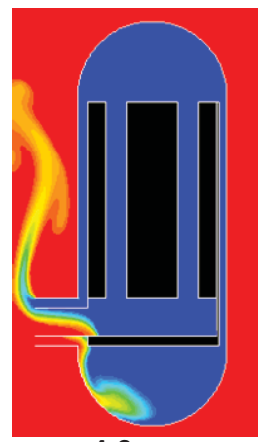

$4.0 \mathrm{sec}$

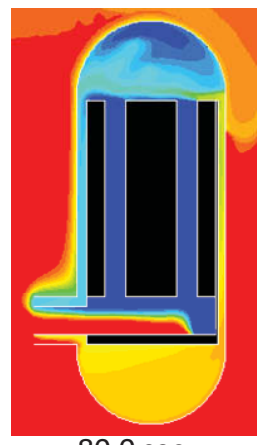

$80.0 \mathrm{sec}$

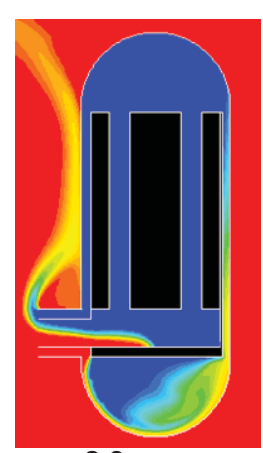

$8.0 \mathrm{sec}$

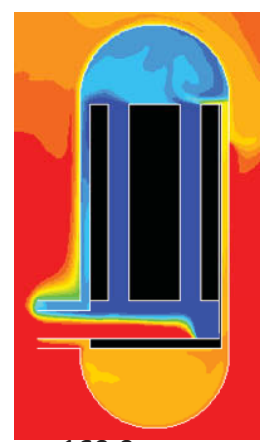

$160.0 \mathrm{sec}$

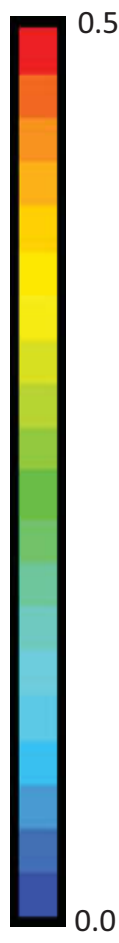

Figure 5-17. Helium injection $\left(V_{i n j}=2.0 \mathrm{~m} / \mathrm{s}\right)$. 


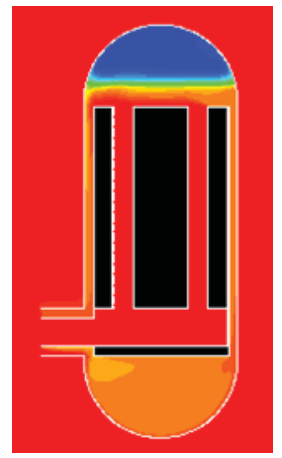

$v_{\text {inj }}=0.0 \mathrm{~m} / \mathrm{s}$

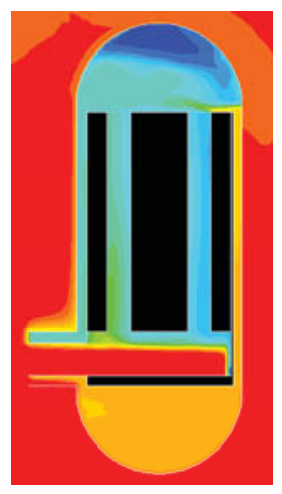

$\mathrm{v}_{\mathrm{inj}}=0.5 \mathrm{~m} / \mathrm{s}$

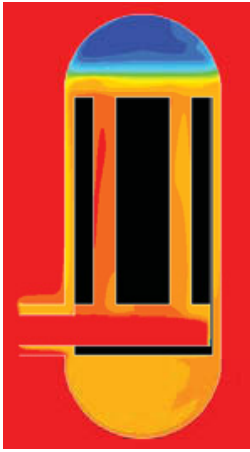

$v_{i n j}=0.05 \mathrm{~m} / \mathrm{s}$

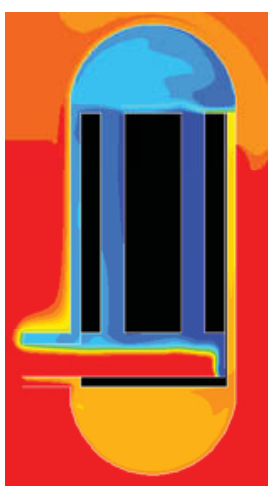

$\mathrm{v}_{\mathrm{inj}}=1.0 \mathrm{~m} / \mathrm{s}$

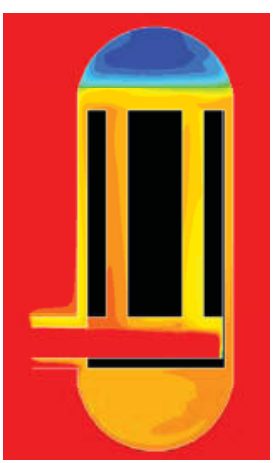

$v_{\text {inj }}=0.1 \mathrm{~m} / \mathrm{s}$

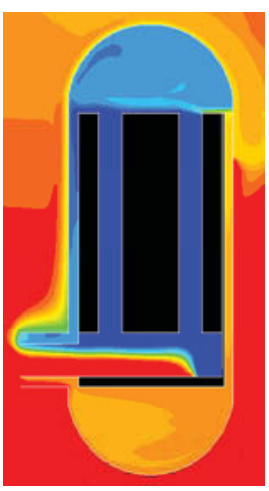

$\mathrm{v}_{\mathrm{inj}}=2.0 \mathrm{~m} / \mathrm{s}$

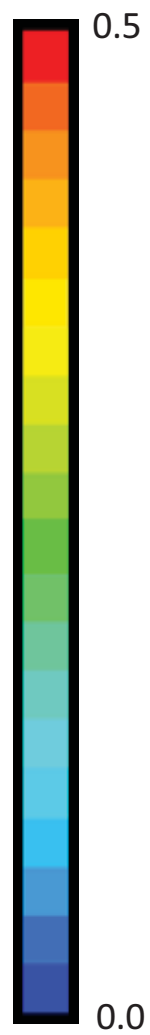

0.0

Figure 5-18. Comparisons on the air distributions for the different injection speed $(\mathrm{t}=200 \mathrm{sec})$.

Two possible locations in the lower plenum for the helium injection are the side and bottom. However, the bottom of the lower plenum is not proposed here because when the helium injected at the bottom is easily mixed with the air inflow, and the separation between the helium and air could not be achieved. For the prismatic type VHTR, the injection location will be at the opposite wall of the inlet pipe.

\subsubsection{Summary and Conclusions}

This section described a method that injects helium flow into the lower plenum for mitigation of the air-ingress. To validate this method, a CFD simulation was performed, which showed that the helium replaces the air flow into the core and significantly reduces the air concentration in the core and bottom reflector, potentially protecting oxidation damage. According to the simulation results, even a small helium flow was sufficient to remove air in the core, effectively mitigating air ingress.

\subsubsection{Validation of Air-ingress Mitigation using Reactor Enclosure Concept}

The previous section proposes opening an enclosure at the bottom of the reactor to mitigate airingress into the VHTR. The basic idea is to enclose the reactor as shown in Figure 5-19. This enclosure is not the pressure boundary and openings are located at the bottom of the enclosure. So, in the depressurization stage, the air in the inside of the enclosure will be discharged through this opening. Once the pressure between the inside and outside is balanced, the flow will stop. In this case, the air contents in the inside of the enclosure are very small after depressurization because most of the air is discharged out of the enclosure. After pressure balance between the inside and outside enclosure is achieved, air ingress into the enclosure is controlled by a molecular diffusion process, which works because the heavy gas is 
located at the lower part and the light gas is located at the upper part, creating a very stable condition. Even though the density gradient flow is generated in the reactor, the air supply is very limited because of the slow diffusion process into the enclosure. This slow diffusion process keeps the air concentration in the enclosure very low, even after ONC. By this method, the air-ingress controlling mechanism can be changed from the density gradient driven flow to the molecular diffusion mechanism. In the confinement, the relief line is recommended to be downward as shown in the figure in order to limit the air-ingress in to the confinement by diffusion, in case the relief valve closing fails.

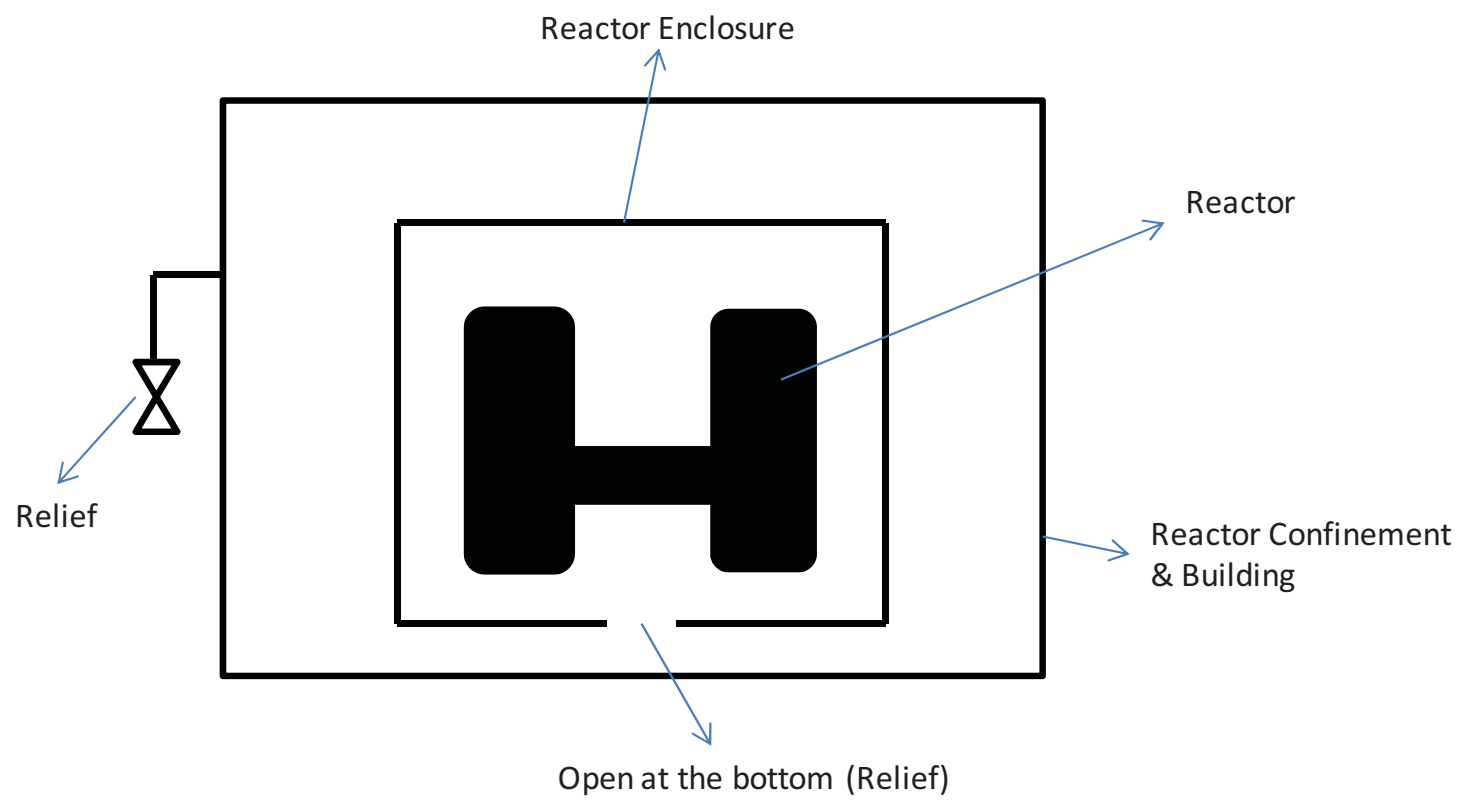

Figure 5-19. Concept of reactor enclosure with opening at the bottom

\subsubsection{Modeling of Reactor Enclosure Using FLUENT Code}

To validate this method, a CFD simulation was performed. The 600 MWth GTMHR was selected as a reference design and the FLUENT 6.3 code was used for simulation. The details are as follows.

\section{Reference System}

The reference system selected here is the $600 \mathrm{MWth}$ GTMHR. This reactor is already explained in the previous section and the schematic is shown in Figure 5-9.

\section{2-D Grid Model and Model Setup}

Figure 5-20 shows the 2-D grid model for the CFD analyses. The grid model was developed by GAMBIT mesh generation software. The geometry was simplified to be 2-D for saving time in computations. The grid mode was divided into seven regions: core, lower plenum, hot-leg, cold leg, vessel inside, enclosure, and confinement. Of these regions, the core was assumed to be the porous media. The porous media parameters were referred to by $\mathrm{Oh}$ et al. (2008). The model was hexagonally meshed by mapping scheme in the inside reactor and the confinement and by pave scheme in the enclosure. The total number of mesh was 85,041 . 


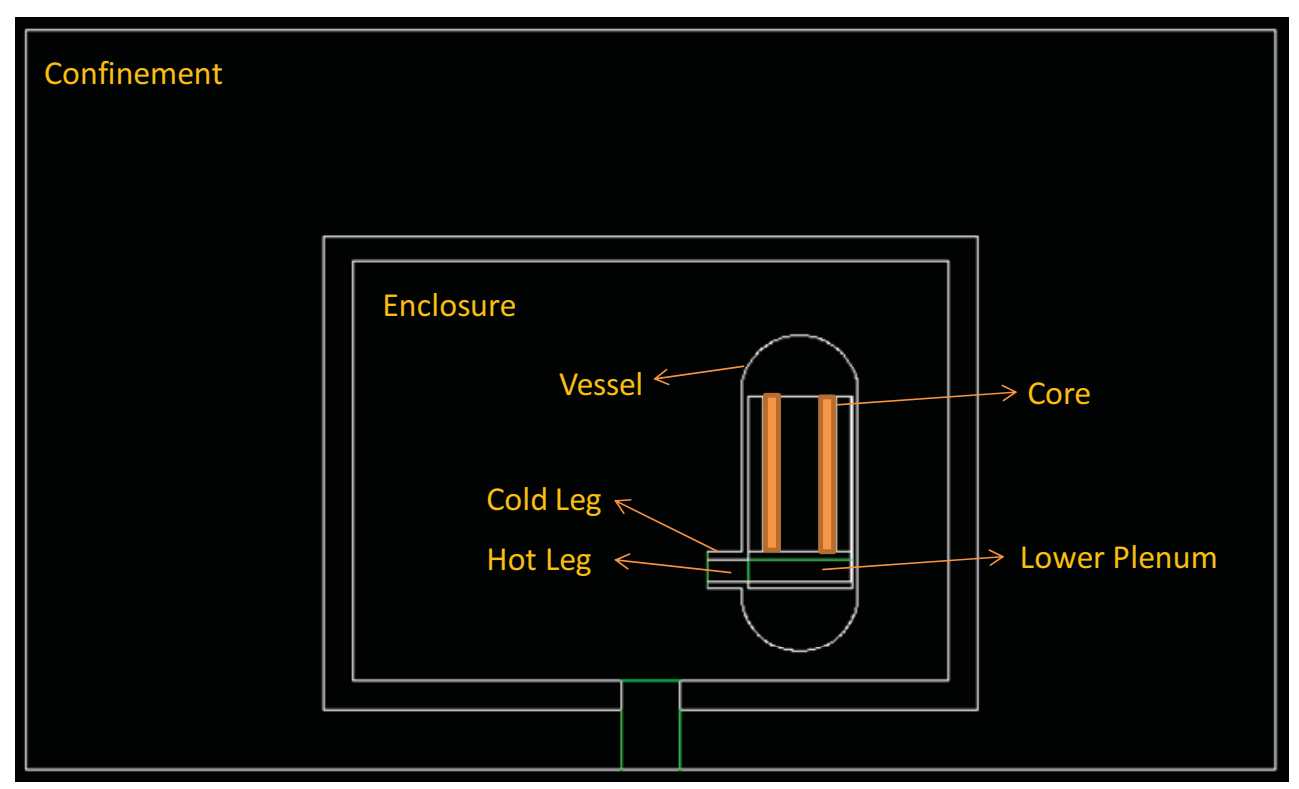

(a) Geometry Summary.

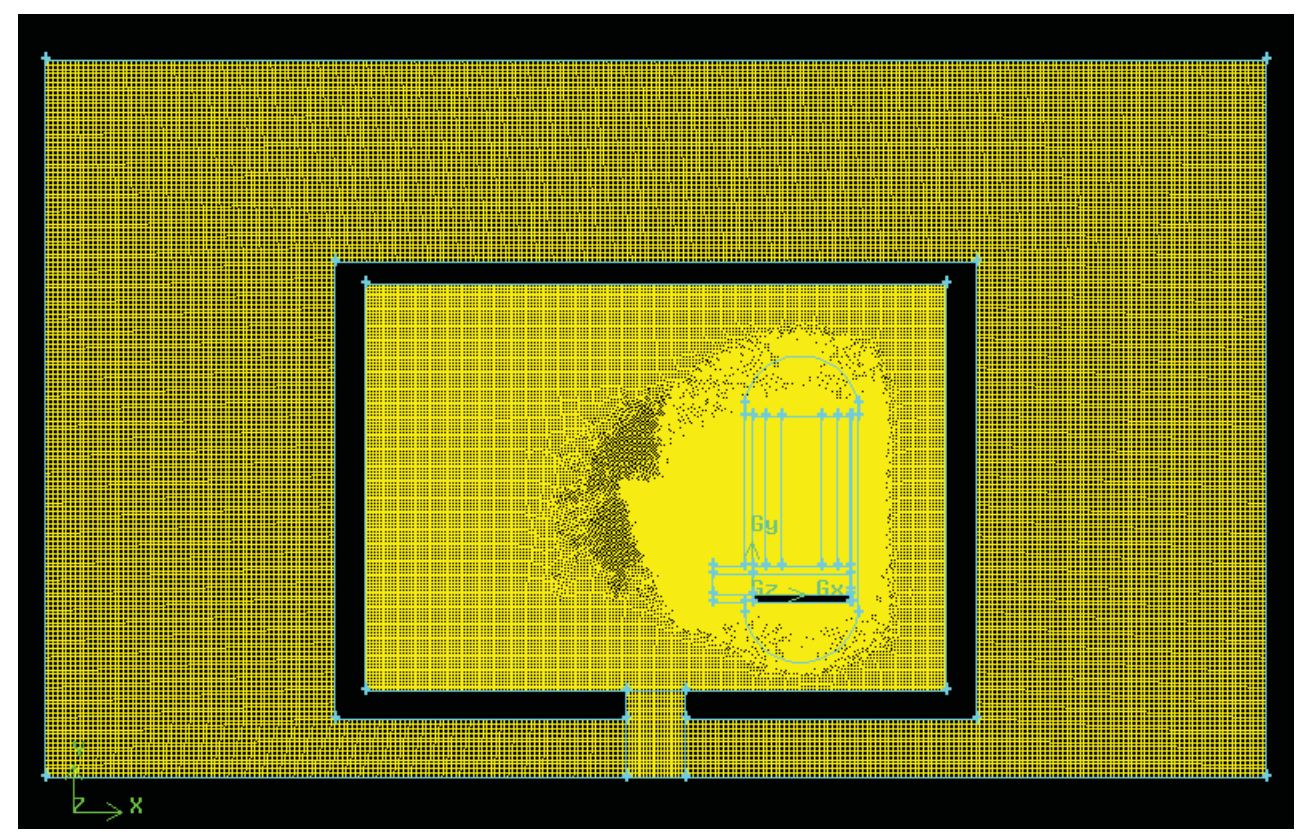

(b) Grid Model (85,041 cells).

Figure 5-20. 2D FLUENT Model (Geometry and Grid Model).

Table 5-4 summarizes the FLUENT solver setup. The FLUENT version used here was 6.3.26. The pressure based solver was selected as a solver type. The model was 2-D, and the time scheme was implicit. This simulation was unsteady and starting after the depressurization process was completed and the pressures were equilibrated. Twenty parallel CPUs were used for the simulations. The viscous model was selected to be laminar model for conservative analyses. Energy and species conservation was also solved here. The two gas species considered in the calculation were air and helium. The incompressible ideal gas law was used for density estimation, and mixing laws were used for other properties. A multicomponent 
diffusion model was used for molecular diffusions. The initial pressure in the whole model was set as $1.0 \mathrm{~atm}$, which represents the condition right after depressurization.

Table 5-4. Reference FLUENT model setup.

\begin{tabular}{|l|l|}
\hline \multicolumn{1}{|c|}{ Parameters } & \multicolumn{1}{c|}{ Settings } \\
\hline Code Version & FLUENT 6.3.26 \\
\hline Solver Type & Pressure Based Solver \\
\hline Time Scheme & Implicit \\
\hline Dimension & 2-D \\
\hline Steady/Unsteady & Unsteady \\
\hline Number of CPUs & 20 \\
\hline Velocity Formulation & Absolute \\
\hline Gradient Option & Node Based \\
\hline Porous Formulation & Physical Velocity \\
\hline Viscous Model & Laminar \\
\hline PressureVelocity Coupling & SIMPLE \\
\hline Energy Equation Solve & Yes \\
\hline Species Equation Solve & Yes \\
\hline Density & Incompressible Ideal Gas Law \\
\hline Heat Capacity & Mixing Law \\
\hline Thermal Conductivity & Mass Weighted Mixing Law \\
\hline Viscosity & Mass Weighted Mixing Law \\
\hline Diffusion & Multi-component \\
\hline &
\end{tabular}

\subsubsection{Initial and Boundary Conditions}

Figure 5-21 shows the initial air concentration and the temperature profile of the simulation. Initially, the air concentration in the inside of the vessel was assumed to be 0.0 since the helium coolant occupies this region before air-ingress. In the enclosure, it was assumed to be 0.5 in mass fraction with a uniform distribution as described in the preceding section. The confinement initial air mass fraction was assumed to be 1.0, which is the most conservative assumption. The outside temperature of the reactor was assumed to be $300 \mathrm{~K}$, and the inside was determined based on the GAMMA code results following the depressurization phase (Oh et al. 2009). The lower plenum temperature profile was also assumed as uniform; the inside structures were not considered here because of the 2-D geometries. The riser temperature at the vessel side was assumed to be $773 \mathrm{~K}\left(300^{\circ} \mathrm{C}\right)$, the enclosure wall temperatures $500 \mathrm{~K}$, and the confinement wall temperature $300 \mathrm{~K}$. The upper and the lower domes of the reactor vessel were assumed to be adiabatic. For other walls, coupled wall heat transfer options were selected to consider heat exchanges through the walls. 


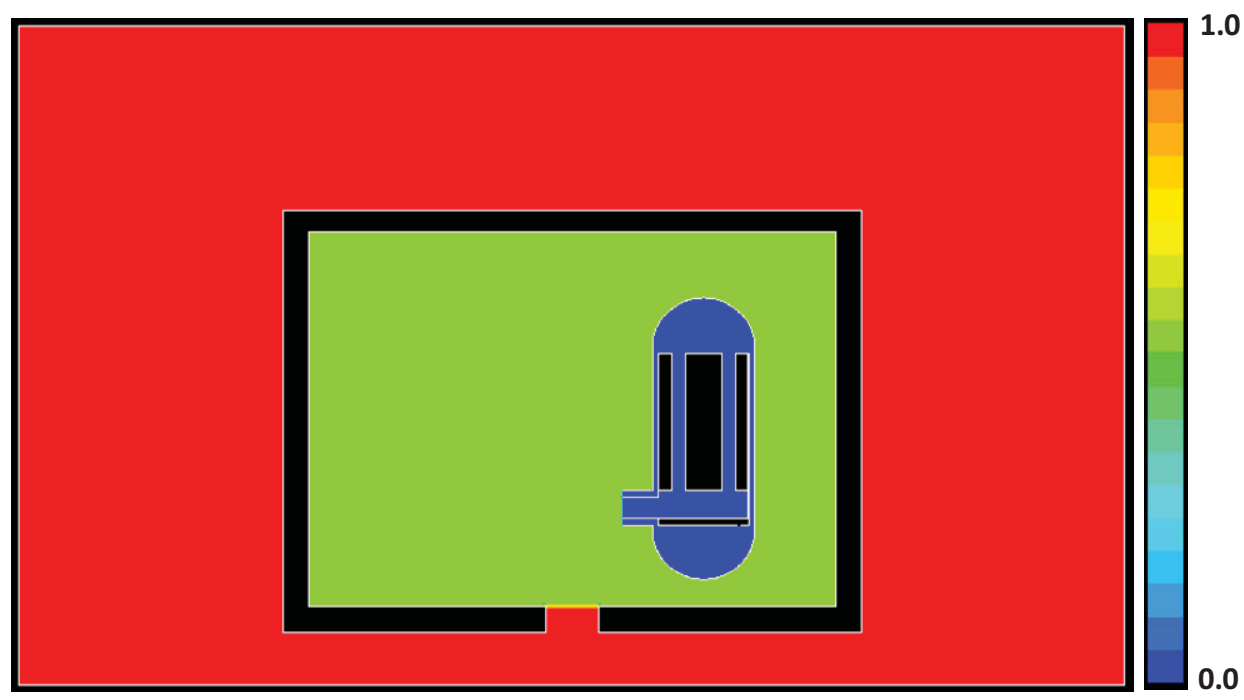

(a) Air mass fraction.

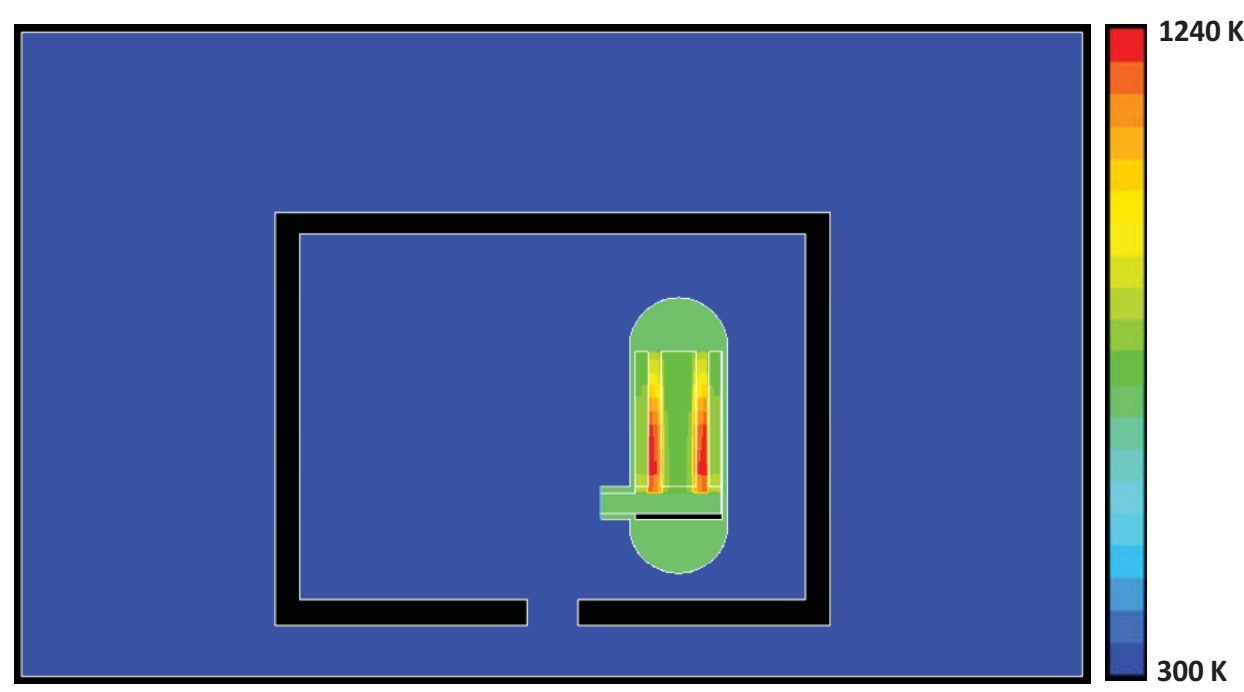

(b) temperature

Figure 5-21. Initial conditions (air mass fraction, temperature).

\subsubsection{CFD Results and Discussions}

Figures 5-22 through 5-29 show the CFD results for different times. Initially, air in the enclosure is ingressed into the reactor by density gradient stratified flow (See Figures 5-23 and 5-24). After the air has filled the lower plenum, it moves into the core by buoyancy force generated by heating (Figures 5-25, 5-26, and 5-27). The air in the cavity moves into the core, flows up towards to the reactor core, and flows out of the vessel through the riser and the cold-leg (Figures 5-27 and 5-28). After that, the global circulation is dominated in the reactor (Figure 5-29) with a tiny air flow showing at the bottom opening slot. One notable point in this simulation result is that the air-ingress at the bottom opening of the enclosure is controlled by molecular diffusion, even though ONC starts. Therefore, the overall air-ingress rate into the reactor inside will be limited by the air diffusion through the opening at the enclosure after the air in the enclosure is initially consumed. 


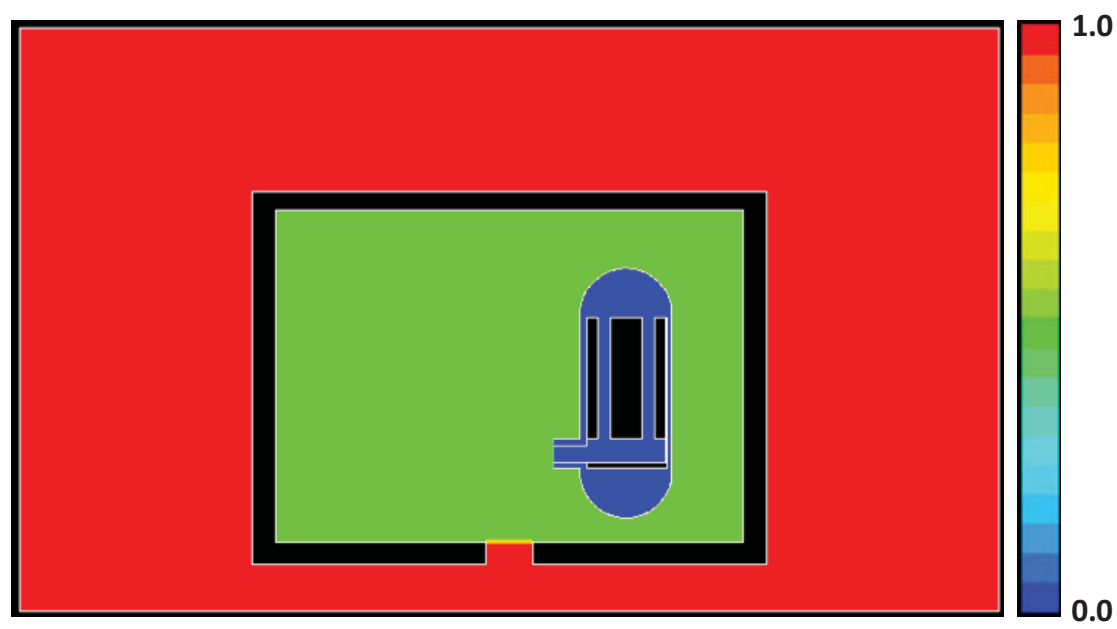

Figure 5-22. Air mass fractions $(0.0 \mathrm{sec})$.

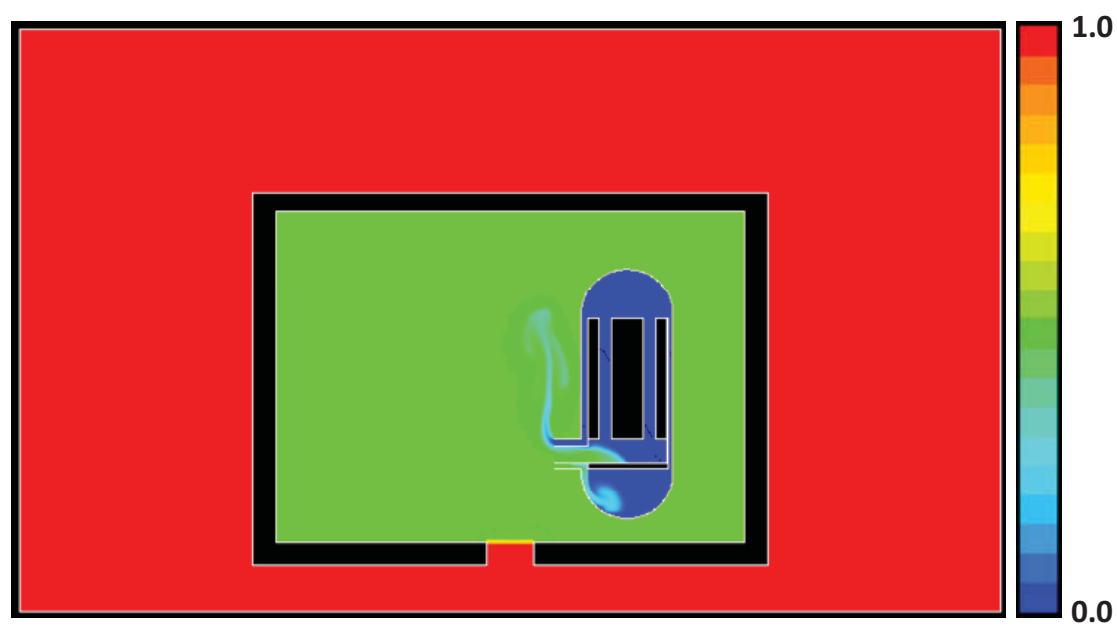

Figure 5-23. Air mass fractions (4.0 sec).

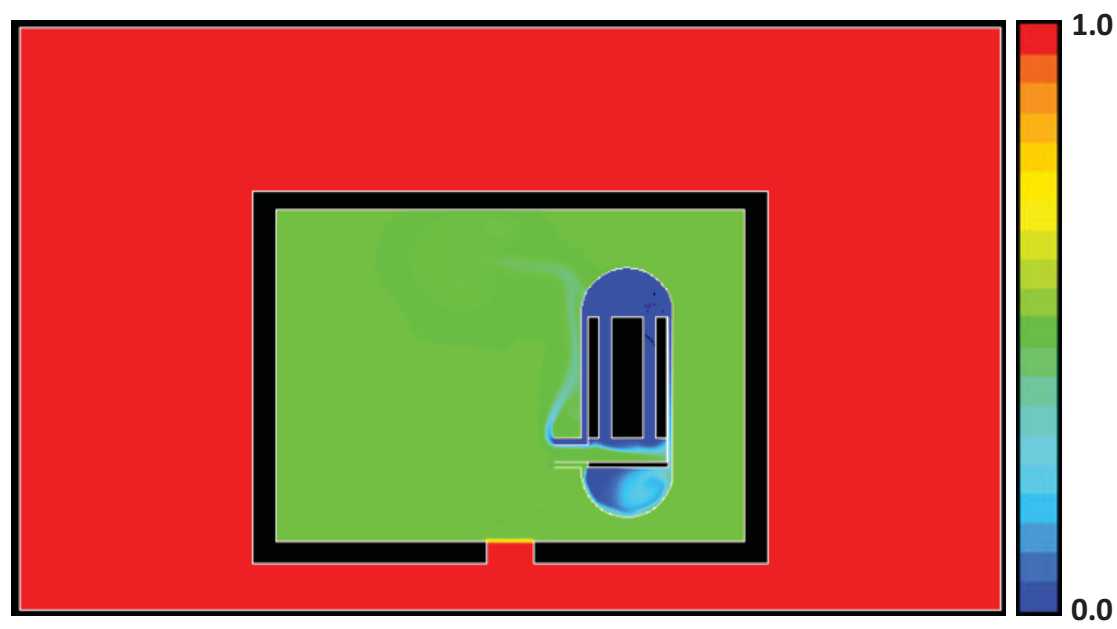

Figure 5-24. Air mass fractions (8.0 sec). 


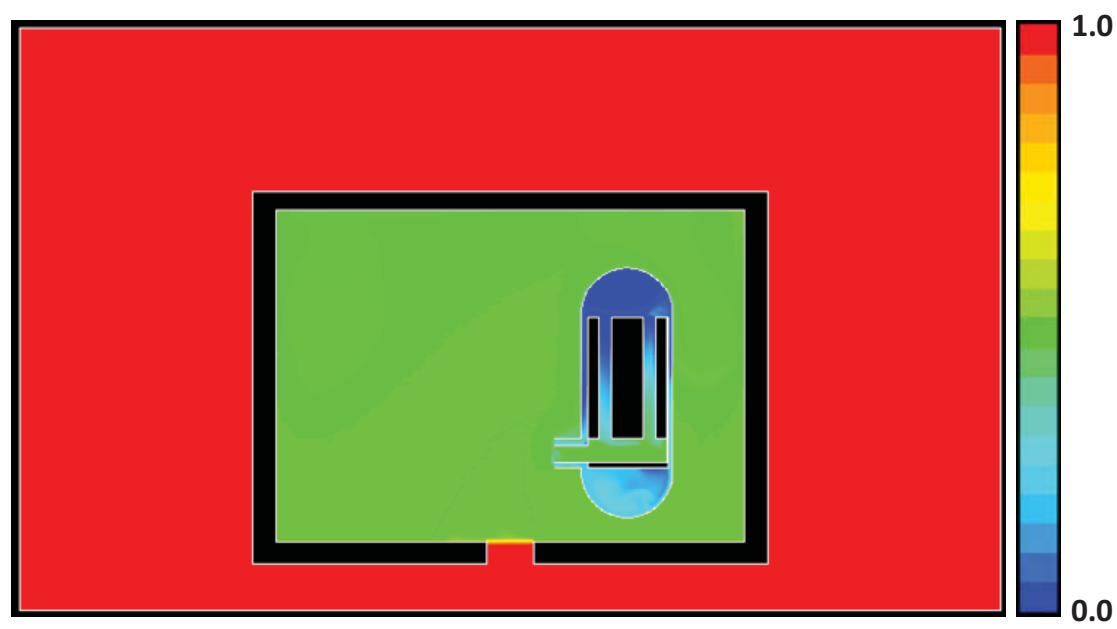

Figure 5-25. Air mass fractions (16.0 sec).

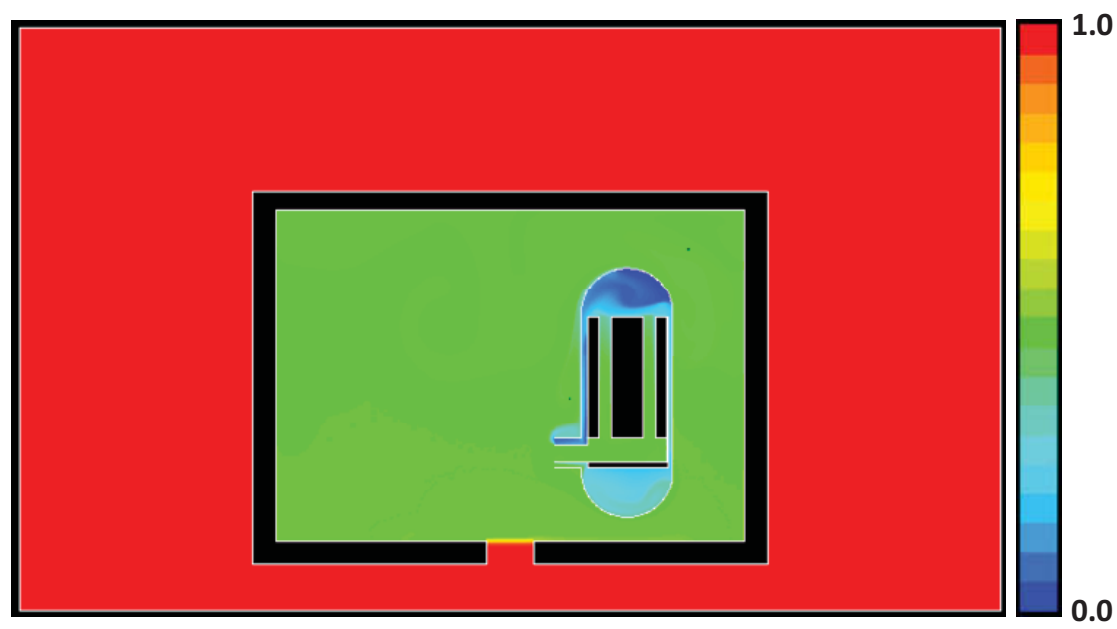

Figure 5-26. Air mass fractions (32.0 sec).

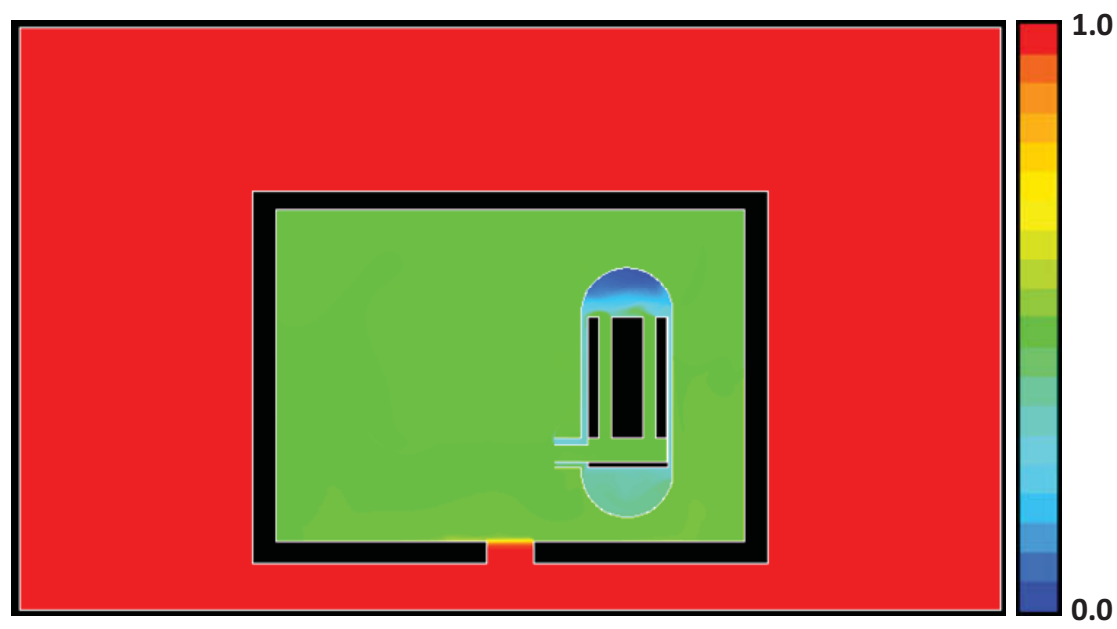

Figure 5-27. Air mass fractions (64.0 sec). 


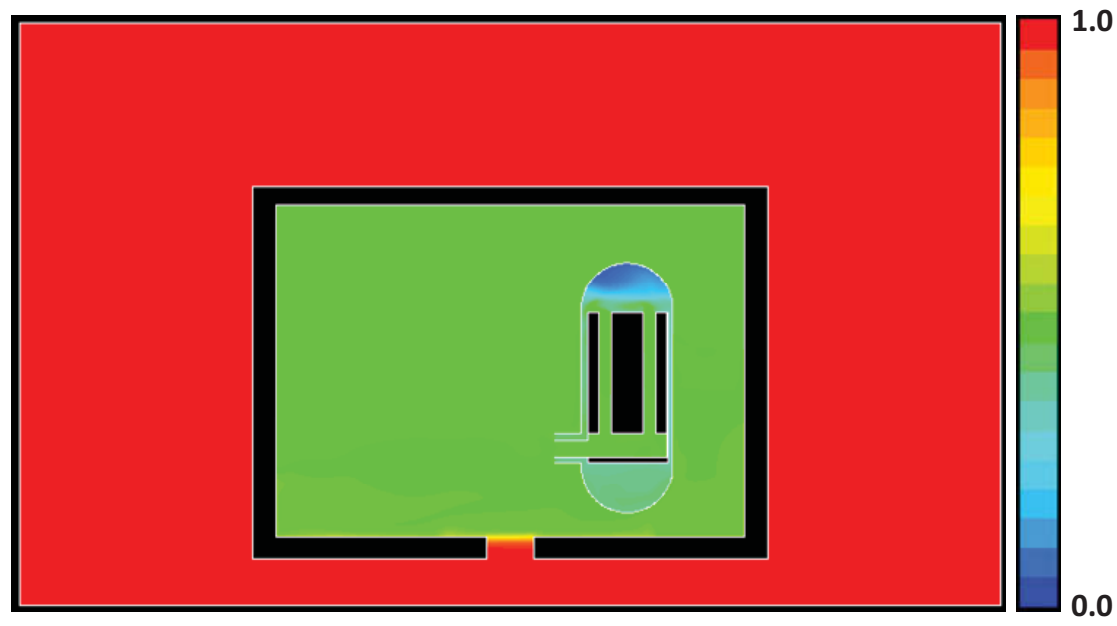

Figure 5-28. Air mass fractions (128.0 sec).

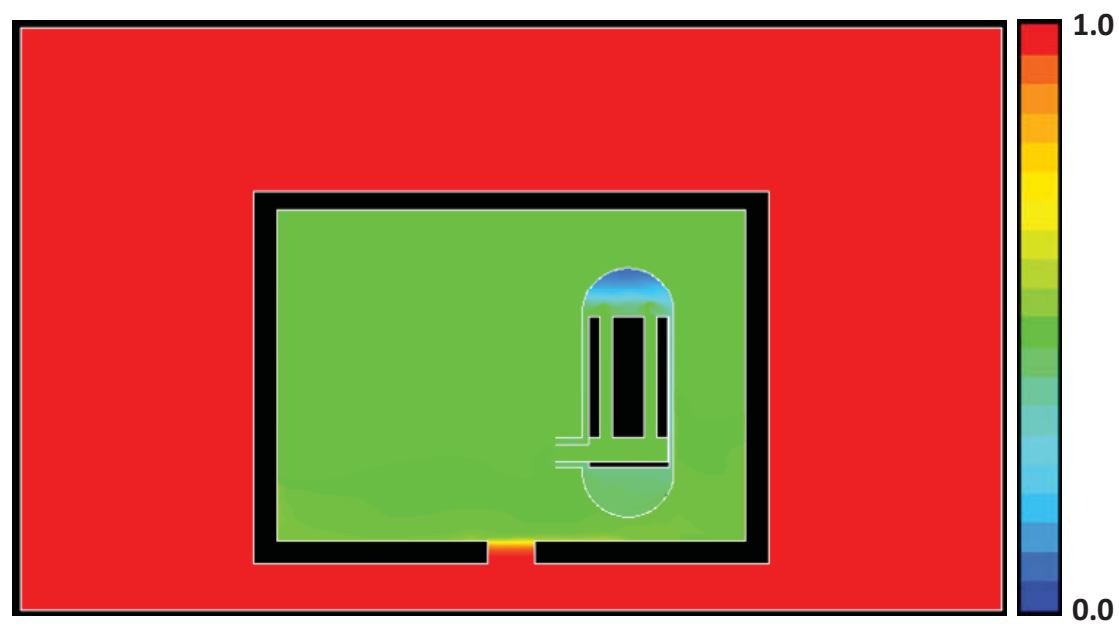

Figure 5-29. Air mass fractions $(216.0 \mathrm{sec})$.

Figure 5-30 shows the details of the air-ingress mechanism when the proposed air-ingress mitigation method is applied. As shown in this figure, air ingress (molecular diffusion) through the opening is not affected by the natural circulation flow in the reactor because the air volume in the enclosure is very small and this air volume does not affect the graphite oxidation much. A more detailed calculation will be made when the final design of the modular HTGR is completed. 


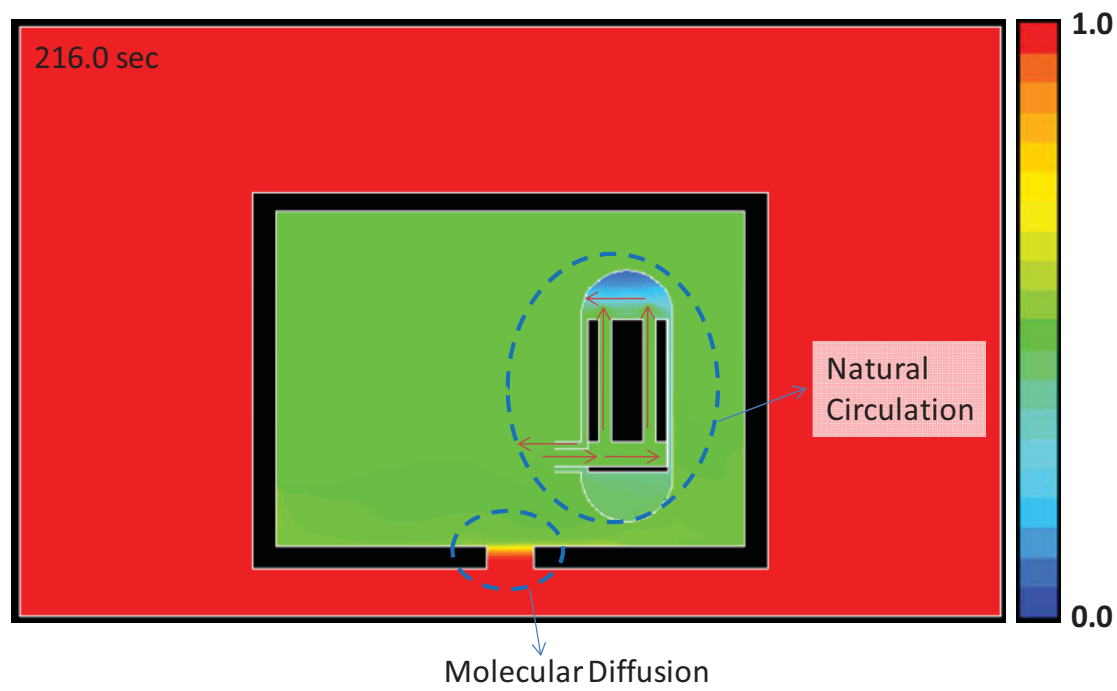

Figure 5-30. Air-ingress mechanism in the proposed air-ingress mitigation method $(\mathrm{t}=216 \mathrm{sec})$.

\subsubsection{Summary}

This section described a method that used the reactor enclosure opened at the bottom to mitigate an air-ingress accident. This method was validated by performing a CFD simulation. The main idea of this method is to change the density-gradient-flow-controlled air-ingress process to the molecular-diffusion controlled process. The simulation results show that the enclosure open at the bottom can successfully mitigate air-ingress into the reactor, even after ONC occurs.

\subsection{Summary of Air Ingress Mitigation}

Section 5 discussed various air-ingress mitigation methods that apply to the VHTR. The previous airingress mitigation concept, which injected helium into the top of the reactor, may not work in the VHTRs for a DEGB situation because:

- The way they modeled does not represent the cross duct of the GTHTR300 because an axi-symmetric assumption was made in their study. Because of the unique characteristics of the GTHTR300 cross duct similar to that of a modular HTGR, the CFD model should use a 180 degree model.

- The momentum created by the helium top injection is 0.062 Newton when compared with the buoyancy forces created in the lower plenum of the VHTR, which is $155 \mathrm{~N}$ (for the DEGB case). Therefore, the buoyancy force is much larger than that of the top injected flow by a factor of 2,500. The top inject concept is therefore not feasible to prevent air flow to the VHTR.

Second, important factors affecting air-ingress consequences were figured out from the root-cause analysis as a starting point of the air-ingress mitigation study. The basic air-ingress ideas were developed from this analysis. The air-ingress can be conceptually mitigated by preventing the main causes identified in the root-cause analysis. The main air-ingress mitigation concepts are to prevent:

- Fracture (under structural degradation or load increase conditions)

- Structural Degradation (under oxidation environment)

- Load Increase (under oxidation environment) 
- Oxidation (under air-ingress and high temperature conditions)

- Air Ingress (under density gradient existing conditions)

- Density Gradient Driven Flow (under density gradient existing conditions)

- Density Gradient (under temperature and molecular mass difference existing conditions)

- Temperature Gradient (between inside and outside of the reactor)

- Molecular Mass Difference (between inside and outside of the reactor)

- High Temperature (in the reactor inside).

From the basic concepts, various air-ingress mitigation methods were proposed. Of those, the following two mitigation methods were strongly recommended.

- Helium injection in the lower plenum: This method injects helium into the lower plenum. The injected helium replaces the air in the core and the lower plenum upper part by buoyancy force. It significantly reduces graphite oxidation in the inside of the reactor.

- Reactor enclosure opened at the bottom: This method encloses the reactor by a nonpressure boundary. Some design modifications of the cavity can be used for this. This enclosure has an opening at the bottom. After depressurization, the air-ingress rate is controlled by molecular diffusion through this opening even after ONC.

Validation of those two air-ingress mitigation methods was conducted by CFD methods. The results show that both methods are effectively mitigating air-ingress process. 


\section{TASK 5: EXPERIMENT OF BURN-OFF IN THE BOTTOM REFLECTOR (KAIST)}

This task measured the oxidation rate and density of the nuclear graphite and developed oxidation models of the bottom reflector that would be directly exposed to the air-ingress event. The main parameters affecting the rate of oxidation and density of the graphite of the bottom reflector are kinetics, mass diffusion, combined effect of kinetics and mass diffusion, moisture, shape and size, and degree of burn-off. Several types of candidate graphite were selected for the experiment.

\subsection{Graphite Selection}

Candidate graphite materials are proposed at the NGNP program: NGNP graphite testing and qualification specimen selection strategy (Robert and Timothy 2005). Some of the candidates were listed in Table 6-1. IG-110 and IG-430, which are isotropic and fine-grained graphite produced by Toyo Tanso, were selected for the experiment. Most of the data for IG-110, except the effect of burn-off on the reaction rate, were obtained from the previous research (Oh et al. 2006). General properties of IG-110 and IG-430 are presented in Table 6-2.

Table 6-1. Graphite selection matrix.

\begin{tabular}{|c|c|c|c|}
\hline Graphite & Vendor & Proposed Use & Remarks \\
\hline IG-110 & Toyo Tanso & $\begin{array}{l}\text { Prismatic fuel element, replaceable } \\
\text { reflector, and core support pedestals }\end{array}$ & $\begin{array}{l}\text { Historical Reference } \\
\text { Currently being used in the HTTR } \\
\text { and HTR-10 }\end{array}$ \\
\hline PCEA & $\begin{array}{l}\text { Graftech } \\
\text { International }\end{array}$ & $\begin{array}{l}\text { Prismatic fuel and replaceable block } \\
\text { Pebble bed reflector and insulation } \\
\text { blocks }\end{array}$ & $\begin{array}{l}\text { AREVA wants to construct the } \\
\text { entire graphite core out of the same } \\
\text { graphite }\end{array}$ \\
\hline NBG-17 & SGL & $\begin{array}{l}\text { Prismatic fuel element and } \\
\text { replaceable reflector } \\
\text { Pebble bed reflector structure and } \\
\text { insulation blocks }\end{array}$ & $\begin{array}{l}\text { AREVA wants to construct the } \\
\text { entire graphite core out of the same } \\
\text { graphite }\end{array}$ \\
\hline IG-430 & Toyo tanso & $\begin{array}{l}\text { Prismatic fuel element, replaceable } \\
\text { reflector, and core support pedestals }\end{array}$ & $\begin{array}{l}\text { Japan Atomic Energy Agency wants } \\
\text { to use this graphite in the GTHTR } \\
300\end{array}$ \\
\hline PGX & $\begin{array}{l}\text { Graftech } \\
\text { International }\end{array}$ & Prismatic large permanent reflector & $\begin{array}{l}\text { AREVA may use this material; } \\
\text { preference is to use PCEA or NBG- } \\
17 \text { for permanent reflector. HTTR } \\
\text { permanent structure }\end{array}$ \\
\hline NBG-25 & SGL & Core support candidate & Isostatic fine grain \\
\hline NBG-10 & SGL & $\begin{array}{l}\text { Prismatic Fuel element and } \\
\text { replaceable reflector } \\
\text { Pebble bed reflector structure and } \\
\text { insulation blocks }\end{array}$ & $\begin{array}{l}\text { pebble bed modular reactor's } \\
(\mathrm{PBMR}) \text { original choice for } \\
\text { replaceable reflector } \\
\text { Price/performance will be the basis } \\
\text { between NBG-18 and NBG-10 }\end{array}$ \\
\hline
\end{tabular}


Table 6-2. Properties of IG-110 and IG-430 manufactured by Toyo Tanso in Japan.

\begin{tabular}{|l|l|l|}
\hline \multicolumn{1}{|c|}{ Material } & \multicolumn{1}{c|}{ IG-110 } & \multicolumn{1}{c|}{ IG-430 } \\
\hline Vendor & Toyo Tanso & Toyo Tanso \\
\hline Bulk Density (g/cm3) & 1.77 & 1.88 \\
\hline Young's Modulus (GPa) & 9.8 & 10.8 \\
\hline Compressive strength (MPa) & 78 & 90 \\
\hline Tensile strength (MPa) & 25 & 37 \\
\hline Hardness (HSD) & 51 & 55 \\
\hline Thermal Conductivity (W/mK) & 120 & 140 \\
\hline
\end{tabular}

\subsection{Graphite Oxidation Model}

\subsubsection{Kinetics Effect}

Kinetics tests were performed in Zone I. where the kinetics effect controls the rate of reaction. Variables were experimentally investigated. A schematic of the experimental facility is shown in Figure 6-1. $\mathrm{He} / \mathrm{O}_{2}$ mixture gas was injected into the heated test section. Injected mixture gas was controlled by a mass flow controller. A $15 \mathrm{~kW}$ induction heater was installed for graphite heating. Gas passing through the test section was cooled and analyzed through a gas analyzer. The reaction rate was calculated by gas component analysis through two gas analyzers (Rosemount NGA2000, Yokogawa IR100). Figure 6-2 shows the picture of the experimental facility.

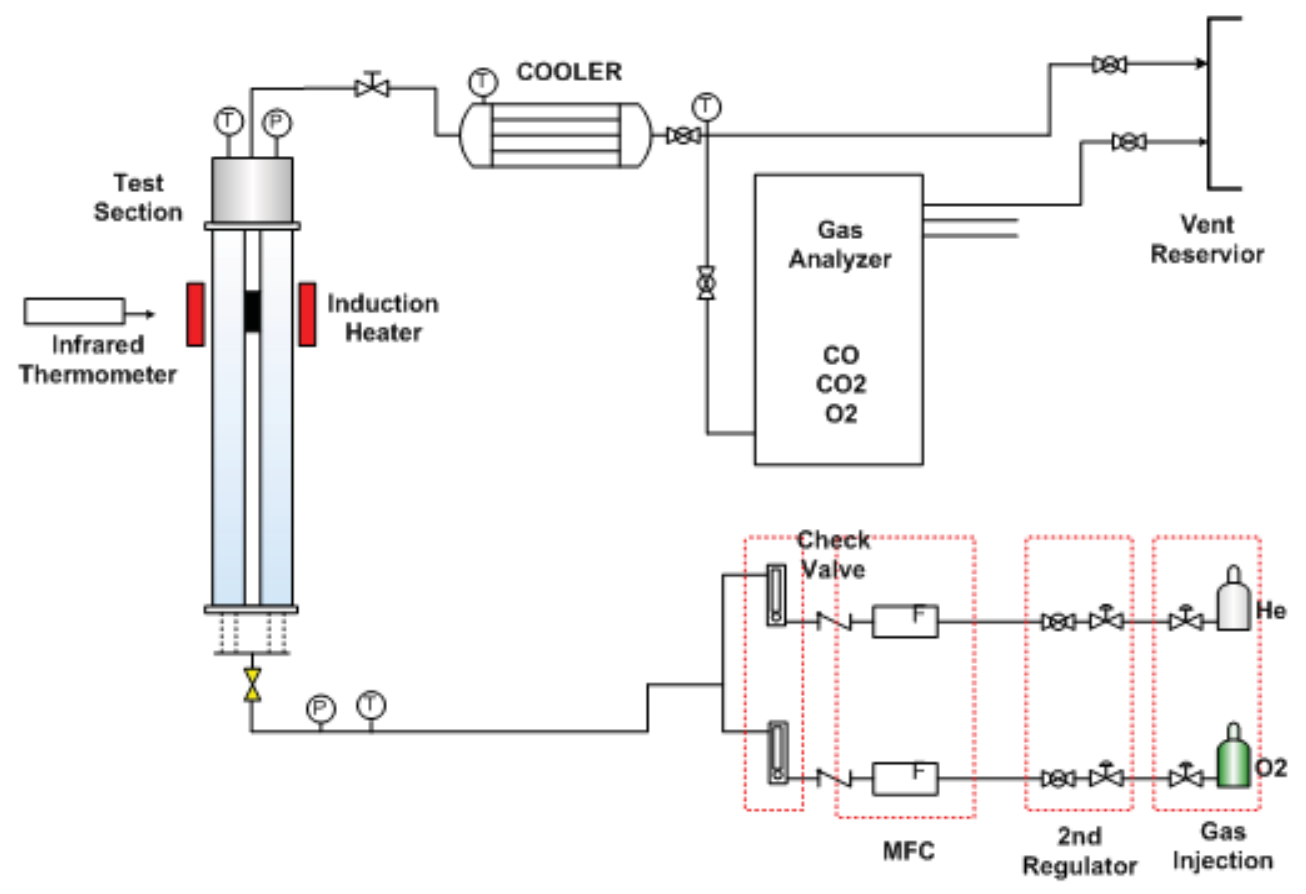

Figure 6-1. Schematic of experimental facility.

The section was surrounded by a cylindrical quartz tube. A long entry length was designed to maintain the fully developed flow. The specimen was $2.1 \mathrm{~cm}$ in diameter and $3 \mathrm{~cm}$ in height and the test section was $7.6 \mathrm{~cm}$ in diameter. An induction heating method was used and the temperature was measured by an infrared thermometer (IRtext Taymatic 10, Raytec Ranger 3). 


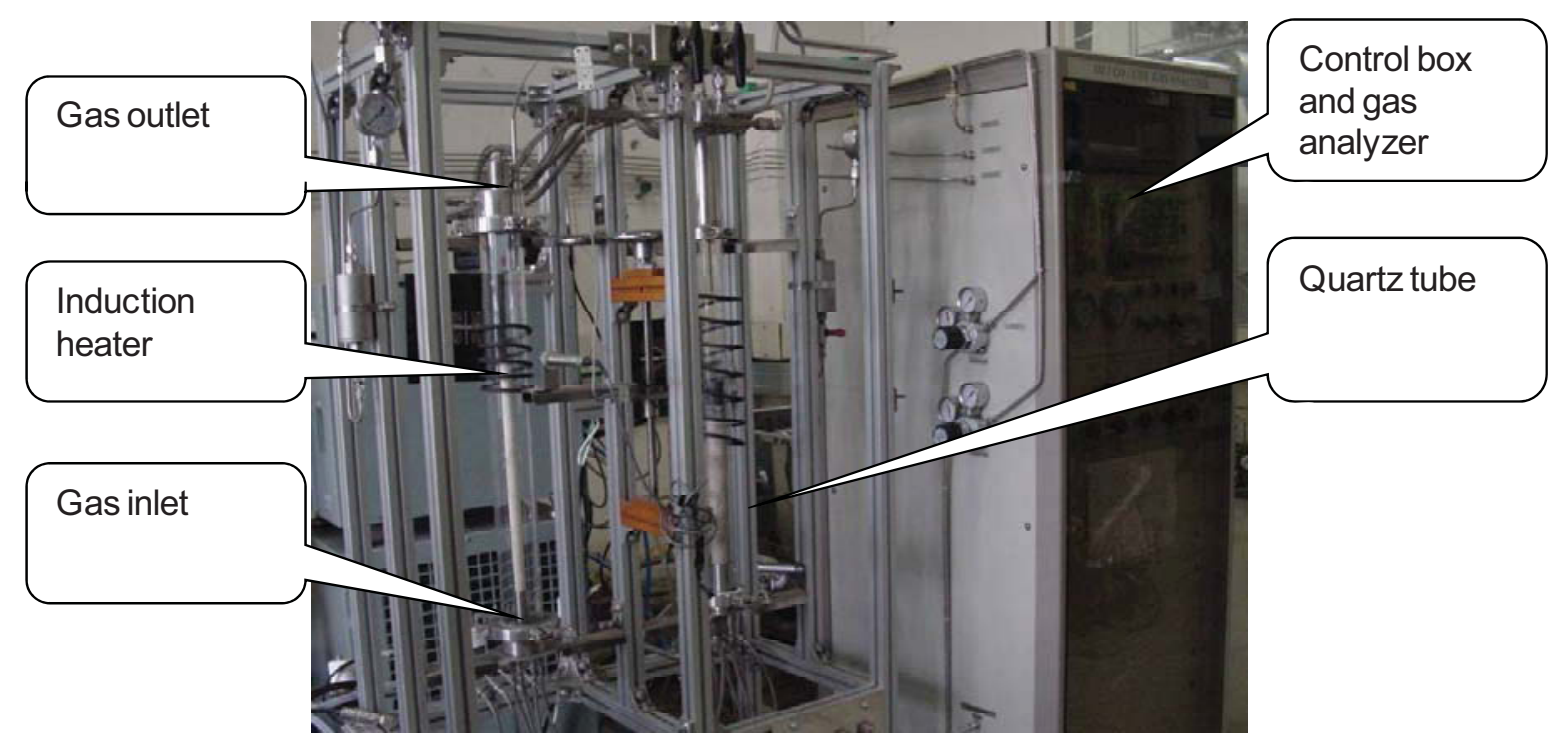

Figure 6-2. Experimental facility.

Temperature, flow rate, and oxygen concentration are summarized in Table 6-3

Table 6-3. Test conditions.

\begin{tabular}{|l|l|}
\hline Temperature $\left({ }^{\circ} \mathrm{C}\right)$ & $540 \sim 800$ \\
\hline Flow rate $($ SLPM) & $\sim$ SLPM $(0.04 \mathrm{~m} / \mathrm{s})$ \\
\hline Oxygen fraction $(\%)$ & $\sim 34 \%$ \\
\hline
\end{tabular}

Data measured at 5.26\% of oxygen concentration were shown in Figure 6-3. They are in good agreement with results predicted by the Arrhenius model. The sensitivity study on this model has been performed in the previous experiment. The activation energy was not affected by oxygen concentration (Oh et al. 2006). The reaction rate at different oxygen concentration was measured to obtain the order of reaction. The effect of oxygen concentration on oxidation rates is shown in Figure 6-4.

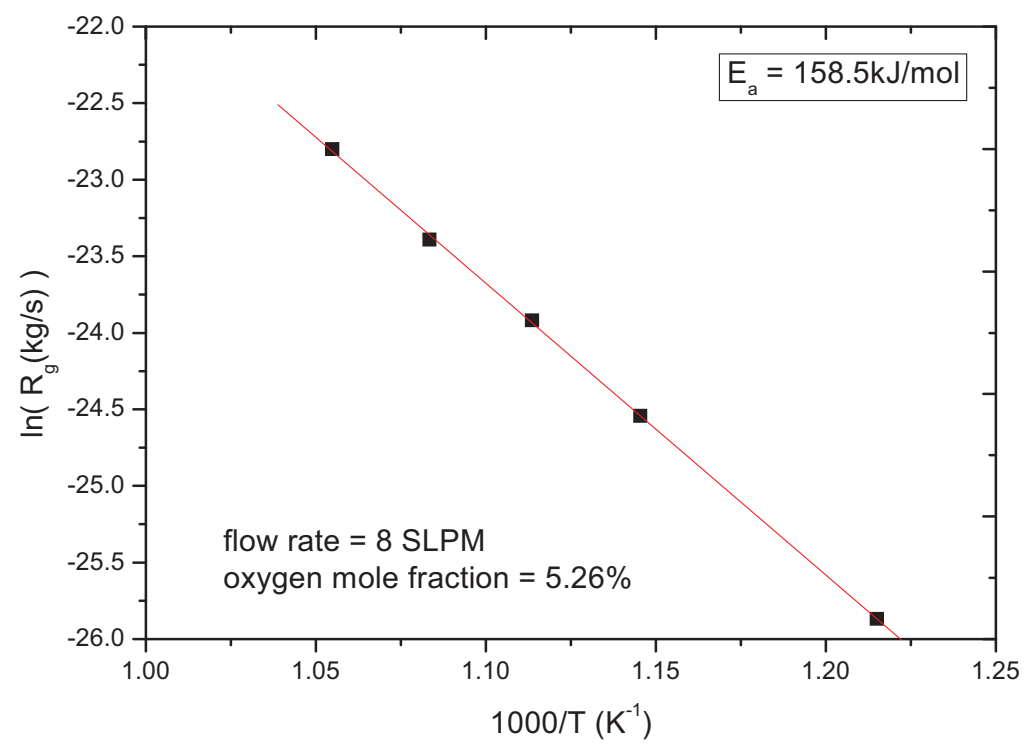

Figure 6-3. Effect of temperature on oxidation rate (IG-430). 


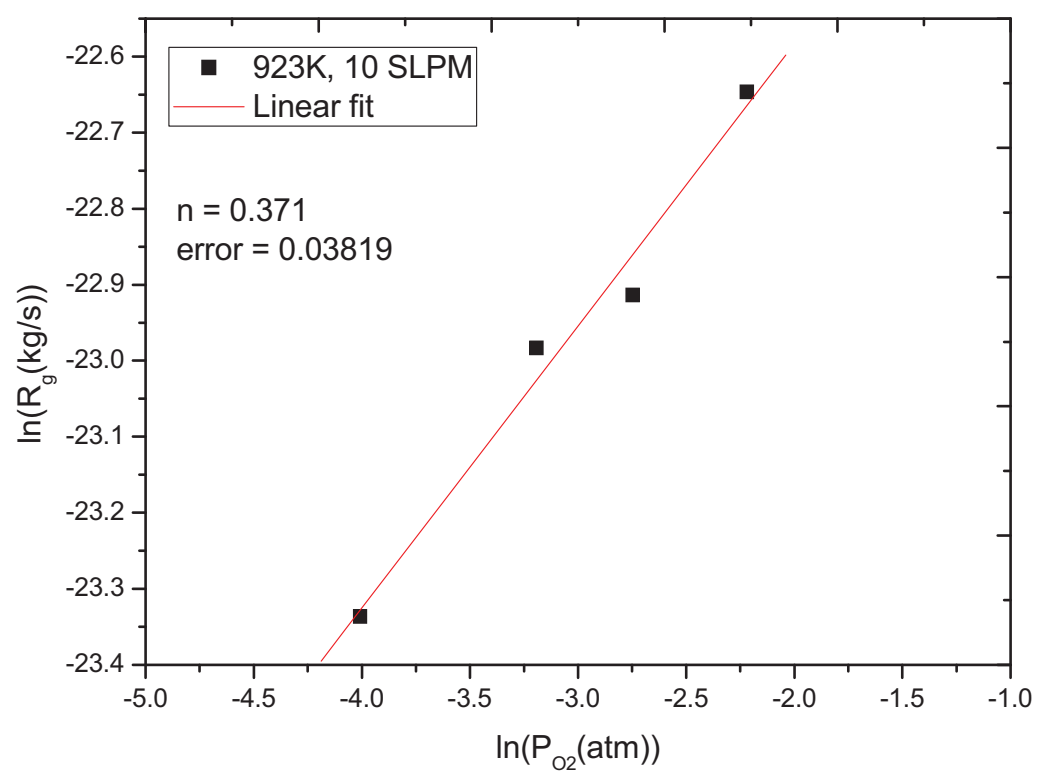

Figure 6-4. Effect of oxygen concentration on oxidation rate (IG-430).

The previous and KAIST kinetics parameters for IG-430 are summarized in Tables 6-4 and 6-5.

Table 6-4. Activation energy and order of reaction.

\begin{tabular}{|l|l|r|}
\hline \multicolumn{1}{|c|}{ Material } & \multicolumn{1}{|c|}{ IG-110 } & IG-430 \\
\hline Activation energy $(\mathrm{kJ} / \mathrm{mol})$ & $218 \pm 4$ & $158.5 \pm 1.5$ \\
\hline Order of reaction, $\mathrm{n}$ & $0.75 \pm 0.15$ & $0.37 \pm 0.04$ \\
\hline
\end{tabular}

Table 6-5. Experimental results on kinetics parameters for IG-430.

\begin{tabular}{|l|l|l|l|l|l|l|}
\hline \multicolumn{1}{|c|}{ Author } & \multicolumn{1}{|c|}{ Temp. $\left({ }^{\circ} \mathbf{C}\right)$} & $\begin{array}{c}\text { Oxygen mole } \\
\text { fraction }\end{array}$ & $\begin{array}{c}\text { Flow rate } \\
\text { (SLPM) }\end{array}$ & Ea (kJ/mol) & \multicolumn{1}{|c|}{$\mathbf{n}$} & \multicolumn{1}{c|}{ Method } \\
\hline Chi $(2008)$ & $608 \sim 808$ & 0.2 & 10 & 167.4 & - & TGA \\
\hline KAIST & $540 \sim 800$ & $0.02 \sim 0.34$ & $8 \sim 10$ & 158.5 & 0.37 & Gas analysis \\
\hline
\end{tabular}

\subsubsection{Mass Diffusion}

Even though the rate of reaction is controlled by chemical parameters at low temperatures, the rate of reaction is limited by a mass diffusion process at high temperatures. The well-proven correlation was therefore used in this study. The correlation was developed for heat transfer through the laminar boundary layer and averaged through the whole length (Welty et al. 1984), which produced good agreement with the experiment data investigated by a previous study (Oh et al. 2006). The heat/mass transfer analogy is applicable for predicting the mass diffusion rate in Zone 3:

$K_{m}=0.664 \frac{D_{i j}}{L} \operatorname{Re}_{L}^{1 / 2} S c^{1 / 3}$

This correlation is made based on the analogy of the heat transfer correlation, which was developed for heat transfer through the laminar boundary layer and averaged through the whole length. 


\subsubsection{Combined Effect of Kinetics and Mass Diffusion}

The good correlation for the combined effect of kinetics and mass diffusion was suggested by the previous I-NERI program (Oh et al. 2006). The following is the correlation for the combined effect:

$$
\frac{1}{R_{g}}=\frac{1}{R_{m b}}+\frac{1}{R_{c b}}
$$

where

$$
\begin{aligned}
& R_{m b}=K_{m} \cdot C_{b, O 2} \cdot A \\
& R_{c b}=K_{0} \cdot e^{-\frac{E a}{R \cdot T}} \cdot P_{O 2, b}^{n} \cdot A_{(n \neq 0) .}
\end{aligned}
$$

The mass transfer coefficient is calculated from the following Graetz solution, which includes the effect of the entrance effect.

$$
S h=\frac{k_{m} \cdot d}{D}=3.66+\frac{0.0668(d / x) \cdot(\operatorname{Re} \cdot S c)}{1+0.04 \cdot[(d / x) \cdot(\operatorname{Re} \cdot S c)]^{2 / 3}}
$$

The following correction was performed for high reaction rate:

$k_{m}{ }^{\text {corrected }}=\theta \cdot k_{m}$

where

$$
\begin{aligned}
& \theta=\frac{\ln \left(B_{m}+1\right)}{B_{m}} \\
& B_{m}=\frac{X_{O 2, b}-X_{O 2,0}}{X_{O 2, b}-1} .
\end{aligned}
$$

The effective diffusion coefficient used instead of a binary diffusion coefficient because of high reaction rate was

$$
D_{O 2, m}=\frac{\left(1-X_{1}\right)}{\sum_{i=2}^{n}\left(X_{i} / D_{O 2, i}\right)}
$$

\subsubsection{Effect of Burn-off}

The reaction rate of oxidation is dependent on the level of burn-off. The effect of burn-off was experimentally investigated. A schematic of experimental facility is shown in Figure 6-5. The temperature of $600^{\circ} \mathrm{C}$ was maintained in the furnace. The detailed conditions are summarized in Table 6-6. The reaction rates of specimens with different volume were measured. The burn-off history is shown in Figure 6-6. The burn-off histories of IG-430 are independent of graphite dimension and volume. The

relation between bulk flow and reaction rate is shown in Figure 6-6 and Figure 6-7. The effect of burn-off is independent of bulk flow. Actually, the oxidation reaction in the temperature of $600^{\circ} \mathrm{C}$ is in Zone 1 . 


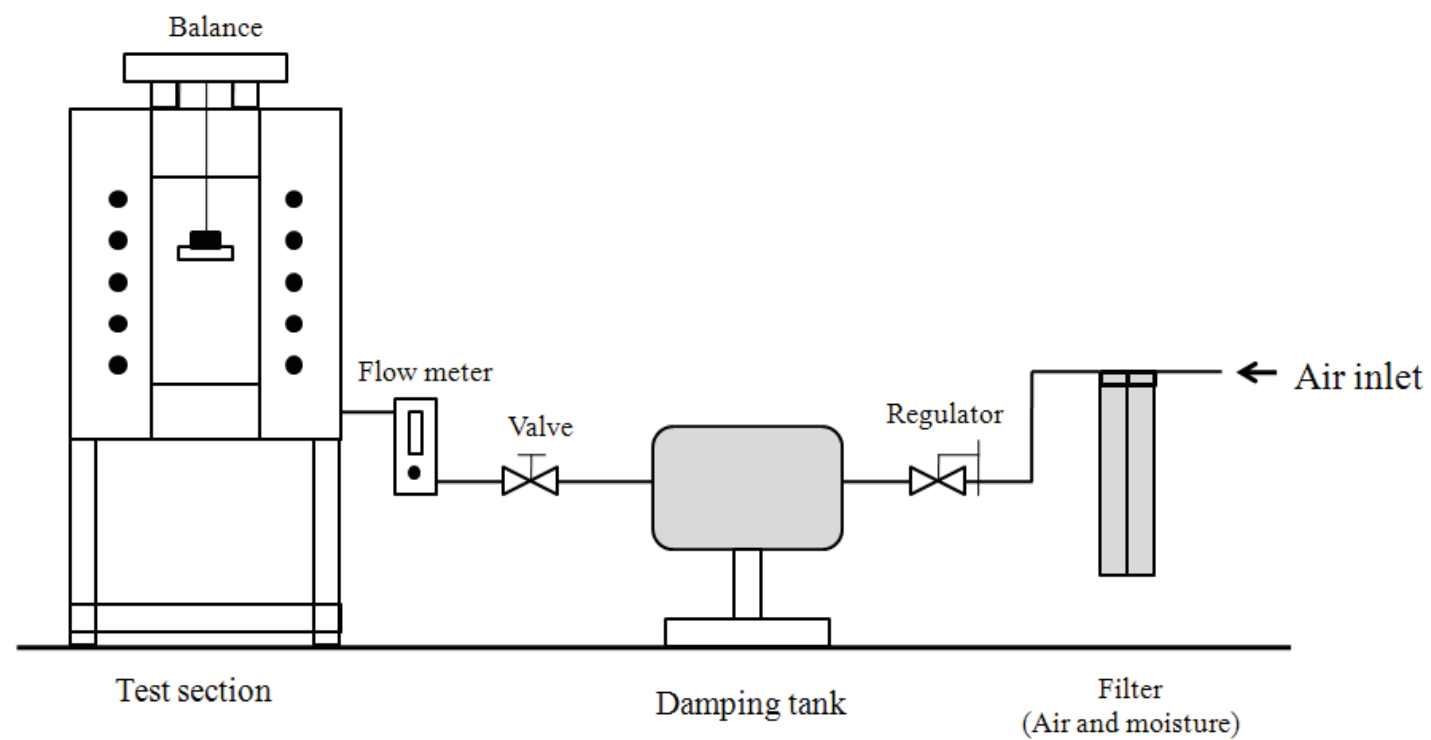

Figure 6-5. Experimental facility for burn-off tests.

Table 6-6 Conditions for burn-off tests.

\begin{tabular}{|l|l|c|l|}
\hline \multicolumn{1}{|c|}{ Material } & \multicolumn{1}{|c|}{ Air } & Volume & Purpose \\
\hline \multirow{2}{*}{ IG-430 } & $5 \mathrm{SLPM}$ & $5126.6 \mathrm{~mm}^{2}$ & Relation between graphite volume and reaction \\
\cline { 2 - 3 } & $5 \mathrm{SLPM}$ & $9922.4 \mathrm{~mm}^{2}$ & \\
\hline \multirow{2}{*}{ IG-110 } & $5 \mathrm{SLPM}$ & $5126.6 \mathrm{~mm}^{2}$ & Relation between bulk flow and reaction \\
\cline { 2 - 3 } & $5 \mathrm{SLPM}$ & $5126.6 \mathrm{~mm}^{2}$ & \\
\hline
\end{tabular}

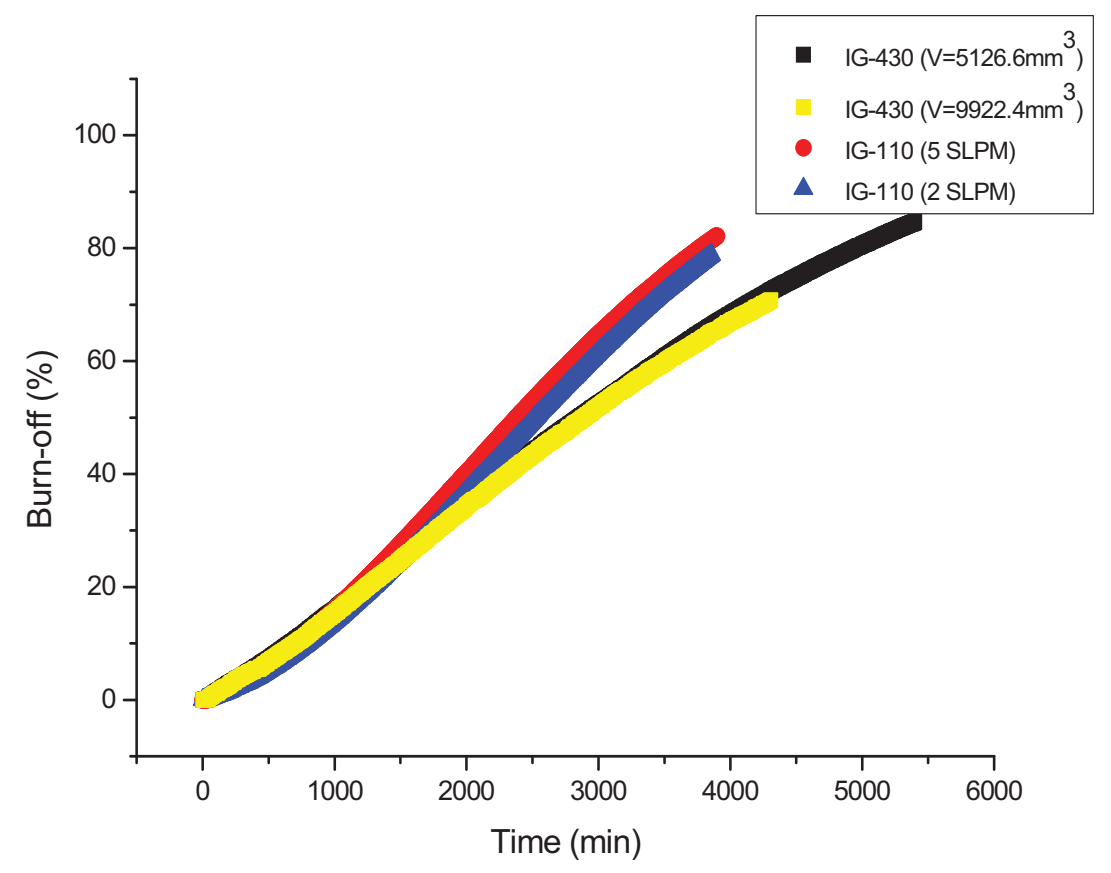

Figure 6-6. Changes of burn-off with time for different conditions. 


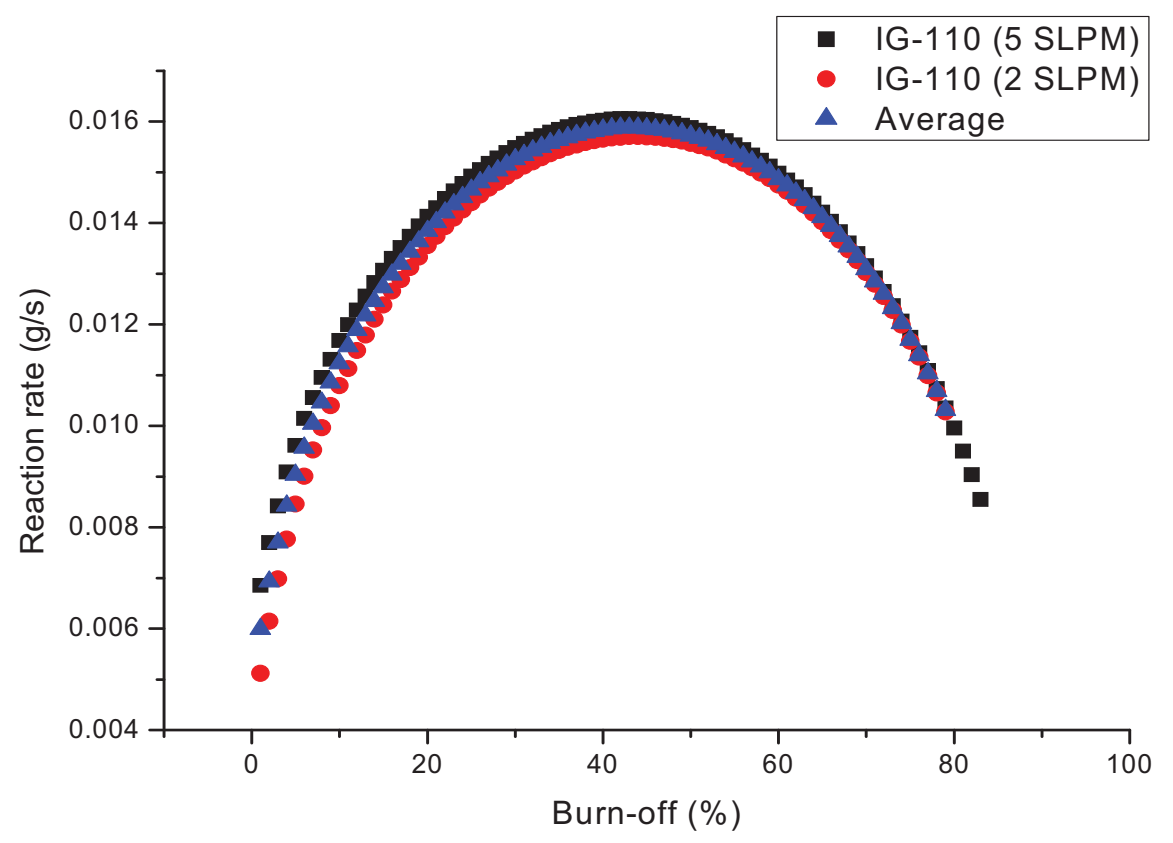

Figure 6-7. Changes of reaction rate with burn-off.

Relation between the burn-off and relative reaction rate was also obtained from the burn-off history. The internal surface area change is caused by oxidation:

$M_{B}=\frac{R_{g}(B)}{R g(0)}=\frac{\theta(B)}{\theta_{0}}$

where $R_{g}$ is the reaction rate $(\mathrm{kg} / \mathrm{s})$ and $\theta(B)$ is internal surface density (m-1) at burn-off (B). According to Fuller and Okoh (1997), the reaction rate with the effect of burn-off can be expressed as

$$
R_{g}=K_{0} \cdot \exp \left(-\frac{E a}{R \cdot T}\right) \cdot P_{O 2}{ }^{n} \cdot \theta(0) \cdot M_{B} \text {. }
$$

The measured $M_{B}$ of IG-110 and IG-430 are shown in Figure 6-8. Both reaction rates of IG-110 and IG-430 in Zone 1 have the maximum reaction rate of about $40 \%$ of burn-off. They are in good agreement with the former results. Even though the ratio of $M_{B}$ has some sensitivity to initial reaction rates, the absolute value of reaction rate is almost the same as shown in Figure 6-7. The effect of burn-off on the reaction rate in IG-110 is much larger than that in IG-430.

\subsubsection{Effect of Moisture}

Most former experiments were performed in dehumidified conditions, but the normal environments always have moisture. This section describes the effect of moisture on oxidation in Zones 1 and 3 . The results of Zone 1 tests were obtained from the facility shown in Figure 6-5. The results of Zone 3 test were obtained from the facility shown in Figure 6-2. Both test facilities had an add-on of water chamber to control humidity. The temperature was controlled from 600 to $1300^{\circ} \mathrm{C}$ and the relative humidity was controlled from 0 to $63.6 \%$. Test material was IG-430. The test conditions are summarized in Table 6-7. 


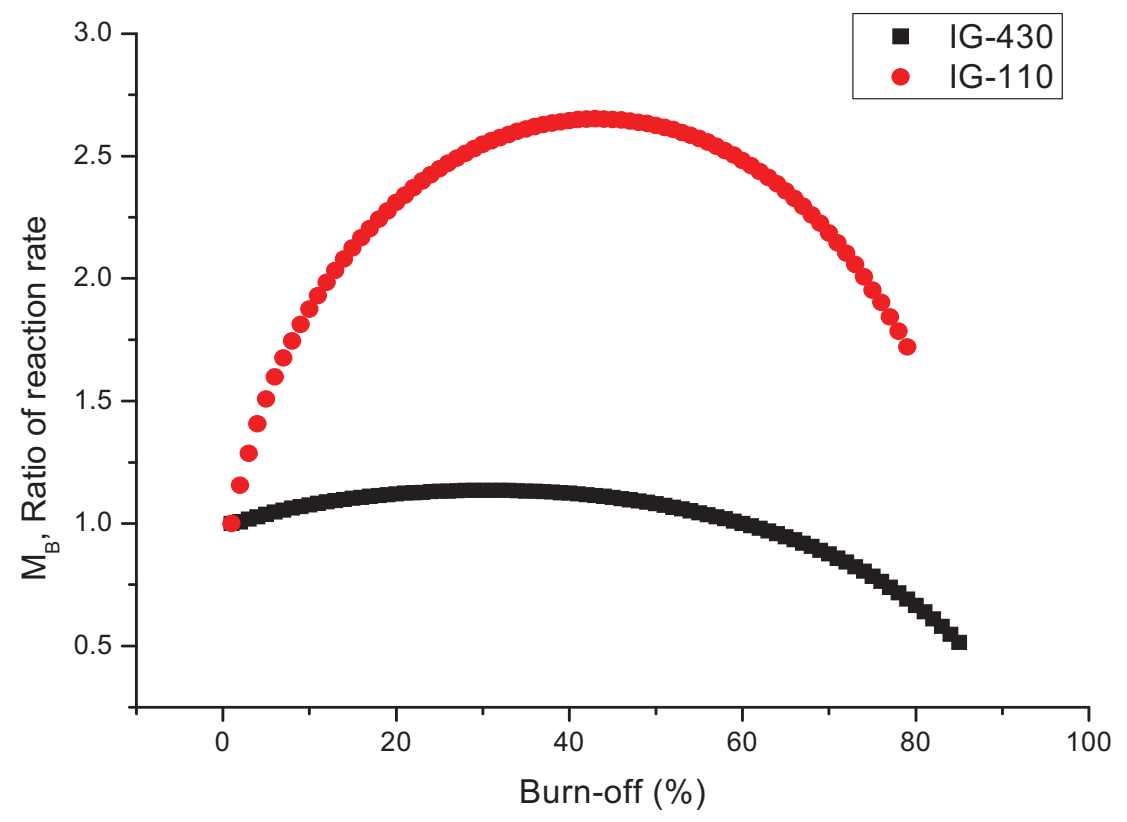

Figure 6-8. Relation between burn-off and ratio of reaction rate.

Table 6-7. Conditions for effect of moisture tests.

\begin{tabular}{|l|l|l|l|l|l|}
\hline $\begin{array}{c}\text { Oxidation } \\
\text { regime }\end{array}$ & Temperature & Relative humidity & $\begin{array}{c}\text { Gas bulk flow } \\
\text { (He+O2) }\end{array}$ & $\begin{array}{c}\text { Oxygen } \\
\text { concentration }\end{array}$ & \multicolumn{1}{c|}{ Method } \\
\hline Zone 1 & $600^{\circ} \mathrm{C}$ & 0 to $63.6 \%$ & 1 SLPM & $20 \%$ & TGA \\
\hline Zone 3 & 1000 to $1300^{\circ} \mathrm{C}$ & 0 to $63.6 \%$ & 8 SLPM & $20 \%$ & Gas analysis \\
\hline
\end{tabular}

The test results of the Zone 1 reaction are shown in Figure 6-9. From the burn-off histories, the effect of moisture is negligible in Zone 1. The internal structure of graphite and the chemical reaction are not affected by moisture in gas.

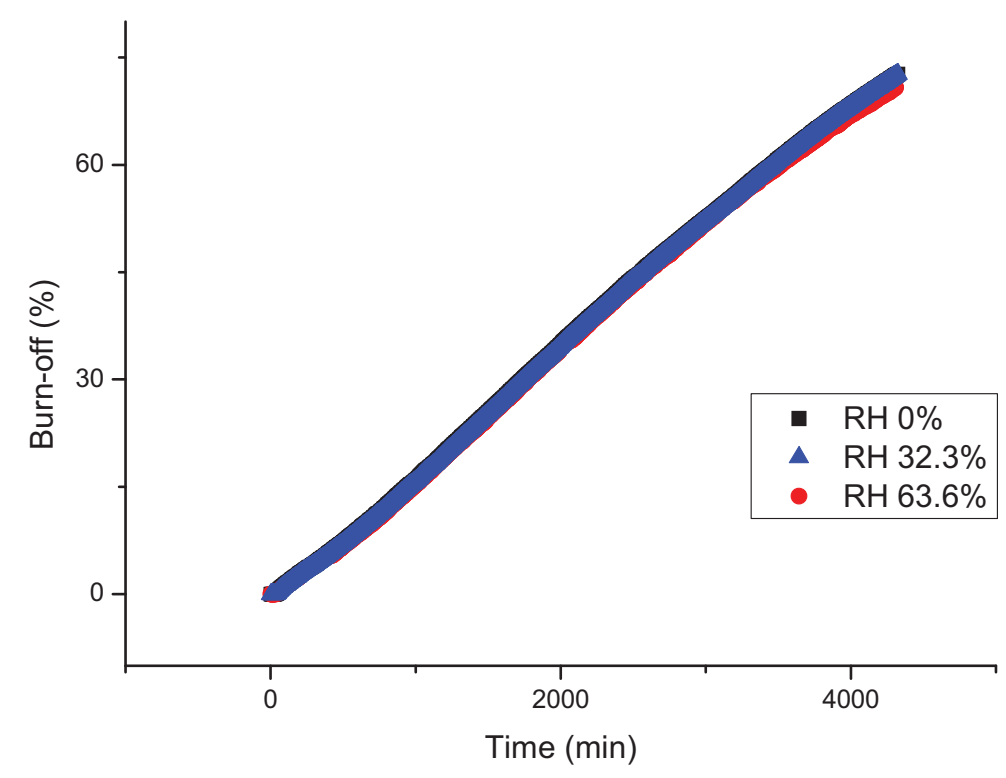

Figure 6-9. Moisture effect on the graphite oxidation in Zone 1. 
E. S. Kim suggested that the mass transfer, including the moisture effect, is half of the rate without moisture effect (Kim 2005).

$$
r_{g, \text { moisuture }}=0.5 \cdot r_{g, d r y}
$$

The graphite oxidation in Zone 3 is controlled by mass transfer. Therefore,

$$
r_{g} \sim R_{g} \text { (Reaction rate) }
$$

and

$$
R_{g, \text { moisture }}=0.5 \cdot R_{g, d r y} .
$$

The experimental results for the effect of moisture in Zone 3 as shown in Figure 6-10 are in good agreement with those from Equation 6-14. The difference between the data is under 5\%.

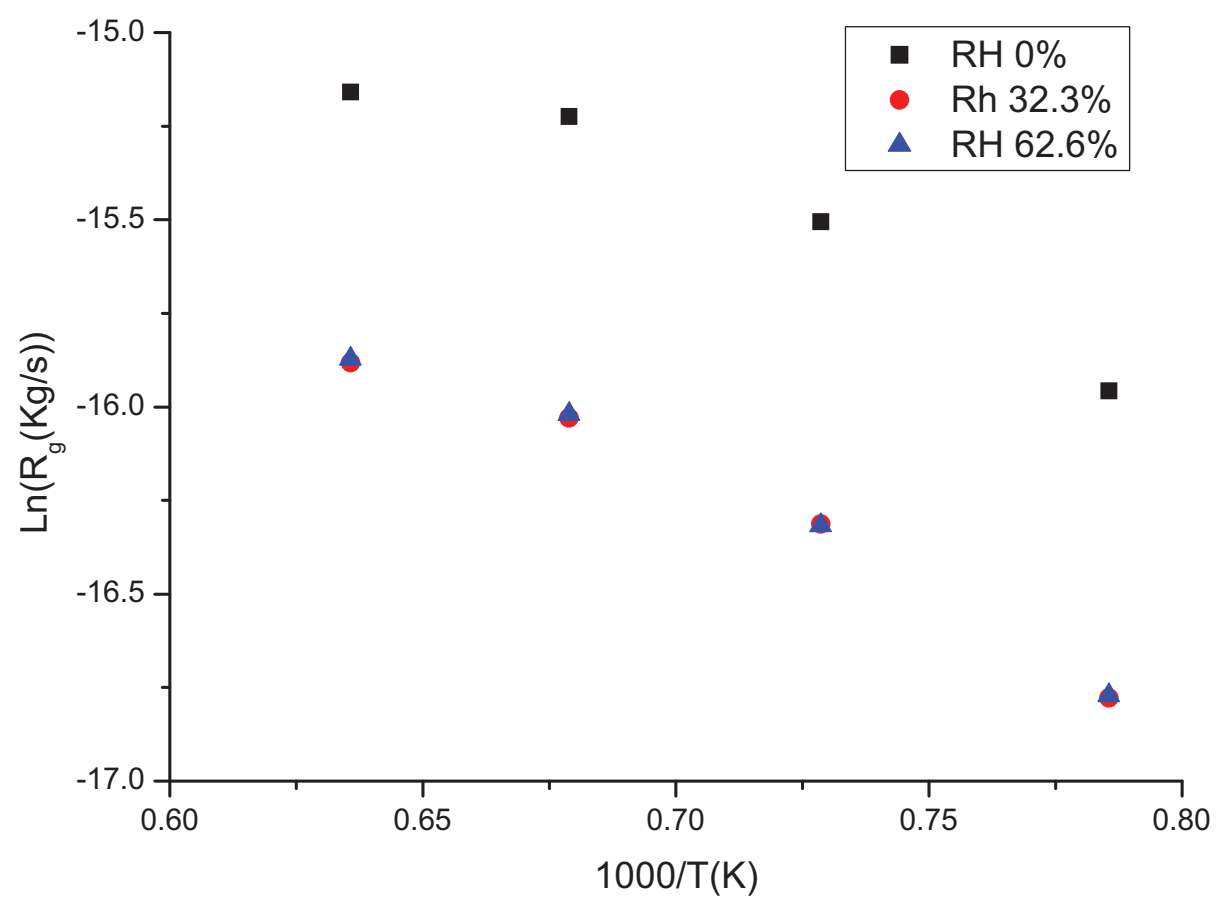

Figure 6-10 Moisture effect on the graphite oxidation in Zone 3 


\section{TASK 6: Structural Tests of Burn-off Bottom reflector}

The objective of this task is to carry out the structural test of the oxidized graphite in order to develop the fracture model for the oxidized bottom reflector and lower plenum. The graphite structures are oxidized and damaged during an air-ingress event. The reactor core is supported by graphite support columns and support blocks. Schematics of the 600 MWth GTMHR (GA 2002) and graphite column are shown in Figure 7-1. As shown, the graphite column is relatively long; the slenderness ratio of the cylindrical column is about 40 . When the air ingress event starts air encounters the graphite column first. Predicting the failure of the oxidized graphite structure is important for the design and safety analysis of the VHTR because oxidation changes the strength and geometry of the graphite.

The oxidation of nuclear graphite is classified into Zones I, II, and III. Zone I is the temperature range below $600^{\circ} \mathrm{C}$ where the nuclear graphite is uniformly oxidized with a bulk density decrease following the volumetric weight loss. The decrease of the bulk density will degrade the strength of the graphite. In Zone III, which generally ranges above $1000^{\circ} \mathrm{C}$, the oxidation of the graphite is dominated by mass diffusion limit so the surface of graphite is mainly corroded, resulting in the maximum stress increase of the graphite structure. In Zone II $\left(600\right.$ to $\left.1000^{\circ} \mathrm{C}\right)$, density decrease and surface corrosion occur at the same time.
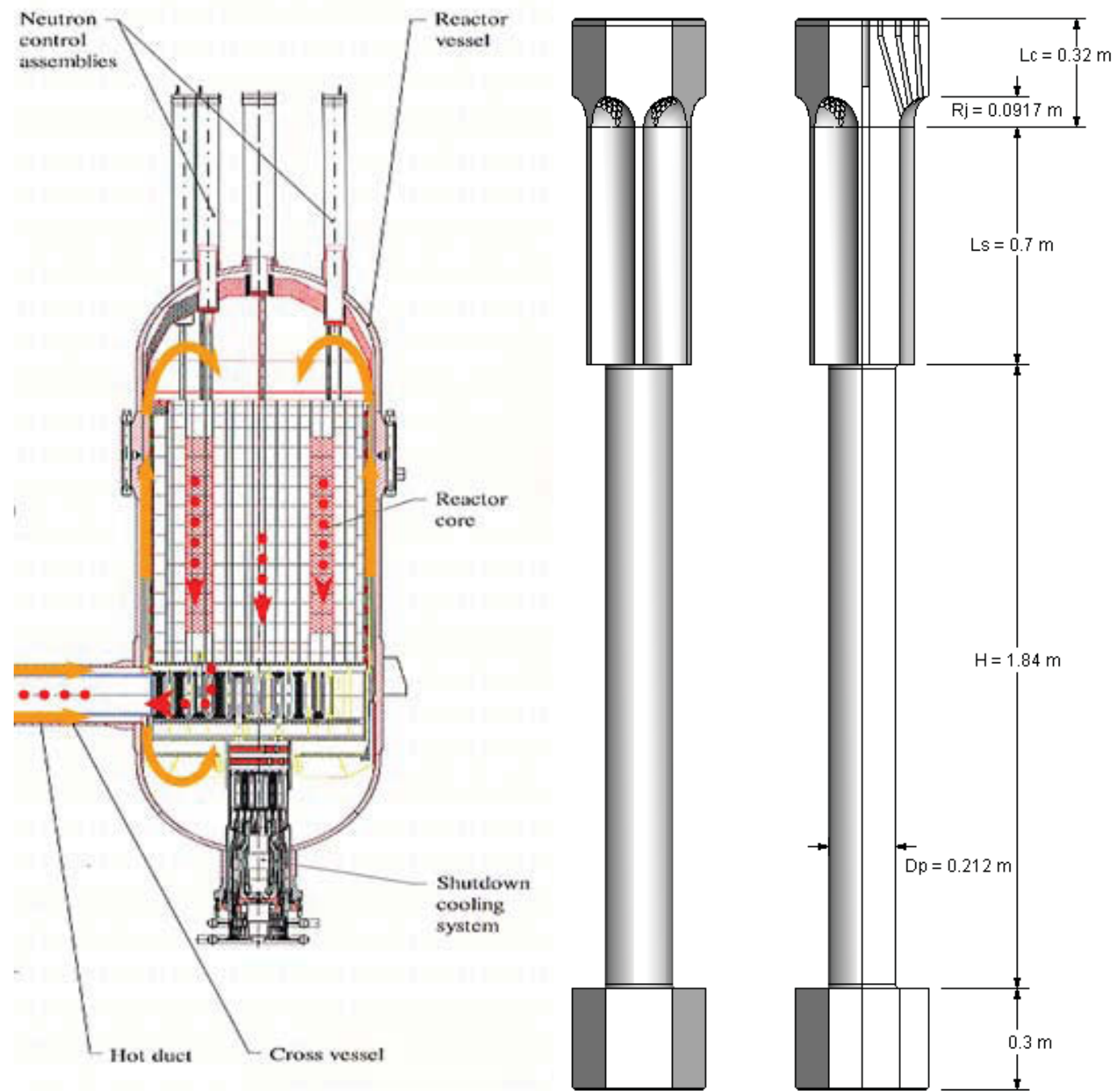

Figure 7-1. Schematics of 600 MWth GTMHR and graphite support column in the lower plenum. 


\subsection{Graphite Oxidation and Fracture Mechanisms}

A simplified graphite column and the oxidation process, where an axial force is loaded on the graphite columns, are shown in Figure 7-2.

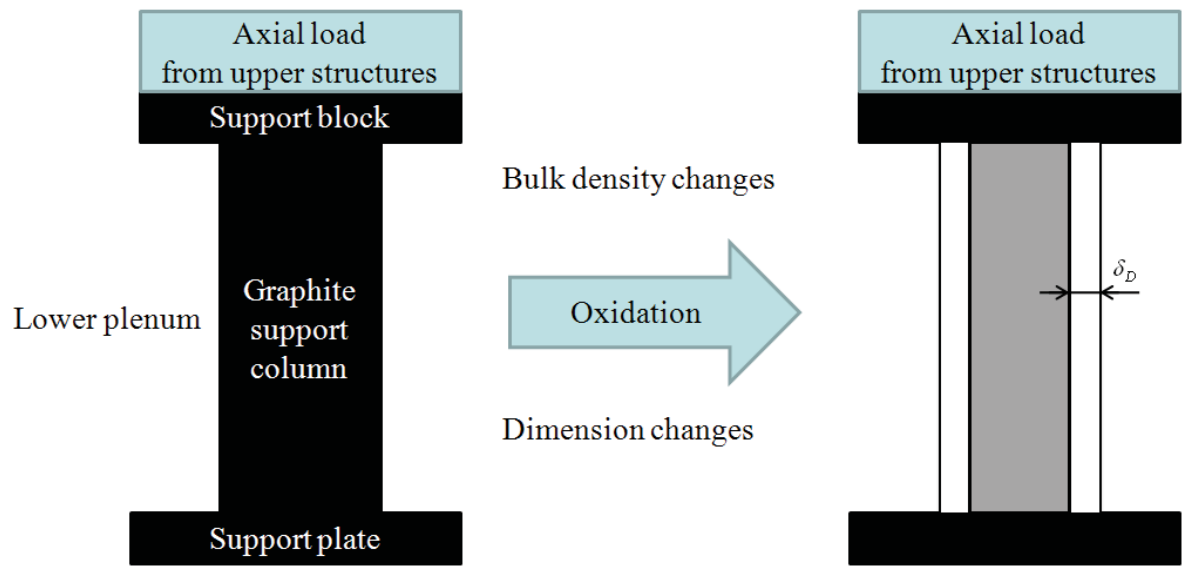

Figure 7-2 Schematic of oxidation in a graphite column.

The graphite oxidation in Zone 1 causes a decrease in bulk density. The compressive strength of the graphite is degraded with a decrease in bulk density. This is well verified by the former experiments of Eto and Growcock (1983), Yoda et al. (1985), Kim et al. (2007), and Neighbour and Hacker (2001). It is believed that the real oxidation is processed over the overall oxidation regimes during an air ingress event (Oh et al. (2006). The graphite column became slender as a result of the oxidation in Zone 2 and Zone 3 , in which case the oxidized graphite column can fail by buckling, a different mechanism from that of the fresh graphite, but this cannot be explained by the former experiments. Therefore, the understanding of the oxidation in Zone 2 and Zone 3 are needed to predict the failure of a graphite column. The graphite column becomes slender as a result of surface oxidation processed in Zones 2 and 3.

The graphite column becomes slender as oxidation is processed per the equation

aspect ratio $=\frac{L}{D} \rightarrow \frac{L-2 \delta_{L}}{D-2 \delta_{D}}$.

Top and bottom surfaces of the graphite column are shielded by a support block and support plate. The lateral surface is easy to contact with air expressed as

$\delta_{L} \leq \delta_{D}$

$L>D$

and

aspect ratio $=\frac{L}{D-2 \delta}$.

Therefore, the two parameters are dominant in predicting the failure strength of graphite columns; the compressive strength of a fresh graphite sample and the slenderness ratio at which graphite columns fail by buckling are also important. 
When the column becomes slender, it becomes easy for the column to fail by buckling. An analysis of the critical stress for long columns was previously developed by Leonhard Euler (Singer and Pytel 1980). The Euler formula for the fixed-fixed column is simply expressed by

$$
\sigma_{c r}=4 \pi^{2} E I / A L^{2}
$$

where $I$ is the moment of inertia for the principal axis about which buckling occurs. When $I$ in Eq. (7-5) is replaced with the following relationship

$$
r=\sqrt{I / A}
$$

Eq. (7-5) becomes

$$
\sigma_{c r}=4 \pi^{2} E /(L / r)^{2}
$$

where $A$ is the cross sectional area and $r$ is the least radius gyration. The ratio $L / r$ is a nondimensional ratio called the slenderness ratio of the column. A column can be classified as either a long or short column based on the slenderness ratio. However, there are limitations to Euler's formula because this formula is only valid in estimating the strength of very long columns. For estimating the strength of a small-slenderness-ratio column, the empirical straight-line formula proposed by T. H. Johnson (Singer and Pytel 1980) was

$$
\sigma_{c r, \text { buckling }}=\sigma_{\text {straight-line }}-C(L / r)
$$

where the constant $\sigma_{\text {straight-line }}$ is the intercept for $\mathrm{L} / \mathrm{r}=0$ and the constant $C$ is the magnitude of slope.

The strength degradation of IG-110, IG-430, and NBG-10 columns, which are oxidized in all Zones, were studied. Isotropic fine-grained IG-110, IG-430, and NBG-10 graphite was selected as a test material. Tests, the detailed dimensions of specimens, and the objectives of experiments are summarized in Table 7-1. 
Table 7-1. Test matrix.

\begin{tabular}{|c|c|c|c|c|c|}
\hline Diameter & Length & $\begin{array}{c}\text { Slenderness } \\
\text { ratio }\end{array}$ & $\begin{array}{c}\text { Burn-off } \\
(\%)\end{array}$ & $\begin{array}{l}\text { Oxidation } \\
\text { regime }\end{array}$ & Objective \\
\hline 15 & 30 & 8 & \multirow{16}{*}{ N/A } & \multirow{16}{*}{ N/A } & \multirow{2}{*}{$\begin{array}{l}\text { Compressive strength of fresh graphite was } \\
\text { measured. }\end{array}$} \\
\hline 25 & 50 & 8 & & & \\
\hline \multirow{11}{*}{25} & 50 & 8 & & & \multirow{11}{*}{$\begin{array}{l}\text { Buckling strength was measured. Empirical } \\
\text { buckling strength formula was also obtained. }\end{array}$} \\
\hline & 75 & 12 & & & \\
\hline & 80 & 12.8 & & & \\
\hline & 85 & 13.6 & & & \\
\hline & 90 & 14.4 & & & \\
\hline & 95 & 15.2 & & & \\
\hline & 100 & 16 & & & \\
\hline & 125 & 20 & & & \\
\hline & 150 & 24 & & & \\
\hline & 200 & 32 & & & \\
\hline & 250 & 40 & & & \\
\hline \multirow{3}{*}{15} & 30 & 8 & & & \multirow{3}{*}{$\begin{array}{l}\text { The applicability for the straight-line formula } \\
\text { in the different diameter was confirmed. }\end{array}$} \\
\hline & 60 & 16 & & & \\
\hline & 120 & 32 & & & \\
\hline 25 & 50 & 8 & $0 \sim 24 \%$ & \multirow{6}{*}{ Zone 1} & $\begin{array}{l}\text { The compressive strength of oxidized } \\
\text { graphite was measured. }\end{array}$ \\
\hline 15 & 60 & 16 & \multirow{5}{*}{$0 \sim 24 \%$} & & \multirow{5}{*}{$\begin{array}{l}\text { The buckling strength of various oxidized } \\
\text { graphite columns was measured. The relation } \\
\text { between strength degradation and failure } \\
\text { mode was found out. }\end{array}$} \\
\hline 15 & 120 & 32 & & & \\
\hline 25 & 50 & 8 & & & \\
\hline 25 & 100 & 16 & & & \\
\hline 25 & 200 & 32 & & & \\
\hline 21 & 42 & 16 & $0 \sim 40 \%$ & Zone 2 & Experiments for oxidation in Zone 2. \\
\hline 15 & 60 & 16 & \multirow{2}{*}{ N/A } & \multirow{2}{*}{ Zone 3} & \multirow{2}{*}{$\begin{array}{l}\text { Experiments for oxidation in Zone } 3 . \\
\text { Buckling strength was measured. }\end{array}$} \\
\hline 15 & 120 & 32 & & & \\
\hline 15 & 60 & 16 & \multirow{3}{*}{$0 \sim 22 \%$} & \multirow{3}{*}{ Zone 1} & \multirow{3}{*}{ Pretests for support block modeling. } \\
\hline \multicolumn{2}{|c|}{ Annular column } & 15.5 & & & \\
\hline \multicolumn{2}{|c|}{ Rectangular column } & 15.5 & & & \\
\hline
\end{tabular}

\subsection{Strength Measurement}

The largest axial force is always loaded on the graphite columns because they are the main component in the core bottom supporter. An INSTRON Model 4204 mechanical testing facility shown in Figure 7-3 was used for this compression test. The maximum loading of this facility was $50 \mathrm{kN}$. The basic test setup and procedures were based on American Society of Testing and Materials Standard C695-91. The load was continuously applied to the sample at the constant rate of crosshead movement, and without shock until ultimate failure. The speed of the cross head movement was constant for all specimens. The strain rate for the shortest specimen, $15 \mathrm{~mm} \Phi \times 30 \mathrm{~mm}$, was $1.11 \times 10^{-4} \mathrm{sec}^{-1}$. 


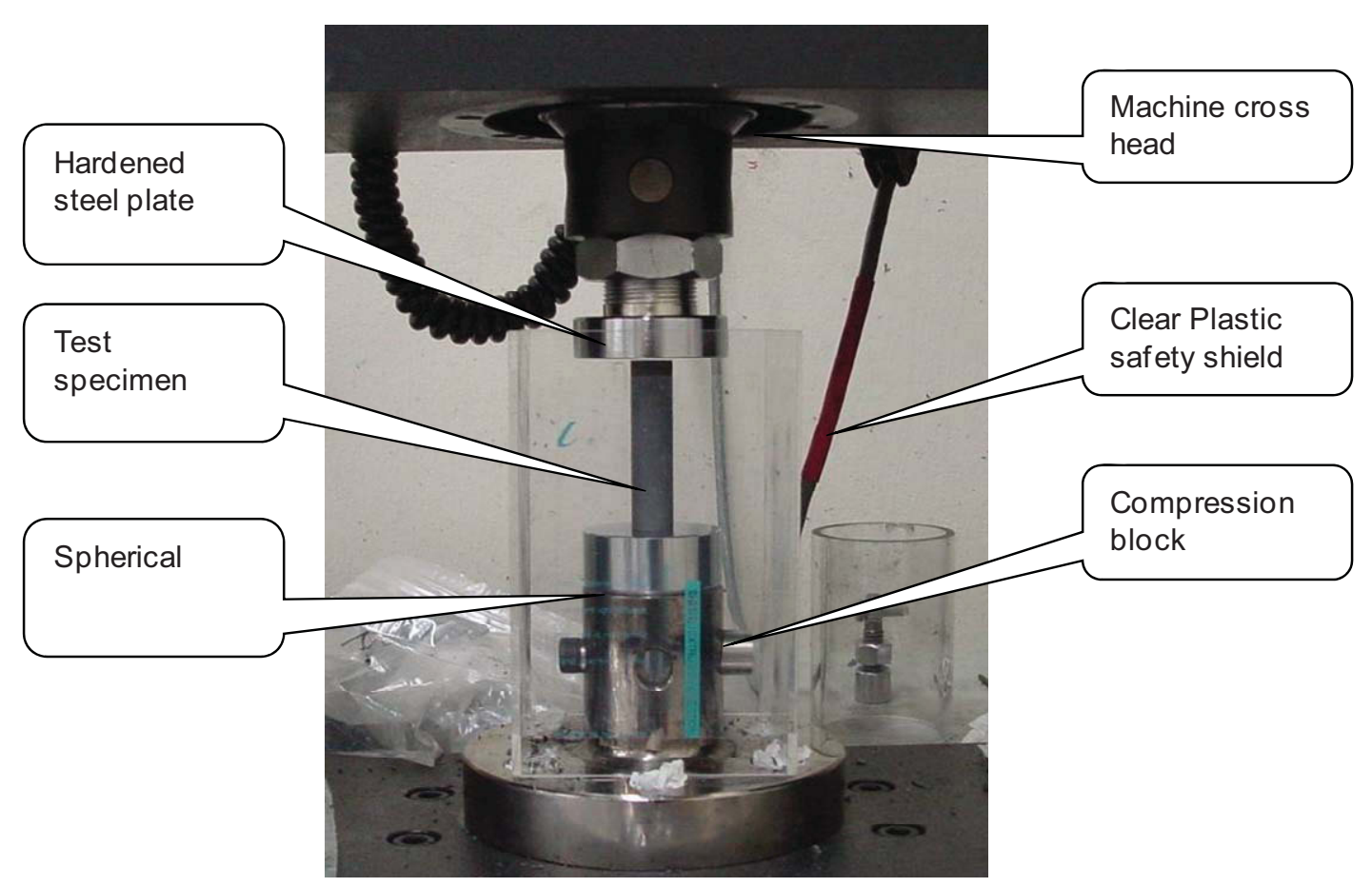

Figure 7-3. Compression test facility, INSTRON Model 4204.

\subsection{Oxidation Treatment}

Oxidation was carried out in an electrical muffle furnace. Filtered dry air was uniformly supplied to the bottom of the furnace and gas was naturally vented out through the top of the furnace. The internal temperature of the furnace was maintained at $600^{\circ} \mathrm{C}$ for Zone 1 oxidation and at $1050^{\circ} \mathrm{C}$ for Zone 3 oxidation. The picture of the experimental facility is shown in Figure 7-4.

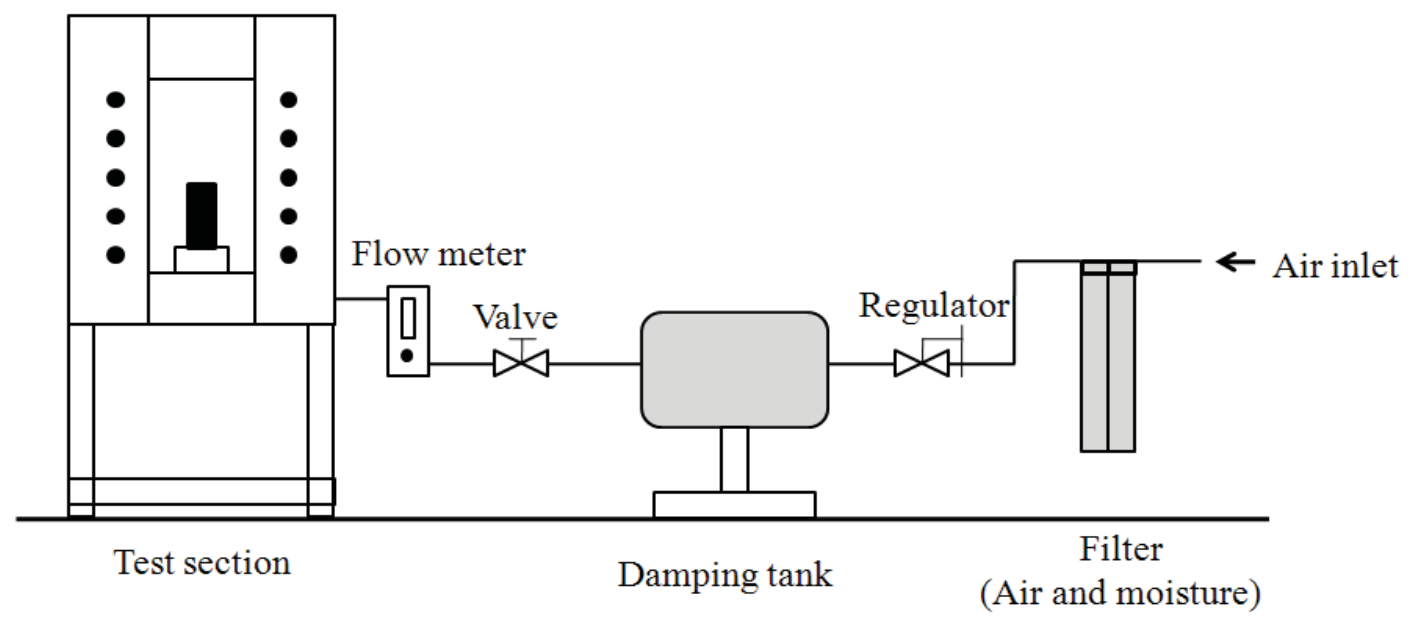

Figure 7-4. Experimental facility for oxidation tests.

Zone 2 Oxidation was carried out in a tube furnace using an induction heater. The test section is surrounded by a quartz tube. Filtered dry air was uniformly supplied into the bottom of the furnace and gas was vented out through the top of the furnace. The internal temperature of the furnace was maintained at $800^{\circ} \mathrm{C}$. The picture of the tube furnace is shown in Figure 7-5 


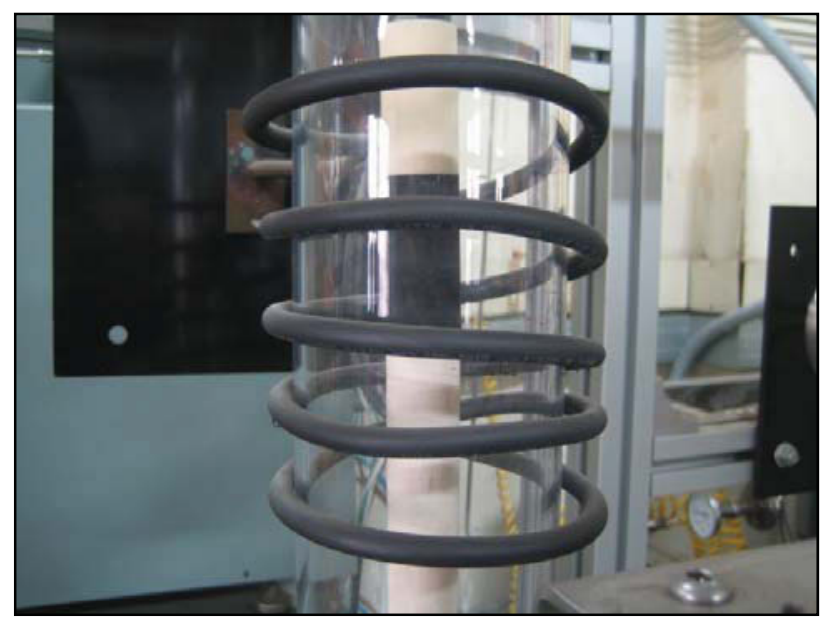

Figure 7-5. Tube furnace.

\subsection{Measurements of Compressive and Buckling Strength of Fresh Graphite Column}

The measured average compressive strengths of IG-110, IG-430, and NBG-10 are listed in Table 7-2, which shows good agreement with typical properties of the graphite. The buckling strength of a graphite column decreases as the slenderness ratio increases. The buckling strength of IG-110 graphite columns is shown Figure 7-6.

Table 7-2. Measured compressive strength.

\begin{tabular}{|c|c|}
\hline Graphite Grade & Compressive Strength \\
\hline IG-110 & $79.5 \mathrm{MPa}$ \\
\hline IG-430 & $90.0 \mathrm{MPa}$ \\
\hline NBG-10 & $58.4 \mathrm{MPa}$ \\
\hline
\end{tabular}

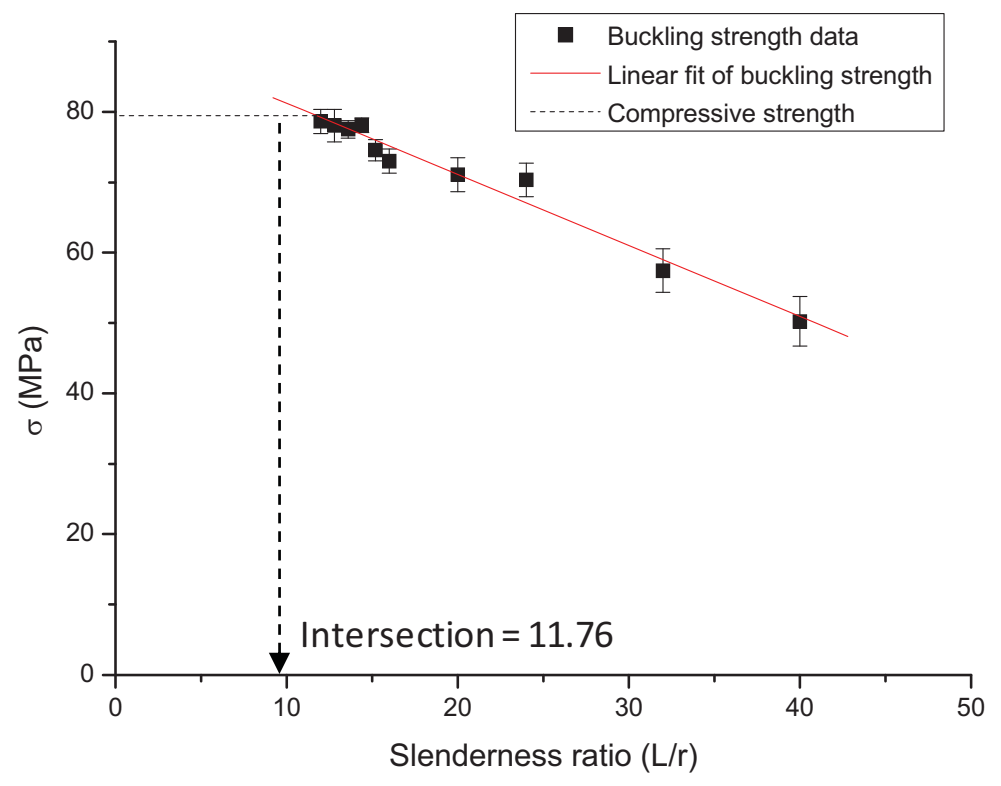

Figure 7-6. Compressive and buckling strength of IG-110 graphite columns. 
The intercept and the magnitude of the empirical formula are obtained from the empirical data as follows:

$$
\sigma_{c r, b u c k l i n g}=91.3-(L / r) \text {. }
$$

The buckling strength of IG-430 graphite columns is also shown in Figure 7-7.

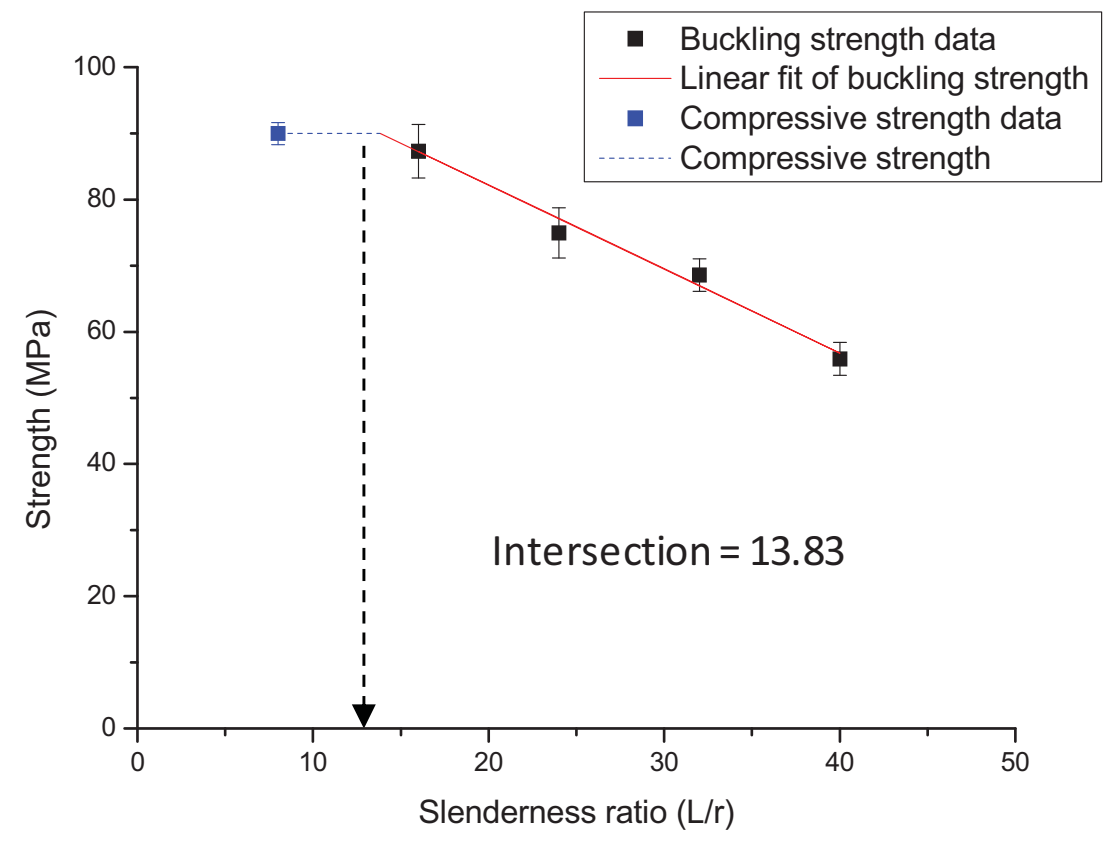

Figure 7-7. Compressive and buckling strength of IG-430 graphite columns.

The obtained empirical formula for IG-430 becomes

$\sigma=107.6-1.3 \cdot(L / r)$

The buckling limit for IG-430 column is 13.8 .

The following is the empirical formula for NBG-10. Figure 7-8 shows compressive and buckling strength of NBG-10 columns.

$\sigma=61.8-0.4 \cdot(L / r)$

The structural strength of a graphite column under compression force can be determined by the slenderness ratio. The graphite columns with the same slenderness ratio also have the same strength. It is believed that the buckling failure occurs over the slenderness ratio, which is the intersection of the compressive strength and the empirical line. The intersection is 11.8 for IG-110, 13.8 for IG-430, and 8 for NBG-10, respectively.

An additional experiment using IG-110 graphite was performed to confirm the proposed applicable range of the slenderness ratio described above. Figure 7-9 shows the measured buckling strength vs. slenderness ratio for the additional tests. This figure shows good agreement between the experimental data and the line obtained from previous buckling tests (Figure 7-6). From this result, the strength of a graphite column can be determined by the slenderness ratio. The graphite columns with the same slenderness ratio have the same strength, but graphite columns have different diameters. 


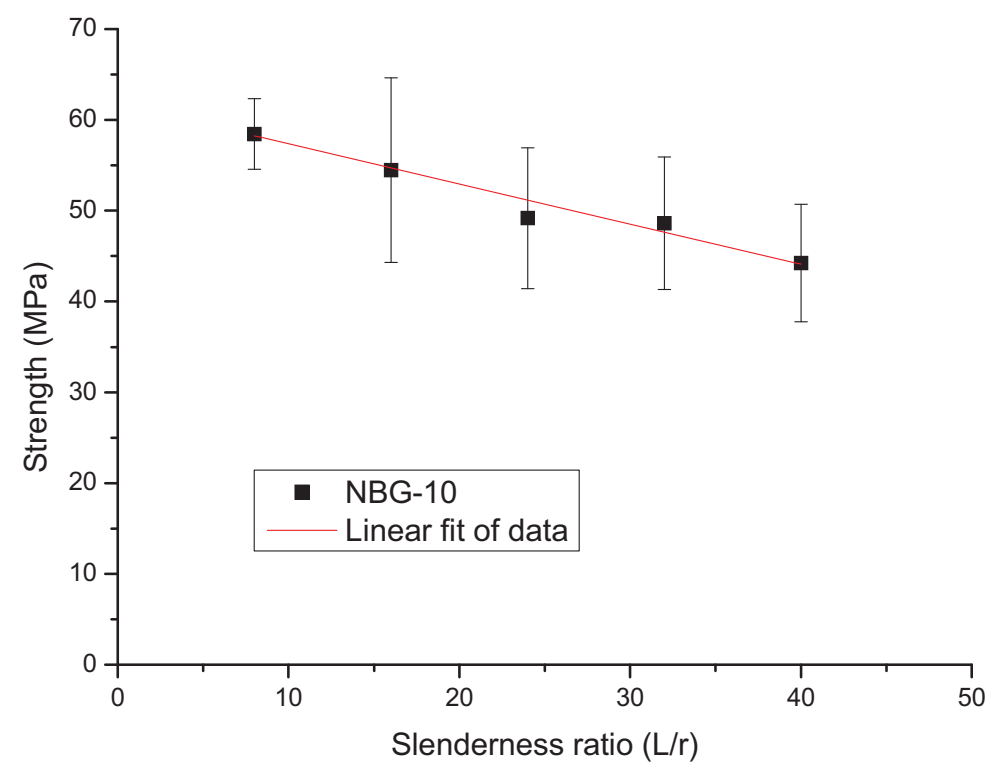

Figure 7-8. Compressive and buckling strength of NBG-10 graphite columns.

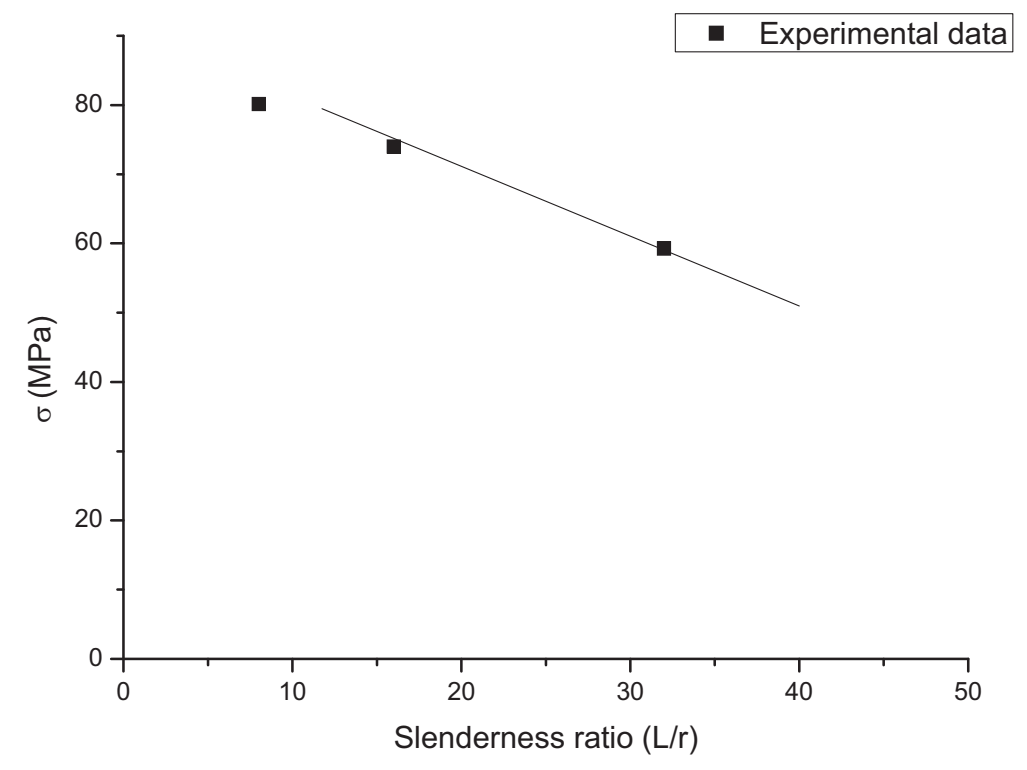

Figure 7-9. Buckling strength of $\Phi 15 \mathrm{~mm}$ columns.

\subsection{Compressive and Buckling Strength Degradation of Graphite Column Oxidized in Zone 1}

The degradation in compressive strength as a function of the decrease in bulk density is shown in Figure 7-10. The dimensions of an oxidized specimen did not change. The percentage decrease in bulk density is the same as the percentage of weight loss. It is also called burn-off and expressed as

$d=\left(m_{0}-m\right) / m_{0} \times 100$ 


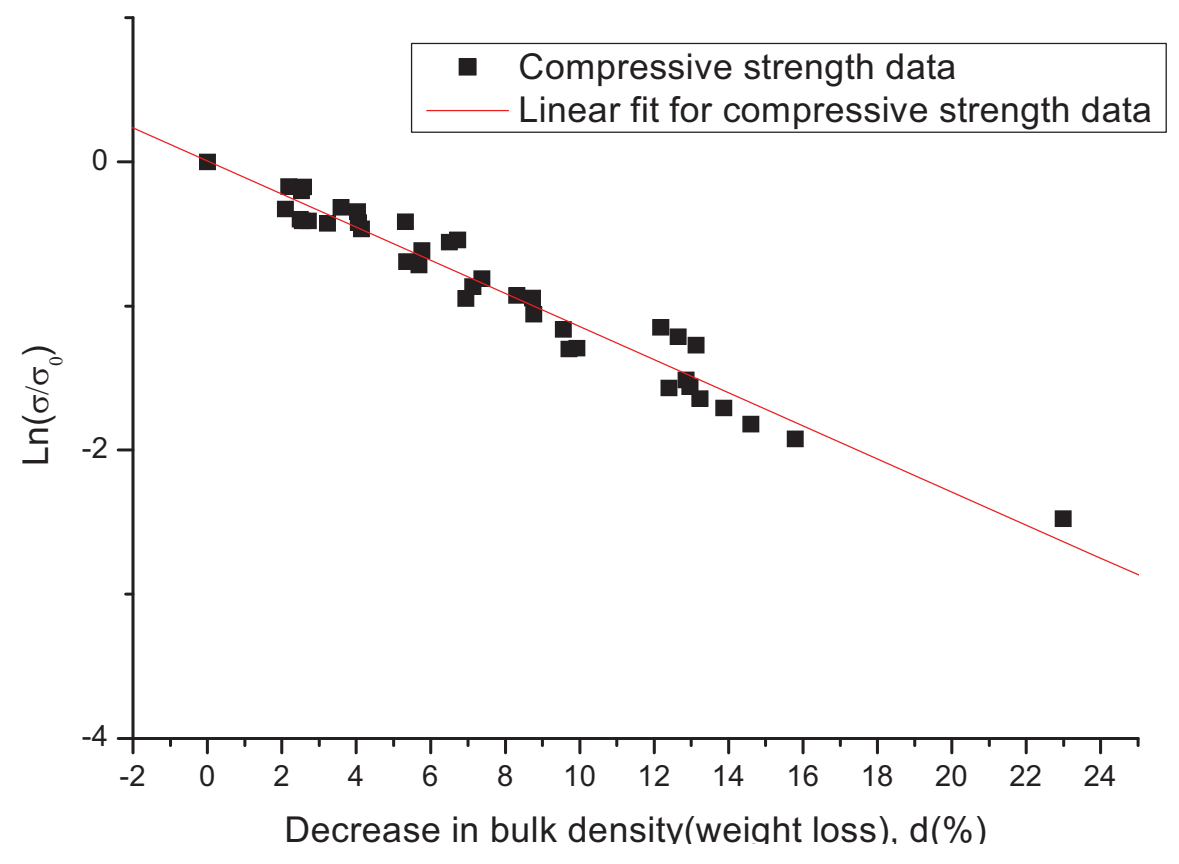

Figure 7-10. Normalized compressive strength of IG-110 oxidized in Zone 1.

The relation between the compressive strength and decrease in bulk density can be expressed by the Knudsen relation (Neighbour and Hacker 2001) as

$$
\begin{aligned}
& \sigma / \sigma_{0}=\exp (-k d) \\
& k=0.114
\end{aligned} .
$$

The buckling strengths of the oxidized columns with various dimensions are shown in Figure 7-11. The buckling strength of the oxidized graphite column decreases as the compressive strength decreases. The relation between the buckling strength and the bulk density is expressed by the Knudsen relation as

$\sigma / \sigma_{0}=\exp (-k d)$

$k=0.111$

The exponents of Eqs. (7-13) and (7-14) were almost the same. From this result, the buckling strength degradation of the oxidized graphite column is found to be independent of the slenderness ratio. The structural strength degradation of the oxidized graphite column only depends on the initial strength and a degree of the decrease in bulk density. Therefore, the strength degradation of an oxidized graphite column under axial compressive load can be expressed conservatively by Eq. (7-13).

Comparison of the Knudsen relation with former researchers' data is shown in Figure 7-12. Test materials, test conditions, and test materials are summarized in Table 7-3. The data obtained from KAIST is more conservative in its results. Kim et al. (2007) obtained $\mathrm{k}=0.089$ in the burn-off range of 0 to $7 \%$. It is possible that the difference mainly comes from the narrow burn-off range and the use of different materials. Eto and Growcock (1983) and Yoda et al. (1985) have reported test results for IG-11. IG-110 and IG-11 are similar kinds of material, but the purity is slightly different. It is believed that the burn-off range has some sensitivity to decide exponent $\mathrm{k}$ from the different results of Eto and Yoda's tests. These researchers tested the same IG-11 but the burn-off ranges were found to be different. 


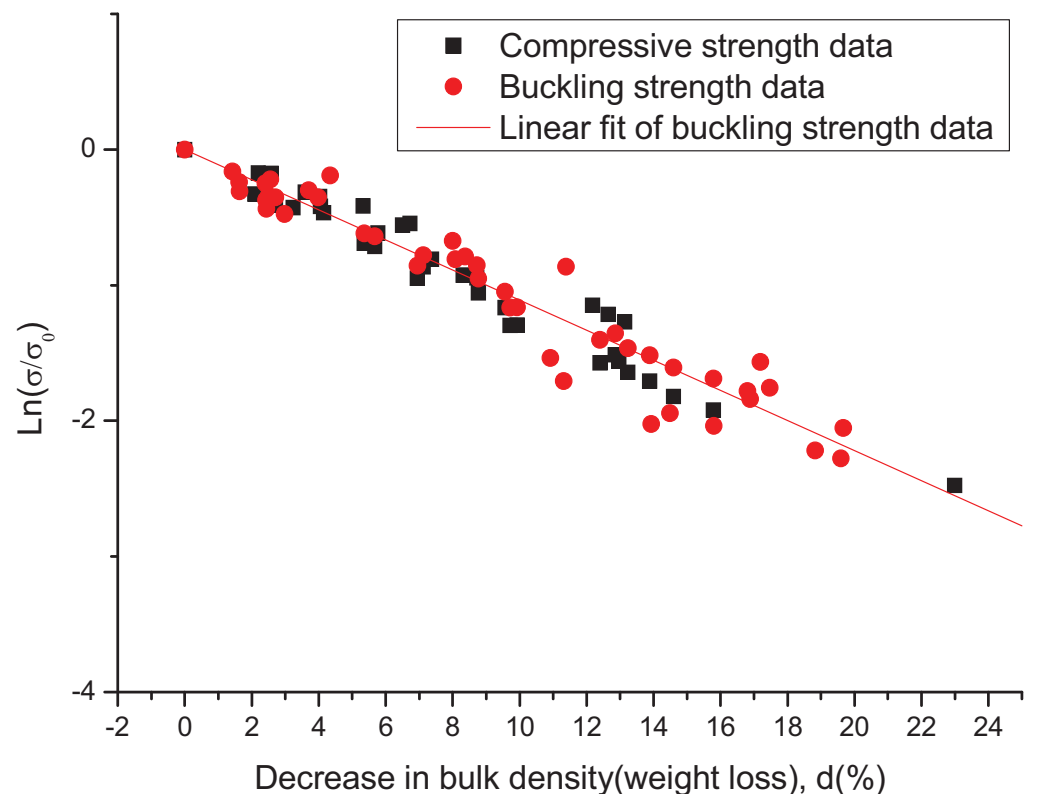

Figure 7-11. Normalized compressive and buckling strength of graphite columns oxidized in Zone 1.

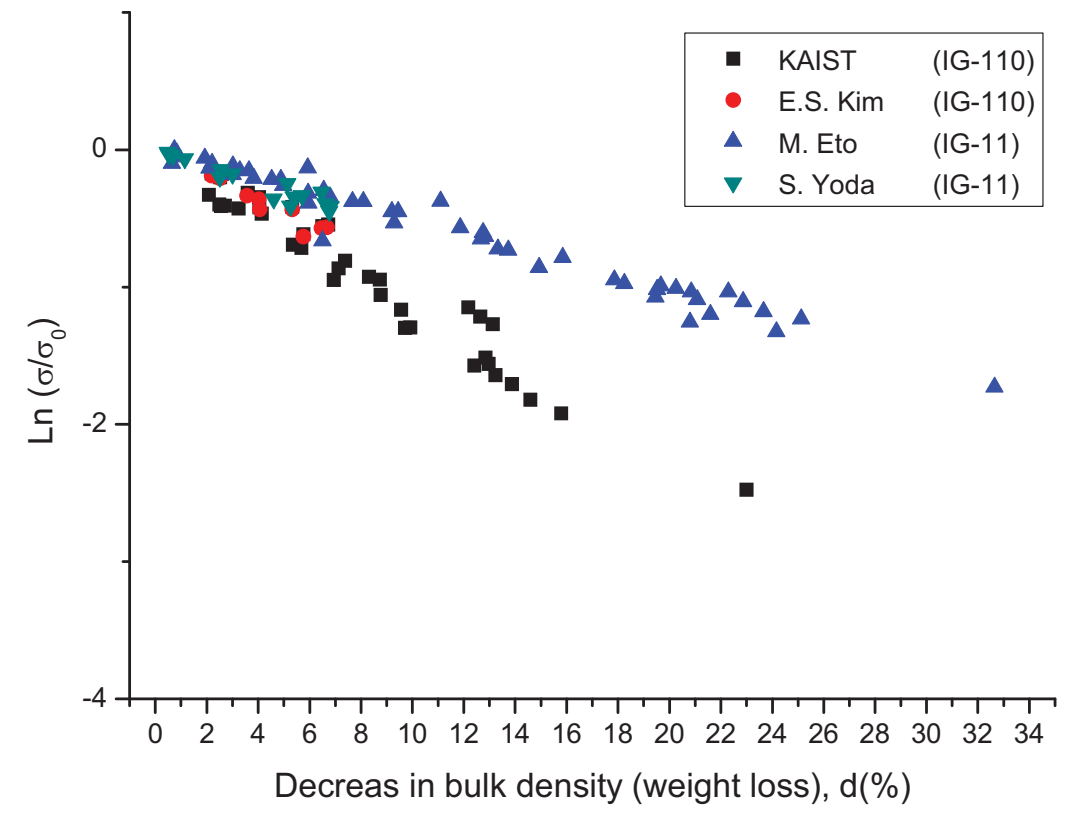

Figure 7-12. Comparison of the experimental results for the compressive strength degradation.

Table 7-3. Comparison of the Knudsen relation.

\begin{tabular}{|l|l|l|l|l|}
\hline \multicolumn{1}{|c|}{ Author } & $\begin{array}{c}\text { Eto and Growcock } \\
(\mathbf{1 9 8 3})\end{array}$ & $\begin{array}{c}\text { Yoda et al. } \\
(\mathbf{1 9 8 5})\end{array}$ & \multicolumn{1}{|c|}{$\begin{array}{c}\text { Kim et al. } \\
(\mathbf{2 0 0 7})\end{array}$} & \multicolumn{1}{c|}{ KAIST } \\
\hline Test material & IG-11 & IG-11 & IG-110 & IG-110 \\
\hline Temperature $\left({ }^{\circ} \mathrm{C}\right)$ & 500 & 500 & 600 & 600 \\
\hline Burn-off $(\%)$ & $0 \sim 6$ & $0 \sim 35$ & $0 \sim 7$ & $0 \sim 23$ \\
\hline exponent k & 0.060 & 0.053 & 0.089 & 0.114 \\
\hline
\end{tabular}


The strength degradation of IG-430 oxidized in Zone 1 was also obtained in the same method. The results are shown in Figure 7-13.

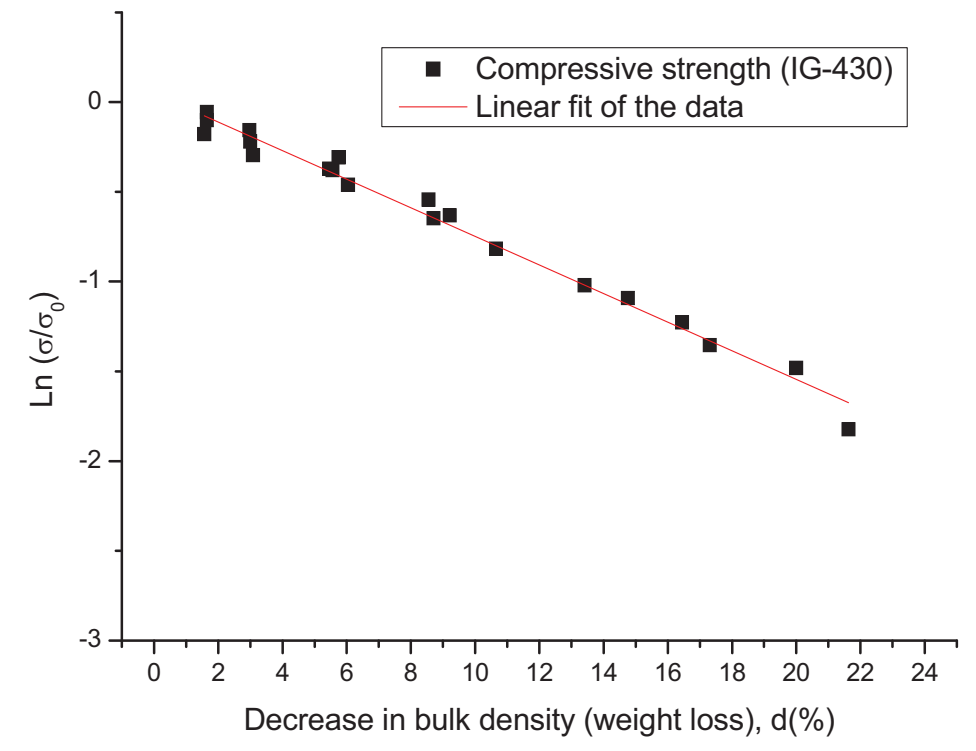

Figure 7-13. Normalized compressive strength of IG-430 oxidized in Zone 1.

The relation between the buckling strength and the bulk density is also expressed by the Knudsen relation as

$\sigma / \sigma_{0}=\exp (-k d)$

$k=0.080$

The strength degradation of NBG-10 oxidized in Zone 1 is shown in Figure 7-14. The exponent k was 0.06 .

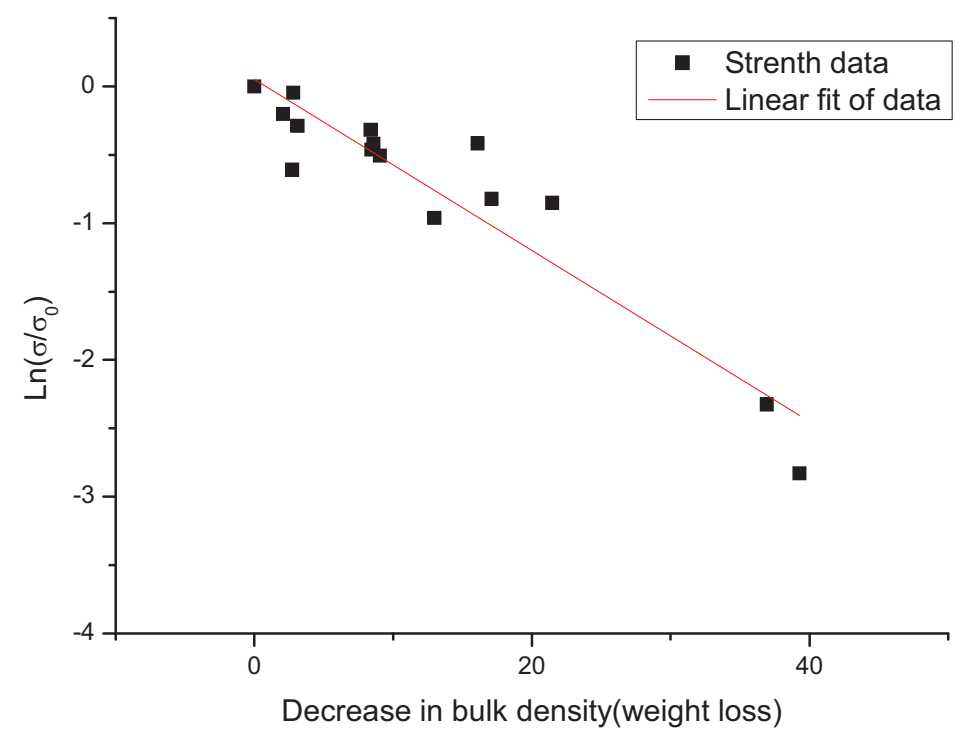

Figure 7-14. Normalized compressive strength of NBG-10 oxidized in Zone 1. 


\subsection{Strength Degradation in Zone 2}

Twenty-two specimens of IG-430 graphite were tested up to $32 \%$ of total burn-off. 5 SLPM of filtered dry air was supplied for 12 specimens and 10 SLPM was supplied for 10 specimens. Both bulk density, which is related to mass loss caused by volume reaction and dimension, and external surface changed during oxidation, which was measured by the ATOS 3-D digitizer as shown in Figure 7-15.
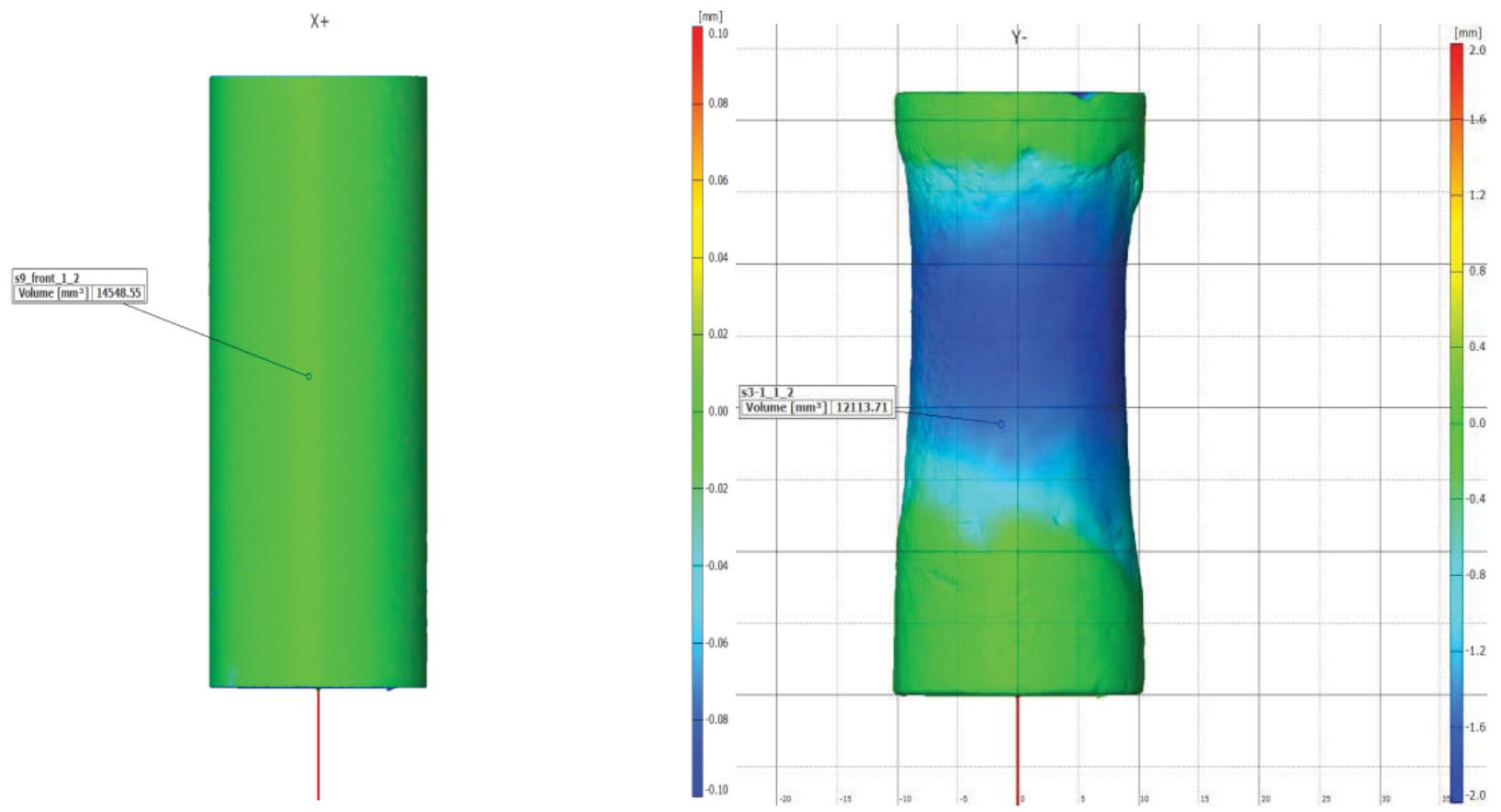

Figure 7-15. Picture of digitized specimen.

Fraction of surface reaction to total reaction in the 5 SLPM case are plotted in Figure 7-16.

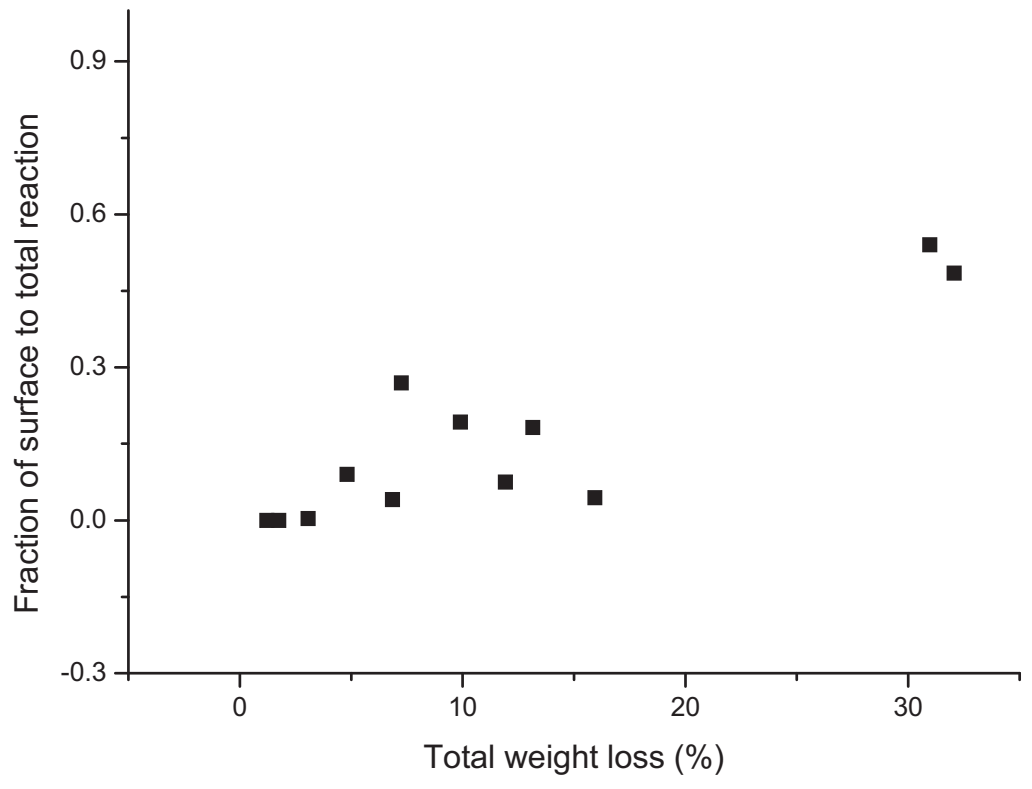

Figure 7-16. Fraction of surface reaction to total reaction (5 SLPM). 
Initially, three fresh specimens were tested to obtain average strength. The obtained average strength was $89.59 \mathrm{MPa}$. It is a good agreement with the compressive strength in Table 7-2.

Strength degradation of graphite oxidized in Zone 2 is also plotted with Knudsen relation to compare the trend of strength degradation in Zones 1 and 2. Data for both Zones are plotted in Figure 7-17.

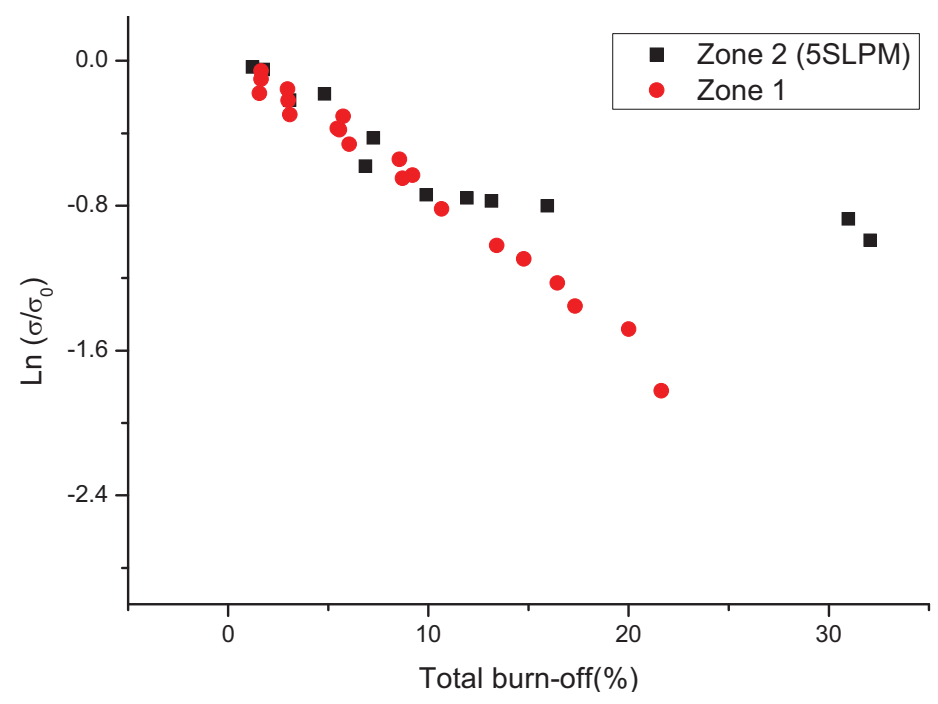

Figure 7-17. Normalized strength of graphite oxidized in Zones 1 and 2 versus total burn-off.

Both volume reaction and surface reaction occurs in the Zone 2 reaction. However, the total burn-off does not show any relation with strength degradation as shown in Figure 7-17. It is therefore assumed that the strength degradation is dependent on mass loss by volume reaction. Corrected burn-off is defined as

$$
d^{\prime}=\frac{\text { mass loss by volume reaction }}{\text { Initial mass }}=\frac{M_{0}-M-\left\{\left(V_{0}-V\right) \rho_{0}\right\}}{M_{0}}=1-\frac{M+\left\{\left(V_{0}-V\right) \rho_{0}\right\}}{M_{0}} .
$$

This corrected burn-off is applicable to both Zone 1 and 2 cases as shown in Eq. (7-16). Normalized strength versus corrected burn-off is plotted in Figure 7-18.

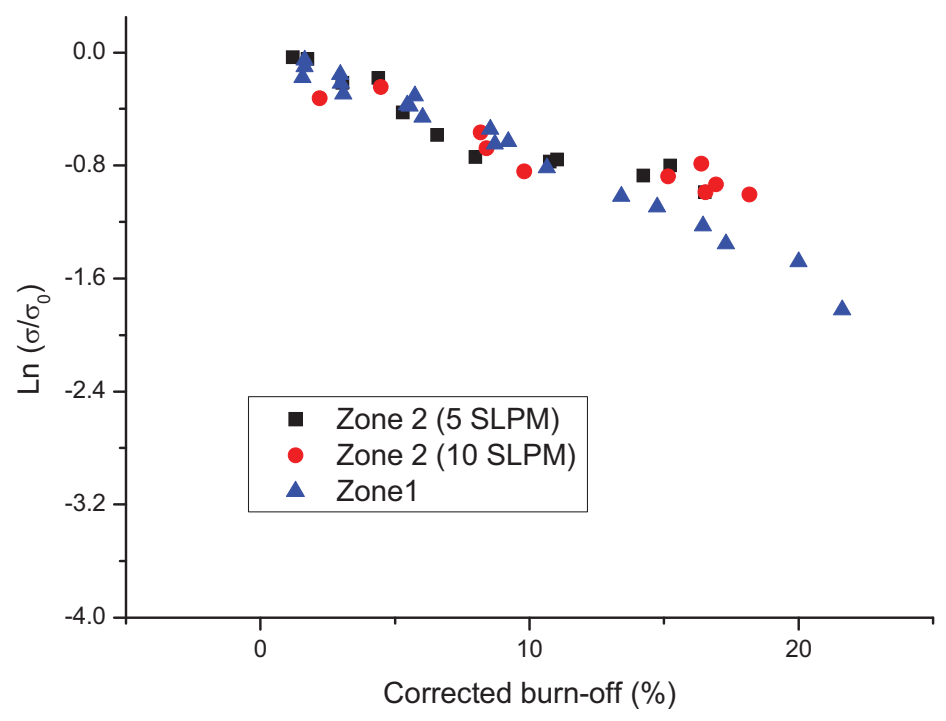

Figure 7-18. Normalized strength versus corrected burn-off. 


\subsection{Buckling Strength Graphite Column Oxidized in Zone 3}

The oxidation of IG-110 test material was carried in the same muffle furnace. Three specimens were oxidized at the same time under the same conditions. The oxidation temperature was $1050^{\circ} \mathrm{C}$. The averaged values of the strength data from the three samples are shown in Figure 7-19. The differences of slenderness ratios in each group were under $1 \%$. The error bar in the figure is a standard deviation for each group. The diameter in axial direction was not uniform because of a graphite cap. The failure occurred in the center of the column with the minimum diameter; slenderness ratio was calculated using the minimum diameter. A cross-section of column is not a perfect circle but elliptical in shape. The difference of two different diameters is under $0.5 \mathrm{~mm}$ for all samples. The measured minimum diameter after oxidation was $12.3 \mathrm{~mm}$. The maximum uncertainty, calculated from the maximum difference and the minimum diameter, in cross-section was under 4\%. The solid line in Figure 7-19 represents the strength of a fresh graphite column as a function of the slenderness ratio. The linear fit of experimental strength data was expressed by an empirical straight-line formula as

$\sigma_{\text {cr,buckling }}=\sigma_{\text {straight-line }}-C(L / r)=86.8 \mathrm{MPa}-0.9 \mathrm{MPa} \cdot(L / r)$.

The deviation between the experimental data and the empirical line for the fresh graphite is about $5 \mathrm{MPa}$. From this result, we can say that the surface oxidation is dominant at temperatures over $1000^{\circ} \mathrm{C}$ and that the strength of the graphite column oxidized at temperatures over $1000^{\circ} \mathrm{C}$ can be predicted by the empirical straight-line formula of Eq. (7-9).

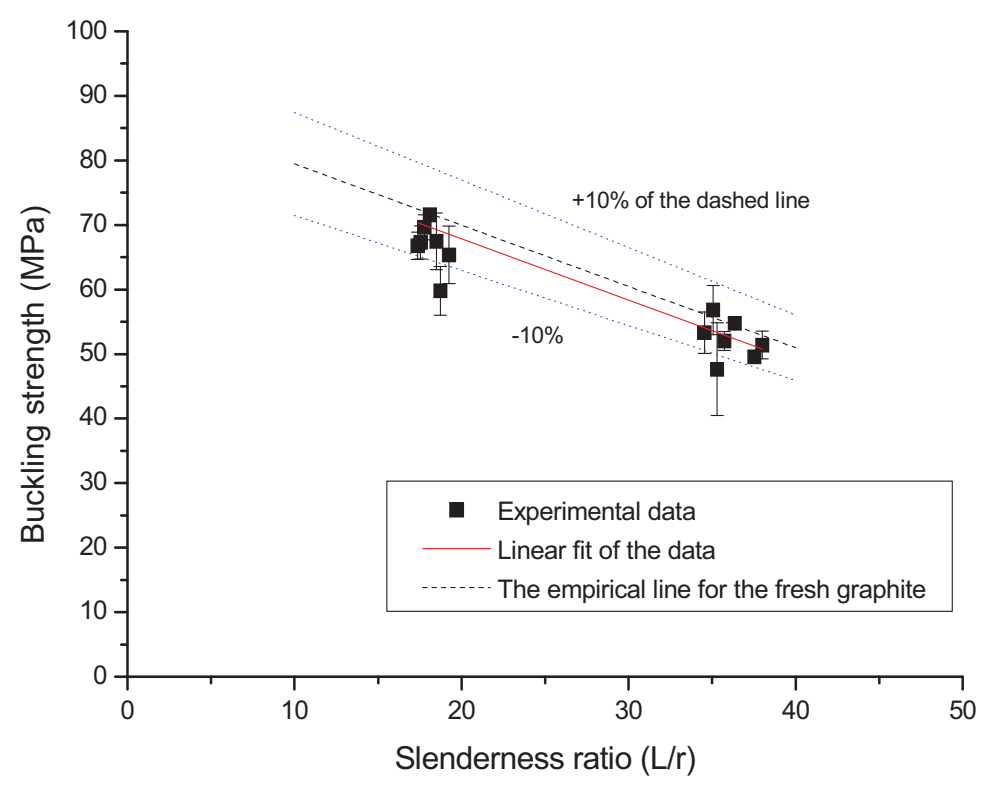

Figure 7-19. Strength of graphite columns oxidized in Zone 3. 


\subsection{Graphite Support Block}

A graphite support block has very complex dimension with a lot of holes. One-sixth of the graphite support block and simplified model are shown in Figure 7-20. The modeling in Figure 7-19 can be a good model for oxidation reaction but it is not a good model for structural tests because they have different structural parameters like a slenderness ratio. It is hard to make a chemically and structurally equivalent model. On the other hand, the graphite support block can also be considered as a short column. It is considered that the strength of the column depends on slenderness ratio by Euler's formula and the empirical straight-line formula. So, graphite columns have the same strength if graphite columns have the same slenderness ratio with different dimensions as confirmed by the following tests. The simplified model, which has the same slenderness ratio with graphite support block, was then used for analyses of graphite the support block.

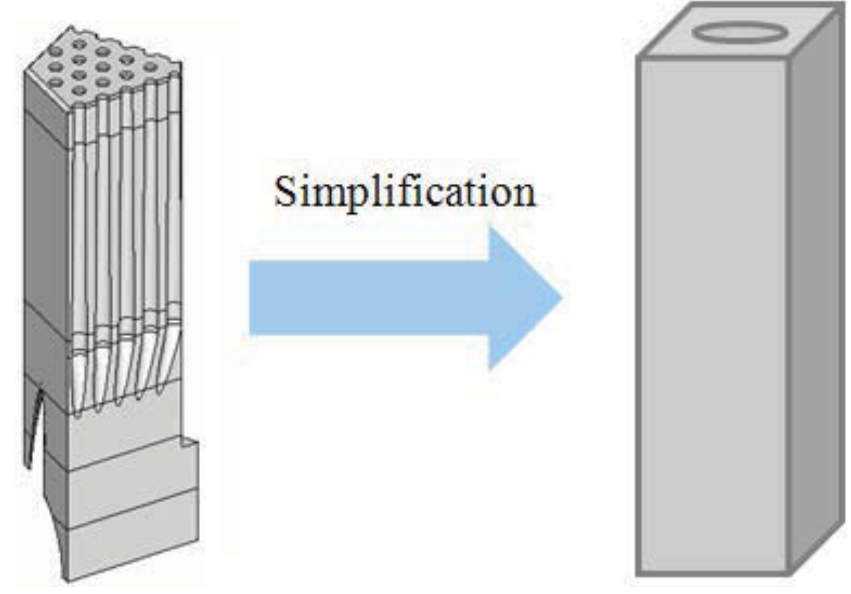

Figure 7-20. One-sixth of the graphite support block and its simplified model.

\subsubsection{Fresh Graphite Tests}

The strength of three specimens was measured. IG-110 and IG-430 were tested. The detailed specification is shown in Table 7-4. Specimens B and C have the same slenderness ratio and crosssectional area, but their dimensions are different.

Table 7-4. Dimensions of graphite specimens for fresh graphite tests.

\begin{tabular}{|c|c|c|c|}
\hline & A & $\mathrm{B}$ & $\mathrm{C}$ \\
\hline Pictures of specimens & . & & \\
\hline Slenderness ratio $(\mathrm{L} / \mathrm{r})$ & 16 & 15.5 & 15.5 \\
\hline Dimension (mm) & $15 \Phi \times 60$ & $\begin{array}{l}15 \Phi \times 60 \\
(3.8 \Phi 1 \text { hole })\end{array}$ & $\begin{array}{l}13.3 \times 13.3 \times 60 \\
(1.9 \Phi 4 \text { holes })\end{array}$ \\
\hline Cross-sectional area $\left(\mathrm{mm}^{2}\right)$ & 176.7 & 165.5 & 165.4 \\
\hline
\end{tabular}


An INSTRON model 4204 mechanical testing facility was also used for the compression test with the same procedures. Seven specimens are tested for each kind of specimen. All the specimens fail by buckling. The strain rate was $5.55 \times 10^{-5} \mathrm{sec}^{-1}$. The experimental results are summarized in Tables 7-5 and 7-6. The strength of Specimens B and $\mathrm{C}$ are almost the same, and it is also proper value as compared with the specimen strengths. The experimental results show good agreement with the calculated buckling strength from the previous empirical buckling strength formulas. Therefore, the empirical buckling strength formula applies to all kinds of columns over the slenderness ratio tested in the previous experiments.

Table 7-5. Experimental results of IG-110 columns.

\begin{tabular}{|l|l|l|l|}
\hline & \multicolumn{1}{|c|}{ A } & \multicolumn{1}{c|}{ B } & \multicolumn{1}{c|}{ C } \\
\hline Strength (MPa) & $74.02 \pm 2.9$ & $74.49 \pm 4.9$ & $75.46 \pm 1.9$ \\
\hline Calculated buckling strength (MPa) & 75.18 & 75.69 & 75.69 \\
\hline Differences & $1.5 \%$ & $1.0 \%$ & $0.3 \%$ \\
\hline
\end{tabular}

Table 7-6. Experimental results of IG-430 columns.

\begin{tabular}{|l|l|l|l|}
\hline & \multicolumn{1}{|c|}{ A } & \multicolumn{1}{c|}{ B } & \multicolumn{1}{c|}{ C } \\
\hline Strength & $87.30 \pm 4.06$ & $87.56 \pm 2.57$ & $87.12 \pm 2.9$ \\
\hline Calculated buckling strength (MPa) & 87.23 & 87.85 & 87.86 \\
\hline Differences & $0.1 \%$ & $0.3 \%$ & $0.8 \%$ \\
\hline
\end{tabular}

\subsubsection{Strength Degradation of Various Columns Oxidized In Zone 1}

The same kinds of IG-430 columns in Table 7-4 were oxidized at the temperature of $600^{\circ} \mathrm{C}$. The strength was measured with the same compression test machine. The obtained data were also compared with the compressive strength degradation of IG-430. The normalized experimental results are shown in Figure 7-21. The results can also be expressed by the Knudsen relation. The exponents for the data are almost the same. So, the strength of graphite column oxidized in Zone 1 is only dependent on the initial strength and decrease in bulk density, even though the graphite columns have the different dimensions and the different failure modes. The same conclusion also applies with the strength degradation in Zone 1 for IG-110. 


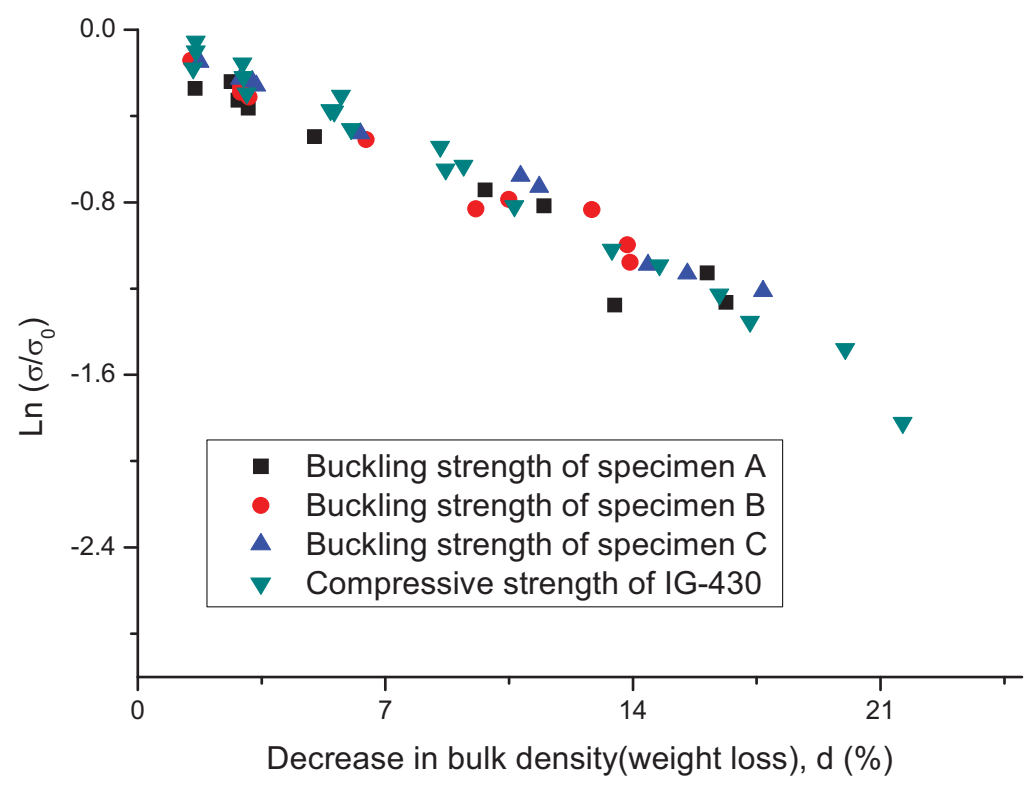

Figure 7-21. Normalized compressive and buckling strength of IG-430 columns.

\subsection{Minimum Burn-off to Lead Failure of Structural Component}

\subsubsection{Failure model of graphite structure}

We suggest the strength degradation of the graphite structure as follows:

$\sigma_{0}=A-B \frac{L}{r}$

and

$\frac{\sigma}{\sigma_{0}}=\exp (-k d), d=1-\frac{M+\left\{\left(V_{0}-V\right) \rho_{0}\right\}}{M_{0}}$

The strength depends only on the slenderness ratio and current bulk density of the graphite. This failure model applies to all the oxidation regimes. The constants (A, B, and the exponent $\mathrm{k}$ ) are different with the graphite grades. Table 7-7 summarizes all the constants.

Table 7-7. Constants for strength degradation.

\begin{tabular}{|l|c|c|c|}
\hline & A & B & K \\
\hline IG-110 & 91.3 & 1 & 011 \\
\hline IG-430 & 107.6 & 1.3 & 0.08 \\
\hline NBG-10 & 61.8 & 0.4 & 0.06 \\
\hline
\end{tabular}




\subsubsection{Minimum Allowable Burn-off}

The followings safety criteria apply for the failure of the graphite structure:

- Stress criterion: $\sigma_{\text {stress }}>\sigma_{\text {strength }}(f, b)$

- Density criterion: $\rho<\rho_{\text {limit }}$

If the strength calculated from the failure model is smaller than applied stress to the graphite structure, the structure fails by the stress criterion. The structure fails by the density criterion if the current density is smaller than the density limit. The density limit was obtained by the INL high burn-off experiment. The density limit is $0.35 p$ for IG-110 (Oh et al. 2008).

The oxidation trend can be expressed in terms of f-value, which is the fraction of surface reaction to total reaction. The dimensional change of oxidized graphite is considered as surface reaction. The f-value of graphite structure oxidized under $600^{\circ} \mathrm{C}$ is 0 . The value of a graphite structure oxidized over $1000^{\circ} \mathrm{C}$ is 1. If the condition of the graphite structure belongs to Zone 2 , the f-value has a value between 0 and 1 .

The loaded weight for a support column of GTMHR is about $1800 \mathrm{~kg}$. The stress is about $0.5 \mathrm{MPa}$. The relation between the allowable total burn-off and f-value is shown in Figure 7-21. It is calculated by using the failure model, assuming that the column has uniform diameter after the oxidation. The graphite structure fails if the current total burn-off is higher than the obtained line in Figure 7-21. The graphite structure is expected to fail according to the stress criterion indicated by the rectangular point in Figure 7-22. The circular point in the figure represents its failure by the density criterion.

Figure 7-23 shows the comparisons of the allowable burn-offs of IG-110, IG-430, and NBG-10 graphite structure. As shown in Figure 7-23, the minimum allowable total burn-off was obtained when the $\mathrm{f}$-value is 0 . Using this minimum allowable total burn-off is recommended for the conservative approach. It was concluded that NBG-10 is best for the graphite structure but the graphite oxidation rate is different. Both structural and chemical characteristics must be considered in selecting the structural materials.

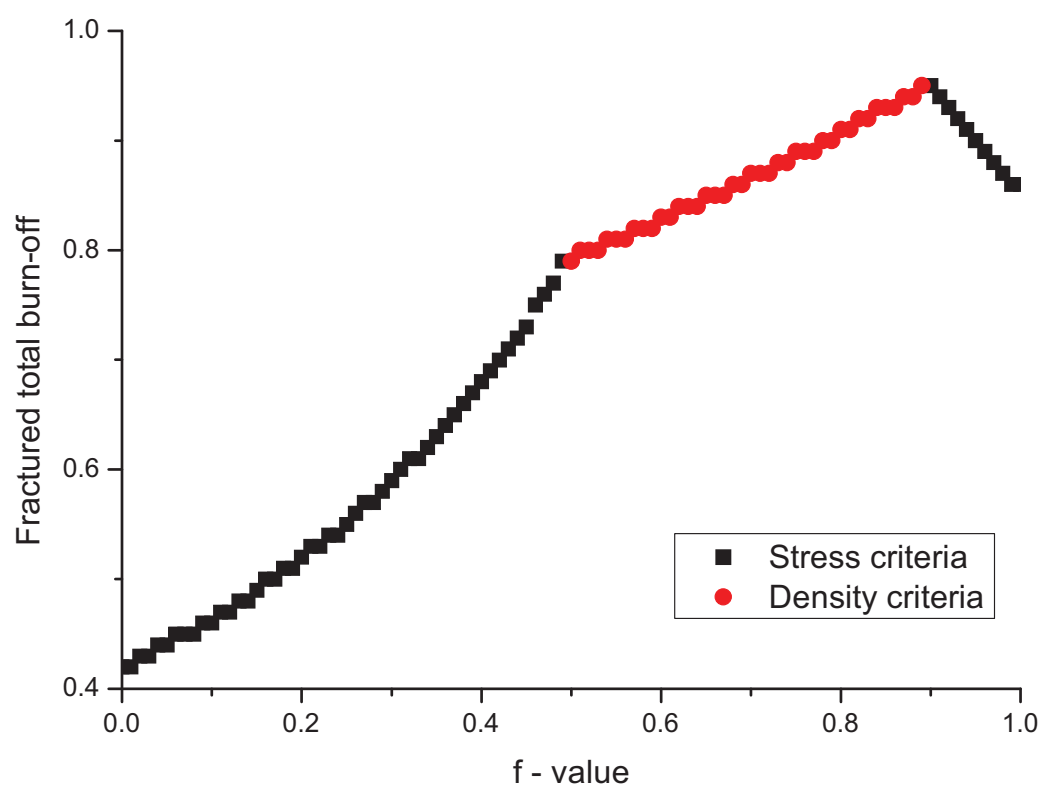

Figure 7-22. Relation between allowable total burn-off and f-value. 


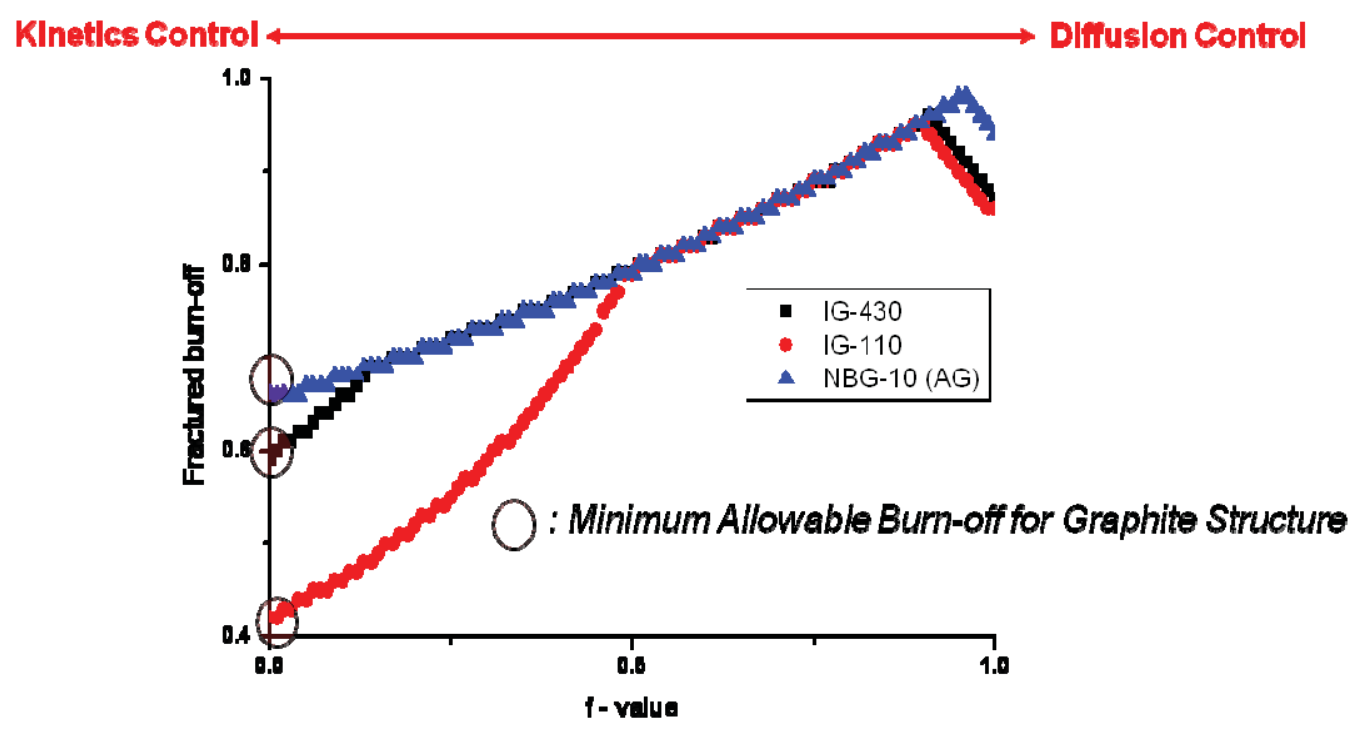

Figure 7-23. Comparison of allowable total burn-offs for graphite structure.

\subsection{Effects of Onset Time of NC in 1D Simulation}

\subsubsection{Maximum Core Temperature}

One of the most important safety criteria of HTGRs is the maximum core temperature. Under any kinds of $\mathrm{NC}$ scenarios, the maximum core temperature should not exceed $1600^{\circ} \mathrm{C}$. Figure $7-24$ shows that the onset time of $\mathrm{NC}$ barely affects extra core temperature rising.

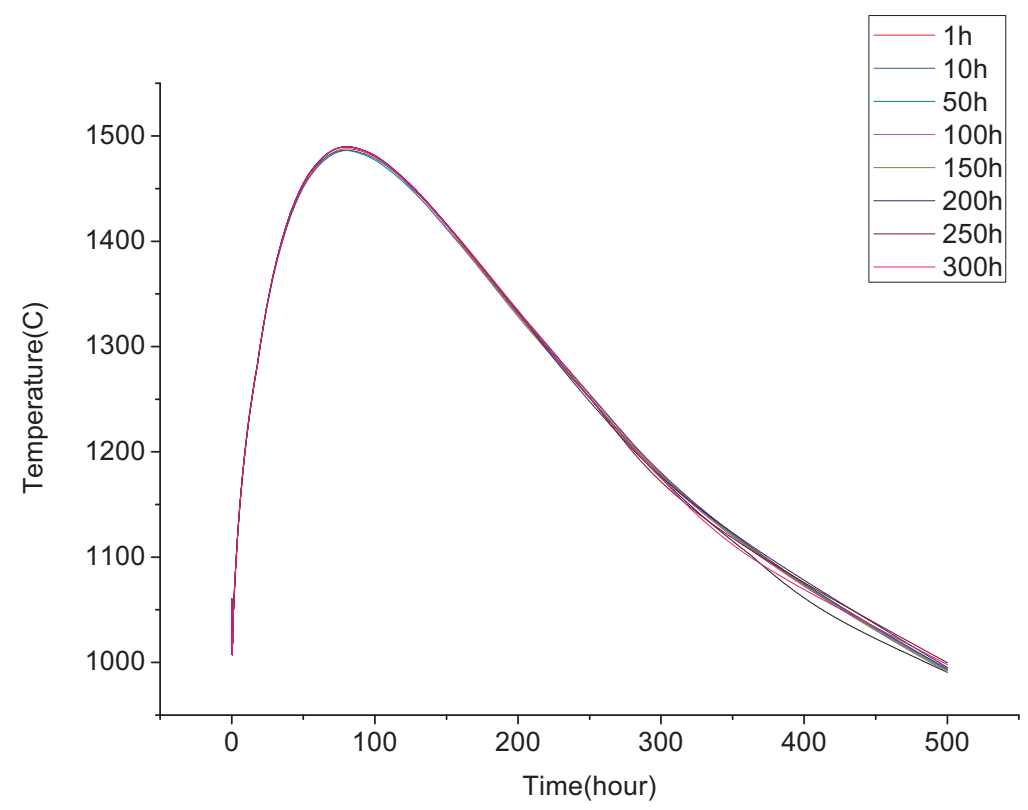

Figure 7-24. Maximum core temperature (GAMMA 1D). 


\subsubsection{Bottom Reflector Temperature}

Variation in the bottom reflector temperature really depends on the onset time of NC. Because of NCdriven massive air invasion, vigorous oxidation takes place and the temperature rebounds when NC starts.

Two groups of trends are shown in Figure 7-25 (onset time of NC under or over 150 hours). When NC happens, there are two competing effects: the cooling effect by the convection of air coming from the cavity, and the heating effect by the exothermic reactions of the graphite oxidation. For earlier NC than 150 hours, due to the high temperature of the bottom reflector, the heating effect is dominant. Therefore, the temperature rebounds as soon as NC starts. However, in the cases that NC starts later than 150 hours (200, 250, or 350 hours), the cooling effect is dominant. This being the case, the temperature goes slightly downward and then rises again. This highly depends on oxidation temperature when NC starts.

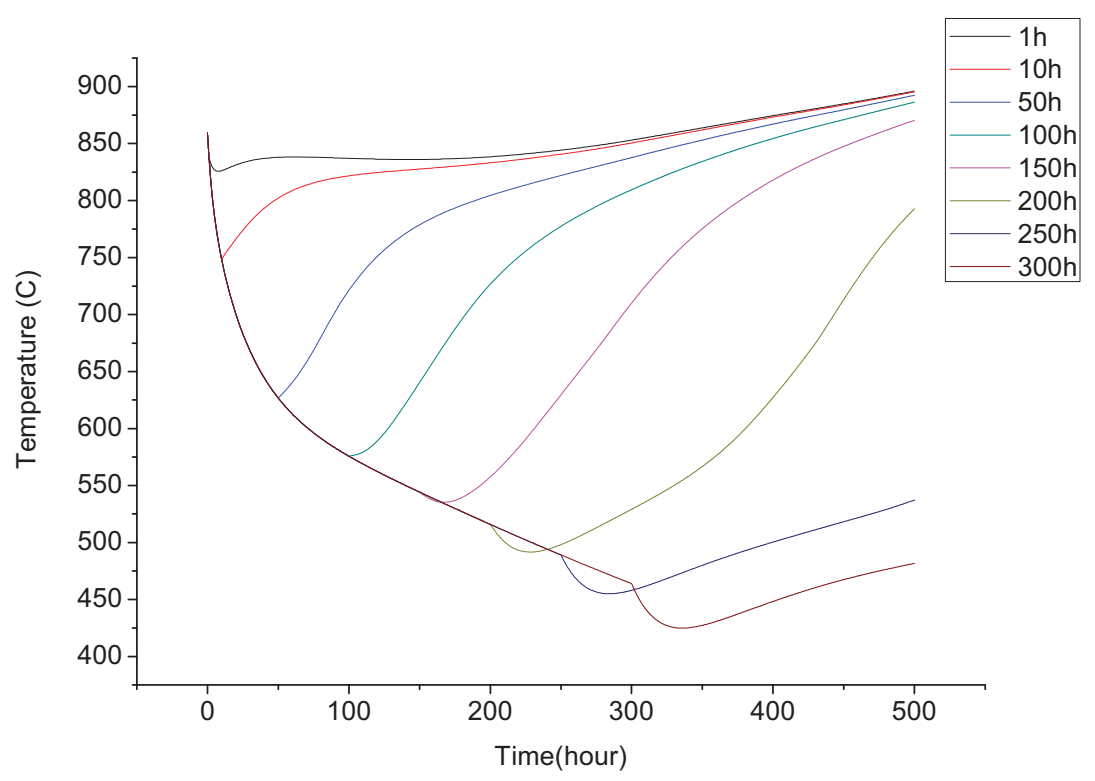

Figure 7-25. Bottom reflector temperature (GAMMA 1D).

\subsection{Effects of Onset Time of NC in 2D Simulation}

Maximum core temperature contours are presented in Figure 7-26. In the legend, 2D stands for 'no forced $\mathrm{NC}$ case' to compare with other 'forced NC cases', where NC starts at 1 hour and 10 hours, respectively. Under any kinds of $\mathrm{NC}$ scenarios, the maximum core temperature is below $1600^{\circ} \mathrm{C}$. Therefore, $2 \mathrm{D}$ results demonstrate that the reactor core is still safe even in air ingress accidents. 


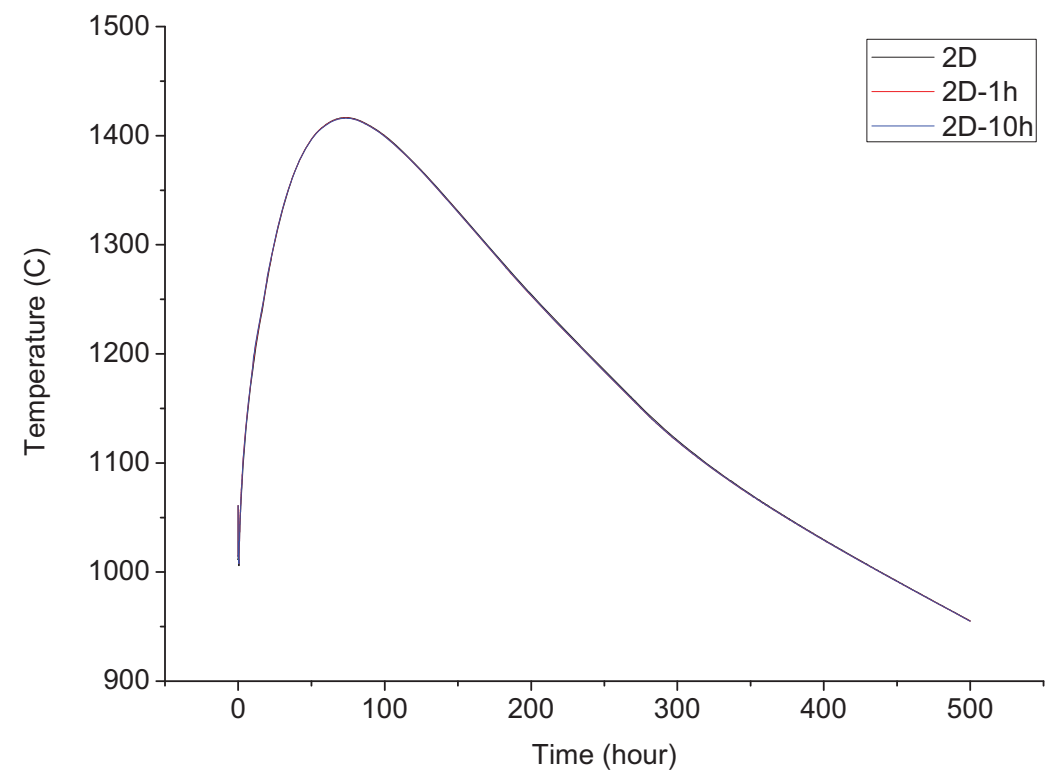

Figure 7-26. Maximum core temperature (GAMMA 2D).

One of the interesting parts of this research is the bottom reflector temperature of $2 \mathrm{D}$ case. For better understanding of this result, 'no forced NC' and 'forced NC' cases are presented in Figure 7-27. At the top, the 1D-1h line represents the bottom reflector temperature from 1D air ingress analysis with the 1 hour forced $\mathrm{NC}$ case. In this case the graphite oxidation driven heating effect is dominant compared with the 1D line - no forced NC. However, when comparing the bottom reflector temperature in the 1 hour NC timing 2D case (2D-1h) with that in 2D no forced NC case, they have quite similar trends with a small difference: the early $\mathrm{NC}$ in $2 \mathrm{D}$ cases does not lead to its early temperature rebound because of the cooling effect.

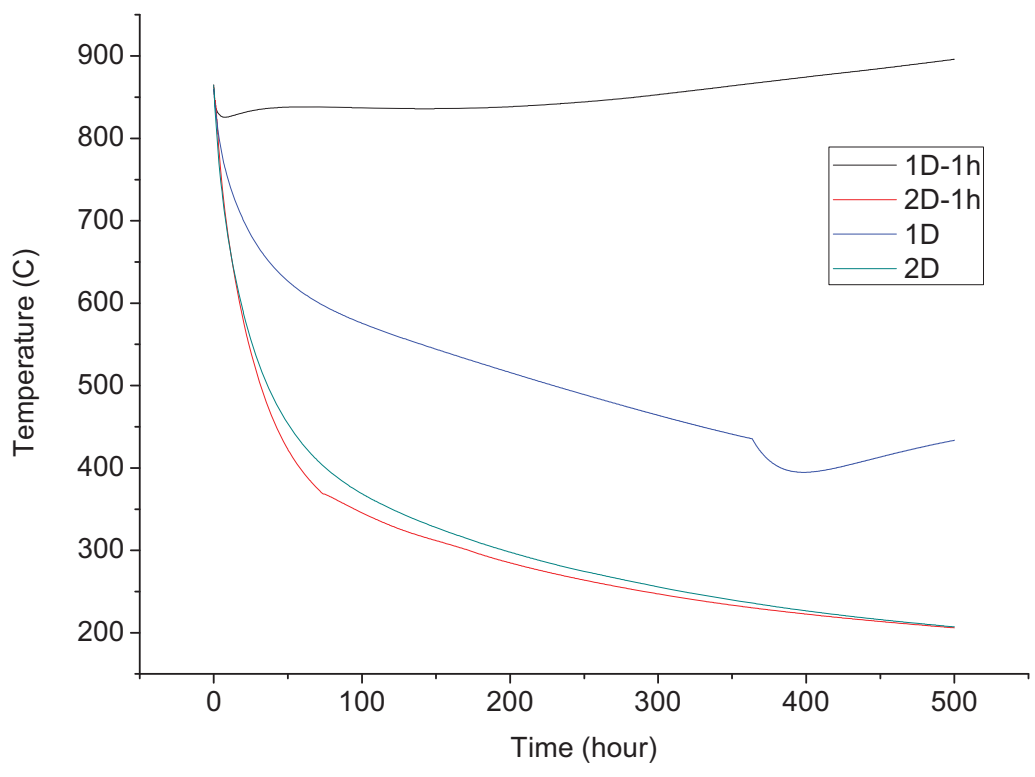

Figure 7-27. Bottom reflector temperature (GAMMA 2D). 


\subsection{Comparison of Minimum Burn-off with GAMMA 1D and 2D Results}

The ratio of the total burn-off of the support post to the minimum allowable burn-off for the graphite structure is given in Figure 7-28. Even in the most severe case (1D-1h), the total burn-off is under the safety limit. For 2D cases, which have a genetically dominant cooling effect, there is no chance to get high burn-off. This big difference comes from the difference of the bottom reflector temperatures between 1D and 2D simulation. In conclusion, the current HTGRs have a considerable safety margin for core collapse because of the degradation of the bottom support material by the graphite oxidation in the event of air-ingress accidents.

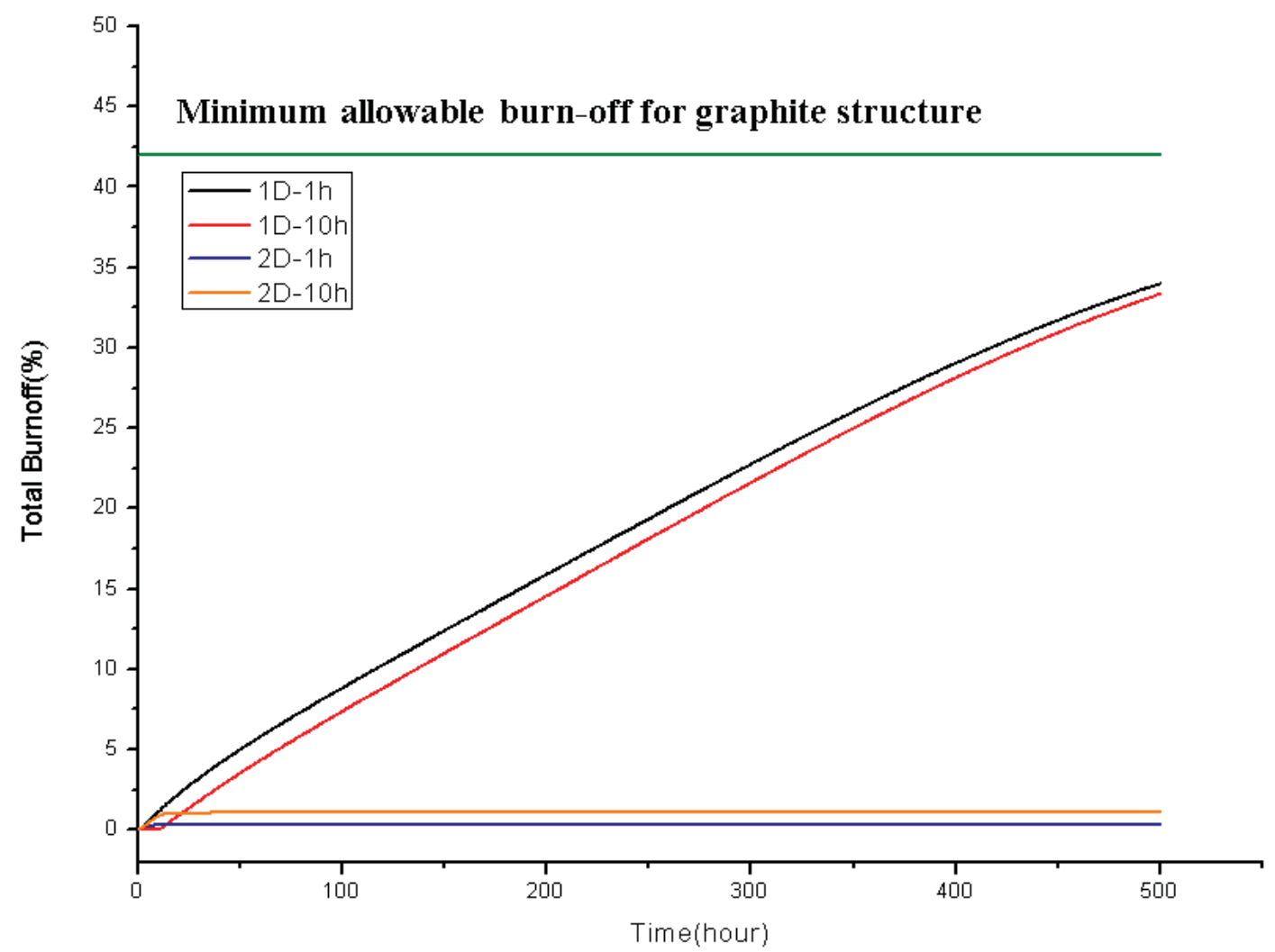

Figure 7-28. Total burn-off (GAMMA 1D and 2D).

\subsection{Validation on the GAMMA Code 2D Simulation for Density Driven Stratified Flow}

The GAMMA code is a system analysis code used to predict the thermal hydraulic and chemical reaction phenomena expected to occur during thermo-fluid transients, including air-ingress accidents in HTGRs. The fluid flow and heat transport in the GAMMA code is solved unsteadily by the thermal nonequilibrium model, which consists of two sets of equations for two media: gas and solid parts. In the gas flow medium, the multidimensional governing equations for a chemically reacting flow consist of the spatially averaged conservation equations for the continuity, momentum, energy of the gas mixture, and the mass of each species. For the present analytical model, the gas mixture contains several types of species ( $\mathrm{He}, \mathrm{H}_{2}, \mathrm{~N}_{2}, \mathrm{O}_{2}, \mathrm{CO}, \mathrm{CO}_{2}, \mathrm{CH}_{4}, \mathrm{H}_{2} \mathrm{O}$, and air), and it is assumed that each gas species and the gas mixture follow the equation of state for an ideal gas. 
The experiment by Grobelbauser et al. (1993) was used to validate GAMMA code 2D simulation, which is based on the simple lock-exchange flows in the rectangular channel, the 2-D assumption is quite valid here. The model consists of two boxes of different sizes: the left one is $0.3 \mathrm{~m}$ high and $3.0 \mathrm{~m}$ long and the right one is $0.3 \mathrm{~m}$ high and $0.8 \mathrm{~m}$ long. The mesh size is $0.1 \mathrm{~m}$ wide and $0.015 \mathrm{~m}$ high for each grid. Figure 7-29 shows the geometry and mesh of the GAMMA code model.

(a) Geometry

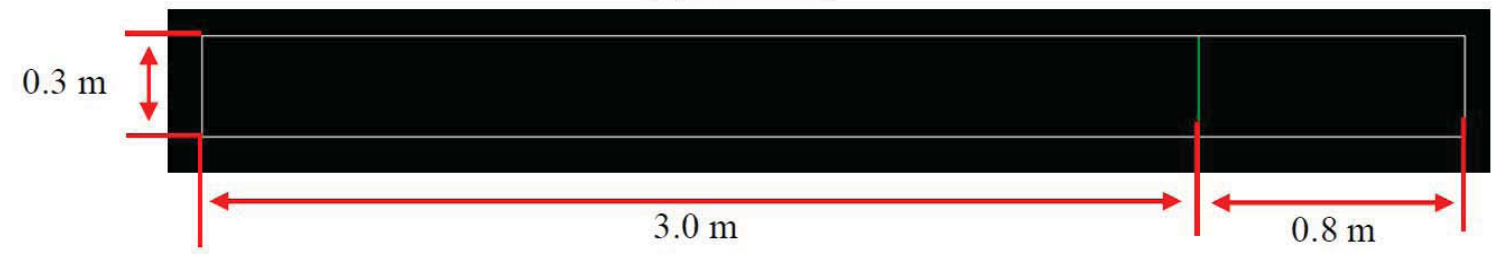

(b) Mesh

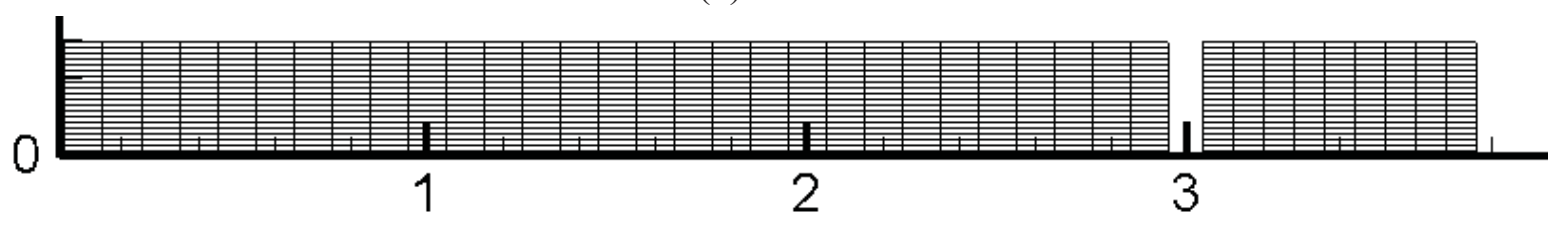

Figure 7-29. Geometry and GAMMA mesh.

Figure 7-30 shows the initial simulation condition. The left hand side was filled with helium and the right hand side with air. Therefore, this simulation demonstrates the heavy fluid intrusion down to the light fluid. Initial temperature was set as $300 \mathrm{~K}$ and pressure at $1 \mathrm{~atm}$.

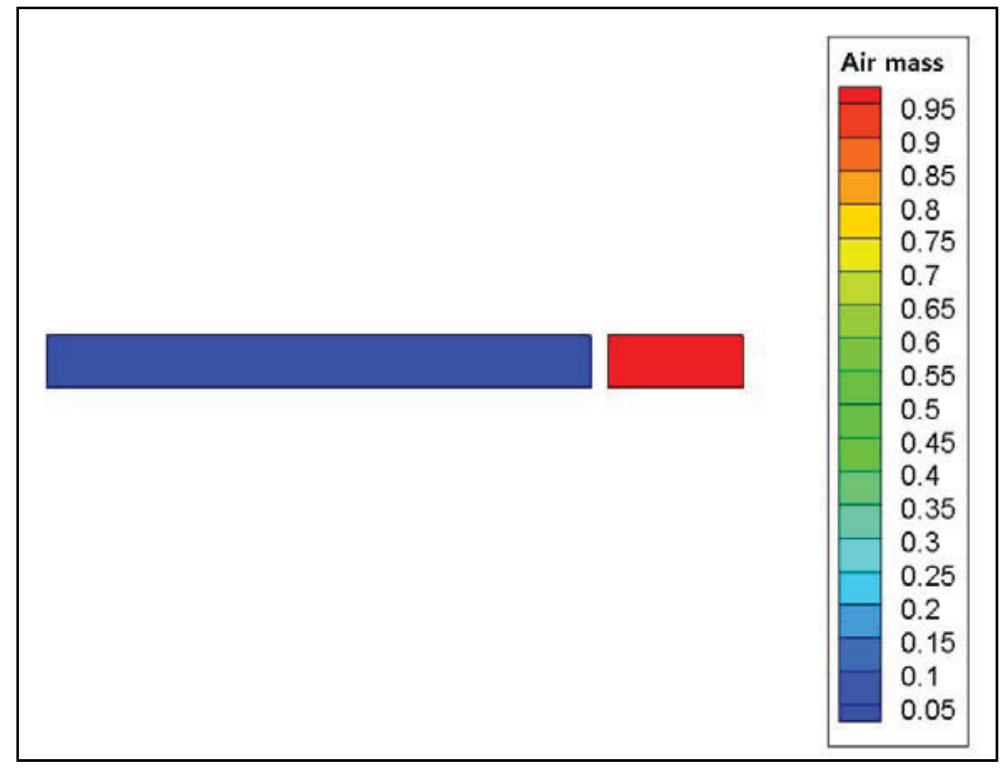

Figure 7-30. Initial air mass fractions.

Figures 7-31, 7-32, and 7-33 show the contour plots of air mass fractions at different times for GAMMA and FLUENT, respectively. FLUENT results include the turbulence model effect (k-w, standard k-e, and RSM). All of FLUENT simulations have been performed by using fine mesh grid and the same model setup as the base calculation except for turbulence models. The four figures show that the overall qualitative flow behaviors are similar. 
FLUENT simulation (air mass fraction, k-w model, fine mesh).

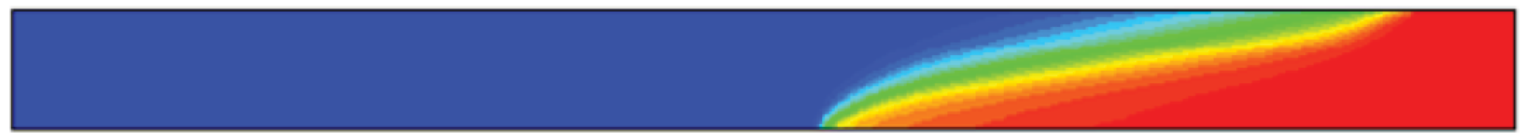

FLUENT simulation (air mass fraction, standard k-e model, fine mesh).

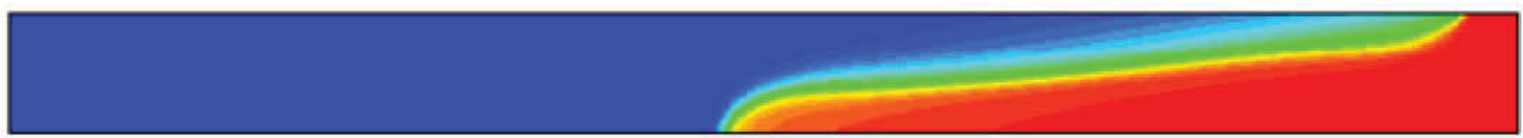

FLUENT simulation (air mass fraction, RSM model, fine mesh).

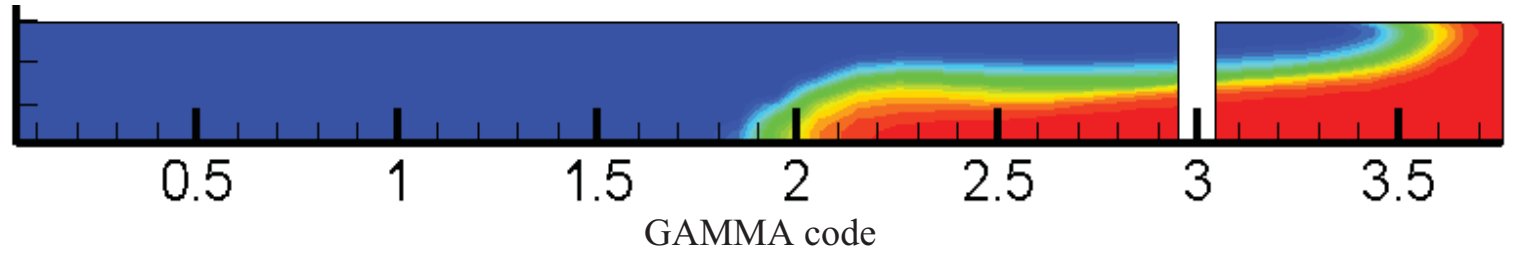

Figure 7-31. FLUENT and GAMMA simulation (1 second).

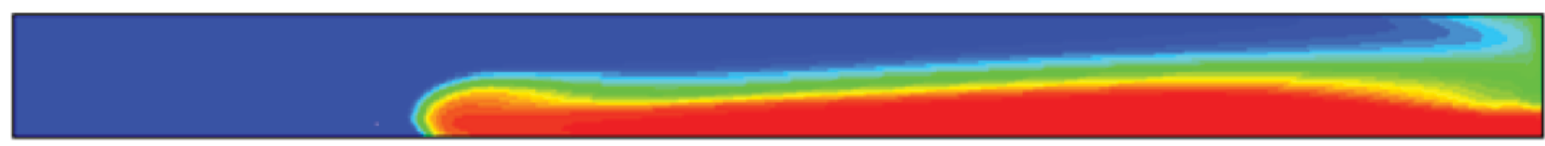

FLUENT simulation (air mass fraction, k-w model, fine mesh).

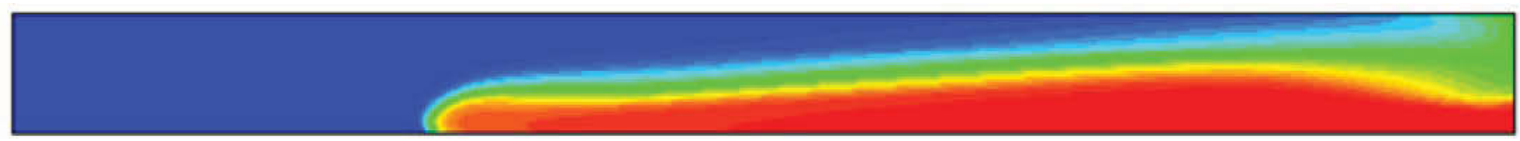

FLUENT simulation (air mass fraction, standard k-e model, fine mesh).

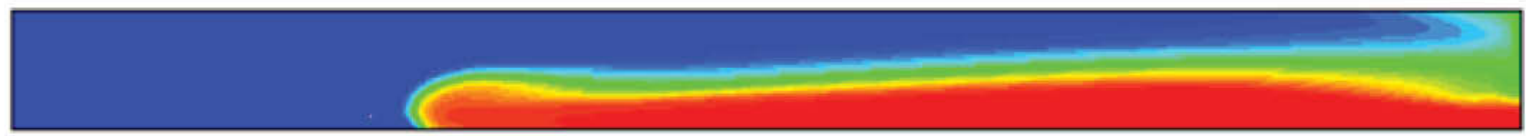

FLUENT simulation (air mass fraction, RSM model, fine mesh).

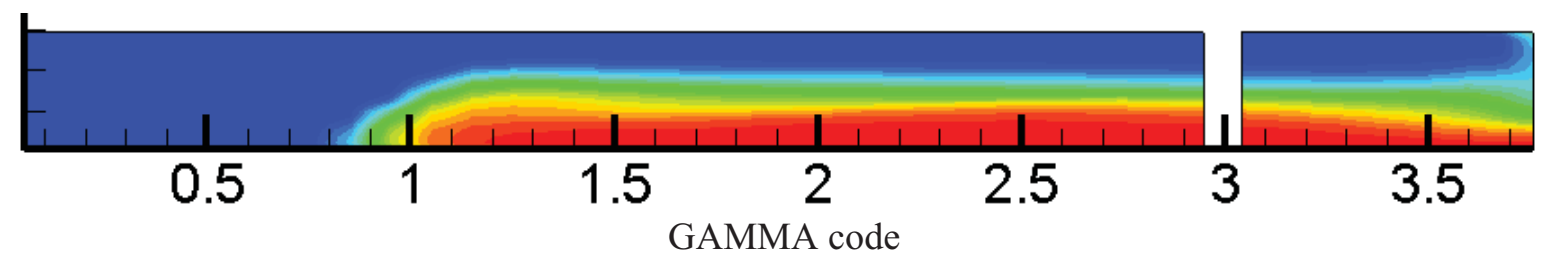

Figure 7-32. FLUENT and GAMMA simulation (2 second). 
FLUENT simulation (air mass fraction, k-w model, fine mesh)

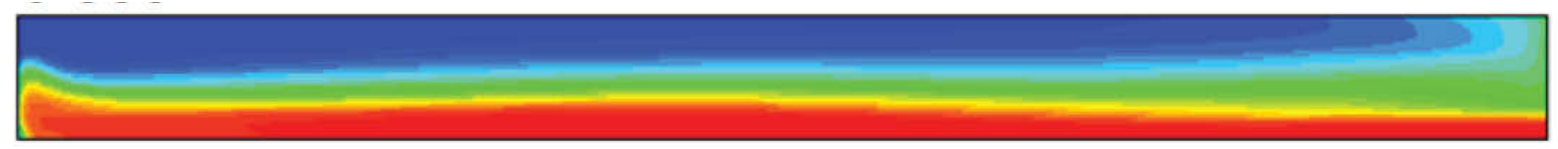

FLUENT simulation (air mass fraction, standard k-e model, fine mesh).

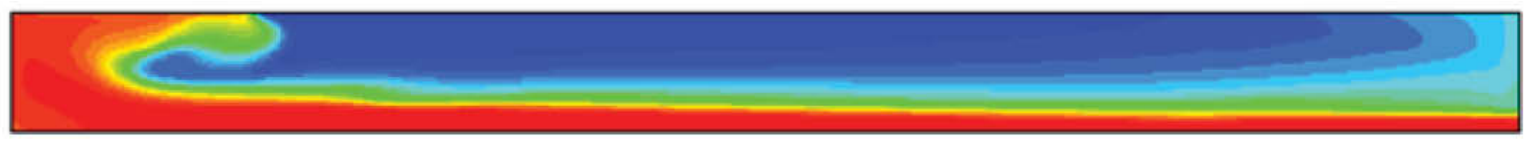

FLUENT simulation (air mass fraction, RSM model, fine mesh).

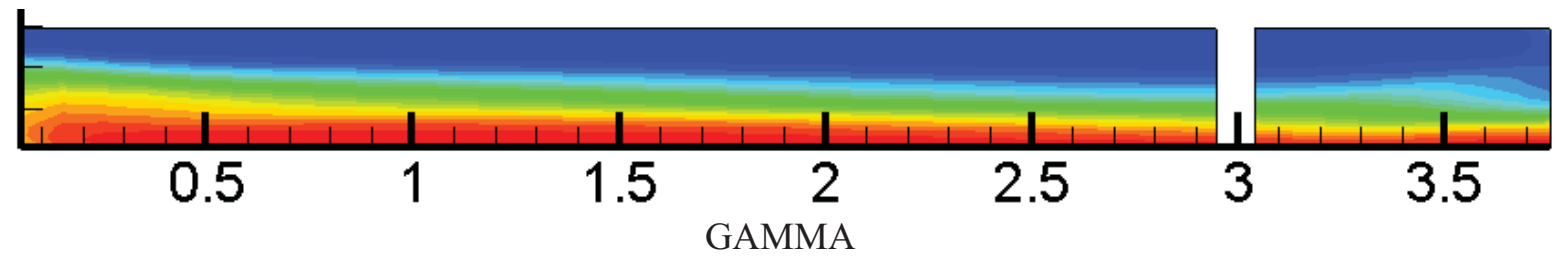

Figure 7-33. FLUENT and GAMMA simulation (3 second).

Figure 7-34 shows the comparisons between the CFD simulation results (Fluent, GAMMA code) and the experimental data. The compared parameter is the front location of the heavy current (air). It also shows that GAMMA code is good at predicting the front locations and that the data in the beginning shows more deviations than those in the later. This is because the opening gate in the actual experiment is not instantaneous and the gate opening process disturbs the flow field. After 1 second, the experimental data and the GAMMA results show quite good agreement.

\subsection{Structural Tests of Bottom Reflector}

\subsubsection{Experiments}

The oxidation and compression test of the one-eighth scaled bottom support system was performed. Test material was IG-11. The number of coolant holes on a support block was scaled down. Originally, the number of coolant holes was over 100 in the GTMHR design. The one-eighth scaled structure has six coolant holes. It is hard to machine over 100 holes on a one-eighth scaled-down support block because of limitations on the machining technique. The slenderness ratio of each part was conserved. As shown in previous results, the buckling strength of a graphite structure depends on the slenderness ratio. Other geometrical parameters are also linearly scaled down. The load applied to the graphite structure was onesixty-forth of the originally applied load.

Heating was carried by the induction heater. The test section was surrounded by the quartz tube. Figure 7-35 shows the test sample and the facility. 


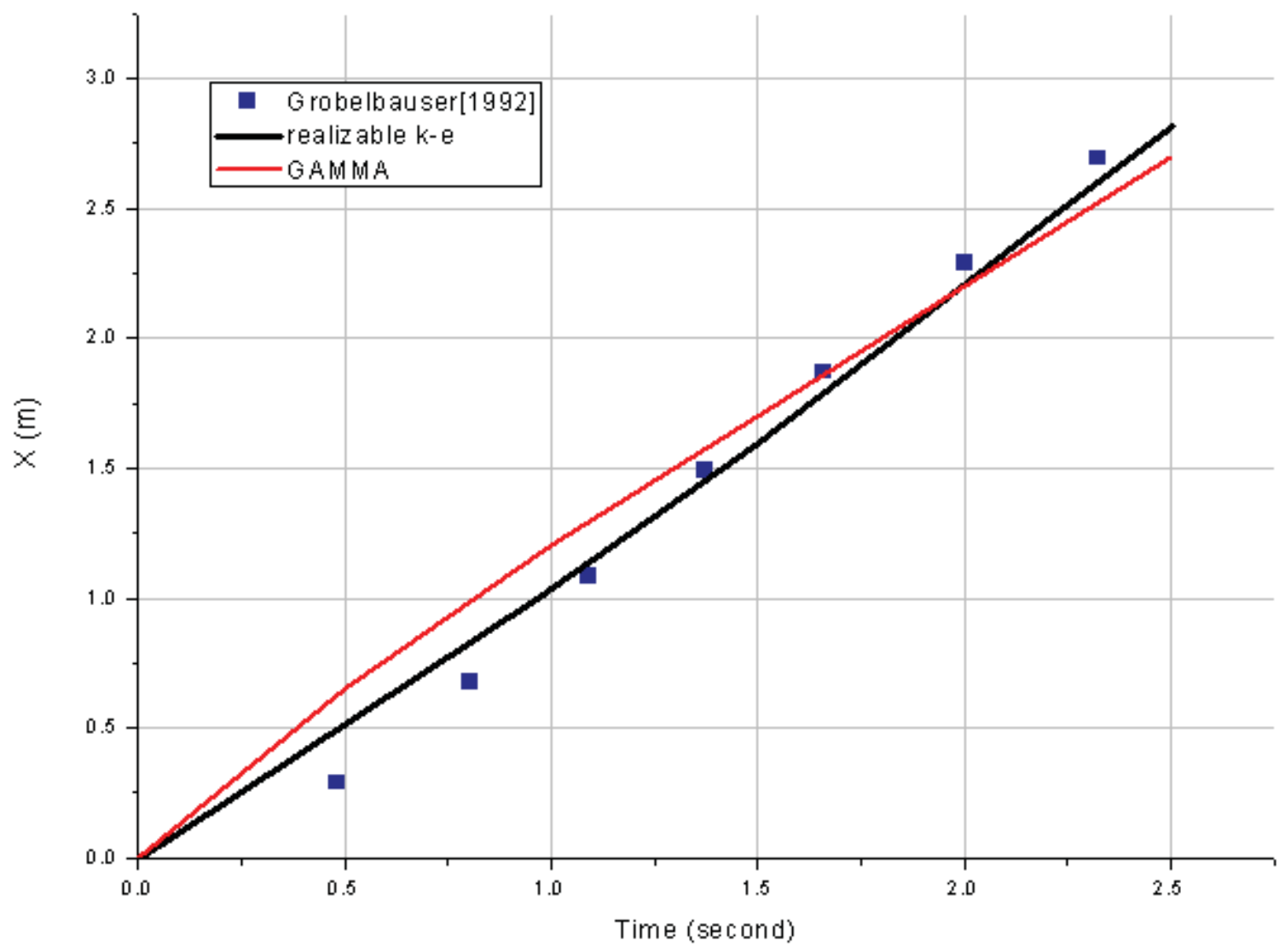

Figure 7-34. Velocity of front location (experimental data, Fluent, GAMMA code).

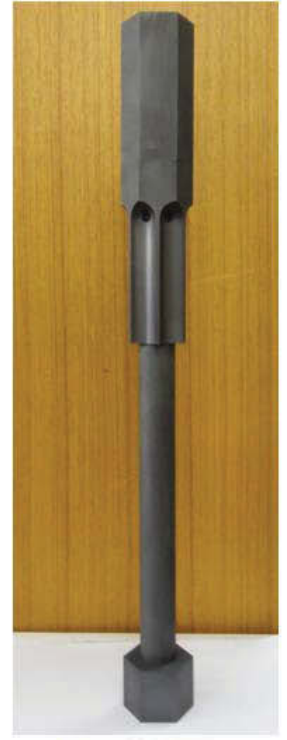

Test sample

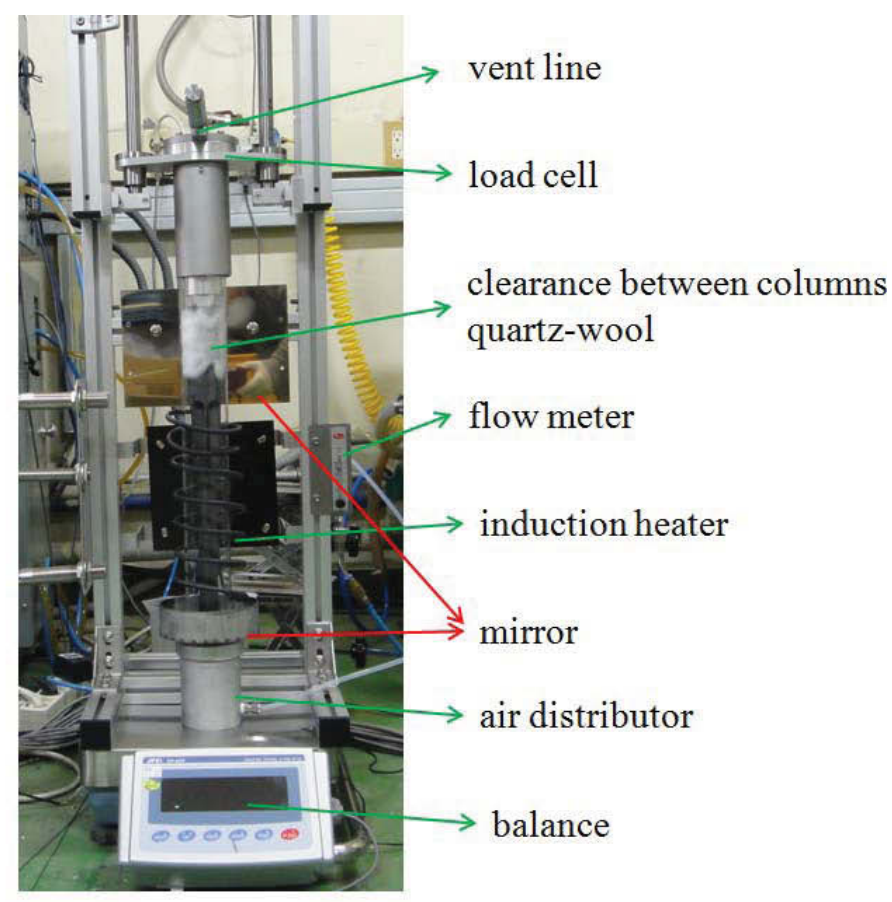

Test facility

Figure 7-35. Test sample and test facility. 
The optical mirror was installed because the induction coil is short. Figure 7-36 and Figure 7-37 show FLUENT calculation results of the temperature difference between the case with the mirror and the case without the mirror. The used radiation model was a DO model. The mirror was polished steel which has 0.1 emissivity. The initial oxidation was carried at 650 and $1050^{\circ} \mathrm{C}$. Heat-up times were 5 and 15 minutes. Filtered dry air was continuously supplied and the flow rate was 20 SLPM. The balance was installed to record the burn-off history, but it did not work properly during the experiment. Burn-off was measured after the test.

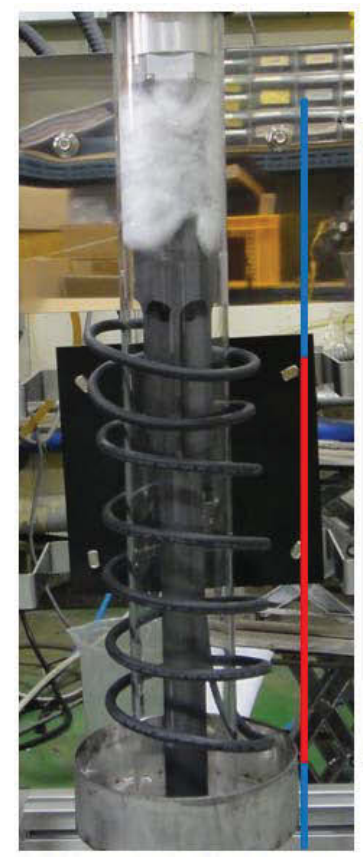

Picture of the test section

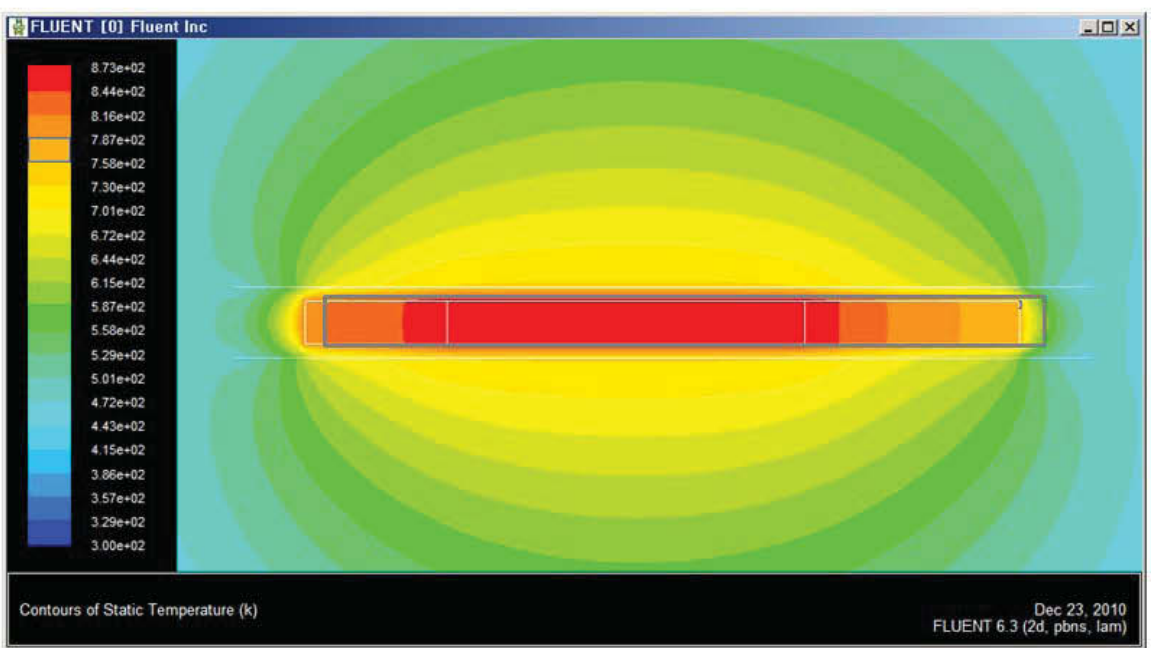

FLUENT calculation result

(DO model, w/o mirror)

Figure 7-36. Test section without the mirror and FLUENT calculation result.

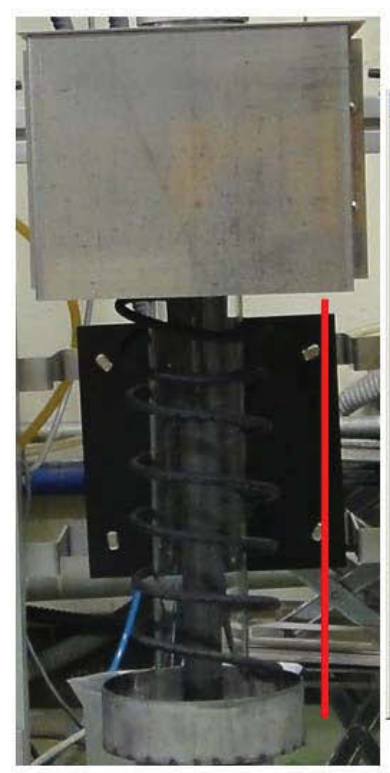

Picture of the test section

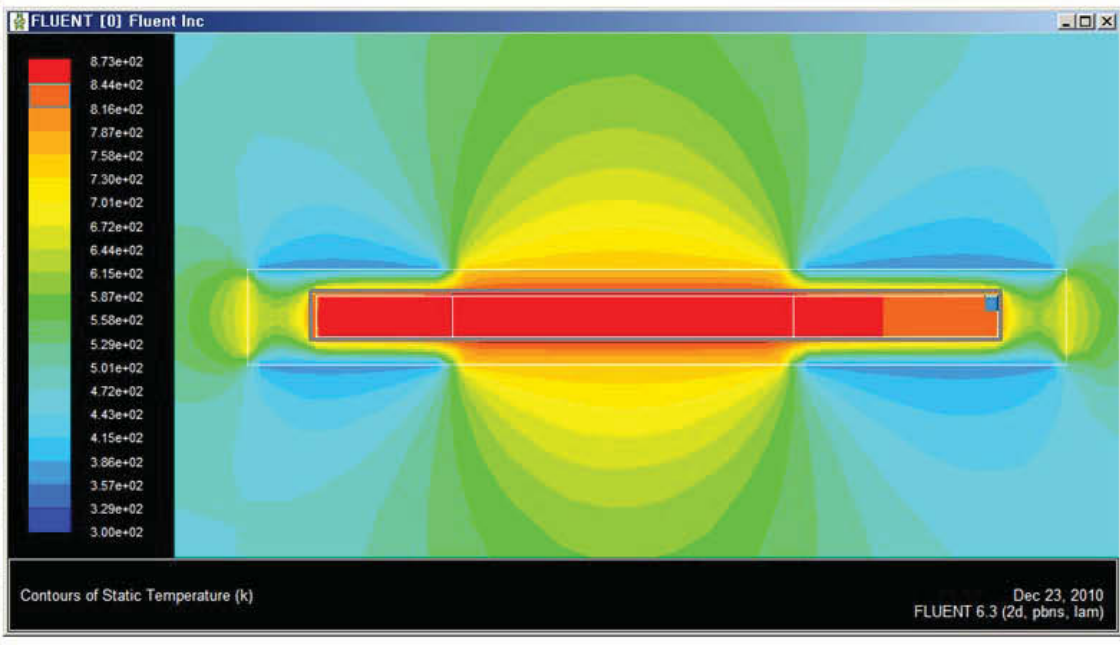

FLUENT calculation result

(DO model, with mirror, emissivity $=0.1$ )

Figure 7-37. Test section with the mirror and FLUENT calculation result. 


\subsubsection{Results}

The graphite surface temperature was initially 650 and $1050^{\circ} \mathrm{C}$, respectively, but it continuously decreased as the graphite lost it weight caused by oxidation. Heating and oxidation stopped at $40.5 \%$ of local burn-off (support column) in the case with the initial temperature of $650^{\circ} \mathrm{C}$. It stopped at $30 \%$ of the local burn-off in the case with the initial temperature of $1050^{\circ} \mathrm{C}$. In both experimental cases, the graphite structure did not fail at that burn-off. Table 7-8 shows the test conditions and local burn-off of each graphite structure. The f-value is defined as the ratio of surface reaction to total reaction.

Table 7-8. Test condition and local burn-off of graphite structure.

\begin{tabular}{|l|c|c|}
\hline \multicolumn{1}{|c|}{ Initial temperature } & $\mathbf{6 5 0}^{\circ} \mathbf{C}$ & $\mathbf{1 0 5 0}^{\circ} \mathbf{C}$ \\
\hline Support block & $0 \%$ & $0 \%$ \\
\hline Chamfer & $11.9 \%$ & $12.0 \%$ \\
\hline Support column & $40.5 \%$ & $30.0 \%$ \\
\hline Seat & $0 \%$ & $0 \%$ \\
\hline f-value & 0.02 & 0.28 \\
\hline
\end{tabular}

IG-11 fractured burn-off was obtained using former researchers' data (Kikuchi et al. 1984 and Eto et al. 1983). Figure 7-38 shows the fractured burn-off and experimental burn-off data and GAMMA calculation. The GAMMA calculation results indicate the burn-off at 500 hours after the air-ingress event.

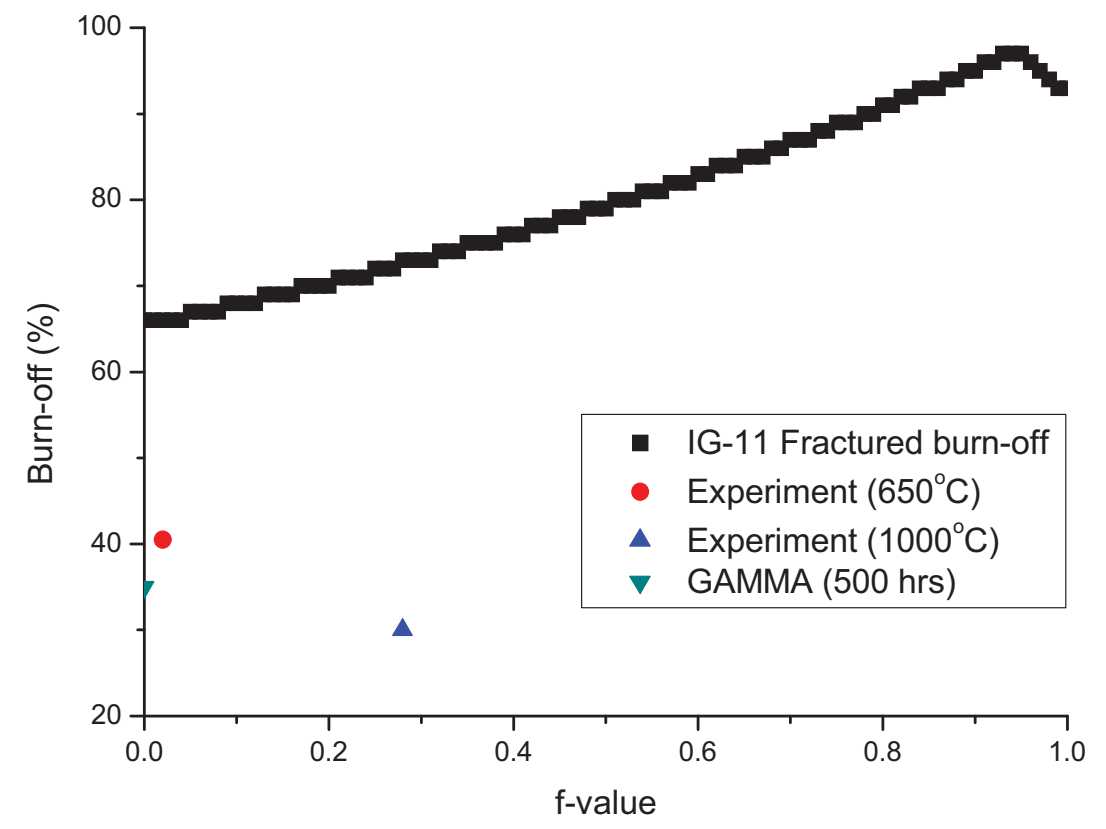

Figure 7-38. Relation between fractured burn-off and f-value

It is confirmed that the bottom structure is safe to at least $40 \%$ of the local burn-off. Figure 7-39 shows a picture of the severely oxidized point in the case with the initial temperature of $650^{\circ} \mathrm{C}$. 


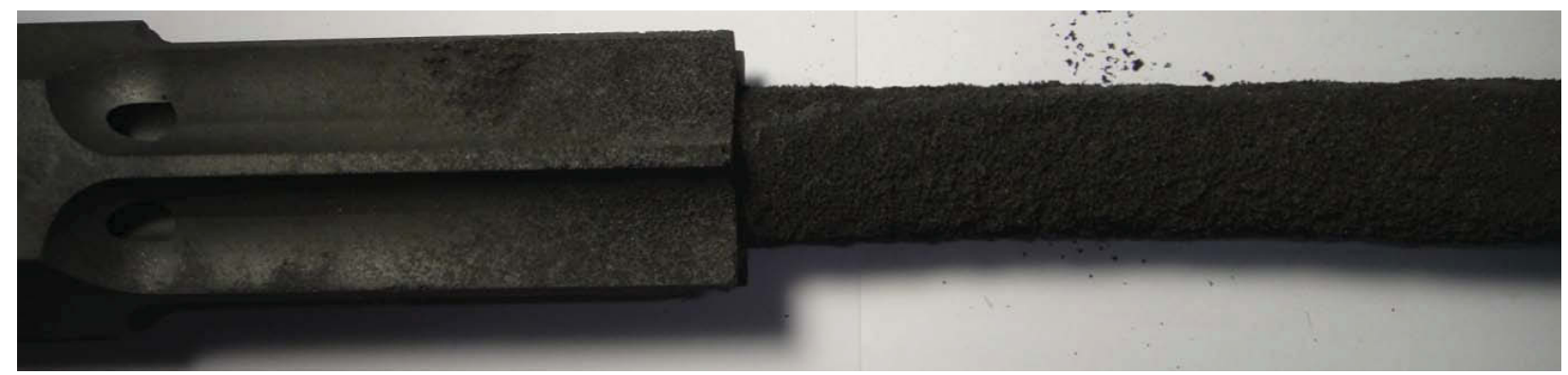

Figure 7-39. Oxidized graphite structure in the case with initial temperature of $650^{\circ} \mathrm{C}$.

Figure 7-40 shows a picture of the oxidized graphite structure, which is severely oxidized in the initial case with the temperature of $1050^{\circ} \mathrm{C}$.

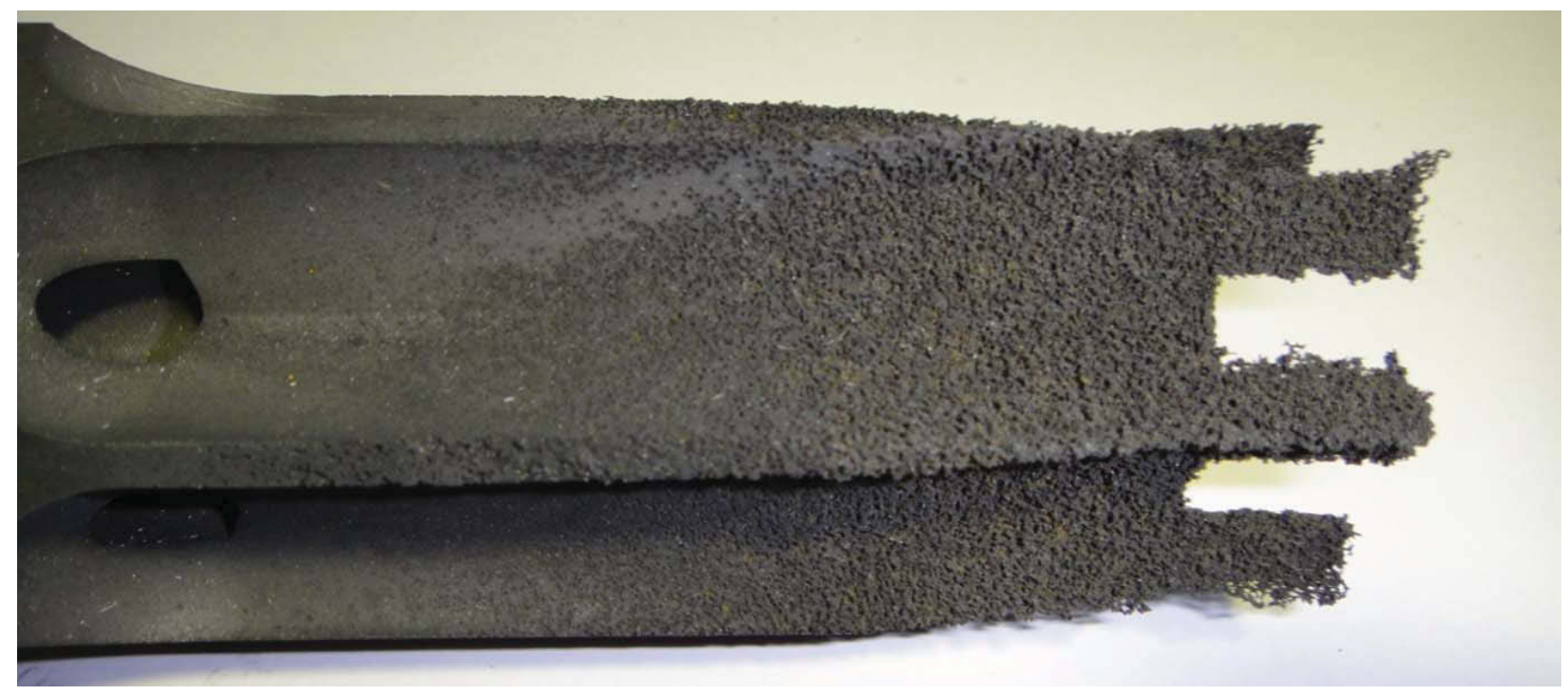

Figure 7-40. Oxidized graphite structure in the case with initial temperature of $1050^{\circ} \mathrm{C}$.

As shown in Figure 7-40, the joint part between the cylindrical column and the chamfered end is relatively thin and is therefore easily oxidized. When a uniaxial load condition was applied to the experiment, the structure was safe. However, the oxidized structure seemed to be weak to multiaxial load.

\subsection{Recommendations}

\subsubsection{Structural strength degradation}

Figure 7-41 shows the fractured burn-off versus the f-value. NBG-10 shows the best material in the view point of strength degradation. The applied assumption is that their density limit criterion is the same.

Density criterion is $\rho<\rho_{\text {limit }}, \rho_{\text {limit }}=0.65 \rho$.

As shown in Figure 7-38, GAMMA calculation, and Figure 7-41, experimental results, the strength margin is quite high. Therefore, other aspects such as kinetics and degradation caused by irradiation should be also considered. 


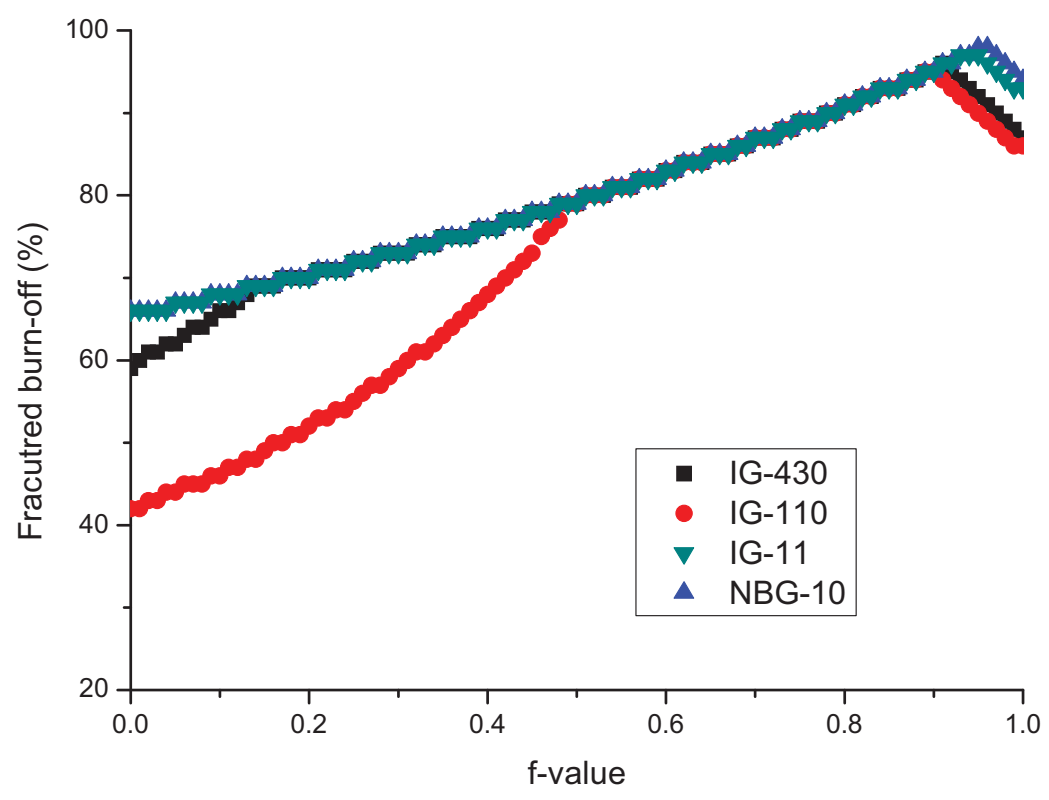

Figure 7-41. Fractured burn-off vs. f-value

\subsubsection{IG-110 vs. IG-430}

The kinetics of IG-11 and NBG-10 are not measured in this study and are not yet clear. IG-110 is historical material used in HTR-10 and the HTTR. JAEA plans to use IG-430 in the GTHTR. IG-110 and IG-430 are compared in this study. On the other hand, the minimum allowed off of IG-430 is $20 \%$ higher than that of IG-110. Therefore, IG-430 is the recommended material for the graphite structure rather than IG-110. 


\section{TASK 7: COUPLING NEUTRONIC-THERMAL HYDRAULIC TOOLS}

This task involved the enhancement of thermal-hydraulic capability of GAMMA code. Thermal power distribution is needed in the reactor core to improve the reliability of thermal-hydraulic analysis. The knowledge of accurate thermal distribution is also necessary to generate an exact cross-section of nuclei. This task investigated neutronics/thermal-hydraulics feedback effects and implemented the feedback parameters into an upgraded GAMMA code. The activities carried out during this task are described in this section.

\subsection{Setup Parameters for Neutronics/Thermal-Hydraulics Code Coupling}

The GAMMA code should transfer following these parameters to the COREDAX code:

- Node[icell].Temp0 : Fluid temperature $\left({ }^{\circ} \mathrm{K}\right)$

- Node[icell].rhoo : Fluid density $\left(\mathrm{kg} \mathrm{m}^{3}\right)$

- W3D[kji].Temp0 : Block temperature ( ${ }^{\circ} \mathrm{K}$ )

- Wblk[QC[m].num] giho : Block mixture density (kgim

These parameters determine coupling data in the GAMMA code and are determined in one data variable ' $\mathrm{QC}$ ' as follows and are then sent to the COREDAX code:

- QC[m].Tfuel : fuel temperature $\left({ }^{\circ} \mathrm{K}\right)$

- $Q C[\mathrm{~m}]$ Dfuel : fuel density $\left(\mathrm{kg} / \mathrm{m}^{3}\right)$

- QC[m].Tmod: Coolant temperature $\left({ }^{\circ} \mathrm{K}\right)$

- QC[m].Dnod : Coolant density $\left(\mathrm{kg} \mathrm{m}^{3}\right)$

- QC[m].Prel : Node relative power

In the COREDAX code, QC data are determined Mapth and Mapn variables for the calculation node. Mapth is for TH calculation node and Mapn is neutronics calculation node variable as follows:

- Mapth[1th].n : nettronics node number in lth TH node.

- Mapth[lth].id[lthn] : nentronics node W in Ith TH node

- Mapth[lth].frac[lthn] : nettronics node fraction.

- Mapn[m].n : TH ndoe number in mentronics node

- Mapn[m].id[imn : TH node ID in m enutronics node.

- Mapn[m].frac[im] : TH node fraction.

In the COREDAX code, subroutine 'exdata' perform as a data transfer function.

\subsection{Calculation Node Mapping Between the COREDAX and GAMMA Code}

Calculation node between the COREDAX and GAMMA code are much different and should be mapped node by node. This calculation node mapping was determined by hand and given by the input file: 


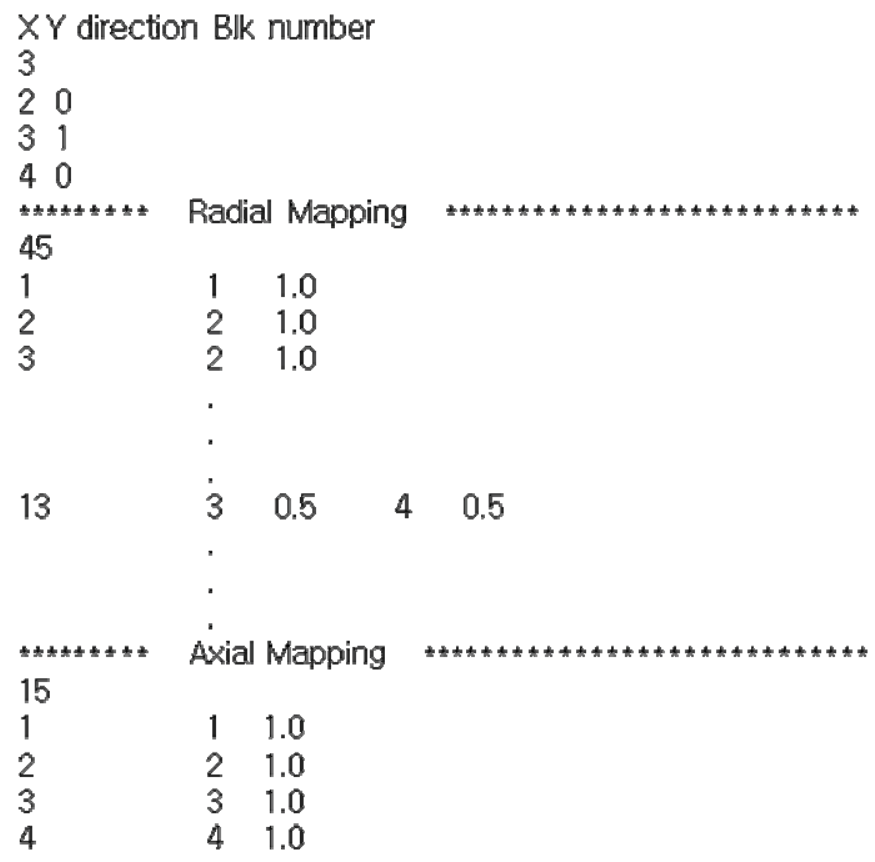

Radial and axial calculation node matching sequence and node-by-node mapping is done by the subroutine 'genmap' in the COREDAX code as shown if Figure 8-1.

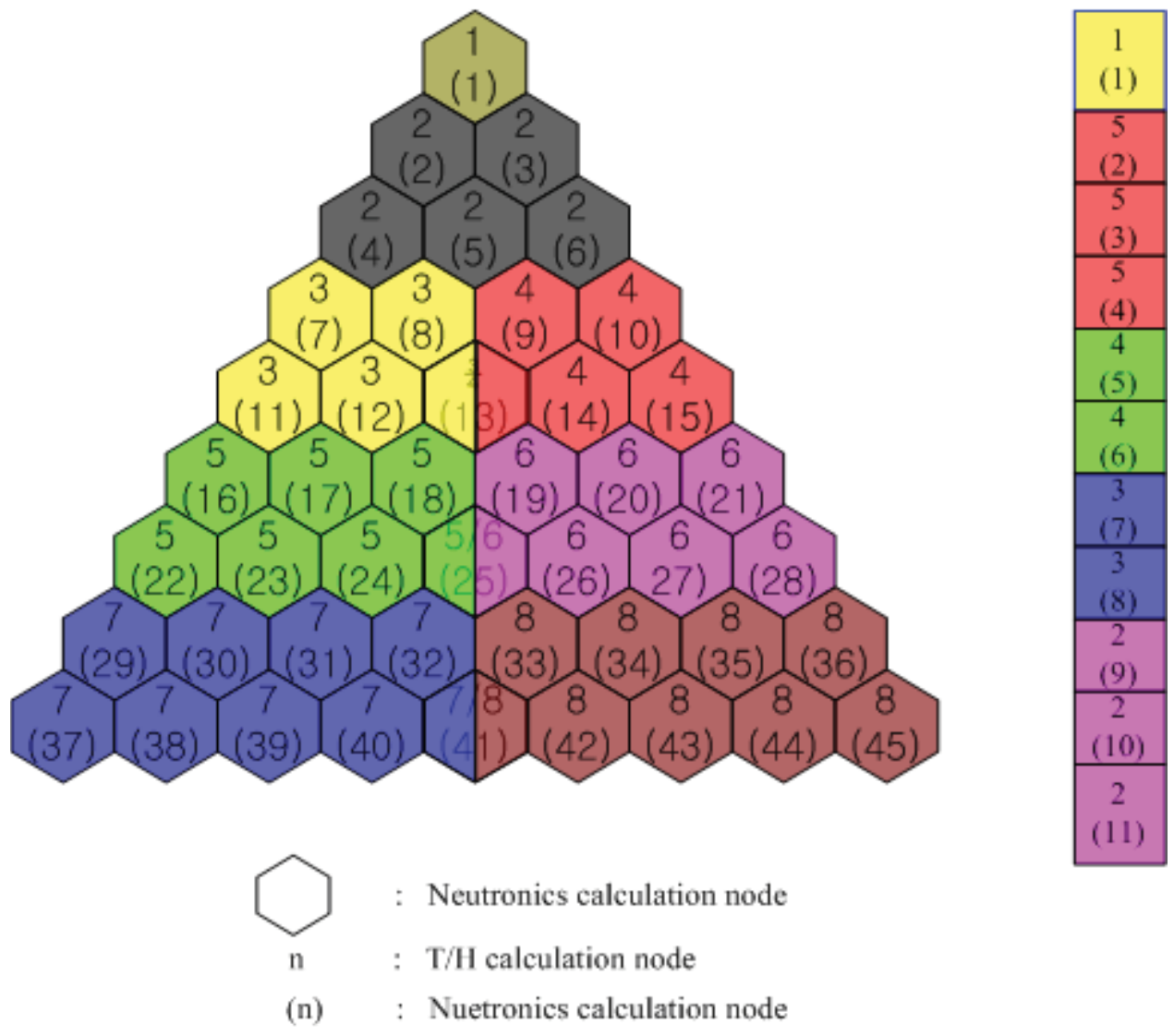

Figure 8-1. Subroutine 'genmap' in the COREDAX code. 


\subsection{Code Coupling of COREDAX with GAMMA}

Before the GAMMA code calculates power distribution by subroutine 'point kinetics,' it is replaced by subroutine 'COREDAX_coupling ' for both steady and transient states as follows:

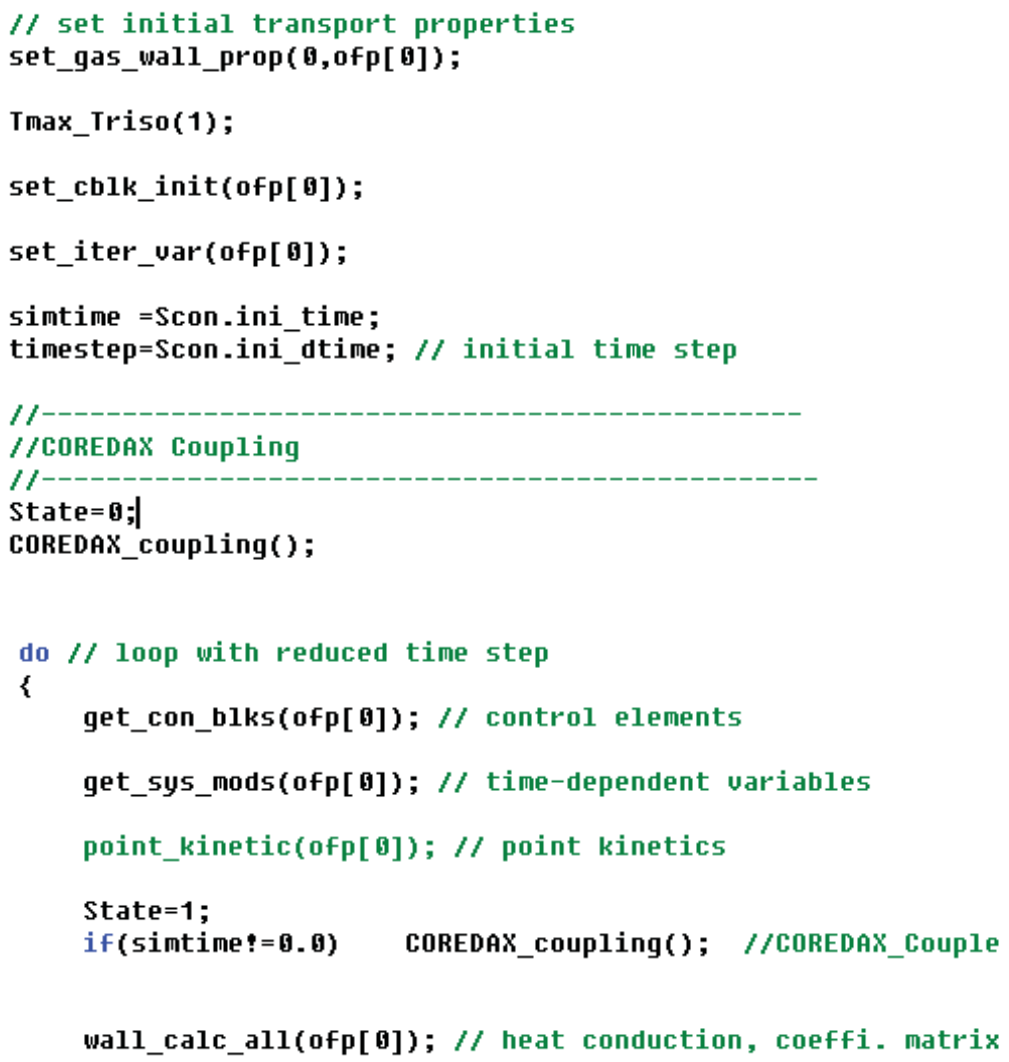

\subsection{Calculation Procedure in a Coupling System}

Generally, steady-state calculation is done first to take a transient calculation. When several coupled variables are transmitted, a proper calculation state to the neutronics code is determined. The neutronics code has four different calculation modes: initialization mode, steady-state mode, transient mode, and output mode. The calculation scheme described in Figure 8-2 shows the neutronics part in an envisioned coupled code system. 


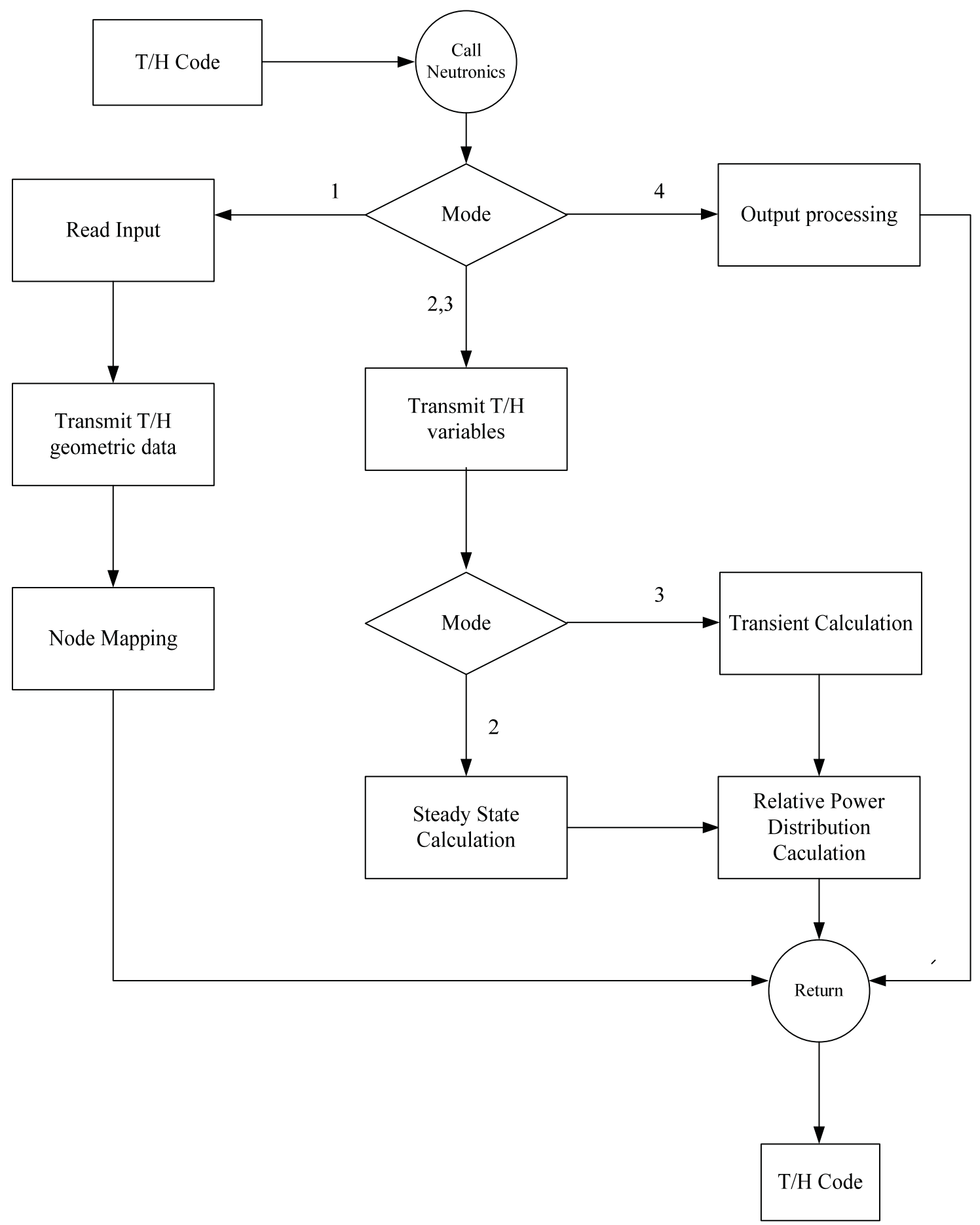

Figure 8-2. Calculation procedure in neutronics code. 


\subsection{The GAMMA/COREDAX Test Calculation}

The GAMMA/COREDAX code coupling was tested with the simplified 600 MWth GTMHR. The simplified GTMHR 600 benchmark problem and its results are described in Figures 8-3 and 8-4, respectively. The calculation results between GAMMA with COREDAX results and GAMMA/COREDAX code are exactly matched.
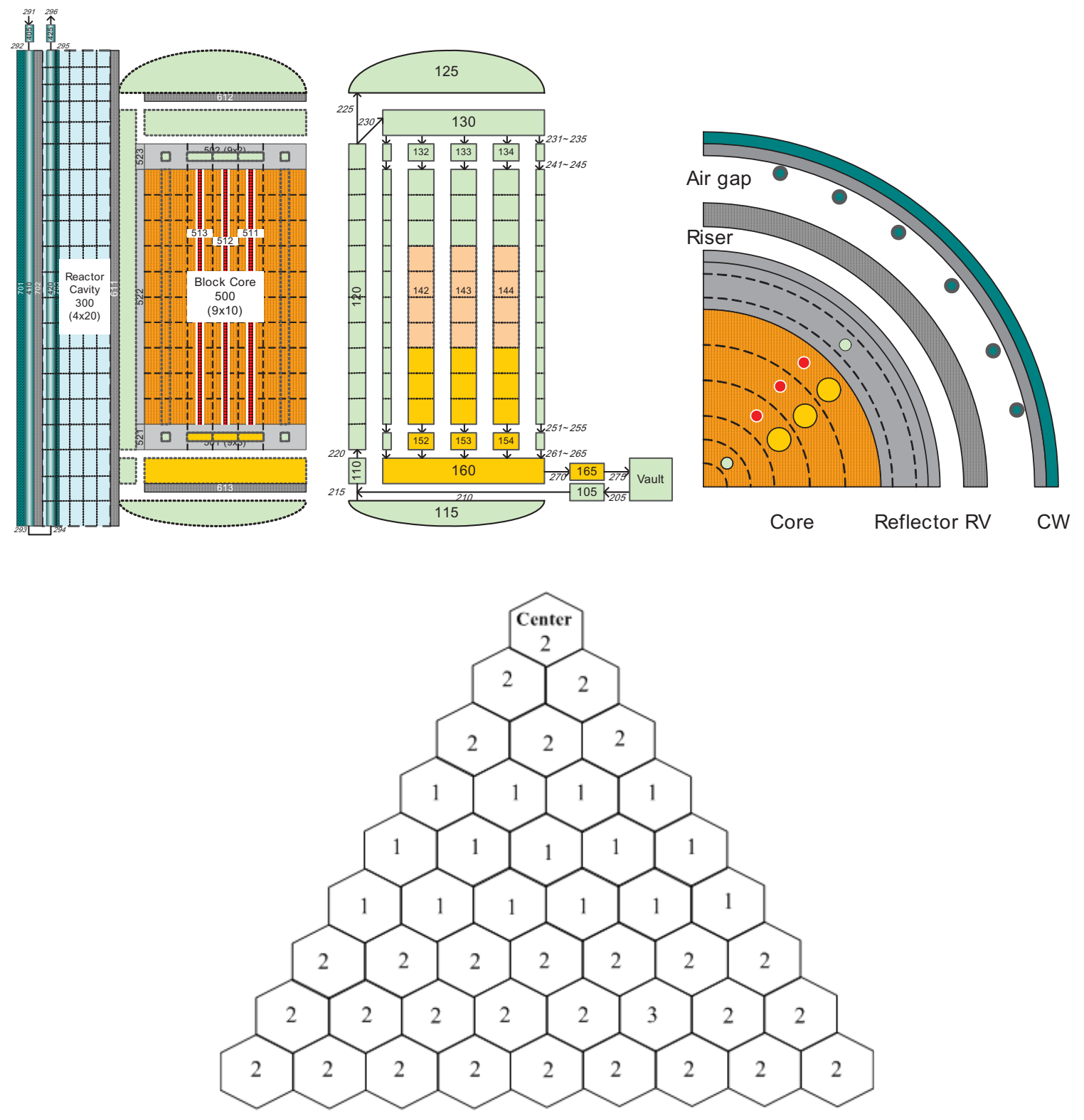

Figure 8-3: Simplified GTMHR 600 benchmark problem in the GAMMA/COREDAX code. 


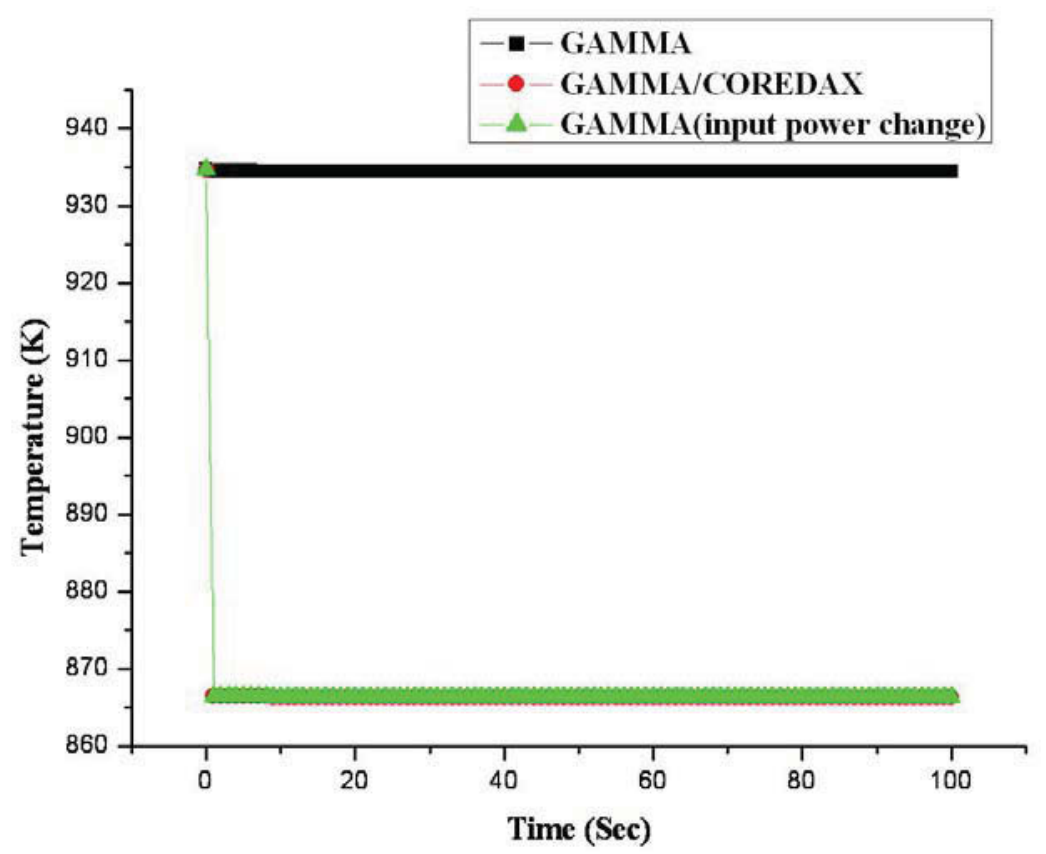

Figure 8-4. Calculation results of GAMMA/COREDAX code.

\subsection{Homogenized Cross-section table}

A homogenized cross-section table was made to analyze the VHTR core. The homogenized crosssection was linearly interpolated from the precalculated TABLESET based on fuel temperature, graphite temperature, and coolant density. Figures 8-5 and 8-6 show the format of the homogenized cross-section TALBESET for coupling calculation.

\begin{tabular}{|c|c|c|c|c|}
\hline $\mathrm{T}_{\mathrm{f} 1}$ & $\mathrm{~T}_{\mathrm{f} 2}$ & $\mathrm{~T}_{\mathrm{f} 3}$ & $\mathrm{~T}_{\mathrm{f} 4}$ & $\mathrm{~T}_{\mathrm{f} 5}$ \\
\hline$\rho_{\mathrm{m} 1}$ & $\rho_{\mathrm{m} 2}$ & $\rho_{\mathrm{m} 3}$ & $\rho_{\mathrm{m} 4}$ & $\rho_{\mathrm{m} 5}$ \\
\hline$\rho_{\mathrm{m} 6}$ & $\Sigma_{1}$ & $\Sigma_{2}$ & $\cdots$ & \\
\hline & & $\cdots$ & $\Sigma_{29}$ & $\Sigma_{30}$ \\
\hline
\end{tabular}

Where:

$-\mathrm{T}_{\mathrm{f}}$ is the Doppler (fuel) temperature $\left({ }^{\circ} \mathrm{K}\right)$

$-\rho_{\mathrm{m}}$ is the moderator density $\left(\mathrm{kg} / \mathrm{m}^{3}\right)$

Macroscopic cross-sections are in units of $\mathrm{cm}^{-1}$

Figure 8-5. Homogenized cross-section form in the COREDAX code. 


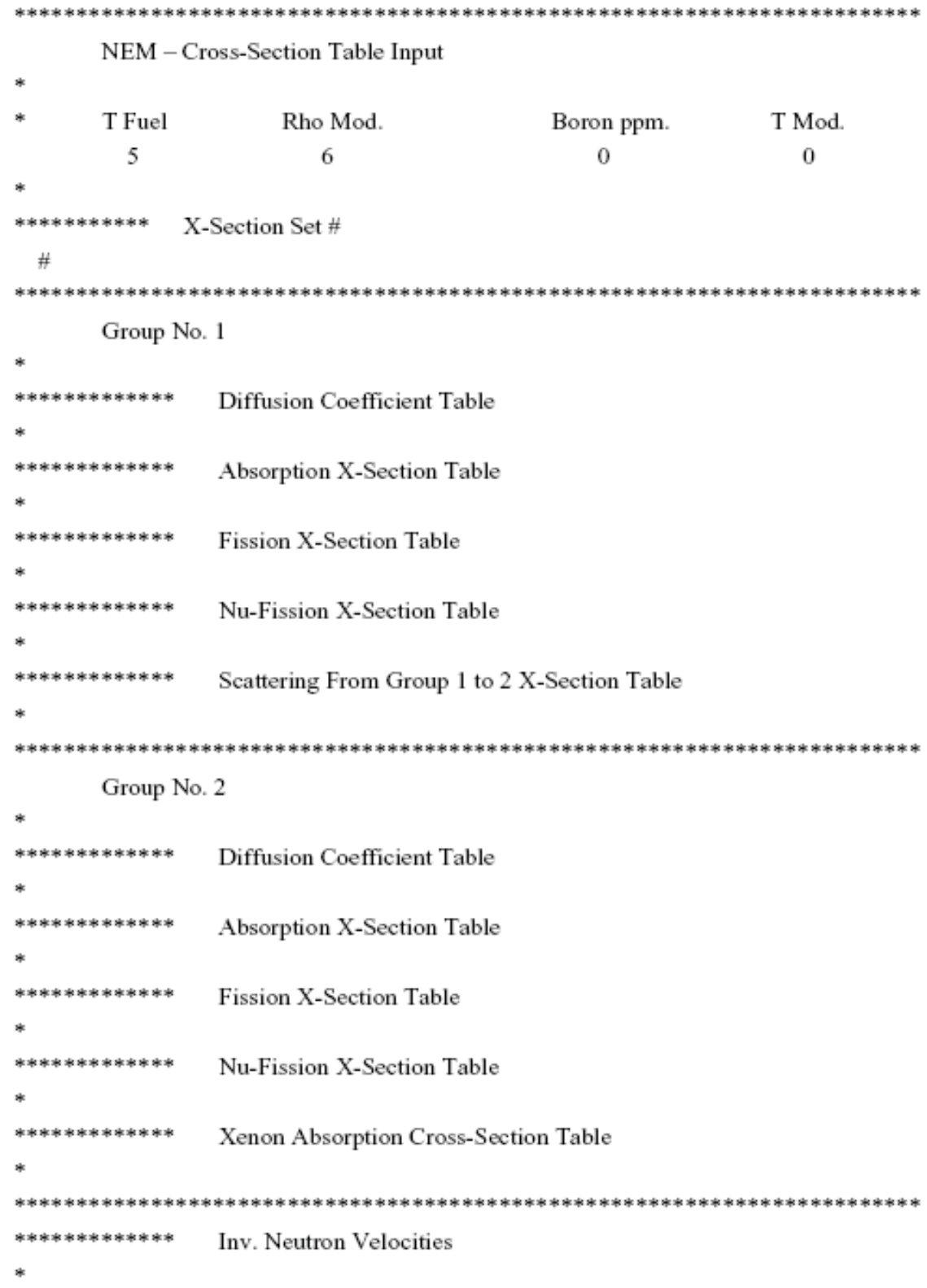

Figure 8-6. Homogenized cross-section sample in the COREDAX code. 


\section{TASK 8: CORE NEUTRONIC MODEL}

The AFEN methodology in hexagonal 3-D geometry is developed in both steady-state and transient conditions.

The AFEN method is described from the following 3-D hexagonal-z geometry multigroup diffusion equations:

$$
-[D] \nabla^{2} \vec{\phi}+[A] \vec{\phi}=\frac{\vec{\chi}}{k_{\text {eff }}} \vec{F}^{T} \vec{\phi}
$$

where:

$[D]=$ diffusion coefficient matrix

$[\mathrm{A}]=$ removal and scattering cross section matrix

$\vec{F}=$ fission production cross section vector

$\vec{\chi}=$ fission spectrum vector

$k_{\text {eff }}=$ multiplication factor.

The solution of the diffusion equation for nod $\mathrm{n}$ is expressed as

$$
\begin{aligned}
\vec{\phi}^{n}(x, y, z) & =\vec{\varphi}_{1}^{n}(x, y, z)+\vec{\varphi}_{2}^{n}(x, y, z)+\vec{\varphi}_{3}^{n}(x, y, z) \\
& +\vec{\varphi}_{4}^{n}(x, y, z)
\end{aligned}
$$

where

$$
\begin{aligned}
\vec{\varphi}_{l}^{n}(x, y, z)= & \sinh \left(\sqrt{\Lambda^{n}} x_{l}\right) \vec{a}_{1 l}^{n}+\cosh \left(\sqrt{\Lambda^{n}} x_{l}\right) \vec{a}_{2 l}^{n} \\
& +y_{l} \sinh \left(\sqrt{\Lambda^{n}} x_{l}\right) \vec{b}_{1 l}^{n}+y_{l} \cosh \left(\sqrt{\Lambda^{n}} x_{l}\right) \vec{b}_{2 l}^{n} \\
& +z \sinh \left(\sqrt{\Lambda^{n}} x_{l}\right) \vec{c}_{1 l}^{n}+z \cosh \left(\sqrt{\Lambda^{n}} x_{l}\right) \vec{c}_{2 l}^{n}, \quad l=1,2,3 \\
\vec{\varphi}_{4}^{n}(x, y, z)= & \sinh \left(\sqrt{\Lambda^{n}} z\right) \vec{d}_{10}^{n}+\cosh \left(\sqrt{\Lambda^{n}} z\right) \vec{d}_{20}^{n}
\end{aligned}
$$

and

$\left[\Lambda^{n}\right]=\left[D^{n}\right]^{-1}\left([A]-\frac{\vec{\chi}}{k_{\text {eff }}} \vec{F}^{T}\right)^{n}$

The coordinates $x_{1}, x_{2}$, and $x_{3}$ are defined in Figure 9-1. Note that each of the 20 terms in Eq. (9-2) is an analytic solution of Eq. (9-1). Note in particular that Eq. (9-2) includes terms of transverse gradient basis functions. Evaluation of the matrix functions is facilitated by spectral decomposition in functional theory of linear operators. 


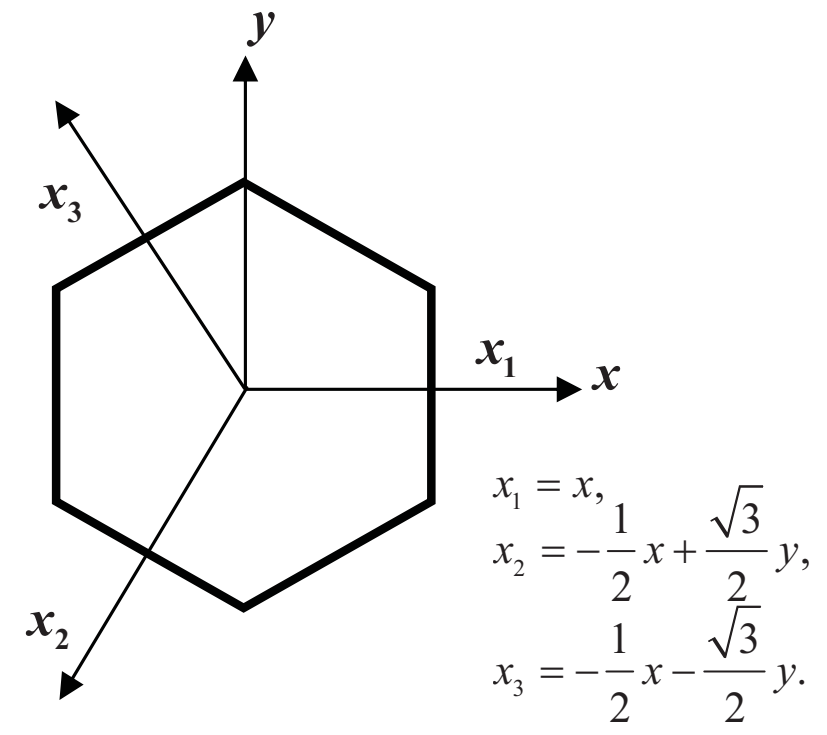

Figure 9-1. Coordinates in a hexagon.

The coefficients in the Eq. (9-2) flux expansion are expressed in terms of nodal unknowns, such as the node average flux, the node-interface fluxes, and the node-interface flux moments. In particular, the 12 node-interface flux moments (y-and z-weighted average fluxes by six radial interfaces) are included as nodal unknowns in this research. All the nodal unknowns are defined as follows:

- $\quad$ The COREDAX code is developed based on the AFEN method.

- The COREDAX code is verified by several benchmark problems:

- VVER-440 benchmark problem.

The VVER-440 benchmark problem is a hexagonal-z 3-D two-group VVER-400 core. It has onetwelfth reflective symmetry geometry and the outer boundary conditions are vacuum. Table 9-1 shows the results, compared with those of the PARCS code, the COREDAX code, and the reference solution. Note that the COREDAX code provides very accurate nodal solutions.

Table 9-1. Results of VVER-440 benchmark problem. ${ }^{a}$

\begin{tabular}{|l|l|l|}
\hline \multirow{2}{*}{$\begin{array}{c}\text { No. of } \\
\text { planes }\end{array}$} & \multicolumn{1}{|c|}{$\begin{array}{c}\text { PARCS }\left(\boldsymbol{k}_{\text {eff }} \% \text { error) }\right. \\
\text { (Node power maximum \% } \\
\text { error })\end{array}$} & \multicolumn{1}{|c|}{$\begin{array}{c}\text { COREDAX }\left(\boldsymbol{k}_{\text {eff }} \% \text { error }\right) \\
\text { (Node power maximum \% } \\
\text { error })\end{array}$} \\
\hline \multirow{2}{*}{12} & $1.010297(-0.101)$ & $1.01126696(-0.0052)$ \\
\cline { 2 - 3 } & $(21.71)$ & $(1.28)$ \\
\hline 60 & $1.010918(-0.040)$ & $1.01125753(-0.0062)$ \\
\hline
\end{tabular}

a. Reference solution $k_{\text {eff }}=1.01132$ from DIF3D-FD runs extrapolation

- A “simplified" VVER-1000 benchmark problem.

The data of the original VVER-1000 benchmark problem (every fuel assembly has differing cross sections) is too big to fit into the VENTURE code for the reference solution. Therefore, a simplified core data structure was constructed maintaining the same geometry. We just changed the material data numbers from 283 to seven such that the core contains five radially different 
nodes and 12 axial planes with two reflector and 10 fuel planes. There was no change in axial node components. The core has one-sixth reflective symmetry and the outer boundary conditions are zero flux. The side length of each assembly is $13.6 \mathrm{~cm}$, the core temperature is $552.15^{\circ} \mathrm{K}$ and the coolant density is $767.1 \mathrm{~kg} / \mathrm{m}^{3}$. Table $9-2$ shows the results on $k_{\text {eff }}$ compared with the

VENTURE reference. The reference was obtained by the VENTURE code using 384 triangles $\times$ 20 axial meshes per hexagonal prism node. Two sets of results are compared; one using flux zero boundary condition and the other using incoming current zero boundary condition. Both results show that COREDAX gives very accurate solutions. This is because the axial calculational modeling (via interface flux moments) uses an enlarged set of analytic functions to provide more accurate axial dependence.

Table 9-2. Results on $k_{\text {eff }}$ of simplified VVER-1000 benchmark problem.

\begin{tabular}{|c|c|c|}
\hline Boundary conditions & $\begin{array}{l}\text { VENTURE (ref.) } \\
(384 \times 20 \text { per node) }\end{array}$ & COREDAX (\% error) \\
\hline Flux zero B.C. & 1.0000503 & $1.0000055(-0.0045)$ \\
\hline Incoming current zero B.C. & 1.0000534 & $1.0000476(-0.0006)$ \\
\hline
\end{tabular}

- SNR-400 benchmark problem.

Finally, SNR-300 was solved to test the multigroup extension in the COREDAX code. SNR-300 is a four-group problem modeling a small liquid-metal fast breeder reactor core. ${ }^{1}$ The core has one-sixth reflective symmetry and the outer boundary condition is vacuum. The results are shown in Table 9-3. The reference solution was obtained with DIF3D-FD.

Table 9-3. Results of SNR-300 benchmark problem.

\begin{tabular}{|l|l|l|}
\hline \multicolumn{1}{|c|}{ Code } & \multicolumn{1}{c|}{$\begin{array}{c}\text { Keff } \\
\text { Number of axial nodes }\end{array}$} & \multicolumn{1}{c|}{ (\% error) } \\
\hline Reference (DIF3D-FD) & Richardson extrapolation & 1.00989 \\
\hline DIF3D Nodal & 8 & $1.01151(0.160)$ \\
\cline { 2 - 3 } $\begin{array}{l}\text { COREDAX } \\
\text { (Transformation matrix) }\end{array}$ & 18 & $1.01125(0.135)$ \\
\hline COREDAX & 16 & $1.01376(0.383)$ \\
(Matrix function theory) & 8 & $1.01134(0.185)$ \\
\hline
\end{tabular}

- VVER-1000 benchmark problem.

The COREDAX code was verified by comparing the results of the COREDAX code with the other participants in the VVER-1000 benchmark problem. The core configuration of the 3-D VVER-1000 benchmark problem is described in Figure 9-2. 

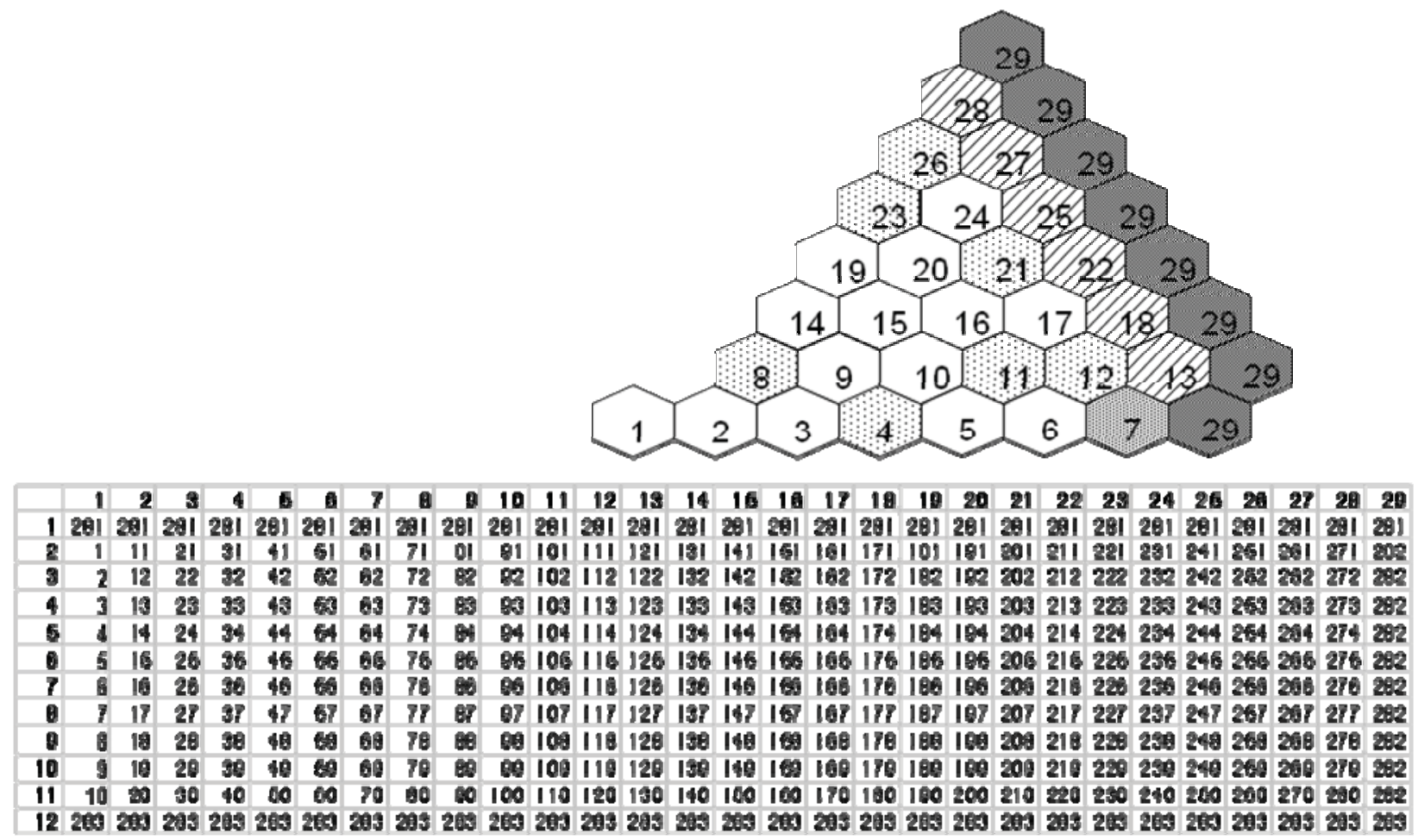

Figure 9-2. Core configuration of 3-D VVER-100 benchmark problem.

The steady-state results are shown in Figure 9-3 and Table 9-4. The Hot Zero Power condition results show that the COREDAX code results are well matched with other participants of the benchmark problem and the relative power deviation from the participants' results is the smallest of the participants.

HZP Keff

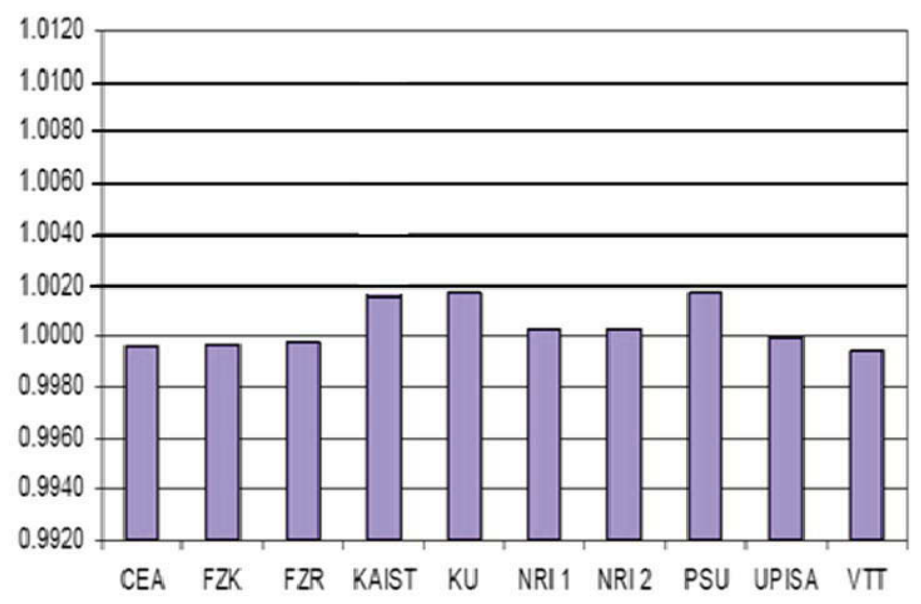

Figure 9-3. Results of VVER-100 benchmark problem. 
Table 9-4. Relative power deviation from mean (\%).

\begin{tabular}{|c|c|c|}
\hline Part.: & Max & Min \\
\hline CEA & 1.16 & -1.0 \\
\hline FZK & 1.07 & -0.82 \\
\hline FZR & 0.62 & -0.71 \\
\hline NRI1 & 1.70 & -2.17 \\
\hline NRI2 & 1.70 & -2.17 \\
\hline IPISA & 0.43 & -0.86 \\
\hline VTT & 2.20 & -1.65 \\
\hline KAIST & 0.39 & -0.27 \\
\hline
\end{tabular}

To test transient calculation ability of the COREDAX code, we suggest a transient scenario with control rod. The control rod X is withdrawn with $8 \mathrm{~cm} / \mathrm{sec}$ at 0 second after 20 second control rod VI is inserted with $8 \mathrm{~cm} / \mathrm{sec}$ for $50 \mathrm{sec}$. The control rods are described in Figure 9-4. The power change in time is depicted in Figure 9-5.

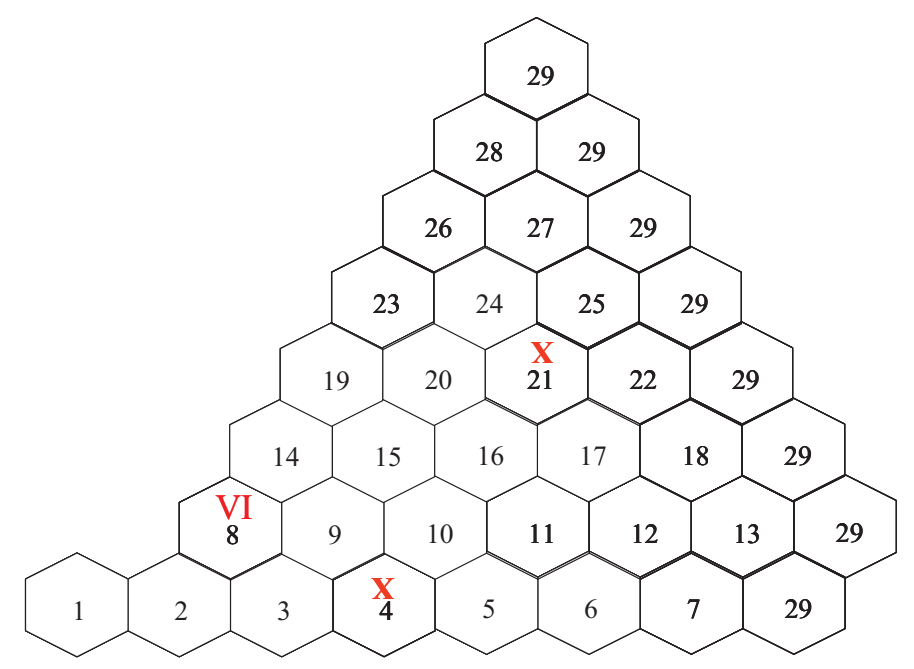

Figure 9-4. Control rod position in VVER-1000. 


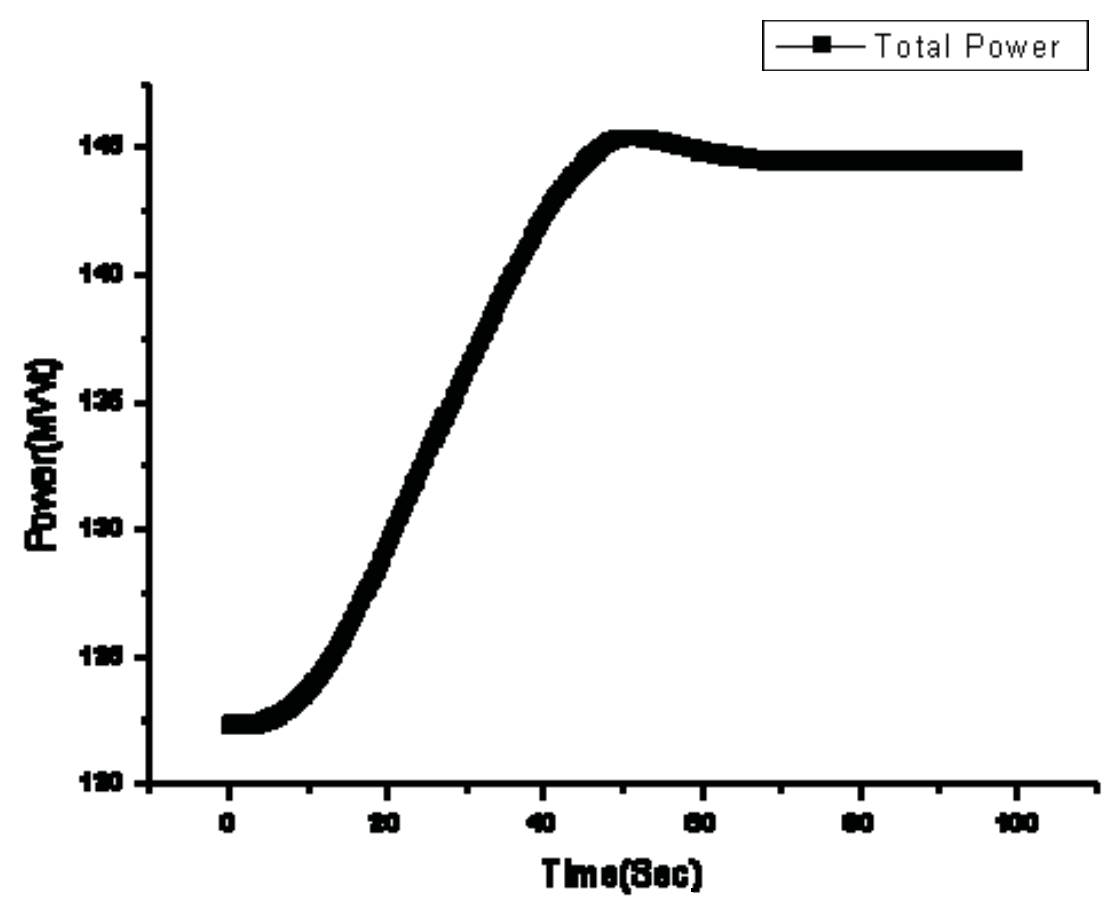

Figure 9-5.Total power change in time.

- GTMHR-600 benchmark problem

A neutronics calculation of a simple GTMHR core model was performed in preparation for $\mathrm{V} \& \mathrm{~V}$ of the coupled code .

The GTMHR is one of the most significant HTGR gas turbine plant designs currently under investigation. The GTMHR is being developed on an international basis to optimize the HTGR capabilities and resources of many countries.

The GTMHR reactor core (Figure 9-6) represents an annular stack of hexahedral prismatic fuel assemblies with $36 \mathrm{~cm}$ across flats size as shown in Figures 9-7 and 9-8, which form 102 columns $800 \mathrm{~cm}$ high consisting of 10 fuel assemblies (FAs) stacked axially in each of these columns. The FA columns are arranged with a $0.25 \mathrm{~cm}$ gap to ensure performance of FA reloads during the reactor core life. The active core is enclosed by graphite reflector as shown in Figure 9-6. The core is surrounded by radial reflectors (RR), and the core internal surface is contiguous with the internal reflector (IR). The upper axial reflector (UAR) and lower axial reflector (LAR) are located above and beneath the core, respectively. The UAR is $130 \mathrm{~cm}$ high consisting of stacks of columns assembled from hexahedral prismatic graphite blocks (GBs). These are located above the core and are separated by a gap of $0.25 \mathrm{~cm}$.

As the GTMHR benchmark problem gives nuclear data as nuclide density, homogenized cross sections had to be generated for nodal calculation. Generating homogenized cross sections is an involved and distinct task that is outside the scope of this project. In this research, simple 10group homogenized cross sections were used for GTMHR FAs, which were obtained informally from KAERI (and that are incomplete, e.g., cross sections for rodded blocks are not available).

The FA radial configuration is described in Figure 9-9, in which FA 1 to 3 are fuels blocks and 4 and 5 are graphite blocks. Test calculation results of the COREDAX code are shown in Table 9-5. 


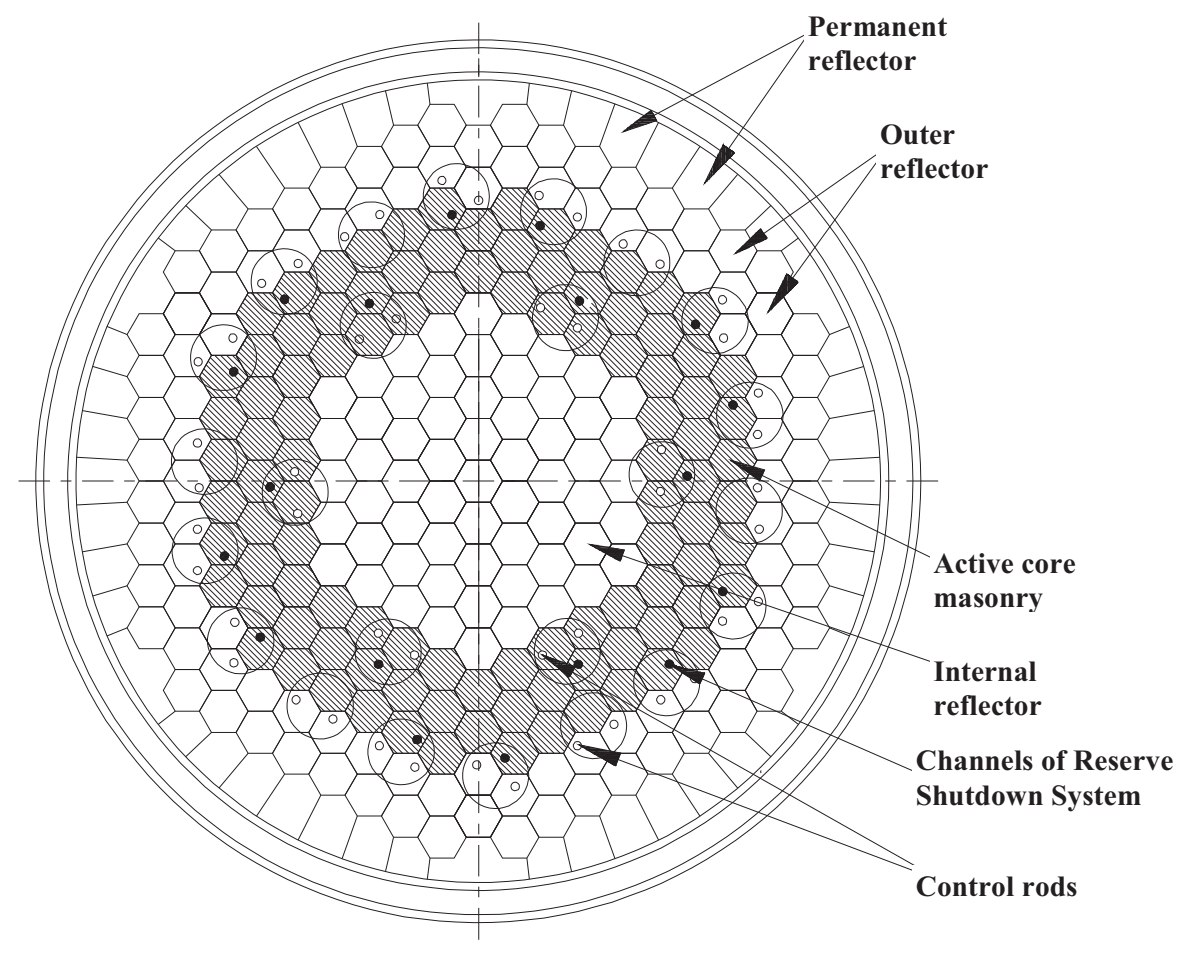

Figure 9-6. Arrangement of active core and its components.

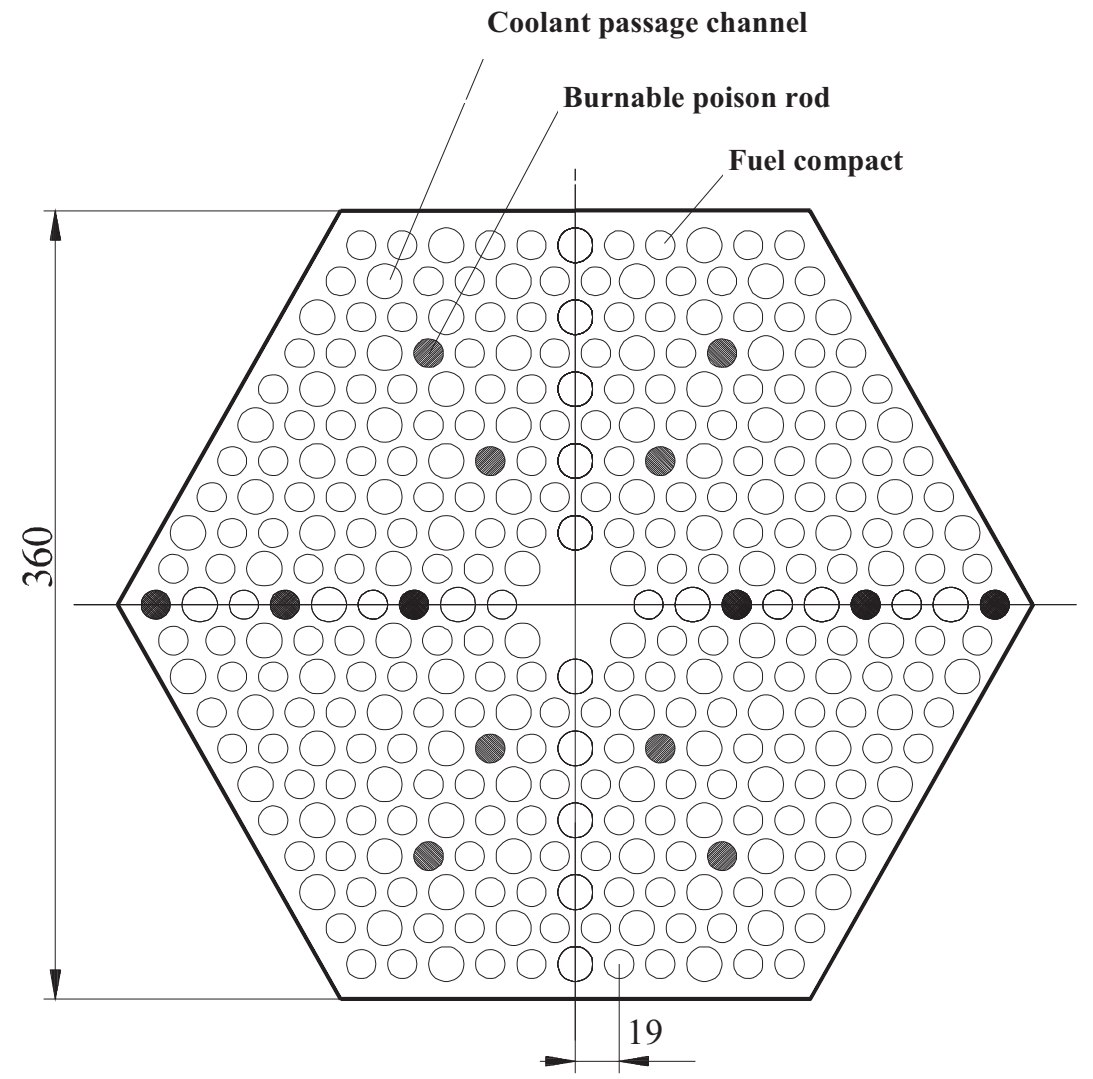

Figure 9-7. Fuel block cell, Type 1FA. 


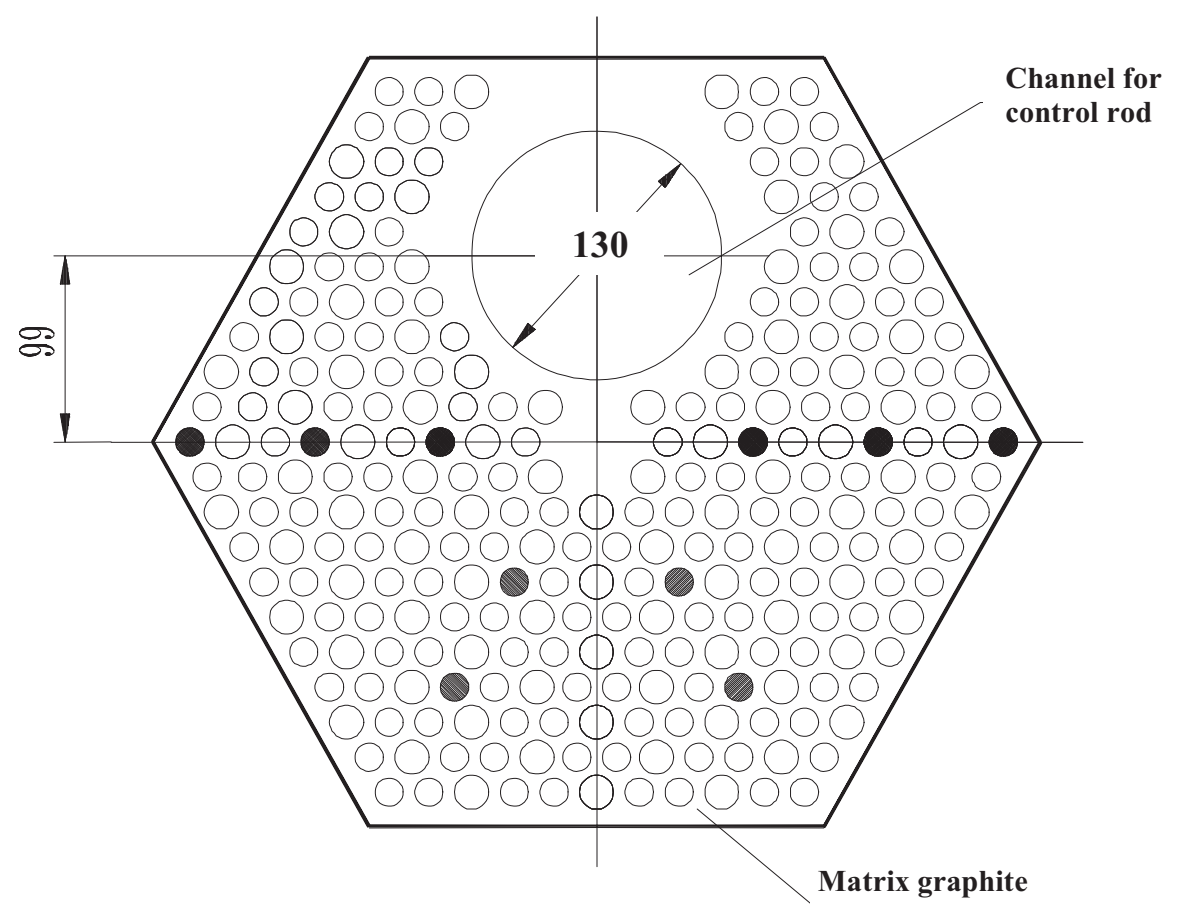

Figure 9-8. Fuel block with cavity for control rod or reserve shutdown system, Type 2FA.

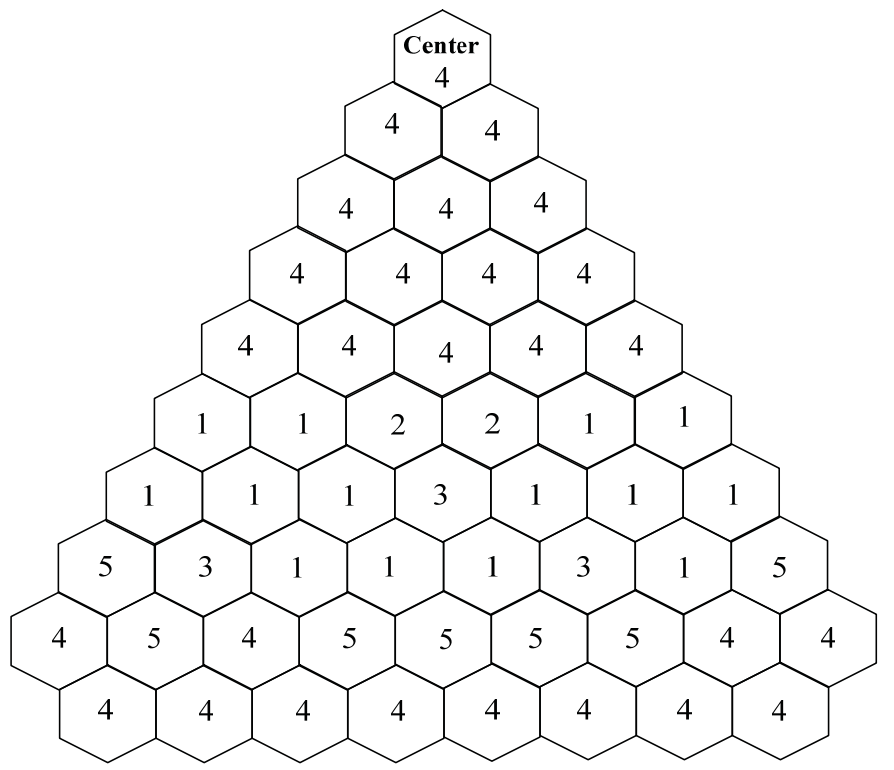

Figure 9-9. Radial core configuration of simplified 3-D GTMHR.

Table 9-5. Results on $\boldsymbol{k}_{\text {eff }}$ of simplified GTMHR benchmark problem.

\begin{tabular}{|l|c|c|}
\hline $\begin{array}{c}\text { Boundary } \\
\text { conditions }\end{array}$ & $\begin{array}{c}\text { VENTURE (ref.) } \\
\text { (per node) }\end{array}$ & COREDAX (\% error) \\
\hline All Rods Out & & 2.90801 \\
\hline Fuel Region Rods In & & 1.47786 \\
\hline All Rods In & & 1.44863 \\
\hline
\end{tabular}




\section{TASK 9: COUPLED CORE MODEL V\&V}

\subsection{V\&V of COREDAX (Steady State/Transient Calculation)}

For V\&V of the COREDAX code, the VVER-1000 benchmark problem has been tested and the result is compared with those of other core analysis codes. Core configuration of VVER-1000 is shown in Figure 10-1. This benchmark problem consists of hexagonal assemblies and has a full 3-D configuration.
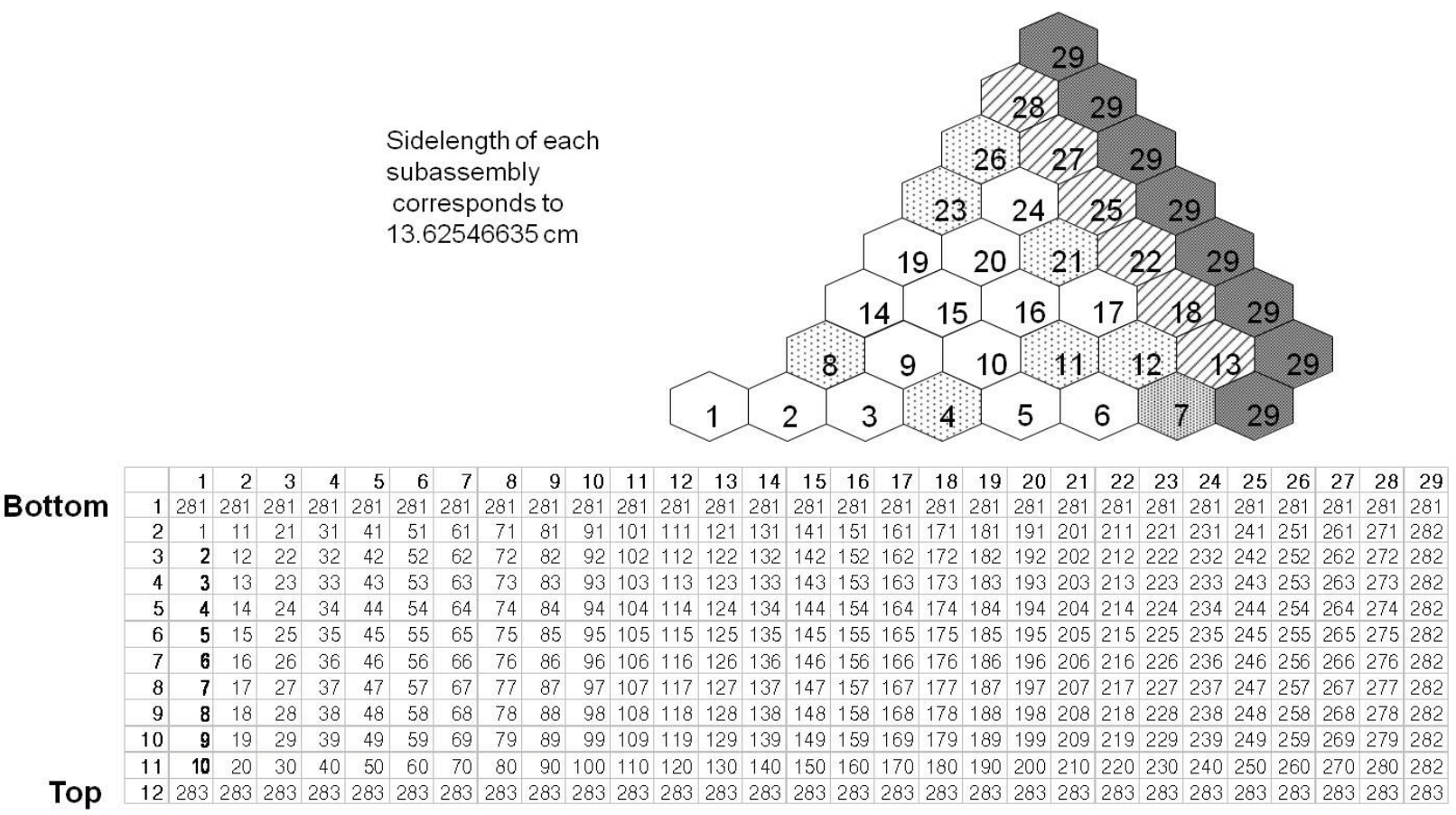

Figure 10-1. VVER-1000 core configuration.

First, the steady-state is tested and the test result (k-eff) of the COREDAX code is compared with those of other core analysis codes in Figure 10-2.

\section{HP-SS Keff}

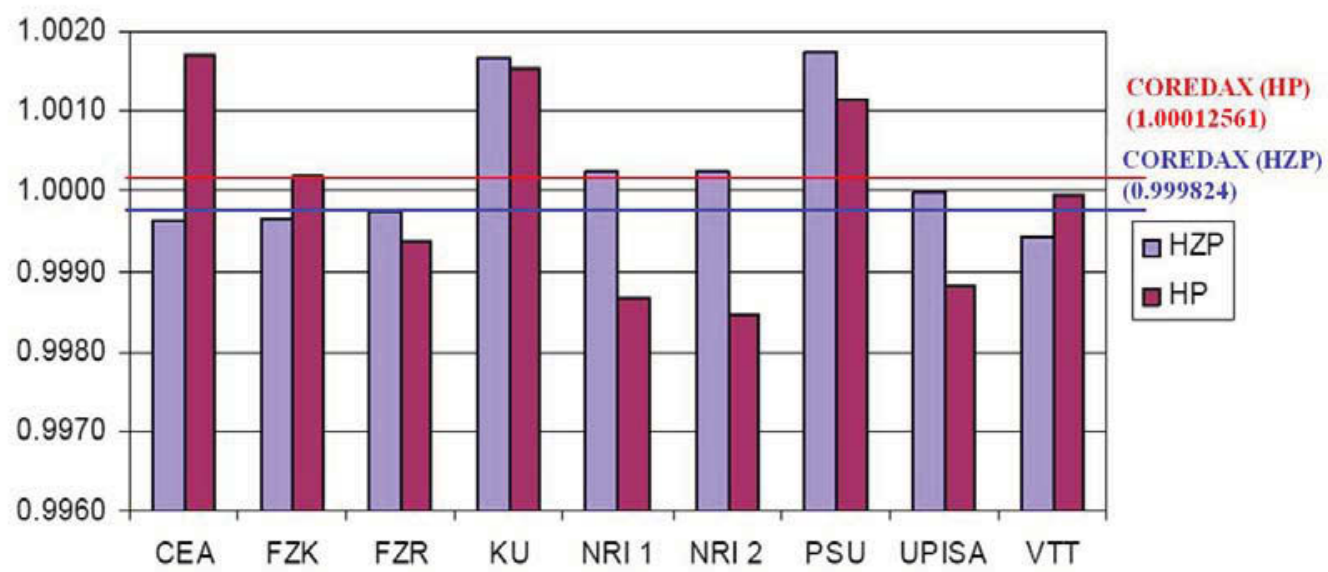

Figure 10-2. VVER-1000 benchmark test results (k-eff). 
Conditions for the transient test are:

- At reactor power $27.46 \% \mathrm{~N}_{\text {nom }}$ (HP Steady state), MCP\#3 is switched on.

- The reactor power gradually increases to $29.8 \% \mathrm{~N}_{\text {nom }}$.

- The pressurizer water level decreases from 744 to $728 \mathrm{~cm}$.

- The water level in the Steam Generator \#3 decreases by $9 \mathrm{~cm}$.

- EHTC is supporting the pressure in MSH at level 6.0 to 0.05 MPA when the TG power is 1645.0 MW.

- The flow rate in loop \#3 reverses back to normal at the $13^{\text {th }}$ second of the switching on of MCP\#3; the timing is consistent with reactivity increase observed through the reactor power set points.

- Mass flow rates of each node are given as B.C condition.

- T_in is almost same with initial condition.

- Pressure in reactor is assumed as constant.

The transient case results are shown in Figures 10-3 and 10-4.
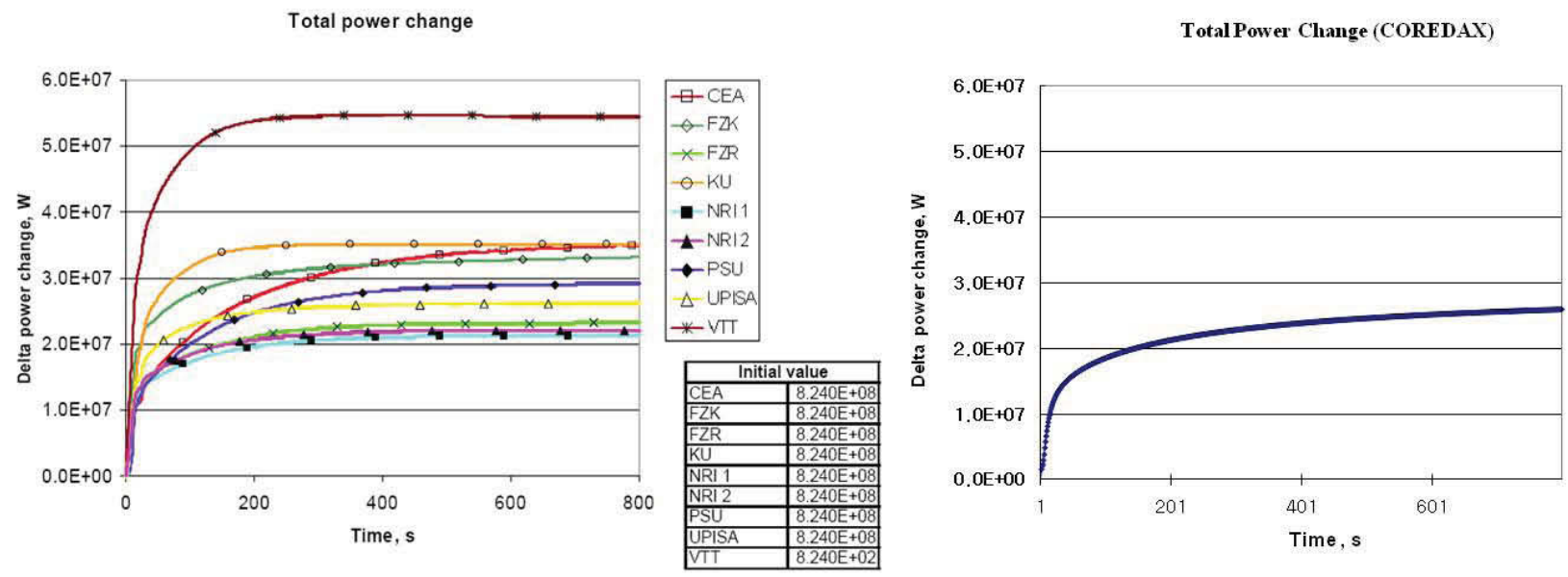

Figure 10-3. Comparisons of total power change between COREDAX and other codes.
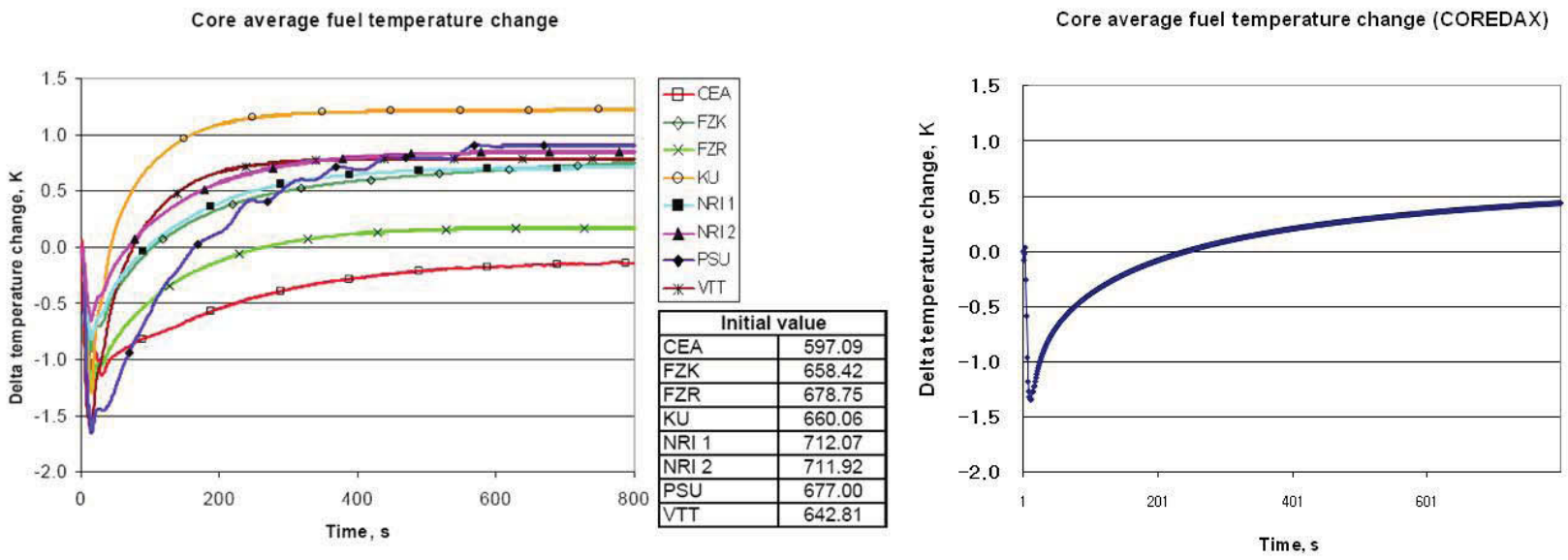

Figure 10-4. Comparisons of core average fuel temperature between COREDAX and other codes. 


\subsection{Generation of Cross-Section Table Set for the GTMHR-600.}

Double heterogeneity in GTMHR assemblies makes it difficult to generate homogenized and condensed cross-section tables using conventional deterministic lattice physics code. To remedy this difficulty, the modified MCNP5 code is employed to generate following cross sections:

$$
\begin{aligned}
& \sum_{x, g}^{h o m}=\frac{\int_{E_{g}} d E \int d \vec{r} \sum_{x}^{h e t}(\vec{r}, E) \phi^{h e t}(\vec{r}, E)}{\int_{E_{g}} d E \int d \vec{r} \phi^{h e t}(\vec{r}, E)}, x=\gamma, f, \\
& \sum_{s, g^{\prime} \rightarrow g}^{h o m}=\frac{\left\langle\sum_{s}^{h e t}\left(\vec{r}, E^{\prime} \rightarrow E, \vec{\Omega}^{\prime} \rightarrow \vec{\Omega}\right) \psi^{h e t}\left(\vec{r}, E^{\prime}, \vec{\Omega}^{\prime}\right)\right\rangle}{\int_{E_{g^{\prime}}} d E^{\prime} \int d \vec{r} \phi^{h e t}\left(\vec{r}, E^{\prime}\right)}, \\
& v \sum_{f, g}^{h o m}=\frac{\int_{E_{g}} d E \int d \vec{r} v(\vec{r}, E) \Sigma_{f}^{h e t}(\vec{r}, E) \phi^{h e t}(\vec{r}, E)}{\int_{E_{g}} d E \int d \vec{r} \phi^{h e t}(\vec{r}, E)}, \\
& \sum_{t, g}^{h o m}=\sum_{\gamma, g}^{h o m}+\sum_{f, g}^{h o m}+\sum_{g^{\prime}} \sum_{s, g \rightarrow g^{\prime}}^{h o m}, \\
& \sum_{s 1, g^{\prime} \rightarrow g}^{h o m}=\frac{\left\{\left(\vec{\Omega}{ }^{\prime} \cdot \vec{\Omega}\right) \sum_{s}^{h e t}\left(\vec{r}, E^{\prime} \rightarrow E, \Omega^{\prime} \cdot \vec{\Omega}\right) \phi^{h e t}\left(\vec{r}, E^{\prime}\right)\right\}}{\int_{E_{g^{\prime}}} d E^{\prime} \int_{d \vec{r}} \phi^{h e t}\left(\vec{r}, E^{\prime}\right)} \\
& D_{g}^{h o m}=\left[3\left(\sum_{t, g}^{h o m}-\sum_{g^{\prime}} \sum_{s 1, g^{\prime} \rightarrow g}^{h o m} \frac{\phi_{g^{\prime}}^{h e t}}{\phi_{g}^{h e t}}\right)\right]^{-1}, \\
& \text { where }
\end{aligned}
$$

As shown in Figure 9-2, the core configuration of GTMHR requires many assembly lattice calculations because of the leakage effect of neighboring assembly types. However, this study considers three types of fuel assembly models and three types of moderator assembly models. In the description of fuel channel, Coarse Lattice with Centered Sphere (CLCS) distribution of fuel particles is used. The radial configurations of the MCNP5 model are shown in Figures 10-5 to 10-10. The axial boundary condition of fuel assembly model is reflective (or zero leakage condition), while the axial boundary condition of moderator assembly model is shown in Figure 10-11. 


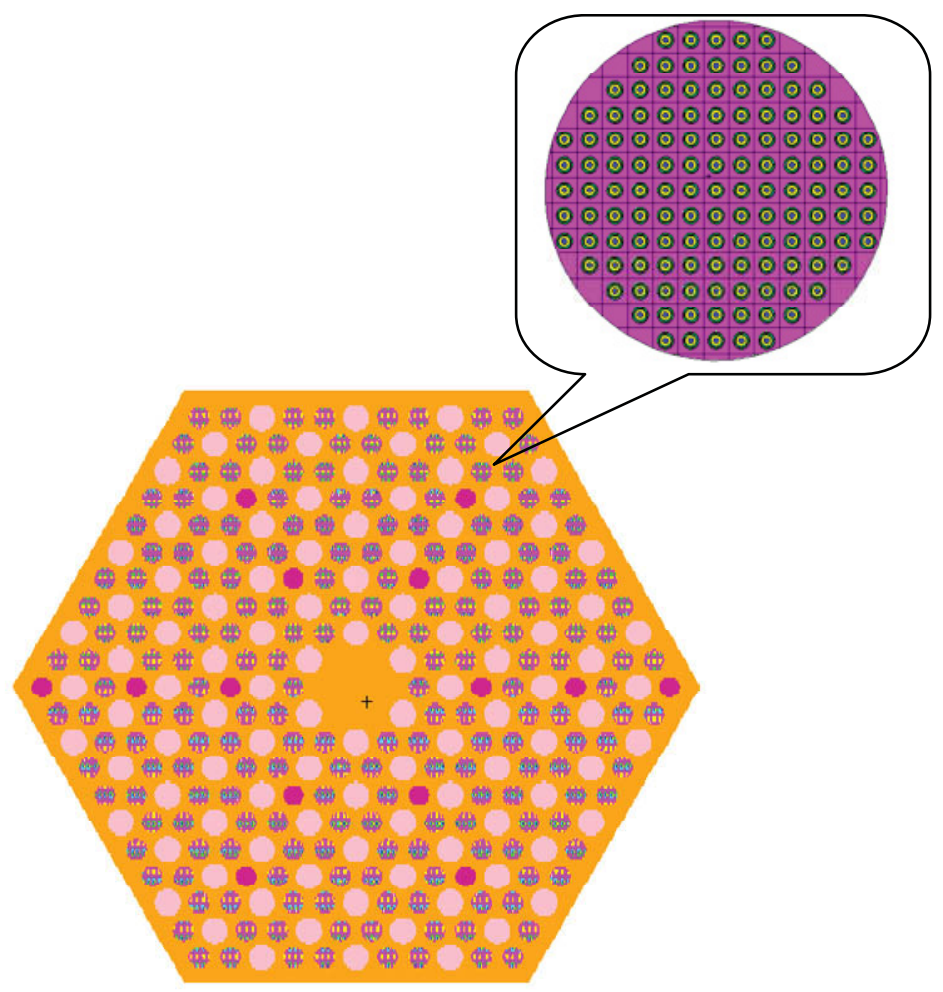

Figure 10-5. Radial configuration of type 1 fuel assembly model.

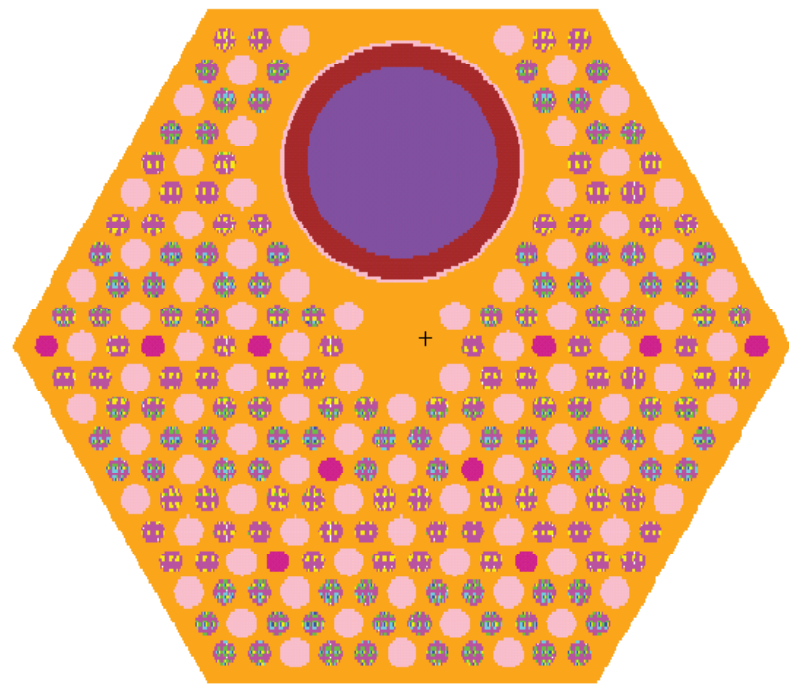

Figure 10-6. Radial configuration of type 2 fuel assembly with control rod model.

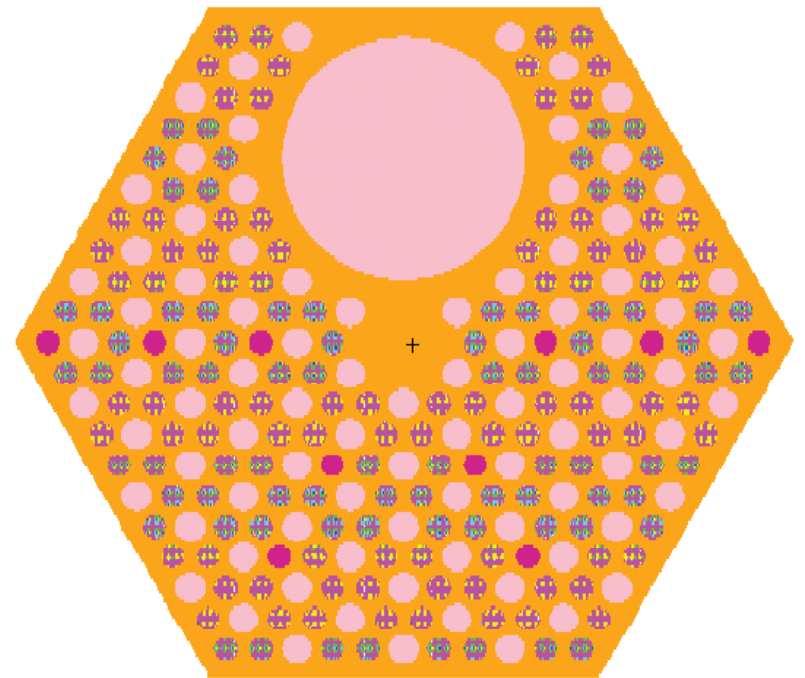

Figure 10-7. Radial configuration of type 2 fuel assembly without control rod model. 


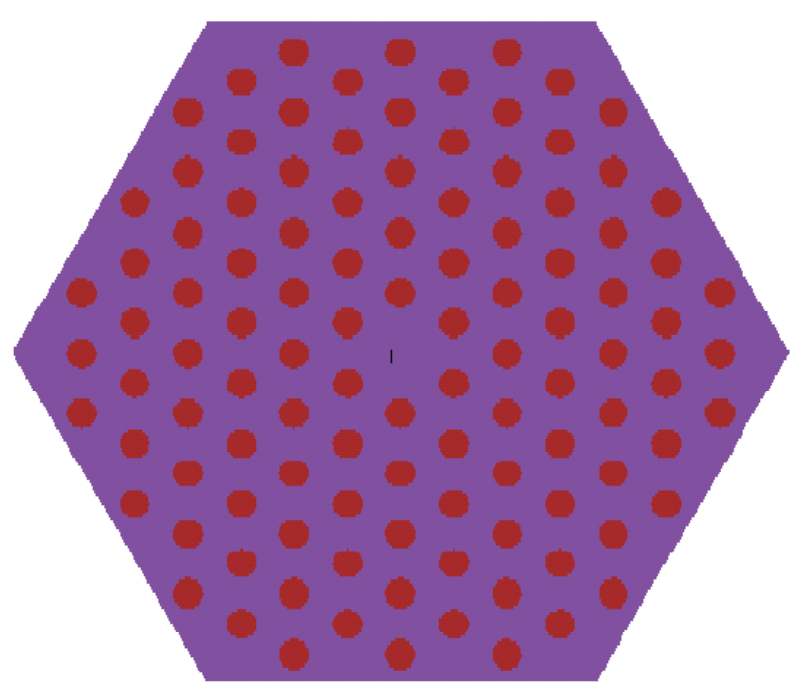

Figure 10-8. Radial configuration of type 1 moderator assembly model.

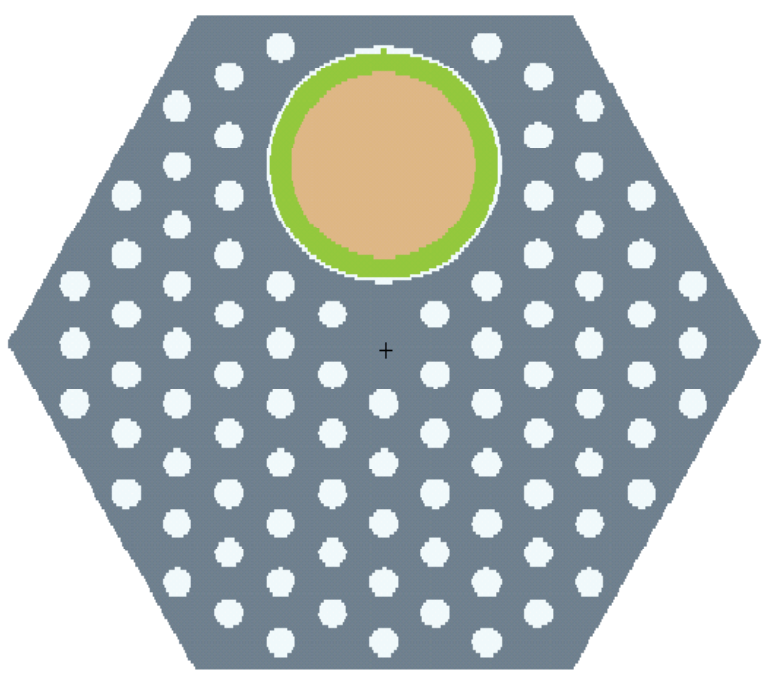

Figure 10-9. Radial configuration of type 2 moderator assembly with control rod model.

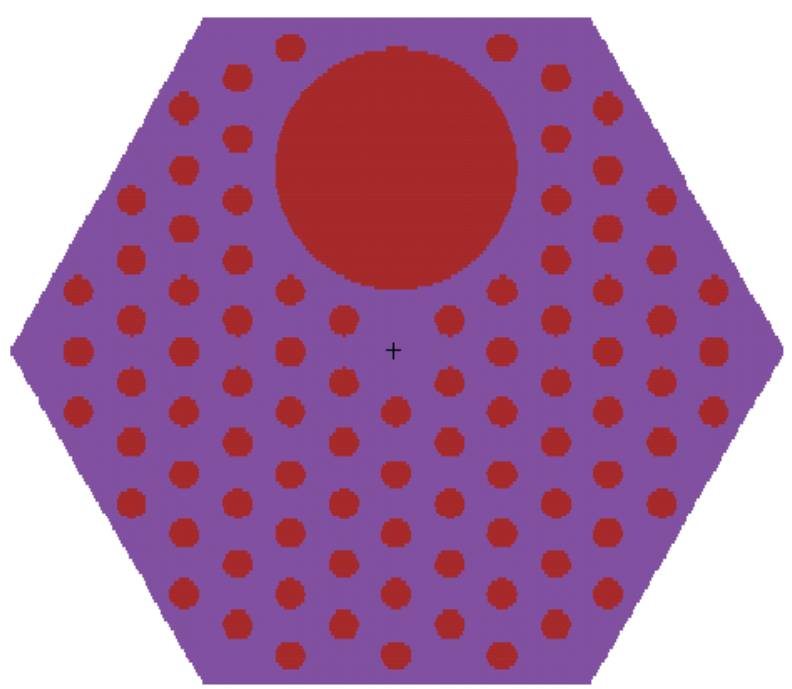

Figure 10-10. Radial configuration of type 2 moderator assembly without control rod model. 
Reflective B.C.

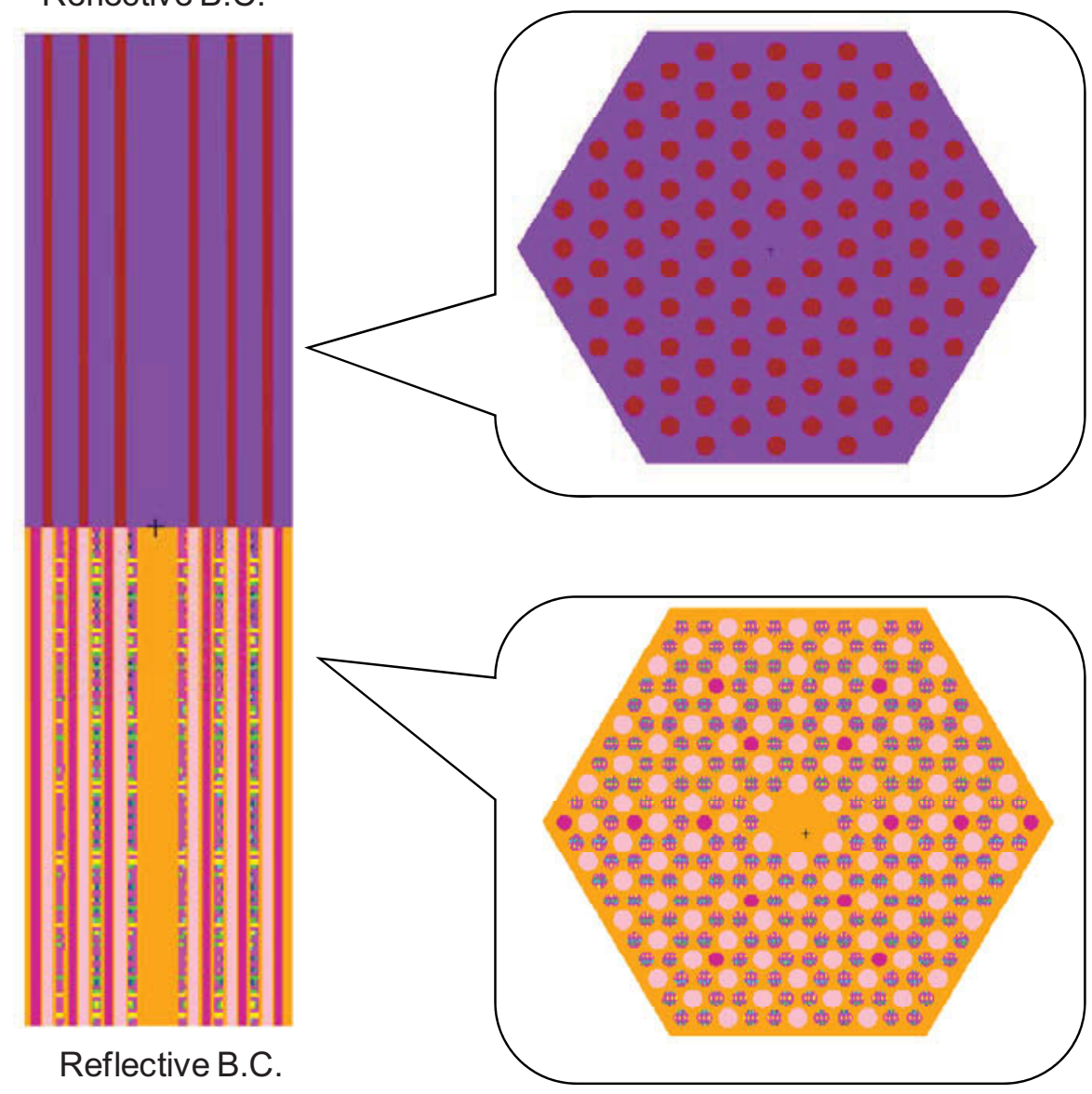

Figure 10-11. Axial configuration of type 1 moderator assembly model.

The MCNP5 calculation used 50,000 histories per generation, 100 inactive/400 active generations, and continuous-energy ENDF-B/VII cross section. Four temperatures $\left(500,1000,1500\right.$, and $\left.2000^{\circ} \mathrm{K}\right)$ are considered as fuel channel temperature and moderator graphite temperature. The behaviors of $\mathrm{k}_{\text {inf }}$ and generated cross sections in Type 1 fuel assembly for various temperatures are shown in Figures 10-12 to Figure 10-16. 


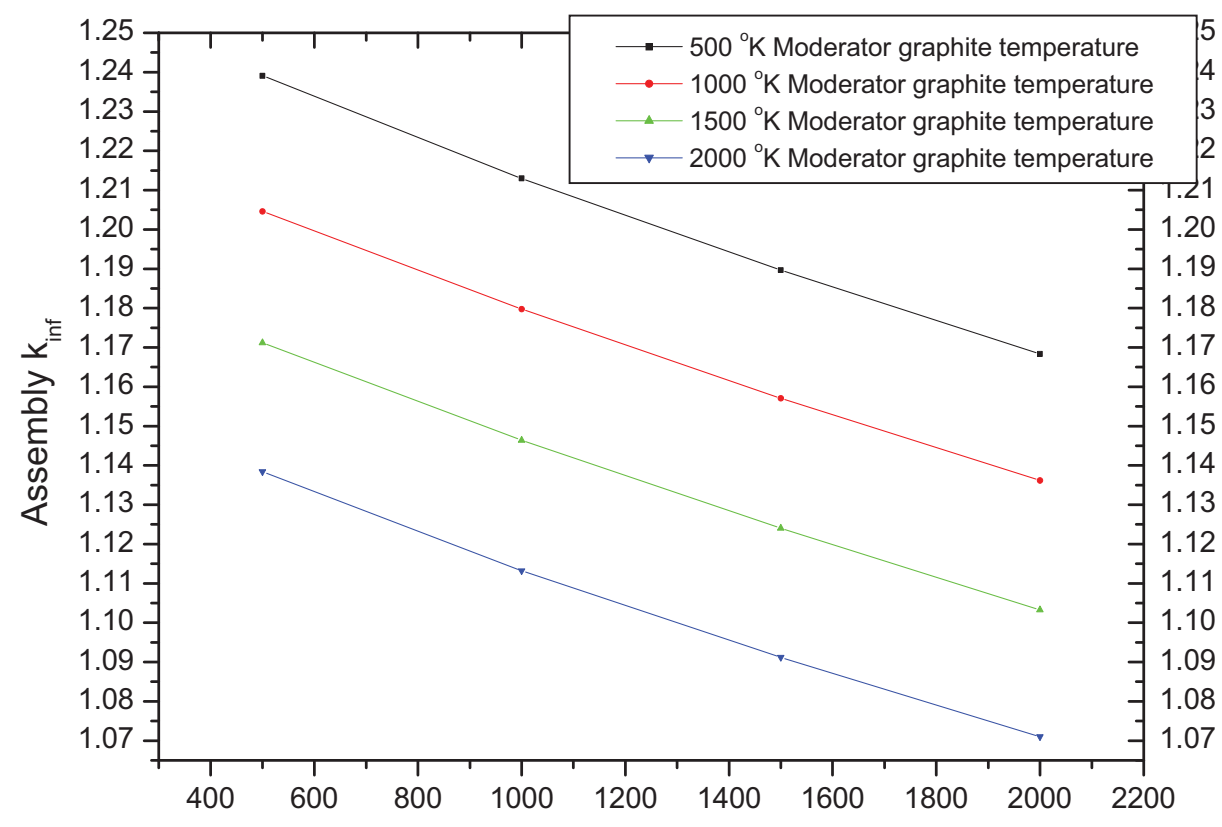

Fuel compact temperature

Figure $10-12 . k_{\text {inf }}$ for various fuel and moderator temperatures in type 1 fuel assembly.

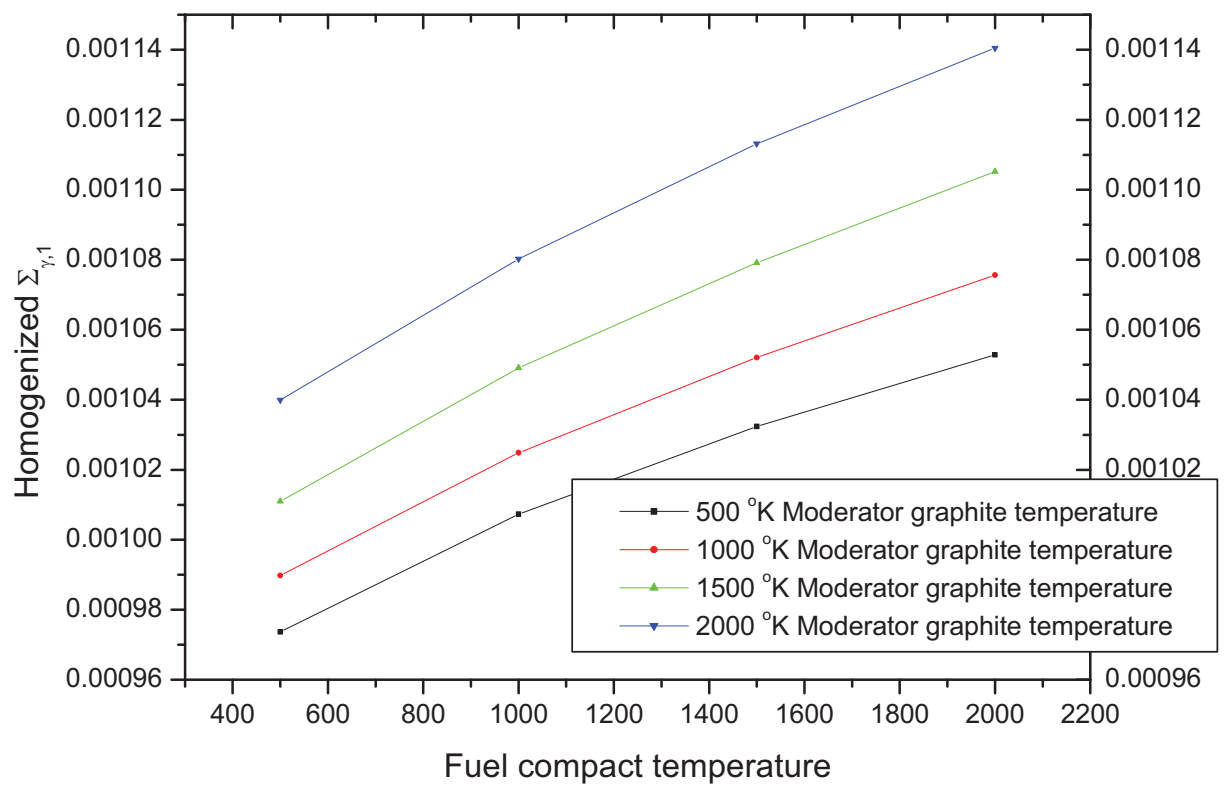

Figure 10-13. Generated fast-group capture cross-section for various temperature calculation in type 1 fuel assembly. 


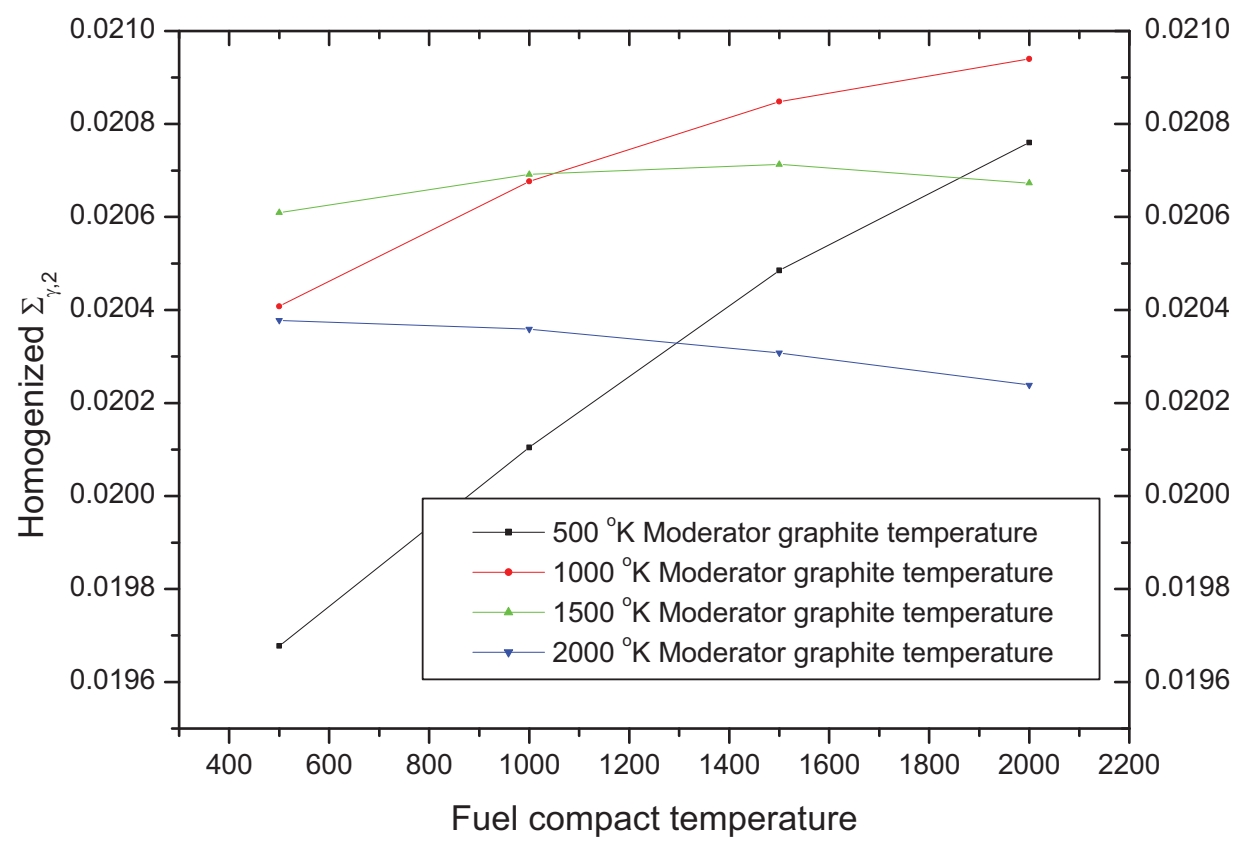

Figure 10-14. Generated thermal-group capture cross-section for various temperature calculation in type 1 fuel assembly

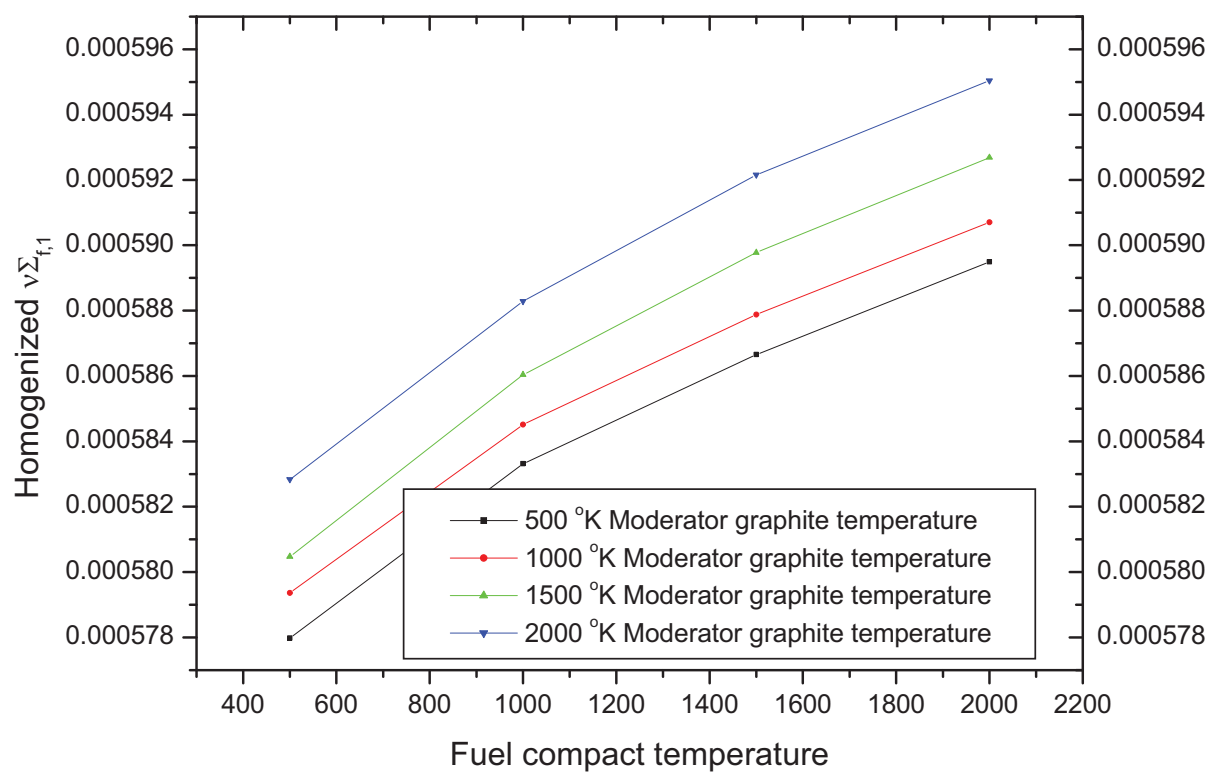

Figure 10-15. Generated fast-group $v \Sigma_{f}$ cross-section for various temperature calculation in type 1 fuel assembly 


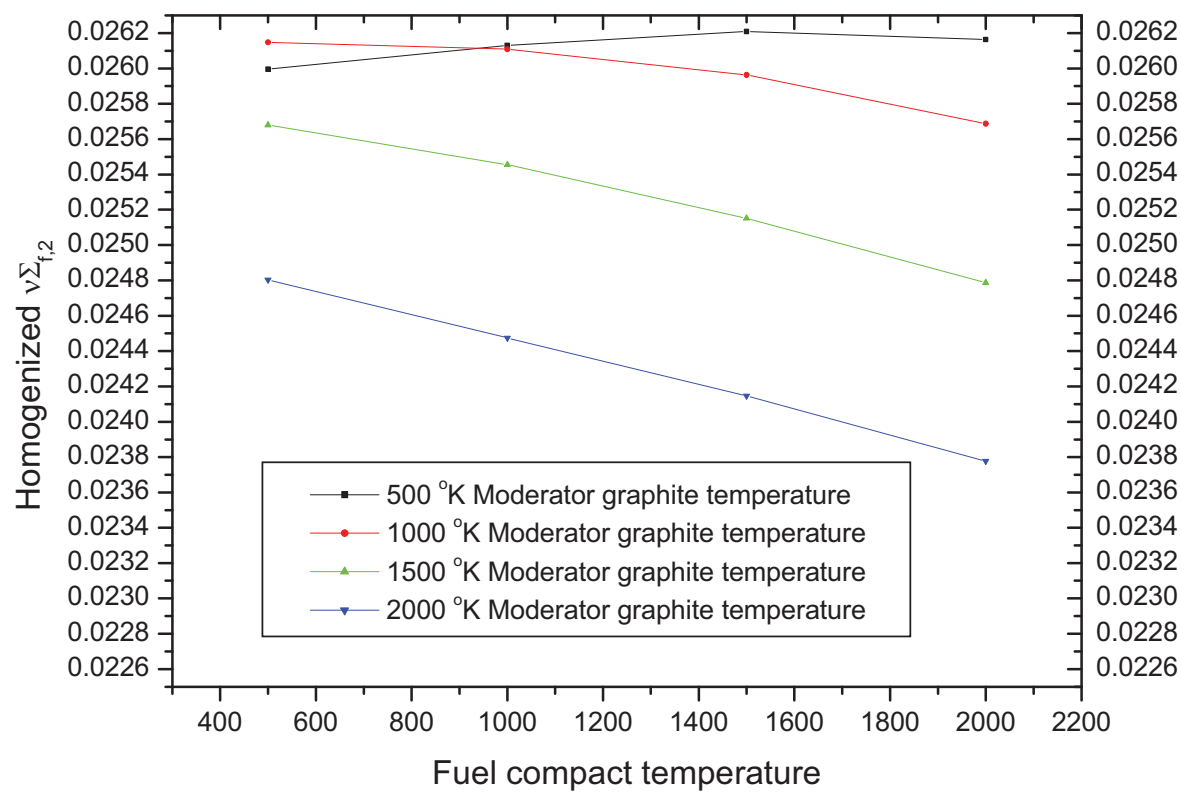

Figure 10-16. Generated thermal-group $v \Sigma_{f}$ cross-section for various temperature calculation in type 1 fuel assembly.

The difference of $\mathrm{k}_{\text {inf }}$ in a Type 1 fuel assembly for the air-ingress accident calculation is shown in Table 10-1. For this calculation, the coolant is changed from helium to air with different temperatures, while fuel compact and moderator temperatures are conserved. The discrepancy in $\mathrm{k}_{\text {inf }}$ owing to the airingress accident is less than 50 [pcm], while the two standard deviations of $\mathrm{k}_{\text {inf }}$ is $42 \sim 46$ [pcm].

Table 10-1. $\boldsymbol{k}_{\text {inf }}$ of type 1 fuel assembly in air-ingress accident calculation.

\begin{tabular}{|c|c|c|c|}
\hline & $\mathrm{k}_{\text {inf }}$ & $\begin{array}{c}\text { Standard } \\
\text { deviation [pcm] }\end{array}$ & $\square \mathrm{k}[\mathrm{pcm}]$ \\
\hline Without Air & 1.23909 & 21.06 & \\
\hline Air-ingression [500 K] & 1.23894 & 22.30 & 15 \\
\hline Air-ingression [942 K] & 1.23883 & 23.54 & 26 \\
\hline Air-ingression [300 K] & 1.23940 & 21.07 & -46 \\
\hline
\end{tabular}

\subsection{Calculation of Steady-State by the GAMMA/COREDAX Code with the Generated GTMHR-600 Data}

Cross-section tables for selected temperatures are generated by the MCNP code for homogenized assemblies. Using this cross-section data, the GAMMA/COREDAX code performed a steady-state calculation for the GTMHR-600 core. The GTMTR-600 core and core mapping between GAMMA and COREDAX are described in Figure 10-17.

The calculation results of GAMMA/COREDAX are compared with those of GAMMA for all rods in, all rods out, and rod half-insertion cases. Power distribution in the GAMMA/COREDAX result shows higher peaking factors for every case in Figures 10-18 through 10-20. 


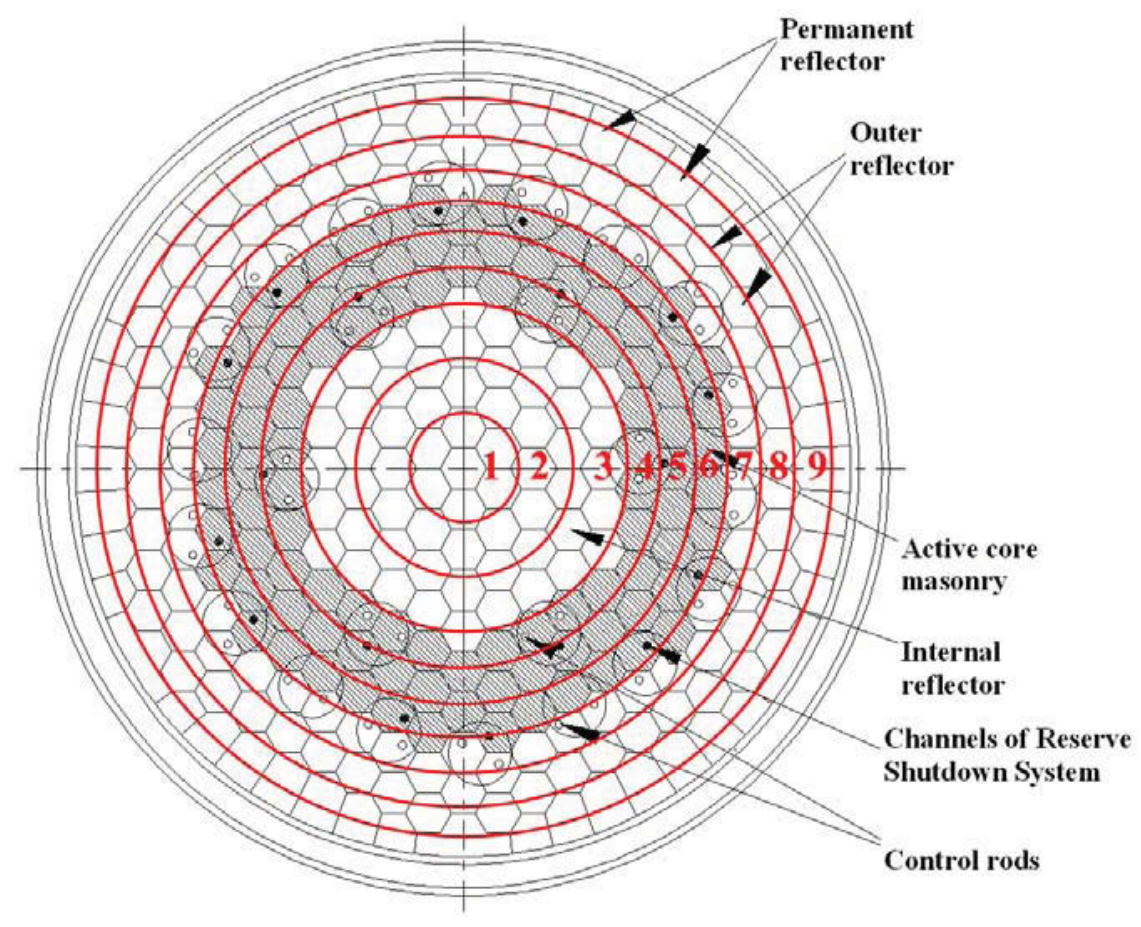

Figure 10-17. GTMHR core mapping configuration.

3.938968

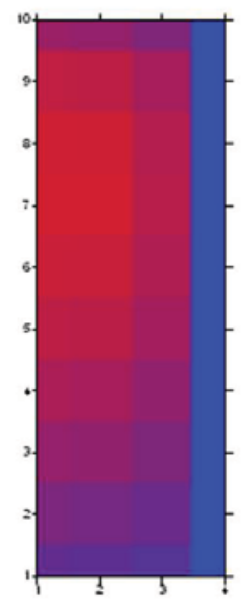

GAMMA INPUT
Peaking Factor

4.795

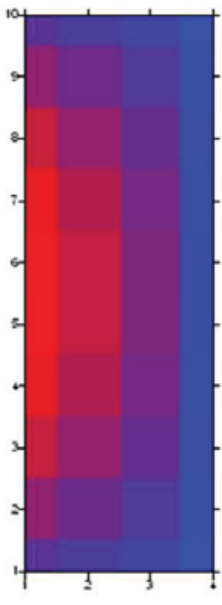

$\mathrm{HZP}$
4.692

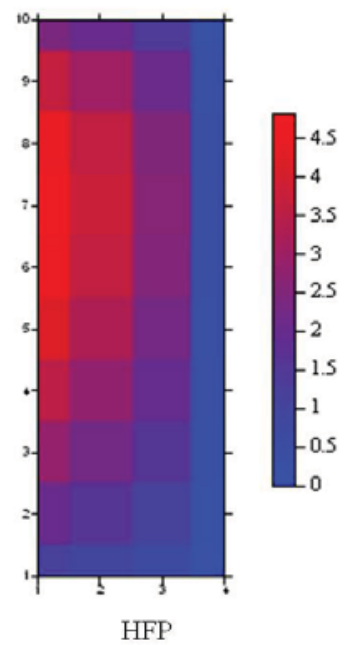

Figure 10-18. Block power distribution compared to GAMMA for all rods in (ARI) case. 


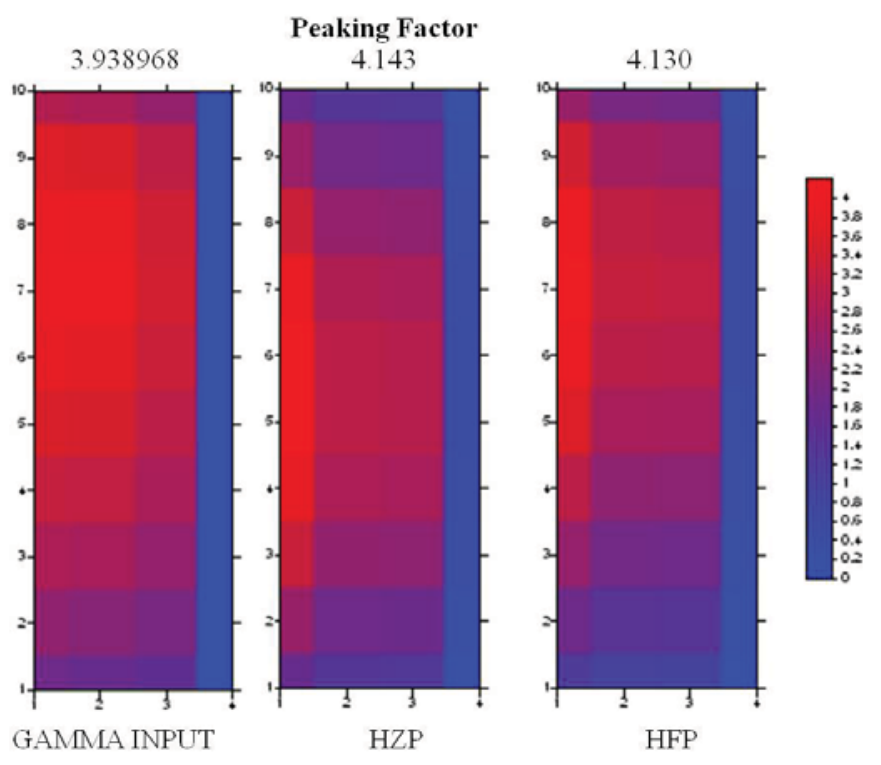

Figure 10-19. Block power distribution compared to GAMMA for all rods out (ARO) case.

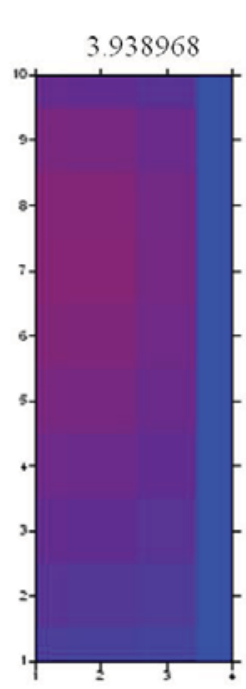

GAMMA INPUT
Peaking Factor

7.279

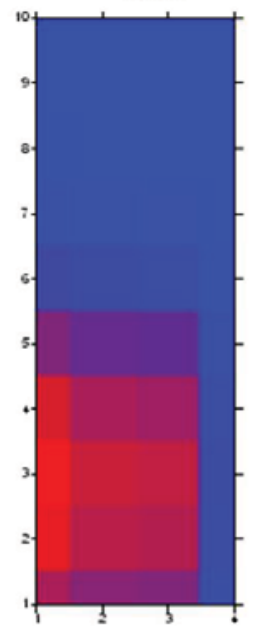

$\mathrm{HZP}$

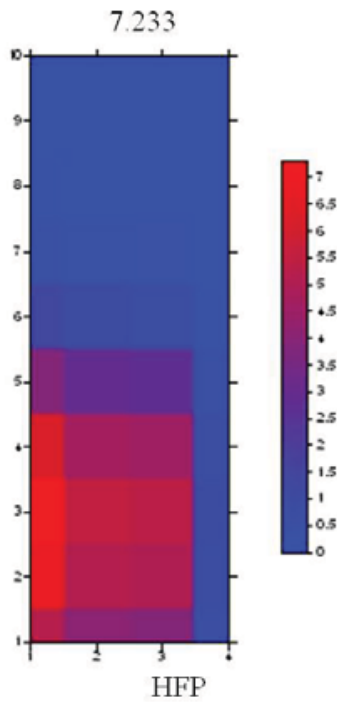

Figure 10-20. Block power distribution compared to GAMMA for rod half insertion case.

\subsection{Calculation of 'Air-ingress' Accident by GAMMA/COREDAX}

To verify the GAMMA/COREDAX code, the results of the GAMMA/COREDAX code were compared with the results of the GAMMA code for the air ingress accident. When an air ingress accident occurs, all control rods fall down to shut down the reactor. This circumstance and 600 MWth are initial conditions for analyzing an air ingress accident. The GAMMA code only considers decay heat as a heat source after an air ingress accident. However, the GAMMA/COREDAX code takes both decay heat and heat generation from the shutting down fission into account. Decay heat data for the GAMMA/COREDAX is taken from "Decay Heat Power in Light Water Reactors," ANSI/ANS-5.1-2005. 
The core nodalization of the 3-D GTMHR-600 reactor is described in Figure 10-21. The results in total power are shown in Figure 10-22 and other parameters in comparison with GAMMA are shown in Figure 10-23 up to 2,100 seconds after shutdown. The results show that total power from GAMMA is lower than GAMMA/COREDAX because of the (shutting down) fission energy. GAMMA/COREDAX predicts significantly increasing fuel temperature after shutdown while fuel temperature from GAMMA increases in much lower speed, since fission energy remains inside the core without heat removal, and the peaking factor is higher in the GAMMA/COREDAX result.

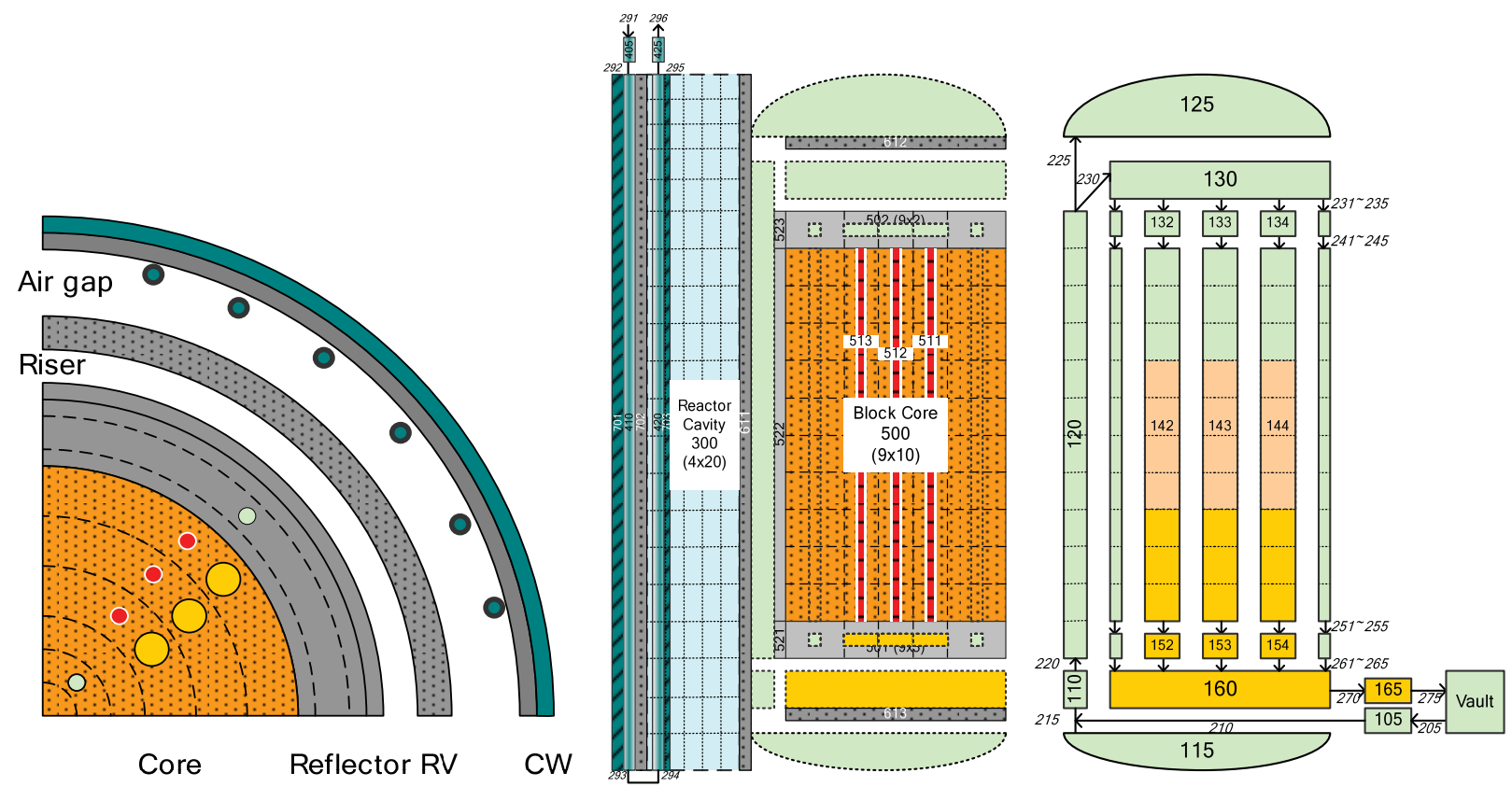

Figure 10-21. GAMMA nodal scheme of 600 MWtg GTMHR.

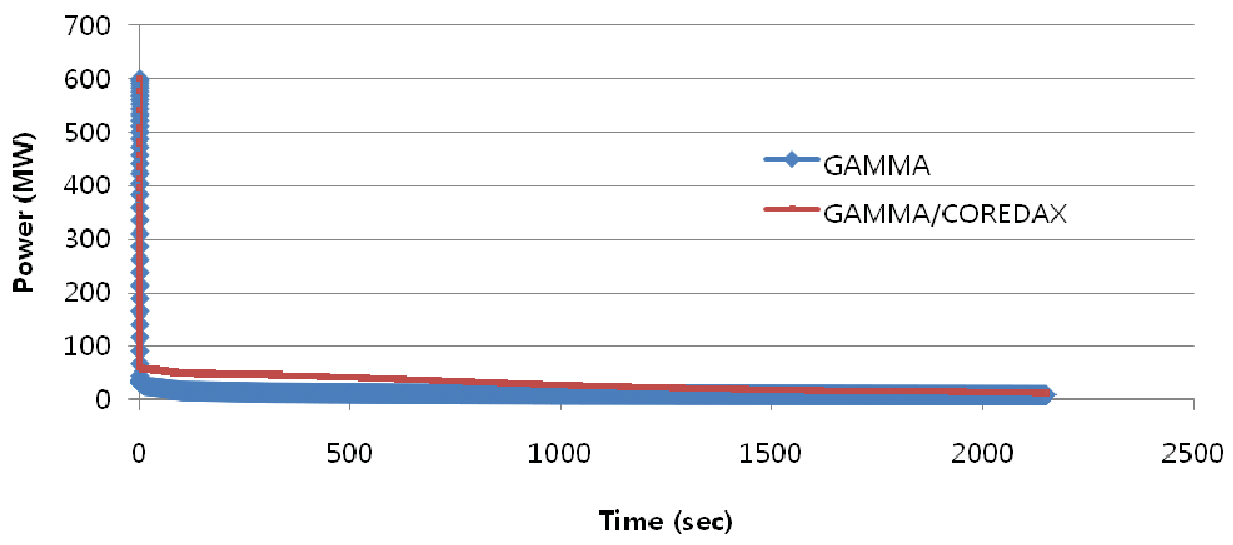

Figure 10-22. Total Power change in time. 


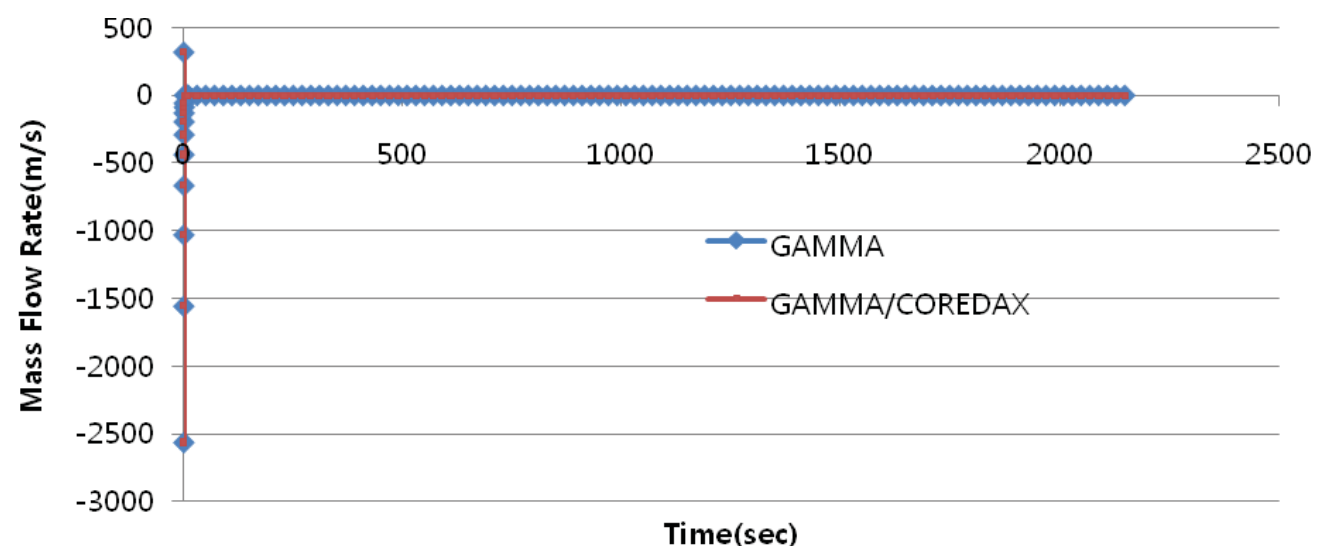

(a) Mass flow rate change in time

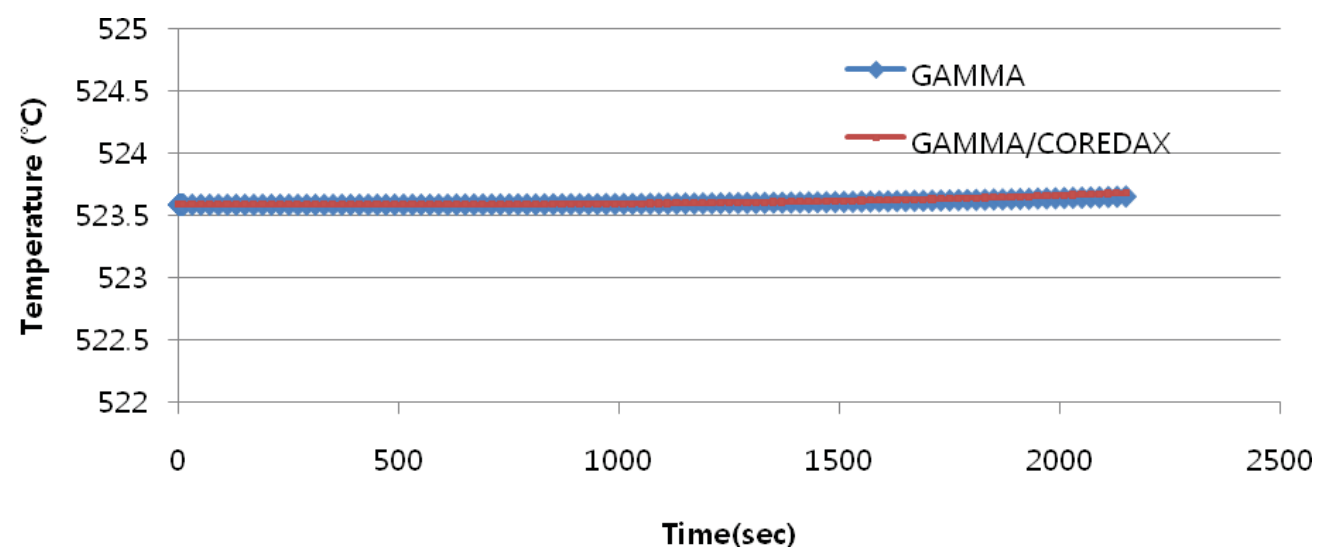

(b) Graphite temperature at block 514

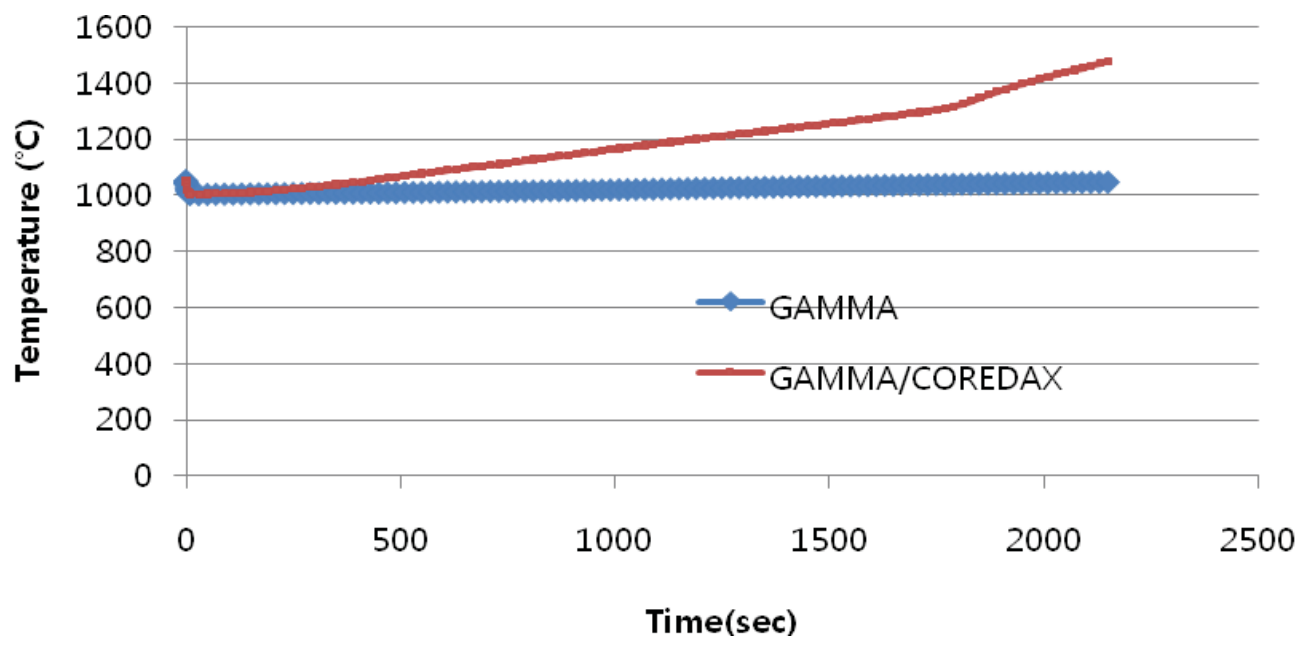

(c) Fuel Compact temperature at block 514

Figure 10-23. Comparisons of Parameters between GAMMA and GAMMA/COREDAX code. 


\section{OVERALL SUMMARY}

Several important issues associated with the VHTR air-ingress accident were investigated from FY 2009 to FY 2010. This section summarizes the key accomplishments of the 3-year project.

\subsection{Task 1}

Task 1 activities consisted of both theoretical and computational work based on analytical estimations and CFD analyses. All of Task 1 results conclude that density gradient-driven stratified flow dominates the air-ingress process in the simplified model of the GTMHR, and the effect of molecular diffusion is generally negligible. The air ingress phenomena depend on the geometry, location, and size of the break.

A new air-ingress scenario based on the density gradient driven flow has been established. This scenario is divided into four steps; (1) depressurization, (2) stratified flow - Stage 1, (3) stratified flow Stage 2 (or molecular diffusion), and (4) global natural circulation. Based on this scenario, the relative importance of two air-ingress mechanisms (molecular diffusion versus density gradient driven flow) has been estimated on a theoretical basis for each step of the accident scenario. A significant decrease in the time scale of air ingress was observed. Time-scale comparisons between stratified flow and diffusion showed that Stage 1 is clearly dominated by density gradient driven flow. The speed of the stratified flow is estimated to be about 800 times faster than the diffusion process in the GTMHR design. Two different designs were considered in Stage 2: 600 MWth GTMHR and the NACOK experiment. The results showed that the pressure build-up in the GTMHR design was larger than the static head, but the pressure build-up in the NACOK experiment was much smaller than the static head, indicating that the $600 \mathrm{MW}$ th GTMHR design will be dominated by density gradient driven flow, while the NACOK experiment will be dominated by the diffusion process in Stage 2. Stage 2 time-scale comparisons for the $600 \mathrm{MWth}$ GTMHR design also showed that density gradient driven flow dominates air-ingress into the core. The speed of convective flow is estimated to be about 600 times faster than the diffusion in the GTMHR design.

Various preliminary CFD analyses were performed in Task 1. Both 2-D and 3-D CFD simulation showed the density gradient driven flow to be the dominant mechanism in the whole air-ingress scenario. Preliminary analyses for the chemical reaction effect on air-ingress were also performed with the FLUENT code using a 2-D simplified geometry based on the GTMHR design. It showed that the chemical reaction will accelerate the air-ingress process by producing $\mathrm{CO}$ in the flow channel. Various preliminary analyses for the partial break accident have been performed with FLUENT using a 2-D simplified geometry based on the GTMHR design with a steam generator. This accident assumed the pressure relief line fracture at the top of the stream generator. The analysis showed that the partial break is also controlled by a density gradient driven flow. One notable result is that the small break air-ingress mechanism is significantly affected by the break size, break angle, and density difference.

The natural circulation pattern in the post ONC was investigated using 3-D simulation. The objective of this simulation was to validate the 1-D single channel natural circulation pattern assumed in the previous air-ingress analysis. The 3-D simulation reveals different flow patterns. According to the 3-D analyses, actual natural circulation consists of the following two flow paths:

- Recirculation flow: This flow occurs in the hot-leg and the lower plenum. The circulation speed is about 1.5 to $2 \mathrm{~m} / \mathrm{s}$. The main driving force is the temperature gradient between the inside and outside of the reactor vessel.

- Global natural circulation flow: This flow occurs from the reactor core to the cold-leg, which is a general natural circulation pattern assumed in the previous studies. The natural circulation speed is about 0.1 to $0.2 \mathrm{~m} / \mathrm{s}$. 
The recirculation flow pattern rapidly draws air in the reactor inside. The air-ingress speed is about an order faster than the previously assumed molecular diffusion, indicating that the air will be ingressed into the reactor about 10 times faster than previously estimated. For this reason, 1-D analysis is not recommended for the air-ingress accident.

\subsection{Task 2}

Task 2 activities consisted of designing and conducting isothermal experiments. The objectives of the isothermal experiments were two-fold: (1) to understand stratified flow phenomena in the VHTR, and (2) to provide experimental data for validating computer codes.

The isothermal experiment focused on three flow characteristics unique in the VHTR air-ingress accident: (1) stratified flow in the horizontal pipe, (2) stratified flow expansion at the pipe and vessel junction, and (3) stratified flow around supporting structures.

Brine and sucrose were used as heavy fluids and water was used as the light fluid. The density ratios were changed between 0.99 and 0.7 .

The experiment shows clear stratified flow between heavy and light fluids, even for the low-density differences.

The stratified flow experimental data based on the circular pipe was compared with the previous theoretical model based on the rectangular channel. Results are in good agreement with the experimental data within a $10 \%$ deviation.

Some blind CFD calculations were carried out for comparisons with the experimental data. FLUENT 6.3.26 was used for the simulation. The simulation results showed good agreement with the experimental data as the mesh size decreases, indicating that the current CFD code and physical models are appropriate for predicting stratified flow phenomena. As a part of the CFD validation, previous air/helium lock exchange experiments were also compared to the CFD simulations. This comparison also showed that the CFD methods are predicting density gradient driven stratified flow speed for both heavy and light current intrusions.

\subsection{Task 3}

Task 3 activities consisted of experimental investigation of graphite oxidation in air-ingress, especially focusing on graphite structure collapse because of corrosion. The following characteristics of graphite were considered to predict this phenomenon: (1) effect of oxidation degree on the graphite strength, (2) effect of oxidized graphite density on the oxidation rate, and (3) surface area density in the graphite internal pores. The relationship between oxidation and graphite strength is well documented, using measurements at burn-off below 10\%. The actual collapse of the graphite structure in the accident is expected to occur at burn-off above $50 \%$, so the previous correlations needed to be validated. To achieve that goal, a new methodology was developed that measured the degree of burn-off at which the graphite loses its mechanical strength. That measurement was been performed for nuclear graphite IG-110 and H451. The obtained data was compared to the previous correlations, which confirmed their validity. Finally, a new correlation containing the data from low burn-off to high burn-off was developed. These new correlations predict the oxidized graphite strength more conservatively than the previous correlations. The effect of burn-off on the oxidation rate was previously investigated for the same graphite. Using that previously published data, the relationships between burn-off and oxidation rate were obtained for other graphite: NBG-10, NBG-18 and V484T. These relationships can be used in transient analysis of the later air-ingress analysis. The surface area densities of some nuclear graphite have been calculated from the published data about BET surface areas by previous investigators. 
In parallel with the experimental works, an analysis was performed to predict the graphite collapse time in the air-ingress accident. This analysis used GAMMA code to calculate changes of graphite oxidation and corrosion and implemented the corrosion and oxidation data to the ABAQUS stress analysis code. The graphite supporting structure was analyzed for the $600 \mathrm{MWth}$ GTMHR design based on the new air-ingress scenario described in Task 1. Both internal and external corrosion of the graphite supports were estimated using GAMMA system analyses code, and structural stress was estimated using ABAQUS code. The graphite strength and the maximum stress were then compared for different times. The structural fracture time was defined as being when the maximum stress exceeds the strength.

Computational methods were used to investigate the maximum allowable burn-off for maintaining graphite structural integrity. Both internal and external corrosions were estimated using a graphite oxidation model, and the resulting stress and strength were compared. The ratio of internal and external corrosion was randomly chosen for each time-step to cover all the possible air-ingress scenarios. As a result, the maximum allowable burn-offs for IG-110 and H451 graphite are estimated to be 0.58 and 0.59 , respectively.

\subsection{Task 4}

Task 4 activities consisted of investigating air-ingress mitigation methods. This task reviewed a wellknown previous idea using helium injection at the top, and found that this idea may not work in real situations for several reasons. This top injection method was based on the counter-diffusion mechanism for air-ingress mitigation. This method can mitigate molecular diffusion but cannot prevent buoyancy force generated in the lower plenum by heating. CFD methods were used to validate this idea, but the model was based on the molecular diffusion air-ingress mechanism and did not consider density gradient driven flow, which is an important air-ingress mechanism. Therefore, the top injection model in the literature did not represent real physics.

Important factors affecting air-ingress consequences were investigated from root-cause analyses as a starting point of the air-ingress mitigation study. The basic air-ingress ideas were developed from this analysis. Various air-ingress mitigation methods were investigated based on these ideas. Among them, the following two mitigation methods were strongly recommended:

- Helium injection in the lower plenum. This method injects helium into the lower plenum, which replaces the air in the core and the lower plenum upper part by buoyancy force, significantly reducing graphite oxidation inside the reactor.

- Reactor enclosure opened at the bottom. This method encloses the reactor in a nonpressure boundary. Some design modifications of the cavity can be used for this. This enclosure has an opening at the bottom. After depressurization, the air-ingress rate is controlled by molecular diffusion through this opening.

Validation of the air-ingress mitigation method was conducted by CFD methods. The results of the newly developed air ingress mitigation methods show that both methods are effectively mitigating the airingress process.

\subsection{Task 5}

Task 5 activities consisted of performing graphite oxidation tests for IG-110 and IG-430. Kinetics parameters, dimensional effect, effect of burn-off, and effect of moisture were also investigated. The activation energy of IG-430 was $158.5 \mathrm{~kJ} / \mathrm{mol}$ and the order of reaction was 0.37 . Both reaction rates of IG-110 and IG-430 in Zone 1 have the maximum reaction rate of about $40 \%$ of burn-off. The effect of burn-off on the reaction rate in IG-110 is much larger than that in IG-430. It is confirmed that the mass transfer in Zone 3, including the moisture effect, is half of the rate without moisture effect. 


\subsection{Task 6}

Task 6 activities consisted of KAIST investigating graphite oxidation and mechanical behaviors of selected graphite materials (IG-110, IG-430, and NBG-10). According to the experiment, the graphite mechanical fracture was mainly affected by slenderness ratio and oxidation burn-off. Based on the results of this study, two correlations were finally suggested for predicting graphite fracture in the VHTRs:

$$
\sigma_{0}=A-B \frac{L}{r}
$$

and

$$
\frac{\sigma}{\sigma_{0}}=\exp (-k d), d=1-\frac{M+\left\{\left(V_{0}-V\right) \rho_{0}\right\}}{M_{0}}
$$

Allowable total burn-offs for IG-110, IG-430, and NBG-10 graphite were estimated, which can be used for conservative graphite fracture criteria. The estimates showed that the allowable burn-off is predicted when the reaction is dominated by internal pore reactions ( $\mathrm{f}$-value $=0$ ).

Graphite oxidation models were implemented into the GAMMA code and various air-ingress analyses were conducted using 1-D and 2-D modeling. These analyses showed that core maximum temperature is not affected by the onset natural circulation and type of models (1-D or 2-D). However, predications of bottom reflector temperature and oxidation patterns were significantly different between 1-D and 2-D modeling because of different flow patterns. Validation on the GAMMA code 2-D simulation for density driven stratified flow was performed.

Finally, a one-eighth scaled-down bottom structure was tested. Main characteristics of the test are that heating and loading are applied at once. It is confirmed that the bottom structure is safe for at least $40 \%$ of the local burn-off.

\subsection{Task 7}

Task 7 activities consisted of providing thermal power distributions in the reactor core to GAMMA code by COREDAX(nodal diffusion code) for realistic thermal-hydraulics analysis. Thermal feedback from GAMMA affected the cross-section, and these effects were reflected on the next time step neutronics calculation by COREDAX. This procedure was included in coupled code GAMMA/COREDAX and tested on GTMHR-600 core with various transient situations.

\subsection{Task 8}

Task 8 activities consisted of completing advanced neutronics code development based on analytic function expansion method (AFEN).This code provides accurate results compared with well known PARCS code as shown in the VVER-440 benchmark problem test result.

\subsection{Task 9}

Task 9 activities consisted of analyzing realistic situations in a nuclear reactor. To accomplish this, homogenized cross-sections of GTMHR-600 assemblies were generated by MCNP calculation and tabulated for representative temperatures. From this data, cross-section values were interpolated for required temperature and provided to GAMMA/COREDAX code. 
The air-ingress accident was then analyzed using the GAMMA/COREDAX code, and the important parameters were calculated and compared with the results of GAMMA, which used the point kinetics method for power generation feedback. GAMMA and GAMMA/COREDAX code results showed that the fuel compact temperature progressed in different directions. 


\section{REFERENCES}

\section{Executive Summary and Chapter 1 (Introductions)}

Oh, C. H., Davis, C., Siefken, L., Moore, R., NO, H. C., Kim, J., Park, G. C., Lee, J. C. and Martin, W. R., 2006, Development of Safety Analysis Codes and Experimental Validation for a Very High Temperature Gas-Cooled Reactor, Final Report, Idaho National Laboratory, INL/EXT-06-01362, March 2006.

Schultz et al., 2006, Next Generation Nuclear Plant Methods Technical Program Plan, INL/EXT-0611804, Rev 0, September 2006.

\section{Chapter 2 (Task 1)}

ANSYS, 2009, CFX-12 Manual.

ANSYS, 2008, ICEM CFD-11.0, Manual.

Barr, D. I. H., 1967, "Densimetric Exchange Flows in Rectangular Channels," La Houille Blanche, Vol. 22, pp. 619-631.

Benjamin, T. B., 1968, "Gravity Currents and Related Phenomena," J. Fluid Mechanics, Vol. 31, pp. 209-248.

Bergelin, O. P., Colburn, A. P., and Hull, H. L., 1950, "Heat Transfer and Pressure Drop during Viscous Flow across Unbaffled Tube Banks," Engineering Experimental Station, Bulletin No.2, University of Delaware, Newark, DE.

Birman, V. K., Martin, J. E., and Meiburg, E., 2005, “The Non-Boussinesq Lock-Exchange Problem. Part 2. High-resolution Simulations," J. Fluid Mechanics, Vol. 537, pp. 125-144.

Britter, R. E., and Simpson, J. E., 1978, “A Note on the Structure of a Gravity Current Head," J. Fluid Mechanics, Vol. 112, pp. 459-466.

Chen, G. Q., and Lee, J. H., 1999, “Turbulent Gravity Current of Lock Release Type: a Numerical Study,” Envir. Hyd., Balkema, pp. 449-454.

Chilton, T. H. and Generaux, R. P., 1933, "Pressure Drop Across Tube Banks," Trans. Am. Inst. Chem. Eng., 29, pp. 161-173.

Damerow, W. P., Murtaugh, J. C., and Burgraf, F., 1972, "Experimental and Analytical Investigation of the Coolant Flow Characteristics in Cooled Turbine Airfoils," NASA, CR-120883.

Etienne, J., Hopfinger, E. J., and Saramito, P., 2005, "Numerical Simulation Of High Density Ratio LockExchange Flows," Physics of Fluids, Vol. 17.

Eugeny, V. E., and Gavrilov, N. V., 2007, “A Note on the Propagation Speed of a Weakly Dissipative Gravity Current,” J. Fluid Mechanics, Vol. 574, pp. 393-403.

FLUENT Inc., Lebanon, N. H., 2007, FLUENT 6.3 User's Guide.

Gaddis, E. S. and Gnielski, V., 1985, "Pressure Drop in Horizontal Cross Flow across Tube Bundles," Int. Chem. Eng., 25s1-D, pp. 1-15.

Gardner, G. C., and Crow, I. G., 1970, “The Motion of Large Bubbles in Horizontal Channels," J. Fluid Mechanics, Vol. 112, pp. 459-466.

General Atomic, 1997,“Internal Design Review Presentation Material,” December 8-12.

General Atomics Co., 1996, Gas Turbine-Modular helium Reactor (GT-MHR) Conceptual Design Description, Report 910720, Revision 1, July 1996. 
Grobelbauer, H. P., Fannelop, T. K., and Britter, R. E., 1993, “The Propagation of Intrusion Fronts of High Density Ratios,” J. Fluid Mechanics, Vol. 31, pp. 669-687.

Gunter, A. Y. and Shaw, W. A., 1945, "A General Correlation of Friction Factors for Various Types of Surfaces in Cross Flow,” Mech. Eng. Am. Soc. Mech. Eng., 67, pp. 643-660.

Hartel, C., Meiburg, E., and Neckar, F., 2000, "Analysis and Direct Numerical Simulation of the Flow at a Gravity Current Head, Part 1. Flow Topology and Front Speed for Slip and Non-Slip Boundaries"., J. Fluid Mechanics, Vol. 269, pp. 169-198.

Jacob, M., 1938, "Discussion-Heat Transfer and Flow Resistance in Cross Flow of Gases Over Tube Banks," Mech. Eng. Am. Soc. Mech. Eng., Vol. 60, pp. 381-392.

Johnson, R. W., 2008, "Modeling Strategies for Unsteady Turbulent Flows in the Lower Plenum of the VHTR," Nuclear Engineering and Design, Vol. 238, pp. 482-491.

Kang, H. S., 2006, "CFD Analysis for the Turbulent Flow in the $3 \times 3$ Hybrid Vane," FLUENT User Group Meeting, Kyungju, Korea.

Keller, J. J. and Chyou, Y. P., 1991, "On the Hydraulic Lock-Exchange Problem,” J. Applied Mathematics and Physics, Vol. 41.

Kim, E., NO, H.C., Kim, B., and Oh, C. H., 2007, "Estimation of Graphite and Mechanical Strength Variation of VHTR during Air-Ingress Accident," In press to Nuclear Engineering and Design.

Klemp, J. B., Rotunno, R., and Skamarock, W. C., 1994, "On the Dynamics of Gravity Currents in a Channel,” J. Fluid Dynamics, Vol. 269, pp. 169-198.

Kosar, A., Mishra, C. and Peles, Y., 2005, Laminar Flow Across a Bank of Low Aspect Ratio Micro Pin Fins, J. Fluid Engineering, Vol. 127, pp 419-423.

Kuelegan, G. H., 1958, “The Motion of Saline Fronts in Still Water,” Natl Bur. Stnd. Rep. 5813.

Lindeburg, M. R., 1997, Mechanical Engineering Reference Manual, Professional Publications, INL, ISBN 1-888577-13-4.

Linden, P. F. and Simpson, J. E., 1986, "Gravity-driven flows in turbulent fluid," Journal of Fluid Mechanics, Vol. 172, pp. 481-497.

Liou, C. P., Schultz, R. R., and Kukita, Y., 1997, "Stably Stratified Flows in Closed Conduits," ICONE52-24, Proceedings of the $5^{\text {th }}$ International Conference on Nuclear Engineering, ASME/SFEN/JSME, May, Nice, France.

Liou, C. P., Parks, D. L., Schultz, R. R., and Williams, B. G., 2005, "Stratified Flows in Horizontal Piping of Passive Emergency Core Cooling Systems," 13th International Conference on Nuclear Engineering, ICONE 13-50450, May 16-20, Beijing, China.

Lowe, R. J., Linden, P. E., and Rottman, J. W., 2002, “A Laboratory Study of the Velocity Structure in an Intrusive Gravity Current," J. Fluid Mechanics, Vol. 456, pp. 22-48.

Lowe, R. J., Rottman, J. W., and Linden, P. F., 2005, “The Non-Boussinesq Lock-Exchange Problem. Part 1. Theory and Experiments," J. Fluid Mechanics, Vol. 537, pp.101-124.

McEligot, D. M. and McCreery, G. E., 2004, Scaling Studies and Conceptual Experiment Designs for NGNP CFD Assessment, INEEL/EXT-04-02502.

Metzger, D. E., Fan, Z. N., and Shepard, W. B., 1982, "Pressure Loss and Heat Transfer Through Multiple Rows of Short Pin Fins," Journal of Heat Transfer, Vol. 3, edited by U. Grigull et al., Hemisphere, Washington, pp.137-142. 
Mok, K. M., IEONG, K. K. and Yeh, H., 2003, "Experimental Observation of the Flow Structures at Gravity Current Front," International Conference on Estuaries and Coasts, November 9-11, Hangzhou, China, 2003.

Moores, K. A. and Joshi, Y. K., 2003, "Effect of Tip Clearance on the Thermal and Hydrodynamic Performance of a Shrouded Pin Fin Array," J. Heat Transfer, Vol. 125, pp. 999-1006.

NIST Chemistry Webbook, http://webbook.nist.gov/chemistry/fluid/

NO, H. C., Lim, H. S., Kim, J., Oh, C. H., Siefken, L., and Davis, C., 2007, “Multicomponent Diffusion Analysis and Assessment of GAMMA Code and Improved RELAP 5 Code," Nuclear Engineering and Design, Vol. 237, pp. 997-1008.

Oh, C. H., Davis, C., Siefken, L., Moore, R., NO, H. C., Kim, J., Park, G. C., Lee, J. C. and Martin, W. R., 2006, Development of Safety Analysis Codes and Experimental Validation for a Very High Temperature Gas-Cooled Reactor, Final Report, Idaho National Laboratory, INL/EXT-06-01362, March.

Oh, C. H., Kim, E. S., NO, H. C., and Cho, N. Z., 2008, Experimental Validation of Stratified Flow Phenomena, Graphite Oxidation, and Mitigation Strategies of Air Ingress Accident, INL/EXT-0814840, December.

Parson, J. D., and Garcia, M. H., 1998, "Similarity of Gravity Current Fronts," Phys. Fluids, Vol. 10, pp. 3209-3213.

Schaaf, T., Frohling, W., Hohn, H., Struth, S., 1998, "The NACOK Experimental Facility for Investigating an Air Ingress into the Core of a High Temperature Reactor, Kerntechnik, Vol. 63, Issue 3 , pp. 107-112.

Schultz et al., 2006, Next Generation Nuclear Plant Methods Technical Program Plan, INL/EXT-0611804, Rev 0.26, September 2006.

Shin, J. O., Dalziel, S. B., and Linden, P. F., 2004, “Gravity Currents Produced By Lock Exchange,” J. Fluid Mechanics, Vol. 521, pp. 1-34.

Short, Jr., B. E., Price D. C., and Raad P. E., 2004, "Design of Cast Pin Fin Cold walls for Air-Cooled Electronic Systems,” J. Electron. Packag., 126, pp. 67-73.

Simpson, J. E., 1999, "Gravity Currents in the Environment," PINSA, Vol. 65, No. 1, pp.1-25.

Sparrow, E. M. and Grannis, 1985, "Longitudinally-finned Cross Flow Tube Banks and their Heat Transfer and Pressure Drop Characteristics," Int. J. Heat Mass Transfer, 28s 2d, pp. 339-350.

Taborek, J., 1983, "Shell-and-Tube Heat Exchangers: Single phase Flow," Handbook of Heat Exchanger Design, Hemisphere, New York, Chap. 3.3.

Takeda T., 1997, Mixing Process of a Binary Gas in a Density Stratified Layer, JAERI-Research 97-061, Japan Atomic Energy Research Institute.

Takeda, T. and Hishida, M., 1996,"Studies on Molecular Diffusion and Natural Convection in a Multicomponent Gas System," International Journal of Heat and Mass Transfer, Vol. 39, No. 3, pp. $527-536$.

Vassallo, P. and Symolon, P., 2007, Friction Factor Measurements in an Equally Spaced Triangular Tube Array, LM-07K012, March 19.

Von Karman, T., 1940, “The Engineer Grapples with Nonlinear Problems," Bull. Am. Math. Soc., Vol. 46, pp.615-683. 
Welty, J. R., Wicks, C. E. and Wilson, R. E., 1984, Fundamentals of Momentum, Heat and Mass Transfer, John Wiley \& Sons, Inc.

Wilkinson, D. L., 1982, "Motion of Air Cavities in Long Horizontal Ducts, J. Fluid Mechanics, Vol. 118, pp. 109-122.

Yih, C. S., 1965, Dynamics of Nonhomogeneous Fluids, Macmillan.

Yih, C. S., 1980, Stratified Flows, Academic Press.

\section{Chapter 3 (Task 2)}

ANAYS FLUENT 6.3, 2008, User Guide.

Benjamin, T. B., 1968, "Gravity Currents and Related Phenomena," J. Fluid Mechanics, Vol. 31, pp. 209-248.

Britter, R. E. and Simpson, J. E., 1978, “A Note on the Structure of a Gravity Current Head,” J. Fluid Mechanics, Vol. 112, pp. 459-466.

Grobelbauer, H. P., Fannelop, T. K., and Britter, R. E., 1993, “The Propagation of Intrusion Front of High Density Ratios, J. Fluid Mech., Vol. 250, pp. 669-687.

Lowe, R. J., Rottman, J. W., and Linden, P. F., 2005, "The Non-Boussinesq Lock-Exchange Problem. Part 1. Theory and Experiments," J. Fluid Mechanics, Vol. 537, pp.101-124.

NIST Chemistry Webbook, http://webbook.nist.gov/chemistry/fluid/

Roache, P.J., 1998, Verification and Validation in Computational Science and Engineering, Hermosa Publishers, Albuquerque, New Mexico.

Simpson, J. E., 1999, “Gravity Currents in the Environment,” PINSA, Vol. 65, No. 1, pp.1-25.

\section{Chapter 4 (Task 3)}

ABAQUS, Inc, 2007, “Getting Started with ABAQUS (ver. 6.75)."

Beer, F. P., Dewolf, J. T., Johnston, E. R., 2006, Mechanics of Materials, (4th ed). New York: McGraw-Hill.

Bratton, R. L., Burchell, T. D., Corwin, W. R., Hayner, G. O., Katoh, Y., Klett, J. W., 2005, Next Generation Nuclear Plant Materials Research and Development Program Plan, INL/EXT-05-00758 Rev. 2, September.

Bruauer, S., Emmett, P. H., Teller, T., 1938, “Adsorption of gases in multimolecular layers,” J. Amer. Chem. Soc., Vol. 60, pp. 309-319.

Burchell, T. D., Eto, M., Ishiyama, S., Strizak, J. P., 1996, “The Effect of high Fluence Neutron Irradiation on the Properties of a Fine-Grained Isotropic Nuclear Graphite," Journal of Nuclear Materials, Vol.230, pp. 1-7.

Burchell, T. D., Romanoski, G. R., 1991, "The Effects of Specimen Geometry and Size on the Fracture Toughness of Nuclear Graphites," ORNL CONF-9109266-1, January.

Cocheme, F. G., 2004, “Assessment of Passive Decay Heat Removal in the General Atomic Modular Helium Reactor," Unpublished master's thesis, Texas A\&M University, Texas, U.S.A..

Contescu, C., 2008, "Current R\&D Activities on Graphite Oxidation at ORNL," personal communication, NGNP R\&D Technical Review Meeting, Idaho National Laboratory, May 5-8. 
Contescu, C. I., Azad, S., Miller, D., Lance, M. J., Baker, F. S., and Burchell, T. D., 2008, "Practical aspects for characterizing air oxidation of graphite," Journal of Nuclear Materials, in press (available online), 2008.

Engle, G. B., 1997, General Atomics Co. Report, GA-A14690.

Eto, M. and Growcock, F. B., 1983, "Effect of Oxidizing Environment on the Strength of H451, PGX and IG-110 Graphites," Carbon, Vol. 21, No. 1, pp. 135-147.

Eto, M., Konishi, T., Oku, T., 1986, "High Temperature Young's Modulus of IG-110 Graphite,” JAERI, Conference article from Specialists' meeting on graphite component structural design, JAERI Tokai (Japan), September 8-11, 1986, conducted by the International Atomic Energy Agency, Vienna (Austria). International Working Group on Gas-Cooled Reactors. IWGGCR-11, pp. 133-137.

Fagerlund, G., 1973, "Determination of specific surface by BET method," Materjaux et Constructions, Vol. 6, No. 33, pp. 239-245.

Fuji, K., Nakano, J., Yamada, R., 1997, "Mechanical Properties of Oxidation-Resistant SiC/C Compositionally Graded Graphite Materials," Journal of the American Ceramic Society, Vol. 80, No. 11, pp. 2897-2902.

Fuller, E. L., Okoh, J. M., 1997, "Kinetics and Mechanisms of the Reaction of Air with Nuclear Grade Graphites: IG-110,” J. Nuclear Materials, Vol. 240, pp. 241-250.

General Atomics, 1997, International GT-MHR Project Internal Design Review Presentation Material.

General Atomics, 2003, Screening Tests for Selection of VHTR Advanced Fuel, Contract No. DE-AC0301SF22343, Revision 0.

Hinssen, H. K., Kuhn, K., Moorman, R., Schlogl, B., Fechter, M. and Mitchell, M., 2008, "Oxidation experiments and theoretical examinations on graphite materials relevant for the PBMR," Nuclear Engineering and Design, in press (accessible on the website).

Ishihara, M., Iyoku, T., Oku, T., Shibata, T., Sumita, J., 2004, "Principle Design and Data of Graphite Components," Nuclear Engineering and Design, Vol. 233, pp. 251-260.

Kim, E. S. and NO, H. C., 2006, "Experimental Study on the Oxidation of Nuclear Graphite and Development of an Oxidation Model, Journal of Nuclear Materials, Vol. 349, pp. 182-194.

McCreery, G. E., McEligot, D. M., 2004, Scaling Studies and Conceptual Experiment Designs for NGNP CFD Assessment, INEEL/EXT-04-02502, November.

Moorman, R., Alberici, S., Hinssen, H. K., Krussenberg, A. K., Wu, C. H., 1999, "Oxidation behavior of carbon-based materials used for HTGRs and fusion reactors," Adv. Sci. Technol., Vol. 24, pp. 331339.

Ogawa, M, 1993, "Mass Transfer with Graphite Oxidation in Gas Mixture Laminar Flow through Circular Tube,” J. At. Energy Soc. Jpn., Vol. 35, Issue No. 3, p. 245.

Oh, C. H., Davis, C., Siefken, L., Moore, R., NO, H. C., Kim, J., Park, G. C., Lee, J. C. and Martin, W. R., 2006, Development of Safety Analysis Codes and Experimental Validation for a Very High Temperature Gas-Cooled Reactor, INL/EXT-06-01362, March.

Oh, C. H., Kim, E. S., NO, H. C., and Cho, N. Z., 2008, Experimental Validation of Stratified Flow Phenomena, Graphite Oxidation, and Mitigation Strategies of Air Ingress Accident, INL/EXT-0814840, December.

Rasmussen, K., 2001, Full-range Stress-strain Curves for Stainless Steel Alloys, Published Ph.D research, Report No R811, University of Sydney, Sydney, Australia. 
Shenoy, 2007, "Modular Helium Reactor Design, Technology and Applications," http://cer.ucsd.edu/SEMINARS/Shenoy.ppt.

Velasquez. C, Hightower, G., and Burnette, R., 1978, "The oxidation of H451 graphite by steam, Part 1: reaction kinetics," General Atomics Report GA-A14951.

Wichner, R. P., 1976, Oak Ridge National Laboratory Report, ORNL/TM-5534.

\section{Chapter 5 (Task 4)}

Yan et al., "A Study of Air Ingress and Its Prevention in HTGR," Nuclear Technology, Vol.163, Sep. 2008.

\section{Chapter 6 10 (Task $5 \sim 9$ )}

Bratton, R., and Burchell, T., 2005, NGNP Graphite Testing and Qualification Specimen Selection Strategy, INL/EXT-05-00269, pp. 8-14, May.

Chang H. Oh et al., 2006, Development of Safety analysis Codes and Experimental Validation for a Very High Temperature Gas Cooled Reactor, INL/EXT-06-01362, pp 112-141, March.

Welty, J. R., Wicks, C. E., Wilson, R. E., 1984, Fundamentals of Momentum, Heat and Mass Transfer, John Wiley and Sons, pp. 615-618.

Fuller, E. L., and Okoh, J. M., 1997, Kinetics and mechanisms of the reaction of air with nuclear grade graphites: IG-110, J. Nuclear Materials, Vol. 240, pp. 241-250.

Chi, Se-H., and Kim, Gen-C., 2008, "Comparison of the oxidation rate and degree of graphitization of selected IG and NBG nuclear graphite grades," J. Nuclear Material, Vol. 381, pp. 9-14.

KIM, E. S., 2006, Development of Graphite Oxidation Models for Air-ingress Analysis in HTGR, pp. 52, KAIST.

Eto, M., and Growcock, F. B., 1983, "Effect of oxidizing environment on the strength of H451, PGX and IG-11 graphite," Carbon, Vol. 21, No. 2, pp. 135-147.

Yoda, S., et al., 1985, "Effect of oxidation on compressive deformation behavior of nuclear-grade isotropic graphite," Carbon, Vol. 23, No. 1, pp. 33-38.

Kim, E. S., and Kim, Y. W., 2007, "Effect of a thermal oxidation on the compressive strengths of selected nuclear graphites," Transactions of the Korean Nuclear Society Autumn Meeting, Korea.

Neighbour, G. B., and Hacker, P. J., 2001, "The variation of compressive strength of AGR moderator graphite with increasing thermal weight loss," Material Letter, Vol. 51, pp. 307-314.

Idaho National Laboratory, 2006, Development of Safety Analysis Codes and Experimental Validation for a Very High Temperature Gas-Cooled Reactor, INL/EXT-06-01362, March.

Singer, F. L., and Pytel, A., 1980, Strength of Material, pp. 439-453, Harper \& Row, New York.

ASTM, 2005, Int'l, Standard Test method for Compressive strength of carbon and graphite, C695-91, Reapproved 2005.

K. Kikuchi et al., 1984, "Fracture of graphite short bars with hemispherical seats,"

Engineering Fracture Mechanics, Vol. 19, Issue 6, pp. 1013-1024.

Eto, M., and Growcock, F. B., 1983, "Effect of oxidizing environment on the strength of H451, PGX and IG-11 graphite,” Carbon, Vol. 21, No. 2, pp. 135-147. 
Appendix A

\section{Isothermal Stratified Flow Experiment Facility Schematics}




\section{Appendix A - Isothermal Stratified Flow Experiment Facility Schematics}

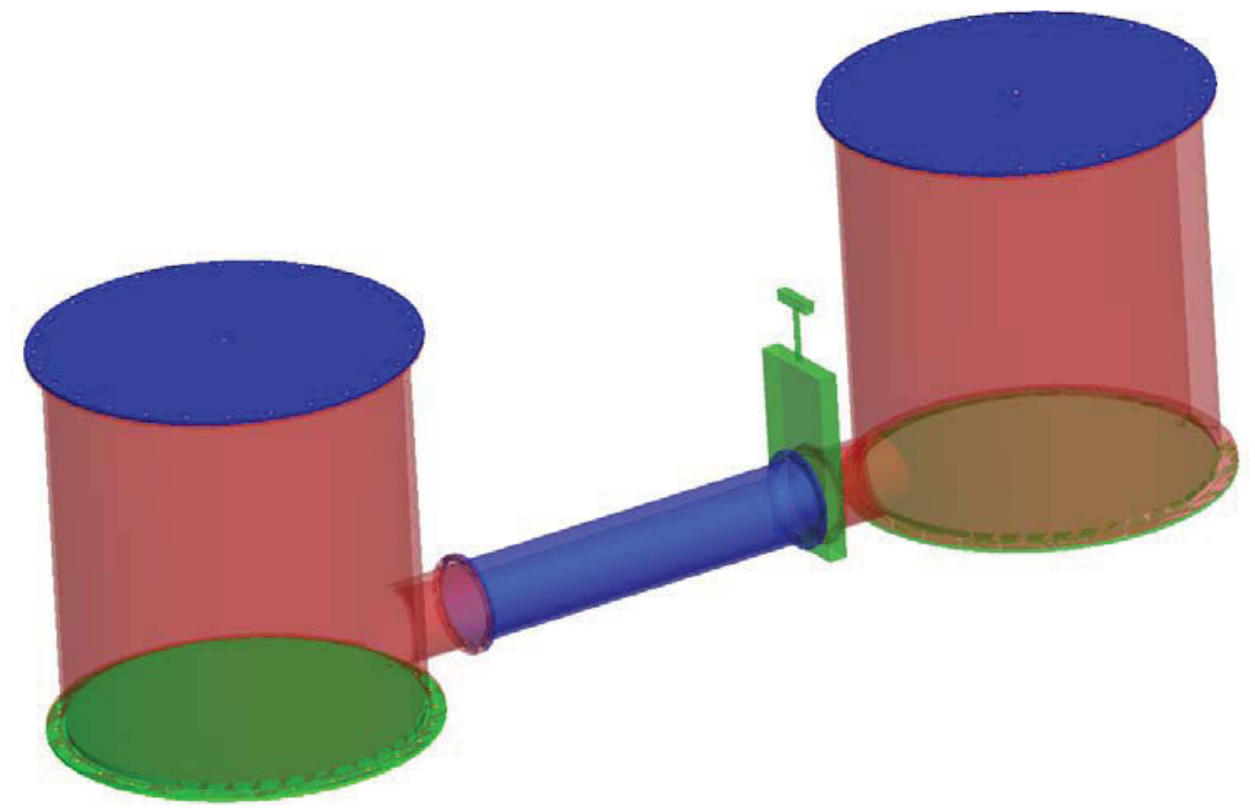

Figure A-1. Double-ended guillotine break facility assembly with knife gate valve.
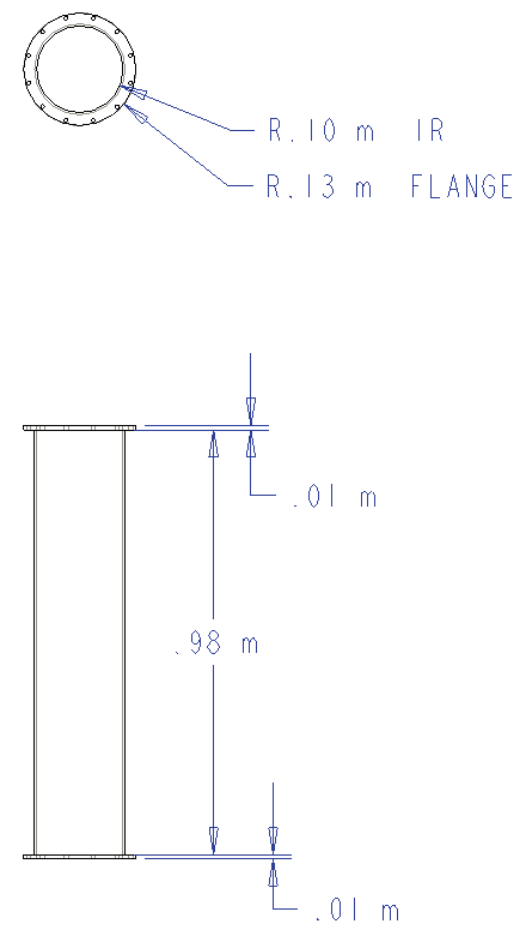

$5 \mathrm{~mm}$ PIPE THICKNESS

PIPE FLANGE HAS I 2 EQUALLY SPACED

25/64" HOLES $0.122 \mathrm{~m}$ FROM AXIS

Figure A-2. Horizontal pipe dimensions. 

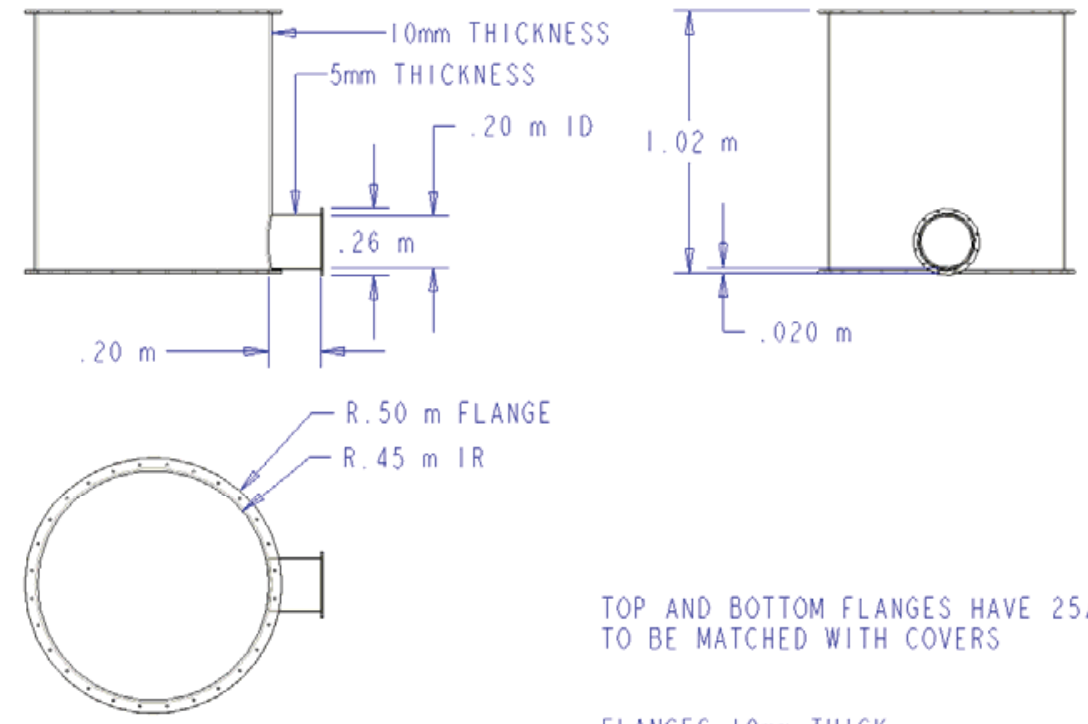

TOP AND BOTTOM FLANGES HAVE 25/64" HOLES

TO BE MATCHED WITH COVERS

FLANGES $10 \mathrm{~mm}$ THICK

CUT AN O-RING GROVE IN THE TOP FLANGE

OR SPEC APPROPRIATE GASKET

OUTLET PIPE I.D. IS TO BE $20 \mathrm{~mm}$ FROM

BOTTOM OF FLANGE

Figure A-3. Flanged tank dimensions. Tanks have covers on both top and bottom to facilitate cleaning.

1/4" hOLES ARE AT 22.5 DEgREES From the X AXIS

25/64" HOLES OFSET BY 6.4 DEGREES FROM X AXIS
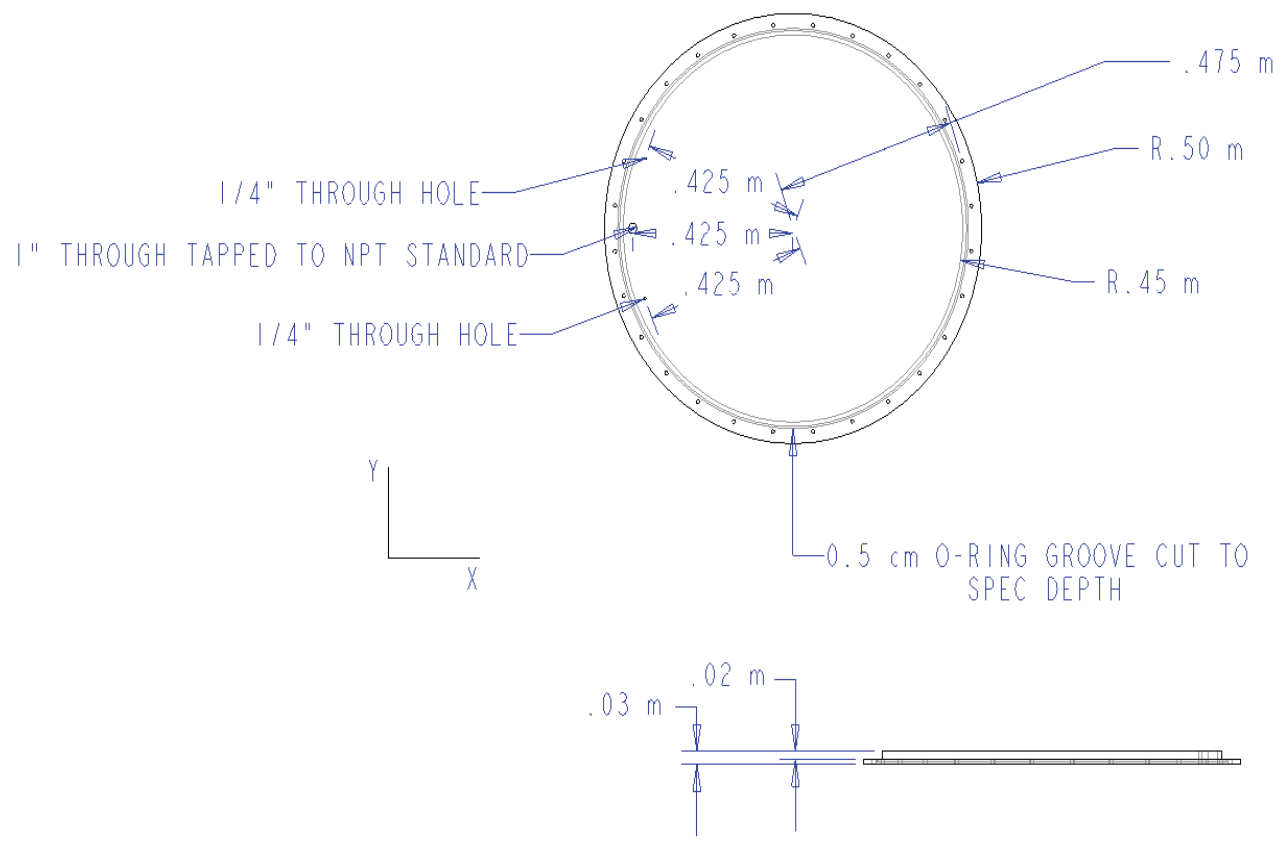

Figure A-4. Bottom tank cover. 


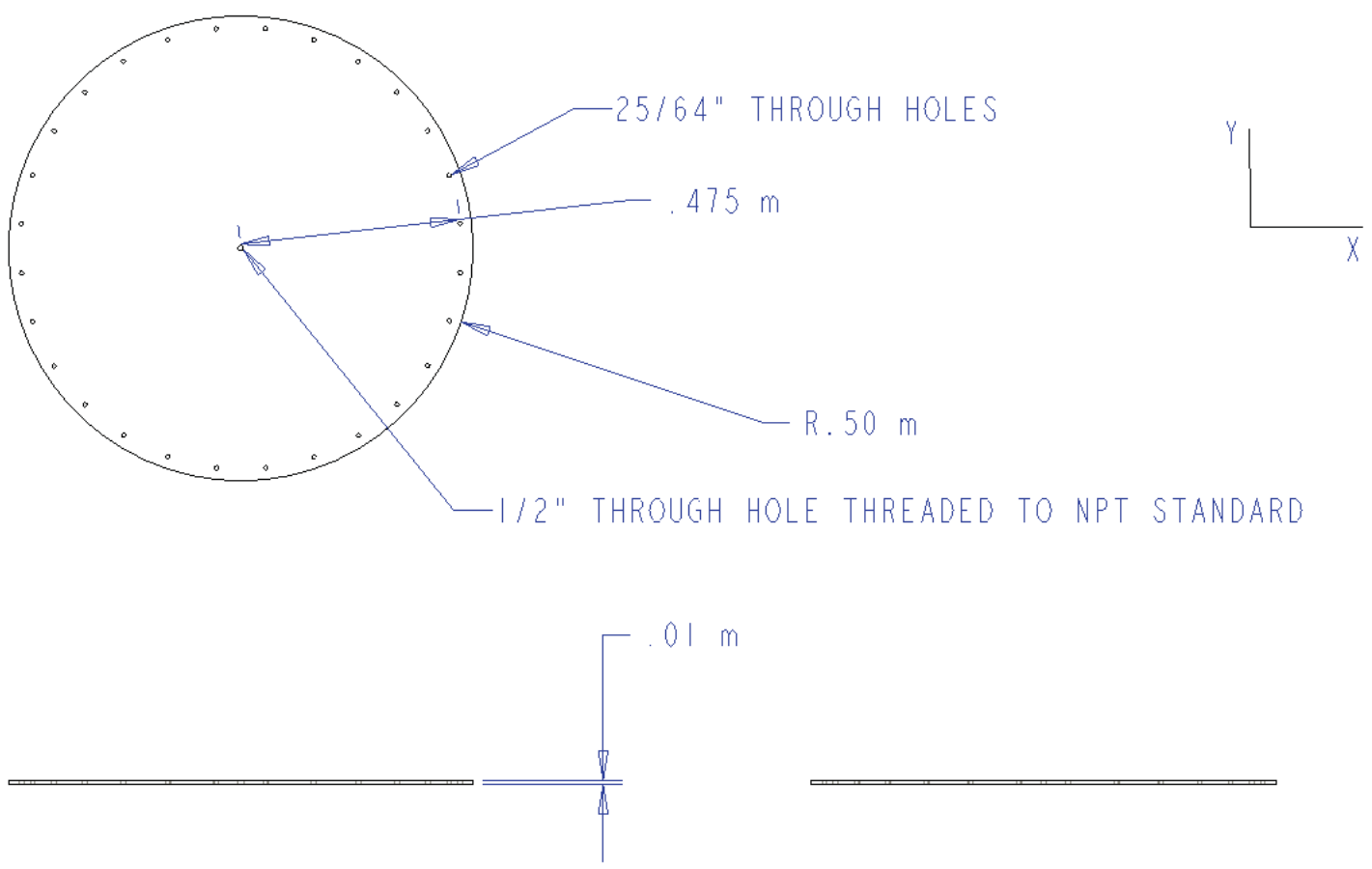

Figure A-5. Top tank cover. 
Appendix B

\section{Knife Valve Supporter Load Analysis}




\section{Appendix B-Knife Valve Supporter Load Analysis}

This perfunctory analysis includes basic load calculations to determine the resultant forces that would be seen at the floor bolts because of a $200 \mathrm{lbf}$ lateral force at the top of the valve, which represents a worst-case load induced by an average-sized person leaning against the valve. It also includes a finite element analysis to determine the stresses in the assembly and a weld analysis to determine the length of weld required at the assembly interfaces.

The Knive Valve Brace assembly is shown in Figure B-1. The brace is built around a three-quarter in. A36 steel plate which mounts to the knife valve flange [A]. Six one-half inch bolts [B] are used to sandwich the plate between two $4 \times 4 \times 1 / 4$ inch A36 steel L-beams [C]. These L-beams are welded to two more A36 L-beams [D] which have through holes on the base flange for a $24 \times 24$ inch bolt pattern. These outer L-beams are then attached, via nuts and washers sandwiching the upper and lower faces of the lower L-beam flange, to four one-half inch bolts [E] anchored into the concrete floor with Hilti Kwik Bolt 3 expansion anchors. The bolted connection to the base flange allows for height adjustment on all four corners of the brace.

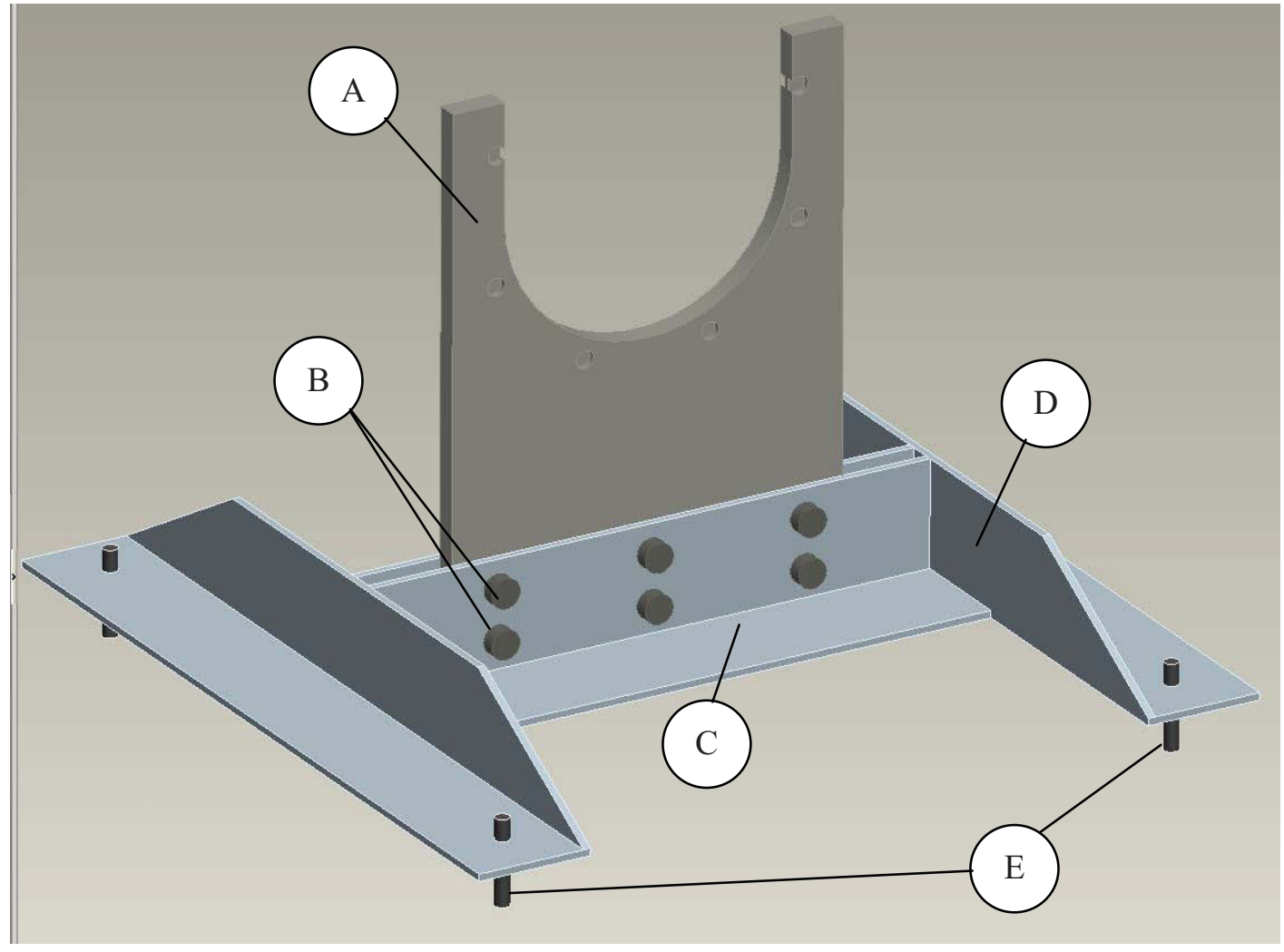

Figure B-1. Knife valve brace.

The part was modeled and analyzed in Pro/Engineer and Pro/Mechanica, respectively. Also, various hand calculations of simplified models were performed to compare against the Pro/Mechanica model.

For example, one scenario would be applying a 4,800 in-lbf moment (400 ft-lbf) to the frame and checking the reaction forces at the corners. It is easily seen that for a 24-inch base bolt pattern, the reactions at each bolt would be $100 \mathrm{lbf}$ (see Figure B-2 and Eqs.B-1, B-2). 


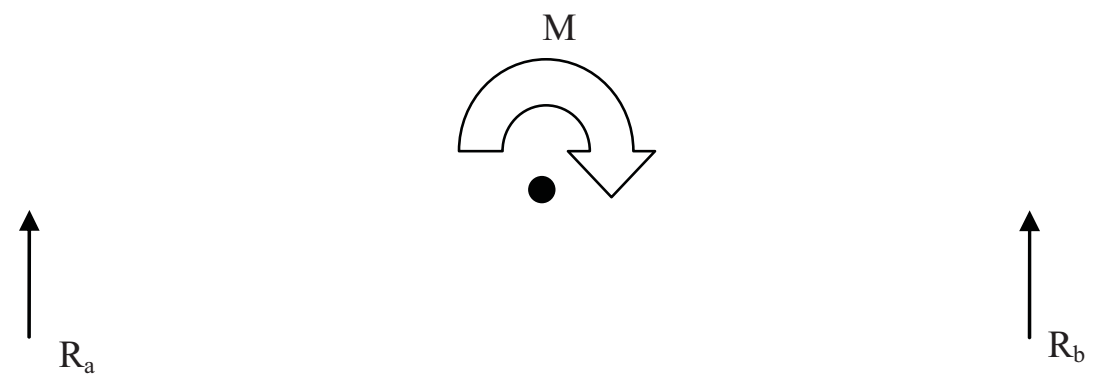

Figure B-2. Simple moment diagram.

$R_{a}=\frac{M}{l}($ Blodgett and Omer (1991))

$R_{b}=\frac{-M}{l}$

Applying the same moment (4,800 in-lbf) to the Pro/E model yields the reaction forces shown in Figure B-3. In summary, there is tension on the bolts nearest the person-loading of approximately $150 \mathrm{lbf}$ vertical, and the two bolts furthest from the person-loading the frame see a compressive force of $\sim 197 \mathrm{lbf}$. Each bolt also sees a shear force of $\sim 50 \mathrm{lbf}$.

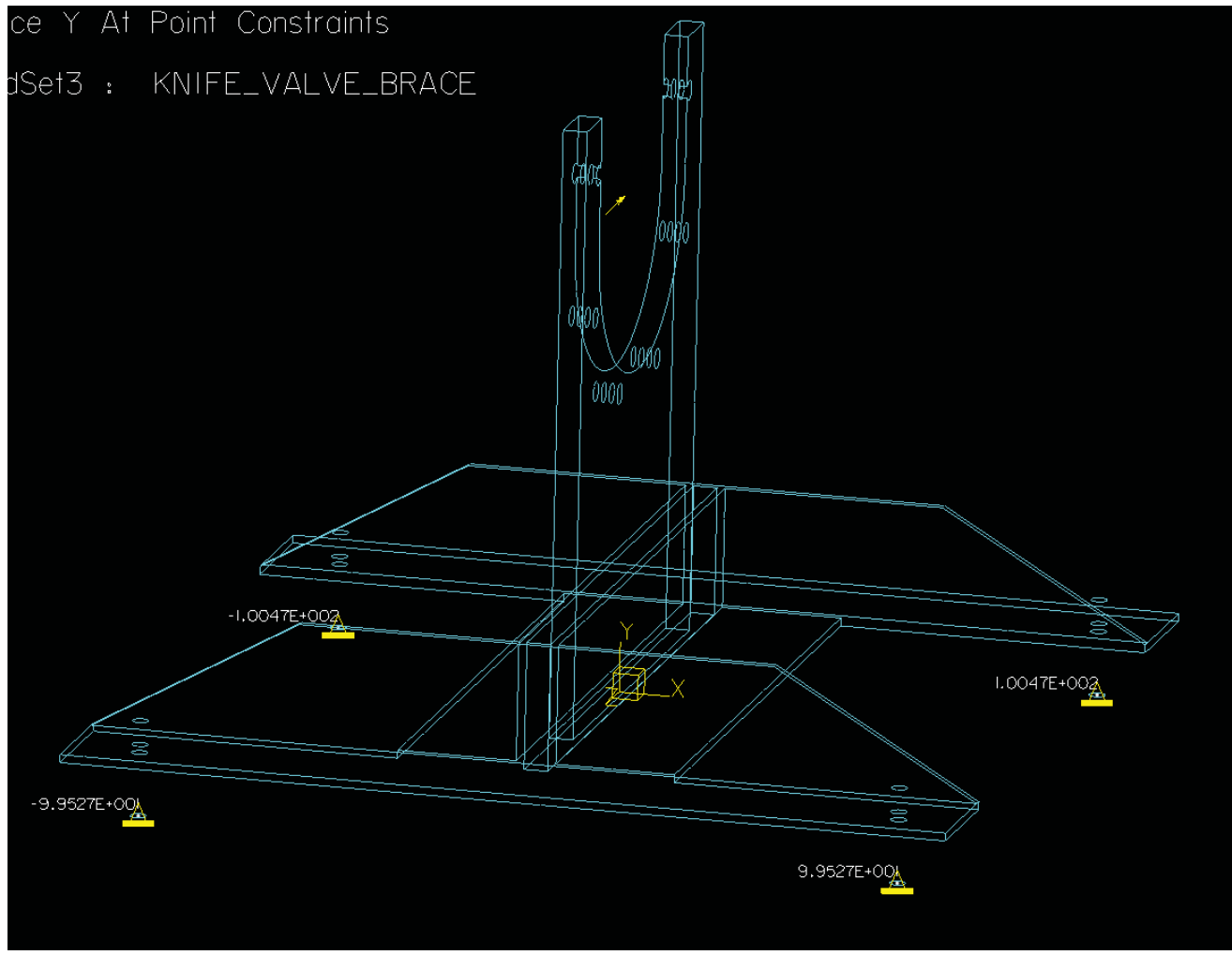

Figure B-3. Simple moment resultant forces (lbf).

Next, applying the lateral force of $200 \mathrm{lbf}$ at the top of the valve (with the corresponding moment of 4,800 in-lbf) and an additional $100 \mathrm{lbf}$ vertical load from the valve itself, the resultant forces seen by the 
anchor bolts are shown in Figure B-4. It can be seen that this is nearly identical to the previous load case because of the symmetric bolt pattern.

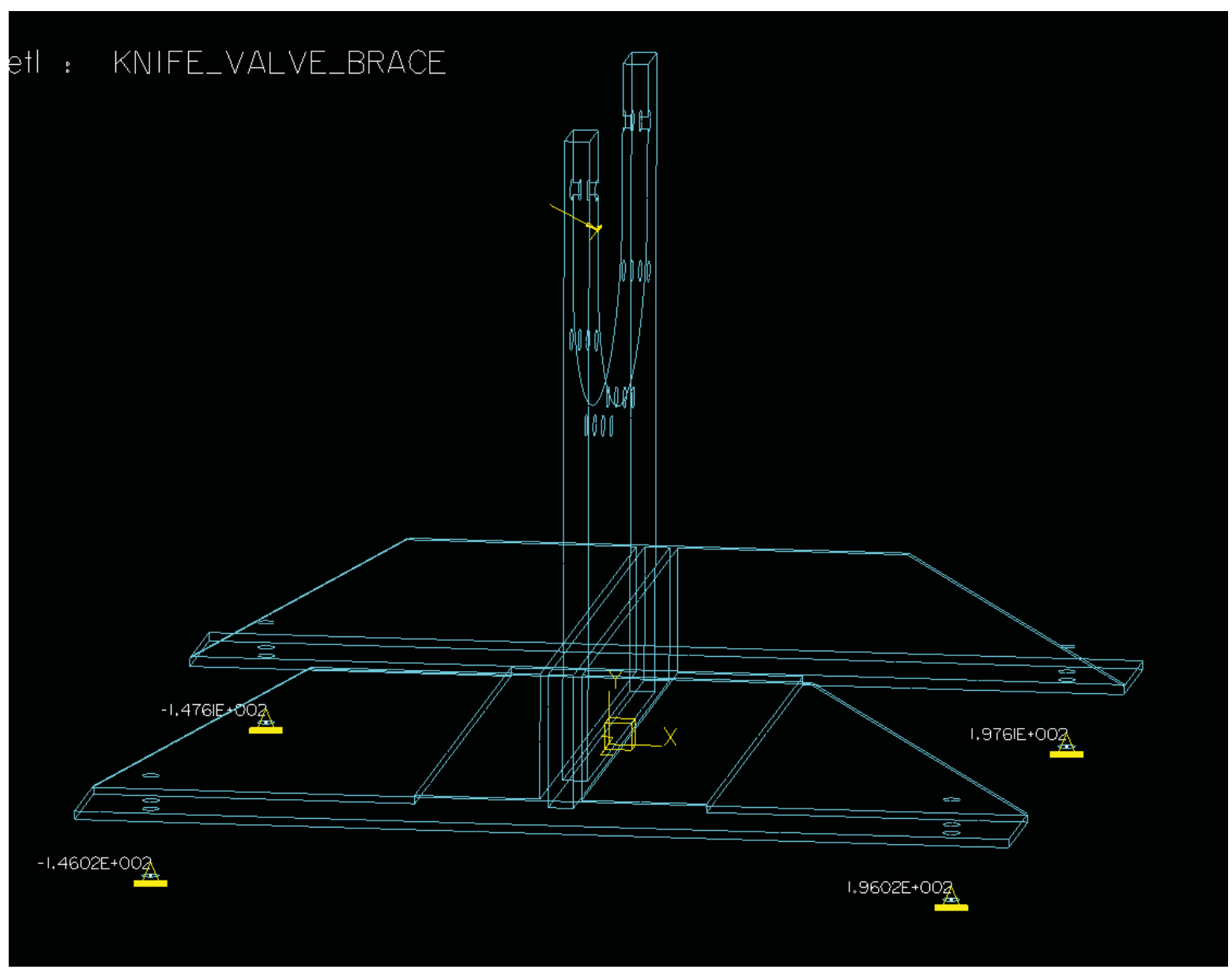

Figure B-4. Resultant from simulated loading (lbf).

A weld analysis was performed per Blodgett to determine the minimum weld length required at the interface between the lateral L-beams [C] and the longitudinal L-beams [D]. It was determined that the minimum weld length for one $4 \times 4$ inch L-beam under the simulated load is 0.07 inches. Therefore we will call out an intermittent $1 / 4$ " fillet weld in two places on each face of the L-beam.

A bolt pull-out analysis was performed using the Hilti anchor design guide (2005) as a reference. Using a Hilti Kwik Bolt 3 expansion anchor with a one-half inch bolt diameter and an embedded depth of $2-1 / 4$ inches, and assuming 2,000 psi concrete, the maximum allowable load is seen to be 1,235 $\mathrm{lbf}$ in tension and 2235 in shear. Given the simulated loading condition of a load being applied at the top of the valve body (36 inches above the base), it would require an $800 \mathrm{lbf}$ lateral force to generate this load.

Figure B-5 shows the stresses in the brace as a result of the applied simulated loading. The stresses in the vertical plate where it meets the lateral supports can be seen to be approximately 5,000 psi. This is compared to stresses calculated using first principles $(\mathrm{Mc} / \mathrm{I})$ to be 5,640 psi. It is evident that there is agreement between the analytical and computational models. 


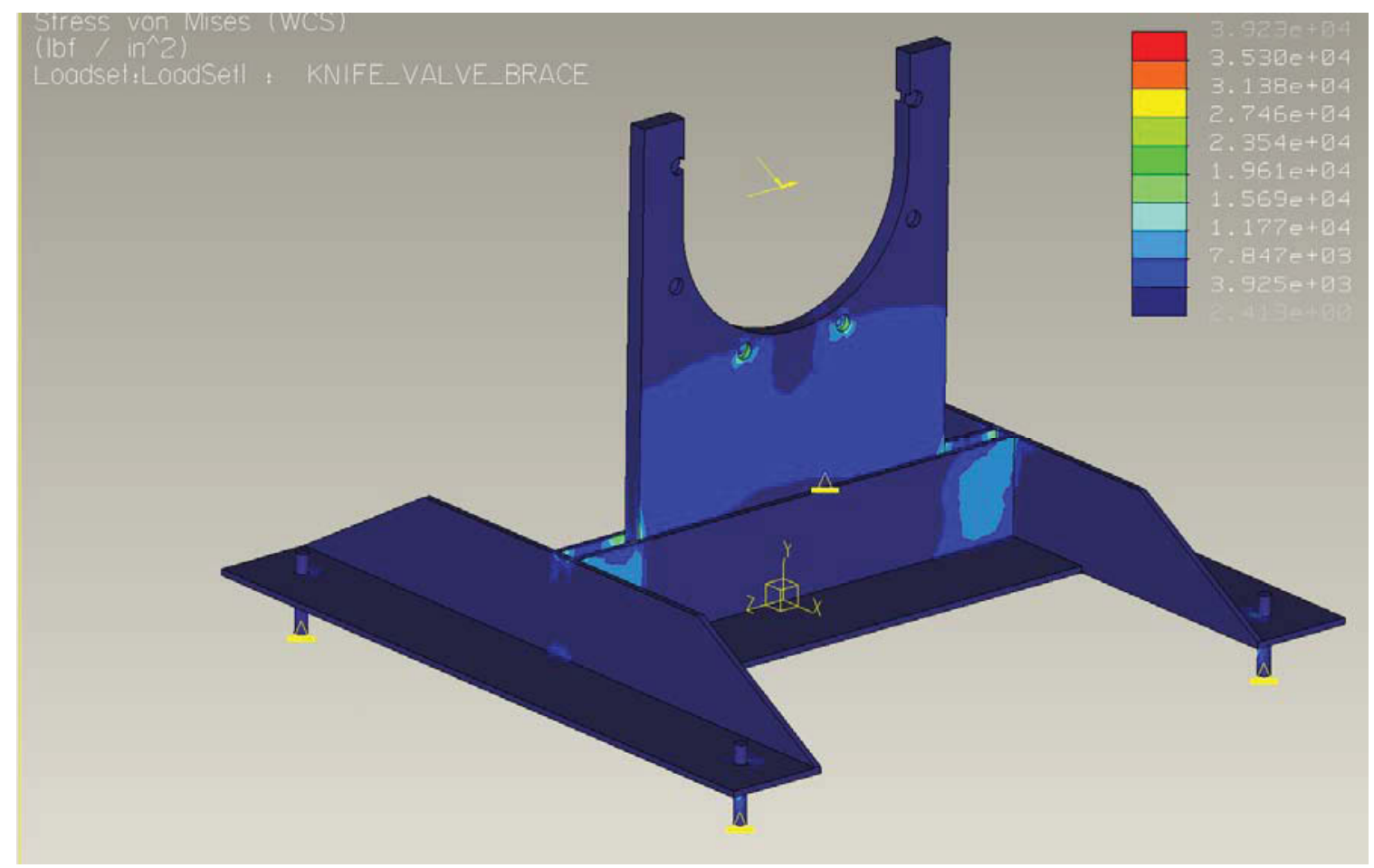

Figure B-5. Local Frame Stresses from Simulated Load (psi).

As all components and interfaces adequately carry the assumed worst-case load, this frame is seen to be adequate for the purpose of holding the knife valve for the Air Ingress Experiment.

\section{References}

Blodgett, Omer W., Design of Welded Structures, The James F. Lincoln Arc Welding Foundation, Cleveland, Ohio, $14^{\text {th }}$ printing, 1991.

Hilti, North America Kwik Bolt 3 Product Technical Guide Supplement, 2005, Hilti (Canada)

Corporation. 